Alexander Brandl

Candidate

Department of Physics and Astronomy

Department

This dissertation is approved, and it is acceptable in quality and form for publication on microfilm:

Approved by the Dissertation Committee:

, Chairperson

Accepted:

Dean, Graduate School

Date 


\title{
Comparison of Three-jet Events to Predictions from a Next-to-leading Order Calculation
}

\author{
by
}

Alexander Brandl

B.S., Physics, Mathematics, University of New Mexico, 1996

M.S., Physics, University of New Mexico, 1999

\author{
Dissertation \\ Submitted in Partial Fulfillment of the \\ Requirements for the Degree of \\ Doctor of Philosophy \\ Physics \\ The University of New Mexico \\ Albuquerque, New Mexico
}

November 2002 
(C)2002, Alexander Brandl 


\section{Acknowledgments}

I thank my wife, Katherine, and my family for their continued support and patience in the course of this dissertation and the Austrian Research Centers Seibersdorf, in particular Chris Schmitzer, for allowing me the time for completion of this work. I thank the members of my Dissertation Committee, Sally Seidel, Bernd Bassalleck, John Matthews, Alejandro Aceves, and Philip Burrows, for their help and patience. Many ideas and solutions in this project came from the valuable interaction with Eric Moore, Nichelle Bruner, and Martin Hoeferkamp, who I thank for their input. This research project was funded by the DoE and in part through the NASA PURSUE program, I am thankful for their financial support until December 2000 and the help of the PURSUE undergraduate students, in particular Gavin Mendel-Gleason who helped with the analytical unsmearing procedure. I thank Igor Gorelov and Tim Thomas for all their help with research and computing. This work would not have been possible had it not been for the QCD group at CDF who provided experience and input in every aspect of this research and the High Performance Computing Education Resource Center where most of the extensive computational work has been achieved. As the next-to-leading order calculation is an integral part of this dissertation, I want to thank its authors, William Kilgore and Walter Giele, for providing the computer code. I also thank Kevin Cahill, Michael Gold, and Marc Price for their input and 
support in problematic phases of this dissertation. 


\title{
Comparison of Three-jet Events to Predictions from a Next-to-leading Order Calculation
}

\author{
by
}

Alexander Brandl

\author{
Abstract of Dissertation \\ Submitted in Partial Fulfillment of the \\ Requirements for the Degree of \\ Doctor of Philosophy \\ Physics
}

The University of New Mexico

Albuquerque, New Mexico

November 2002 


\title{
Comparison of Three-jet Events to Predictions from a Next-to-leading Order Calculation
}

\author{
by
}

\begin{abstract}
Alexander Brandl
B.S., Physics, Mathematics, University of New Mexico, 1996

M.S., Physics, University of New Mexico, 1999

Ph.D., Physics, University of New Mexico, 2002
\end{abstract}

\begin{abstract}
The properties of three-jet events in data of integrated luminosity $86 \pm 4 \mathrm{pb}^{-1}$ from CDF Run $1 \mathrm{~b}$ and with total transverse energy greater than $175 \mathrm{GeV}$ have been analyzed and compared to predictions from a next-to-leading order perturbative QCD calculation. Special emphasis has been placed on analysis of the Dalitz variables. The average $\chi^{2}$ between CDF data and the prediction is used to make the comparison. The data are examined for the possibility to measure the strong coupling constant, $\alpha_{S}$, by means of a newly developed method. The Dalitz variables are not sufficiently sensitive to changes in $\alpha_{\mathrm{S}}$ for a successful measurement at the statistical accuracy obtained in this analysis.
\end{abstract}




\section{Contents}

List of Figures $\quad$ xi

List of Tables $\quad$ xxvii

1 Theoretical Introduction 1

1.1 Particle Physics . . . . . . . . . . . . . . . . 1

1.2 The Standard Model ..................... 3

1.2.1 The Fermions ................... 3

1.2.2 The Bosons ..................... 6

1.3 Gauge Field Theories . . . . . . . . . . . . . . . 8

1.3.1 The Interactions of the Standard Model . . . . . . . . . . 10

1.3.2 Quantum Chromodynamics ............ 15

1.4 Jet Physics . . . . . . . . . . . . . . . . 24

1.4.1 Parton Density Evolution ............. 25

1.4.2 Jets in High Energy Collisions . . . . . . . . . . . 26

1.4.3 Three-jet Events . . . . . . . . . . . . . 29

2 The CDF Detector at the Tevatron Collider 31

2.1 The Accelerator . . . . . . . . . . . . . . . 32

2.2 CDF, the Collider Detector at Fermilab . . . . . . . . . . . 34

2.3 Detector Components .................. 36

2.3.1 The Tracking System . . . . . . . . . . . . 38 
2.3 .2 The Solenoid . . . . . . . . . . . . . . . . . . 39

2.3 .3 The Calorimeters . . . . . . . . . . . . . . . . . 39

2.3 .4 The Muon Detectors . . . . . . . . . . . . . 48

2.4 The Data Acquisition System . . . . . . . . . . . . . . 49

2.5 Trigger and Data Analysis Systems $\ldots \ldots \ldots \ldots \ldots$

3 Comparison of Inclusive Three-jet Data to a Next-to-leading Order Calculation 53

3.1 Three-jet Variables . . . . . . . . . . . . . . . . . 54

3.2 Data Sample . . . . . . . . . . . . . . . . 56

3.2 .1 Level 2 Trigger $\ldots \ldots \ldots \ldots \ldots \ldots \ldots$

3.2 .2 Level 3 Trigger . . . . . . . . . . . . . . . 57

3.2 .3 Offline Selection . . . . . . . . . . . . 57

3.3 Jet Corrections and Offline Event Selection . . . . . . . . . . 59

3.4 Detection and Offline Cut Efficiencies . . . . . . . . . . . 62

3.5 Monte Carlo Prediction . . . . . . . . . . . . . . . . 72

3.6 Comparison of Data to Predictions from the Next-To-Leading Order

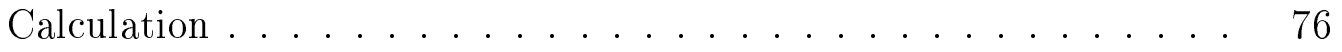

3.7 Measurement of the Strong Coupling Constant . . . . . . . . 77

4 Unsmearing the Data $\quad 81$

$4.1 \quad$ True and Measured Values of an Observable . . . . . . . . . 82

4.2 True Level Clustering . . . . . . . . . . . . . . . . . 82

4.3 The Monte Carlo Unsmearing Procedure . . . . . . . . . . 83

4.4 Unsmearing the Jet Transverse Energy $\ldots \ldots \ldots \ldots$

$\begin{array}{lll}5 & \text { Systematic Uncertainties } & 97\end{array}$

5.1 Systematic Uncertainties on the Dalitz Variables . . . . . . . . 97

5.1.1 Absolute Jet Energy Scale . . . . . . . . . . . . . . 98

5.1 .2 Relative Jet Energy Scale . . . . . . . . . . . . . . 99 
5.1.3 Total Integrated Luminosity and z-vertex Cut Efficiency . . 108

5.1.4 Smearing Correction Factor . . . . . . . . . . 111

5.2 The Total Systematic Uncertainty on the Dalitz Variables . . . . . . 111

6 Results

115

6.1 The Inclusive Three-jet Comparison . . . . . . . . . . . . . . 115

6.2 Discussion and Cross Checks . . . . . . . . . . . . 141

7 Results from CTEQ4 Parton Distribution Functions 148

7.1 CTEQ4A Results . . . . . . . . . . . . . 148

7.2 CTEQ4HJ Results . . . . . . . . . . . . 169

$\begin{array}{ll}\text { Conclusions } & 181\end{array}$

A The Smearing Correction Factor in All Kinematically Allowed Bins in the Dalitz Plane

B The Analytical Unsmearing Procedure $\quad 191$

C Summary of the $\mathrm{E}_{\mathrm{T}}$ Smearing Correction Factors 209

D Summary of the Systematic Uncertainties on the Dalitz Variables 211

E The Dalitz Distributions After Variation of the Cut Parameters 222

F The Cross Section in All Kinematically Allowed Bins in the Dalitz $\begin{array}{ll}\text { Plane } & 237\end{array}$

G The CDF Collaboration $\quad 245$

$\begin{array}{ll}\text { References } & 250\end{array}$ 


\section{List of Figures}

1.1 Examples of gauge boson exchanges. a) Strong interaction: a gluon $(g)$ is exchanged; b) electromagnetic interaction: a photon $(\gamma)$ is exchanged; and c) weak interaction: a $W^{ \pm}$or $Z^{0}$ is exchanged. . . . . 11

1.2 A typical parton distribution function parameterization for $u$ and $d$ valence quarks, $\bar{u}$ and $s$ sea quarks, and gluons inside a hadron. (Figure taken from Reference $[23].) \ldots \ldots \ldots \ldots$

1.3 Polarization of a dielectric medium by a charge. (Figure taken from

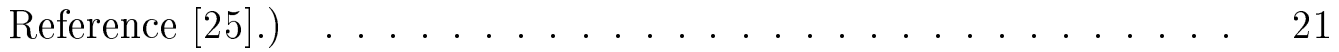

1.4 Second-order corrections to strong interaction observables. a) a quarkantiquark loop; and b) a gluon loop. . . . . . . . . . . . . . 22

1.5 The deceasing $\mu$ dependence in a cross section calculation from a leading order ("Born") to a next-to-leading order ("Full") calculation. (Figure taken from Reference [28].) . . . . . . . . . . . . . . 23

1.6 The running of the strong coupling as a function of the 4-momentum transfer, Q, for $\Lambda=0.1 \mathrm{GeV}$. (Figure taken from Reference [29].) . . 25

1.7 Schematic of the color lines of force between two partons, as described in the Lund string model. . . . . . . . . . . . . . . . . 28

1.8 Schematic view of jet fragmentation, as described by the Lund string

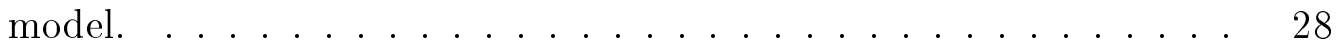

1.9 A three-jet event observed in the CDF detector at the Tevatron. . . 30 
2.1 Schematic of the accelerators. . . . . . . . . . . . 33

2.2 Photograph of the CDF detector at the Tevatron. . . . . . . . . 35

2.3 Schematic view of the CDF detector, showing the detector components. 36

2.4 A schematic side view of a quadrant of the CDF detector. . . . . . 37

2.5 Schematic view of one module of the central electromagnetic calorimeter. (Figure taken from Reference [35].) . . . . . . . . . . 42

2.6 Schematic view of a) the PEM proportional tubes, plastic anodes, and wire supports; b) one proportional layer which is sandwiched between copper clad G-10 panels. (Figure taken from Reference [35].) . . . .

3.1 Definitions of $\theta_{3}^{*}$ and $\psi^{*}$ for the three-jet final state in the three-jet rest frame. (Figure taken from Reference [48].) . . . . . . . . . 56

3.2 The predicted jet cross section as a function of the cone size, R. (Figure taken from Reference [51].) . . . . . . . . . . 58

3.3 The fraction of two jets merged into a single jet as a function of $\Delta R$. (Figure taken from Reference [50].) . . . . . . . . . . 60

3.4 The distribution of the Dalitz variables of events rejected by the requirement that $\Delta \mathrm{R}>1.0 \ldots \ldots \ldots \ldots 6 \ldots \ldots \ldots \ldots$

3.5 The number of events as a function of instantaneous luminosity. . . 64

3.6 The fraction of resolved multiple interactions as a function of instan-

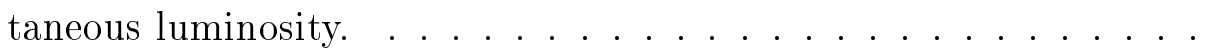

3.7 The jet multiplicities as a function of instantaneous luminosity for the samples with resolved and unresolved multiple interactions (Figure courtesy of Igor Gorelov.) . . . . . . . . . . . . . . 66

3.8 The expected total number of events after rejection of multiple interactions as a function of instantaneous luminosity. . . . . . . . .

3.9 The change of the number of vertices in the data due to the rejection of multiple interactions. . . . . . . . . . . . . . 
3.10 The distribution of $\left|\cos \theta_{3}^{*}\right|$ versus $m_{3 \mathrm{~J}}$ after all the cuts have been applied to the data. . . . . . . . . . . . . .

3.11 The distribution of $\psi^{*}$ versus $\cos \theta_{3}^{*}$ after all the cuts have been applied to the data. ....................... 70

3.12 The distribution of $\mathrm{X}_{4}$ versus $\mathrm{X}_{3}$ after all the cuts have been applied to the data.

4.1 The smearing correction factor, $\mathrm{K}$, for bins of size $0.02 \times 0.02$ in the Dalitz plane, as calculated with the Monte Carlo method. . . . . .

4.2 The smearing correction factor, $\mathrm{K}$, for bins of size 0.02 in $\mathrm{X}_{3}$, as calculated with the Monte Carlo method. . . . . . . . . . .

4.3 The smearing correction factor, $\mathrm{K}$, for bins of size 0.02 in $\mathrm{X}_{4}$, as calculated with the Monte Carlo method. . . . . . . . . . . 86

4.4 The size of the smearing correction factor, $\mathrm{K}$, as calculated with the Monte Carlo method. . . . . . . . . . . . . . .

4.5 Comparison of the smearing correction factor, K, for bins of size 0.02 in $\mathrm{X}_{3}$ and $\mathrm{X}_{4}$, as calculated with the Monte Carlo method for a minimum $\mathrm{p}_{\mathrm{T}}$ of $60 \mathrm{GeV}$ and $150 \mathrm{GeV} \ldots \ldots \ldots$

4.6 The fractional difference between the unsmeared measured and the true $\mathrm{X}_{3}$ distributions. . . . . . . . . . . . . 90

4.7 The fractional difference between the unsmeared measured and the true $\mathrm{X}_{4}$ distributions. . . . . . . . . . . . . . . 91

4.8 The difference between the unsmeared measured and the true $\mathrm{X}_{3}$ distributions, scaled by its uncertainty. . . . . . . . . . .

4.9 The difference between the unsmeared measured and the true $\mathrm{X}_{4}$ distributions, scaled by its uncertainty. . . . . . . . . . .

4.10 The fractional difference between the unsmeared measured and the

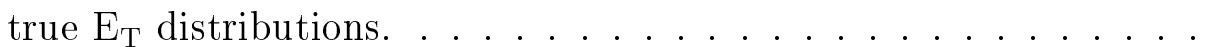


4.11 The difference between the unsmeared measured and the true $\mathrm{E}_{\mathrm{T}}$ distributions, scaled by its uncertainty. . . . . . . . . . 96

5.1 The systematic uncertainty on the Dalitz variables due to the uncertainty on the detector calibration as a function of $\mathrm{X}_{3}$ for $0.74<\mathrm{X}_{4} \leq 0.98 .100$

5.2 The systematic uncertainty on the Dalitz variables due to the uncertainty on the detector calibration as a function of $\mathrm{X}_{3}$ for $0.50<\mathrm{X}_{4} \leq 0.74 .101$

5.3 The systematic uncertainty on the Dalitz variables due to the uncertainty on the jet fragmentation as a function of $\mathrm{X}_{3}$ for $0.74<\mathrm{X}_{4} \leq 0.98 .102$

5.4 The systematic uncertainty on the Dalitz variables due to the uncertainty on the jet fragmentation as a function of $\mathrm{X}_{3}$ for $0.50<\mathrm{X}_{4} \leq 0.74 .103$

5.5 The systematic uncertainty on the Dalitz variables due to the uncertainty on the detector stability as a function of $\mathrm{X}_{3}$ for $0.74<\mathrm{X}_{4} \leq 0.98 .104$

5.6 The systematic uncertainty on the Dalitz variables due to the uncertainty on the detector stability as a function of $\mathrm{X}_{3}$ for $0.50<\mathrm{X}_{4} \leq 0.74$. 105

5.7 The systematic uncertainty on the Dalitz variables due to the uncertainty on the underlying event correction as a function of $\mathrm{X}_{3}$ for $0.74<\mathrm{X}_{4} \leq 0.98 \ldots \ldots \ldots \ldots \ldots \ldots$

5.8 The systematic uncertainty on the Dalitz variables due to the uncertainty on the underlying event correction as a function of $\mathrm{X}_{3}$ for $0.50<\mathrm{X}_{4} \leq 0.74$

5.9 The systematic uncertainty on the Dalitz variables due to the uncertainty on the relative jet energy scale as a function of $\mathrm{X}_{3}$ for $0.74<\mathrm{X}_{4} \leq 0.98$.

5.10 The systematic uncertainty on the Dalitz variables due to the uncertainty on the relative jet energy scale as a function of $\mathrm{X}_{3}$ for $0.50<\mathrm{X}_{4} \leq 0.74$.

5.11 The total systematic uncertainty on the Dalitz variables as a function of $\mathrm{X}_{3}$ for $0.74<\mathrm{X}_{4} \leq 0.98 \ldots \ldots \ldots \ldots \ldots \ldots \ldots$ 
5.12 The total systematic uncertainty on the Dalitz variables as a function of $\mathrm{X}_{3}$ for $0.50<\mathrm{X}_{4} \leq 0.74 \ldots \ldots \ldots \ldots \ldots$

6.1 The transverse energy distributions for the three jets in unsmeared data, the NLO prediction, and HERWIG. . . . . . . . . . . . 117

6.2 The number of jets in each event for the data and for HERWIG with $\mathrm{p}_{\mathrm{T}}=60 \mathrm{GeV} \ldots \ldots \ldots \ldots \ldots \ldots \ldots$

6.3 The $\sum \mathrm{E}_{\mathrm{T}}$ per event after all the cuts have been applied to the data and to HERWIG Monte Carlo with $\mathrm{p}_{\mathrm{T}}=60 \mathrm{GeV} . \ldots . . . . .119$

6.4 The $\mathrm{E}_{\mathrm{T}}$ distribution of the three leading jets in all data events as the cut on $\sum \mathrm{E}_{\mathrm{T}}$ is varied. . . . . . . . . . . . . . 120

6.5 The three-jet mass distribution for data and HERWIG with $\mathrm{p}_{\mathrm{T}}=$ $60 \mathrm{GeV}$, using a different set of cuts $\left(\mathrm{m}_{3 \mathrm{~J}}>500 \mathrm{GeV}\right.$ and $\mathrm{X}_{3}<0.9$. $)$

6.6 The $\mathrm{X}_{3}$ distribution for data and HERWIG with $\mathrm{p}_{\mathrm{T}}=60 \mathrm{GeV}$, using a different set of cuts $\left(\mathrm{m}_{3 \mathrm{~J}}>500 \mathrm{GeV}\right.$ and $\left.\mathrm{X}_{3}<0.9.\right) \ldots \ldots 122$

6.7 The $\mathrm{X}_{4}$ distribution for data and HERWIG with $\mathrm{p}_{\mathrm{T}}=60 \mathrm{GeV}$, using a different set of cuts $\left(\mathrm{m}_{3 \mathrm{~J}}>500 \mathrm{GeV}\right.$ and $\left.\mathrm{X}_{3}<0.9.\right) \ldots 123$

6.8 The transverse energy distributions for the three jets in unsmeared data from the Jet100 trigger, the NLO prediction, and HERWIG with $\mathrm{p}_{\mathrm{T}}=60 \mathrm{GeV}$.

6.9 The event density in the Dalitz plane as predicted by the NLO calculation with $\alpha_{\mathrm{S}}=0.1118 \ldots \ldots \ldots \ldots \ldots$

6.10 The event density in the Dalitz plane as predicted by the NLO calculation with $\alpha_{\mathrm{S}}=0.1133 . \ldots \ldots \ldots \ldots . \ldots \ldots$

6.11 The event density in the Dalitz plane as predicted by the NLO calculation with $\alpha_{\mathrm{S}}=0.1160 . \ldots \ldots \ldots \ldots . \ldots \ldots$

6.12 The event density in the Dalitz plane as predicted by the NLO calculation with $\alpha_{\mathrm{S}}=0.1185 . \ldots \ldots \ldots \ldots . \ldots \ldots$

6.13 The event density in the Dalitz plane for the data. . . . . . . . . 129 
6.14 The fractional difference between unsmeared data and the NLO prediction, using the CTEQ3 parton distribution function, as a function of $\mathrm{X}_{3}$ for $0.84<\mathrm{X}_{4} \leq 0.94 \ldots \ldots \ldots \ldots \ldots$. . . . . . . . 131

6.15 The fractional difference between unsmeared data and the NLO prediction, using the CTEQ3 parton distribution function, as a function of $\mathrm{X}_{3}$ for $0.74<\mathrm{X}_{4} \leq 0.84 \ldots \ldots \ldots \ldots \ldots \ldots$

6.16 The fractional difference between unsmeared data and the NLO prediction, using the CTEQ3 parton distribution function, as a function of $\mathrm{X}_{3}$ for $0.64<\mathrm{X}_{4} \leq 0.74 \ldots \ldots \ldots \ldots$

6.17 The fractional difference between unsmeared data and the NLO prediction, using the CTEQ3 parton distribution function, as a function of $\mathrm{X}_{3}$ for $0.54<\mathrm{X}_{4} \leq 0.64 \ldots \ldots \ldots \ldots \ldots$. . . . . . . . . 134

6.18 The area enclosed by the lines indicates the bins in $\mathrm{X}_{3}-\mathrm{X}_{4}$ space that are used in the analysis and plotted in Figures 6.14 through 6.17. Bins with a theoretical error $>0.2$ are excluded. . . . . . . . 135

6.19 The average fractional difference for the three-jet sample as a function

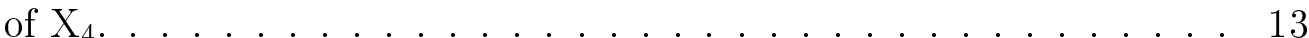

$6.20 \chi^{2}$ between the data and the NLO calculation for different values of $\alpha_{\mathrm{S}}$, using appropriate parton distribution functions from the CTEQ3

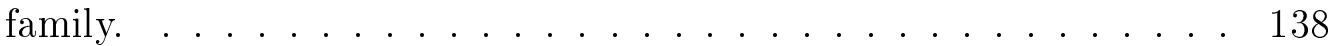

6.21 The total three-jet production cross section predicted by the NLO calculation as a function of $\alpha_{\mathrm{S}}$ compared to the data. . . . . . . . 143

6.22 The circles represent $\left(\chi^{\prime}\right)^{2}$ between the NLO calculation and events that have been generated by HERWIG, passed through QFL, and then unsmeared by the Monte Carlo approach. The squares represent $\left(\chi^{\prime}\right)^{2}$ between the NLO calculation and hadron-level HERWIG events. The results are consistent, a confirmation of the validity of the unsmearing procedure. The $\left(\chi^{\prime}\right)^{2}$ factor is defined in Section 6.2. . . . . . . . 144 
$6.23\left(\chi^{\prime \prime}\right)^{2}$ between the NLO calculation with $\alpha_{\mathrm{S}}=0.1160$ and the NLO calculation for other values of $\alpha_{\mathrm{S}}$, using appropriate parton distribution functions from the CTEQ3 family. The $\left(\chi^{\prime \prime}\right)^{2}$ factor is defined in

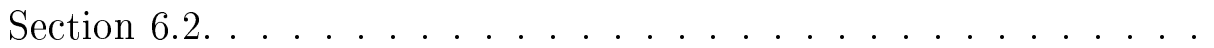

$6.24 \chi^{2}$ between the data and the NLO calculation for different values of $\alpha_{\mathrm{S}}$, using appropriate parton distribution functions from the CTEQ3 family. The cut on the statistical uncertainty on the NLO calculation is not applied. . . . . . . . . . . . . . . . 146

$6.25\left(\chi^{\prime \prime}\right)^{2}$ between the NLO calculation with $\alpha_{\mathrm{S}}=0.1160$ and the NLO calculation for other values of $\alpha_{\mathrm{S}}$, using appropriate parton distribution functions from the CTEQ3 family. The $\left(\chi^{\prime \prime}\right)^{2}$ factor is defined in Section 6.2. The cut on the statistical uncertainty on the NLO calculation is not applied. . . . . . . . . . . . . . . 147

7.1 Comparison between the CTEQ3 and CTEQ4 parton distribution functions for the quarks (Figure taken from Reference [67].) . . . . . 149

7.2 Comparison between the CTEQ3 and CTEQ4 parton distribution functions for the gluons (Figure taken from Reference [67].) . . . . . 150

7.3 The transverse energy distributions for the three jets in unsmeared data, the NLO prediction using the CTEQ4M parton distribution function, and HERWIG. . . . . . . . . . . . . . . 152

7.4 The ratio of the jet $\mathrm{E}_{\mathrm{T}}$ distributions obtained by using CTEQ3 and

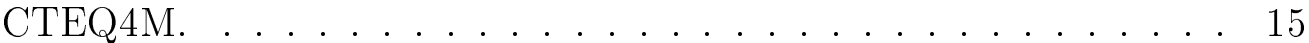

7.5 The transverse energy distributions for the three jets in unsmeared data from the Jet100 trigger, the NLO prediction using the CTEQ4M parton distribution function, and HERWIG with $\mathrm{p}_{\mathrm{T}}=60 \mathrm{GeV}$. . . 154

7.6 The event density in the Dalitz plane as predicted by the NLO calcu-

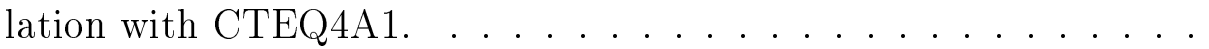


7.7 The event density in the Dalitz plane as predicted by the NLO calcu-

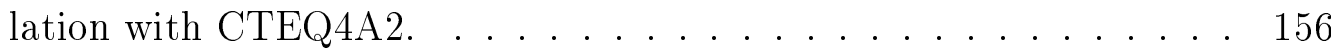

7.8 The event density in the Dalitz plane as predicted by the NLO calculation with CTEQ4M. . . . . . . . . . . . 157

7.9 The event density in the Dalitz plane as predicted by the NLO calculation with CTEQ4A4. . . . . . . . . . . 158

7.10 The event density in the Dalitz plane as predicted by the NLO calcu-

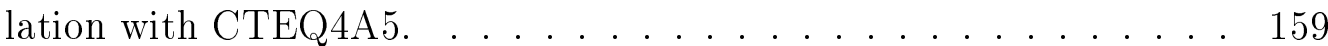

7.11 The fractional difference between unsmeared data and the NLO prediction, using the CTEQ4M parton distribution function, as a function of $\mathrm{X}_{3}$ for $0.84<\mathrm{X}_{4} \leq 0.94 \ldots \ldots \ldots \ldots$. . . . . . . . 160

7.12 The fractional difference between unsmeared data and the NLO prediction, using the CTEQ4M parton distribution function, as a function of $\mathrm{X}_{3}$ for $0.74<\mathrm{X}_{4} \leq 0.84 \ldots \ldots \ldots \ldots 16 \ldots$

7.13 The fractional difference between unsmeared data and the NLO prediction, using the CTEQ4M parton distribution function, as a function of $\mathrm{X}_{3}$ for $0.64<\mathrm{X}_{4} \leq 0.74 \ldots \ldots \ldots \ldots$

7.14 The fractional difference between unsmeared data and the NLO prediction, using the CTEQ4M parton distribution function, as a function of $\mathrm{X}_{3}$ for $0.54<\mathrm{X}_{4} \leq 0.64 \ldots \ldots \ldots \ldots$

7.15 The area left of the line indicates the bins in $\mathrm{X}_{3}-\mathrm{X}_{4}$ space that are used in the analysis and plotted in Figures 7.11 through 7.14 . . . . 164

7.16 The average fractional difference for the three-jet sample as a function of $\mathrm{X}_{4} \ldots \ldots \ldots \ldots \ldots \ldots \ldots \ldots$

$7.17 \chi^{2}$ between the data and the NLO calculation for different values of $\alpha_{\mathrm{S}}$, using appropriate parton distribution functions from the CTEQ4

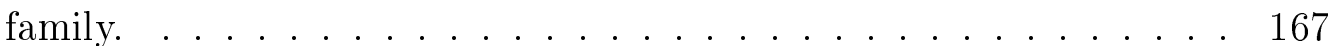


7.18 The circles represent $\left(\chi^{\prime}\right)^{2}$ between the NLO calculation and events that have been generated by HERWIG, passed through QFL, and then unsmeared by the Monte Carlo approach. The squares represent $\left(\chi^{\prime}\right)^{2}$ between the NLO calculation and hadron-level HERWIG events. 169

$7.19\left(\chi^{\prime \prime}\right)^{2}$ between the NLO calculation with $\alpha_{\mathrm{S}}=0.1155$ and the NLO calculation for other values of $\alpha_{\mathrm{S}}$, using appropriate parton distribution functions from the CTEQ4 family. . . . . . . . . . . . 170

7.20 The transverse energy distributions for the three jets in unsmeared data, the NLO prediction using the CTEQ4HJ parton distribution function, and HERWIG. . . . . . . . . . . . . . . . . . 172

7.21 The ratio of the jet $\mathrm{E}_{\mathrm{T}}$ distributions obtained by using CTEQ3 and

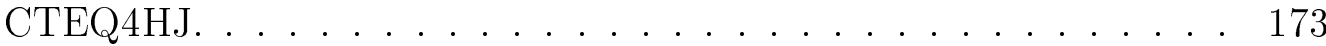

7.22 The transverse energy distributions for the three jets in unsmeared data from the Jet100 trigger, the NLO prediction using the CTEQ4HJ parton distribution function, and HERWIG with $\mathrm{p}_{\mathrm{T}}=60 \mathrm{GeV}$. . . 174

7.23 The event density in the Dalitz plane as predicted by the NLO calcu-

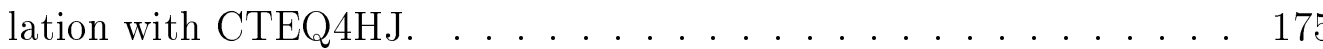

7.24 The fractional difference between unsmeared data and the NLO prediction, using the CTEQ4HJ parton distribution function, as a function of $\mathrm{X}_{3}$ for $0.84<\mathrm{X}_{4} \leq 0.94 \ldots \ldots \ldots \ldots$

7.25 The fractional difference between unsmeared data and the NLO prediction, using the CTEQ4HJ parton distribution function, as a function of $\mathrm{X}_{3}$ for $0.74<\mathrm{X}_{4} \leq 0.84 \ldots \ldots \ldots \ldots$

7.26 The fractional difference between unsmeared data and the NLO prediction, using the CTEQ4HJ parton distribution function, as a function of $\mathrm{X}_{3}$ for $0.64<\mathrm{X}_{4} \leq 0.74$. . . . . . . . . . . . 178 
7.27 The fractional difference between unsmeared data and the NLO prediction, using the CTEQ4HJ parton distribution function, as a function of $\mathrm{X}_{3}$ for $0.54<\mathrm{X}_{4} \leq 0.64 \ldots \ldots \ldots \ldots \ldots$

7.28 The average fractional difference for the three-jet sample as a function of $\mathrm{x}_{4} \ldots \ldots \ldots \ldots \ldots \ldots \ldots \ldots \ldots$

B.1 The measured $\mathrm{X}_{3}$ distributions for $\mathrm{X}_{j}^{\text {true }}=0.72,0.80,0.85,0.90$, and 0.93, and the detector response function, R. . . . . . . 195

B.2 The measured $\mathrm{X}_{4}$ distributions for $\mathrm{X}_{j}^{\text {true }}=0.53,0.60,0.67,0.74,0.81$, 0.88, and 0.95, and the detector response function, R. . . . . 196

B.3 The $\mathrm{X}_{3}^{\text {true }}$ distribution and $\mathrm{T}$, which is parameterized by polynomials of varying degrees. . . . . . . . . . . . . . . . 198

B.4 The $\mathrm{X}_{4}^{\text {true }}$ distribution and $\mathrm{T}$, which is parameterized by polynomials of varying degrees. . . . . . . . . . . . . . . . . 199

B.5 The $\mathrm{X}_{3}$ data distribution and the convolution integral, O. . . . . 201

B.6 The $\mathrm{X}_{4}$ data distribution and the convolution integral, O. . . . . . 202

B.7 The smearing correction factor, $\mathrm{K}$, for bins of size 0.02 in $\mathrm{X}_{3}$, as calculated with the analytical approach. . . . . . . . . 203

B.8 The smearing correction factor, $\mathrm{K}$, for bins of size 0.02 in $\mathrm{X}_{4}$, as calculated with the analytical approach. . . . . . . . . . . 204

B.9 The fractional difference between the number of Monte Carlo events (which have been generated by HERWIG, propagated through QFL, then unsmeared) and their true number, as calculated with the analytical approach, versus $\mathrm{X}_{3} \ldots \ldots \ldots 207$

B.10 The fractional difference between the number of Monte Carlo events (which have been generated by HERWIG, propagated through QFL, then unsmeared) and their true number, as calculated with the ana-

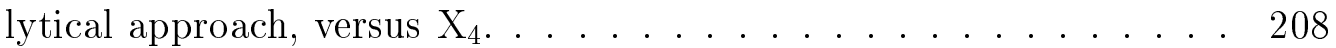

E.1 The Dalitz distribution after variation of the cone overlap cut by -0.05 .223$ 
E.2 The Dalitz distribution after variation of the cone overlap cut by +0.05 .224$

E.3 The Dalitz distribution after variation of the $\eta$ cut by $-0.075 \ldots$. . 225

E.4 The Dalitz distribution after variation of the $\eta$ cut by +0.075 . . . 226

E.5 The Dalitz distribution after variation of the jet $\mathrm{E}_{\mathrm{T}}$ cut by $-5 \mathrm{GeV} .227$

E.6 The Dalitz distribution after variation of the jet $\mathrm{E}_{\mathrm{T}}$ cut by $+5 \mathrm{GeV} .228$

E.7 The Dalitz distribution after variation of the geometrical acceptance

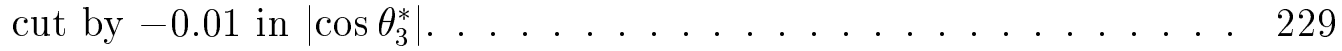

E.8 The Dalitz distribution after variation of the geometrical acceptance cut by +0.01 in $\left|\cos \theta_{3}^{*}\right| \ldots \ldots \ldots \ldots$. . . . . . . . . 230

E.9 The Dalitz distribution after variation of the $\sum \mathrm{E}_{\mathrm{T}}$ cut by $-10 \mathrm{GeV} .231$

E.10 The Dalitz distribution after variation of the $\sum \mathrm{E}_{\mathrm{T}}$ cut by $+10 \mathrm{GeV} .232$

E.11 The Dalitz distribution after variation of the number of tracks per vertex by $-1 . \ldots \ldots \ldots \ldots \ldots \ldots \ldots \ldots \ldots$

E.12 The Dalitz distribution after variation of the number of tracks per vertex by $+1 . \ldots \ldots . \ldots . \ldots . \ldots . \ldots 234$

E.13 The Dalitz distribution after variation of the distance between two resolved vertices by $-1 \mathrm{~cm} . \ldots \ldots \ldots . \ldots . \ldots . \ldots 235$

E.14 The Dalitz distribution after variation of the distance between two resolved vertices by $+1 \mathrm{~cm} \ldots \ldots . \ldots . \ldots 236$ 


\section{List of Tables}

1.1 The fermions of the Standard Model. . . . . . . . . . . . 4

1.2 The masses of the fermions of the Standard Model. . . . . . . . . . 4

1.3 The bosons of the Standard Model. . . . . . . . . . . . 7

1.4 The ranges, strengths, and typical lifetimes associated with the three Standard Model interactions, and with gravity. . . . . . . . 8

$2.1 \quad$ Summary of the CDF calorimeter properties. . . . . . . . . . 41

3.1 Summary of $\alpha_{\mathrm{S}}$ measurements. (Table taken from Reference [27].) . 78

5.1 Uncertainty on the jet energy measurement in the three different detector regions. . . . . . . . . . . . . . . . 108

5.2 The average total systematic uncertainty on the Dalitz variables in every $\mathrm{X}_{4}$ bin. . . . . . . . . . . . . . . . . . 114

6.1 The jet multiplicities in the data and in HERWIG, using a minimum

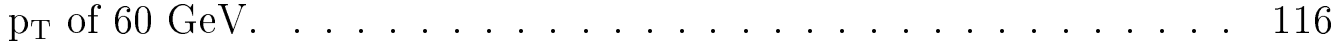

6.2 The $\chi^{2}$ between the data and the scaled Dalitz distributions after the introduction of the various analysis cuts. . . . . . . . . 137

6.3 The $\chi^{2}$ between the data and the NLO calculation for different values of $\alpha_{S}$, using appropriate parton distribution functions from the CTEQ3 family. . . . . . . . . . . . . . . . . . . . . . 139 
6.4 The three-jet production cross section of events in the NLO templates, for different values of $\alpha_{\mathrm{S}}$, using the appropriate parton distribution functions from the CTEQ3 family. The uncertainties are statistical

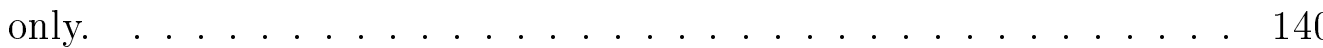

7.1 The $\chi^{2}$ between the data and the NLO calculation for different values of $\alpha_{\mathrm{S}}$, using appropriate parton distribution functions from the

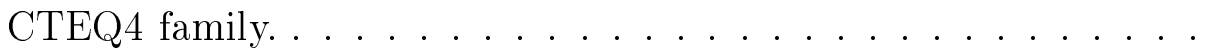

7.2 The three-jet production cross section of events in the NLO templates, for different values of $\alpha_{\mathrm{S}}$, using the appropriate parton distribution functions from the CTEQ4 family. The uncertainties are statistical and theoretical, due to the changes of the renormalization and factor-

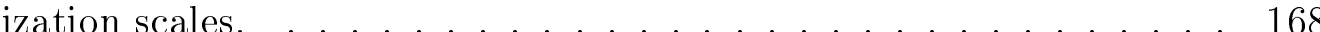

A.1 The fractional difference between the unsmeared measured and the true Dalitz distributions in every kinematically allowed bin in the Dalitz plane as a function of $\mathrm{X}_{3}$ and $\mathrm{X}_{4} \ldots \ldots \ldots \ldots$

A.2 The fractional difference between the unsmeared measured and the true Dalitz distributions in every kinematically allowed bin in the Dalitz plane as a function of $\mathrm{X}_{3}$ and $\mathrm{X}_{4} \ldots \ldots \ldots$

A.3 The fractional difference between the unsmeared measured and the true Dalitz distributions in every kinematically allowed bin in the Dalitz plane as a function of $\mathrm{X}_{3}$ and $\mathrm{X}_{4} \ldots \ldots \ldots \ldots$

A.4 The fractional difference between the unsmeared measured and the true Dalitz distributions in every kinematically allowed bin in the Dalitz plane as a function of $\mathrm{X}_{3}$ and $\mathrm{X}_{4} \ldots \ldots \ldots 185$

A.5 The fractional difference between the unsmeared measured and the true Dalitz distributions in every kinematically allowed bin in the Dalitz plane as a function of $\mathrm{X}_{3}$ and $\mathrm{X}_{4} \ldots \ldots \ldots 186$ 
A.6 The fractional difference between the unsmeared measured and the true Dalitz distributions in every kinematically allowed bin in the Dalitz plane as a function of $\mathrm{X}_{3}$ and $\mathrm{X}_{4} \ldots \ldots \ldots$. . . . . 186

A.7 The fractional difference between the unsmeared measured and the true Dalitz distributions in every kinematically allowed bin in the Dalitz plane as a function of $\mathrm{X}_{3}$ and $\mathrm{X}_{4} \ldots \ldots \ldots 187$

A.8 The fractional difference between the unsmeared measured and the true Dalitz distributions in every kinematically allowed bin in the Dalitz plane as a function of $\mathrm{X}_{3}$ and $\mathrm{X}_{4} \ldots \ldots \ldots \ldots$

A.9 The fractional difference between the unsmeared measured and the true Dalitz distributions in every kinematically allowed bin in the Dalitz plane as a function of $\mathrm{X}_{3}$ and $\mathrm{X}_{4} \ldots \ldots \ldots \ldots$

A.10 The fractional difference between the unsmeared measured and the true Dalitz distributions in every kinematically allowed bin in the Dalitz plane as a function of $\mathrm{X}_{3}$ and $\mathrm{X}_{4} \ldots \ldots \ldots 188$

A.11 The fractional difference between the unsmeared measured and the true Dalitz distributions in every kinematically allowed bin in the Dalitz plane as a function of $\mathrm{X}_{3}$ and $\mathrm{X}_{4} \ldots \ldots \ldots . \ldots 189$

A.12 The fractional difference between the unsmeared measured and the true Dalitz distributions in every kinematically allowed bin in the Dalitz plane as a function of $\mathrm{X}_{3}$ and $\mathrm{X}_{4} \ldots \ldots \ldots 189$

A.13 The fractional difference between the unsmeared measured and the true Dalitz distributions in every kinematically allowed bin in the Dalitz plane as a function of $\mathrm{X}_{3}$ and $\mathrm{X}_{4} \ldots \ldots \ldots . \ldots . . \ldots 190$

A.14 The fractional difference between the unsmeared measured and the true Dalitz distributions in every kinematically allowed bin in the Dalitz plane as a function of $\mathrm{X}_{3}$ and $\mathrm{X}_{4} \ldots \ldots \ldots$. . . . . . . 190 
C.1 The unsmearing correction factor for the $\mathrm{E}_{\mathrm{T}}$ distributions and their uncertainties for $20 \mathrm{GeV}<\mathrm{E}_{\mathrm{T}}<180 \mathrm{GeV}$........... 209

C.2 The unsmearing correction factor for the $\mathrm{E}_{\mathrm{T}}$ distributions and their uncertainties for $180 \mathrm{GeV}<\mathrm{E}_{\mathrm{T}}<340 \mathrm{GeV}$. . . . . . . . . . 210

C.3 The unsmearing correction factor for the $\mathrm{E}_{\mathrm{T}}$ distributions and their uncertainties for $340 \mathrm{GeV}<\mathrm{E}_{\mathrm{T}}<500 \mathrm{GeV}$. . . . . . . . . 210

D.1 The total systematic uncertainty, and the main contributions, on the Dalitz variables for $0.96<\mathrm{X}_{4} \leq 0.98 . \ldots \ldots . \ldots . \ldots . . \ldots 211$

D.2 The total systematic uncertainty, and the main contributions, on the Dalitz variables for $0.94<\mathrm{X}_{4} \leq 0.96 . \ldots \ldots . . . \ldots 211$

D.3 The total systematic uncertainty, and the main contributions, on the Dalitz variables for $0.92<\mathrm{X}_{4} \leq 0.94 \ldots \ldots \ldots . \ldots . . \ldots 212$

D.4 The total systematic uncertainty, and the main contributions, on the

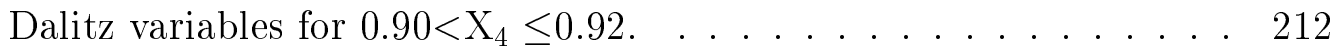

D.5 The total systematic uncertainty, and the main contributions, on the

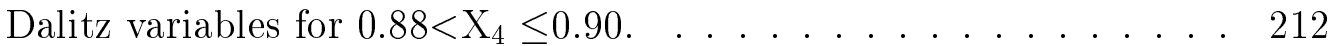

D.6 The total systematic uncertainty, and the main contributions, on the Dalitz variables for $0.86<\mathrm{X}_{4} \leq 0.88$. . . . . . . . . . 213

D.7 The total systematic uncertainty, and the main contributions, on the Dalitz variables for $0.84<\mathrm{X}_{4} \leq 0.86$. . . . . . . . . . 213

D.8 The total systematic uncertainty, and the main contributions, on the Dalitz variables for $0.82<\mathrm{X}_{4} \leq 0.84$. . . . . . . . . . . . . 214

D.9 The total systematic uncertainty, and the main contributions, on the

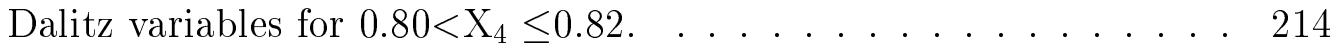

D.10 The total systematic uncertainty, and the main contributions, on the Dalitz variables for $0.78<\mathrm{X}_{4} \leq 0.80 \ldots \ldots \ldots . . \ldots 215$

D.11 The total systematic uncertainty, and the main contributions, on the

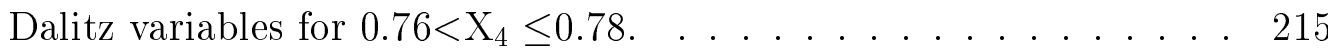


D.12 The total systematic uncertainty, and the main contributions, on the Dalitz variables for $0.74<\mathrm{X}_{4} \leq 0.76 . \ldots \ldots . \ldots . \ldots 216$

D.13 The total systematic uncertainty, and the main contributions, on the Dalitz variables for $0.72<\mathrm{X}_{4} \leq 0.74 . \ldots \ldots . \ldots . . \ldots 216$

D.14 The total systematic uncertainty, and the main contributions, on the

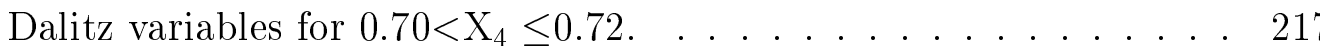

D.15 The total systematic uncertainty, and the main contributions, on the

D.16 The total systematic uncertainty, and the main contributions, on the

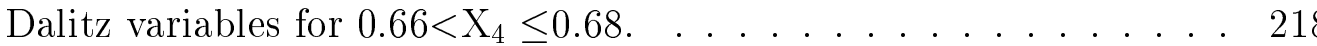

D.17 The total systematic uncertainty, and the main contributions, on the Dalitz variables for $0.64<\mathrm{X}_{4} \leq 0.66$.............. 218

D.18 The total systematic uncertainty, and the main contributions, on the Dalitz variables for $0.62<\mathrm{X}_{4} \leq 0.64$. . . . . . . . . . . 219

D.19 The total systematic uncertainty, and the main contributions, on the Dalitz variables for $0.60<\mathrm{X}_{4} \leq 0.62 . \ldots \ldots . . . . . . . .220$

D.20 The total systematic uncertainty, and the main contributions, on the Dalitz variables for $0.58<\mathrm{X}_{4} \leq 0.60$. . . . . . . . . . . 220

D.21 The total systematic uncertainty, and the main contributions, on the Dalitz variables for $0.56<\mathrm{X}_{4} \leq 0.58$. . . . . . . . . . . . 220

D.22 The total systematic uncertainty, and the main contributions, on the Dalitz variables for $0.54<\mathrm{X}_{4} \leq 0.56$. . . . . . . . . . . 221

D.23 The total systematic uncertainty, and the main contributions, on the Dalitz variables for $0.52<\mathrm{X}_{4} \leq 0.54 \ldots \ldots \ldots 221$

D.24 The total systematic uncertainty, and the main contributions, on the Dalitz variables for $0.50<\mathrm{X}_{4} \leq 0.52 \ldots \ldots \ldots \ldots . \ldots . \ldots . \ldots 221$

F.1 The three-jet production cross section in every kinematically allowed bin in the Dalitz plane as a function of $\mathrm{X}_{3}$ and $\mathrm{X}_{4} \ldots \ldots . . .238$ 
F.2 The three-jet production cross section in every kinematically allowed bin in the Dalitz plane as a function of $\mathrm{X}_{3}$ and $\mathrm{X}_{4} \ldots \ldots . . . .238$

F.3 The three-jet production cross section in every kinematically allowed bin in the Dalitz plane as a function of $\mathrm{X}_{3}$ and $\mathrm{X}_{4} \ldots \ldots . \ldots 239$

F.4 The three-jet production cross section in every kinematically allowed bin in the Dalitz plane as a function of $\mathrm{X}_{3}$ and $\mathrm{X}_{4} \ldots \ldots . . . .239$

F.5 The three-jet production cross section in every kinematically allowed bin in the Dalitz plane as a function of $\mathrm{X}_{3}$ and $\mathrm{X}_{4} \ldots \ldots$. . . . . 240

F.6 The three-jet production cross section in every kinematically allowed bin in the Dalitz plane as a function of $\mathrm{X}_{3}$ and $\mathrm{X}_{4}$. . . . . . . . . 240

F.7 The three-jet production cross section in every kinematically allowed bin in the Dalitz plane as a function of $\mathrm{X}_{3}$ and $\mathrm{X}_{4} \ldots \ldots$. . . . . 241

F.8 The three-jet production cross section in every kinematically allowed bin in the Dalitz plane as a function of $\mathrm{X}_{3}$ and $\mathrm{X}_{4} \ldots \ldots . . . .241$

F.9 The three-jet production cross section in every kinematically allowed bin in the Dalitz plane as a function of $\mathrm{X}_{3}$ and $\mathrm{X}_{4} \ldots \ldots$. . . . . . 242

F.10 The three-jet production cross section in every kinematically allowed bin in the Dalitz plane as a function of $\mathrm{X}_{3}$ and $\mathrm{X}_{4}$. . . . . . . . . 242

F.11 The three-jet production cross section in every kinematically allowed bin in the Dalitz plane as a function of $\mathrm{X}_{3}$ and $\mathrm{X}_{4} \ldots \ldots . . . .243$

F.12 The three-jet production cross section in every kinematically allowed bin in the Dalitz plane as a function of $\mathrm{X}_{3}$ and $\mathrm{X}_{4}$. . . . . . . . 243

F.13 The three-jet production cross section in every kinematically allowed bin in the Dalitz plane as a function of $\mathrm{X}_{3}$ and $\mathrm{X}_{4}$. . . . . . . . . 244

F.14 The three-jet production cross section in every kinematically allowed bin in the Dalitz plane as a function of $\mathrm{X}_{3}$ and $\mathrm{X}_{4}$. . . . . . . . . 244 


\section{Chapter 1}

\section{Theoretical Introduction}

\subsection{Particle Physics}

The goal of science in the history of humankind has been to describe and predict observations in the universe that surrounds us. Before the $20^{\text {th }}$ century, most scientific contributions were restricted to a macroscopic scale, resulting in individual theories for a large number of phenomena that seemingly had little in common.

Significant successes in connecting some observations to more complete theories were achieved in the nineteenth century. Mendeleev ordered the chemical elements into the Periodic Table [1], accounting for the pattern of similar chemical properties for certain elements with different atomic weights; and Maxwell succeeded in writing a set of equations that links electric and magnetic fields [2]. Understanding of physics at an even more fundamental level, however, required the investigation of matter at the microscopic scale.

The beginning of particle physics, the quest for the understanding of the physical world at the most fundamental level, can be associated with the discovery of the electron by J.J. Thompson in 1897 [3]. Atoms, therefore, were found to be composite objects; and E. Rutherford showed in 1911 that they consisted of a hard positively charged core surrounded by a cloud of negatively charged electrons [4]. With the 
discovery of the neutron by J. Chadwick in 1932 [5], the question of the structure of matter seemed to have been solved.

Only one year later, the positron, an antiparticle which had been postulated by P.A.M. Dirac in 1928 [6], was experimentally found by C.D. Anderson [7]. Further efforts by experimental physicists then lead to the discovery of the muon [8], the neutrino [9], and an ever increasing number of other particles, the hadrons.

An explanation for this abundance of different hadrons was found by M. GellMann [10] and G. Zweig [11] independently in 1964, when they proposed that hadrons were composed of even more elementary particles, named quarks by Gell-Mann. Using a method almost comparable to Mendeleev's proposal for the Periodic Table of Elements, Gell-Mann and Zweig significantly simplified classification of the hadrons. The inexplicably high number of hadrons reduced to combinations of only three quarks. All hadrons known at the time could be categorized by these combinations. In subsequent years, hadrons produced in interactions at higher energies required the extension of the model from three to six quarks. Including the six leptons, and the antiparticles of quarks and leptons, the foundations for the Standard Model of particle physics were laid.

In the Standard Model, the fundamental interactions between particles, with the exception of gravity, are described as the exchange of a class of particles called the gauge bosons. The Standard Model is the most promising model of nature today. It was strongly confirmed by the discoveries of the gauge bosons of the weak interaction at CERN in 1983 [12, 13] and of the top quark at Fermilab in 1994 [14, 15]. Extensions of the Standard Model, though mathematically appealing, have not been verified experimentally to date. 


\subsection{The Standard Model}

The Standard Model describes all interactions of particles, with the exception of gravity. All particles in the Standard Model are considered to be elementary; spatial structure is not observable in them. Classically speaking, they are point-like. There are two distinct classes of elementary particles in nature, the half-integer-spin fermions, leptons and quarks, and the integer-spin bosons. While the fermions make up matter in the universe, the bosons mediate the interactions between particles.

The Standard Model is based on group theory. The interactions are described as transformations within a group. Group symmetries give rise to conservation laws; every interaction has an associated conserved quantity, the conserved current in the interaction.

\subsubsection{The Fermions}

There are two kinds of fermions in the Standard Model, the quarks and the leptons. These are the fundamental constituents of matter.

The quarks are subject to all four forces of the Standard Model: the strong, the electromagnetic, and the weak forces, and gravity. The leptons, however, do not interact strongly. They are subject only to the electromagnetic and the weak forces, as well as gravity.

Six quarks, six leptons, their respective antiparticles, and the Higgs bosons which are needed to explain the masses of the fermions are described by the Standard

Model. All quarks carry fractional electric charges of $+\frac{2}{3}|e|$ and $-\frac{1}{3}|e|$; the leptons are either neutral or carry one unit of electric charge, $-|e|$. The quantum number that distinguishes the six quarks is called flavor; the six flavors are: up, down, charm, strange, top, and bottom $(u, d, c, s, t$, and $b)$. The leptons are the electron $(e)$, the muon $(\mu)$, the tau $(\tau)$, and their respective neutrinos, $\nu_{e}, \nu_{\mu}$, and $\nu_{\tau}$. The Standard Model divides quarks and leptons into three generations, each generation containing 
both a quark and a lepton doublet. Only the masses of the members of one generation distinguish it from the others; apart from that, the three generations appear to be the same. Table 1.1 lists the fermions of the Standard Model and shows their classification into three generations. The masses of the Standard Model fermions are shown in Table 1.2, illustrating their increase from one generation to the next.

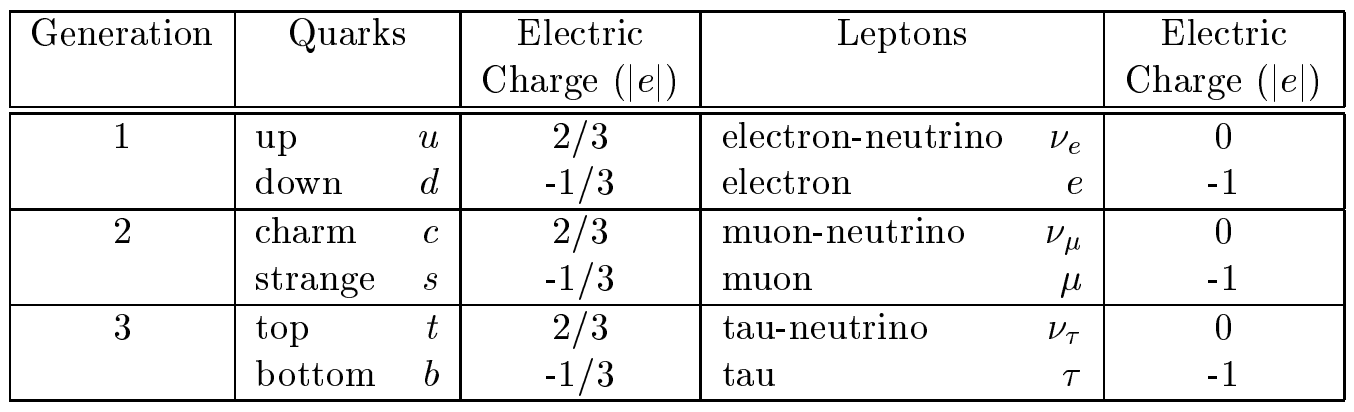

Table 1.1: The fermions of the Standard Model.

\begin{tabular}{|c|c|c|c|c|}
\hline Generation & Quarks & $\begin{array}{c}\text { Mass } \\
\left(\mathrm{GeV} / \mathrm{c}^{2}\right)\end{array}$ & Leptons & $\begin{array}{c}\text { Mass } \\
\left(\mathrm{GeV} / \mathrm{c}^{2}\right)\end{array}$ \\
\hline \hline 1 & $u$ & 0.0015 to 0.005 & $\nu_{e}$ & $<1 \times 10^{-8}$ \\
& $d$ & 0.003 to 0.009 & $e$ & $5.11 \times 10^{-4} \pm 2 \times 10^{-10}$ \\
\hline 2 & $c$ & 1.1 to 1.4 & $\nu_{\mu}$ & $<0.0002$ \\
& $s$ & 0.06 to 0.17 & $\mu$ & $0.106 \pm 3 \times 10^{-8}$ \\
\hline 3 & $t$ & $173.8 \pm 5.2$ & $\nu_{\tau}$ & $<0.02$ \\
& $b$ & 4.1 to 4.4 & $\tau$ & $1.7771 \pm 3 \times 10^{-4}$ \\
\hline
\end{tabular}

Table 1.2: The masses of the fermions of the Standard Model.

Fermions are half-integer-spin particles $\left(\frac{1}{2} \hbar, \frac{3}{2} \hbar, \ldots\right)$; as such they obey FermiDirac statistics. If a wavefunction $\Psi$ describes two identical particles under interchange, then the square of the wavefunction, $|\Psi|^{2}$, which gives the probability of the first particle at one coordinate and the second particle at another, will be unaltered by that interchange. Thus, for the particle interchange $1 \leftrightarrow 2$,

$$
|\Psi|^{2} \stackrel{1 \leftrightarrow 2}{=} 1
$$


which implies

$$
\Psi \stackrel{1 \leftrightarrow 2}{=} \pm \Psi
$$

The positive solution describes the interchange of identical bosons, while identical fermion interchange is described by the negative eigenvalue. The wavefunction is symmetric for boson interchange and antisymmetric for fermion interchange.

A well-known application of the antisymmetry of the wavefunction of two identical fermions under interchange is the Pauli Exclusion Principle. Any two-particle wavefunction can be written as the product of symmetric or antisymmetric combinations of the quantum numbers (i.e., spatial coordinates, spin) of the two particles. Suppose two identical particles are in the same quantum mechanical state. The wavefunction for that state is necessarily symmetric, since all factors in $\Psi$ are identical, making it impossible to find an antisymmetric combination of any part. This violates the observation that two identical fermions need to have an antisymmetric $\Psi$ under interchange. Hence they cannot exist in the same quantum state. The Pauli Principle does not apply to bosons, as their $\Psi$ is symmetric under interchange of two identical particles; there is no restriction on the number of identical bosons that can exist in the same quantum mechanical state.

Fermions and antifermions can only be created or destroyed in pairs. Mathematically, this can be expressed as a conservation law. If leptons and antileptons are assigned a lepton number of +1 and -1 , respectively, then this lepton number is a conserved quantity in any interaction. Similarly, the baryon number is conserved, if quarks and antiquarks are assigned a baryon number of $+1 / 3$ and $-1 / 3$, respectively.

The theory of the interquark forces, quantum chromodynamics (QCD), described in the Standard Model, accounts for the fact that to date quarks and antiquarks have not been observed by themselves in nature. Only two kinds of quarkantiquark combinations occur. From these, all strongly interacting particles, the hadrons, are built. Mesons and baryons are the two different classes of hadrons.

A meson is a bound state of a quark-antiquark $(q \bar{q})$ pair. They have a total 
spin of 0 or 1 , and therefore are bosons. The discovery of two mesons, the pion $(\pi)$ and the kaon $(K)$, in cosmic rays in 1947 marked the onset of the discovery of an abundance of hadrons that led to the quark-parton model proposed by Gell-Mann and Zweig. An example of a meson is the positively charged pion, $\pi^{+}$, which consists of a combination of a $u$ quark and a $\bar{d}$ antiquark.

A baryon is a combination of three quarks $(q q q)$, or three antiquarks $(\overline{q q q})$. They carry a total spin of $1 / 2$ or $3 / 2$ and are fermions. Nuclear matter, protons $(u u d)$ and neutrons $(u d d)$, is built from baryons. The baryon numbers assigned to the quarks combine for baryons and antibaryons, which have a baryon number of +1 and -1 , respectively.

\subsubsection{The Bosons}

Classical physics describes interactions at a distance in terms of potentials or fields due to one particle acting on another. In quantum physics, interactions, or forces, between particles are described as the exchange of quanta of the fields, the gauge bosons. Each interaction has its specific set of gauge bosons, which carry momentum and energy among other things (also L, color, etc.). They carry integral spin of 1. The interactions conserve energy and momentum, but for a time limited by the Heisenberg Uncertainty Principle, that is, $\Delta \mathrm{E} \cdot \Delta \mathrm{t} \leq \hbar$, the bosons can be off massshell, violating the conservation laws. Transient field quanta that do not conserve energy and momentum are called virtual.

Two particles exerting a force on each other continuously emit and absorb gauge bosons, carrying momentum q. This change in momentum results in the force, F, through

$$
\mathbf{F} \equiv \frac{d \mathbf{q}}{d \mathrm{t}}
$$

Unlike the fermions which carry half-integer spin, the bosons are integer-spin particles. The Pauli Exclusion Principle does not apply to bosons, so any number of identical bosons can occupy the same quantum mechanical state. This gives rise to 
macroscopic manifestations, i.e., coherent light as it is generated by a laser.

The most familiar field quanta are the bosons responsible for the electromagnetic interaction, the photons $(\gamma)$. The strong interaction is mediated by the gluons $(g)$, and the weak interaction gauge bosons are the $W^{+}$, the $W^{-}$, and the $Z^{0}$. For the electromagnetic interaction, $\mathbf{q}=\mathrm{q} \hat{\mathbf{r}}$, where $\mathrm{q}$ is the magnitude of $\mathbf{q}$ and $\hat{\mathbf{r}}$ is the unit vector in the radial direction away from a charged particle. From the Heisenberg Uncertainty Principle, $\mathrm{qr} \simeq \hbar$. Momentum is transferred by a virtual photon over a time period $\mathrm{t}=\mathrm{r} / c, c$ being the speed of light. The force between two charges then becomes $\mathrm{F}=\hbar c / \mathrm{r}^{2}$. The number of photons exchanged is assumed to be proportional to the product of the two charges such that the classical Coulomb law, $\mathrm{F}=\mathrm{Q}_{1} \mathrm{Q}_{2} / \mathrm{r}^{2}$, is obtained.

The Standard Model gauge bosons can also be observed as real particles, rather than solely as virtual exchange quanta. They then acquire definite mass, and energy and momentum are conserved in their production. Table 1.3 summarizes the gauge bosons for the Standard Model interactions. It also lists the masses of the real bosons.

\begin{tabular}{|c|c|c|c|c|}
\hline Interaction & Acting on & Gauge Boson & $\begin{array}{c}\text { Mass } \\
\left(\mathrm{GeV} / \mathrm{c}^{2}\right)\end{array}$ & Electric Charge \\
\hline \hline \multirow{2}{*}{ Weak } & Quarks, Leptons & $W^{+}$ & 80.4 & +1 \\
& & $W^{-}$ & 80.4 & -1 \\
& & 91.187 & 0 \\
\hline Electromagnetic & Quarks, charged Leptons & Photon $(\gamma)$ & 0 & 0 \\
\hline Strong & Quarks, Gluons & 8 Gluons $(g)$ & 0 & 0 \\
\hline
\end{tabular}

Table 1.3: The bosons of the Standard Model.

The range of the forces is determined by the mass of the mediating gauge bosons. A minimum of energy is necessary to create a virtual massive gauge boson, such that only a limited lifetime, $\tau$, can be expected for this virtual gauge boson by Heisenberg's Uncertainty Principle. An upper limit on the lifetime of the gauge boson restricts the range of the force, as information cannot be mediated beyond a distance $c \cdot \tau_{v i r t}$, where $c$ is the speed of light and $\tau_{v i r t}$ is the lifetime of the virtual gauge 
boson. The gluons mediating the strong force are massless, yet its range is finite. This is due to a mechanism, called confinement, which is explained in detail in later sections. Table 1.4 summarizes the ranges of the three Standard Model interactions and of gravity and shows typical lifetimes for particles coupled by these forces.

\begin{tabular}{|c|c|c|c|c|}
\hline Interaction & Coupling Constant & Range $(\mathrm{m})$ & Typical Lifetime $(\mathrm{s})$ & Strength \\
\hline \hline Strong & $\alpha_{\mathrm{S}}$ & $\approx 10^{-15}$ & $10^{-23}$ & 0.1 \\
Electromagnetic & $\alpha$ & $\infty$ & $10^{-20}-10^{-16}$ & $\frac{1}{137}$ \\
Weak & $\mathrm{G}_{\mathrm{F}}$ & $<10^{-18}$ & $\geq 10^{-12}$ & $1 \times 10^{-5}$ \\
Gravity & $\mathrm{G}$ & $\infty$ & $\infty$ & $6 \times 10^{-39}$ \\
\hline
\end{tabular}

Table 1.4: The ranges, strengths, and typical lifetimes associated with the three Standard Model interactions, and with gravity.

The forces can by classified according to the strength of the coupling between the gauge bosons and the interacting fermions. This coupling is measured by the coupling constant associated with each force. Table 1.4 lists the coupling constants for the three Standard Model interactions and for gravity. The strong interaction is the strongest force, followed by the electromagnetic and the weak interaction. Newton's gravitational constant, G, is several orders of magnitude smaller than any of the Standard Model coupling constants. The effects of gravity can be neglected in the study of particle interactions at energies obtainable in modern particle physics experiments.

\subsection{Gauge Field Theories}

The three fundamental interactions described by the Standard Model, the electromagnetic, the weak and the strong interactions, belong to a class of theories known as gauge field theories. Construction of a field theory begins by examining classical Lagrangian formalism and the variational principle in the context of a relativistic quantum theory. The Lagrangian density, $\mathcal{L}$, is a functional of the field $\phi(x)$ and its 
four-gradient $\partial_{\mu} \phi(x) \equiv \partial \phi(x) / \partial x^{\mu}$,

$$
\mathcal{L} \equiv \mathcal{L}\left(\phi(x), \partial_{\mu} \phi(x)\right)
$$

The spatial integral of $\mathcal{L}$ takes part of the classical Lagrangian, L,

$$
\mathrm{L} \equiv \int \mathrm{d}^{3} \mathbf{x} \mathcal{L}\left(\phi, \partial_{\mu} \phi\right)
$$

such that the action, $\mathrm{S}$, can be written as

$$
\mathrm{S} \equiv \int_{\mathrm{t}_{1}}^{\mathrm{t}_{2}} \mathrm{dt} \int \mathrm{d}^{3} \mathbf{x} \mathcal{L}\left(\phi, \partial_{\mu} \phi\right)
$$

According to Hamilton's principle of least action, the variation

$$
\delta \mathrm{S}=\delta \int_{\mathrm{t}_{1}}^{\mathrm{t}_{2}} \mathrm{~d}^{4} x \mathcal{L}\left(\phi, \partial_{\mu} \phi\right)=0
$$

subject to the constraint that the variations in the fields at the endpoints, $t_{1}$ and $t_{2}$, vanish. This requirement of least action is satisfied by the Euler-Lagrange equations for $\mathcal{L}$,

$$
\frac{\partial \mathcal{L}}{\partial \phi(x)}=\partial_{\mu} \frac{\partial \mathcal{L}}{\partial\left(\partial_{\mu} \phi(x)\right)} .
$$

From these, explicit equations of motion for the fields can be obtained. In the case of several fields, $\phi_{i}$, the variational principle is applied separately to each field, leading to a set of equations of motion.

In gauge field theories, invariance of the Lagrangian of an interaction under local gauge transformations is required in order to account for conservation laws and constants of the motion observed in experiments. These experimental observations are imposed as symmetries of the Lagrangian, thereby restricting and defining its functional form. The theoretical basis for the equivalence of a conservation law and a symmetry of the Lagrangian is found in Noether's theorem [16]. In order for the Lagrangian to be invariant under local gauge transformations, the 4-potential of the interaction has to transform appropriately, such that changes in the 4-potential compensate for local gauge changes. 
General physical considerations and experimental observations (e.g., conservation of energy and momentum, conservation of angular momentum, etc.) lead to additional requirements upon the total Lagrangian of an interaction. In the end, local gauge invariance, Lorentz invariance, invariance under spatial inversion and time reversal, and renormalizability define an interaction Lagrangian. In quantum field theories, unphysical infinite contributions often arise in the calculations. In a renormalizable theory, these infinities can be consistently eliminated.

\subsubsection{The Interactions of the Standard Model}

The Standard Model describes with the most fundamental building blocks in nature. Therefore, it inherently requires quantum mechanical treatment. The discussion of gauge field theories has, so far, been classical. The transition from a classical field theory to a quantum field theory can be made if the classical fields are regarded as operators. By imposing canonical commutation relations between these operators, the field theory can be quantized [17].

After the quantization, the fields of a gauge theory can be associated with the particles undergoing the interaction, and the 4-potential of the interaction becomes the expression for the mediators of the force. These mediators are the spin-1 gauge bosons. The interactions are modeled as a continuous exchange of gauge bosons between the particles participating in the interactions. Examples of gauge boson exchanges are shown in Figure 1.1.

If a symmetry is exact, then the gauge bosons that mediate the interaction are necessarily massless. Only broken symmetries, symmetries that are not exact, but approximate rather, allow for massive gauge bosons, such as the $W^{ \pm}$and $Z^{0}$, through the Higgs [18] or other mechanisms.

Exact symmetries observed in nature include symmetries of the Lagrangian under translation in space and time and under rotation. These symmetries are considered geometrical symmetries because they involve coordinate transformations. The 


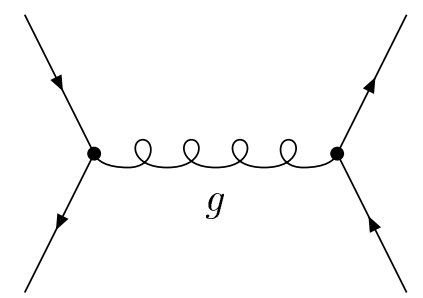

a)

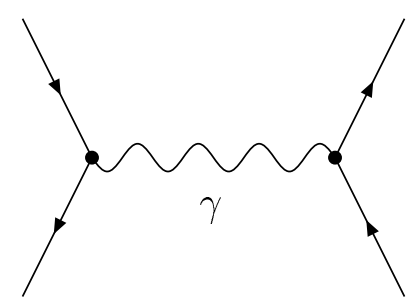

b)

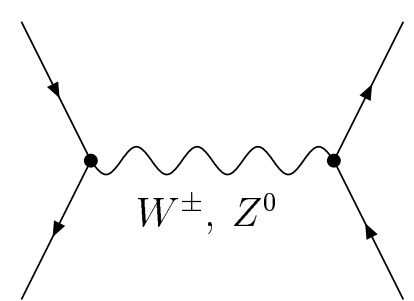

c)

Figure 1.1: Examples of gauge boson exchanges. a) Strong interaction: a gluon $(g)$ is exchanged; b) electromagnetic interaction: a photon $(\gamma)$ is exchanged; and $c)$ weak interaction: a $W^{ \pm}$or $Z^{0}$ is exchanged.

conservation laws associated with these symmetries are conservation of energy and momentum and conservation of angular momentum.

Internal symmetries involve conservation of internal degrees of freedom in an interaction, such as conservation of electric charge. The underlying symmetry in that case is due to the gauge invariance of electrodynamics.

The mathematical basis for the treatment of symmetries is given by group theory. The three interactions of the Standard Model are invariant under different group transformations, depending on the nature of the underlying gauge symmetry. The strong interaction is invariant under local SU(3) transformation, the electromagnetic interaction under $\mathrm{U}(1)$, and the weak interaction under $\mathrm{SU}(2)$. The properties of the gauge bosons can be determined by group-theoretical considerations.

\section{The Electromagnetic Interaction}

The earliest developed and most accurate quantum gauge theory is the theory of the electromagnetic interaction, quantum electrodynamics (QED). Exact conservation of electric charge implies the existence of a long-range field coupled to the charge. This requires the photon to be massless.

Quantum electrodynamics is invariant under local U(1) transformations, meaning the group of all one-dimensional unitary matrices. A unitary matrix, U, is defined 
by:

$$
\mathrm{UU}^{\dagger}=\mathrm{U}^{\dagger} \mathrm{U}=\mathrm{I}
$$

where $I$ is the identity matrix and ${ }^{\dagger}$ denotes the Hermitian conjugate. In the onedimensional case of $\mathrm{U}(1), \mathrm{U}$ is a single complex number. The covariant derivative, $\mathcal{D}_{\mu}$, is defined by the gauge transformation of the operator $\partial_{\mu}$,

$$
\partial_{\mu} \longrightarrow \mathcal{D}_{\mu}=\partial_{\mu}+i e A_{\mu}
$$

where $A_{\mu}$ is the electromagnetic 4-potential and $e$ is the electron charge. If the covariant derivative replaces any term $\partial_{\mu}$ in the Lagrangian, and $A_{\mu}$ is required to transform as

$$
A_{\mu} \longrightarrow A_{\mu}^{\prime}=A_{\mu}+\partial_{\mu} \Lambda
$$

then the fermion field, $\Psi$, transforms as

$$
\Psi(x) \longrightarrow \Psi^{\prime}(x)=e^{-i e \Lambda(x)} \Psi(x)
$$

and is locally invariant under the transformation. The arbitrary gauge variable, $\Lambda$, introduced in Equations 1.11 and 1.12, transforms $\Psi$ infinitesimally in the vector space spanned by the generators of the group. Successive U(1) transformations commute, so Quantum Electrodynamics is an Abelian gauge theory.

Quantum electrodynamics allows for extremely accurate predictions of many experimental observations. Among these are the magnetic moments of the leptons and the bound-state energy levels of hydrogen and other atoms, of muonium $\left(\mu^{+} e^{-}\right)$, and of positronium $\left(e^{+} e^{-}\right)$. Its success spurred attempts to describe the other Standard Model interactions in terms of gauge field theories.

\section{The Weak Interaction}

The weak interaction acts on all quarks and leptons; it is the only interaction in the Standard Model that takes place between all its fundamental fermions. Since neutrinos are electrically neutral, and since as leptons, they also do not interact 
strongly, they can only take part in the weak interaction. The charge the weak interaction couples to is flavor.

Weak interaction cross sections are small compared to strong and electromagnetic cross sections. Thus, the lifetimes for weak decays $\left(\simeq 10^{-10} \mathrm{~s}\right)$ are typically longer than for decays due to one of the other two interactions (strong decays typically limit particle lifetimes to $\simeq 10^{-23} \mathrm{~s}$, electromagnetic decays to $\simeq 10^{-19} \mathrm{~s}$ ).

The weak interaction was first observed in nuclear $\beta$-decay, in which neutrons decay into protons and leptons, or, equivalently, a $d$-quark decays into a $u$-quark, an electron, and an antineutrino. It is the only known interaction that can change quark flavors. On a cosmic scale, the weak interaction is important as it is responsible for the synthesis of heavy elements in the early universe and for the first stage of energy production in the hydrogen of a star's core.

In most interactions, weak effects are swamped by the much stronger strong or electromagnetic couplings. Only in circumstances where the faster strong or electromagnetic decays are forbidden by a conservation law can it be observed.

The mass of these bosons explains the short range of the interaction. It is described by an SU(2) symmetry, the group of all two-dimensional special unitary matrices. Special unitary matrices obey Equation 1.9 and have a unit determinant.

\section{The Electroweak Model}

Local gauge invariance in a gauge field theory requires massless gauge bosons for an interaction. The short-range nature of the weak interaction, however, requires the gauge bosons to be massive. The mechanism of spontaneous symmetry breaking, for example the Higgs mechanism, is able to combine local gauge invariance and the appearance of massive gauge bosons.

The electroweak model proposed by Glashow, Weinberg, and Salam [19] in the 1960's makes use of the Higgs mechanism to explain the massiveness of the weak gauge bosons and to unify the weak and electromagnetic theories. The symmetry 
group describing the electroweak model is $\mathrm{SU}(2) \times \mathrm{U}(1)$.

The fundamental bosons in this theory are the three massless bosons, $W^{+}$, $W^{-}$, and $W^{0}$ for the $\mathrm{SU}(2)$ symmetry, and the massless $B^{0}$ for the $\mathrm{U}(1)$ symmetry. The interaction Lagrangian can be written as

$$
\mathcal{L}=g \mathbf{J} \cdot \mathbf{W}+g^{\prime} J^{Y} B^{0}
$$

where $\mathbf{W}=\left(W^{+}, W^{0}, W^{-}\right), \mathbf{J}$ and $J^{Y}$ are conserved currents in the interaction, and $g$ and $g^{\prime}$ are the couplings to $\mathbf{W}$ and $B^{0}$, respectively. The couplings are analogous to the charge $e$ in the electromagnetic interaction (see Equations 1.10 and 1.12).

The Higgs mechanism gives mass to the components of $\mathbf{W}$. The electrically charged components can be identified as the $W^{+}$and the $W^{-}$of the weak interaction. The two neutral gauge bosons observed in nature are the photon and the $Z^{0}$. A quantum mechanical superposition of the two neutral gauge boson states of the electroweak interaction, the $W^{0}$ and the $B^{0}$, results in the two observable neutral bosons, the photon and the $Z^{0}$. The degree of mixing in this superposition is determined by the electroweak mixing, or Weinberg, angle, $\theta_{W}$. The Weinberg angle is related to the couplings through

$$
\begin{aligned}
g / g^{\prime} & =\tan \theta_{W}, \text { and } \\
e & =g \sin \theta_{W} .
\end{aligned}
$$

The Higgs mechanism introduces at least one more gauge boson to the theory, the Higgs boson. While the masses of the $W^{ \pm}$, the $Z^{0}$, and the photon can be predicted in the electroweak model, the mass of the Higgs boson is a free parameter. It has to be determined by experiment. The question of the existence and mass of the Higgs boson is one of the most important unresolved experimental challenges in physics today. 


\section{The Strong Interaction}

Similarly to the cases of the weak and the electromagnetic interactions, the strong interaction can be described in terms of a symmetry group, $\mathrm{SU}(3)$. The gauge field theory underlying this group-theoretical approach is known as quantum chromodynamics (QCD). Quantum chromodynamics is a non-Abelian gauge theory; successive gauge transformations do not commute.

The strong interaction acts on all quarks; the charge it couples to is an internal quantum number, color. The gauge bosons of the strong interaction are eight massless gluons, $g$.

As the analysis presented in this thesis compares experimental data to predictions from quantum chromodynamics, the theory is of crucial importance and will be discussed in more detail in the next section.

\subsubsection{Quantum Chromodynamics}

Quantum Chromodynamics is the gauge field theory of the strong interaction of quarks and gluons, partons, the constituents of all hadrons. The non-Abelian nature of its $\mathrm{SU}(3)$ symmetry group, and the fact that the strong interaction is strong, lead to properties of the theory that significantly distinguish it from the other gauge field theories of the Standard Model. The effect of color confinement at low energies and asymptotic freedom at high energies are described by it. One important observation in strong interactions is the appearance of jets, well collimated streams

of hadrons emitted from a particle collision. Jets are a unique feature of the strong interaction and are not observed in electroweak interactions. A main concern of this analysis is the investigation of jet properties. 


\section{The Static Quark Model}

By the 1960's, particle collider experiments had discovered a plethora of hadrons, strongly interacting particles. Among others, the spin-3/2 $\Delta^{++}$baryon was found. The $\Delta^{++}$is thought to consist of three $u$ quarks and to be in its lowest mass state, so that the quarks are assumed to be in a spatially symmetric ground state. To account for the total spin of $J=3 / 2$, the three quark spins have to be parallel. At the time of its discovery, the question arose as to how this system could exist without violating the Pauli Exclusion Principle, since the three quarks appear to be in the same quantum state.

To resolve this conflict, an additional degree of freedom for quarks was proposed. This additional quantum number is called color. Every quark can exist in one of three color states, red, blue, or green. It is proposed that experiments are not able to observe color, so that all hadrons have to appear in color singlet states (they have zero net color). Mesons are composed of a color-anticolor combination, with the antiquark carrying the anticolor corresponding to the quark's color, and each one of the three quarks in a baryon carries a different color, such that the combination again is colorless.

Analogously to positive and negative electric charges being the source of the electromagnetic force, the three color charges are the source of the strong force. The particles mediating the strong interaction are the eight massless gluons. Because gluons carry color, they are also subject to strong interactions. The explanation of this effect is given in detail below. Leptons and the other gauge bosons of the Standard Model are colorless; equivalently, they do not interact strongly.

This static quark model is supported experimentally by several measurements. The first experimental evidence of quark color was found in the measurement of the ratio of cross sections, $\mathrm{R}$, between $e^{+} e^{-} \longrightarrow$ hadrons and $e^{+} e^{-} \longrightarrow \mu^{+} \mu^{-}$. R can be written as

$$
\mathrm{R}=\frac{\sigma\left(\mathrm{e}^{+} \mathrm{e}^{-} \longrightarrow \text { hadrons }\right)}{\sigma\left(\mathrm{e}^{+} \mathrm{e}^{-} \longrightarrow \mu^{+} \mu^{-}\right)}=\sum_{\mathrm{a}} \mathrm{q}_{\mathrm{a}}^{2}
$$


where $\mathrm{q}_{\mathrm{a}}$ is the charge of quark type a. At energies below the $t$ quark threshold, $R=11 / 9$ if every quark can only exist in one color state, and $R=11 / 3$ if three color states are possible. Experiments measure $\mathrm{R}=4.17 \pm 0.09$ (stat.) \pm 0.42 (syst.) [20]. A summary of the experimental data with references can be found in Reference [21].

An experimental indication for gluons is three-jet events in high energy particle collisions. Three-jet events can be modeled as the production of a quark-antiquark pair, one of which radiates a gluon, similar to bremsstrahlung in electromagnetic interactions.

\section{The Quark-Parton Model}

Additional experimental data on the structure of nuclei lead to the development of a dynamical, rather than static, model of the structure of hadrons. Deep inelastic scattering of electrons, muons, and neutrinos shows that about $50 \%$ of a nucleon's momentum is carried by electrically neutral nucleon constituents [22]. Gluons are thought to be these additional particles in the nucleon.

Mesons and baryons do not consist of only the two or three quarks that were introduced in the static quark model, the valence quarks. A sea of virtual particles, gluons and quark-antiquark pairs, the sea quarks, is also part of the hadron's composition. The lifetimes of the virtual partons are limited by the Heisenberg Uncertainty Principle. In the quark-parton model, the massless and point-like partons inside the hadron move collinearly with it.

The probability, $f_{i}\left(x_{i}\right)$, of finding a parton, $i$, with a momentum fraction, $x_{i}$, in a hadron is given by a parton distribution function (PDF). Parton distribution functions are determined experimentally from deep inelastic scattering data. Analogously to the Rutherford experiment by which the existence of a nucleus in the atom was established, high energy scattering of leptons on a nucleon reveals the internal distribution of partons. Several parameterizations for PDF's are available. The data analysis described in later chapters considers the differences in QCD predictions that 
use specific PDF parameterizations. Figure 1.2 [23] shows a typical parton distribution for $u$ and $d$ valence quarks, $\bar{u}$ and $s$ sea quarks, and for gluons, as a function of momentum fraction, $x$.

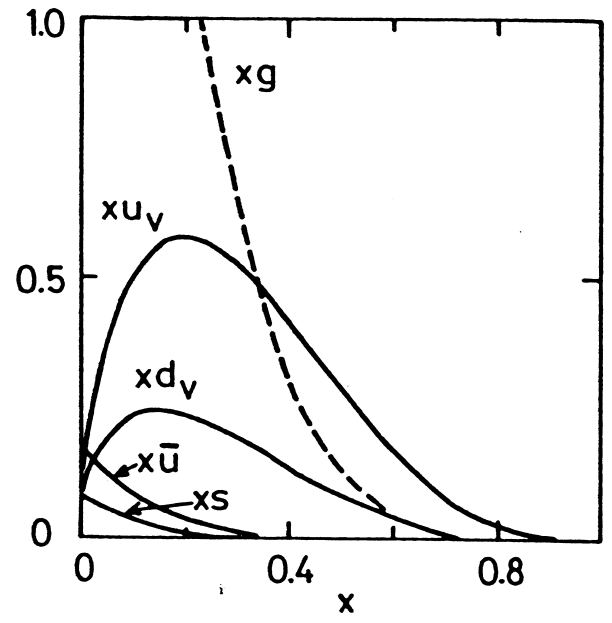

Figure 1.2: A typical parton distribution function parameterization for $u$ and $d$ valence quarks, $\bar{u}$ and $s$ sea quarks, and gluons inside a hadron. (Figure taken from Reference [23].)

Quantum Chromodynamics, The Quantum Field Theory of the Strong Force

The color symmetry underlying Quantum Chromodynamics is described by the group $\mathrm{SU}(3)$. The Lagrangian of the interaction has the form

$$
\mathcal{L}=\bar{\Psi}\left(\imath \gamma^{\mu} \mathcal{D}_{\mu}-\mathrm{m}\right) \Psi-\frac{1}{2} \operatorname{Tr}\left(\mathrm{F}_{\mu \nu} \mathrm{F}^{\mu \nu}\right)
$$


where $\Psi$ are the fermion fields, $\gamma^{\mu}$ are the four Dirac $\gamma$-matrices (see Reference [24]), $\mathrm{m}$ is the fermion mass, $\mathcal{D}$ the covariant derivative, and $\mathrm{F}_{\mu \nu}$ is the gauge field-strength tensor. The gauge field-strength tensor is defined as

$$
\mathrm{F}_{\mu \nu} \equiv \partial_{\nu} \mathrm{B}_{\mu}-\partial_{\mu} \mathrm{B}_{\nu}+\imath \mathrm{g}\left[\mathrm{B}_{\nu}, \mathrm{B}_{\mu}\right]
$$

Here $\mathrm{g}$ is the magnitude of the strong coupling and $\mathrm{B}_{\mu}$ is a three-by-three matrix in color space formed from the eight color gauge fields, $\mathrm{b}_{\mu}^{l}$, and the generators of the $\mathrm{SU}(3)$ group, $\lambda^{l} / 2$, the Gell-Mann matrices [25],

$$
\mathrm{B}_{\mu}=\frac{1}{2} \lambda \cdot \mathbf{b}_{\mu}=\frac{1}{2} \lambda^{l} \mathrm{~b}_{\mu}^{l}
$$

As with all gauge field theories, Quantum Chromodynamics is required to be invariant under local gauge transformations

$$
\Psi \longrightarrow \Psi^{\prime}=\mathrm{e}^{\frac{2}{2} \lambda \cdot \alpha(\mathbf{x})} \cdot \Psi
$$

where $\alpha(\mathbf{x})$ is an arbitrary parameter denoting the magnitude of the gauge transformation. Gauge invariance restricts the covariant derivative to

$$
\mathcal{D}_{\mu} \equiv \partial_{\mu}+\imath \mathrm{gB}_{\mu}
$$

QCD is a non-Abelian gauge theory. The commutator $\left[\mathrm{B}_{\nu}, \mathrm{B}_{\mu}\right] \neq 0$ in the expression for the gauge field-strength tensor, $\mathrm{F}_{\mu \nu}$ (Equation 1.18). The Lagrangian

then depends on third- and fourth-order terms with $\mathrm{B}_{\mu}$ in $\mathrm{F}_{\mu \nu} \mathrm{F}^{\mu \nu}$. This gives rise to the possibility of three- and four-gauge-boson vertices, and with that to gluon-gluon coupling in quantum chromodynamics. In this way QCD is significantly different from the electroweak interaction, in which the gauge bosons do not carry charge and cannot couple to each other.

\section{Confinement, Asymptotic Freedom, and the Running Coupling}

A familiar phenomenon in classical electrodynamics is the polarization of dielectric media. If a test charge is placed in a dielectric, the surrounding medium will polarize 
as indicated in Figure 1.3. At any distance, $r$, from the test charge (if $r$ is larger than the molecular scale), the electric field in the medium is smaller than the electric field of that charge in a vacuum. The proportionality factor between the electric field in a vacuum and the electric field in the dielectric is $\epsilon$, the dielectric constant,

$$
\mathrm{E}_{\text {medium }} \equiv \mathrm{E}_{\text {vacuum }} / \epsilon
$$

with $\epsilon \geq 1$. Gauss's Law determines the magnitude of the effective charge seen by an observer outside a sphere of radius $\mathrm{r}$,

$$
\mathrm{Q}_{\text {effective }} \equiv \mathrm{Q}_{\text {charge }} / \epsilon \text {. }
$$

The test charge is screened by the dielectric medium, as the effective charge is smaller than the test charge in the medium. The smaller the distance to the test charge becomes, the smaller is the screening effect. At distances smaller than the molecular scale, screening cannot occur, and the effective charge is equal to the magnitude of the test charge.

In quantum gauge theories, the vacuum behaves as a polarizable medium. All carriers of charge (e.g., color charge, electric charge, flavor charge) polarize the surrounding vacuum in which other (virtual) charges appear. Consequently, the effective charge also depends on the distance scale.

All Standard Model interactions are modeled as continuous exchanges of gauge bosons, the leading order versions of some of which are shown in Figure 1.1. For the calculation of observables such as cross sections, higher accuracy requires inclusion of higher-order vacuum polarization terms. These higher-order contributions to the observable can be depicted graphically as in Figure 1.4, which shows second-order corrections to strong interaction calculations. For every loop, a closed line in the picture, the correction is higher by one order; second-order corrections are drawn with one loop. Similar vacuum polarization corrections exist for electromagnetic or weak observables. 


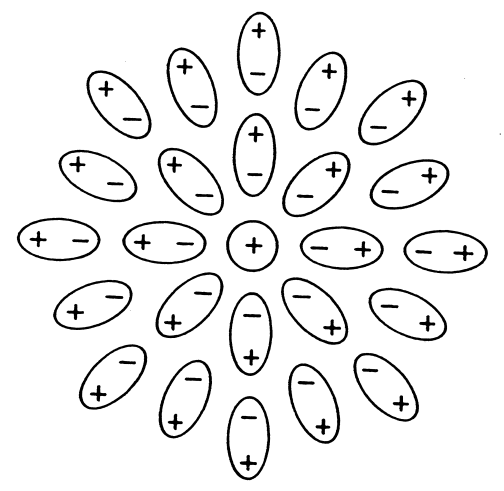

Figure 1.3: Polarization of a dielectric medium by a charge. (Figure taken from Reference [25].)

Calculations that include these higher-order terms lead to infinities in quantum gauge theories when the contributions are integrated over all possible momentum transfers. Physically meaningful, finite results can only be obtained if the theory is renormalized, such that different divergences cancel exactly, leaving only finite residuals. The Standard Model gauge theories are renormalizable [26]. The procedure for renormalization includes the introduction of an arbitrary parameter, the renormalization scale $\mu$. Any observable has to be independent of $\mu$, a fact that can be expressed mathematically in the Renormalization Group Equation. A dimensionless observable, $\mathrm{R}$, generally depends on the ratio $\mathrm{Q}^{2} / \mu^{2}$ of the energy scale of the interaction, $\mathrm{Q}^{2}$, and $\mu^{2}$ and on the coupling constant of the strong interaction, $\alpha_{S}$. This implies a Renormalization Group Equation of the form [27]

$$
\mu^{2} \frac{d}{d \mu^{2}} \mathrm{R}\left(\mathrm{Q}^{2} / \mu^{2}, \alpha_{\mathrm{S}}\right) \equiv\left[\mu^{2} \frac{\partial}{\partial \mu^{2}}+\mu^{2} \frac{\partial \alpha_{\mathrm{S}}}{\partial \mu^{2}} \frac{\partial}{\partial \alpha_{\mathrm{S}}}\right] \mathrm{R}=0
$$




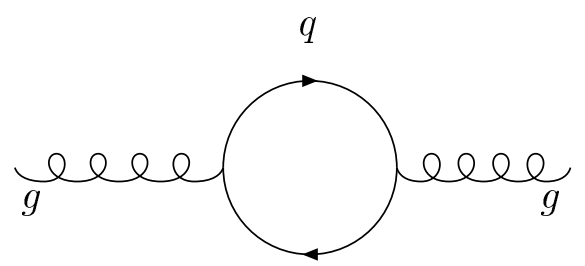

$\bar{q}$

a)

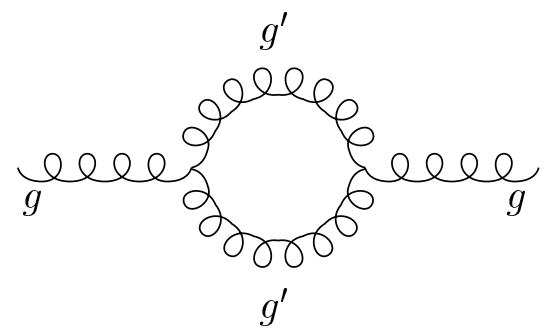

b)

Figure 1.4: Second-order corrections to strong interaction observables. a) a quarkantiquark loop; and b) a gluon loop.

The solution to this equation is a differential equation for renormalizing $\alpha_{\mathrm{S}}$ as a function of $\mathrm{Q}^{2}$, depending on the order in perturbation theory. In practice, observables can only be calculated to a finite order of corrections, since the number of possible diagrams and the complexity of the calculations involved rises significantly with the order of the calculation. In all practical calculations, the series is truncated at a particular order in perturbation; the calculational result then explicitly depends on $\mu$. This dependence on the renormalization scale is postulated to decrease with increasing computed order of corrections. Figure 1.5 shows the decreasing $\mu$ dependence in a cross section calculation from a leading order ("Born") to a next-to-leading order ("Full") calculation.

The non-Abelian nature of QCD results in gluon self-coupling which, in turn, gives rise to anti-screening. Unlike the screening effect in Quantum Electrodynamics, which always results in smaller effective charges, vacuum polarization in QCD results in an increase of the effective charge for larger distances. The coupling strength of an interaction depends on the magnitudes of the charges of the interacting particles. The effect of vacuum polarization can be included in the gauge theory if the coupling strength is allowed to vary with distance due to screening and anti-screening. The coupling strength is not constant, but is exchanged for a running coupling instead. As the negative square of the 4-momentum transfer in the interaction, $Q^{2}$, is equivalent 


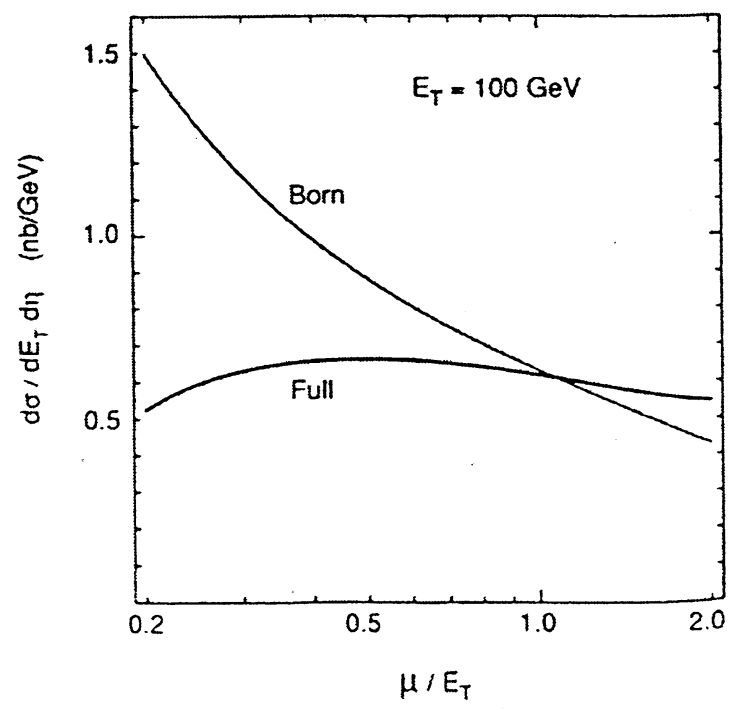

Figure 1.5: The deceasing $\mu$ dependence in a cross section calculation from a leading order ("Born") to a next-to-leading order ("Full") calculation. (Figure taken from Reference [28].)

to a measurement of the distance between the interacting particles, it is convenient to describe the running of the coupling in terms of $\mathrm{Q}^{2}$. Similar to the fine structure constant in electromagnetism, a coupling for the strong force, $\alpha_{\mathrm{S}}$, is defined as

$$
\alpha_{\mathrm{S}} \equiv \frac{\mathrm{g}}{4 \pi}
$$

where $\mathrm{g}$ is the magnitude of the coupling introduced in Equation 1.18 and replaces $e$ in electromagnetic interactions. The strong coupling to first-order in the perturbative 
expansion in $\mu$ then is

$$
\alpha_{\mathrm{S}}\left(\mathrm{Q}^{2}\right)=\frac{12 \pi}{(33-2 \mathrm{f}) \ln \left(\frac{\mathrm{Q}^{2}}{\Lambda^{2}}\right)},
$$

where $\mathrm{f}$ is the number of active quark flavors at $\mathrm{Q}^{2}$ and $\Lambda$ is an arbitrary cut-off at which the perturbation expansion is assumed to diverge (generally between 0.1 and $0.5 \mathrm{GeV}$ ). Anti-screening is possible in Quantum Chromodynamics for energy regimes with less than seventeen active quark flavors. For more than seventeen quark flavors, Equation 1.26 changes sign; this marks the onset of screening. Accessible energies have revealed only six quark flavors to date, and anti-screening has been observed for strong interactions. Figure 1.6 shows the running of the strong coupling as a function of of $\mathrm{Q}^{2}$.

The running of the strong coupling, and its functional form, account for the phenomena that distinguish strong interactions from the other two interactions of the Standard Model. At low energies, or equivalently large distances, $\alpha_{\mathrm{S}}$ is large, which

gives rise to color confinement. Experimentally, free color has not been observed: the strength of the strong coupling does not permit separation larger than the nucleon scale between colored objects. As $\mathrm{Q}^{2}$ increases, $\alpha_{\mathrm{S}}$ decreases. This is called asymptotic freedom. A value for $\alpha_{\mathrm{S}}$ is usually quoted at $\mathrm{Q}^{2}=\mathrm{M}_{\mathrm{Z}}^{2}$, the square of the mass of the $\mathrm{Z}^{0}$ boson. The current world average is [21]

$$
\alpha_{\mathrm{S}}\left(\mathrm{M}_{\mathrm{Z}}^{2}\right)=0.1185 \pm 0.0020
$$

\subsection{Jet Physics}

Well-collimated streams of particles observed in a detector are called jets. The particles assigned to jets are mainly hadrons, which indicates that jet formation is a strong interaction phenomenon. Jet formation is a consequence of the non-Abelian nature of the gauge group through the running of the coupling constant and color confinement. As color confinement is a phenomenon of the energy regime in which $\alpha_{\mathrm{S}}$ is large, 


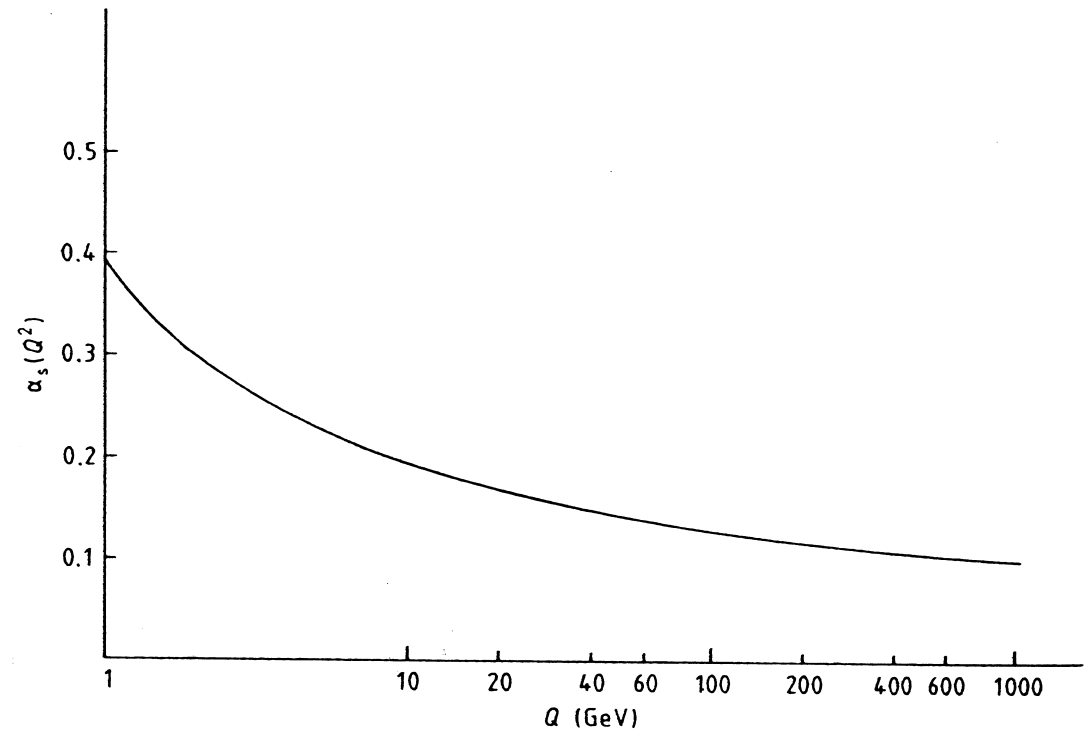

Figure 1.6: The running of the strong coupling as a function of the 4-momentum transfer, Q, for $\Lambda=0.1 \mathrm{GeV}$. (Figure taken from Reference [29].)

QCD models for low-energy, non-perturbative strong interactions are important for jet physics.

\subsubsection{Parton Density Evolution}

Through the electromagnetic interaction, a charged particle when accelerated can emit a photon, a process called bremsstrahlung. By a similar process, any color charge carrier, a quark or a gluon, can radiate a gluon before or after an interaction. Parton distribution functions acquire an explicit dependence on $\mathrm{Q}^{2}$ through these 
gluon bremsstrahlung processes. A mathematical description of the PDF evolution as a function of $\mathrm{Q}^{2}$ is given by the DGLAP (Dokshitzer-Gribov-Lipatov-AltarelliParisi) evolution equations [30]. As an example, if the quark distribution at $\mathrm{Q}^{2}=\mathrm{Q}_{0}^{2}$ is denoted by $f\left(x, \mathrm{Q}_{0}^{2}\right)$, then at $\mathrm{Q}^{2}>\mathrm{Q}_{0}^{2}$ it is modified by the radiation of a gluon. The quark evolution can be written as

$$
\frac{\mathrm{d} f\left(x, \mathrm{Q}^{2}\right)}{\mathrm{d} \ln \mathrm{Q}^{2}}=\frac{\alpha_{\mathrm{S}}\left(\mathrm{Q}^{2}\right)}{2 \pi} \int_{x}^{1} f\left(y, \mathrm{Q}^{2}\right) P_{\mathrm{QQ}}\left(\frac{x}{y}\right) \frac{\mathrm{d} y}{y} .
$$

A quark with momentum fraction $x$ can be thought of as having originated from a quark with momentum fraction $y$ which radiated a gluon with momentum fraction $y-x$. The probability for radiation to occur is proportional to $\alpha_{\mathrm{S}}$, and the splitting function, $P_{\mathrm{QQ}}$, describes the probability for a radiating quark to retain the momentum fraction $z=x / y$. For the radiation of a vector boson by a fermion, $P_{\mathrm{QQ}}$ has the form

$$
P_{\mathrm{QQ}}(z)=\frac{4}{3}\left(\frac{1+z^{2}}{1-z}\right) .
$$

Equation 1.28 is valid for the evolution of a valence quark. Sea quark evolution is described by an analogous expression, but a term that accounts for the possibility for the gluon to split into a $q \bar{q}$ pair has to be added [24].

\subsubsection{Jets in High Energy Collisions}

Jets were first observed in high energy $e^{+} e^{-}$collisions, in which electron-positron annihilation can result in the production of a $q \bar{q}$ pair [31]. As a direct result of color confinement, the quark and the antiquark cannot exist by themselves, but rather hadronize. Real quark-antiquark pairs emerge from the vacuum and clothe the bare quarks through a non-perturbative process not yet fully understood, until only colorless hadrons remain. The energy necessary for the creation of these $q \bar{q}$ pairs reduces the kinetic energy of the original parton produced in the interaction. As the source of the hadrons in a jet is a single quark or antiquark with large kinetic energy, they move in a near-collinear fashion, resulting in the collimation of the jet. Quarks and 
gluons can be observed only indirectly, through jets. Jet studies, therefore, provide a means to test QCD predictions.

The production of jets in hadron-hadron collisions involves the hard scattering of a single parton in one hadron with one parton inside the second hadron. The remaining partons are considered to be spectators that do not participate in the interaction. The collision breaks up the hadrons; the non-interacting remnants generally move along the beamline and may not be detected, while the partons involved in the hard scattering often acquire significant momentum transverse to the beam. Decay of heavy hadrons into lighter ones leads to jets which can be studied with the detector.

Hadronization, or jet fragmentation, is a process that occurs at energies of a few $\mathrm{GeV}$. Perturbative methods cannot be employed to describe it due to the magnitude of the strong coupling in that regime. Instead, non-perturbative, semi-empirical models serve to approximate the process. One model used to describe jet fragmentation is the Lund string model [32]. Since it is part of a Monte Carlo event generator used to study the hadronization of partons in this analysis, it is described here.

In the Lund string model, the potential between the quark and the antiquark in a color-neutral pair is approximated by

$$
V=k r
$$

where $k$ is a constant with value $k \approx 1 \mathrm{GeV} / \mathrm{fm}$ and $r$ is the distance between the two partons. The constant $k$ can be estimated from the size of hadrons. A typical hadron has mass of about $1 \mathrm{GeV}$ and a radius of approximately $1 \mathrm{fm}$. Because of the strong self-interaction between gluons, the force field between $q$ and $\bar{q}$ is confined to a narrow tube, or string. That string has a constant tension, $k$, independent of $r$. A schematic picture of quark and string formation as quarks separate, according to the Lund string model, is shown in Figure 1.7.

If two partons are separated, the potential energy between them rises linearly with $r$. When the potential energy in the string is large enough for the creation of a new $q \bar{q}$ pair, it breaks, and two additional partons emerge from the vacuum. This 

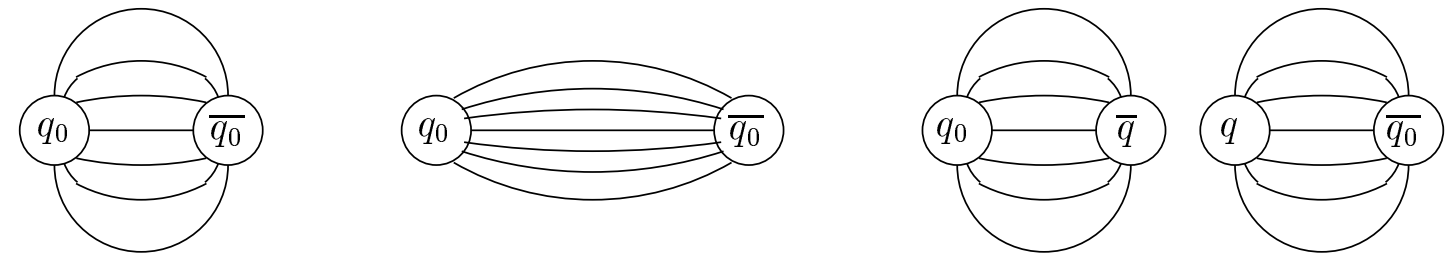

Figure 1.7: Schematic of the color lines of force between two partons, as described in the Lund string model.

process is repeated until the available energy is not sufficient to produce another $q \bar{q}$ pair. Figure 1.8 shows a schematic view of jet fragmentation by means of the Lund string model.

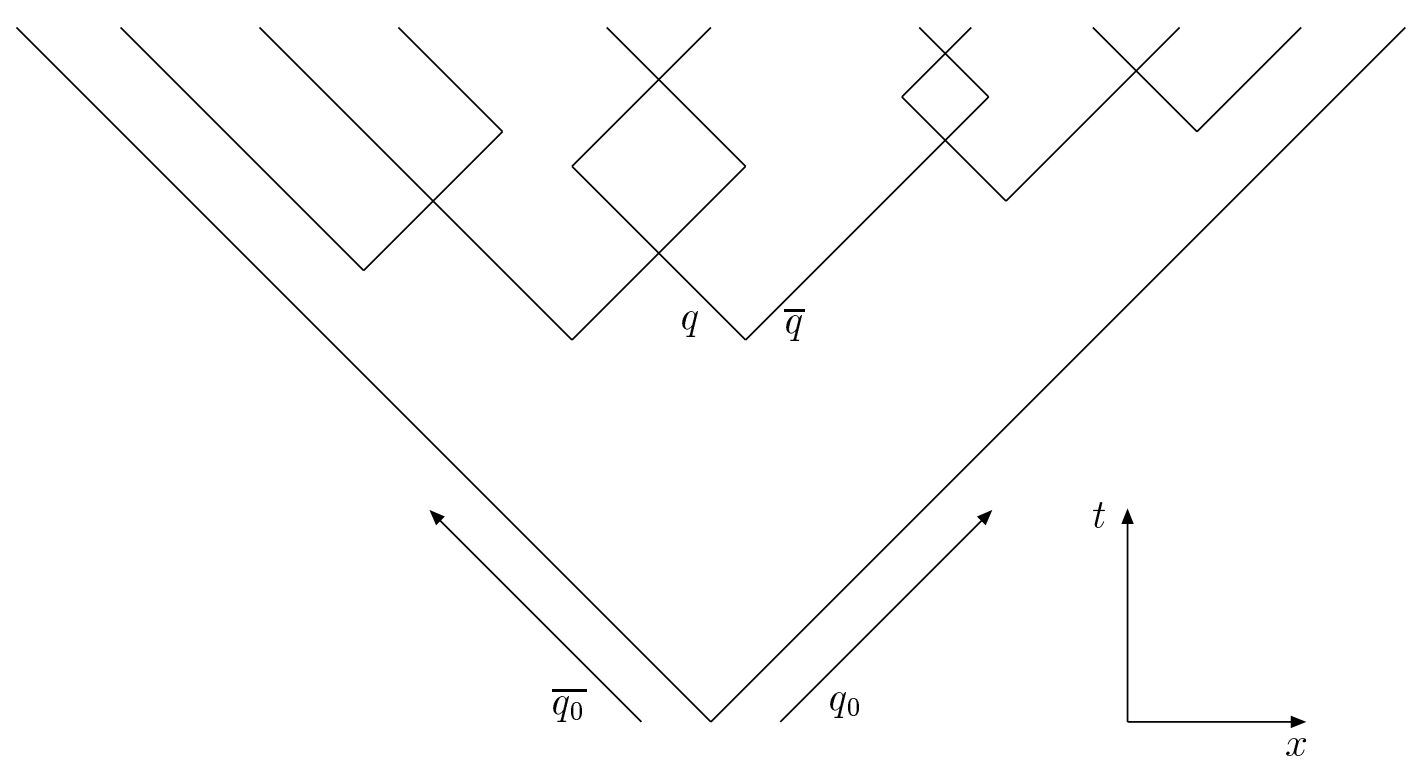

Figure 1.8: Schematic view of jet fragmentation, as described by the Lund string model. 


\subsubsection{Three-jet Events}

When hadrons are created in colliders, they predominantly appear in two-jet events in the detector. The $q \bar{q}$ pair hadronizes to two jets with approximate momentum balance. In the center-of-mass frame, they appear back-to-back.

In collisions with sufficient center-of-mass energy, some events display a third jet. One of the partons in the interaction radiates a gluon which subsequently hadronizes similarly to the two quarks. When three-jet events were first observed [33], they were viewed as experimental indications for the existence of gluons. Since the third jet cannot be produced by the leptons in the collision, only gluon bremsstrahlung can be responsible for an additional hadronizing color charge in the event.

Three-jet events are observed in hadron-hadron collisions. Unlike in $e^{+} e^{-}$ collisions, where only the final state partons can radiate a gluon, the initial and the final state partons can contribute the third jet in hadron collisions. Figure 1.9 shows a three-jet event observed in the CDF detector at the Tevatron (Fermi National Laboratory, Batavia, Illinois).

If the center-of-mass energy in a collision is sufficiently high, four and more jets can occur. Since the probability for gluon bremsstrahlung is proportional to $\alpha_{\mathrm{S}}$, these are observed less frequently than two- or three-jet events.

The analysis described in this thesis is a study of three-jet events at the Tevatron. Characteristics and kinematics of three-jet events are discussed in detail in later sections. 


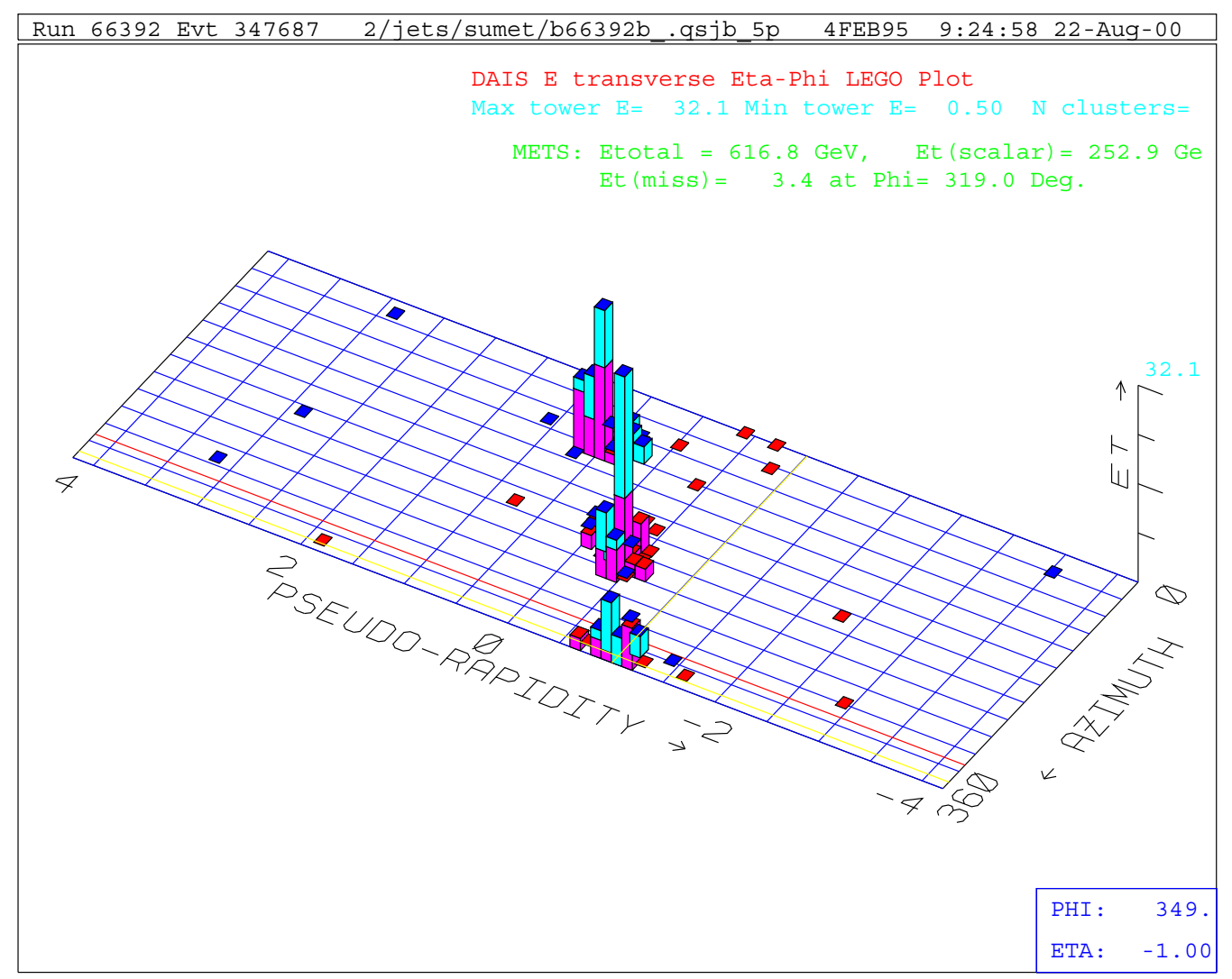

Figure 1.9: A three-jet event observed in the CDF detector at the Tevatron. 


\section{Chapter 2}

\section{The CDF Detector at the Tevatron Collider}

The data used in this study were obtained at the Tevatron collider at the Fermi National Accelerator Laboratory in Batavia, Illinois. Fermilab was founded in 1968 and is located about $65 \mathrm{~km}$ west of Chicago, Illinois. The site, of size approximately $28 \mathrm{~km}^{2}$, houses numerous particle physics experiments and, currently, the world's highest-energy particle accelerator, which are used and maintained by more than 2200 scientists from 20 countries. The Tevatron is a proton-antiproton, $p \bar{p}$, collider with a center-of-mass energy, $\sqrt{s}$, of $1.8 \mathrm{TeV}$.

At two interaction points, detectors are installed, allowing two independent experiments to observe $p \bar{p}$ collisions. The two principal experiments at the Tevatron are the Collider Detector at Fermilab (CDF) and D0. Since this study uses data recorded at $\mathrm{CDF}$, the components of the detector are explained in more detail in this chapter. The main focus is placed on the discussion of the system of calorimeters, as it is most relevant to this study. The data were taken during Run $1 \mathrm{~b}$ at the Tevatron, from 1994 until 1995. 


\subsection{The Accelerator}

The Tevatron $p \bar{p}$ collider started operation in the fall of 1985. A synchrotron accelerator with a length of $6.3 \mathrm{~km}$, it accelerates protons and antiprotons to energies of $900 \mathrm{GeV}$, resulting in a center-of-mass energy of $\sqrt{s}=1.8 \mathrm{TeV}$. The $p$ and $\bar{p}$ beams move in opposite directions in the synchrotron ring. In two interaction regions, the areas where the two particle detectors, CDF and D0, are located, the beams cross. The results of $p \bar{p}$ interactions are recorded by the detectors. The Tevatron tunnel has an inner diameter of $3 \mathrm{~m}$ and is located $6 \mathrm{~m}$ underground.

Proton and antiproton acceleration at Fermilab requires several steps. Five accelerators are used to achieve the final energies. The first acceleration stage is the Cockroft-Walton accelerator, where negative hydrogen ions are accelerated to an energy of $750 \mathrm{keV}$ by a positive potential. After leaving the Cockroft-Walton, the $\mathrm{H}^{-}$ions enter a linear accelerator stage (LINAC). In the approximately $150 \mathrm{~m}$ long tube, they are accelerated to $400 \mathrm{MeV}$ by oscillating electric fields. Leaving the LINAC, the $\mathrm{H}^{-}$ions pass through a carbon foil. The electrons are stripped from the ions, allowing only protons to enter the third stage, the Booster. Located about $6 \mathrm{~m}$ underground, the booster is a synchrotron which accelerates the protons to $8 \mathrm{GeV}$. It sends twelve proton bunches into the Main Ring, the next accelerator stage. The Main Ring is another proton synchrotron, housed in the $6.3 \mathrm{~km}$ long circular Tevatron tunnel. Conventional copper-coil magnets are used to bend and focus the beam, which there reaches $150 \mathrm{GeV}$. The bunches are then injected into the Tevatron, the final accelerating stage, a third synchrotron where the protons obtain their final energy of $900 \mathrm{GeV}$. The Tevatron is located directly below the Main Ring. Superconducting magnets at an operating temperature of $5 \mathrm{~K}$ keep the beam in a circular orbit; liquid helium cools the magnets to the required temperature. The superconducting magnets produce a magnetic field of $4.4 \mathrm{~T}$.

To produce the antiprotons, protons are accelerated in the Main Ring to $120 \mathrm{GeV}$, extracted, and guided to a target area. Collision with a nickel target 
produces a large number of hadrons, including antiprotons. These are focused using a lithium lens. In the Debuncher, $\bar{p}$ bunches are reduced in size through stochastic cooling [34]. Further cooling and storage is provided in the Accumulator. When a sufficient number of antiprotons has accumulated, they are injected back into the Main Ring, and later the Tevatron, where they are accelerated in the direction opposite to the proton beam until they also reach $900 \mathrm{GeV}$. Figure 2.1 shows a schematic of the Fermilab accelerator system.

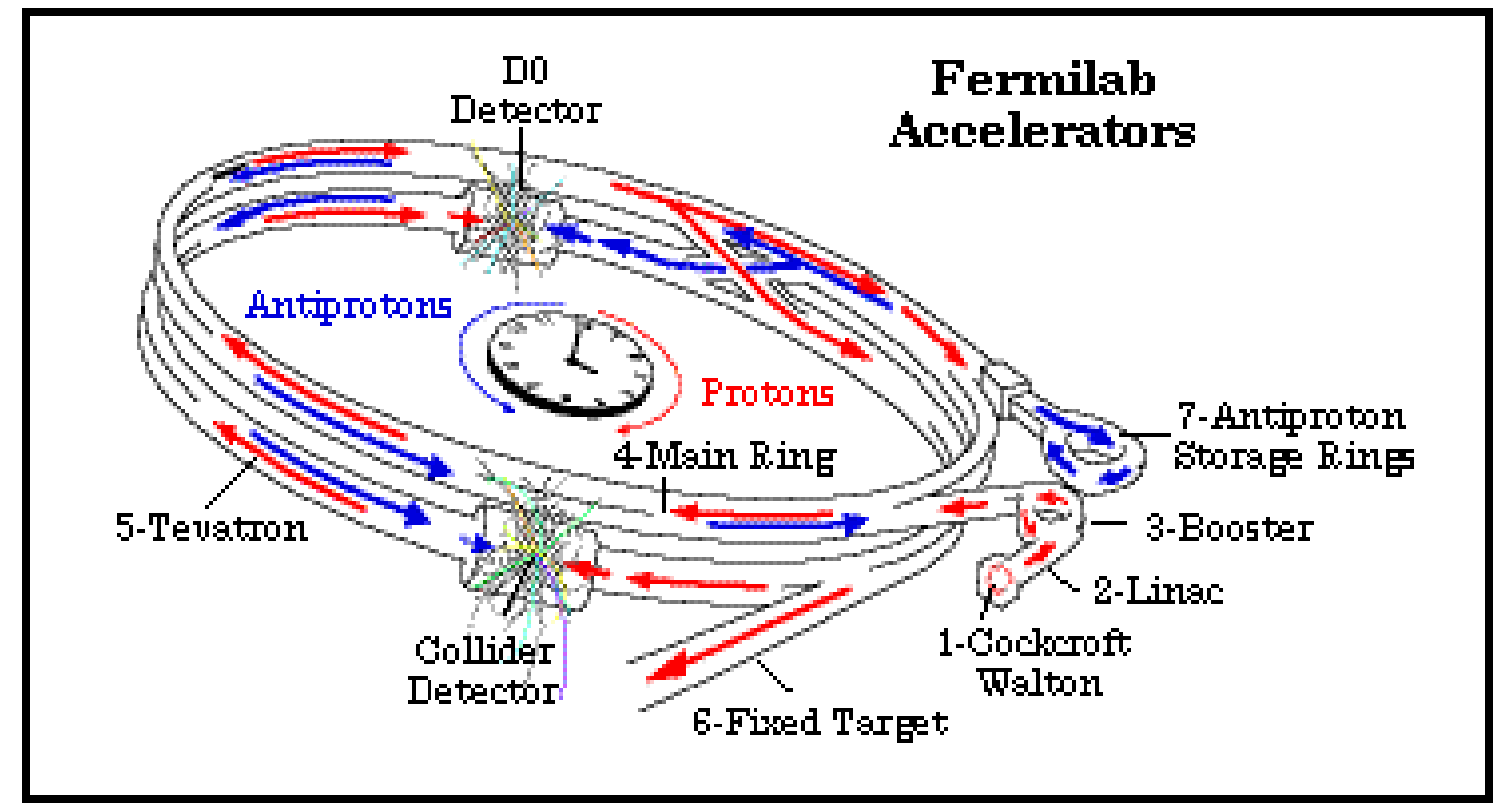

Figure 2.1: Schematic of the accelerators.

In Run 1, six bunches of protons and antiprotons were accelerated simultaneously in the Tevatron. Proton bunches consist of about $2 \times 10^{11}$ particles, while antiproton bunches contain about $6 \times 10^{10}$ particles; the temporal spacing between bunches is $3.5 \mu \mathrm{s}$. With these bunches, the Tevatron achieved instantaneous luminosities in excess of $2 \times 10^{31} \mathrm{~cm}^{-2} \mathrm{~s}^{-1}[35]$. 


\subsection{CDF, the Collider Detector at Fermilab}

The CDF detector is a multi-purpose detector for studying hadronic collisions at the Tevatron. A fraction of the $p \bar{p}$ collisions results in final state particles with significant momentum transverse to the beam, $\mathrm{p}_{\mathrm{T}}$. These are recorded in the detector and subsequently examined in data analyses.

The size and complexity of today's particle physics experiments, and the vast number of $p \bar{p}$ interactions occurring at the Tevatron, made it necessary to conduct the experiment in a large collaboration. The CDF collaboration includes more than 525 scientists from 52 institutions, universities, and laboratories in 11 countries. The collaboration is divided into smaller groups that work on individual tasks. During the design, development, and construction of the detector, some groups choose different components of the 5000 ton, $16 \mathrm{~m}$ high detector. Other groups focus on the development and maintenance of software necessary to control, collect, and classify the data during a run. Physics groups collaborate in the analysis of specific physics topics.

A complete description of the CDF detector is given in Reference [36]. The basic layout is discussed briefly here.

The 2000 t movable central detector includes tracking chambers, a $1.5 \mathrm{~T}$ solenoid magnet, a steel yoke, electromagnetic shower counters, hadron calorimeters, and muon chambers. The two identical plug and forward detectors consist of segmented time-of-flight counters, electromagnetic shower counters, hadron calorimeters, and muon toroidal spectrometers. The total length of the CDF detector is $26.2 \mathrm{~m}$. A photograph of the CDF detector is presented in Figure 2.2. Its layout is shown in Figure 2.3.

The steel yoke serves as support for the superconducting coil and the calorimeterized end plugs. It forms a box that is $9.4 \mathrm{~m}$ high, $7.6 \mathrm{~m}$ wide, and $7.3 \mathrm{~m}$ long. The superconducting coil has a diameter of $3 \mathrm{~m}$ and a length of $5 \mathrm{~m}$. The central calorimeter consists of 48 wedge-shaped modules that are assembled into four self-supporting 


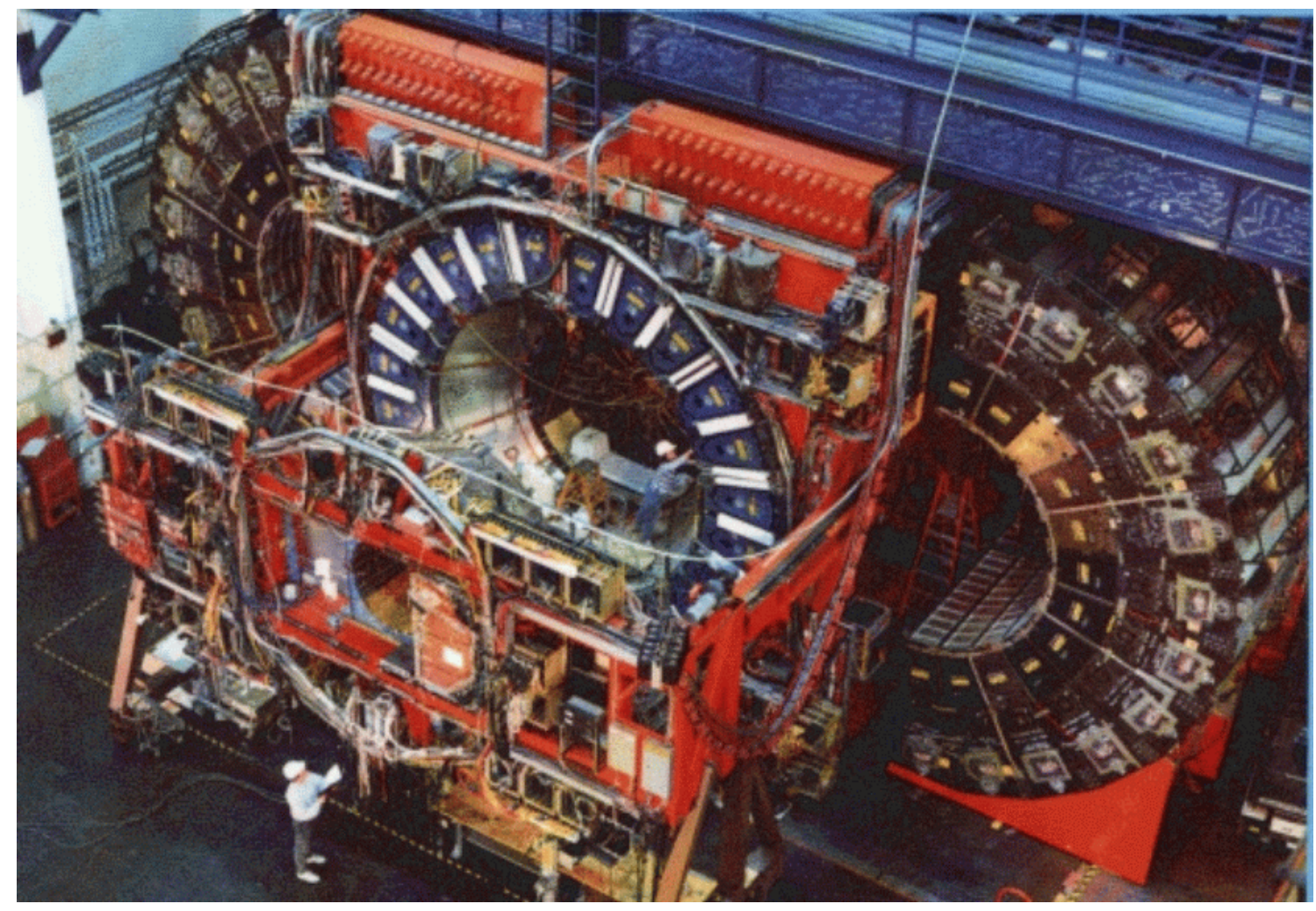

Figure 2.2: Photograph of the CDF detector at the Tevatron.

arches. These arches rest on the steel yoke and can be retracted for service of the modules.

The polar angle, $\theta$, is measured from the beamline. The right-handed coordinate system in the detector is chosen such that the $z$-axis is in the direction of the proton beam, the $x$-axis points radially outward in the Tevatron plane, and the $y$-axis points up. The central detector, which includes the endplugs, covers the range from $10^{\circ}$ to $170^{\circ}$ in $\theta$. The endplugs form the pole pieces for the solenoid and cover the angles $10^{\circ}<\theta<30^{\circ}$ and $150^{\circ}<\theta<170^{\circ}$. Particles that leave the interaction point at angles $<10^{\circ}$ or $>170^{\circ}$ exit a conical hole in the endplug and are detected in the forward calorimeters. These calorimeters are followed by two toroidal magnets of diameter $7.6 \mathrm{~m}$ which are used as muon spectrometers. Scintillation counters at the faces of the forward electromagnetic shower counters serve as luminosity monitors and 


\section{CDF Detector}

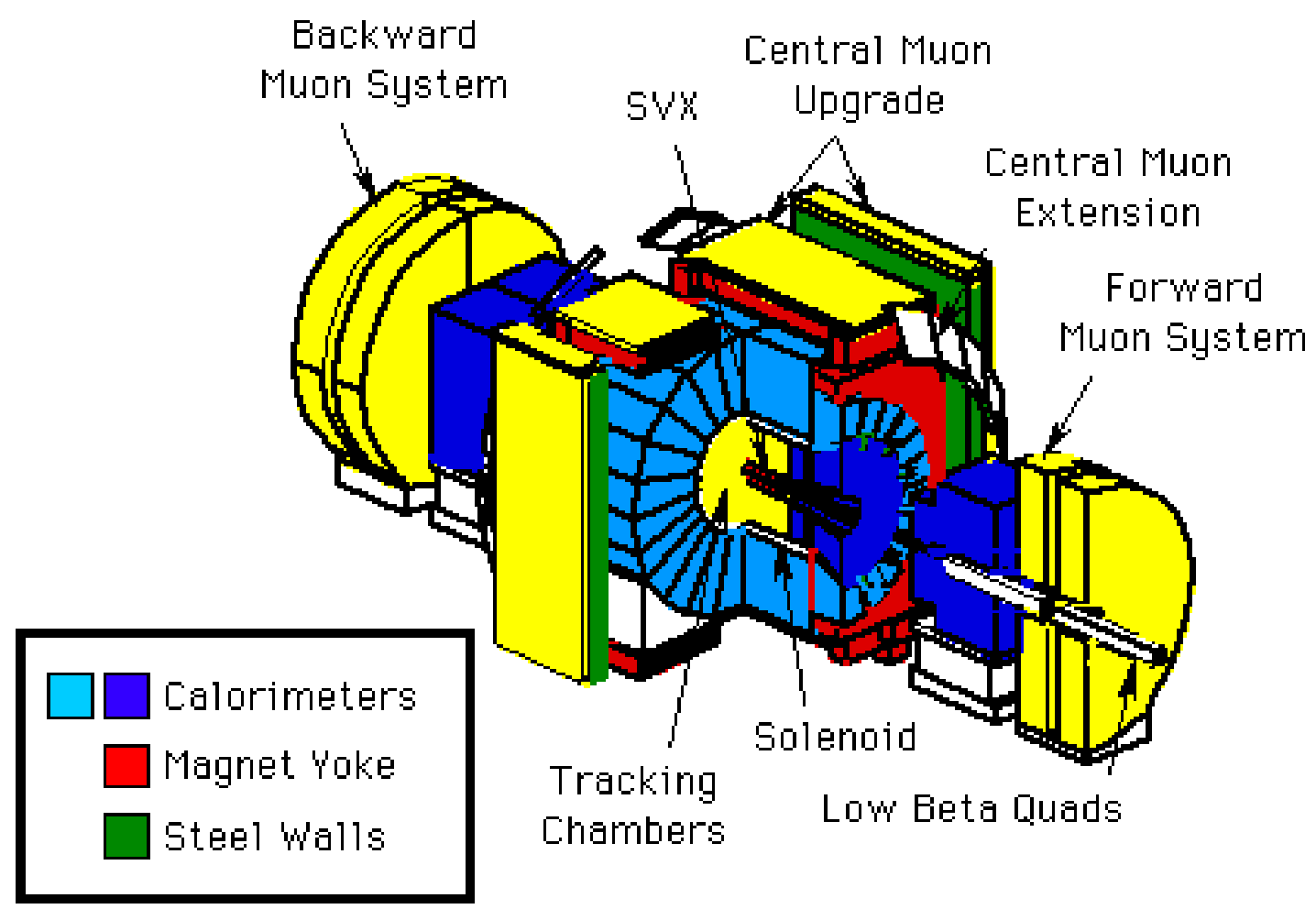

Figure 2.3: Schematic view of the CDF detector, showing the detector components.

are used as minimum bias triggers. When a scintillation counter registers particles off the beamline, an interaction has occurred and data are recorded.

\subsection{Detector Components}

The main purpose of the CDF detector is to measure energy and momentum of particles produced in the Tevatron collider. Where possible, particle identification is done also. To achieve that goal, the CDF detector is built in layers of different detector components surrounding the interaction region and is designed to cover as large a fraction of the solid angle as possible. Closest to the interaction point is the 
tracking system, followed by the solenoid, the calorimeters, and the muon detectors. A schematic side view of one quadrant of the CDF detector is shown in Figure 2.4.

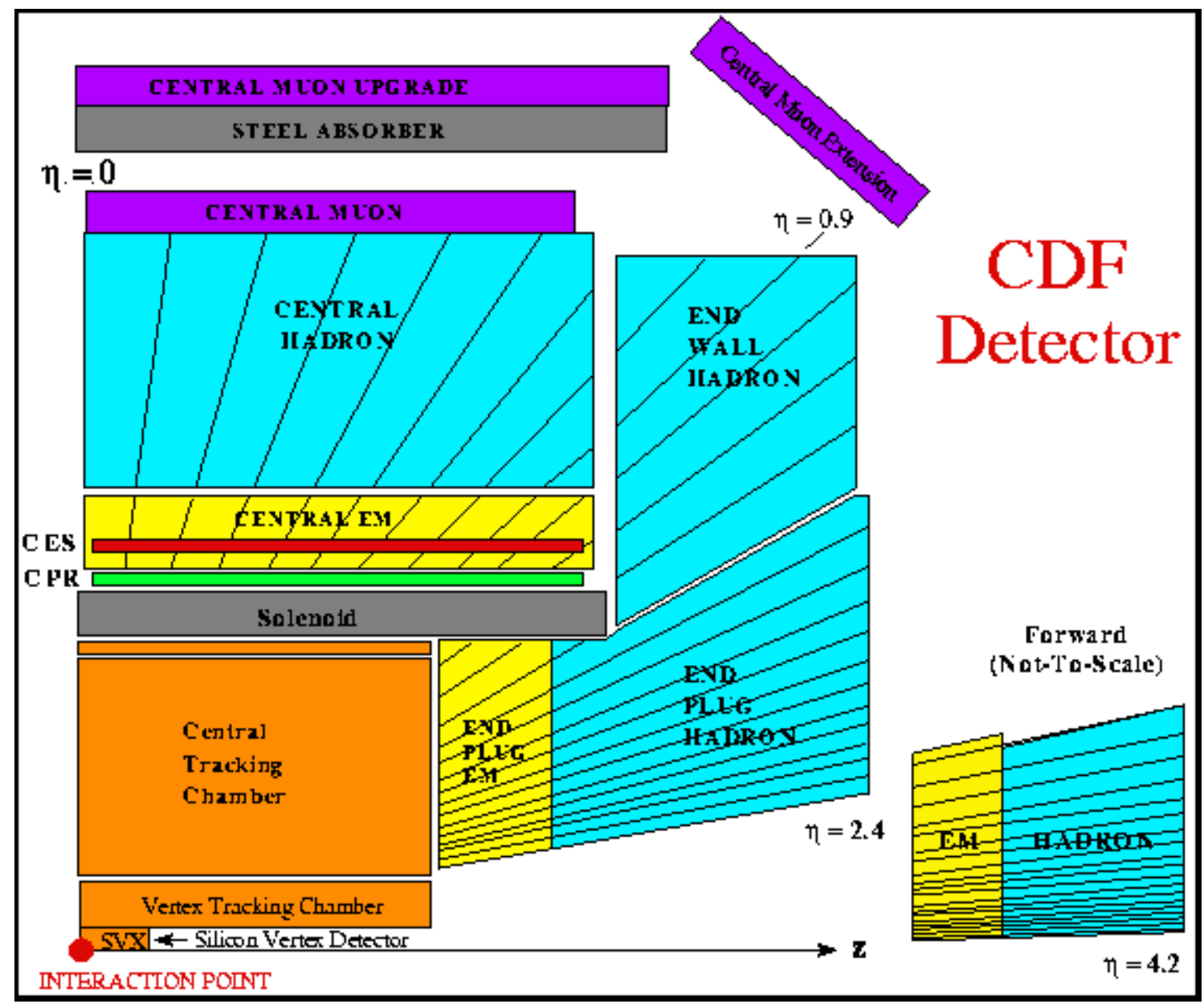

Figure 2.4: A schematic side view of a quadrant of the CDF detector.

High energy hadron collisions are expected to result in a final state particle distribution that is roughly uniform in pseudo-rapidity, $\eta$, and azimuth, $\phi$, where $\eta$ is defined as

$$
\eta \equiv-\ln \left(\tan \frac{\theta}{2}\right) .
$$

For that reason, the CDF detector was designed with approximate cylindrical symmetry, and the detector components are segmented uniformly in $\eta$. In the discussion 
of the data analysis in later chapters, the event pseudo-rapidity and the detector pseudo-rapidity will have to be distinguished. The $\eta$ associated with a particle or jet is the event pseudo-rapidity and takes as its origin the interaction vertex, while the detector pseudo-rapidity, denoted as $\eta_{d}$, assumes a vertex of $z=0$. If an interaction occurs at $z=0$, the two quantities are equal, but they differ by about \pm 0.2 for a vertex at $z= \pm 60 \mathrm{~cm}$.

Brief descriptions of the detector components, starting closest to the interaction point, follow. Emphasis is placed on the description of the central detector, including the central, the end wall, and the endplug calorimeters, as these are most relevant to the data analysis in this thesis.

\subsubsection{The Tracking System}

Closest to the interaction point is the tracking system, which consists of three components, the silicon vertex detector, the vertex tracking chamber, and the central tracking chamber. They are located within the magnetic field of the solenoid to permit determination of momentum of charged particles from radius of curvature. Before the start of Run 1b, the silicon vertex detector was replaced by the silicon microstrip vertex detector, and the vertex tracking chamber was removed.

The beam pipe is surrounded by four layers of semiconductors, the silicon microstrip vertex detector $\left(\mathrm{SVX}^{\prime}\right)$ [37]. The inner $\mathrm{SVX}^{\prime}$ layer is mounted $2.9 \mathrm{~cm}$ from the beamline and is followed by layers at distances of $4.2,5.7$, and $7.9 \mathrm{~cm}$. The layers are arranged as two cylindrical barrels that meet at $z=0$; a barrel is $51 \mathrm{~cm}$ long. Tracks are reconstructed from information recorded with axial and small-angle stereo microstrips in the silicon. The single-hit reconstruction precision is $13 \mu \mathrm{m}$, and the impact parameter resolution is $17 \mu \mathrm{m}$ [35].

The vertex drift chambers (VTX) provide the next stage of particle tracking.

The cylindrical VTX extends from just outside the last SVX' layer to a distance of $22 \mathrm{~cm}$ from the beam line. Track measurements by the VTX are used to reconstruct 
the $p \bar{p}$ interaction vertex with a precision of $1 \mathrm{~mm}$ in the $z$ direction.

The SVX' and the VTX are surrounded by the central tracking chamber (CTC). The $3.2 \mathrm{~m}$ long drift chamber covers angles $40^{\circ}<\theta<140^{\circ}$, or correspondingly $-1<\eta_{d}<1$. The outer radius of the $\mathrm{CTC}$ is $1.3 \mathrm{~m}$. The chamber contains 84 layers of sense wires which are grouped into nine "superlayers." The sense wires are arranged parallel (axial) to the beamline in five of these superlayers; for the other four superlayers, the sense wires are tilted by $\pm 3^{\circ}$ relative to the beam direction. The superlayers with tilted sense wires are called stereo layers. The axial layers provide accurate tracking information in the $r-\phi$ plane. Together, the axial and the stereo layers are used for tracking in the $r-z$ plane. The spatial resolution of the CTC is better than $200 \mu \mathrm{m}$ in the $r-\phi$ plane and better than $6 \mathrm{~mm}$ in $z$. The two-track resolution is $3.5 \mathrm{~mm}$.

\subsubsection{The Solenoid}

The solenoid provides a uniform 1.5 T magnetic field for precision momentum measurements for charged particles in the CTC. The magnetic field is oriented along the beam line. It is produced by a superconducting solenoidal coil that is $5 \mathrm{~m}$ long and has a diameter of $3 \mathrm{~m}$. The coil is made of 1164 turns of a superconductor and cooled by liquid helium.

\subsubsection{The Calorimeters}

Precision energy measurements are obtained with the calorimeters. The calorimeters are divided into the central detector and the forward detectors. The central detector, in turn, has three parts: the central, the endwall, and the endplug regions. The calorimeters have two components: the electromagnetic (EM) calorimeters and the hadronic calorimeters. While the EM calorimeters are used primarily to measure leptonic energies, the hadronic calorimeters provide energy measurements for hadrons. The $\eta_{d}$ coverage of the different parts of the central detector is shown in Table 2.1; 
the forward detectors cover $2.2<\left|\eta_{d}\right|<4.2$ in the case of the EM calorimeters, and the hadronic calorimeters cover $2.3<\left|\eta_{d}\right|<4$.2. Full azimuthal coverage is provided by all calorimeter components.

In $p \bar{p}$ collisions, final state hadronic jets are important for many physics analyses. For that reason, a "tower" geometry was chosen for the calorimeters. A tower is a stack of calorimeter modules oriented at constant $\eta_{d}$ along a radius of the detector from the interaction point. The towers are projective: they point at the interaction region. Each tower covers 0.1 unit in $\eta_{d}$. Segmentation in $\phi$ is $15^{\circ}$ in the central region and $5^{\circ}$ in the plug and forward regions. Each tower consists of an electromagnetic shower counter followed by the hadronic calorimeter. This allows for a detailed comparison between electromagnetic and hadronic energy on a tower-by-tower basis. The physical tower sizes vary according to their position; they range from $24.1 \mathrm{~cm} \times 46.2 \mathrm{~cm}$ in $\eta_{d}$ and $\phi$, respectively, in the central region to $1.8 \mathrm{~cm} \times 1.8 \mathrm{~cm}$ in the forward region. Table 2.1 summarizes the calorimeter properties for the central, the endwall, and the endplug regions. The forward detectors are not included in detail, as the data analysis presented in this thesis is restricted to energy information provided by the central detector.

The hadronic section of the central calorimeter is directly outside the electromagnetic section. Figure 2.5 shows a schematic view of one module of the central electromagnetic calorimeter and the space provided for the central hadronic section.

Lead sheets are interleaved with scintillators as the active detector medium in the electromagnetic shower counters in the central region. In the endplug, and in the forward detectors, the active media are proportional tube chambers with cathode pad readout. The electromagnetic shower counters provide a spatial resolution of $\approx 2 \mathrm{~mm}$ over the complete solid angle. The central shower counter consists of a single sample in depth; an additional measurement with high spatial resolution transverse to the shower is provided by a proportional chamber. In the endplug shower counters, there are three samples in depth, and the forward shower counters provide two samples in 


\begin{tabular}{|c|c|c|c|c|c|}
\hline & \multicolumn{2}{|c|}{ Central } & Endwall & \multicolumn{2}{c|}{ Endplug } \\
\cline { 2 - 6 } & EM & Hadron & Hadron & EM & Hadron \\
\hline \hline$\eta_{d} \mid$ coverage & $0.0-1.1$ & $0.0-0.9$ & $0.7-1.3$ & $1.1-2.4$ & $1.3-2.4$ \\
\hline $\begin{array}{c}\text { Tower Size } \\
\left(\Delta \eta_{d} \times \Delta \phi\right)\end{array}$ & $\approx 0.1 \times 15^{\circ}$ & $\approx 0.1 \times 15^{\circ}$ & $\approx 0.1 \times 15^{\circ}$ & $\approx 0.09 \times 5^{\circ}$ & $\approx 0.09 \times 5^{\circ}$ \\
\hline $\begin{array}{c}\text { Active } \\
\text { Medium }\end{array}$ & $\begin{array}{c}\text { polysterene } \\
\text { scintillator }\end{array}$ & $\begin{array}{c}\text { acrylic } \\
\text { scintillator }\end{array}$ & $\begin{array}{c}\text { acrylic } \\
\text { scintillator }\end{array}$ & $\begin{array}{c}\text { proportional tube chambers } \\
\text { with cathode pad readout }\end{array}$ \\
\hline $\begin{array}{c}\text { Scintillator Thickness, } \\
\text { or Proportional } \\
\text { Tube Size (cm) }\end{array}$ & 0.5 & 1.0 & 1.0 & $0.7 \times 0.7$ & $1.4 \times 0.8$ \\
\hline Number of Layers & 31 & 32 & 15 & 34 & 20 \\
\hline Absorber & Pb & Fe & Fe & Pb & Fe \\
\hline Absorber Thickness $(\mathrm{cm})$ & 0.32 & 2.5 & 5.1 & 0.27 & 5.1 \\
\hline $\begin{array}{c}\text { Energy Resolution } \\
(\sigma / \text { E) at } 50 \text { GeV }(\%)\end{array}$ & 2 & 11 & 14 & 4 & 20 \\
\hline $\begin{array}{c}\text { Position Resolution } \\
\text { at } 50 \text { GeV }\left(\mathrm{cm}^{2}\right)\end{array}$ & $0.2 \times 0.2$ & $10 \times 5$ & $10 \times 5$ & $0.2 \times 0.2$ & $2 \times 2$ \\
\hline
\end{tabular}

Table 2.1: Summary of the CDF calorimeter properties.

depth. The wire planes in the endplug and the forward detectors are digitized by quadrant, giving additional detailed information about the shower profile.

Instead of lead sheets, steel plates are alternated with the active detector materials in the hadronic calorimeter components. The active detectors in the central and endwall regions are plastic scintillators, while gas proportional chambers are used in the endplug and the forward detectors. Due to the geometry of the solenoid, the hadronic calorimeters have slightly different sharing of the coverage in $\eta_{d}$ (see Table 2.1). The endwall part of the central detector is attached to the yoke. All hadron calorimeters have only one depth sample, but the endplug and the forward calorimeters digitize each wire plane in a quadrant for shower profile information.

The scintillator-based central calorimeter components are calibrated by $50 \mathrm{GeV}$ electrons and charged pions from a test beam and with cosmic ray muons. Long term gain variations are monitored by ${ }^{137} \mathrm{Cs}$ radioactive sources which can be moved through the module by remote control. Short term gain changes are monitored by a Xe lamp system in the EM calorimeters and with a nitrogen laser in the hadron calorimeters. With this system, gain changes can be tracked to an accuracy of better 


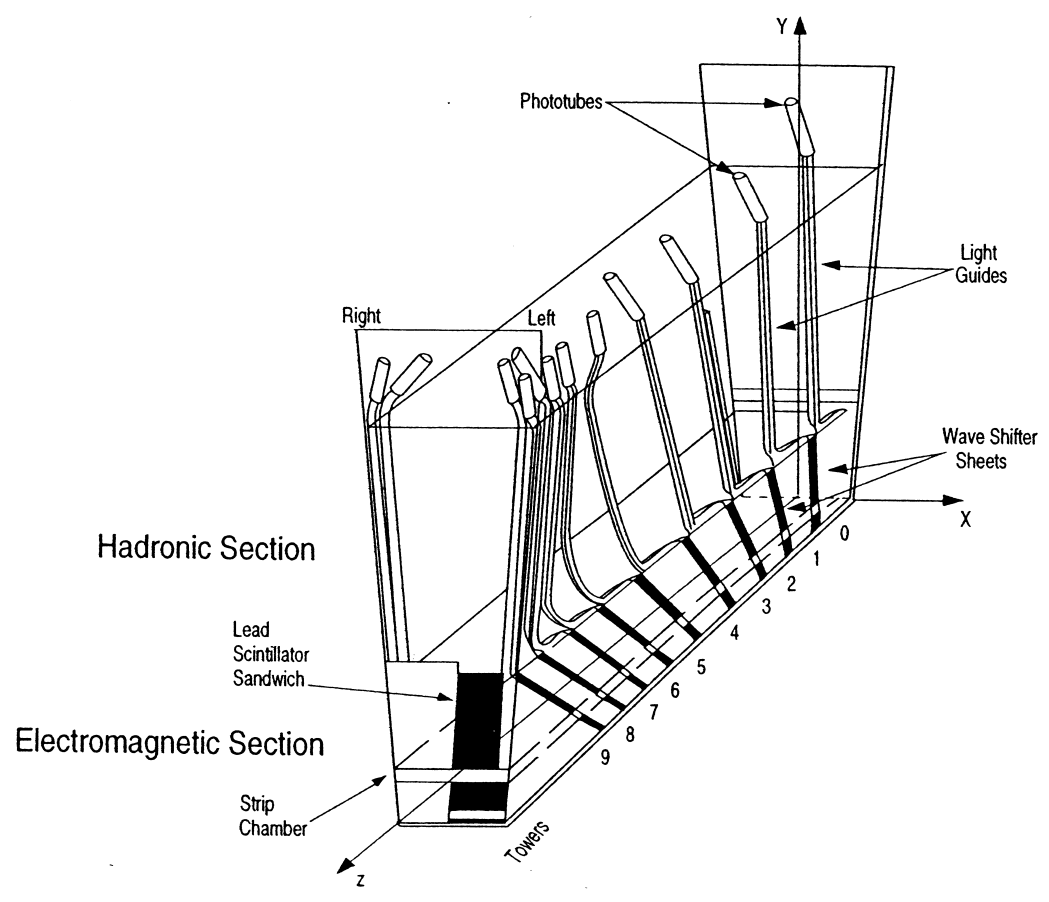

Figure 2.5: Schematic view of one module of the central electromagnetic calorimeter. (Figure taken from Reference [35].)

than $2 \%$.

The gain in the gas-based calorimeters changes with pressure, temperature, and gas composition. Calibrated monitor tubes provide continuous information about changes in the gain. Weather-induced gain changes of up to $25 \%$ have been measured, but the tracking accuracy of the monitor tubes is about $2 \%$.

\section{The Central Electromagnetic Calorimeter}

A detailed description of the central electromagnetic calorimeter is given in Reference [38]. The design and main features are briefly explained here.

The central electromagnetic calorimeter (CEM) covers a range of $0.0<\left|\eta_{d}\right|<$ 
1.1. It consists of 48 wedge-shaped modules; each module holds ten towers. The CEM employs a hybrid design with scintillators alternating with absorbers for energy measurements and an embedded strip chamber for position and longitudinal shower development information. This hybrid design combines the energy resolution of the scintillators with the spatial resolution capabilities of the gas strip chambers.

A CEM module has a $1.4 \mathrm{~mm}$ thick aluminum base plate as its innermost part. This base plate is located $173 \mathrm{~cm}$ from the beamline. The 31 layers of $5 \mathrm{~mm}$ thick SCSN-38 polystyrene scintillators are interleaved with 30 layers of $0.3 \mathrm{~mm}$ thick lead. The individual scintillator pieces are wrapped in two layers of vellum drawing paper, and the lead is clad on both sides with $0.4 \mathrm{~mm}$ thick aluminum. The pieces are assembled into ten projective towers, each subtending a region of $0.1 \times 15^{\circ}$ in $\eta_{d^{-}} \phi$ space. The two sides of each tower are covered by $1.9 \mathrm{~mm}$ thick steel plates. The gap between the scintillator/absorber sandwiches and the steel cover plates is filled with wavelength shifters that collect the light emitted by the scintillators. The calorimeter signals are read out by two photomultiplier tubes per tower, one on each side.

The proportional strip chambers are inserted near the shower maximum, between the eighth lead layer and the ninth scintillator layer. The strips are on copperbacked $1.6 \mathrm{~mm}$ PC boards; high voltage wires are strung orthogonally to the strips. Charge deposition on the wires and strips gives shower position and transverse development information.

The energy resolution achieved by the CEM was measured to be $\sigma / \mathrm{E}_{\mathrm{T}}=$

$13.5 \% / \sqrt{\mathrm{E}_{\mathrm{T}}} \oplus 2 \%$, where $\mathrm{E}_{\mathrm{T}}$ is transverse energy, $\mathrm{E}_{\mathrm{T}} \equiv \mathrm{E} \sin \theta$, and $\theta$ is the angle between the beam direction and the jet axis or outgoing particle direction. The spatial resolution is $2 \mathrm{~mm}$ at $50 \mathrm{GeV}$.

\section{The Central Hadronic Calorimeter}

The central hadronic calorimeter (CHA) [39] covers $0.0<\left|\eta_{d}\right|<0.9$ and $2 \pi$ in $\phi$. It is a pure sampling calorimeter without strip chambers for shower development 
determination. Each CHA tower matches the corresponding central electromagnetic calorimeter tower, also covering 0.1 unit in $\eta_{d}$ and $15^{\circ}$ in $\phi$. The towers consist of 32 layers of $2.5 \mathrm{~cm}$ thick steel absorbers and $1 \mathrm{~cm}$ thick PMMA-based scintillator material. Analogously to the CEM, the scintillator signals are collected by wavelength shifters and read out by photomultiplier tubes.

The initial calibration of the CHA was done with a $50 \mathrm{GeV}$ pion test beam. For pions that were minimum ionizing in the CEM, the CHA response was linear in the energy range between 10 and $150 \mathrm{GeV}$. The energy resolution for the CHA was measured to be $\sigma / \mathrm{E}_{\mathrm{T}}=75 \% / \sqrt{\mathrm{E}_{\mathrm{T}}} \oplus 3 \%$.

\section{The Endwall Hadronic Calorimeter}

The endwall hadronic calorimeter (WHA) [39] bridges the gap between the central and the plug calorimeters in the region $0.7<\left|\eta_{d}\right|<1.3$. There is no electromagnetic section; only the hadronic calorimeter exists, as the central and the endwall parts form a single hadronic calorimeter. The WHA consists of two pieces, one in either $z$ direction from the interaction point. They plug into cavities in the magnetic yoke, serving as part of the magnetic flux return path. Of the 24 modules in each $15^{\circ}$ slice in the central detector, 12 are totally in the central hadron calorimeter, 6 are totally in the endwall hadron calorimeter, and 6 are shared between the two. As in the CHA, WHA towers are projective and cover a region of $0.1 \times 15^{\circ}$ in $\eta_{d^{-}} \phi$ space.

The WHA towers consist of PMMA-based scintillators interleaved with steel absorbers. The 15 layers have scintillator thicknesses of $1.0 \mathrm{~cm}$ and steel thicknesses of $5 \mathrm{~cm}$. Signal collection is similar to that in the CHA.

The CHA and the WHA modules are calibrated together. The energy res-

olution, larger by about a factor of $\sqrt{2}$, is $\sigma / \mathrm{E}_{\mathrm{T}}=105 \% / \sqrt{\mathrm{E}_{\mathrm{T}}} \oplus 5 \%$. This loss in energy resolution is expected because of the lower sampling fraction due to the thicker absorber plates. 


\section{The Endplug Electromagnetic Calorimeter}

Both ends of the $3 \mathrm{~m}$ diameter and $5 \mathrm{~m}$ long solenoid are covered by the endplug electromagnetic calorimeter (PEM) [40]. The PEM is hermetic with the exception of a concentric conical hole with an opening angle of $10^{\circ}$ to the beam axis in either $z$ direction. Angular coverage ranges over $1.1<\left|\eta_{d}\right|<2.4$. Both PEM pieces occupy a cylindrical volume with an outer diameter of $280 \mathrm{~cm}$ and are coaxial with the beam line. Their longitudinal coverage is $173 \mathrm{~cm}<|z|<226 \mathrm{~cm}$.

Due to the large magnetic field in the endplug region, and due to mechanical constraints that make it difficult to machine scintillators for projective geometry towers, the active elements in the PEM are proportional gas chambers. These are read out by cathode pads. The 16 projective towers cover $5^{\circ}$ in $\phi$, and their segmentation in $\eta_{d}$ varies [35]. At $\eta_{d}=2.4$, the first tower covers 0.09 units in $\eta_{d}$. Each of the next four towers spans only half that range; the remaining eleven towers have a size of 0.09 units in $\eta_{d}$ again. Usually the four smaller towers are combined offline to form two standard sets of towers.

Each of the two PEM modules consists of four quadrants, each spanning $90^{\circ}$ in $\phi$. In one quadrant, 34 layers of proportional tube arrays are interleaved with $2.7 \mathrm{~mm}$ thick lead absorber plates. The innermost layer of the PEM is a $1.3 \mathrm{~cm}$ thick steel plate, the first electromagnetic absorber plate; the back is covered by a $4.5 \mathrm{~cm}$ thick steel plate which serves as the first absorber for the endplug hadron calorimeter. The proportional tubes consist of gold plated tungsten wires centered in resistive plastic tubes of $0.7 \times 0.7 \mathrm{~cm}^{2}$ cross section. The planes of tubes are assembled into fan-shaped quadrants in azimuth, with all tubes in a plane perpendicular to the beam axis. The tube planes are sandwiched between two $1.6 \mathrm{~mm}$ thick copper clad G-10 panels. Schematic views of the proportional tubes and the fan-shaped quadrants are provided in Figure 2.6. The tungsten wires in the center of the tubes serve as anodes, while the copper clad panels are the cathodes. For the read-out, the panels are subdivided into pads on one side, with readout traces to the edge of the quadrant on 
the opposite side. The tower signal is the sum of all longitudinal signal contributions.

a)

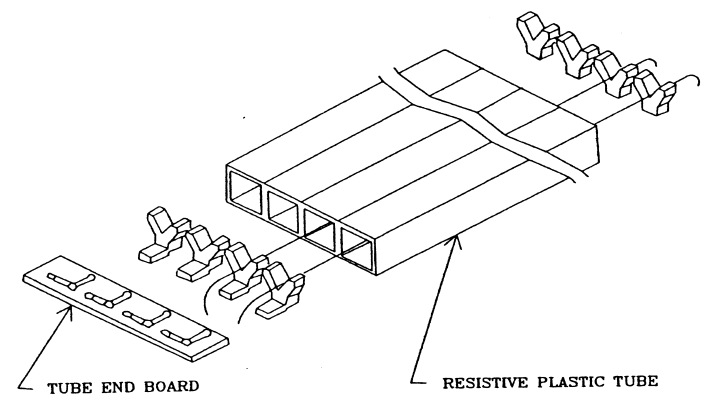

b)

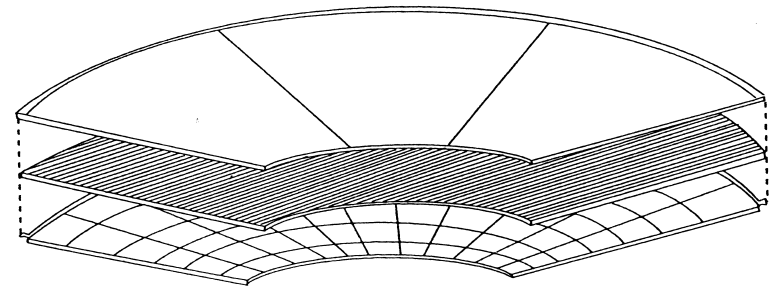

Figure 2.6: Schematic view of a) the PEM proportional tubes, plastic anodes, and wire supports; b) one proportional layer which is sandwiched between copper clad G-10 panels. (Figure taken from Reference [35].)

The gas used in the proportional tubes is a $50 \%-50 \%$ argon-ethane mixture. A small amount of isopropyl alcohol has been added to quench discharge when a large dose of radiation illuminates one spot.

Calibration of the PEM was achieved with a $100 \mathrm{GeV}$ electron beam. The PEM response to electrons was found to be linear within $3 \%$ over the range 20 to $200 \mathrm{GeV}$. The energy resolution was measured to be $\sigma / \mathrm{E}_{\mathrm{T}}=28 \% / \sqrt{\mathrm{E}_{\mathrm{T}}} \oplus 2 \%$. 


\section{The Endplug Hadronic Calorimeter}

The endplug hadronic calorimeter (PHA) [35] covers the regions with $1.3<\left|\eta_{d}\right|<2.4$. Twelve $30^{\circ}$ sectors provide full azimuthal coverage. Individual towers span $0.09 \times 5^{\circ}$ in $\eta_{d^{-}} \phi$ space.

The 20 layers of proportional chambers are sandwiched between $215.1 \mathrm{~cm}$ thick steel plates, except for the steel plate after the fourth proportional layer which is $6.4 \mathrm{~cm}$ thick. The proportional tubes use gold plated tungsten wires centered in resistive plastic tubes and have dimensions of $0.8 \times 1.4 \mathrm{~cm}^{2}$. The tungsten wires act as anodes, and the copper clad G-10 plates that hold the tubes serve as cathodes. The inner sides of the G-10 plates hold 72 copper pads that are electrically connected to the outer side. From there, copper traces provide signal transmission to amplifiers outside the detector.

Calibration of the PHA by $200 \mathrm{GeV}$ pions resulted in an energy resolution measurement of $\sigma / \mathrm{E}_{\mathrm{T}}=90 \% / \sqrt{\mathrm{E}_{\mathrm{T}}} \oplus 4 \%$. In the range from 20 to $200 \mathrm{GeV}$, the response to pions is linear.

\section{The Forward Calorimeters}

Information from the forward calorimeters is not used in the analysis presented in this thesis. For completeness, a brief description is provided. More detailed discussion of the forward calorimeters can be found in References [41] and [42].

The small angle regions $2.2<\left|\eta_{d}\right|<4.2$ of the detector are covered by the forward calorimeters. The forward electromagnetic calorimeters, FEM, are box shaped and located $6.5 \mathrm{~m}$ from the interaction point [41] in either $z$ direction.

The forward electromagnetic calorimeters consist of 30 layers of proportional tube chambers interleaved with lead absorber sheets. The FEM are divided into four quadrants which can be removed individually for repairs. Each quadrant covers an azimuth of $90^{\circ}$. Each projective tower in the calorimeters covers 0.1 unit in $\eta_{d}$ and $5^{\circ}$ in $\phi$. Signals are read out longitudinally. 
Consistent with the FEM, the forward (FHA) hadronic calorimeters cover a region of $2.2<\left|\eta_{d}\right|<4.2$ [42]. Steel absorber plates and proportional chambers are alternated in 27 layers. They are arranged into four quadrants, each with $90^{\circ}$ azimuthal coverage. The 20 projective towers each cover 0.1 unit in $\eta_{d}$ and $5^{\circ}$ in $\phi$.

\section{Uninstrumented Regions in the Calorimeter}

Mechanical constraints on the design and construction of the calorimeter result in small uninstrumented regions, gaps. Particle or jet measurements have to be corrected for energy deposited in these regions.

At $\theta=90^{\circ}$ for $z=0$, two CEM modules, each bounded by $2.5 \mathrm{~cm}$ thick steel plates, connect with an air gap of about $0.5 \mathrm{~cm}$. In addition, a $1.6 \mathrm{~cm}$ gap which contains the support lines separates the scintillator/absorber sandwiches from the steel endplate. The uninstrumented region due to these gaps is called the $90^{\circ}$ crack. The $5.1 \mathrm{~cm}$ gap that houses light guides, and that separates the $5.1 \mathrm{~cm}$ steel end plate from the CEM wedges at $\theta=38^{\circ}$, is referred to as the $30^{\circ}$ crack. The corrections associated with these gaps are discussed in later sections.

\subsubsection{The Muon Detectors}

In the interaction with matter, relativistic electrons lose most of their energy by bremsstrahlung. Muons have a much larger mass than electrons, which results in their significantly smaller cross section for radiation. Muons, therefore, penetrate matter to a higher degree than do electrons. For that reason, the muon detection system in the CDF detector is located in the outer region, allowing the components closer to the interaction point (the tracking system, the solenoid, and the calorimeters) to filter out the majority of electrons and hadrons. CDF has two separate muon detection systems.

The central muon detector (CMU) [43] consists of four layers of rectangular drift cells on the outside of each central hadronic calorimeter wedge. The CMU has 
an inner radius of $3.47 \mathrm{~m}$ from the beamline and covers the region $\left|\eta_{d}\right|<0.65$. A central muon upgrade detector (CMP) [44] was installed. It surrounds the central region behind an additional shield of $630 \mathrm{t}$ of steel which reduces the false muon background associated with hadrons punching through the central calorimeter. The CMP's active elements are four planes of single-wire drift tubes. Additional coverage for muon detection is provided by the central muon extension (CMX), which covers the region $0.65<\left|\eta_{d}\right|<1.0$. The CMX consists of 1536 proportional drift cells.

The forward muon detectors (FMU) [45] consist of muon spectrometers, large magnetized steel toroids with drift chamber planes and scintillation counters. The toroids have a diameter of $7.82 \mathrm{~m}$ and are $1 \mathrm{~m}$ thick; the inner diameter is $0.914 \mathrm{~m}$. The magnetic field decreases from $2.0 \mathrm{~T}$ at the inner radius to $1.6 \mathrm{~T}$ at the outer radius and is created by four coils. Muon trajectories are measured by three layers of drift chambers, and trigger information is provided by two layers of scintillation counters. The FMU cover the regions $1.96<\left|\eta_{d}\right|<3.64$.

\subsection{The Data Acquisition System}

There are approximately 100,000 electronics channels in the CDF detector, providing signals from silicon strip detectors, photomultiplier tubes, wire, strip, and pad chambers, and drift chambers. Most of these channels, about 60,000 of them, are used by the calorimetry. A special crate-based analog front-end system, RABBIT, was developed to manage the calorimeter information. The main challenge for the RABBIT system comes from the large dynamic signal range required by the calorimeters, which provide information from a few tens of $\mathrm{MeV}$ to several hundreds of $\mathrm{GeV}$. To avoid pileup and common mode noise, RABBIT measures two voltage levels, one before the expected interaction time, one immediately after. This is possible because the beam bunches at the Tevatron cross at a regular time interval of $3.5 \mu \mathrm{s}$. The first measurement provides a reference voltage; the difference between the two voltage lev- 
els is proportional to the signal charge. This procedure is referred to as before-after sampling. The RABBIT signals are digitized in the crates, read out by scanners, and subsequently interfaced to a Fastbus data acquisition system.

The remaining electronics channels are mostly used by the drift chambers. Their signals are shaped at the detector and transferred from the collision hall to the counting room by commercial Fastbus TDC modules.

The Fastbus network comprises the data acquisition (DAQ) system. The nominal event size is 100 kBytes. Events are passed from the initial hardware triggers to higher levels of event selection and read out by the DAQ.

\subsection{Trigger and Data Analysis Systems}

Proton-antiproton bunch crossing during Run I at the Tevatron occurred every $3.5 \mu \mathrm{s}$, i.e., with a frequency of $286 \mathrm{kHz}$. Data at CDF can be written to tape at a maximum rate of about $10 \mathrm{~Hz}$. A trigger system that is capable of handling the full $p \bar{p}$ interaction rate, and that selects interesting physics events with a reduction ratio of almost 30,000:1, was developed. The CDF trigger system uses three consecutive triggers. Each trigger level reduces the amount of data transferred to the next higher level, allowing additional time for more sophisticated event selection at that level.

The trigger system is designed to exploit the projective tower geometry of the CDF detector. Trigger towers of size $0.2 \times 15^{\circ}$ in $\eta_{d^{-}} \phi$ space are created by summing hadronic and electromagnetic calorimeter tower energies. The signals are weighted by $\sin \theta$ (where $\theta$ is measured relative to the beam line) to provide the transverse energy, $\mathrm{E}_{\mathrm{T}}$, deposited in the towers. Dedicated trigger cables transfer the trigger signals to the trigger electronics. The information is stored until a Level 1 decision is reached. If the event is rejected at Level 1, a reset is sent in time for the next beam crossing, so no deadtime is introduced at this level.

At Level 1, an individual trigger tower is required to have an energy of $8 \mathrm{GeV}$ in 
the central electromagnetic calorimeter, $11 \mathrm{GeV}$ in the central hadronic calorimeter, $11 \mathrm{GeV}$ in the plug electromagnetic calorimeter, or $51 \mathrm{GeV}$ in either the plug hadronic calorimeter or in one of the forward calorimeters. The Level 1 acceptance rate for an instantaneous luminosity of $\mathcal{L}=5 \times 10^{30} \mathrm{~cm}^{-2} \mathrm{~s}^{-1}$ is approximately $1 \mathrm{kHz}$ [46].

If an event is accepted by Level 1, the Level 2 trigger starts. While the Level 1 trigger requires less than $3.5 \mu \mathrm{s}$, the time between beam crossings, the Level 2 trigger requires about $15 \mu \mathrm{s}$. The increased processing time allows simple calculations to be performed, using the Level 1 information. A hardware cluster finder assigns trigger towers to transverse energy clusters. A trigger tower whose transverse energy in any single electromagnetic or hadronic calorimeter tower exceeds $3 \mathrm{GeV}$ is identified as a seed. The neighboring towers are then examined for transverse energy $>1 \mathrm{GeV}$, the shoulder threshold. All contiguous towers above the shoulder threshold are included in the cluster. The sum of the energies of all towers in a cluster gives the cluster energy. A cluster list with information about electromagnetic and hadronic cluster energies is kept. The clusters are matched to tracks in the CTC, along with muons whose momenta are also included in the cluster list. The Level 2 trigger allows event selection on muons, electrons, photons, jets, and missing $\mathrm{E}_{\mathrm{T}}$, different combinations of which can be programmed in parallel. Prescale factors for certain combinations can also be included. The Level 2 acceptance rate for an instantaneous luminosity of $\mathcal{L}=5 \times 10^{30} \mathrm{~cm}^{-2} \mathrm{~s}^{-1}$ is approximately $12 \mathrm{~Hz}$ [46]. All CDF channels are digitized and read out by the DAQ if an event is accepted at Level 2. This information is passed on to the Level 3 processor farm.

The Level 3 trigger is software-based, executing FORTRAN-77 filter algorithms. It consists of a "computer farm" of 64 commercially available Silicon Graphics processors capable of parallel processing of the events. At Level 3, the event rate is reduced by a factor of two or three, depending on the instantaneous luminosity.

The "consumer server," a process running on a dedicated Silicon Graphics Challenge L machine, receives the events accepted by the Level 3 trigger. Diag- 
nostic applications are running, and information about the detector performance is obtained. Accepted events are written onto local disks by data logger programs, and subsequently they are stored on 8-mm tapes by a tape-staging program. 


\section{Chapter 3}

\section{Comparison of Inclusive Three-jet Data to a Next-to-leading Order Calculation}

Proton-antiproton collisions at the Fermilab Tevatron Collider, which operates at a center-of-mass energy of $1.8 \mathrm{TeV}$, produce events that can be described within the framework of perturbative QCD. During collider Run 1b, CDF recorded data corresponding to an integrated luminosity of $86 \pm 4 \mathrm{pb}^{-1}$. Data presented in this thesis were extracted from that run's Stream 2 data banks for events with total transverse energy $\left(\sum \mathrm{E}_{\mathrm{T}}\right)>175 \mathrm{GeV}$. The transverse energy of a jet or particle is defined as $\mathrm{E}_{\mathrm{T}} \equiv \mathrm{E} \sin \theta$, where $\theta$ is the angle between the beam direction in the laboratory frame (which is the z-axis in the CDF detector) and the jet axis or outgoing particle direction.

In perturbative QCD, hard scattering of the constituent partons in the proton and antiproton results in events with large $\sum \mathrm{E}_{\mathrm{T}}$. The outgoing scattered partons hadronize and so are detected as hadronic jets. Three-jet events can be produced when a hard gluon is radiated from any of the initial, intermediate, or final state partons in an event with two primary outgoing partons. The third jet can have a 
large component $\left(\mathrm{P}_{\mathrm{T}}\right)$ of its momentum transverse to the beam direction; however, the three-jet cross section is expected to be smaller than the two-jet cross section.

In this thesis we describe the analysis of the properties of three-jet events from Run 1b. The results are compared to predictions from a next-to-leading order (NLO) calculation [47] by calculating the difference between the number of data events and the number of expected events in each bin, scaled by the expected number of events. We also calculate the average $\chi^{2}$ between the data and the prediction. Special emphasis has been placed on the analysis of the Dalitz variables.

This analysis is the first to test the NLO prediction against data. Due to its more complex nature, an NLO calculation requires more CPU time than leading order (LO) predictions, but its dependence on the renormalization scale, $\mu$, is expected to be significantly reduced.

\subsection{Three-jet Variables}

We consider systems of three or more massless jets. The three-jet system in the massless parton approximation can be uniquely defined by five independent variables. The choice of parameters is described in Reference [48].

The three leading jets in the laboratory frame are used as a basis of transformation into the three-jet rest frame. In the three-body rest frame, the incoming partons are labeled partons 1 and 2. The highest energy jets in this frame have energies labeled $\mathrm{E}_{3}, \mathrm{E}_{4}$, and $\mathrm{E}_{5}$ and are ordered according to their energies, such that

$$
\mathrm{E}_{3}>\mathrm{E}_{4}>\mathrm{E}_{5}
$$

The outgoing partons associated with these jets are correspondingly labeled partons 3,4 , and 5 . In this frame, $\mathbf{P}_{i}$ is the momentum of parton $i$.

The five independent parameters we choose to describe the three-jet system are:

1. The mass of the three-jet system, $m_{3 \mathrm{~J}}$. 
2. The cosine of the angle between the beam direction and parton 3 in the threejet rest frame, $\cos \theta_{3}^{*}$. In this frame the incoming partons are not necessarily collinear. We define $\cos \theta_{3}^{*}$ with respect to the average beam direction, $\mathbf{P}_{\mathrm{AV}}$, in the three-body rest frame. Thus

$$
\cos \theta_{3}^{*} \equiv \frac{\mathbf{P}_{\mathrm{AV}} \cdot \mathbf{P}_{3}}{\left|\mathbf{P}_{\mathrm{AV}}\right|\left|\mathbf{P}_{3}\right|}
$$

where

$$
\mathbf{P}_{\mathrm{AV}} \equiv \mathbf{P}_{1}-\mathbf{P}_{2}
$$

3. The cosine of the angle between the plane containing the average beam direction and parton 3 and the plane containing partons 3, 4, and 5, which is

$$
\cos \psi^{*} \equiv \frac{\left(\mathbf{P}_{3} \times \mathbf{P}_{\mathrm{AV}}\right) \cdot\left(\mathbf{P}_{4} \times \mathbf{P}_{5}\right)}{\left|\mathbf{P}_{3} \times \mathbf{P}_{\mathrm{AV}}\right|\left|\mathbf{P}_{4} \times \mathbf{P}_{5}\right|}
$$

4. The Dalitz variable for the leading jet, $\mathrm{X}_{3}$. The Dalitz variables, $\mathrm{X}_{i}$, are defined as:

$$
\mathrm{X}_{i} \equiv \frac{2 \cdot \mathrm{E}_{i}}{\mathrm{~m}_{3 \mathrm{~J}}}, \quad(i=3,4,5)
$$

and they are normalized such that:

$$
\sum_{i=3}^{5} \mathrm{X}_{i}=2
$$

5. The Dalitz variable for the next-to-leading jet, $\mathrm{X}_{4}$. The Dalitz variables are used because the density at any point in the Dalitz plane is related to the square of the matrix element for the interaction.

Momentum conservation restricts the ranges of the Dalitz variables to

$$
\begin{aligned}
& \frac{2}{3} \leq \mathrm{X}_{3} \leq 1 \\
& \frac{1}{2} \leq \mathrm{X}_{4} \leq 1, \text { and } \\
& 0 \leq \mathrm{X}_{5} \leq \frac{2}{3}
\end{aligned}
$$

Figure 3.1 illustrates the definitions of the angles $\theta_{3}^{*}$ and $\psi^{*}$. 


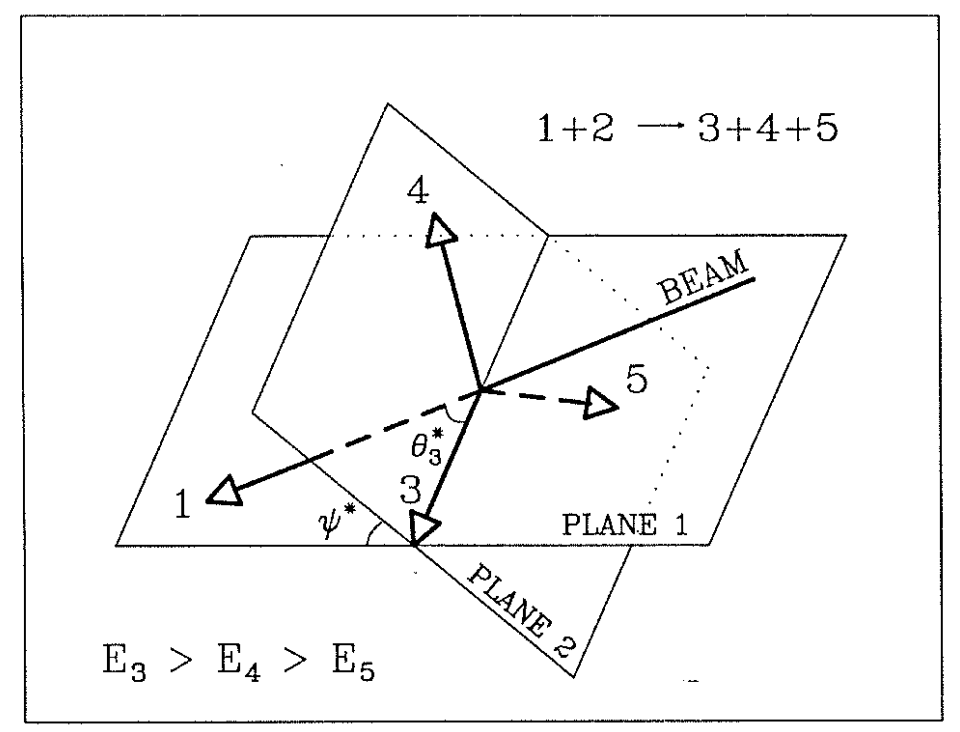

Figure 3.1: Definitions of $\theta_{3}^{*}$ and $\psi^{*}$ for the three-jet final state in the three-jet rest frame. (Figure taken from Reference [48].)

\subsection{Data Sample}

The $\sum \mathrm{E}_{\mathrm{T}}>175 \mathrm{GeV}$ trigger and the event selection requirements are described in Reference [49] and reviewed briefly below.

\subsubsection{Level 2 Trigger}

The trigger hardware performs clustering in $\eta$ - $\phi$ space, where the pseudorapidity, $\eta$, is defined as in Equation 2.1, and $\phi$ is the azimuthal angle. At Level 2, all uncorrected calorimeter clusters with $\mathrm{E}_{\mathrm{T}}>10 \mathrm{GeV}$ in the central, plug, and forward calorimeters are summed for the high- $\sum \mathrm{E}_{\mathrm{T}}$ trigger. Clusters consist of a seed tower with $\mathrm{E}_{\mathrm{T}}>3 \mathrm{GeV}$ and all contiguous towers in $\eta$ and $\phi$ with $\mathrm{E}_{\mathrm{T}}>1 \mathrm{GeV}$. To pass the trigger, an event must have a $\sum \mathrm{E}_{\mathrm{T}}>175 \mathrm{GeV}$. 


\subsubsection{Level 3 Trigger}

Inclusion in the $\sum \mathrm{E}_{\mathrm{T}}$ Stream 2 data sample requires an event to have $\sum \mathrm{E}_{\mathrm{T}}>175 \mathrm{GeV}$, where the sum is over all calorimeter clusters with $\mathrm{E}_{\mathrm{T}}>10 \mathrm{GeV}$ after reconstruction and the CDF jet clustering algorithm [50] is used. A cone size of $\mathrm{R}=0.7$ is used in the jet clustering algorithm, where $\mathrm{R} \equiv \sqrt{(\Delta \eta)^{2}+(\Delta \phi)^{2}}, \Delta \eta=\eta_{2}-\eta_{1}$, and $\Delta \phi=\phi_{2}-\phi_{1}$. The subscripts 1 and 2 correspond to the axis of the cone and the tracks off that axis, respectively. Studies have shown that the effect of the choice of $\mu$ on the theoretical jet cross section prediction is minimized for $\mathrm{R}=0.7$ [51]. Figure 3.2 plots the predicted jet cross section as a function of R. Transverse energies are computed from calorimeter information under the assumption that the event vertex was at $\mathrm{z}=0$.

Background due to high-energy cosmic rays tends to deposit energy either in the electromagnetic calorimeter or in the hadronic calorimeter; this causes the fraction of the total energy in the electromagnetic calorimeter to be small or close to one [52]. Large missing transverse energy, $\notin_{\mathrm{T}}$, defined as

$$
\mathbb{E}_{\mathrm{T}} \equiv\left|\sum_{i} \mathbf{E}_{\mathrm{T}_{i}}\right| \text {, }
$$

is also expected in these events. $\mathbf{E}_{\mathrm{T}_{i}}$ is the vector that points from the interaction point to calorimeter cell $i$; its magnitude is equal to the transverse energy of that cell. The missing transverse energy significance, $\mathrm{S}$, is defined as [53]:

$$
\mathrm{S} \equiv \mathrm{E}_{\mathrm{T}} /\left(\sum \mathrm{E}_{\mathrm{T}}\right)^{1 / 2}
$$

Excluding events with $\mathrm{S}>10$ and a ratio of electromagnetic to total energy $<0.2$ suppresses background due to cosmic rays.

\subsubsection{Offline Selection}

Offline cuts to further reject cosmic rays, beam halo, and calorimeter malfunctions are imposed. Events are removed if: 


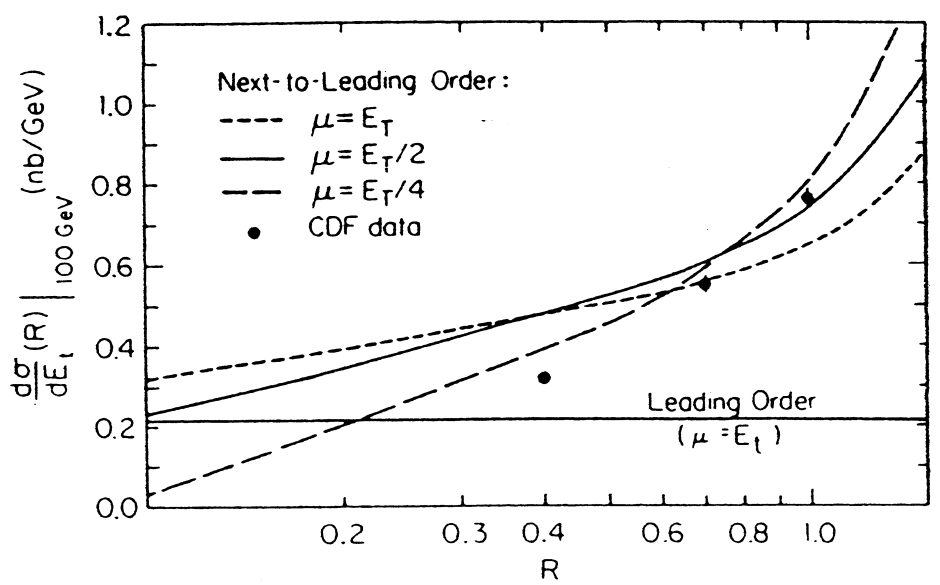

Figure 3.2: The predicted jet cross section as a function of the cone size, R. (Figure taken from Reference [51].)

1. significant energy is deposited in the hadronic calorimeter out of time with the proton-antiproton collision (the module HATFLT is used in the analysis),

2. the total energy of the event exceeds $2000 \mathrm{GeV}$, or

3. $\mathrm{S}>6$.

To guarantee that the projective geometry of the calorimeter towers can be used in the reconstruction, events are rejected if there is no reconstructed primary vertex with $|\mathrm{z}|<60 \mathrm{~cm}$. 
The offline module BADRUN is used to remove events from runs in which any of the calorimeter components or the tracking were not functional.

\subsection{Jet Corrections and Offline Event Selection}

The module JTC96 is used to correct the jet energies for errors in the absolute and relative energy scales and for additional energy associated with the underlying event. The theoretical calculation to which the data are compared treats partons but not jets (see later sections); this means that energy contributions from underlying events are excluded from the theoretical calculation. The data must consequently be corrected to remove the contribution from the underlying event. Since partons that are radiated out of the cone lead to the same losses in the theoretical calculation and in the data, out-of-cone corrections are not applied.

$\mathrm{E}_{\mathrm{T}}$ depends on the position of the primary event vertex. VXPRIM is used to find the vertex with the largest $\sum_{i} \mathrm{P}_{i}$ (where $\mathrm{P}_{i}$ is the total momentum of each particle $i$ leaving the vertex in the event), since this vertex is assumed to correspond to the hard scatter in the event. The jet variables are calculated using this vertex.

All jets in the data are required to have $\mathrm{E}_{\mathrm{T}}>20 \mathrm{GeV}$ and $|\eta|<2.0$. Events with less than three jets are rejected. To avoid collinear instability in the iterative jet algorithm [54], a cone overlap cut is imposed. Events are rejected if the distance $\Delta \mathrm{R}$ in $\eta$ - $\phi$ space between any two axes of the three leading jets is less than 1.0. This particular choice for the cone overlap cut is motivated by measurements of the fraction of two jets that are merged into a single jet as a function of the cone separation [50]. At a cone separation of $\Delta \mathrm{R}=1.0$, no two jets are merged. Figure 3.3 shows the fraction of two jets that are merged into a single jet as a function of $\Delta R$. This cut removes $7 \%$ of the data. Figure 3.4 shows the distribution of the events rejected by this cut in the Dalitz plane.

Data for which the geometrical acceptance is less than about $95 \%$ are excluded. 


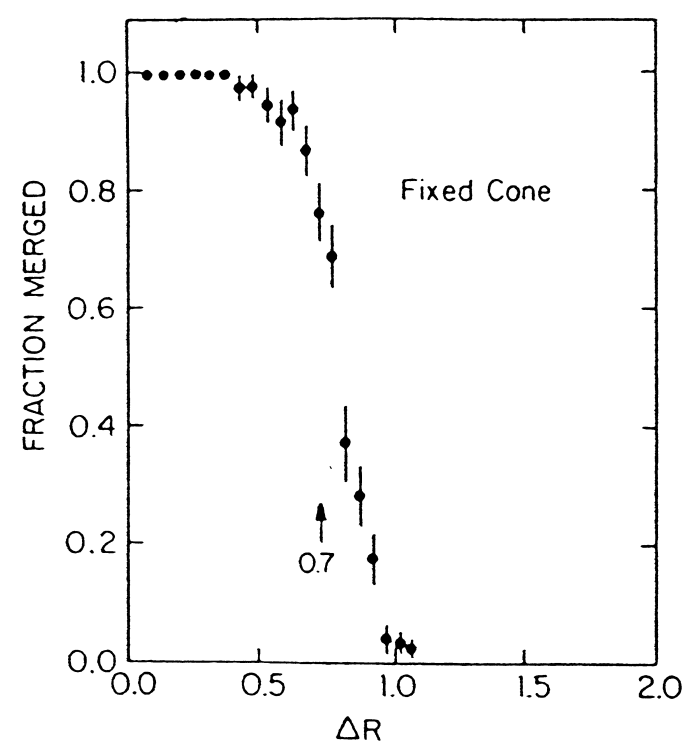

Figure 3.3: The fraction of two jets merged into a single jet as a function of $\Delta R$. (Figure taken from Reference [50].)

For a three-jet system, an approximation for the boundary of the region with full acceptance can be found [55] by considering a two-jet system. If we define the mass of the two-jet system, $\mathrm{m}_{2 \mathrm{~J}}$, to be the mass of the two leading jets in the three-jet system, the region with full acceptance can be approximated by:

$$
\left|\cos \theta_{3}^{*}\right|<\sqrt{1-\left(\frac{\sum \mathrm{E}_{\mathrm{T}}}{\mathrm{m}_{2 \mathrm{~J}}}\right)^{2}}
$$

where $\sum \mathrm{E}_{\mathrm{T}}$ is the minimum total transverse energy $(175 \mathrm{GeV})$ of the event. 


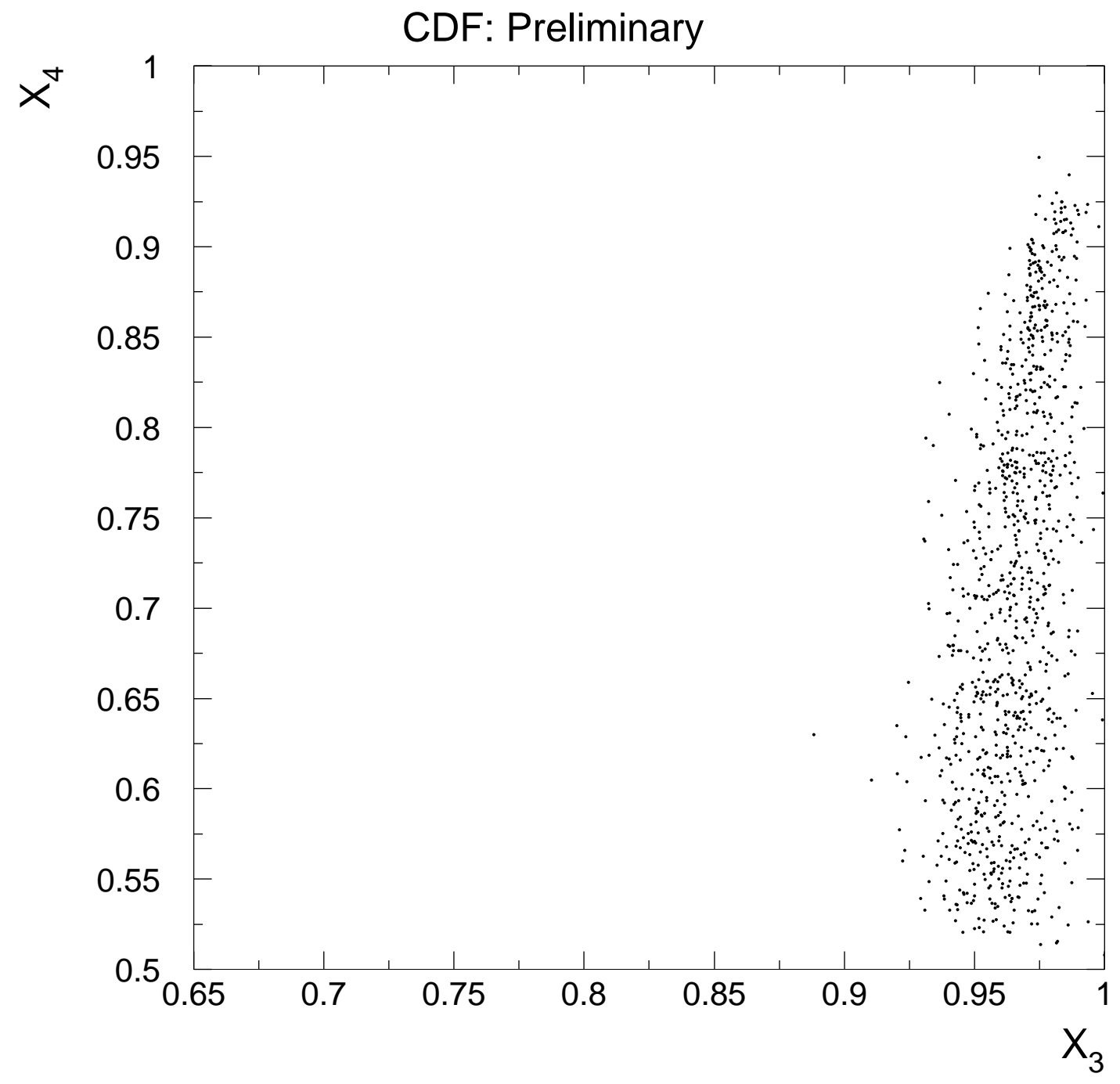

Figure 3.4: The distribution of the Dalitz variables of events rejected by the requirement that $\Delta \mathrm{R}>1.0$. 


\subsection{Detection and Offline Cut Efficiencies}

Since the data selection and the NLO calculation use the same set of cuts before evaluation of the cross section, the efficiencies of all offline cuts are the same in data and Monte Carlo. No correction is necessary for these.

The $\sum \mathrm{E}_{\mathrm{T}}>175 \mathrm{GeV}$ trigger efficiency measurement is described in Reference [56]. Full trigger efficiency is found for $\sum_{\text {jets }} E_{T}>320 \mathrm{GeV}$, where the sum is over all jets with corrected $\mathrm{E}_{\mathrm{T}}>20 \mathrm{GeV}$. We require full trigger efficiency for this analysis and use $\sum_{\text {three leading jets }} \mathrm{E}_{\mathrm{T}}>320 \mathrm{GeV}$. The number of events passing all the online and offline data quality cuts is 53,211.

The $\sum \mathrm{E}_{\mathrm{T}}$ cut used for full trigger efficiency in this analysis provides a bias towards multiple interactions in the event sample [52]. Especially data collected in runs with high instantaneous luminosity have a large fraction of events with more than one interaction per beam crossing [57]. The number of events as a function of instantaneous luminosity is shown in Figure 3.5. To minimize these contributions to the data, we check the event sample for a second interaction reconstructed in the vertex track detector (VTX). We define a second interaction to be present in the data if both vertices have at least 10 associated VTX tracks, and if they are separated by more than $10 \mathrm{~cm}$. This selection is classified as resolved multiple interactions and is removed from the sample. Figure 3.6 shows the fraction of resolved multiple interactions in the data sample as a function of instantaneous luminosity. Removing resolved multiple interactions from the data sample reduces it by $29 \%$. At high instantaneous luminosities the fraction of resolved multiple interactions is underestimated, as it becomes more likely for multiple interactions to be unresolved. To measure the contribution from unresolved multiple interactions, the jet multiplicities in the samples with resolved and unresolved multiple interactions are investigated. The average jet multiplicity as a function of instantaneous luminosity is $3.305 \pm 0.006$ for the unresolved sample and $3.355 \pm 0.006$ for the resolved sample. To calculate the contribution of the unresolved multiple interactions to the three-jet sample, the average jet multiplicities for 
both samples are extrapolated to zero instantaneous luminosity. The extrapolated values are $3.283 \pm 0.008$ and $3.292 \pm 0.014$ for the unresolved and the resolved sample, respectively. Additional contributions from two-jet events that are promoted into the three-jet sample through unresolved multiple interactions are subtracted from these value. Two-jet events contribute $32 \%$ of the events to the $\sum_{\text {jets }} E_{\mathrm{T}}>320 \mathrm{GeV}$ data sample. After correction of CDF data by JTC96, 7\% of the data are found to include $\geq 1$ additional jets with $\mathrm{E}_{\mathrm{T}}>20 \mathrm{GeV}$ which are not attributed to the hard interaction [57]. The two-jet contribution to the three-jet sample is thus estimated to be approximately $2 \%$. The unresolved multiple interaction contribution to the three-jet analysis can be estimated by the relative difference between the two extrapolated and adjusted values. It amounts to $1.0 \pm 0.3 \%$ and is neglected in the remainder of this analysis. The jet multiplicities as a function of instantaneous luminosity for the samples with resolved and unresolved multiple interactions are shown in Figure 3.7. Removing resolved multiple interactions from the data sample reduces the effective total integrated luminosity. The effective total integrated luminosity is estimated by extrapolating the expected total number of events after rejection of multiple interactions as a function of instantaneous luminosity to zero instantaneous luminosity. Figure 3.8 shows the expected total number of events after rejection of multiple interactions as a function of instantaneous luminosity. The ratio of the expected total number of events after rejection to the total number of events in the three-jet sample prior to rejection of multiple interactions multiplies the total integrated luminosity to yield the effective total integrated luminosity of $77 \pm 4 \mathrm{pb}^{-1}$. The change in the number of vertices in the data due to the rejection of multiple interactions is shown in Figure 3.9. After rejection of resolved multiple interactions 33,809 events are left in the data sample.

Figures $3.10,3.11$, and 3.12 show the distributions of $\left|\cos \theta_{3}^{*}\right|$ versus $m_{3 \mathrm{~J}}, \psi^{*}$ versus $\cos \theta_{3}^{*}$, and $\mathrm{X}_{4}$ versus $\mathrm{X}_{3}$ after application of all the event selection criteria to the data. 


\section{CDF: Preliminary}

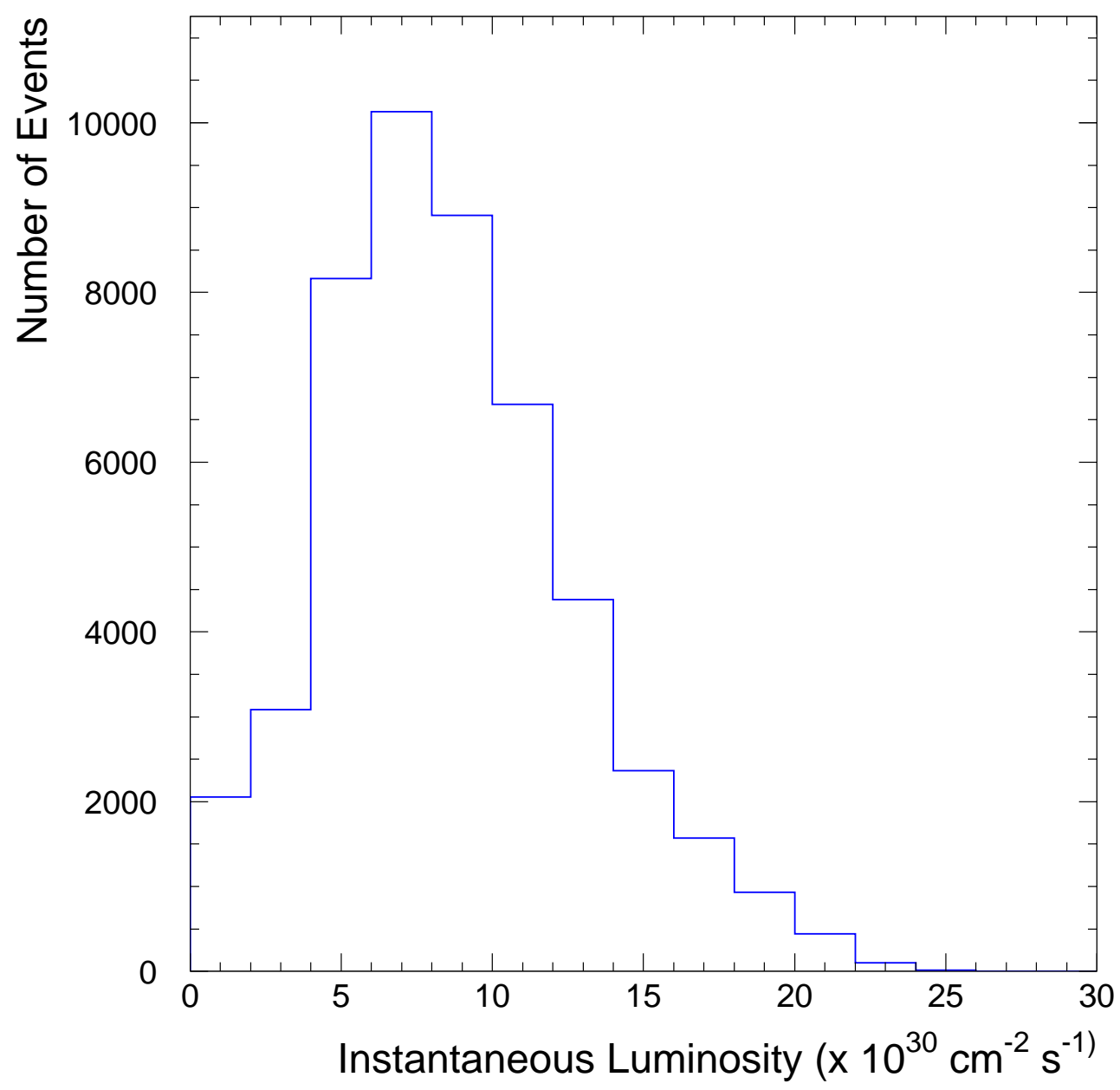

Figure 3.5: The number of events as a function of instantaneous luminosity. 


\section{CDF: Preliminary}

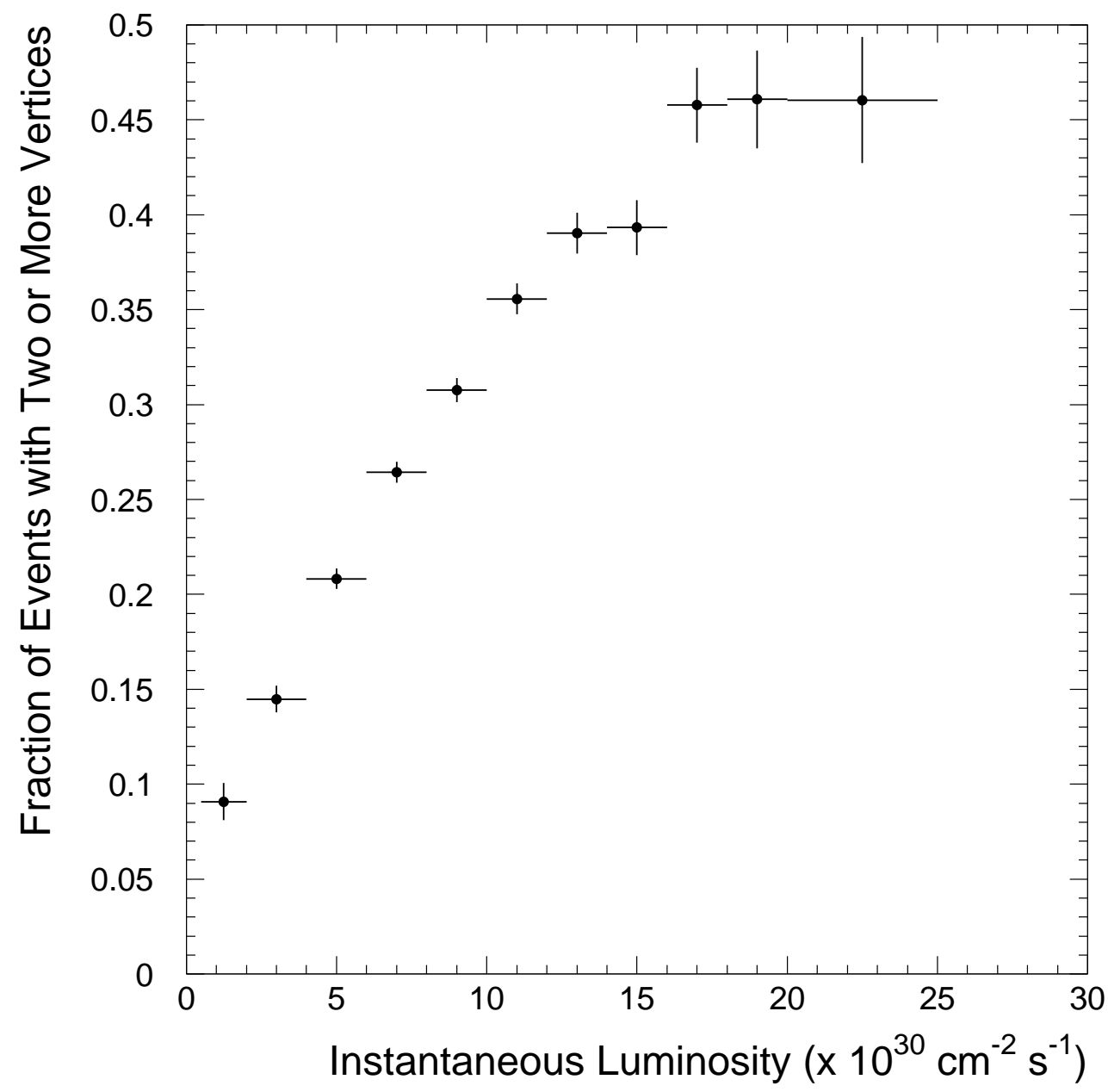

Figure 3.6: The fraction of resolved multiple interactions as a function of instantaneous luminosity. 

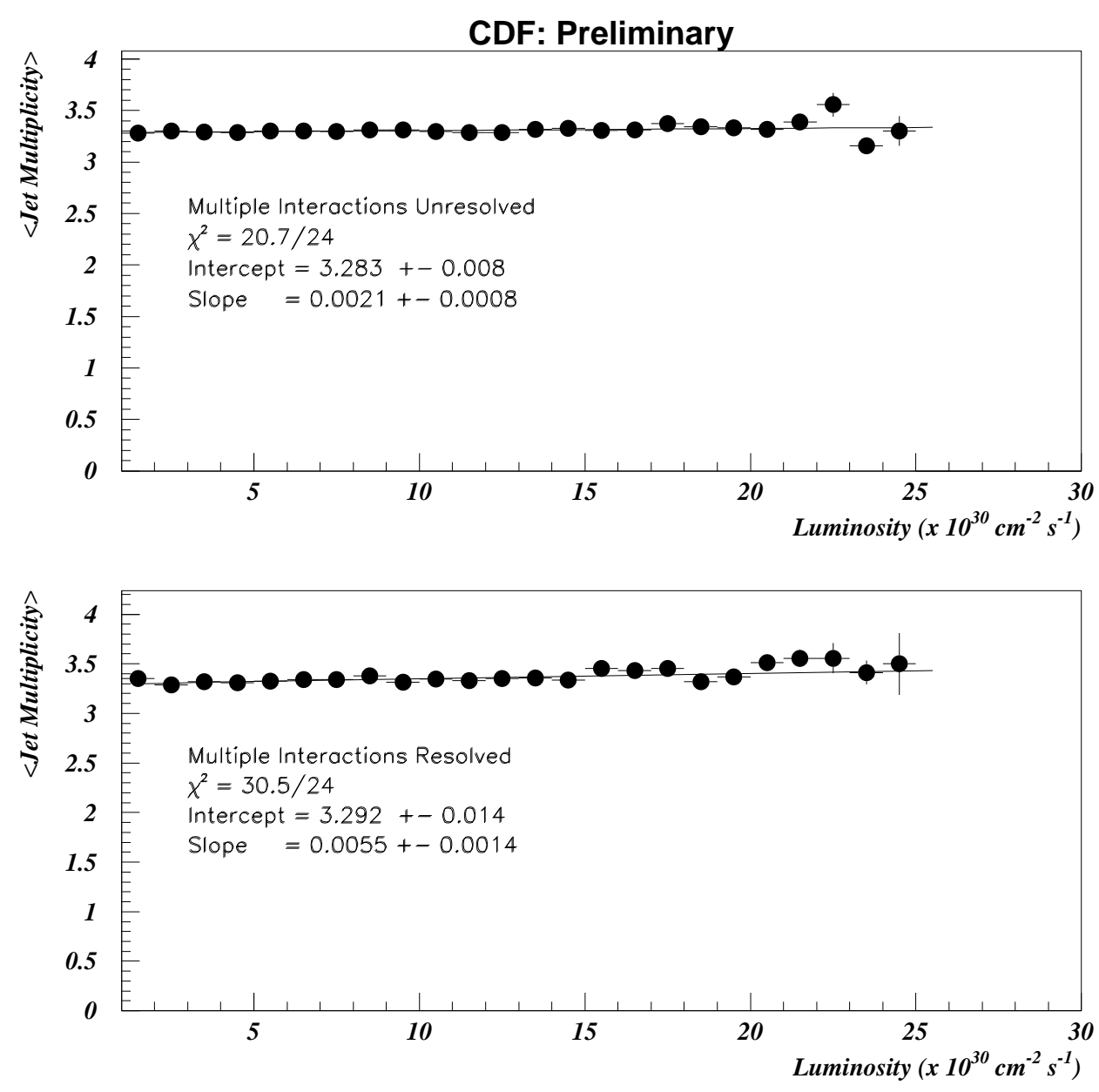

Figure 3.7: The jet multiplicities as a function of instantaneous luminosity for the samples with resolved and unresolved multiple interactions (Figure courtesy of Igor Gorelov.) 


\section{CDF: Preliminary}

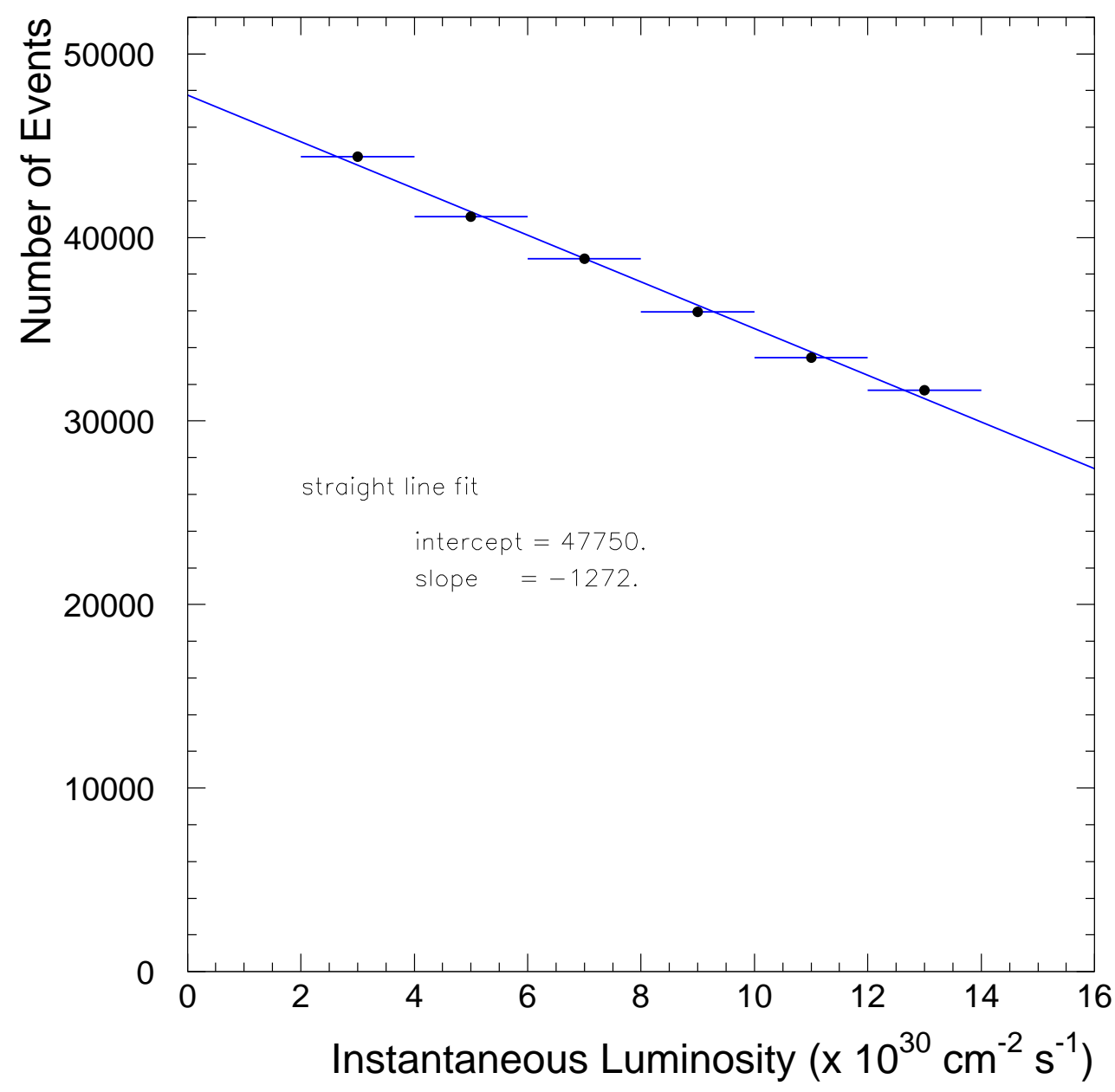

Figure 3.8: The expected total number of events after rejection of multiple interactions as a function of instantaneous luminosity. 
CDF: Preliminary

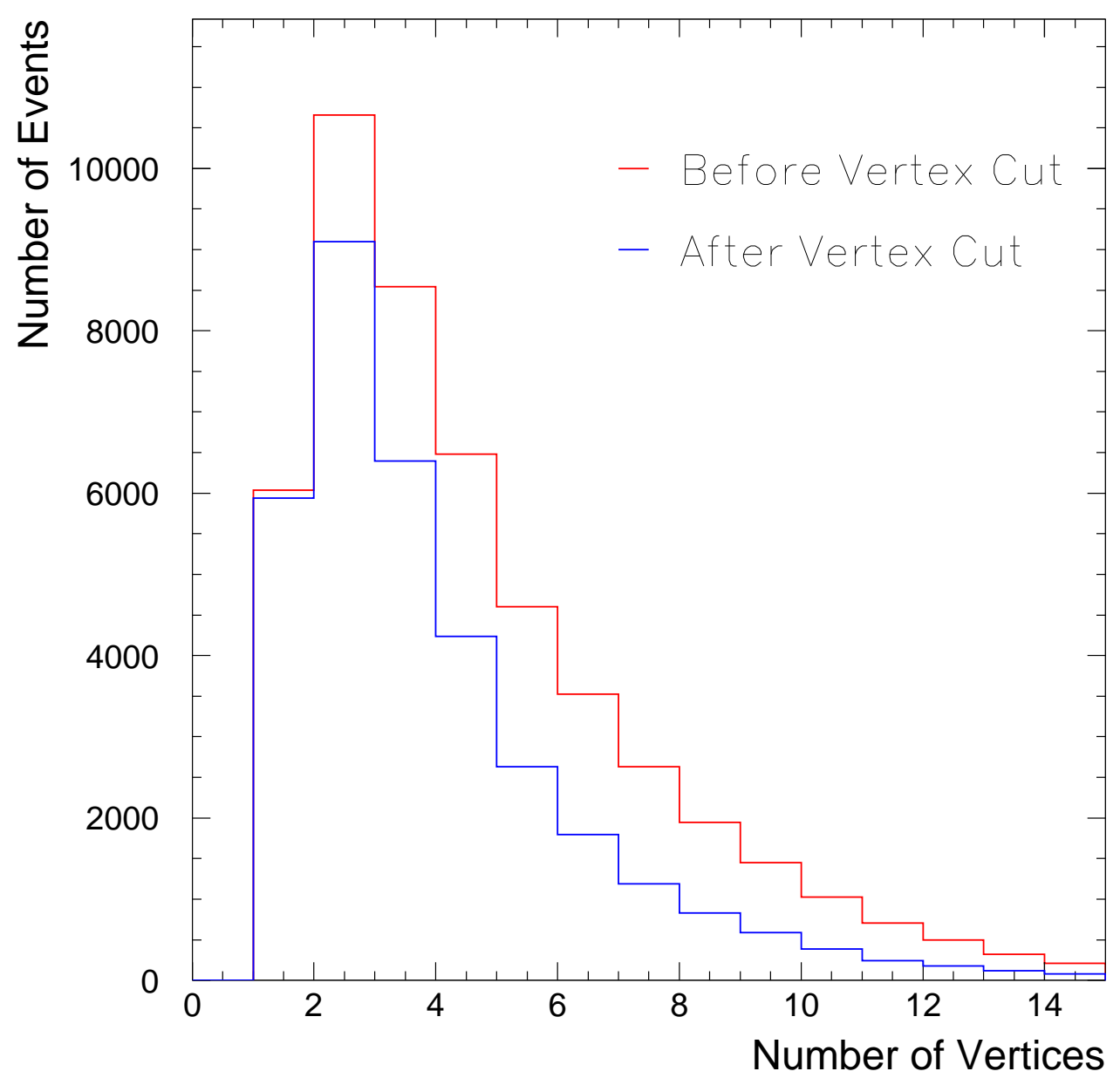

Figure 3.9: The change of the number of vertices in the data due to the rejection of multiple interactions. 


\section{CDF: Preliminary}

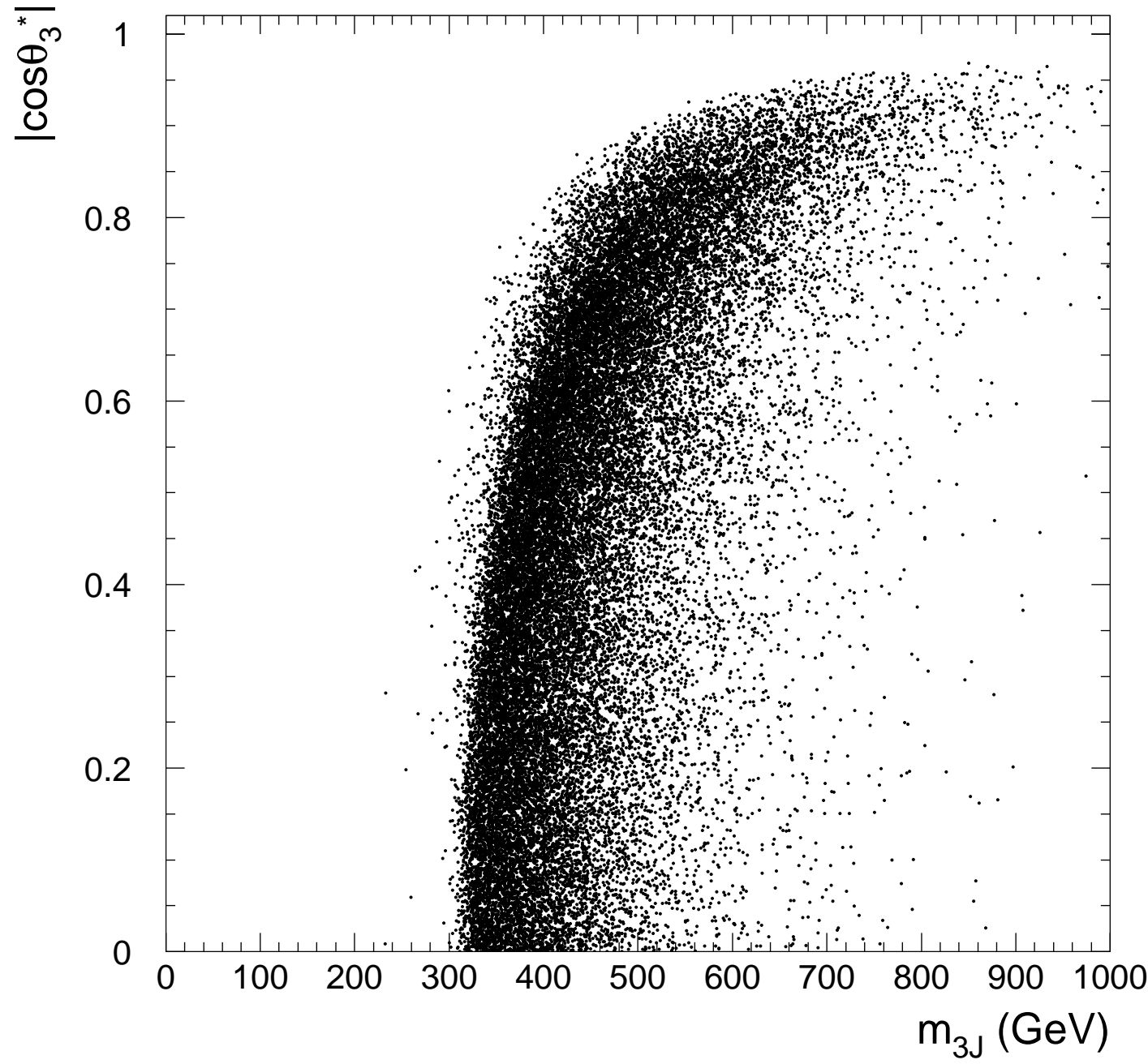

Figure 3.10: The distribution of $\left|\cos \theta_{3}^{*}\right|$ versus $m_{3 \mathrm{~J}}$ after all the cuts have been applied to the data. 


\section{CDF: Preliminary}

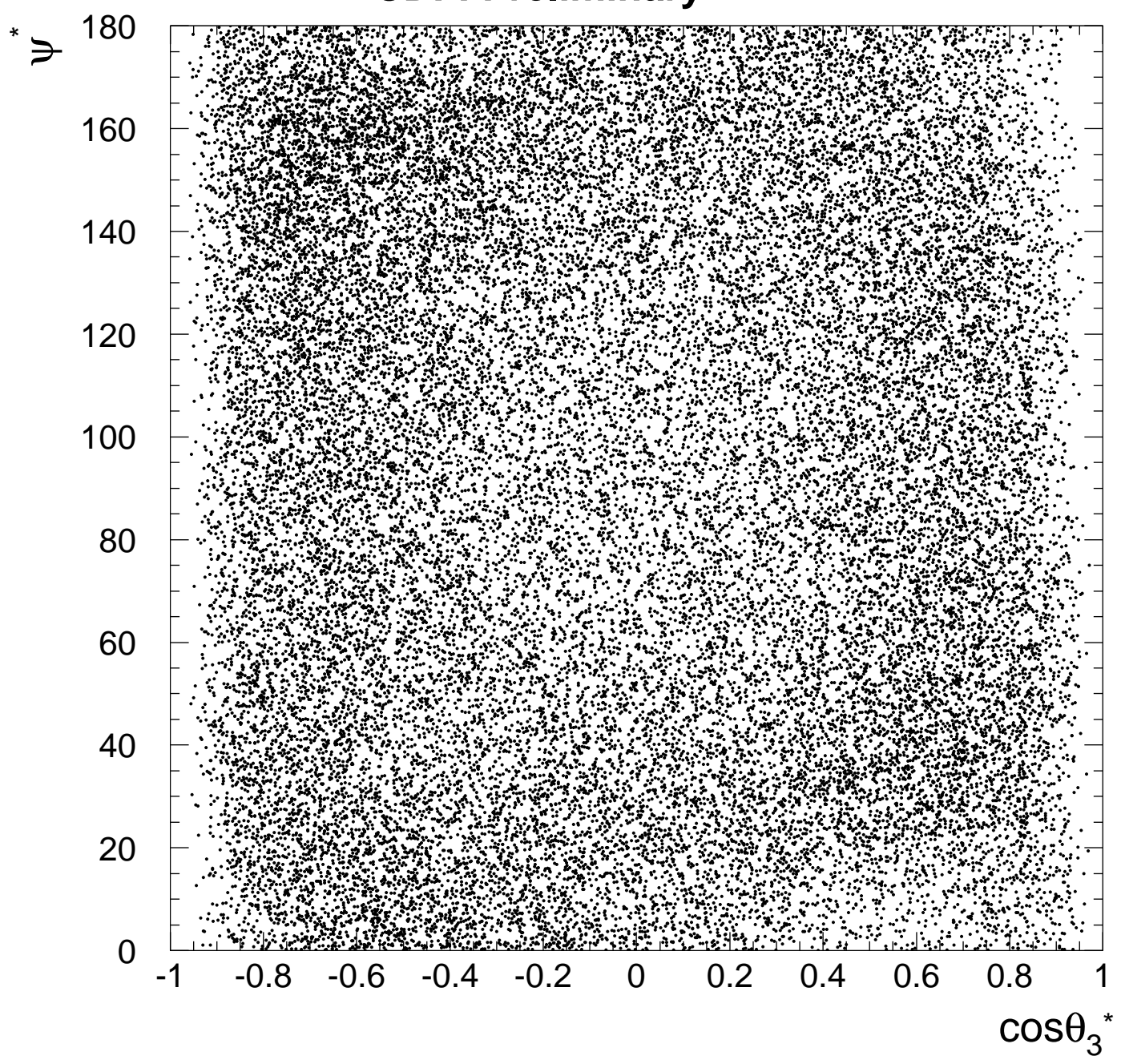

Figure 3.11: The distribution of $\psi^{*}$ versus $\cos \theta_{3}^{*}$ after all the cuts have been applied to the data. 


\section{CDF: Preliminary}

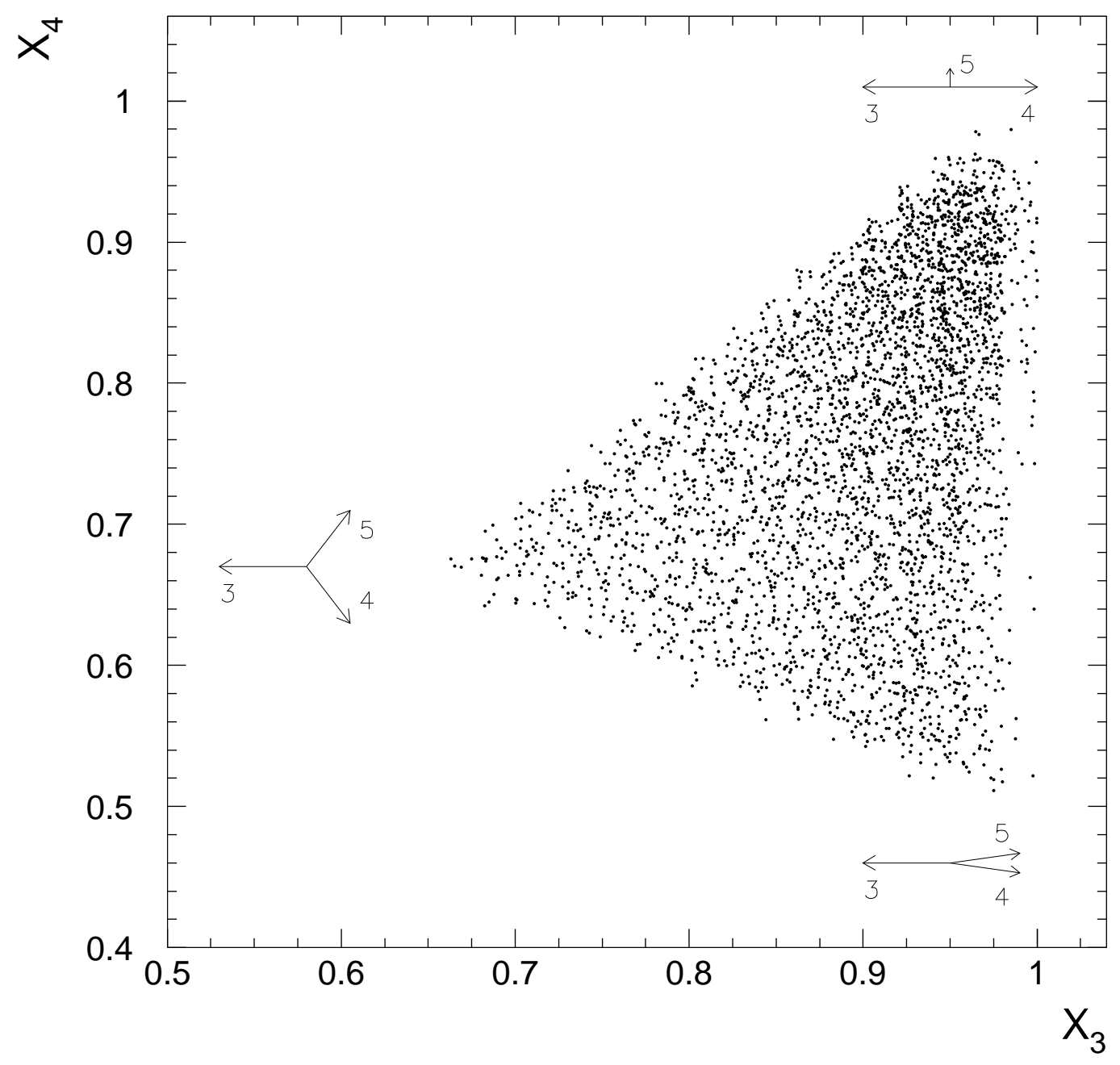

Figure 3.12: The distribution of $\mathrm{X}_{4}$ versus $\mathrm{X}_{3}$ after all the cuts have been applied to the data. 


\subsection{Monte Carlo Prediction}

Unlike in previous analyses where only LO Monte Carlo predictions were available, the data are compared to a NLO Monte Carlo event generator for hadronic threejet production [47] in this analysis. This program is the first to calculate all parton sub-processes to NLO in perturbation theory. It has never before been used by experimentalists and verified against data.

Due to the particular choices of renormalization and factorization scales, LO Monte Carlo predictions have associated systematic uncertainties of the order of $50 \%$. This $\mu$ dependence is expected to decrease significantly at next-to-leading order. One of the LO event generators frequently used at CDF is HERWIG. It has been shown to agree well with CDF data in several analyses, but as it only calculates $2 \longrightarrow 2$ parton processes, hadronization and jet fragmentation need to be simulated by empirical models for events with more than two jets. At NLO, three jets are expected to be predicted more accurately than at LO. In our analysis, HERWIG is used as a cross check for the results obtained by the NLO prediction.

Other QCD event generators used in hadron collider experiments include the LO routine NJETS, and the NLO routine JETRAD. While NJETS calculates LO $2 \longrightarrow \mathrm{n}$ parton processes, JETRAD determines $2 \longrightarrow 2$ parton processes to NLO in perturbative QCD. In addition, another LO routine, PYTHIA, has also been used for comparison with data. All these QCD calculations have been reported to give a good first description of data [58]. However, for three-jet production, LO calculations had to be used or phenomenological approaches to jet fragmentation had to be taken. In this analysis, an NLO event generator is used in which three-jet production is intrinsic, the renormalization scale is chosen such that $\mu=\mathrm{E}_{\mathrm{T}}$.

The NLO calculation consists of two parts: the one-loop $2 \longrightarrow 3$ parton virtual processes and the tree-level $2 \longrightarrow 4$ parton real emission processes. The parton subprocesses calculated to one loop are $g g \rightarrow g g g, \bar{q} q \rightarrow g g g, \bar{q} q \rightarrow \bar{Q} Q g$, and all combinations obtained by crossing symmetry. The ones computed to tree level are 
$g g \rightarrow g g g g, \bar{q} q \rightarrow g g g g, \bar{q} q \rightarrow \bar{Q} Q g g, \bar{q} q \rightarrow \bar{Q} Q \overline{Q^{\prime}} Q^{\prime}$, and all combinations obtained by crossing symmetry. The quark-antiquark pairs $\bar{Q} Q$ may or may not have the same flavors as the $\bar{q} q$ and $\overline{Q^{\prime}} Q^{\prime}$ pairs.

As output the Monte Carlo generator provides binned cross sections for the variables of interest. These cross sections are given in two parts which must be added algebraically; the theoretical errors calculated for each of the two cross section terms are associated with calculation statistics and are added in quadrature.

Construction of an NLO event generator requires careful handling of the cancellation of divergences between soft and collinear contributions and virtual corrections [54]. The soft and collinear divergences are due to the fact that at NLO a parton can only be defined through a resolution criterion. The NLO event generator in this analysis, uses a simple invariant mass resolution criterion, $s_{\min }$. The invariant mass, $\mathrm{M}$, of two partons, 1 and 2 , is defined as $\mathrm{M} \equiv \sqrt{\left(\mathrm{E}_{1}+\mathrm{E}_{2}\right)^{2}-\left(\mathbf{p}_{1}+\mathbf{p}_{2}\right)^{2}}$, where $\mathrm{E}$ and $\mathbf{p}$ are the energies and the momenta of the partons, respectively. Two partons are considered to be unresolvable and treated as a single parton if $\mathrm{M}$ is smaller than $s_{\text {min }}$. The unresolved soft and collinear regions of phase space are isolated from the resolved bremsstrahlung phase space. With that, both the resolved contributions and the combination of the unresolved soft and collinear contributions with the virtual corrections are finite [59].

With this method, the soft and collinear contributions can be calculated. The resolved partonic cross sections are then put together in order to make the NLO jet event generator. The three methods in this step are in increasing order of complexity:

1. The "slicing method," in which both matrix element and phase space are approximated in the soft and collinear regions.

2. The "subtraction method," in which the phase space is approximated in the soft and collinear regions, but the matrix element is exact. The correction factor is added into the matrix element numerically. 
3. The "exact method," in which both the correction factors for the phase space and the matrix element are added in numerically.

The most attractive method is to calculate the matrix element and the phase space with no approximations. All terms in $s_{\min }$ are included in the calculation, and any results are independent of the size of the resolution parameter. As the exact method is difficult to implement, the event generator discussed in this thesis calculates the NLO cross sections using the slicing method.

Only the exact method to generate NLO events is independent of $s_{\min }$, the slicing method and the subtraction method exhibit explicit dependence on the resolution parameter. The cone overlap cut implemented into our analysis (see Section 3.3) significantly reduces this $s_{\text {min }}$ dependence in the calculated cross sections by removing infrared instabilities. The iterative cone algorithm used by CDF is an infrared safe jet algorithm if it is augmented with a jet separation cut.

As the slicing method is implemented in the NLO generator used for this analysis, its mathematical basis is outlined briefly here. The virtual, $V$, and real, $R$, contributions to the NLO cross section can be schematically written as [60]:

$$
\begin{aligned}
& \left(\frac{d \sigma}{d x}\right)_{V}=\frac{1}{2 \epsilon} \delta(1-x), \text { and } \\
& \left(\frac{d \sigma}{d x}\right)_{R}=\frac{1}{1-x},
\end{aligned}
$$

where the parameter $\epsilon$ is introduced through dimensional regularization with a change of the dimensionality of space-time to $d=4-2 \epsilon$, and where $1-x$ represents the energy radiated by a parton. When $x=1$ no radiation is emitted, while for $x=0$ the parton radiates a maximum amount of energy. If the average value $\langle F\rangle$ of a function $F(x)$ denotes a physical observable, then $\langle F\rangle$ at NLO is calculated as:

$$
\begin{aligned}
<F>_{\mathrm{NLO}} & =\int_{0}^{1} d x\left(\frac{d \sigma}{d x}\right)_{V} F(x)+\int_{0}^{1} d x(1-x)^{-2 \epsilon}\left(\frac{d \sigma}{d x}\right)_{R} F(x) \\
& =\frac{1}{2 \epsilon} \int_{0}^{1} d x \delta(1-x) F(x)+\int_{0}^{1} d x(1-x)^{-1-2 \epsilon} F(x) \\
& =\frac{1}{2 \epsilon} F(1)+<F>_{R} .
\end{aligned}
$$


Performing the computation in $d$ dimensions in order to regulate the divergences introduces the factor $(1-x)^{-2 \epsilon}$ in the real cross section contribution. As the virtual part can be integrated analytically, the difficult task of the problem lies in the computation of the real contribution. The real integral is divergent as $\epsilon \rightarrow 0$, where the pole in $1 / \epsilon$ exactly cancels the divergent factor in the virtual contribution.

The slicing and the subtraction method use different approaches to calculate the divergent integral in $\langle F\rangle_{R}$. In the slicing method, $\langle F\rangle_{R}$ is rewritten as:

$$
<F>_{R}=\int_{0}^{1-\delta} d x \frac{F(x)}{(1-x)^{1+2 \epsilon}}+\int_{1-\delta}^{1} d x \frac{F(x)}{(1-x)^{1+2 \epsilon}},
$$

where $\delta$ is an arbitrary parameter, $0<\delta \leq 1$. The first integral in this expression is free of divergences. The term is integrated numerically after setting $\epsilon=0$. The second integral is expanded in a Taylor series in powers of $\epsilon$. Only terms that do not vanish as $\epsilon \rightarrow 0$ are retained, and terms of $\mathcal{O}(\delta)$ are neglected. One is then left with the finite result for the NLO cross section:

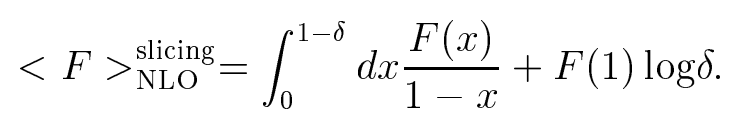

Since a physical result should not depend on the arbitrary parameter $\delta$, it has to be chosen to be small in order for the Taylor expansion and the subsequent approximation to remain valid.

In the subtraction method, no such approximation is used; the final expression for the NLO cross section is independent of $\delta$. In turn, however, the integral for the first term in Equation 3.13 becomes more cumbersome to compute.

To optimize computational performance of the NLO calculation, integrals are evaluated using an adaptive Monte Carlo algorithm, called VEGAS, which is widely used in elementary particle physics for multidimensional integration. The VEGAS routine employs a method, called importance sampling. The integrands are predominantly sampled from active regions in the integral space. Regions where the integrands are relatively constant are evaluated less frequently. Large values of the integrands imply an increased sampling rate. 
In addition to importance sampling, VEGAS also uses stratified sampling to further reduce the amount of computation. The integral space is divided into subregions where the sampling frequency in each region is chosen such that the variance of the integrands is minimized.

Finally, VEGAS is used in an adaptive mode. Several statistically independent iterations are performed to evaluate the desired integrals. These iterations assist each other, since each one is used to refine the sampling grid of the following one. First, the integrals are evaluated with a small number of calls to the random number generator. In the next step, more integrations are performed, but the sampling grid is already improved by accounting for the results from the previous iteration. Performing several steps with increasingly refined sampling grid and increasing numbers of samples optimizes the use of the computational resources available. The results of all iterations are combined into a single best answer in the end.

\subsection{Comparison of Data to Predictions from the Next-To-Leading Order Calculation}

The data and the NLO calculation Data and Monte Carlo predictions are separately binned in $\mathrm{X}_{3}-\mathrm{X}_{4}$ space. The binned cross sections provided by the calculation are multiplied by the total integrated luminosity associated with the data set to predict the number of events in each bin.

Comparison between the data and the NLO predictions is achieved by calculating the difference between the number of events in each bin from the unsmeared data and the NLO calculation. This difference is scaled by the predicted number of events.

To further compare the prediction to the data, we calculate the $\chi^{2}$ between 
the data and the Monte Carlo predictions:

$$
\chi^{2} \equiv \sum_{i}\left(\frac{\left(\mathrm{n}_{\mathrm{MC}_{i}}-\mathrm{n}_{\mathrm{D}_{i}}\right)^{2}}{\sigma_{\text {stat. }_{i}}^{2}+\sigma_{\text {theor. } i}}\right)^{2},
$$

where $\mathrm{n}_{\mathrm{MC}_{i}}$ is the number of scaled Monte Carlo events in bin $i, \mathrm{n}_{\mathrm{D}_{i}}$ is the number of data entries in bin $i$, and $\sigma_{\text {stat. } i}$ and $\sigma_{\text {theor. } i}$ are the statistical and theoretical errors, respectively, on the number of entries in bin $i$.

\subsection{Measurement of the Strong Coupling Constant}

The strong coupling constant, $\alpha_{\mathrm{S}}$, provides the measure of the strength of the strong interaction. Its numerical value is of fundamental importance for our description of nature. Only a large number of independent measurements of $\alpha_{\mathrm{S}}$ at various values of $\mathrm{Q}^{2}$ for better understanding of the running will allow for increasingly accurate theoretical models. For that reason, many different methods to measure $\alpha_{\mathrm{S}}$ have been approached by experimentalists.

The wide variety of experiments that measure the strong coupling include deep inelastic scattering (DIS), $\tau$ lepton decay measurements, lattice gauge theory considerations, $e^{+} e^{-}$annihilation, and hadronic collider experiments. Table 3.1 shows a summary of $\alpha_{\mathrm{S}}$ measurements, the momentum transfer $\mathrm{Q}$ at which $\alpha_{\mathrm{S}}$ is measured, $\alpha_{\mathrm{S}}\left(\mathrm{Q}^{2}\right), \alpha_{\mathrm{S}}$ extrapolated to $\mathrm{M}_{\mathrm{Z}}^{2}$, and the order of the theoretical prediction used in the measurement.

Polarized lepton-nucleon scattering is used to extract a value of $\alpha_{\mathrm{S}}$ from higherorder corrections to the Bjorken sum rule [61], and a similar measurement is based on the Gross-Llewellyn Smith (GLS) sum rule [62] for deep inelastic neutrino-nucleon scattering. As perturbative corrections for these cases have been calculated to nextto-next-to-leading order (NNLO), and since power corrections to deep inelastic scattering are relatively well understood, both measurements are very attractive for determination of $\alpha_{\mathrm{S}}$, even though $\mathrm{Q}^{2}$ is low. Due to the low value of $\mathrm{Q}^{2}$, the theoretical uncertainty in these methods is dominated by power corrections. 


\begin{tabular}{|c|c|c|c|c|}
\hline Process & $\mathrm{Q}(\mathrm{GeV})$ & $\alpha_{\mathrm{S}}\left(\mathrm{Q}^{2}\right)$ & $\alpha_{\mathrm{S}}\left(\mathrm{M}_{\mathrm{Z}}^{2}\right)$ & Theory \\
\hline \hline DIS (BjSR) & 1.58 & $0.375_{-0.081}^{+0.062}$ & $0.122_{-0.009}^{+0.005}$ & NNLO \\
DIS (GLS) & 1.73 & $0.26_{-0.05}^{+0.04}$ & $0.108_{-0.009}^{+0.006}$ & NNLO \\
DIS $(\nu)$ & 5.0 & $0.193_{-0.018}^{+0.019}$ & $0.111 \pm 0.006$ & NLO \\
DIS ( $\mu)$ & 7.1 & $0.180 \pm 0.014$ & $0.113 \pm 0.005$ & NLO \\
DIS (jets) & 22.1 & $0.148 \pm 0.016$ & $0.118 \pm 0.009$ & NLO \\
$\tau$ (R had $)$ & 1.77 & $0.33 \pm 0.03$ & $0.118 \pm 0.004$ & NNLO \\
$\bar{Q} Q$ & 3.6 & $0.219 \pm 0.007$ & $0.115 \pm 0.002$ & LGT \\
$\bar{Q} Q$ & 5.0 & $0.190_{-0.011}^{+0.014}$ & $0.112 \pm 0.004$ & LGT \\
$\bar{Q} Q$ & 10.0 & $0.167_{-0.011}^{+0.015}$ & $0.113_{-0.005}^{+0.007}$ & NLO \\
$e^{+} e^{-}(\sigma$ had $)$ & 31.6 & $0.163 \pm 0.022$ & $0.133 \pm 0.015$ & NNLO \\
$e^{+} e^{-}$(shapes) & 10.5 & $0.165 \pm 0.018$ & $0.113 \pm 0.006$ & NLO \\
$e^{+} e^{-}$(shapes) & 35.0 & $0.140 \pm 0.020$ & $0.119 \pm 0.014$ & NLO \\
$e^{+} e^{-}$(shapes) & 58.0 & $0.130 \pm 0.008$ & $0.122 \pm 0.007$ & NLO \\
$e^{+} e^{-}$(shapes) & 58.0 & $0.132 \pm 0.008$ & $0.124 \pm 0.007$ & resum. \\
$e^{+} e^{-}$(frag.) & $22-91$ & & $0.126 \pm 0.009$ & NLO \\
$Z^{0}$ ( $\left.\Gamma_{\text {had }}\right)$ & 91.2 & $0.123 \pm 0.005$ & $0.123 \pm 0.005$ & NNLO \\
$Z^{0}$ (shapes) & 91.2 & $0.118 \pm 0.006$ & $0.118 \pm 0.006$ & NLO \\
$Z^{0}$ (shapes) & 91.2 & $0.122 \pm 0.005$ & $0.122 \pm 0.005$ & resum. \\
$e^{+} e^{-}$(shapes) & 133 & $0.107 \pm 0.008$ & $0.113 \pm 0.009$ & resum. \\
$\bar{p} p, p p \rightarrow \gamma+X$ & 4.0 & $0.206_{-0.032}^{+0.045}$ & $0.112_{-0.010}^{+0.012}$ & NLO \\
$\bar{p} p \rightarrow \bar{b} b X$ & 20.0 & $0.145_{-0.019}^{+0.018}$ & $0.113 \pm 0.011$ & NLO \\
$\bar{p} p \rightarrow W \rightarrow$ jets & 80.6 & $0.123 \pm 0.025$ & $0.121 \pm 0.024$ & NNLO \\
\hline
\end{tabular}

Table 3.1: Summary of $\alpha_{\mathrm{S}}$ measurements. (Table taken from Reference [27].)

Violation of Bjorken scaling in deep inelastic structure functions provides the most reliable measurements of $\alpha_{\mathrm{S}}$ at intermediate scales $\mathrm{Q}^{2}$. Calculations have been performed to NLO, but the power corrections appear well under control. Muon and neutrino scattering experiments have been used to measure $\alpha_{\mathrm{S}}$ by Bjorken scaling violations.

Jet production rates in deep inelastic scattering allow for $\alpha_{\mathrm{S}}$ measurements at intermediate to high scales. Unlike in $e^{+} e^{-}$annihilation, $\mathrm{Q}^{2}$ can be varied over a wide range in a single deep inelastic scattering experiment at a single beam energy, such that the running of $\alpha_{\mathrm{S}}$ can be mapped more closely. A major contribution to the systematic uncertainty comes from the jet rate dependence on the parton distribution function for a given value of $\alpha_{\mathrm{S}}$. This dependence is also important in our analysis, 
as we are studying hadronic collisions at CDF.

A very carefully studied method to determine $\alpha_{\mathrm{S}}$ is based on the hadronic decay of the $\tau$ lepton. The basic observable measured in this method is the ratio of the hadronic to leptonic decay widths. This determination of $\alpha_{\mathrm{S}}$ shows some of the smallest theoretical uncertainties, assuming small non-perturbative corrections to the calculations. Larger corrections due to confinement effects would significantly increase the theoretical uncertainty in this method.

Non-perturbative calculations of matrix elements are performed in the lattice gauge formulation of QCD. Matching these non-perturbative results to perturbation theory provides a potentially highly accurate determination of $\alpha_{\mathrm{S}}$. Small enough lattice spacing for perturbative behavior to be seen at short distances and well controlled systematic uncertainties in the calculated hadron masses are necessary for lattice gauge theory considerations.

The original measurements of $\alpha_{\mathrm{S}}$ in $e^{+} e^{-}$annihilation use the total hadronic production cross section. Results based on the hadronic width of the $Z^{0}$ boson are more recent.

Determinations of $\alpha_{\mathrm{S}}$ are also possible from $e^{+} e^{-}$event shape and jet rate distributions. Theoretical models for these methods are calculated to NLO in $\alpha_{\mathrm{S}}$. Hadronization corrections necessary to provide a connection between perturbative predictions of event shapes and the corresponding quantities derived from experimentally observed hadrons are important contributors to the systematic uncertainties on the measurements.

Scaling violations in jet fragmentation can be used to determine $\alpha_{\mathrm{S}}$ in $e^{+} e^{-}$annihilation. The systematic uncertainties associated with these methods are generally larger than in DIS.

Some results from hadron-hadron collider experiments have been used to determine $\alpha_{\mathrm{S}}$. Direct photon, heavy quark, and $W$ boson plus jet cross sections are compared to data to extract a value for $\alpha_{\mathrm{S}}$, as predictions for these final states are 
available to NLO.

So far, the precision achieved for measurements of $\alpha_{\mathrm{S}}$ in hadron-hadron colliders has been less than in the other methods. The main experimental and theoretical uncertainties are due to the uncertainties in the incoming and outgoing hadrons. The effects of different parton distribution functions and contributions to the final states from spectator partons are important sources of systematic uncertainties.

Ratios of pure jet production rates could potentially reduce these systematic uncertainties by cancellation of some of the contributions. Direct measurements of jet production cross sections have not been possible until now, as only LO predictions were available.

With completion of the new NLO three-jet event generator such measurements can be attempted. Rather than calculating the three- to two-jet production cross section ratio, we attempt to extract a value for $\alpha_{\mathrm{S}}$ by a new method. This method is closely related to the production cross section ratio, as we are investigating the Dalitz variables which strongly depend on the jet multiplicity in an event. We plot the $\chi^{2}$ between data and NLO prediction, as defined in Equation 3.15, as a function of $\alpha_{\mathrm{S}}$. The minimum $\chi^{2}$ in this plot is the desired strong coupling constant. 


\section{Chapter 4}

\section{Unsmearing the Data}

At CDF, particle energies are measured in the electromagnetic and hadronic calorimeters. Both have cylindrical symmetry relative to the beam line. The electromagnetic calorimeter measures energy associated with the electromagnetic showers of electrically charged particles. The hadronic calorimeter measures hadron energies by collecting electromagnetic radiation from nuclear de-excitations that follow interaction of the hadrons with the calorimeter material.

The resolution of the detector is finite and the calorimeter is not hermetic. This results in a smearing of the jet energies, which in turn leads to a shift of the jet variables that are functions of the jet energies. The magnitude and the direction of the shift depend on the detector resolution and the shape of the parton-level distribution. There are two effects that determine the amount of smearing. In most data analyses, the total number of events is binned as a function of the observables of interest. Due to the detector resolution, events are counted in bins other than the one that is associated with the true jet observable. The binned distribution of the observable of interest is not necessarily symmetric about the true bin, more entries are then passed into adjacent bins in one direction than are received in return. If the parton-level distribution changes rapidly between adjacent bins, the passing of a certain fraction

of entries from the bin with the larger number of events to the one with the lower 
number also affects the shape of the observed distribution more than the reverse process.

This chapter treats the unsmearing of the Dalitz variables, $\mathrm{X}_{3}$ and $\mathrm{X}_{4}$, for CDF Run 1b data. This unsmearing is necessary in order to be able to directly compare the data to parton-level Monte Carlo calculations since these detector resolution effects are not included in the predictions.

\subsection{True and Measured Values of an Observable}

In the analysis of the unsmearing correction factors, we carefully distinguish between the true and the measured spectrum of a generic observable, $\mathrm{X}$, that depends on the detector energy measurement. The way we find both spectra is described in Reference [35] and is summarized briefly here. We generate Monte Carlo events with the HERWIG Monte Carlo program. Any other suitable parton-level event generator could also be used. A cone size of $\mathrm{R}=0.7$ is used in the clustering of all final state particles into jets. The true value of $\mathrm{X}$ is a function of the total energies in these clusters. This clustering is done at the parton level, before any detector simulation is applied to the HERWIG output. To compute the measured value of $\mathrm{X}$, the detector simulation QFL is applied to the Monte Carlo events, the same clustering algorithm is used, and the energies of the tracks in the same reconstruction cones serve as the basis to calculate $\mathrm{X}^{\text {meas }}$.

\subsection{True Level Clustering}

The clustering at the parton, or "true," level is a crucial part of the unsmearing procedure. It has been shown in Reference [35] that the module PYTTOW performs well in the true level clustering. It is used in the unsmearing studies of the Dalitz variables. 
PYTTOW uses the GENP information from HERWIG and creates TOWE banks, simulating an "ideal calorimeter." The standard jet algorithm, JETCLU, can be applied to the TOWE banks. JETCLU produces the JETS banks which contain the relevant jet information for jets in a cone of user-specified cone size. It uses the iterative cone algorithm described in Reference [54] to compute the jet axis and associate calorimeter tower information within the cone to the jet.

\subsection{The Monte Carlo Unsmearing Procedure}

For our analysis of the unsmearing of the Dalitz variables, we use a total of 439,313 HERWIG events. The values of $\mathrm{X}^{\text {true }}$ and $\mathrm{X}^{\text {meas }}$ are calculated for all these Monte Carlo events. The Dalitz plane is divided into bins of size $0.02 \times 0.02$.

The unsmearing factor in the Dalitz plane is computed by defining:

$$
\mathrm{K}_{i}^{\mathrm{mc}} \equiv \frac{\text { number of events in bin } i \text { of the smeared distribution }}{\text { number of events in bin } i \text { of the true distribution }} .
$$

This method to calculate $\mathrm{K}$ in every bin is called Monte Carlo approach. Figure 4.1 shows $\mathrm{K}$ for each bin in the Dalitz plane. The projections of $\mathrm{K}$, as computed by the Monte Carlo method, onto $\mathrm{X}_{3}$ and $\mathrm{X}_{4}$ are shown in Figures 4.2 and 4.3. The size of K obtained by the Monte Carlo approach is shown in Figure 4.4.

The Monte Carlo generator, HERWIG, used to calculate the smearing correction factor has a large set of input parameters that can be set by the user. One such input parameter, the minimum $\mathrm{p}_{\mathrm{T}}$ of the two outgoing partons, effects the jet $\mathrm{E}_{\mathrm{T}}$ distributions of the HERWIG sample (see detailed discussion in Chapter 6 below.) The effect of this input parameter on the smearing correction factor is investigated. The smearing correction factor in all the bins used in this analysis is found to be consistent for different settings of the minimum $\mathrm{p}_{\mathrm{T}}$ in HERWIG. This is expected since the smearing of the Dalitz variables is due to the detector response to a signal and the shape of the Dalitz distribution. The detector response is a function of the energy of the jets, regardless of the specifics of the jet $\mathrm{E}_{\mathrm{T}}$ distribution. At the same time, 


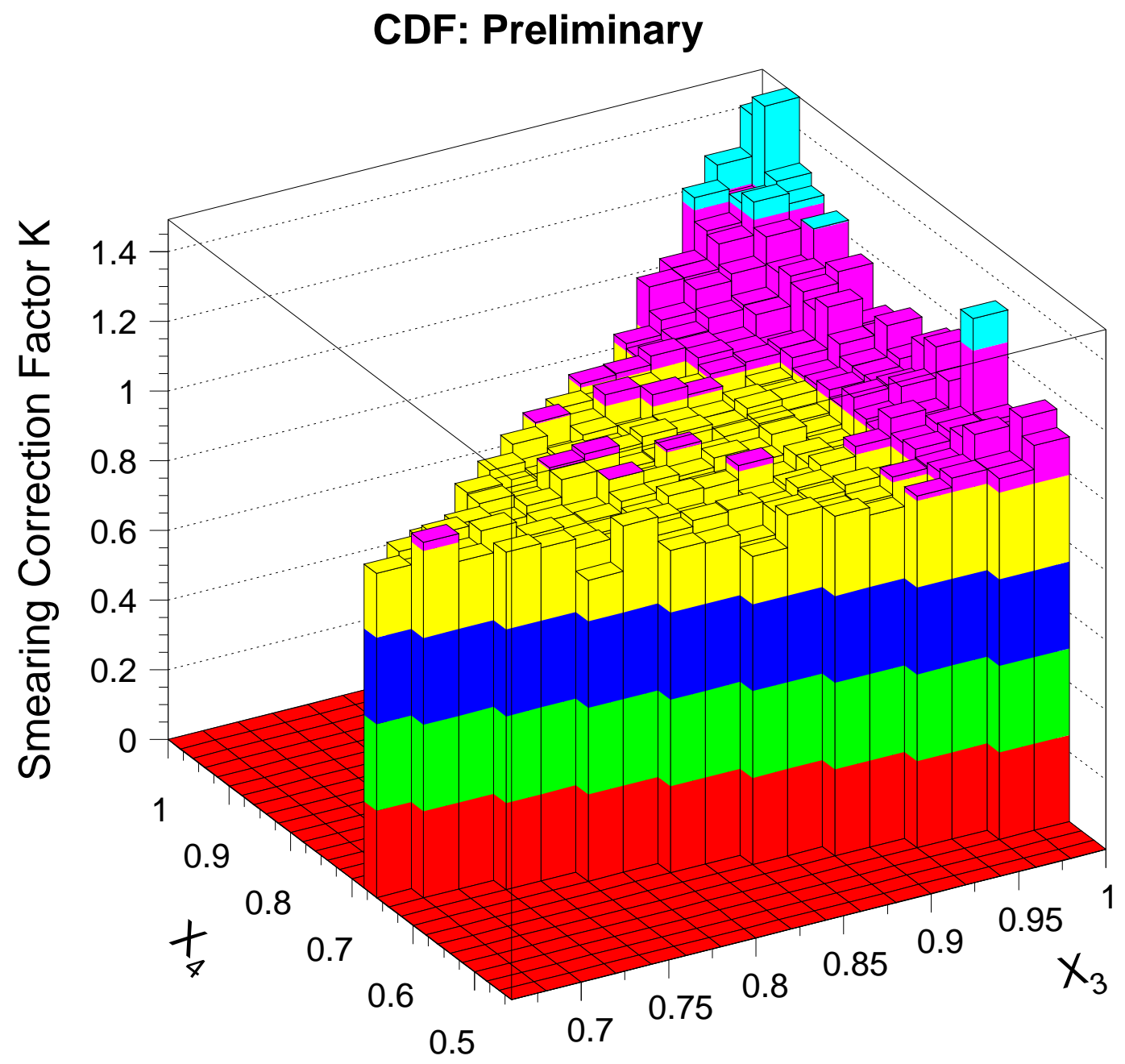

Figure 4.1: The smearing correction factor, K, for bins of size $0.02 \times 0.02$ in the Dalitz plane, as calculated with the Monte Carlo method. 


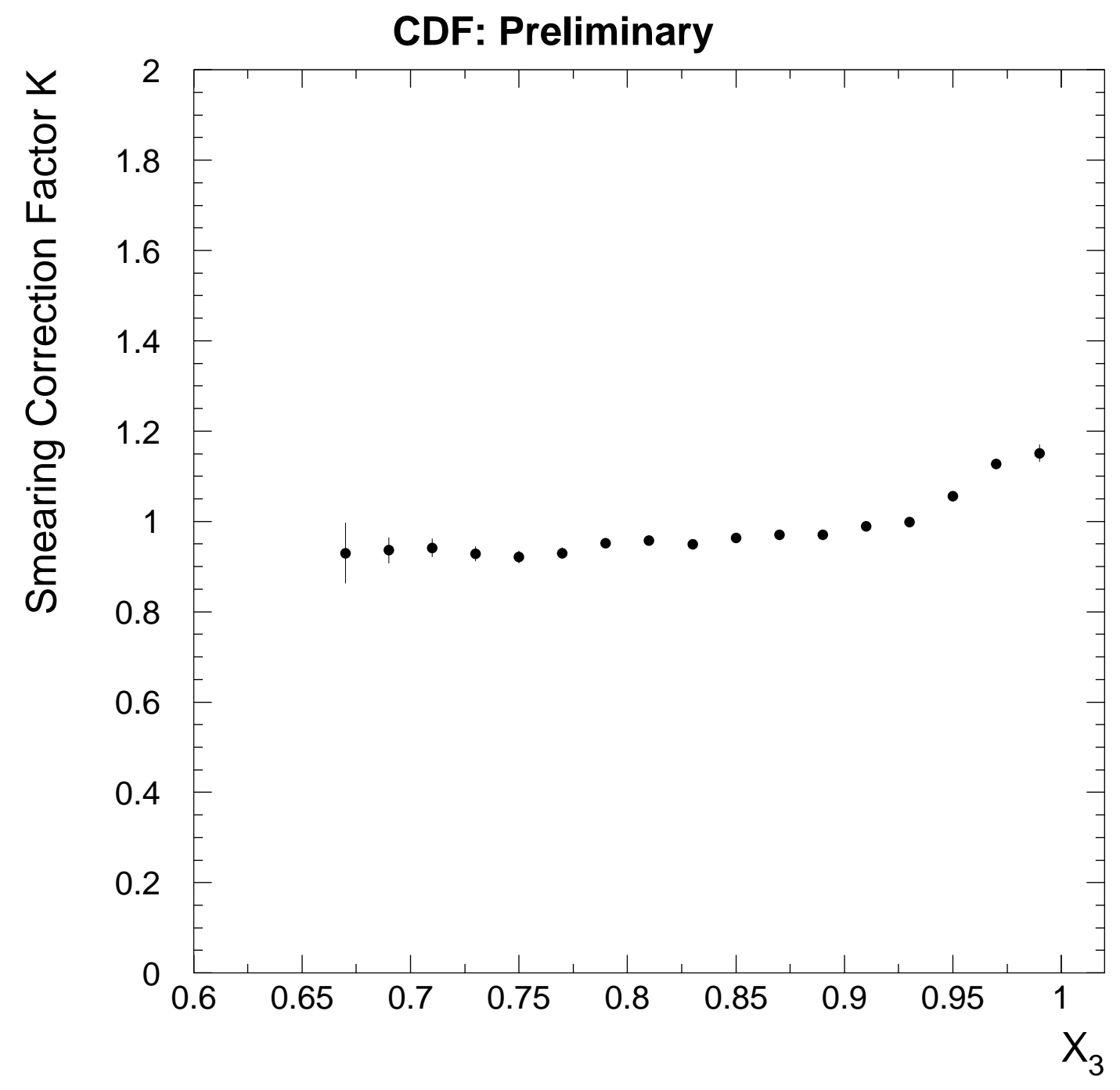

Figure 4.2: The smearing correction factor, $\mathrm{K}$, for bins of size 0.02 in $\mathrm{X}_{3}$, as calculated with the Monte Carlo method. 


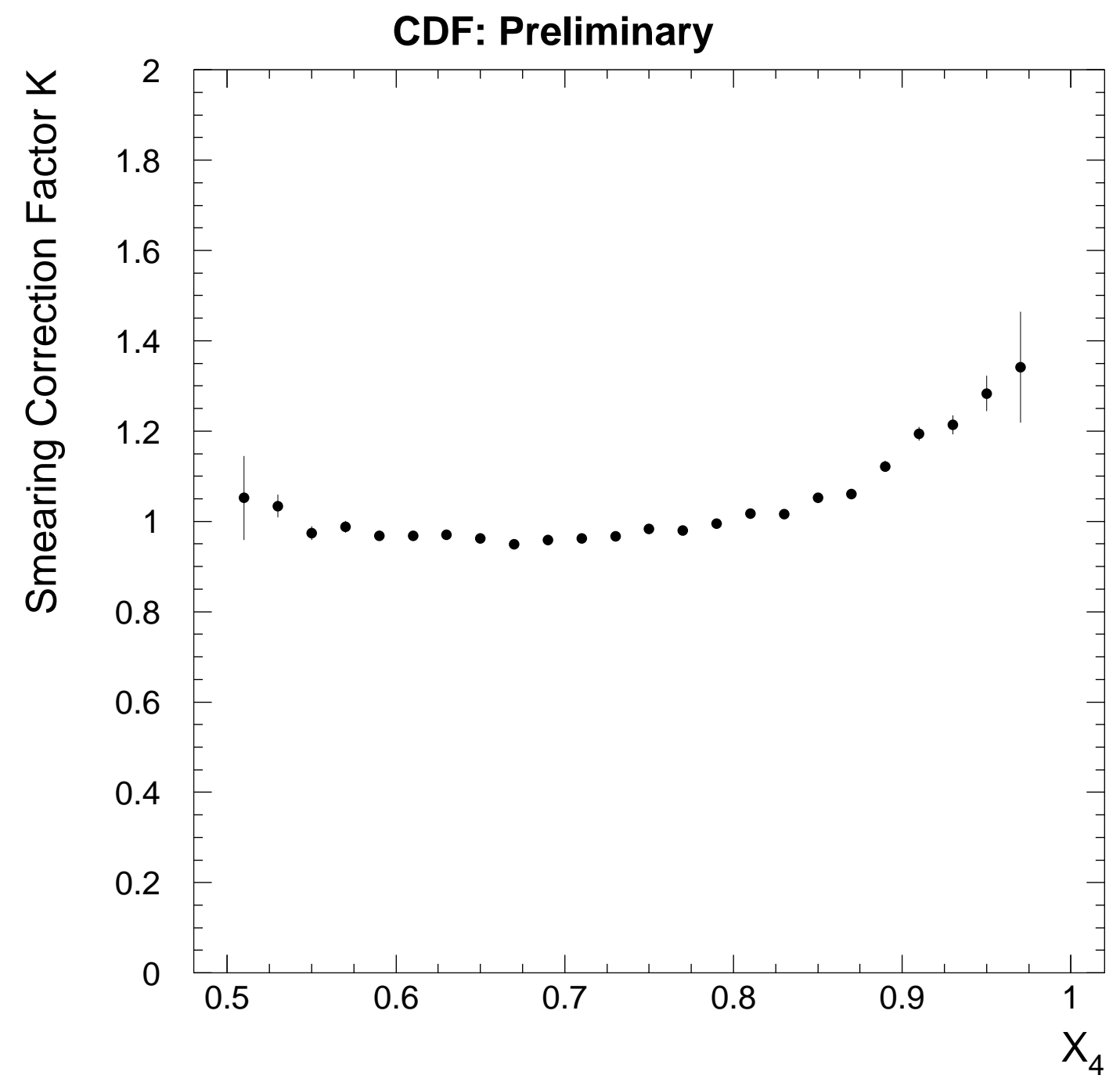

Figure 4.3: The smearing correction factor, $\mathrm{K}$, for bins of size $0.02 \mathrm{in} \mathrm{X}_{4}$, as calculated with the Monte Carlo method. 


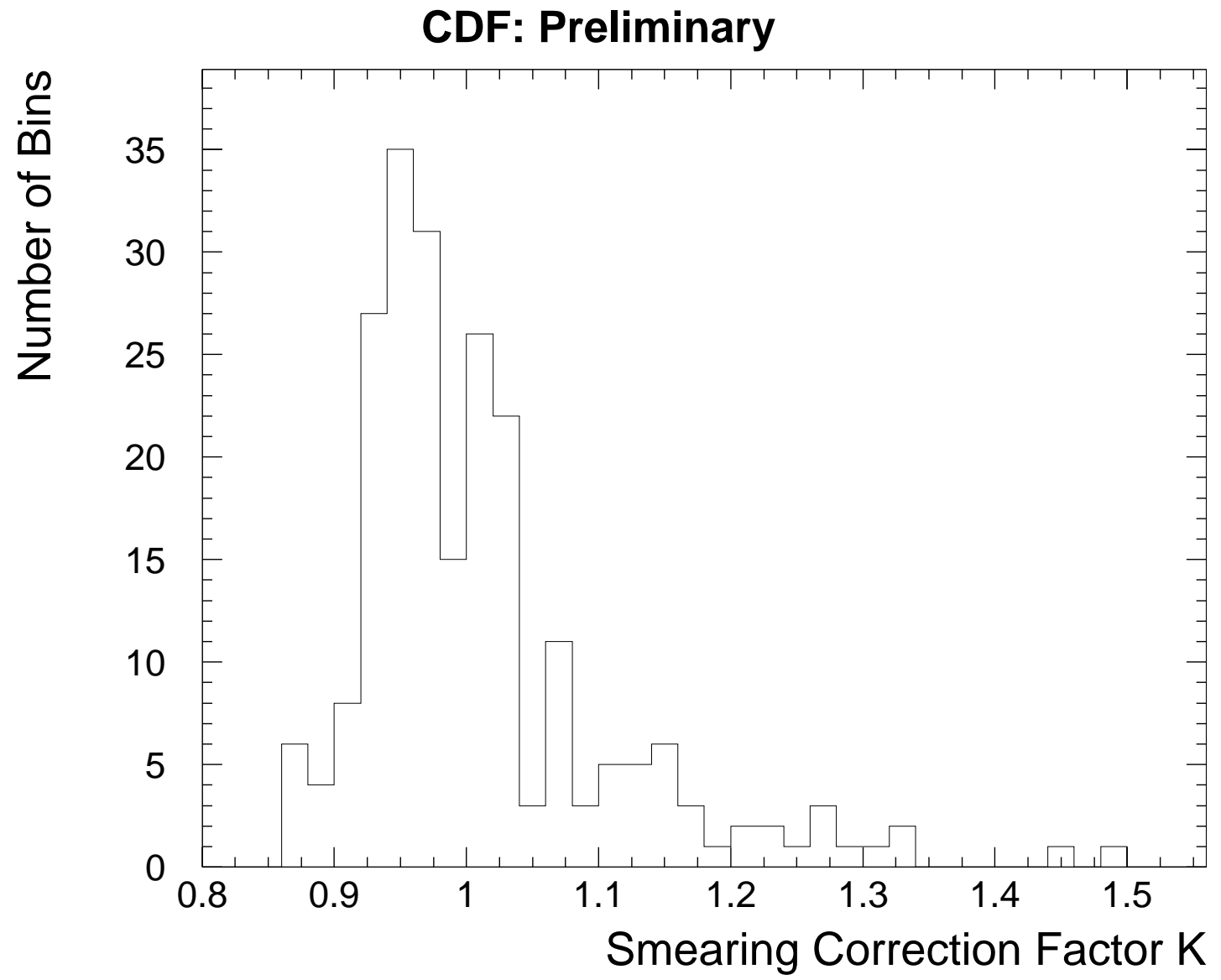

Figure 4.4: The size of the smearing correction factor, K, as calculated with the Monte Carlo method. 
the distribution of the Dalitz variables is not too steep, so that bin-to-bin migration effectively is low. Both these reasons together account for the negligible influence of the minimum $\mathrm{p}_{\mathrm{T}}$ on the smearing correction factor. Figure 4.5 shows the smearing correction factor for bins of size 0.02 in $\mathrm{X}_{3}$ and $\mathrm{X}_{4}$, as calculated with the Monte Carlo method for a minimum $\mathrm{p}_{\mathrm{T}}$ of $60 \mathrm{GeV}$ and $150 \mathrm{GeV}$.

The internal consistency of the unsmearing procedure is checked by an independent HERWIG sample, containing 72,990 events. The fractional difference between the unsmeared measured spectrum and the true spectrum provides a measure of the internal consistency of the procedure. This fractional difference in the Dalitz plane is tabulated in Tables A.1 through A.14 in the Appendix A. The projections of this fractional difference onto the $\mathrm{X}_{3}$ and $\mathrm{X}_{4}$ axes are shown in Figures 4.6 and 4.7. The internal consistency of the method is also demonstrated in Figures 4.8 and 4.9 where the difference between the unsmeared measured and the true Dalitz distributions, scaled by its uncertainty, is shown.

The Monte Carlo approach yields internally consistent smearing correction factors. The smearing correction factors obtained by this method are used in the analysis of the Dalitz variables discussed in the following chapters. An alternative approach, the analytical unsmearing method, which is not internally consistent for this analysis is described in the Appendix B.

\subsection{Unsmearing the Jet Transverse Energy}

Information of particular interest in any high energy data analysis is the jet transverse energy. Transverse energies are generally well modeled by theoretical predictions and are often used in comparing data to models.

As $\mathrm{E}_{\mathrm{T}}$ is a function of the jet energy, it is also subject to smearing by the detector. A smearing correction factor has to be calculated to correctly compare $\mathrm{E}_{\mathrm{T}}$ information from data and prediction. The main emphasis in this thesis is on the 
CDF: Preliminary
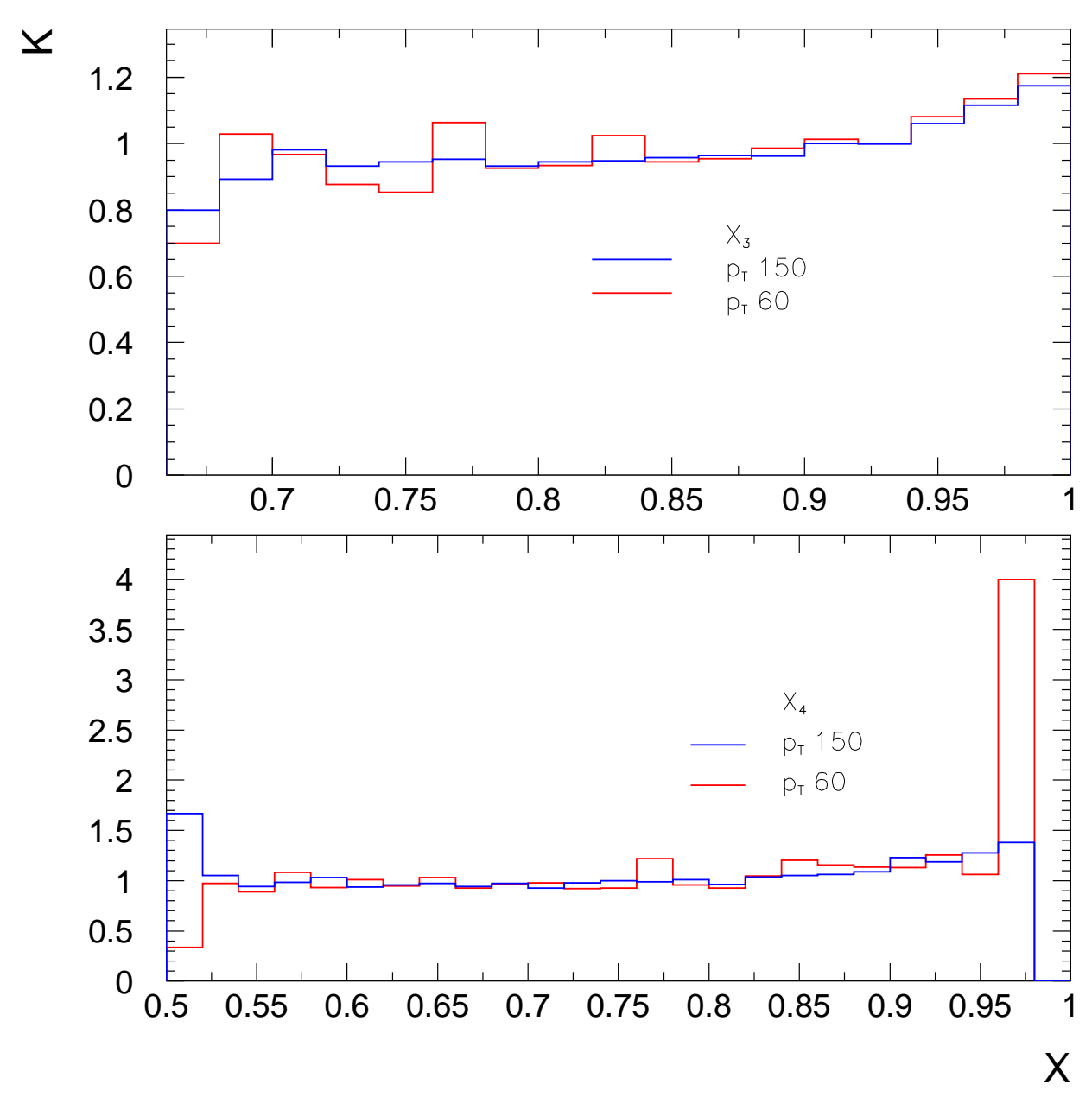

Figure 4.5: Comparison of the smearing correction factor, K, for bins of size 0.02 in $\mathrm{X}_{3}$ and $\mathrm{X}_{4}$, as calculated with the Monte Carlo method for a minimum $\mathrm{p}_{\mathrm{T}}$ of $60 \mathrm{GeV}$ and $150 \mathrm{GeV}$. 


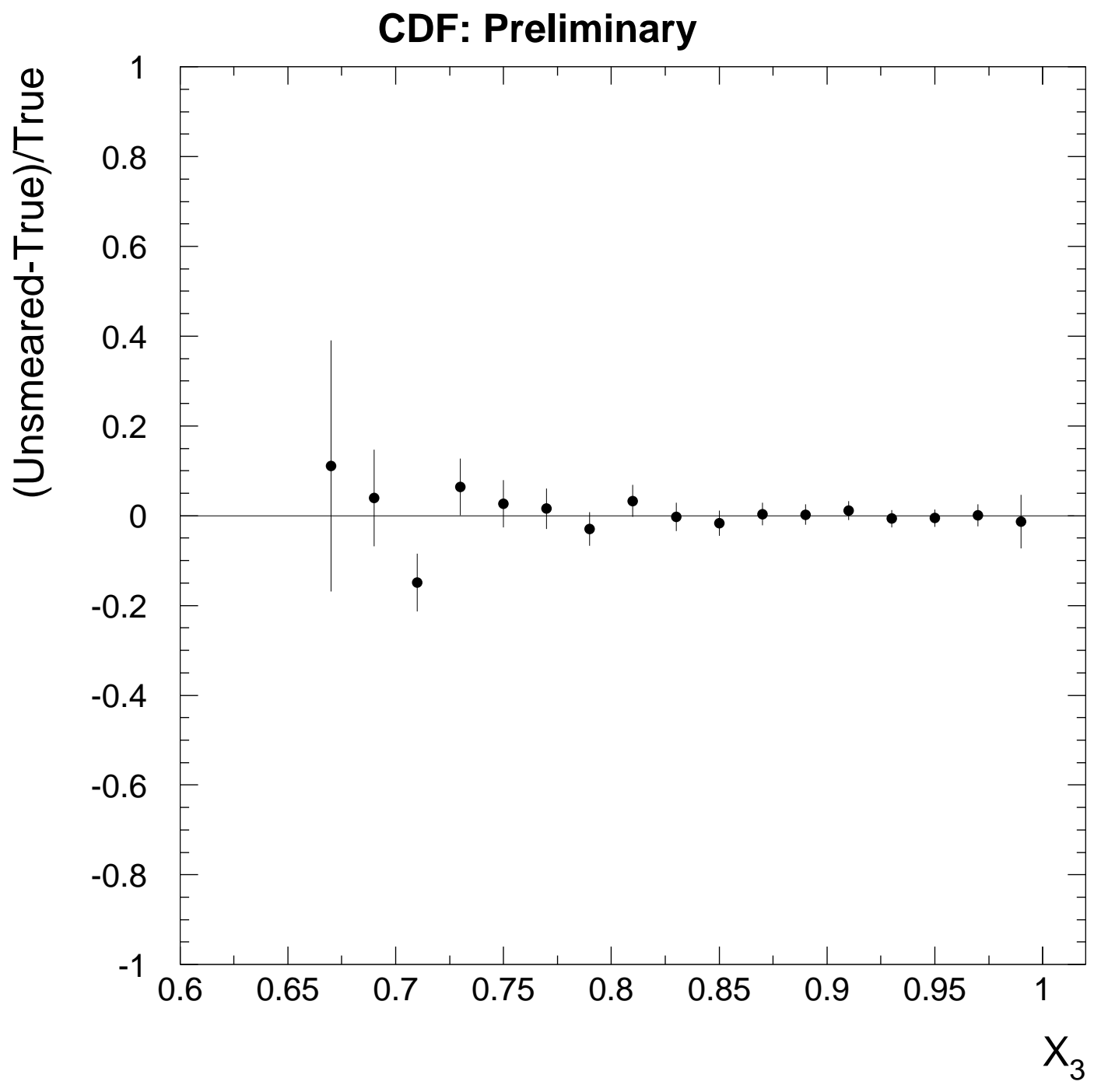

Figure 4.6: The fractional difference between the unsmeared measured and the true $\mathrm{X}_{3}$ distributions. 


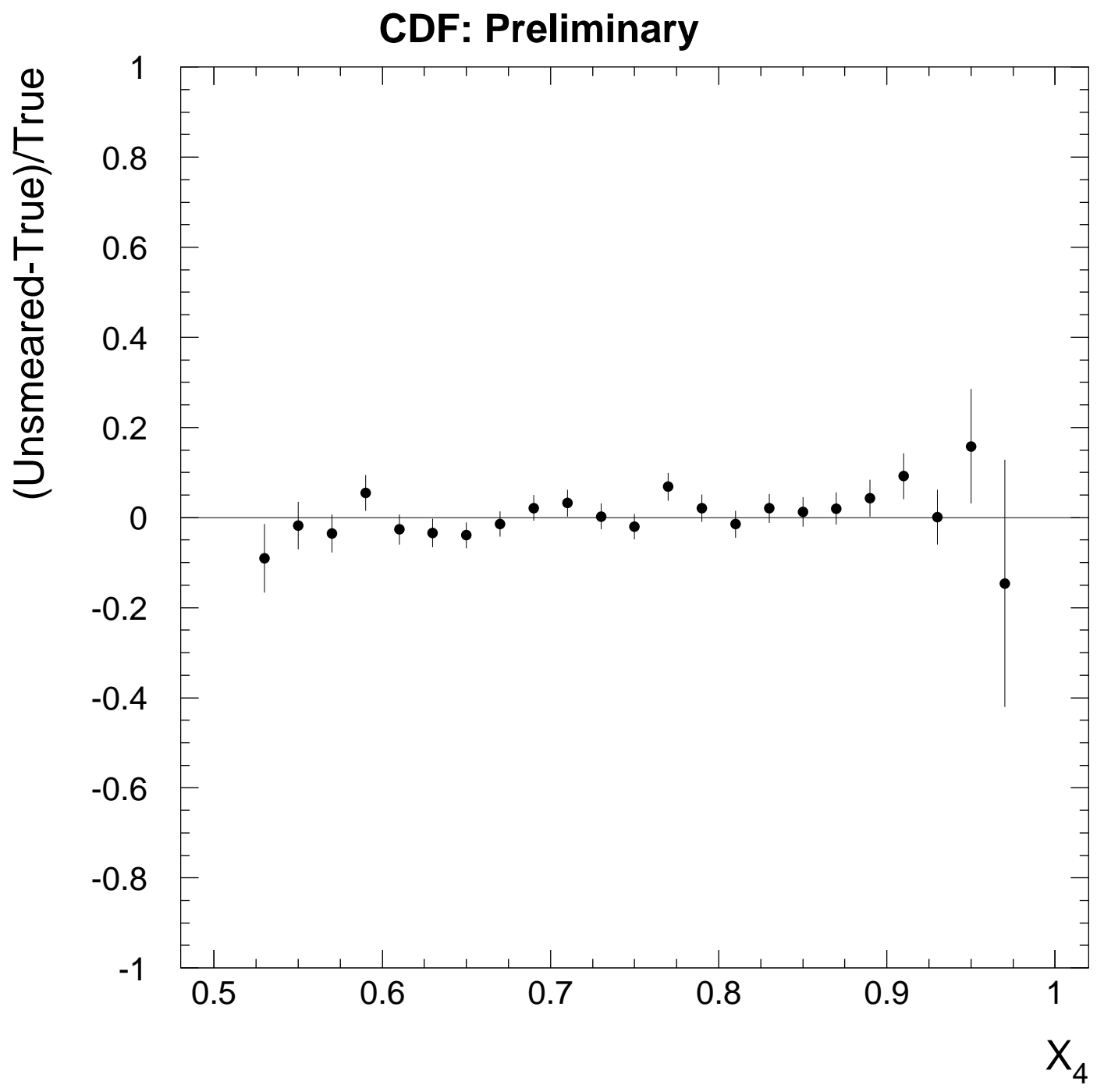

Figure 4.7: The fractional difference between the unsmeared measured and the true $\mathrm{X}_{4}$ distributions. 


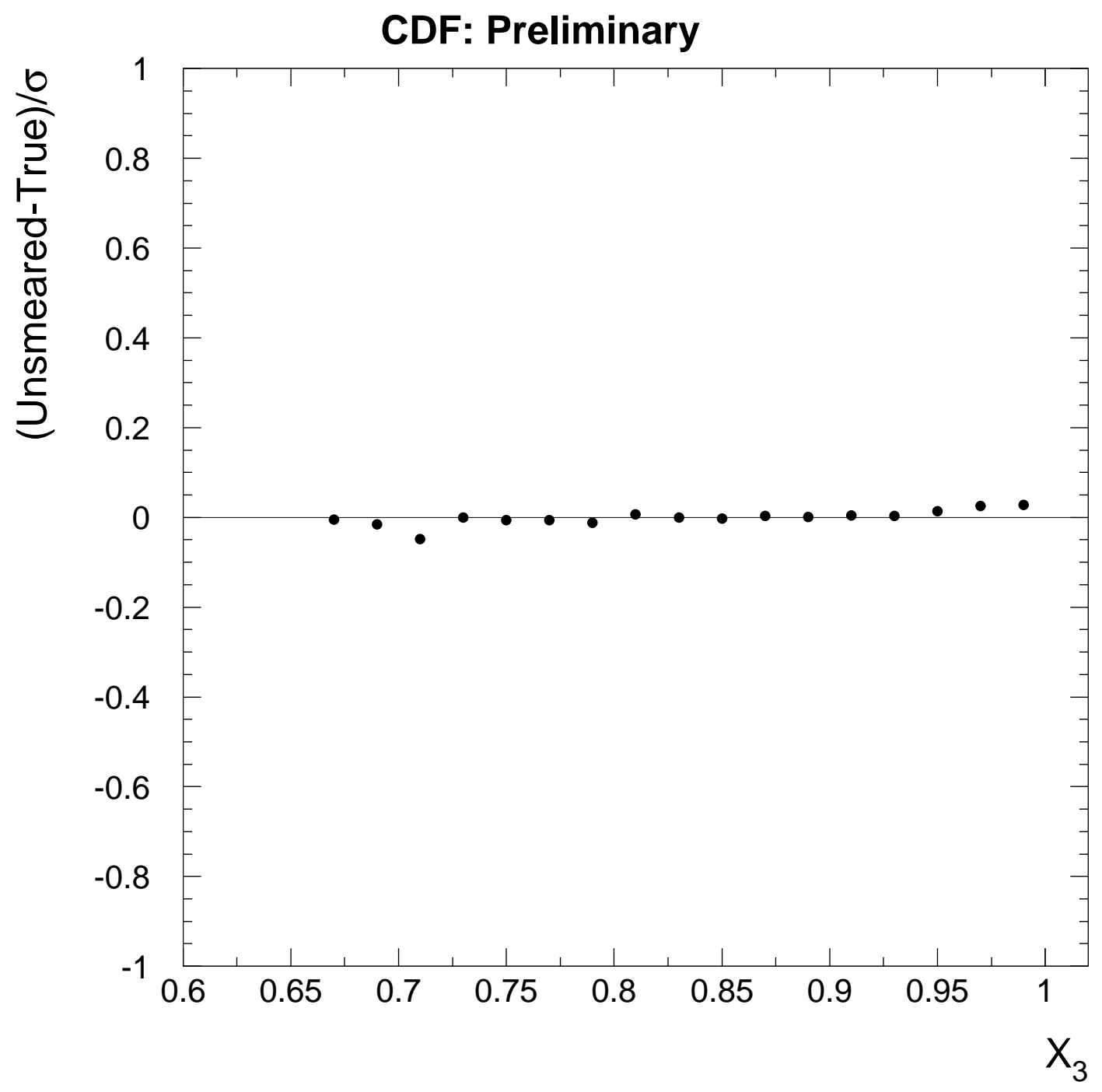

Figure 4.8: The difference between the unsmeared measured and the true $\mathrm{X}_{3}$ distributions, scaled by its uncertainty. 


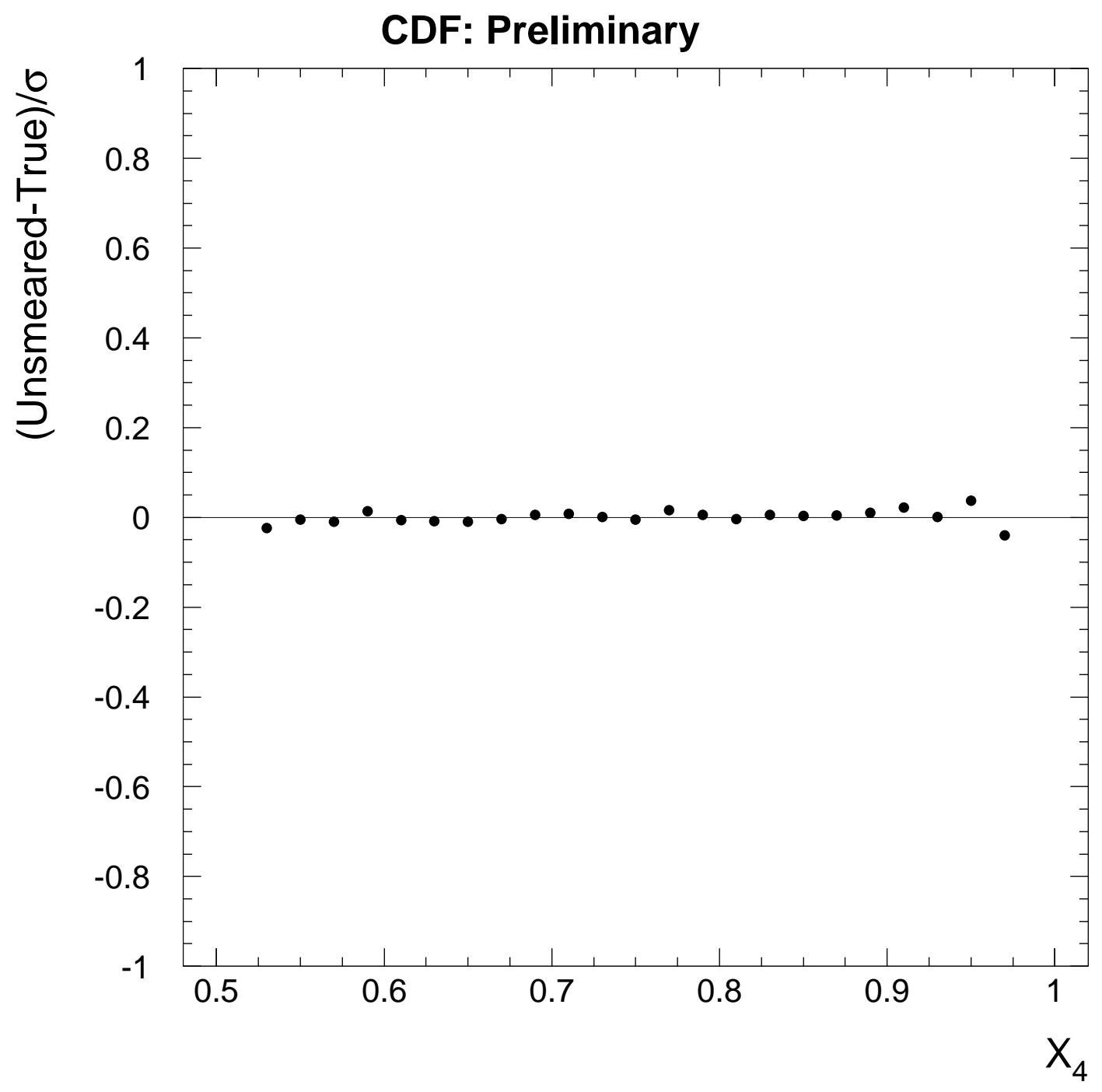

Figure 4.9: The difference between the unsmeared measured and the true $\mathrm{X}_{4}$ distributions, scaled by its uncertainty. 
analysis of the Dalitz variables. The $\mathrm{E}_{\mathrm{T}}$ information is only used for some checks on the results. Unsmearing of the jet $\mathrm{E}_{\mathrm{T}}$ therefore only plays a subordinate role.

The smearing correction factors for the Dalitz variables are not consistent for the analytical and the Monte Carlo method. However, the systematic uncertainty in the Monte Carlo method is found to be small. It is the approach chosen for computation of the $\mathrm{E}_{\mathrm{T}}$ smearing correction factors.

Analogous to the unsmearing procedure for the Dalitz variables, HERWIG is used for unsmearing $\mathrm{E}_{\mathrm{T}}$. We use a total of 439,313 HERWIG events. The values of $\mathrm{E}_{\mathrm{T}}^{\text {true }}$ and $\mathrm{E}_{\mathrm{T}}^{\text {meas }}$ are calculated for the first three jets in these Monte Carlo events. The jet $\mathrm{E}_{\mathrm{T}}$ distributions are binned in bins of $10 \mathrm{GeV}$ increments. The smearing correction factor is calculated by the ratio of the measured and the true $\mathrm{E}_{\mathrm{T}}$ distributions in every bin. Theoretical assumptions in the generation of HERWIG jets do not contribute. As smearing is solely due to detector effects, and since the CDF detector simulation, QFL, is assumed to describe the detector well, the particular mode of generation of a jet does not influence the magnitude of the smearing correction factor. The smearing correction factors calculated for $\mathrm{E}_{\mathrm{T}}$ in this manner are tabulated in Tables C.1 through C.3 in Appendix C.

The internal consistency of the unsmearing procedure is checked by an independent HERWIG sample, containing 72,990 events. The fractional difference between the unsmeared measured spectrum and the true spectrum provides a measure of the internal consistency of the procedure. This fractional difference in the $\mathrm{E}_{\mathrm{T}}$ distribution is shown in Figure 4.10. The internal consistency of the method is also demonstrated in Figure 4.11 where the difference between the unsmeared measured and the true $\mathrm{E}_{\mathrm{T}}$ distributions, scaled by its uncertainty, is shown. 


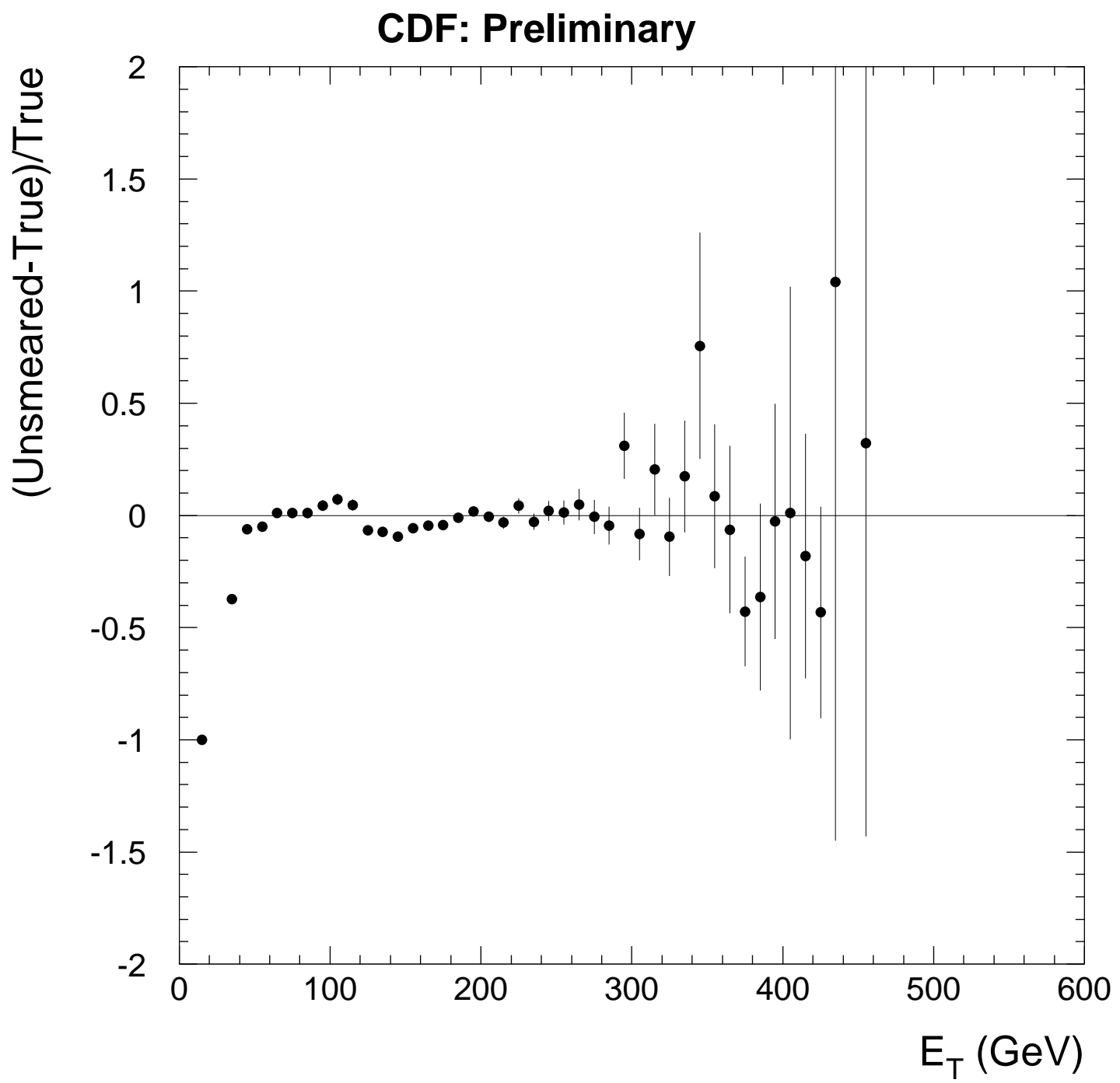

Figure 4.10: The fractional difference between the unsmeared measured and the true $\mathrm{E}_{\mathrm{T}}$ distributions. 


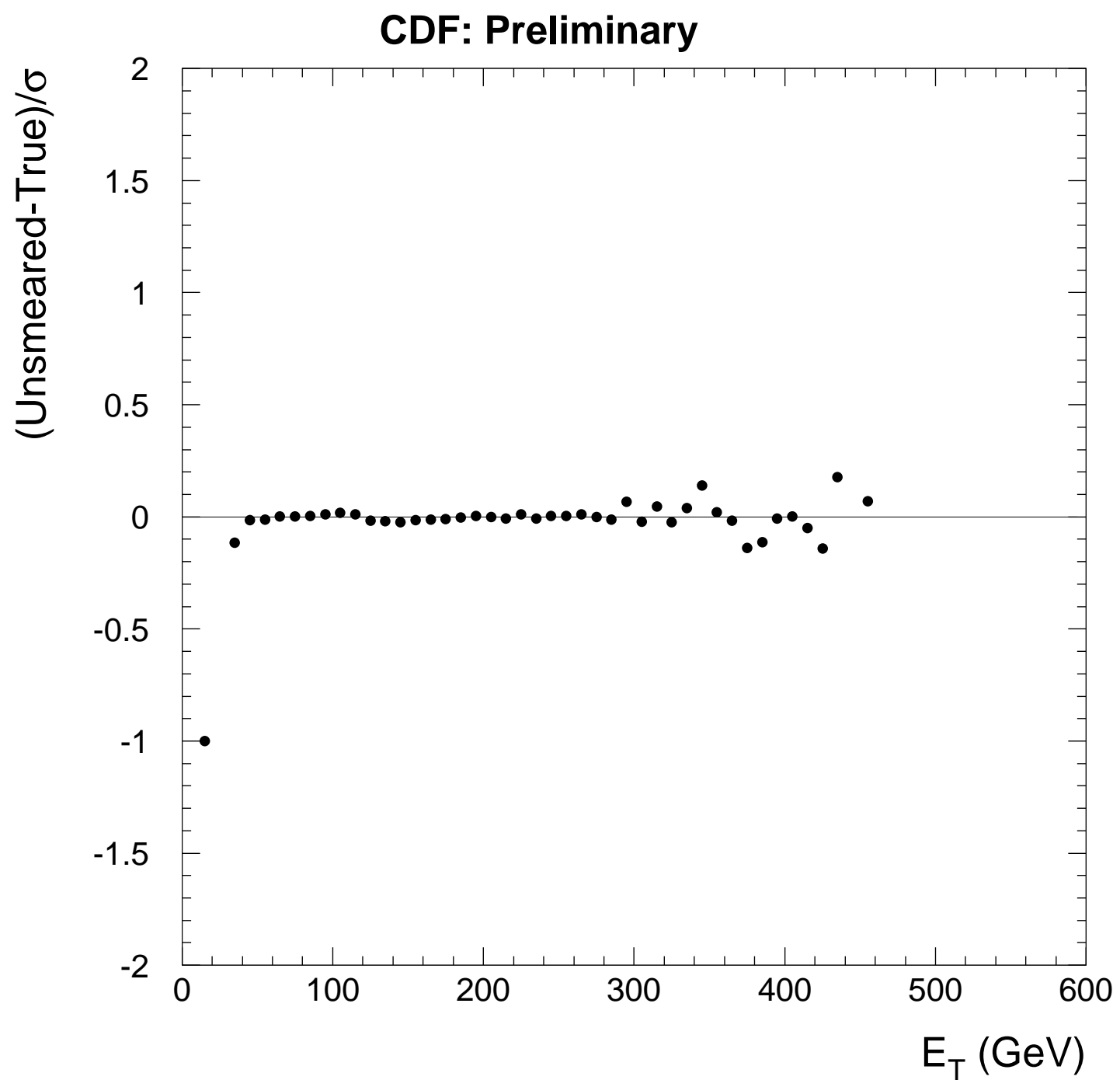

Figure 4.11: The difference between the unsmeared measured and the true $\mathrm{E}_{\mathrm{T}}$ distributions, scaled by its uncertainty. 


\section{Chapter 5}

\section{Systematic Uncertainties}

Energy measurements in the CDF detector are subject to systematic uncertainties associated with the detector response to particle beams used for calibration, detector stability over time, and energy deposited in the detector due to spectator partons. Uncertainties on trigger and measurement efficiencies and on corrections applied to the data contribute to the total systematic measurement uncertainty.

This chapter treats the systematic uncertainties on the Dalitz variables for CDF Run $1 \mathrm{~b}$ data. The Dalitz variables and the other three-jet variables that uniquely describe a three-jet system are defined in earlier chapters.

The systematic uncertainties considered in this chapter are associated with the absolute and relative jet energy scales, the total integrated luminosity, the $z$-vertex cut efficiency, and the systematic uncertainty on the smearing correction factor discussed in the previous chapter.

\subsection{Systematic Uncertainties on the Dalitz Vari- ables}

Five sources of systematic uncertainties on the Dalitz variables are discussed in this chapter. Uncertainties on the absolute and relative ( $\eta$-dependent) jet energy scales 
are due to uncertainties in jet energy measurements. They are the largest contributors to the total systematic uncertainty. The uncertainties on the measurements of the total integrated luminosity and the z-vertex cut efficiency are considered, and the uncertainty on the smearing correction factor is included.

\subsubsection{Absolute Jet Energy Scale}

The absolute jet energy scale uncertainty consists of four components [35]:

1. The uncertainty on the calibration of the central calorimeter is asymmetrical. The high-side uncertainty decreases from $1.8 \%$ to $1.7 \%$ as a function of jet transverse energy, $\mathrm{E}_{\mathrm{T}}$, while the low-side decreases from $1.6 \%$ to $1.3 \%$ [63].

2. The jet fragmentation uncertainty, which is also slightly asymmetrical, decreases from $1.7 \%$ to $1.2 \%$ with increasing jet $\mathrm{E}_{\mathrm{T}}$ [63].

3. The stability of the calorimeter over time is estimated to have an uncertainty of $1 \%$ on the jet $\mathrm{E}_{\mathrm{T}}[35]$.

4. The uncertainty on the underlying event correction in the jet energy correction, JTC96, is $1.16 \mathrm{GeV}$ on the jet $\mathrm{E}_{\mathrm{T}}$.

The estimate of the uncertainty on the underlying event correction has been obtained by following the method outlined in Reference [57]. The average number of vertices per event in CDF data is used as basis to calculate the underlying event correction. For a cone size of 0.7 , the underlying event correction is given by $((1.98 / 1.6) * 1.0+$ $0.91 *($ number of vertices per event -1.0$)) * 1.6$. The relative uncertainty on the underlying event correction is estimated to amount to $30 \%$. The number of vertices in the events is studied, as the cut on the resolved multiple interactions might have changed the average number of vertices per event in the data sample. Investigation of the data sample shows on average of 2.3 vertices per event, reduced from an average number of 2.7 before removal of the resolved multiple interactions. If instantaneous 
luminosities $>15 \times 10^{30} \mathrm{~cm}^{-2} \mathrm{~s}^{-1}$ are neglected, the average number of vertices per event is 2.2. Estimation of the uncertainty on the underlying event correction yields values of $1.34 \mathrm{GeV}, 1.16 \mathrm{GeV}$, and $1.12 \mathrm{GeV}$ for the data samples before and after the removal of resolved multiple interactions and after rejection of the highest instantaneous luminosities, respectively. As, the cut on instantaneous luminosities only reduces the uncertainty on the underlying event correction by $3.4 \%$ but reduces the total number of events in the data sample at the same time, the cut is not implemented in the analysis.

These upper, and lower, limits are added, or subtracted, from the four-momenta of all jets in the data sample to obtain the systematic uncertainties on the Dalitz variables due to each component. Figures 5.1 to 5.8 show the systematic uncertainties on the Dalitz variables as a function of $\mathrm{X}_{3}$ for every bin in $\mathrm{X}_{4}$. The systematic uncertainty due to the detector calibration is plotted in Figures 5.1 and 5.2. Figures 5.3 and 5.4 show the uncertainty on the Dalitz variables due to jet fragmentation, while the uncertainty on the detector stability is shown in Figures 5.5 and 5.6. The effect of the underlying event correction uncertainty on the Dalitz variables is plotted in Figures 5.7 and 5.8. Fluctuations between neighboring bins are largely due to statistical fluctuations in the data.

\subsubsection{Relative Jet Energy Scale}

The systematic uncertainty due to the uncertainty in the relative jet energy scale is calculated following the method described in Reference [35]. Three regions of the detector with different contributions to the systematic uncertainty are identified:

1. the ninety degree crack, for which $\left|\eta_{d}\right|<0.15$,

2. the thirty degree crack, for which $0.9<\left|\eta_{d}\right|<1.4$, and

3. the Plug Calorimeter, for which $\left|\eta_{d}\right|>1.4$. 


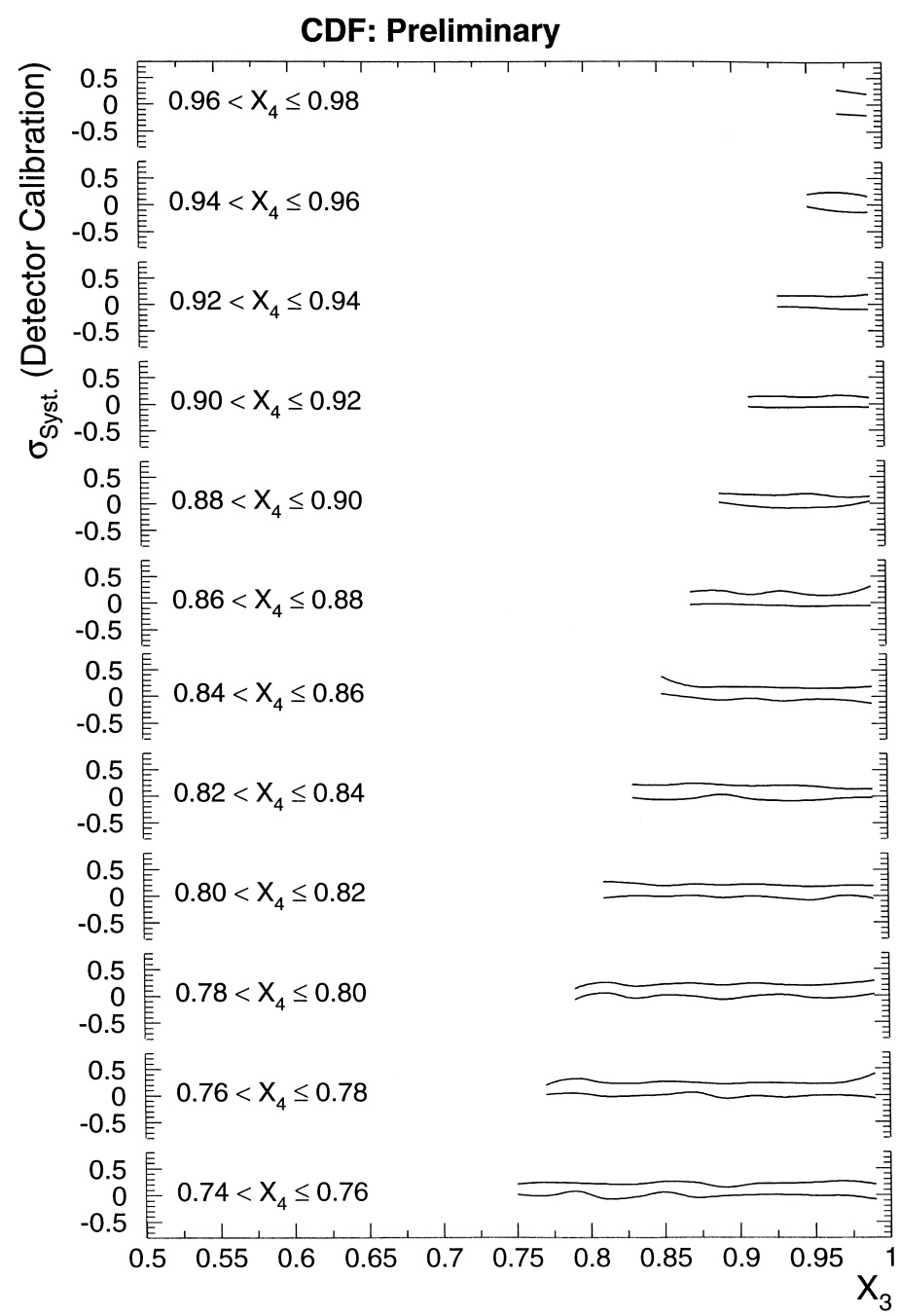

Figure 5.1: The systematic uncertainty on the Dalitz variables due to the uncertainty on the detector calibration as a function of $\mathrm{X}_{3}$ for $0.74<\mathrm{X}_{4} \leq 0.98$. 


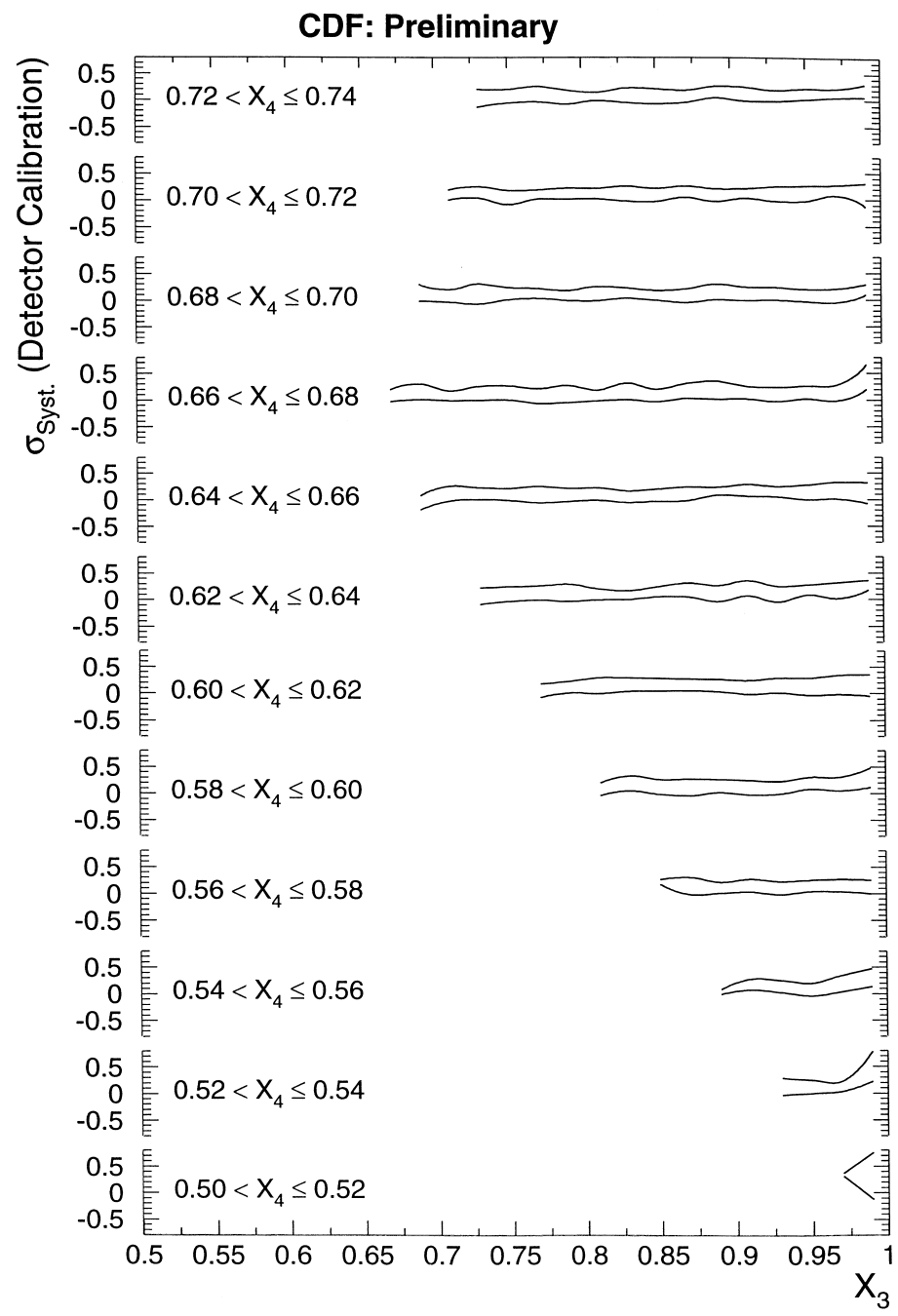

Figure 5.2: The systematic uncertainty on the Dalitz variables due to the uncertainty on the detector calibration as a function of $\mathrm{X}_{3}$ for $0.50<\mathrm{X}_{4} \leq 0.74$. 


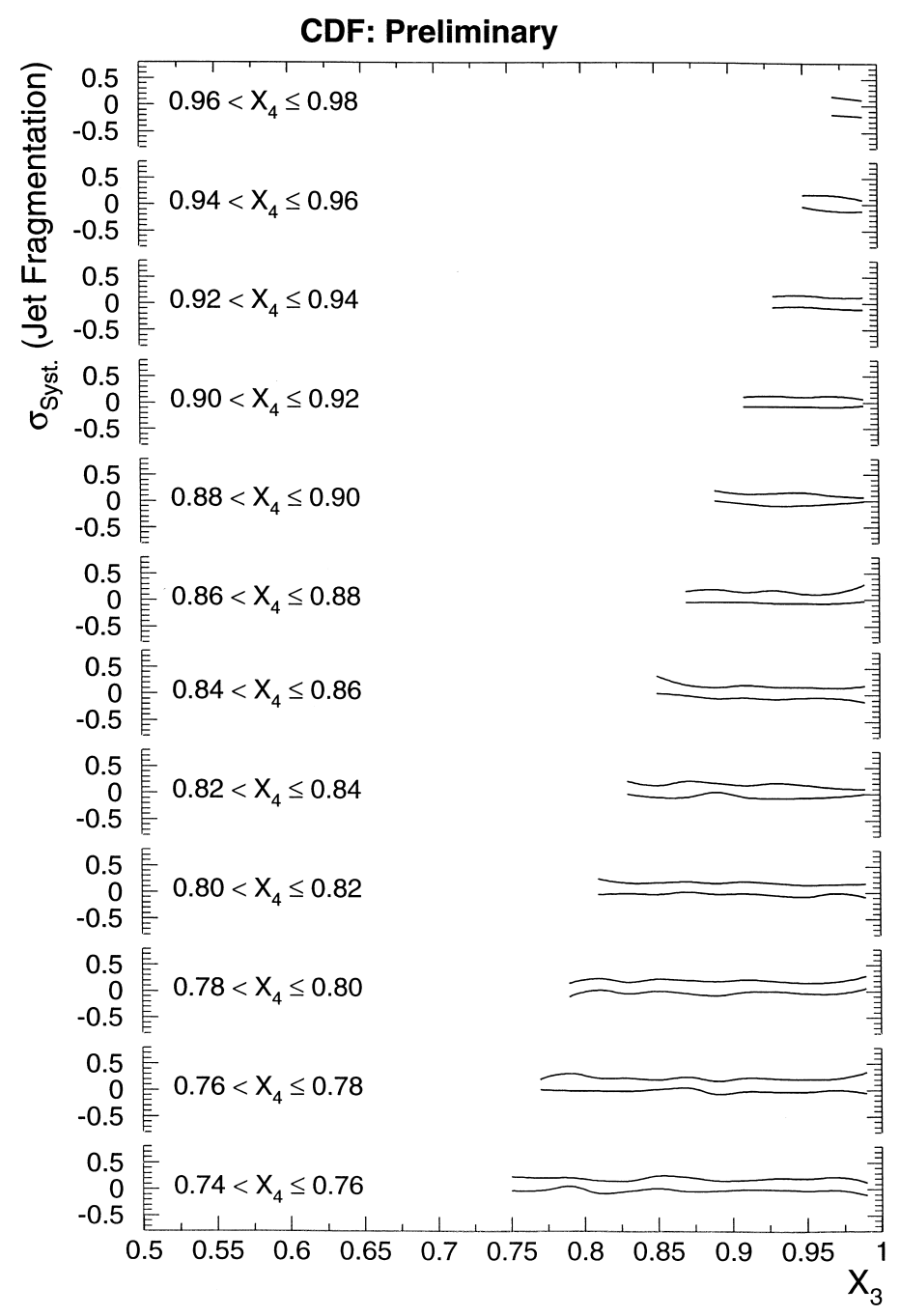

Figure 5.3: The systematic uncertainty on the Dalitz variables due to the uncertainty on the jet fragmentation as a function of $\mathrm{X}_{3}$ for $0.74<\mathrm{X}_{4} \leq 0.98$. 


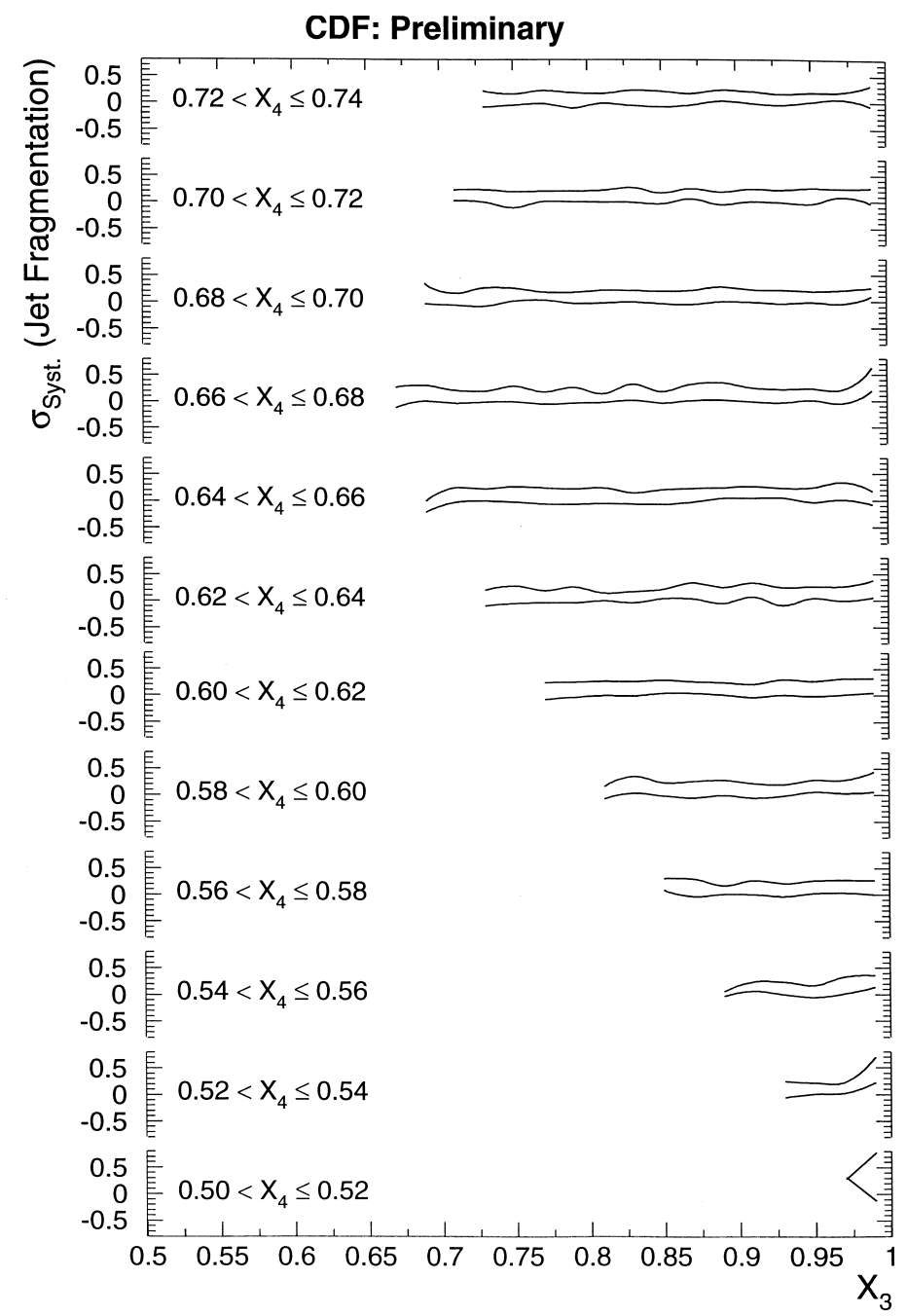

Figure 5.4: The systematic uncertainty on the Dalitz variables due to the uncertainty on the jet fragmentation as a function of $\mathrm{X}_{3}$ for $0.50<\mathrm{X}_{4} \leq 0.74$. 


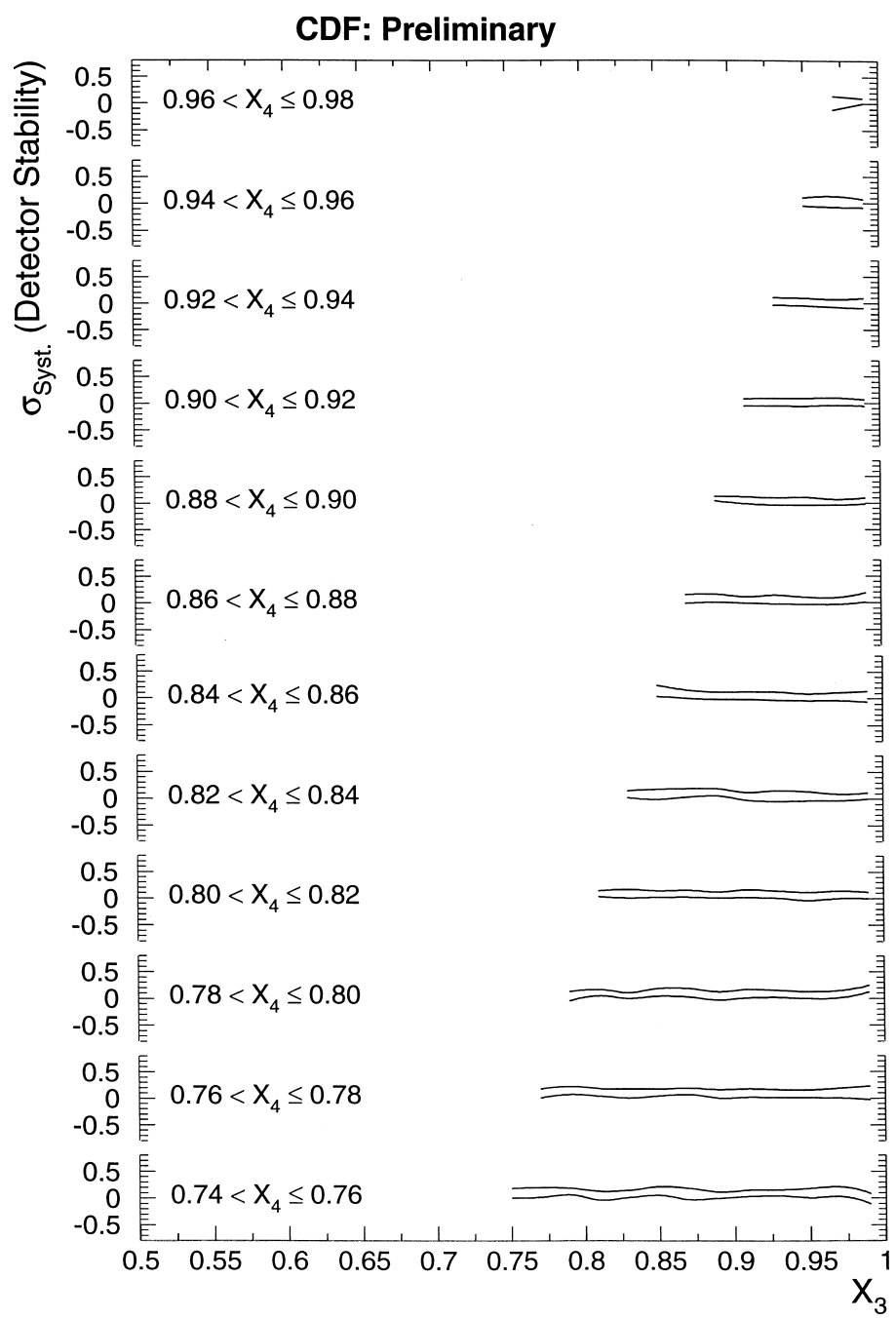

Figure 5.5: The systematic uncertainty on the Dalitz variables due to the uncertainty on the detector stability as a function of $\mathrm{X}_{3}$ for $0.74<\mathrm{X}_{4} \leq 0.98$. 


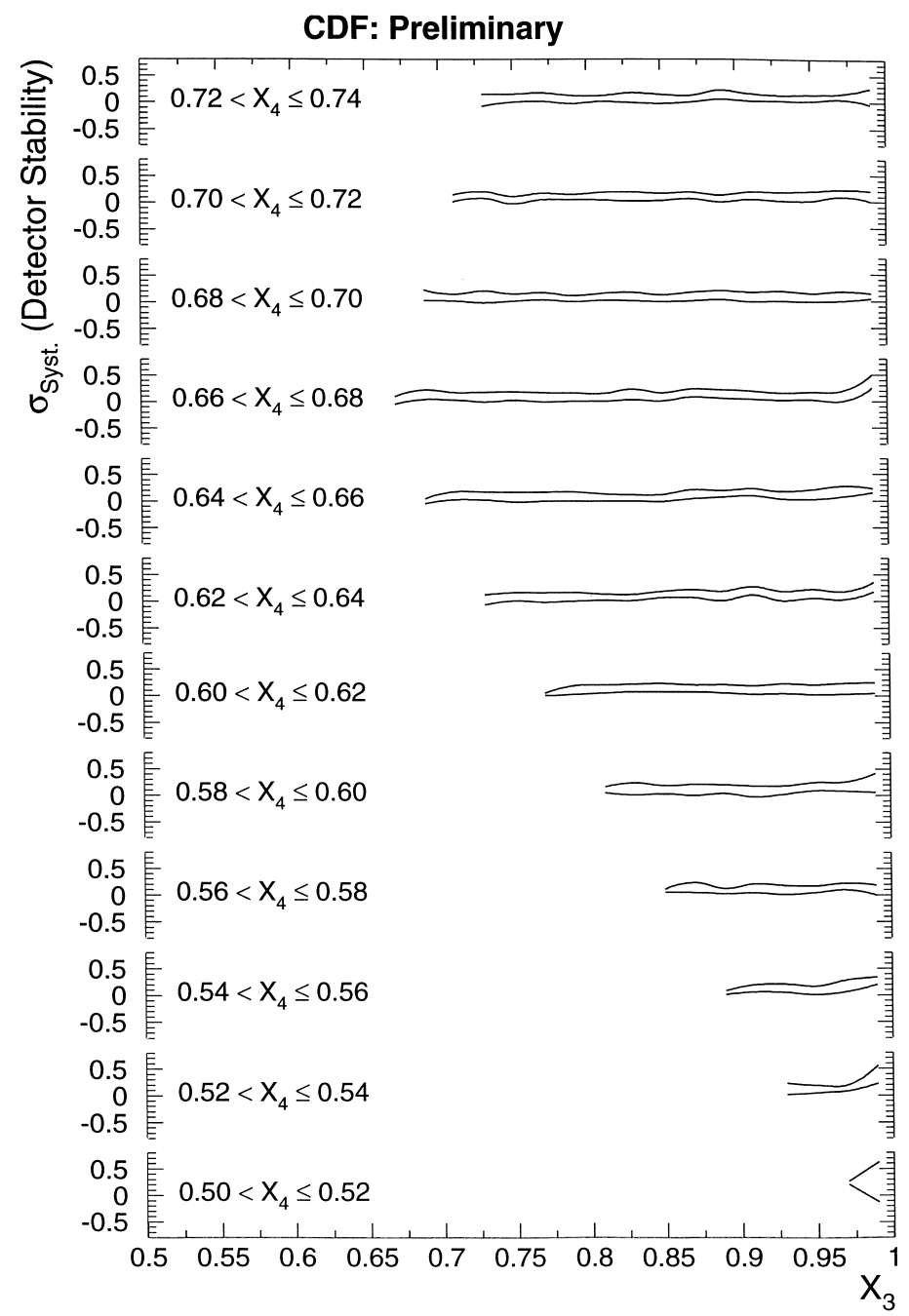

Figure 5.6: The systematic uncertainty on the Dalitz variables due to the uncertainty on the detector stability as a function of $\mathrm{X}_{3}$ for $0.50<\mathrm{X}_{4} \leq 0.74$. 


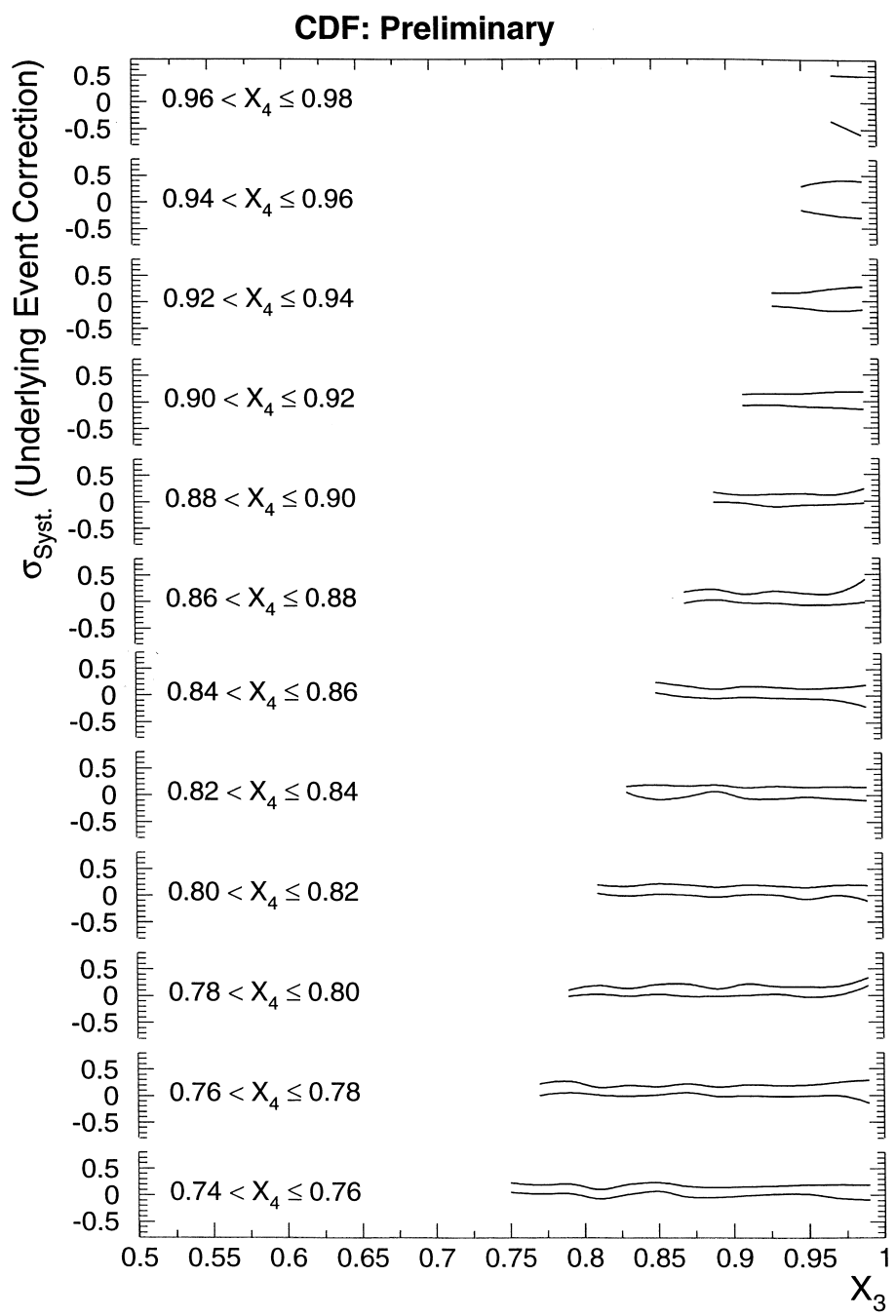

Figure 5.7: The systematic uncertainty on the Dalitz variables due to the uncertainty on the underlying event correction as a function of $\mathrm{X}_{3}$ for $0.74<\mathrm{X}_{4} \leq 0.98$. 


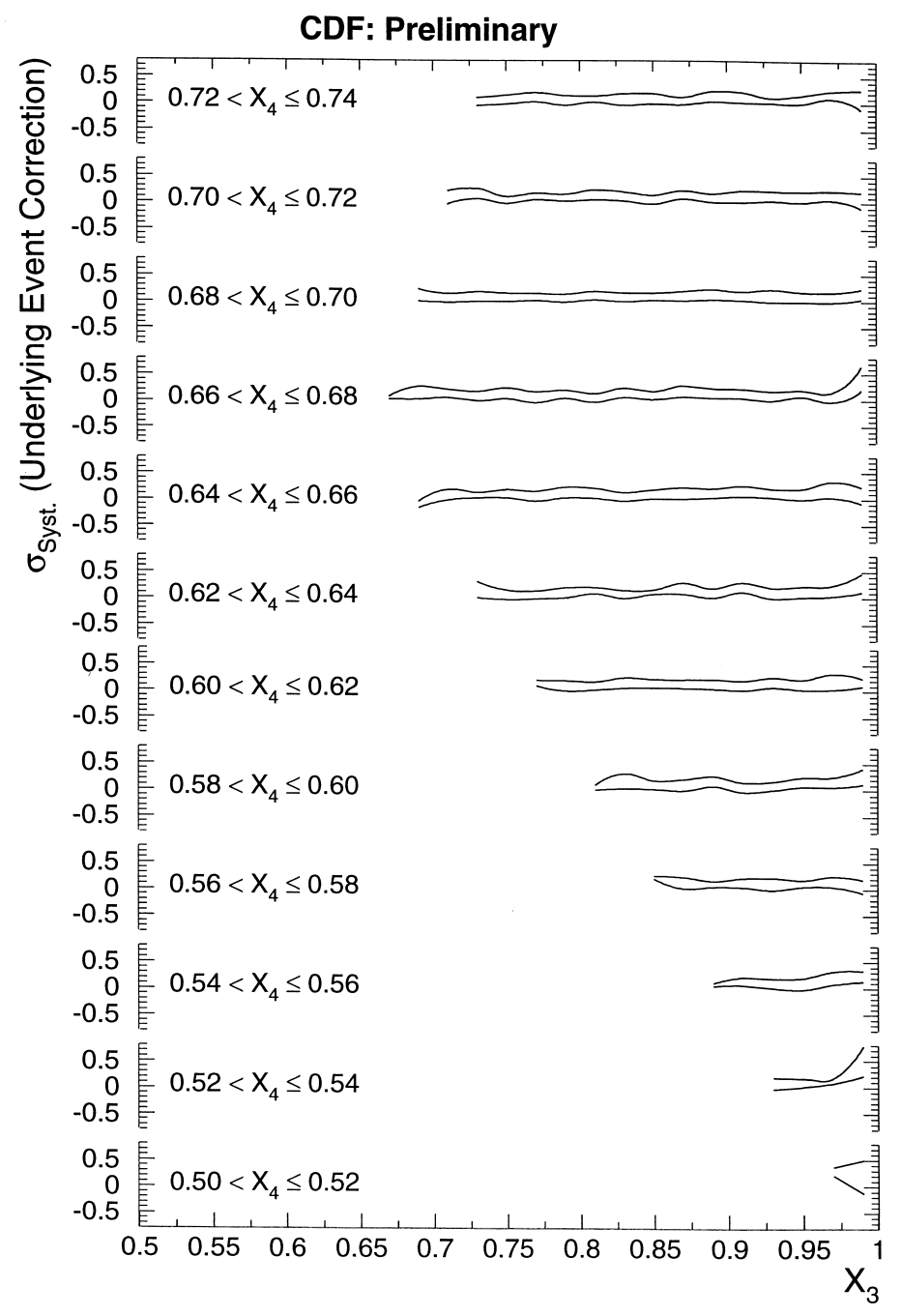

Figure 5.8: The systematic uncertainty on the Dalitz variables due to the uncertainty on the underlying event correction as a function of $\mathrm{X}_{3}$ for $0.50<\mathrm{X}_{4} \leq 0.74$. 
In all of these, $\eta_{d}$ is the $\eta$ relative to the fixed detector coordinate system as shown in Figure 2.4. The uncertainties in the jet energy measurement for these three detector regions are assigned according to Reference [35] and summarized in Table 5.1. They are estimated using dijet balancing [64]. The uncertainties are quoted as a function of dijet mass, $\mathrm{M}_{j j}$, which is defined as $\mathrm{M}_{j j}=\sqrt{\left(\mathrm{E}_{1}+\mathrm{E}_{2}\right)^{2}-\left(\overrightarrow{\mathrm{p}_{1}}+\overrightarrow{\mathrm{p}_{2}}\right)^{2}}$, where $\mathrm{E}_{i}$ and $\overrightarrow{\mathrm{p}_{i}}$ are the energies and three-momenta of the two leading jets in the three-jet system.

\begin{tabular}{|c|c|c|c|}
\hline $\mathrm{M}_{j j}\left(\mathrm{GeV} / \mathrm{c}^{2}\right)$ & $\left|\eta_{d}\right|<0.15$ & $|0.9<| \eta_{d} \mid<1.4$ & $\left|\eta_{d}\right|>1.4$ \\
\hline \hline$\leq 517$ & $3 \%$ & $4 \%$ & $2 \%$ \\
$>517$ & $6 \%$ & $4 \%$ & $2 \%$ \\
\hline
\end{tabular}

Table 5.1: Uncertainty on the jet energy measurement in the three different detector regions.

The systematic uncertainty due to the relative jet energy scale is measured by separate investigation of the three detector regions. The four-momenta of jets in the specific detector region are changed by the amount stated in Table 5.1. The change in the event distribution in the Dalitz plane is recorded. The total contribution to the systematic uncertainty on the Dalitz variables is the quadrature sum of the three individual contributions. Figures 5.9 and 5.10 show the systematic uncertainty on the Dalitz variables due to the relative jet energy scale uncertainty as a function of $\mathrm{X}_{3}$ for all bins in $\mathrm{X}_{4}$.

\subsubsection{Total Integrated Luminosity and $z$-vertex Cut Efficiency}

The uncertainty on the total integrated luminosity for Run $1 \mathrm{~b}$ is $4.1 \%$ [65]. The multijet analysis investigating the Dalitz variables rejects events with multiple interactions, reducing the effective total integrated luminosity of the data sample by $10 \pm 0.5 \%$. The combined uncertainty on the effective total integrated luminosity is $4.2 \%$.

The uncertainty on the $z$-vertex cut efficiency is calculated in Reference [66]. An uncertainty of $2 \%$ is assigned. 


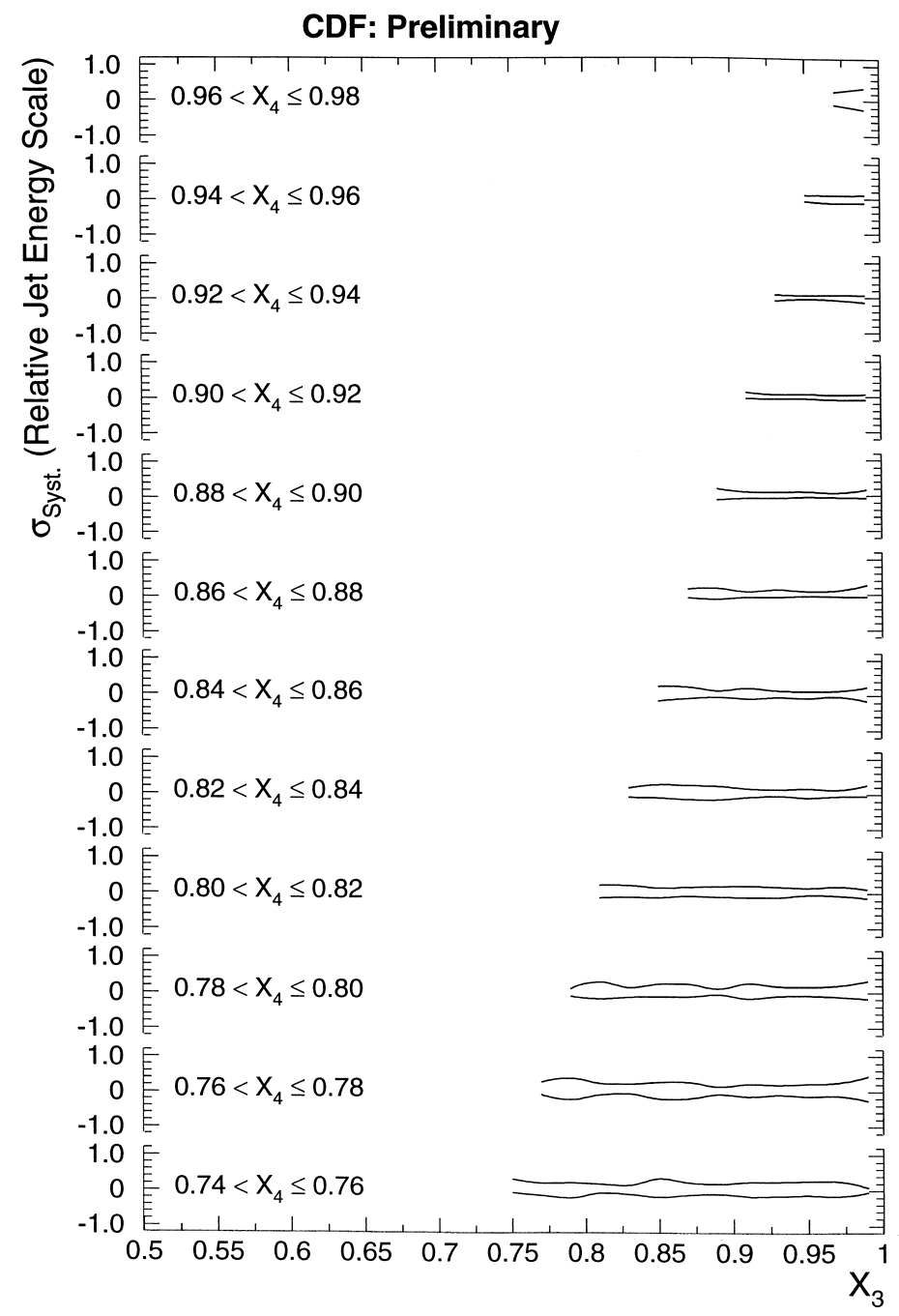

Figure 5.9: The systematic uncertainty on the Dalitz variables due to the uncertainty on the relative jet energy scale as a function of $\mathrm{X}_{3}$ for $0.74<\mathrm{X}_{4} \leq 0.98$. 


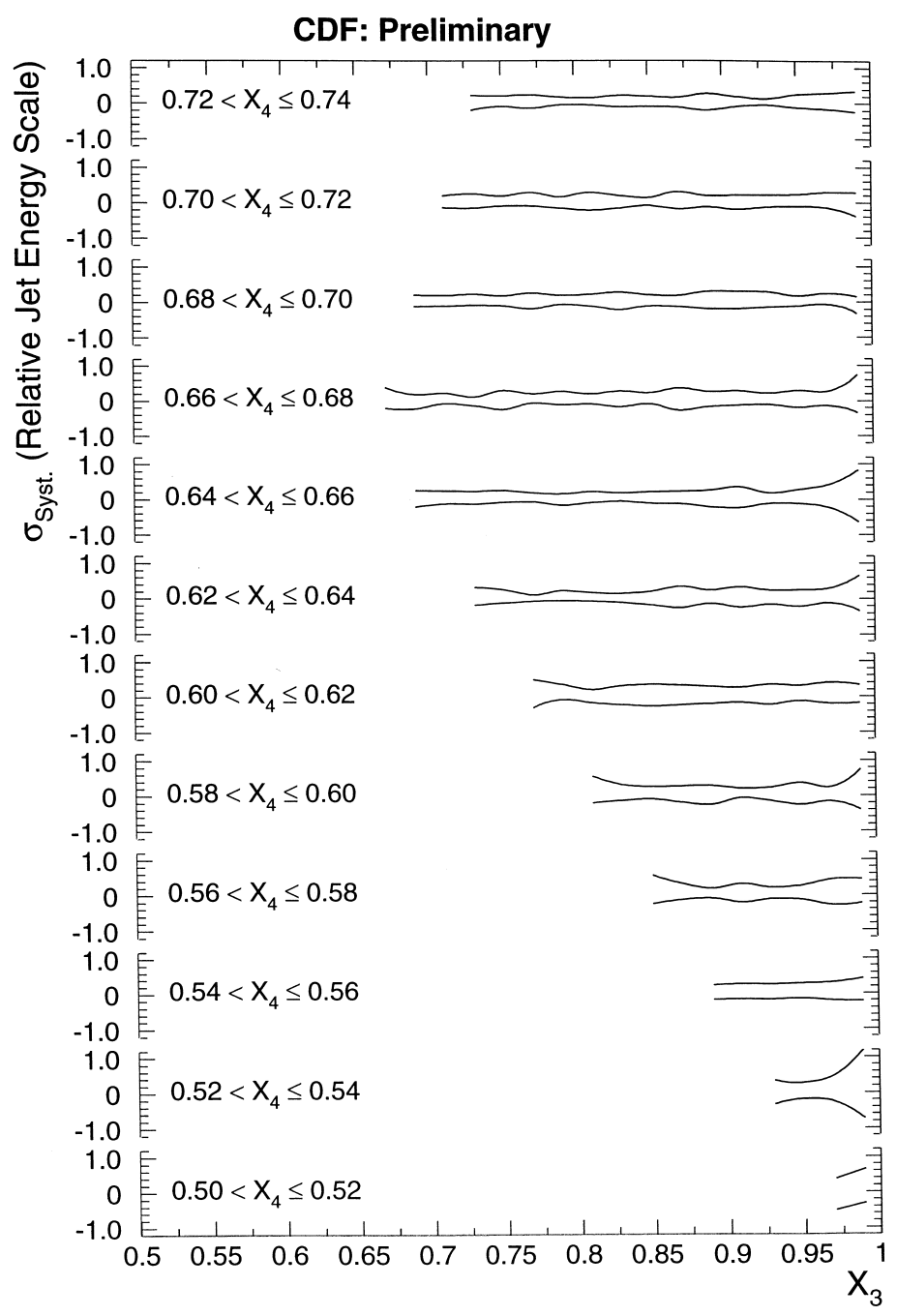

Figure 5.10: The systematic uncertainty on the Dalitz variables due to the uncertainty on the relative jet energy scale as a function of $\mathrm{X}_{3}$ for $0.50<\mathrm{X}_{4} \leq 0.74$. 
Given the size of the systematic uncertainty on the Dalitz variables due the absolute and relative jet energy scales, the systematic uncertainties due to the uncertainties on the total integrated luminosity and on the $z$-vertex cut efficiency are small. Their contributions to the total systematic uncertainty are added in quadrature to the factors obtained in the previous sections.

\subsubsection{Smearing Correction Factor}

The smearing correction factor is obtained by two methods, discussed in the previous chapter. The methods result in comparable smearing correction factors. The uncertainties on all bins used in the multi-jet analysis are $<5 \%$. Regions in the Dalitz plane where the statistical uncertainty on the smearing correction factor would be larger are already excluded from the analysis by the set of cuts applied to the data.

The uncertainty on the smearing correction factor is small compared to the systematic uncertainties due to the absolute and relative jet energy scales. It is added to the other contributions in quadrature.

\subsection{The Total Systematic Uncertainty on the Dalitz Variables}

The total systematic uncertainty on the Dalitz variables is calculated as the squared sum of the individual contributions. Figures 5.11 and 5.12 show the total systematic uncertainty on the Dalitz variables as a function of $\mathrm{X}_{3}$ for all bins in $\mathrm{X}_{4}$. Tables D.1 to D.24 in Appendix D summarize the total systematic uncertainties, and the main contributions, on the Dalitz variables.

The distribution of the total systematic uncertainty on the Dalitz variables as a function of $\mathrm{X}_{3}$ does not vary significantly in each bin in $\mathrm{X}_{4}$. Fluctuations are mainly due to statistical uncertainties. The average total systematic uncertainty in every $\mathrm{X}_{4}$ bin is representative of the values in that bin. It is tabulated in Table 5.2. 


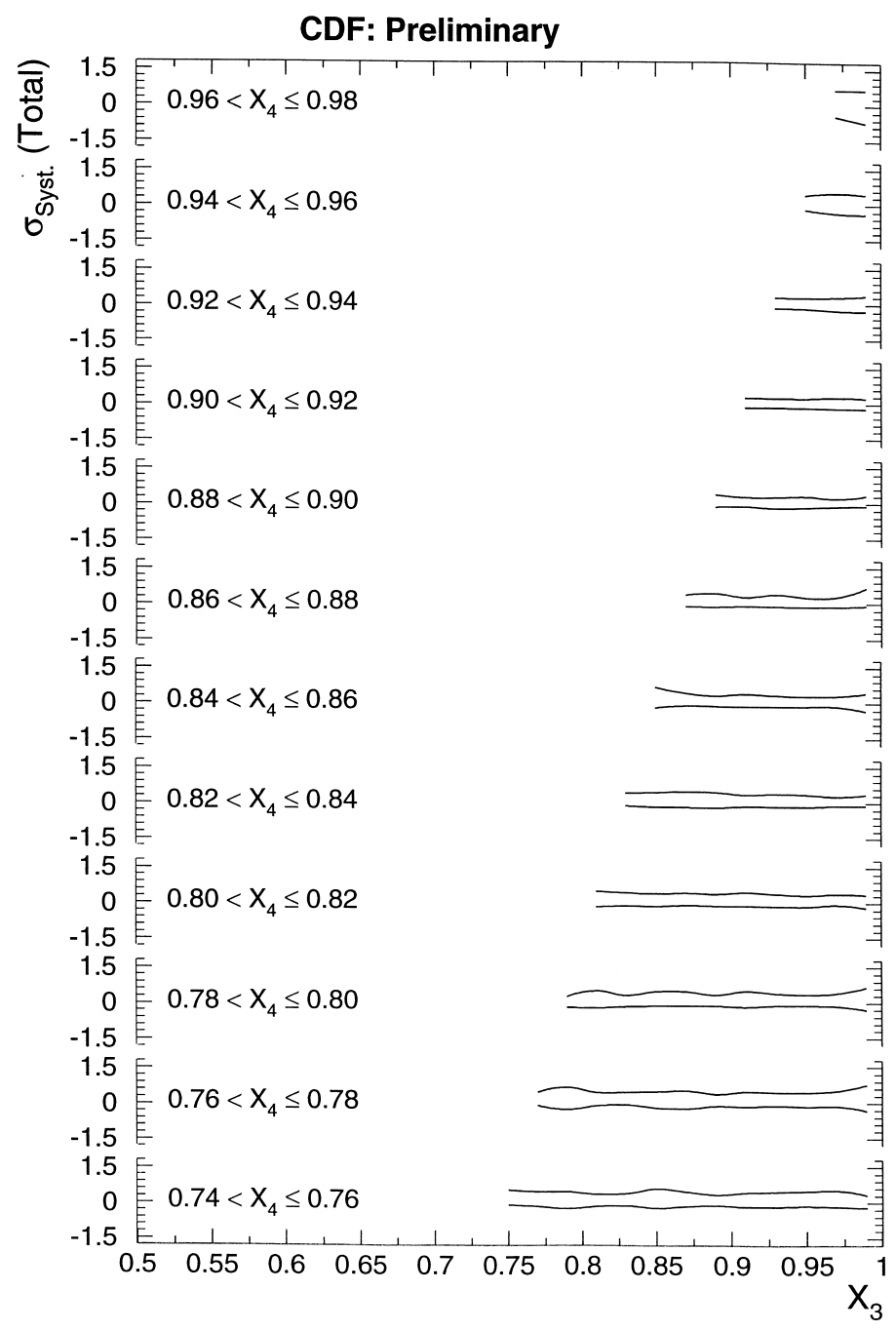

Figure 5.11: The total systematic uncertainty on the Dalitz variables as a function of $\mathrm{X}_{3}$ for $0.74<\mathrm{X}_{4} \leq 0.98$. 


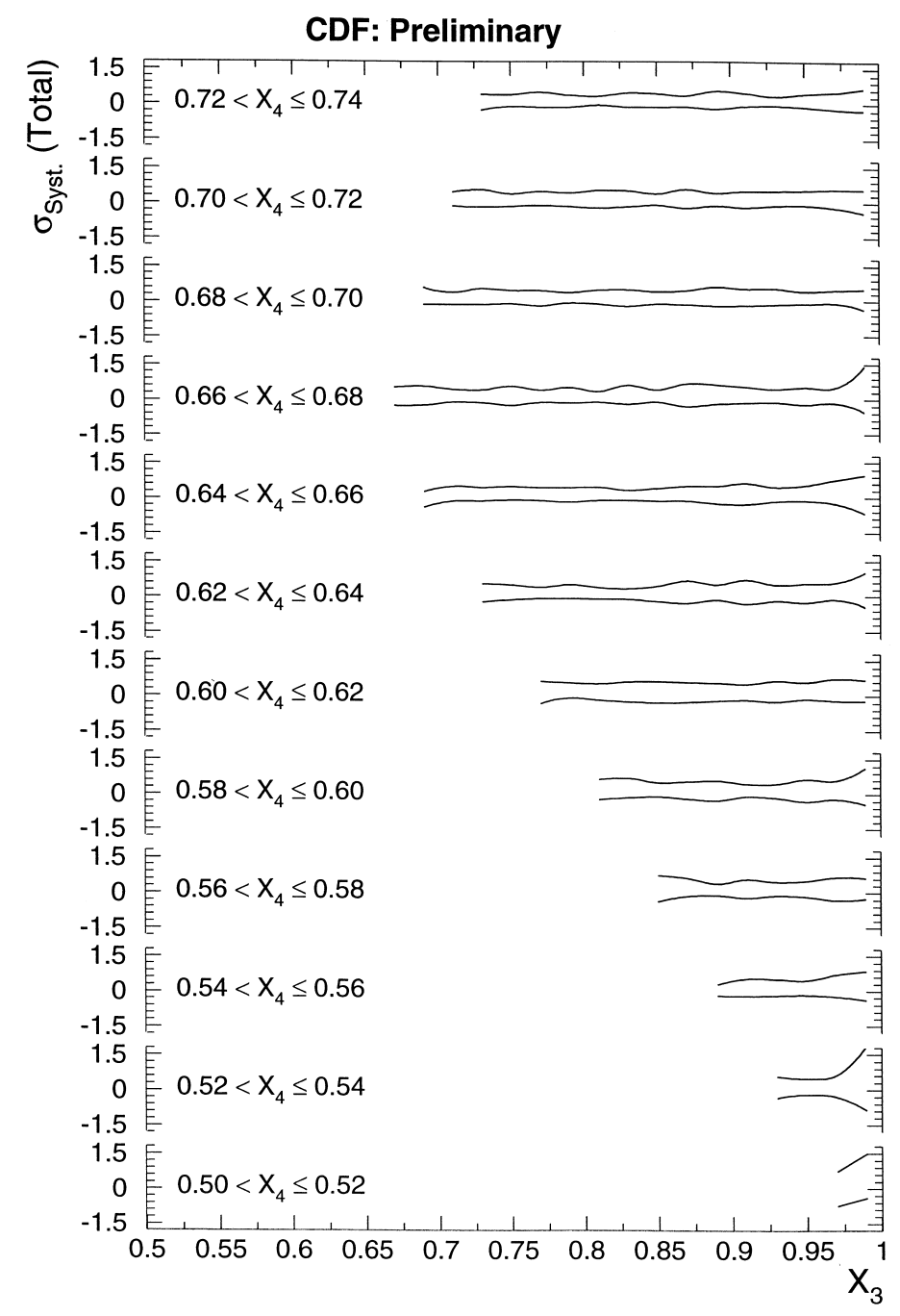

Figure 5.12: The total systematic uncertainty on the Dalitz variables as a function of $\mathrm{X}_{3}$ for $0.50<\mathrm{X}_{4} \leq 0.74$. 


\begin{tabular}{|c|c|c|c|c|c|}
\hline$\overline{\mathrm{X}_{4}}$ & \multicolumn{2}{|c|}{ Average Total (\%) } & $\overline{X_{4}}$ & \multicolumn{2}{|c|}{ Average Total (\%) } \\
\hline $0.96-0.98$ & +68.4 & -59.2 & $0.72-0.74$ & +44.1 & -15.3 \\
\hline $0.94-0.96$ & +46.0 & -30.6 & $0.70-0.72$ & +48.5 & -17.6 \\
\hline $0.92-0.94$ & +33.2 & -20.0 & $0.68-0.70$ & +48.5 & -14.9 \\
\hline $0.90-0.92$ & +28.8 & -16.2 & $0.66-0.68$ & +57.0 & -18.9 \\
\hline $0.88-0.90$ & +31.3 & -12.4 & 0.64-0.66 & +51.4 & -20.0 \\
\hline $0.86-0.88$ & +39.8 & -10.6 & $0.62-0.64$ & +55.2 & -19.1 \\
\hline $0.84-0.86$ & +40.6 & -14.7 & $0.60-0.62$ & +60.5 & -21.0 \\
\hline $0.82-0.84$ & +40.6 & -13.8 & $0.58-0.60$ & +62.5 & -21.3 \\
\hline $0.80-0.82$ & +41.8 & -12.0 & $0.56-0.58$ & +58.1 & -21.2 \\
\hline $0.78-0.80$ & +44.6 & -14.7 & $0.54-0.56$ & +54.8 & -22.4 \\
\hline $0.76-0.78$ & +51.8 & -15.2 & $0.52-0.54$ & +90.7 & -41.5 \\
\hline $0.74-0.76$ & +45.9 & -15.2 & $0.50-0.52$ & +113 & -57.5 \\
\hline
\end{tabular}

Table 5.2: The average total systematic uncertainty on the Dalitz variables in every $\mathrm{X}_{4}$ bin. 


\section{Chapter 6}

\section{Results}

\subsection{The Inclusive Three-jet Comparison}

The transverse energy distributions of all three jets for unsmeared data and the NLO predictions, using the CTEQ3 parton distribution function, are plotted in Figure 6.1 and compared to HERWIG. The HERWIG event sample has to be adjusted for systematic errors in the QFL energy scale (see Reference [35].) A minimum $\mathrm{p}_{\mathrm{T}}$ of $60 \mathrm{GeV}$ is used in the generation of the HERWIG sample. In the HERWIG data sample, the third jet is calculated to be significantly more energetic than the corresponding distributions for the data and the NLO calculation. It is possible that this is due to the fact that HERWIG does not correctly model the jet multiplicities, as shown in Figure 6.2 where the total number of jets in each event is compared between the data sample and HERWIG. For a given value of $\sum \mathrm{E}_{\mathrm{T}}$ in an event, the third jet is more energetic if the total number of jets in the event is less than in the data sample or in the NLO prediction. In Figure 6.3, the $\sum \mathrm{E}_{\mathrm{T}}$ per event is shown for data and HERWIG. Table 6.1 compares the jet multiplicities in the data and the HERWIG sample, where HERWIG is scaled to the total number of jets in the data. The $\mathrm{E}_{\mathrm{T}}$ distributions of the three jets are independent of changes in the cut on $\sum \mathrm{E}_{\mathrm{T}}$. This is demonstrated in Figure 6.4. In this plot, the cut on $\sum \mathrm{E}_{\mathrm{T}}$ is changed in $10 \mathrm{GeV}$ 
increments from $320 \mathrm{GeV}$ to $340 \mathrm{GeV}$; the three jet distributions are normalized to the same number of events to allow comparison of their shapes.

\begin{tabular}{|c|c|c|}
\hline Number of Jets & Data & $\begin{array}{c}\text { HERWIG } \\
\left(\mathrm{p}_{\mathrm{T}}=60 \mathrm{GeV}\right)\end{array}$ \\
\hline \hline 3 & 25,272 & 26,571 \\
4 & 7,265 & 6,230 \\
5 & 1,130 & 927 \\
6 & 121 & 75 \\
7 & 16 & 4 \\
8 & 1 & 0 \\
9 & 1 & 0 \\
10 & 1 & 0 \\
\hline
\end{tabular}

Table 6.1: The jet multiplicities in the data and in HERWIG, using a minimum $\mathrm{p}_{\mathrm{T}}$ of $60 \mathrm{GeV}$.

The distributions of the three-jet mass and the Dalitz variables, $\mathrm{X}_{3}$ and $\mathrm{X}_{4}$, are shown for a different set of cuts, where $\mathrm{m}_{3 \mathrm{~J}}>500 \mathrm{GeV}$ and $\mathrm{X}_{3}<0.9$, in Figures 6.5, 6.6, and 6.7, respectively. Data and the HERWIG sample with $\mathrm{p}_{\mathrm{T}}=60 \mathrm{GeV}$ are compared in these figures.

As an independent check on the data, the jet $\mathrm{E}_{\mathrm{T}}$ distributions obtained by a different trigger are investigated. The main selection criterion for this trigger is at least one jet in each event with $\mathrm{E}_{\mathrm{T}}>100 \mathrm{GeV}$. It is called the Jet100 trigger. Data obtained from the $\sum E_{\mathrm{T}}$ trigger and from the Jet100 trigger agree very well. The jet $\mathrm{E}_{\mathrm{T}}$ distributions in both cases do not differ significantly. Figure 6.8 shows the transverse energy distributions for the three jets in unsmeared data from the Jet100 trigger, the NLO prediction, and HERWIG with $\mathrm{p}_{\mathrm{T}}=60 \mathrm{GeV}$.

In this study, emphasis is placed on the analysis of the Dalitz variables. Figures 6.9 through 6.12 show the Dalitz variable distributions as predicted by the NLO calculation, using the CTEQ3 parton distribution function family, for $\alpha_{\mathrm{S}}=0.1118$ (Figure 6.9), $\alpha_{\mathrm{S}}=0.1133$ (Figure 6.10), $\alpha_{\mathrm{S}}=0.1160$ (Figure 6.11), and $\alpha_{\mathrm{S}}=0.1185$ (Figure 6.12). The Dalitz variable distribution for the data is shown in Figure 6.13. The data and the NLO prediction, using the CTEQ3 parton distribution func- 


\section{CDF: Preliminary}

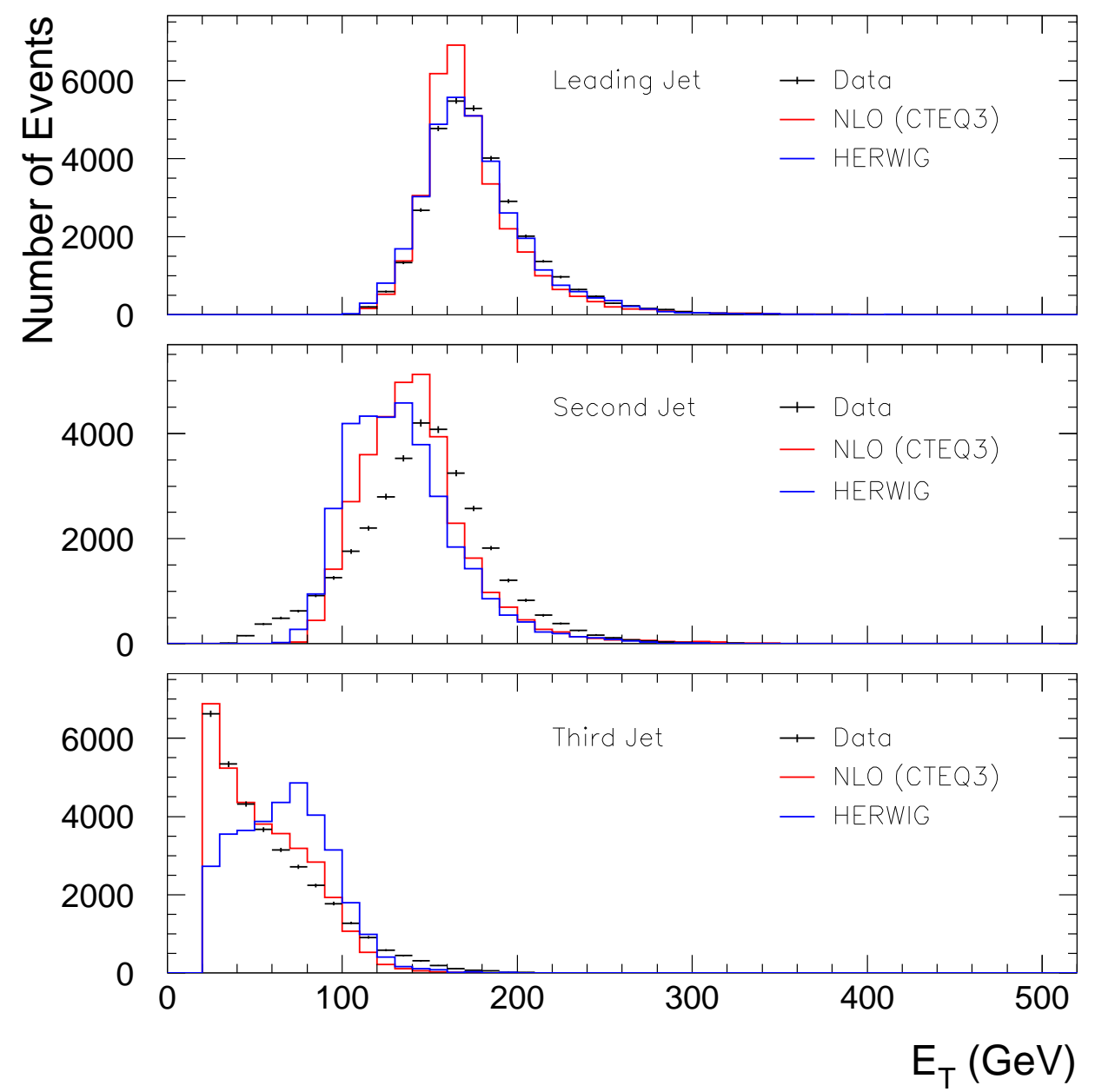

Figure 6.1: The transverse energy distributions for the three jets in unsmeared data, the NLO prediction, and HERWIG. 


\section{CDF: Preliminary}

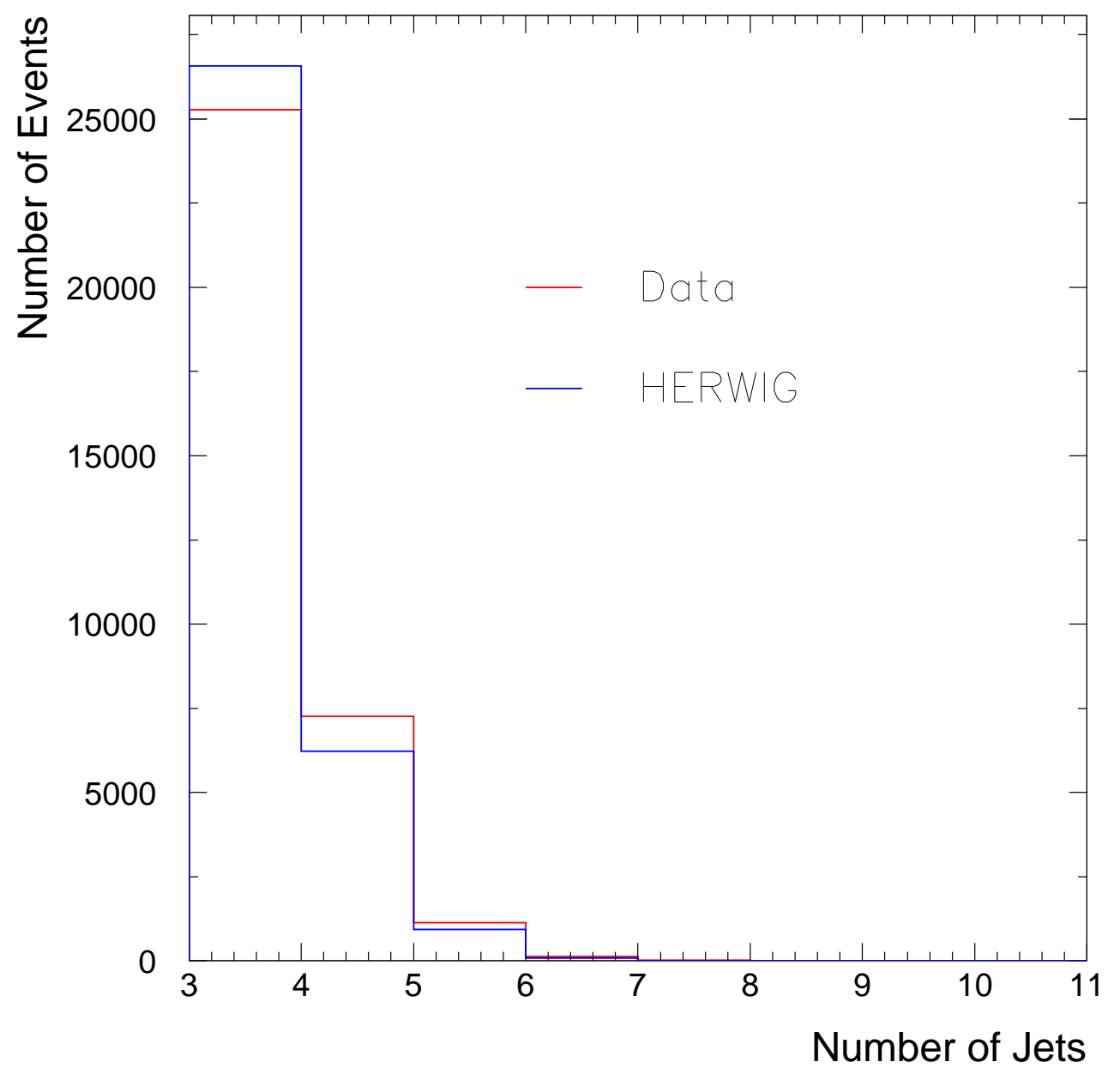

Figure 6.2: The number of jets in each event for the data and for HERWIG with $\mathrm{p}_{\mathrm{T}}=60 \mathrm{GeV}$. 
CDF: Preliminary

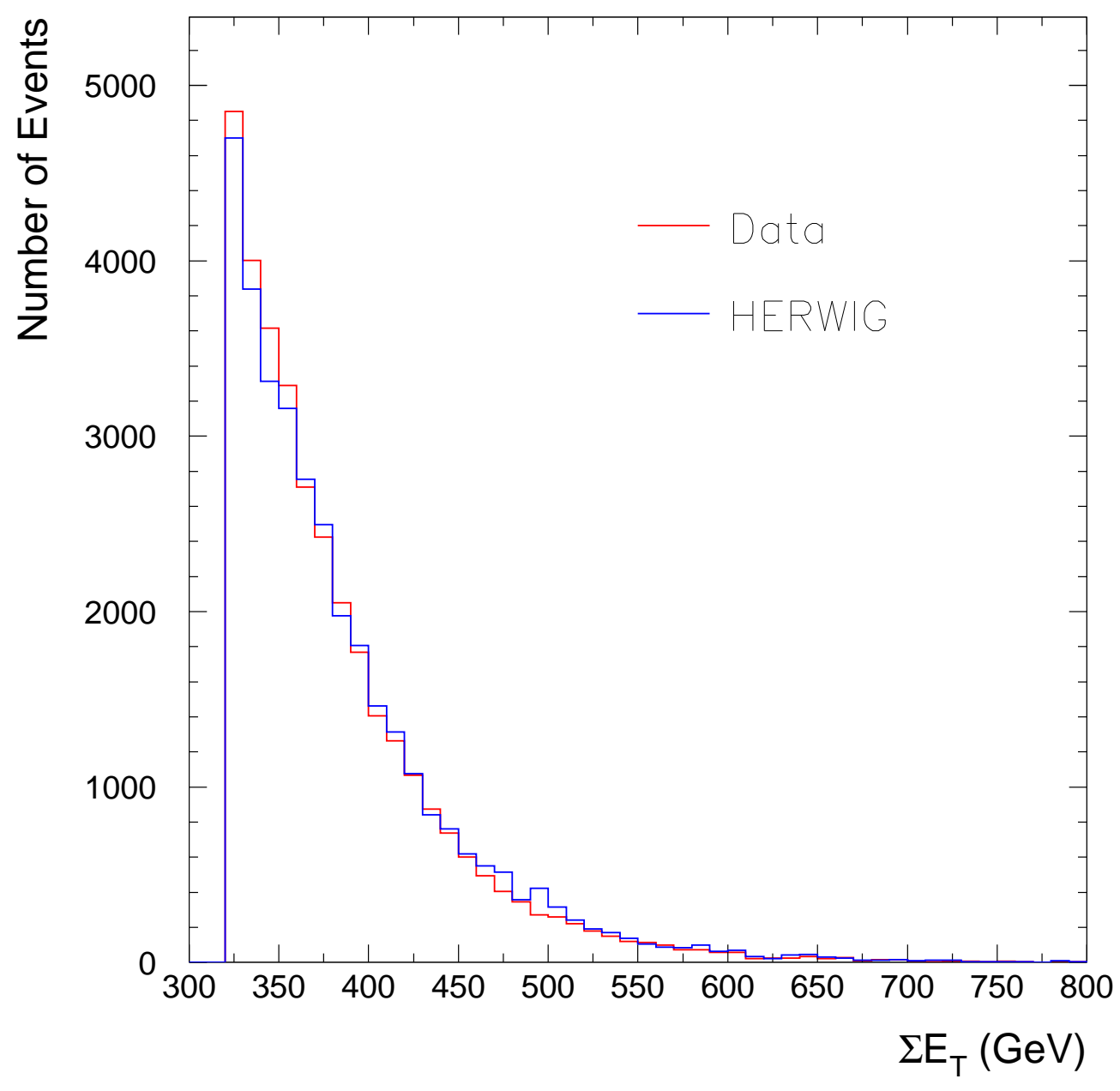

Figure 6.3: The $\sum \mathrm{E}_{\mathrm{T}}$ per event after all the cuts have been applied to the data and to HERWIG Monte Carlo with $\mathrm{p}_{\mathrm{T}}=60 \mathrm{GeV}$. 
CDF: Preliminary

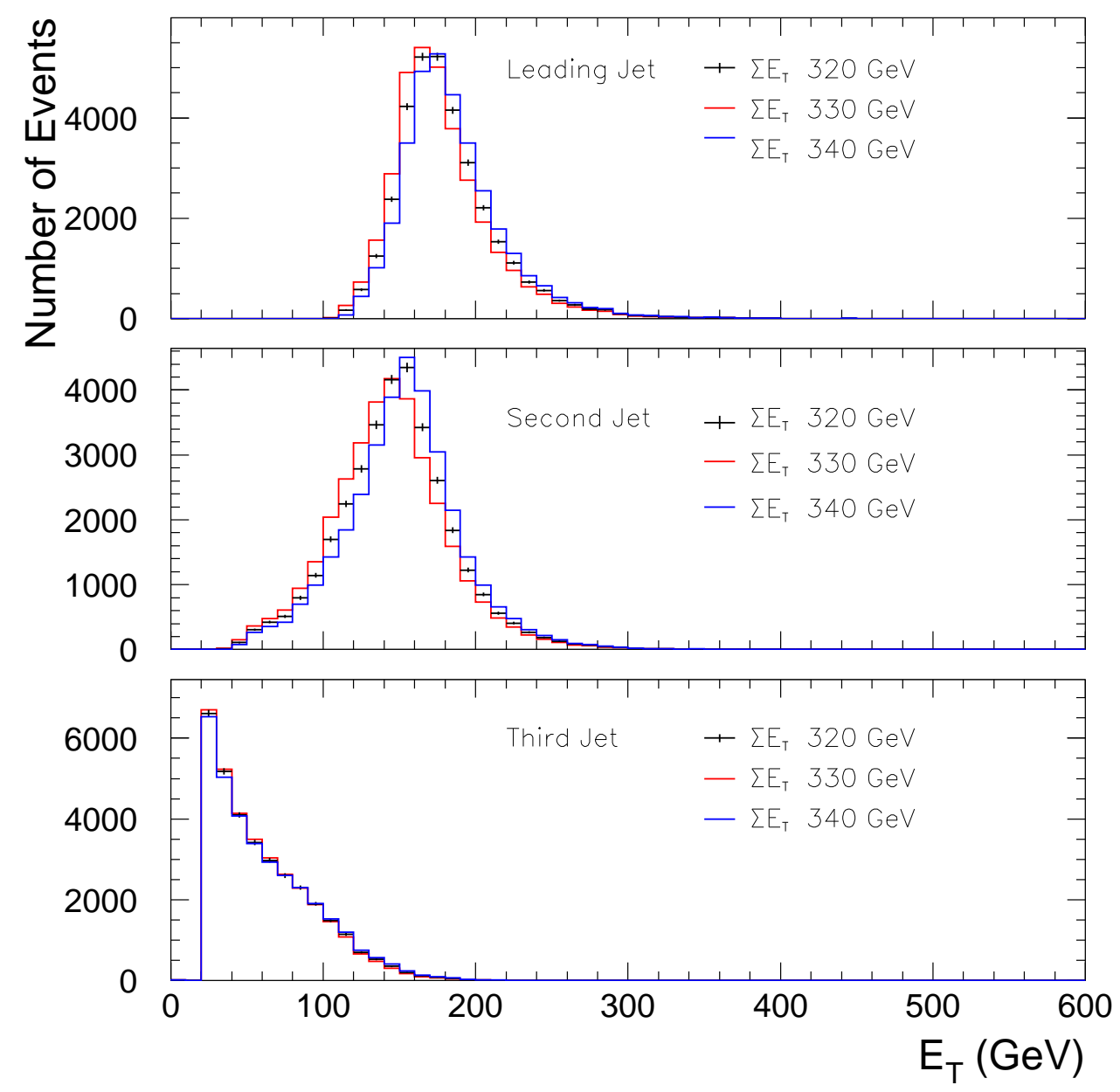

Figure 6.4: The $\mathrm{E}_{\mathrm{T}}$ distribution of the three leading jets in all data events as the cut on $\sum E_{\mathrm{T}}$ is varied. 


\section{CDF: Preliminary}

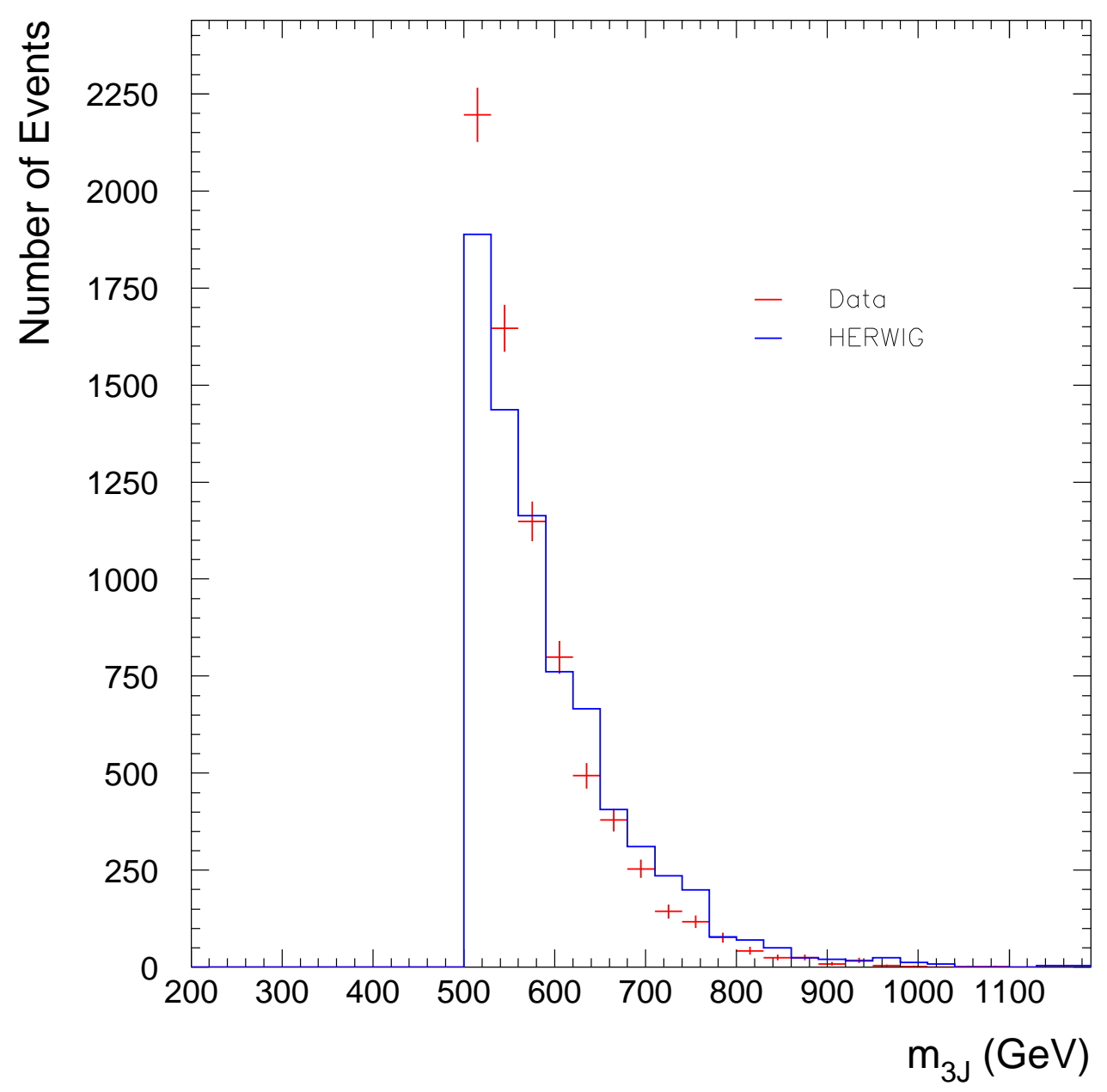

Figure 6.5: The three-jet mass distribution for data and HERWIG with $\mathrm{p}_{\mathrm{T}}=60 \mathrm{GeV}$, using a different set of cuts $\left(\mathrm{m}_{3 \mathrm{~J}}>500 \mathrm{GeV}\right.$ and $\mathrm{X}_{3}<0.9$. $)$ 
CDF: Preliminary

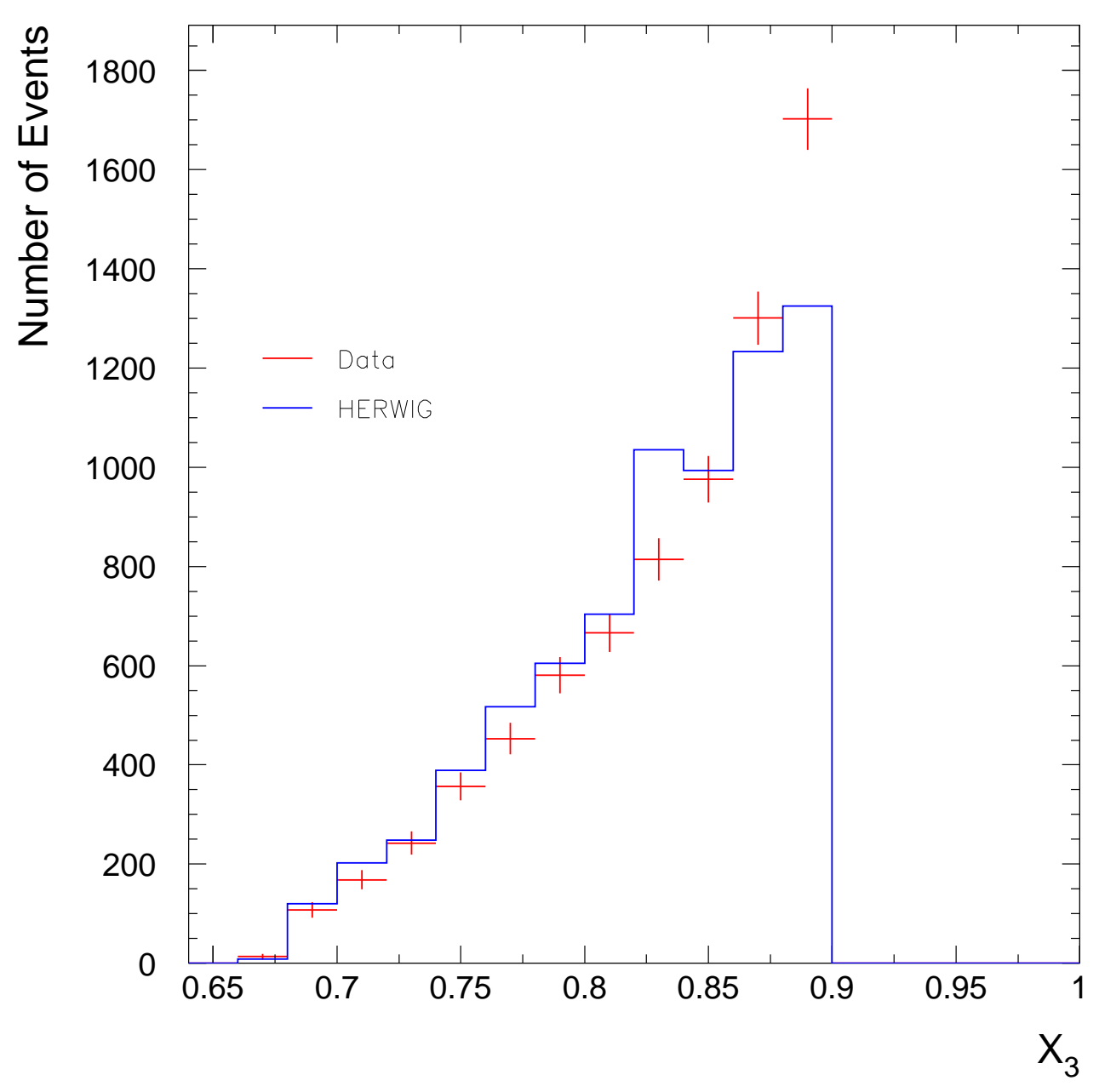

Figure 6.6: The $\mathrm{X}_{3}$ distribution for data and HERWIG with $\mathrm{p}_{\mathrm{T}}=60 \mathrm{GeV}$, using a different set of cuts $\left(\mathrm{m}_{3 \mathrm{~J}}>500 \mathrm{GeV}\right.$ and $\mathrm{X}_{3}<0.9$. $)$ 
CDF: Preliminary

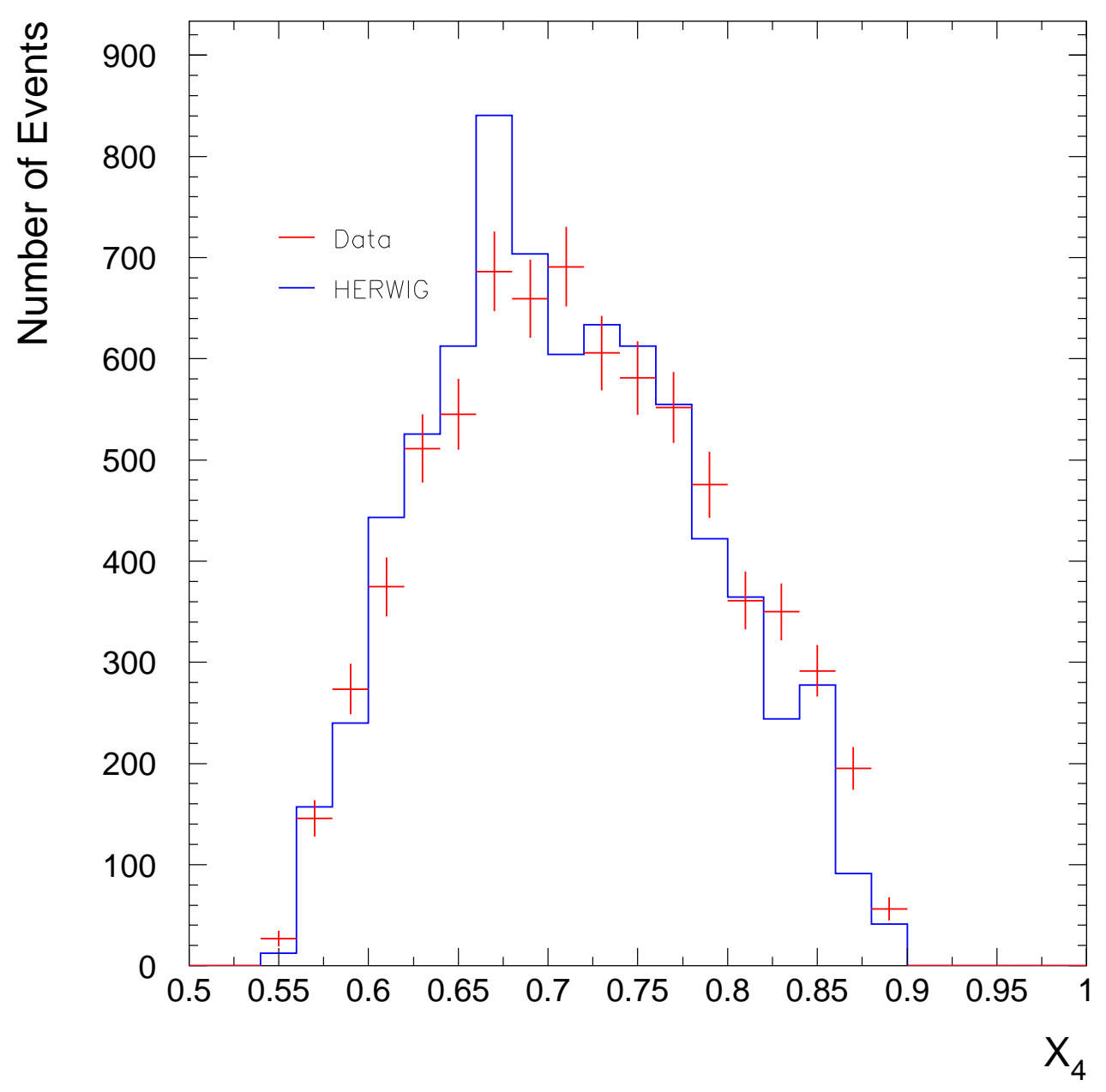

Figure 6.7: The $\mathrm{X}_{4}$ distribution for data and HERWIG with $\mathrm{p}_{\mathrm{T}}=60 \mathrm{GeV}$, using a different set of cuts $\left(\mathrm{m}_{3 \mathrm{~J}}>500 \mathrm{GeV}\right.$ and $\mathrm{X}_{3}<0.9$. $)$ 


\section{CDF: Preliminary}

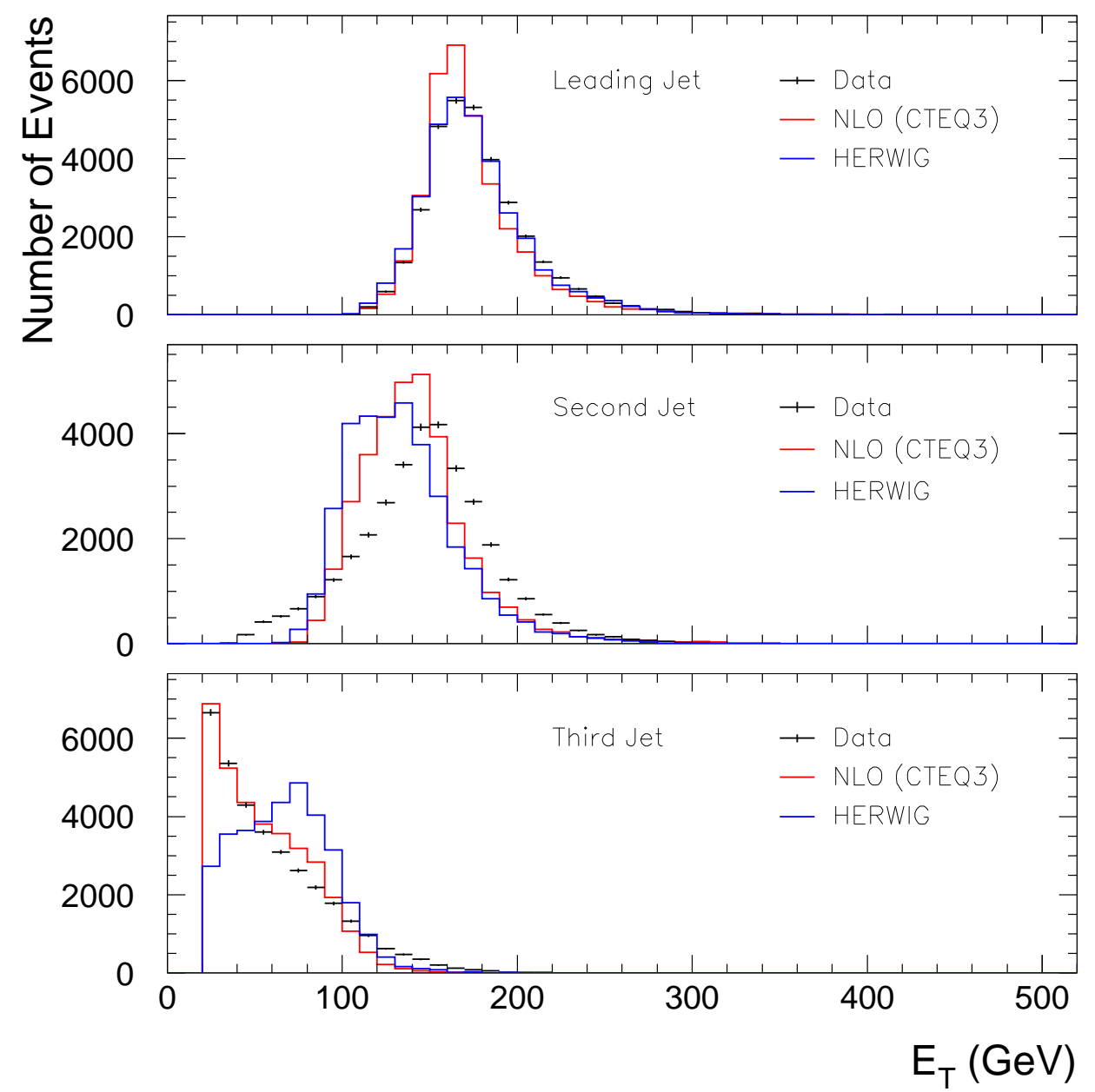

Figure 6.8: The transverse energy distributions for the three jets in unsmeared data from the Jet100 trigger, the NLO prediction, and HERWIG with $\mathrm{p}_{\mathrm{T}}=60 \mathrm{GeV}$. 


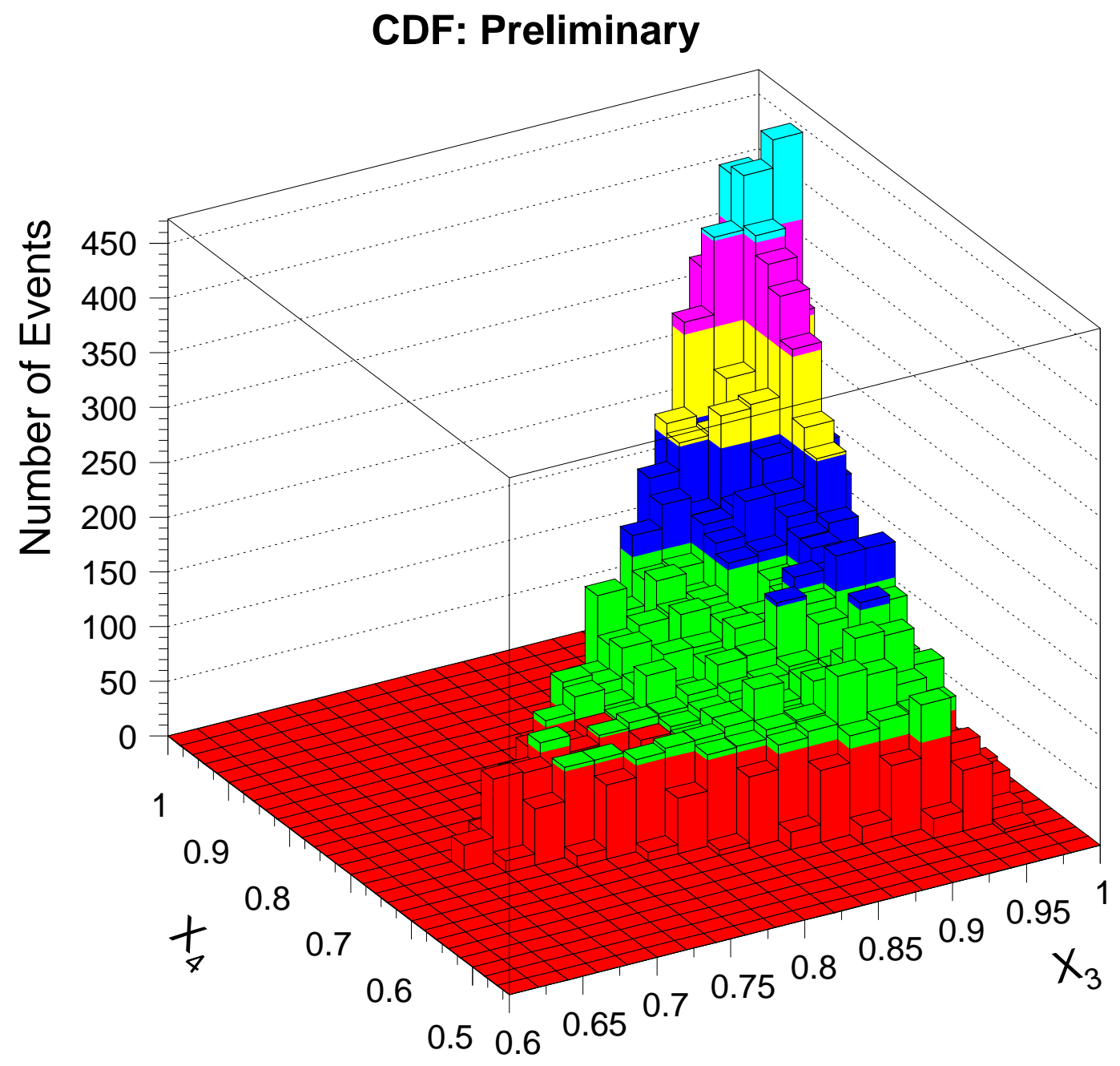

Figure 6.9: The event density in the Dalitz plane as predicted by the NLO calculation with $\alpha_{\mathrm{S}}=0.1118$. 


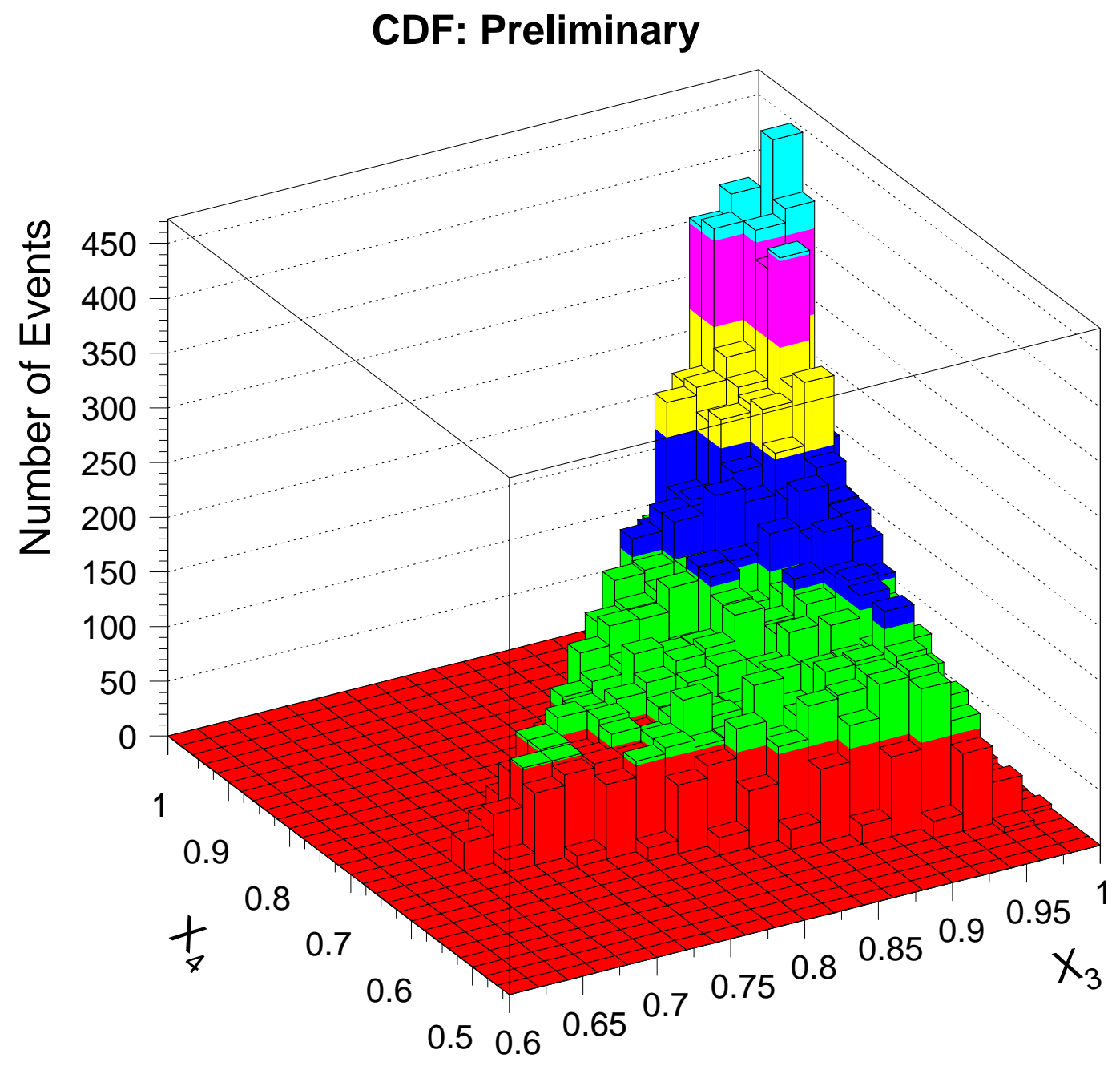

Figure 6.10: The event density in the Dalitz plane as predicted by the NLO calculation with $\alpha_{\mathrm{S}}=0.1133$. 


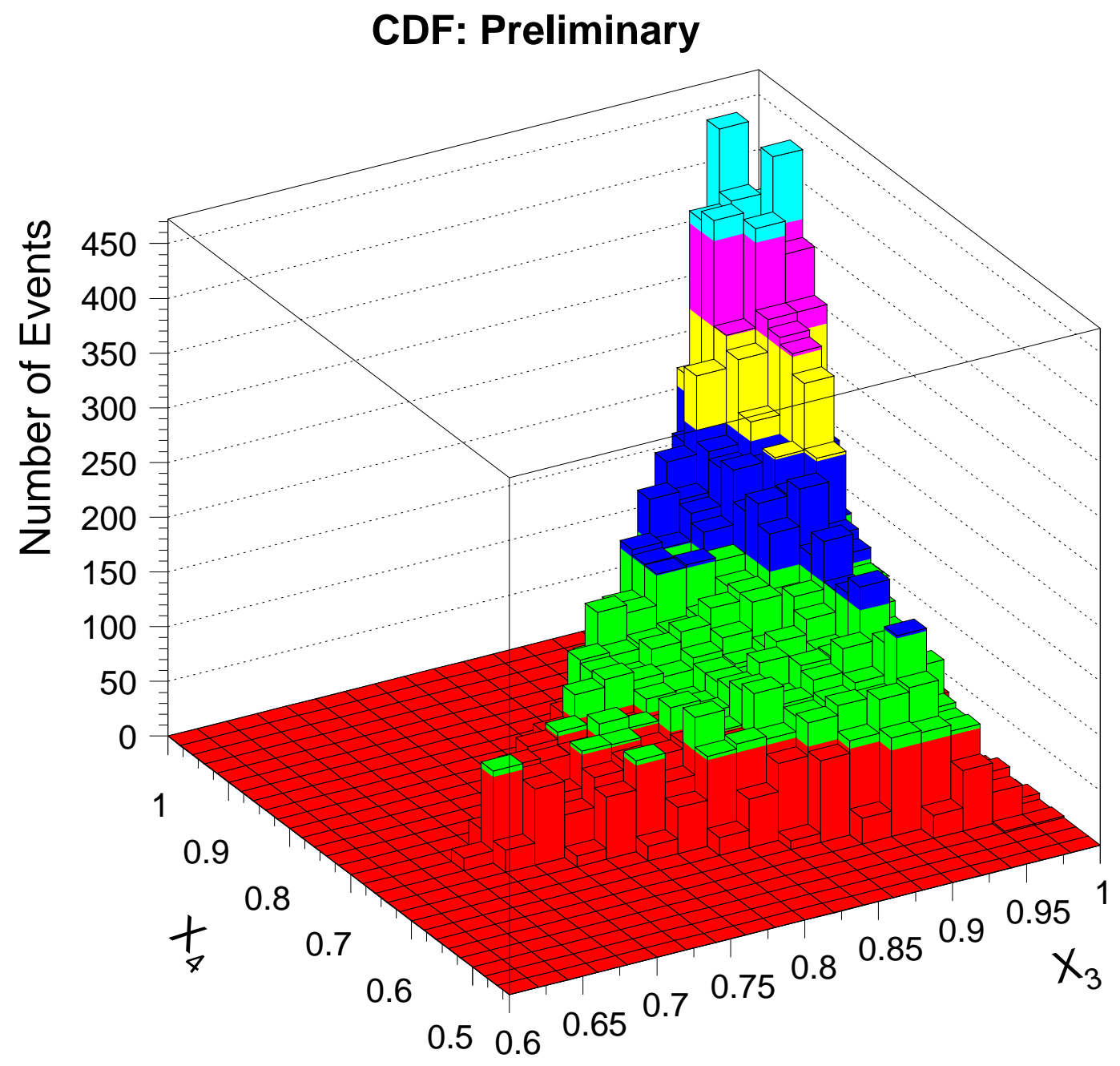

Figure 6.11: The event density in the Dalitz plane as predicted by the NLO calculation with $\alpha_{\mathrm{S}}=0.1160$. 


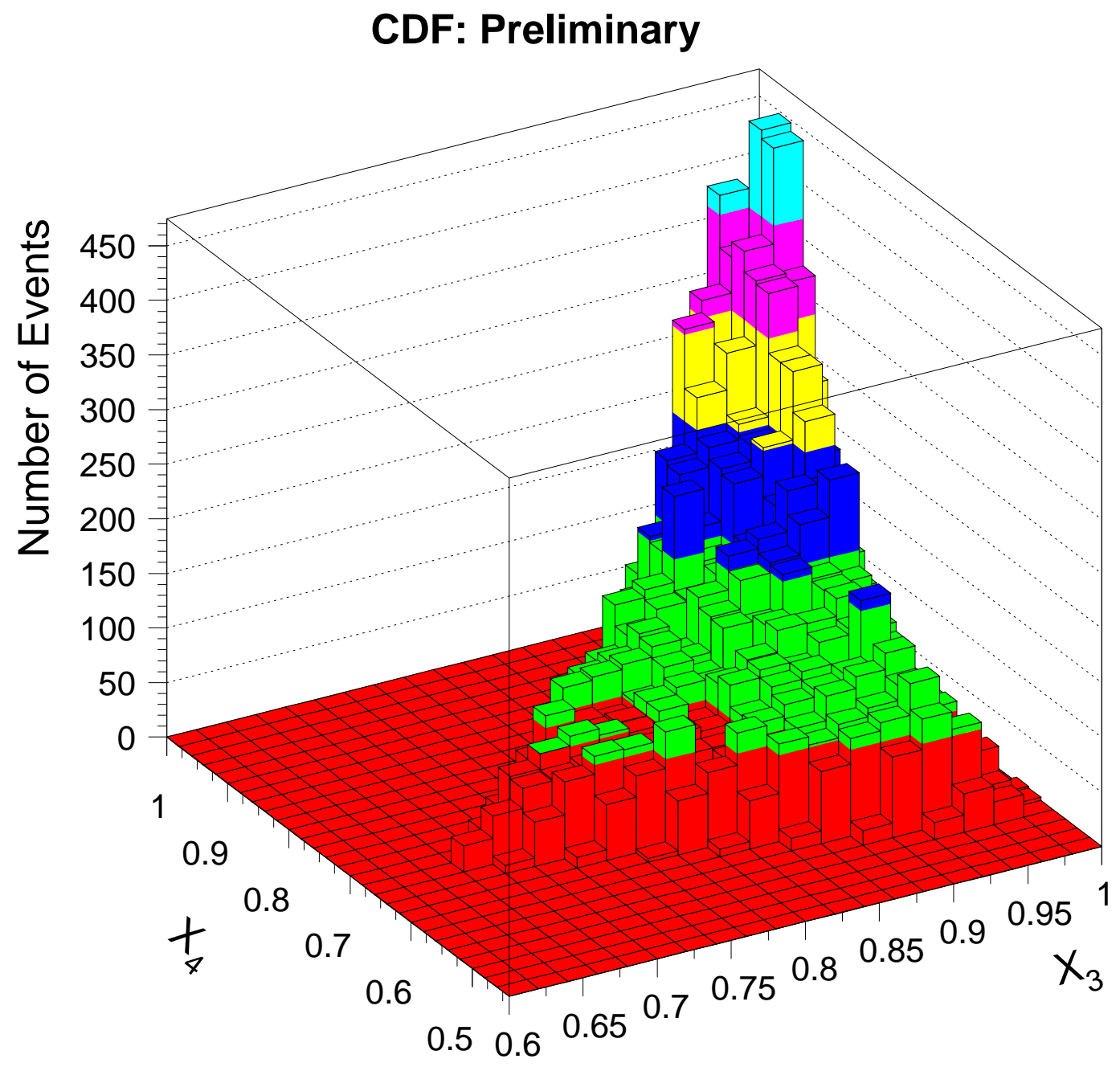

Figure 6.12: The event density in the Dalitz plane as predicted by the NLO calculation with $\alpha_{\mathrm{S}}=0.1185$. 


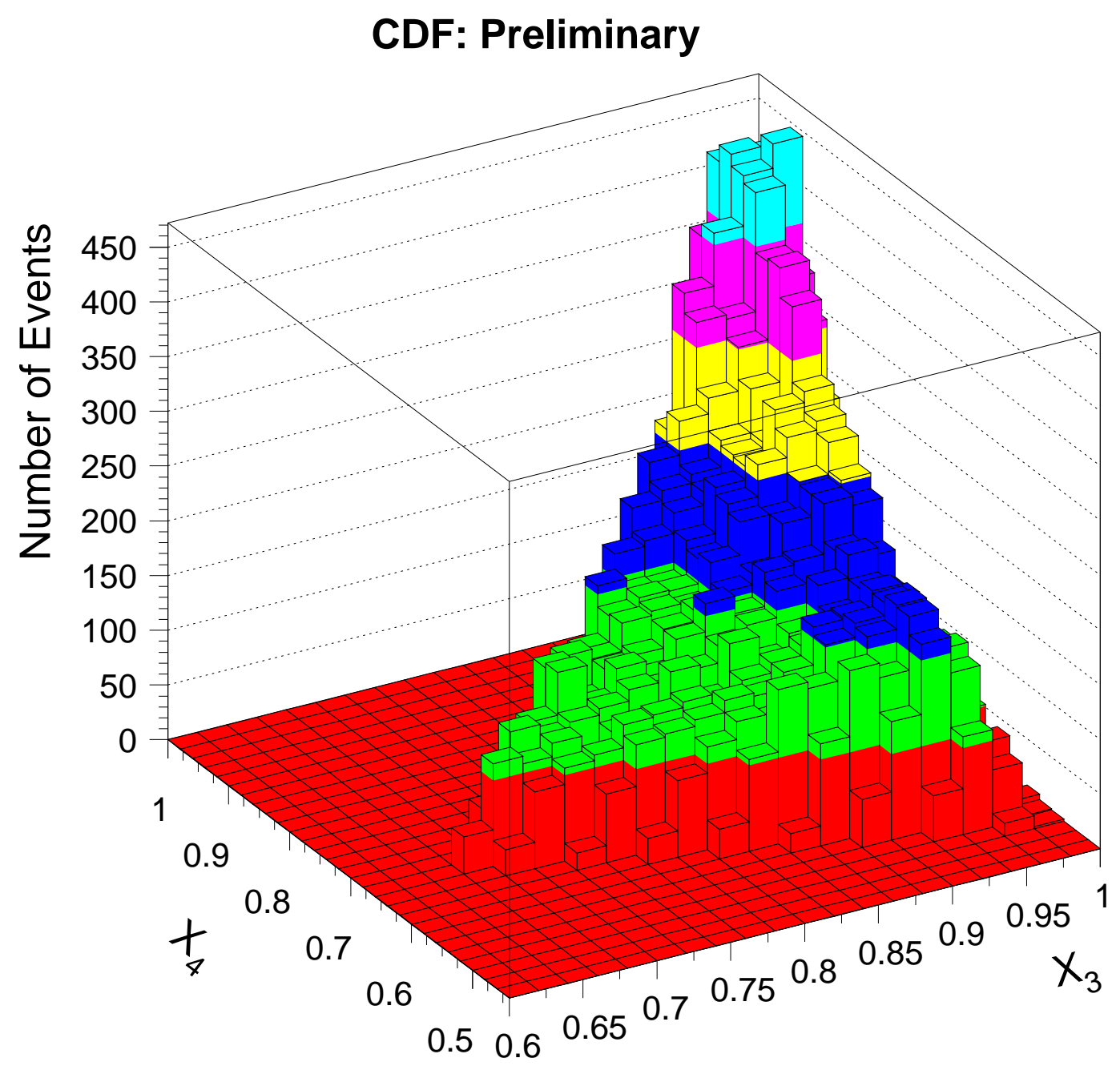

Figure 6.13: The event density in the Dalitz plane for the data. 
tion, are compared by computing the difference between the observed and predicted number of events, scaled by the number of predicted events, in each bin. This fractional difference is plotted in Figures 6.14 through 6.17 as a function of $\mathrm{X}_{3}$, spanning the entire range in $\mathrm{X}_{4}$. Figure 6.14 shows the region with $0.84<\mathrm{X}_{4} \leq 0.94$, $0.74<\mathrm{X}_{4} \leq 0.84$ in Figure 6.15, and Figures 6.16 and 6.17 span $0.64<\mathrm{X}_{4} \leq 0.74$ and $0.54<\mathrm{X}_{4} \leq 0.64$, respectively. Also shown in Figures 6.14 through 6.17 are the total systematic uncertainties on the fractional difference due to systematic uncertainties in the data (see Chapter 5). We limit the analysis to entry bins for which the error on the NLO calculation is $<20 \%$ for every data set; this leaves 163 bins for the comparison between the data and the calculation. The regions in $\mathrm{X}_{3}-\mathrm{X}_{4}$ space that are used in the comparison between the data and the NLO prediction, and which are plotted in Figures 6.14 through 6.17, are shown in Figure 6.18. The lines enclosing the final data set with an error on the NLO prediction $<0.2$ in every data set follow bin boundaries. The data and the NLO prediction are consistent, and the data are stable with respect to the cuts introduced in this analysis. Small changes in cuts do not result in significant changes in the Dalitz distributions. To show this, all cuts are independently changed by $\pm \sigma$. Specifically, the cone overlap cut is changed by \pm 0.05 , the $\eta$ cut by \pm 0.075 , the geometrical acceptance cut by \pm 0.01 in $\left|\cos \theta_{3}^{*}\right|$, the $\sum \mathrm{E}_{\mathrm{T}}$ cut by $\pm 10 \mathrm{GeV}$, the number of tracks for the definition of a resolved multiple interaction by \pm 1 , and the distance between two resolved vertices by $\pm 1 \mathrm{~cm}$. Only the cut on jet $\mathrm{E}_{\mathrm{T}}$ is also changed by $\pm 5 \mathrm{GeV}$, which corresponds to approximately $\pm 5 \sigma$. Variations in the cut on the NLO uncertainty are not shown explicitly, since varying the cut between 0.15 and 0.25 does not result in changes of in the Dalitz variable distribution. The stability of the Dalitz variables with respect to the cuts introduced in the analysis is shown by calculating a $\chi^{2}$ between the data distribution and the scaled distributions obtained after introduction of the cuts. This $\chi^{2}$ is defined in analogy to Equation 3.15, only $n_{M C_{i}}$ is replaced by $n_{D_{i}}$, the scaled number of events in bin $i$ after the introduction of the analysis cuts. Table 6.2 shows the $\chi^{2}$ per bin 
CDF: Preliminary

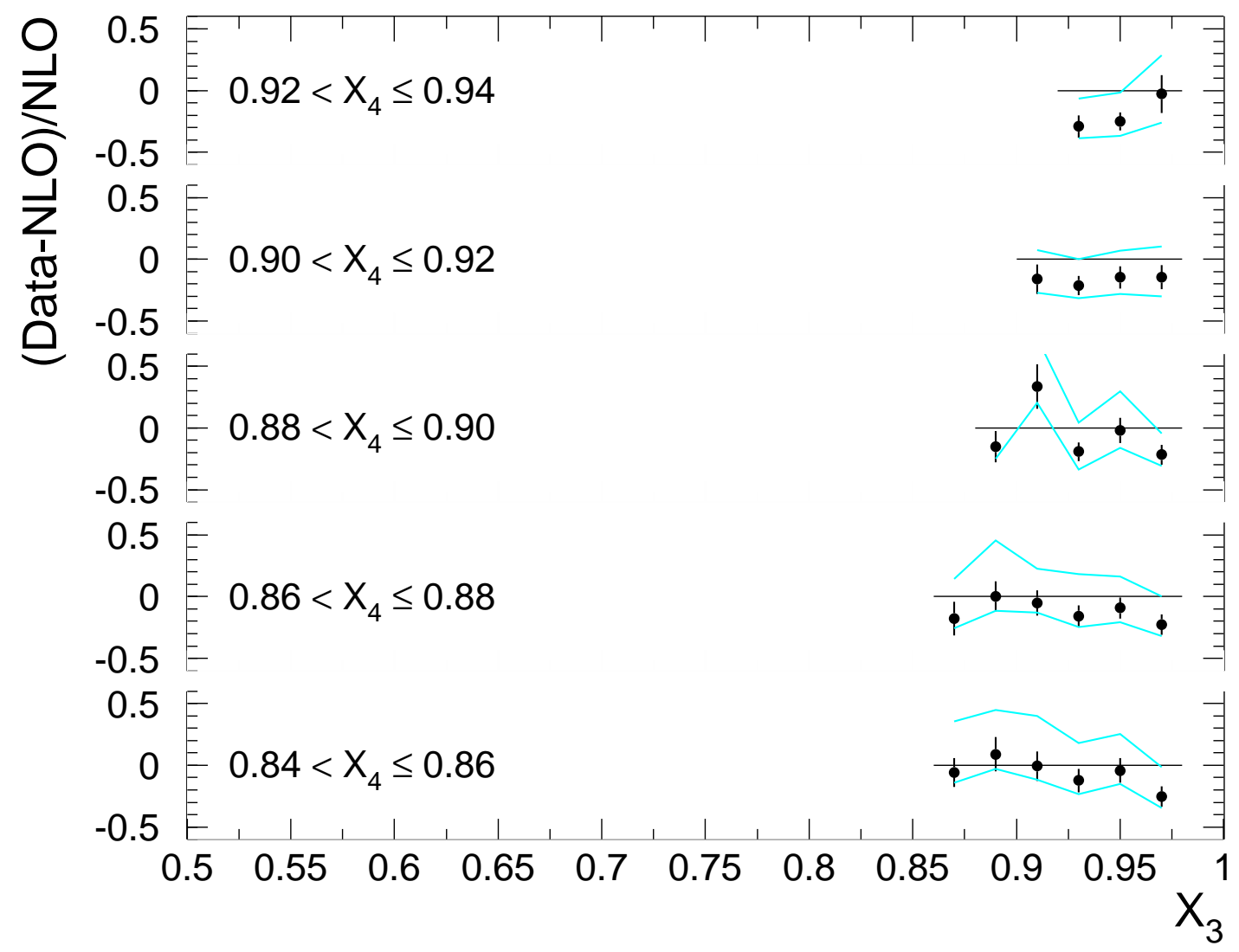

Figure 6.14: The fractional difference between unsmeared data and the NLO prediction, using the CTEQ3 parton distribution function, as a function of $\mathrm{X}_{3}$ for $0.84<\mathrm{X}_{4} \leq 0.94$.

calculated according to this definition. The Dalitz distributions after these variations in the cut parameters are shown in Figures E.1 through E.14 in Appendix E.

Visual comparison of Figures 6.13 and 6.11 indicates a systematic width difference between the Dalitz distributions in the data and the NLO prediction. This difference presents itself in an average fractional difference between data and prediction which is smaller for bins with high $\mathrm{X}_{4}$ compared to bins with low $\mathrm{X}_{4}$. The average fractional difference for the three-jet sample as a function of $\mathrm{X}_{4}$ is shown in Figure 6.19. 
CDF: Preliminary

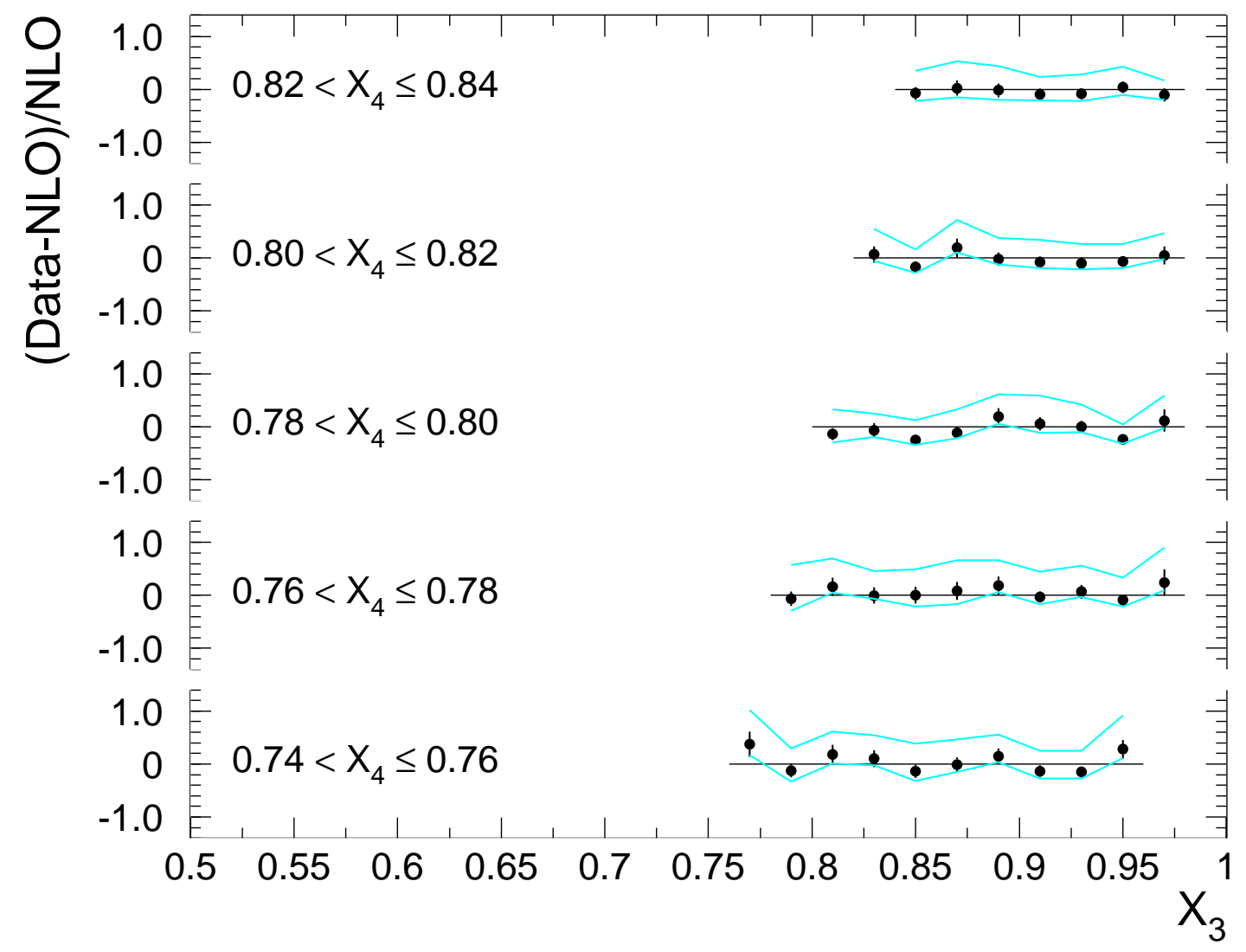

Figure 6.15: The fractional difference between unsmeared data and the NLO prediction, using the CTEQ3 parton distribution function, as a function of $\mathrm{X}_{3}$ for $0.74<\mathrm{X}_{4} \leq 0.84$. 
CDF: Preliminary

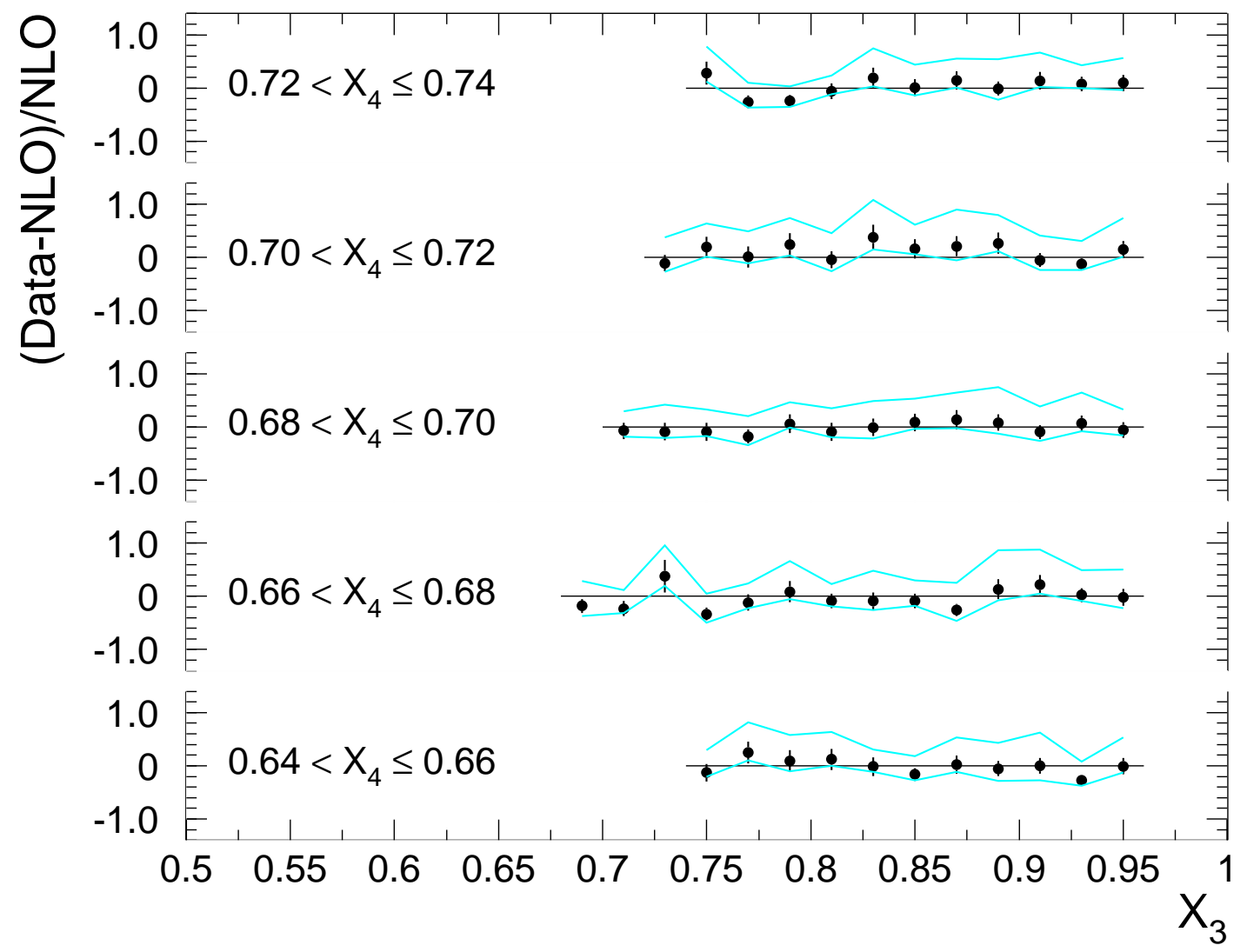

Figure 6.16: The fractional difference between unsmeared data and the NLO prediction, using the CTEQ3 parton distribution function, as a function of $\mathrm{X}_{3}$ for $0.64<\mathrm{X}_{4} \leq 0.74$. 
CDF: Preliminary

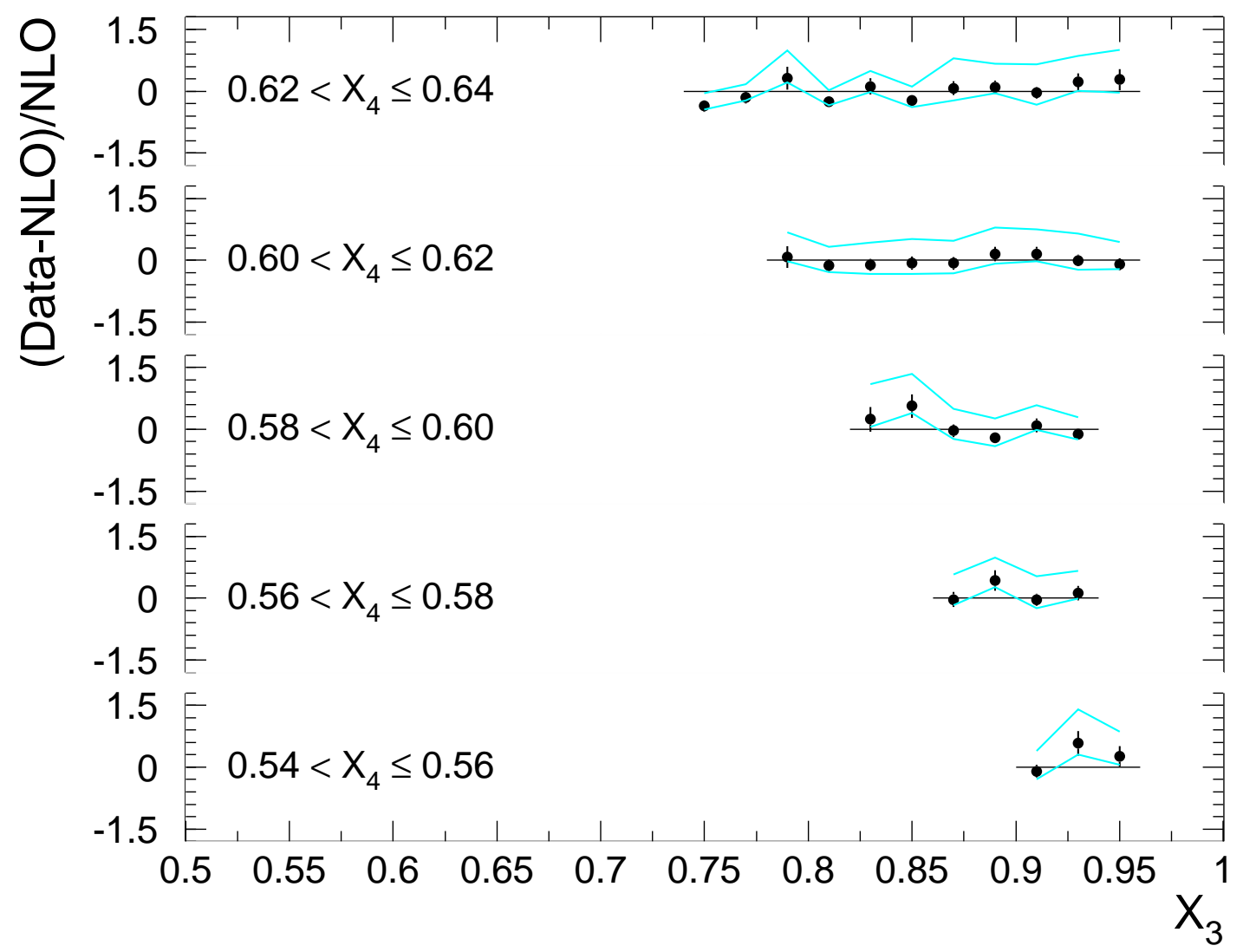

Figure 6.17: The fractional difference between unsmeared data and the NLO prediction, using the CTEQ3 parton distribution function, as a function of $\mathrm{X}_{3}$ for $0.54<\mathrm{X}_{4} \leq 0.64$. 


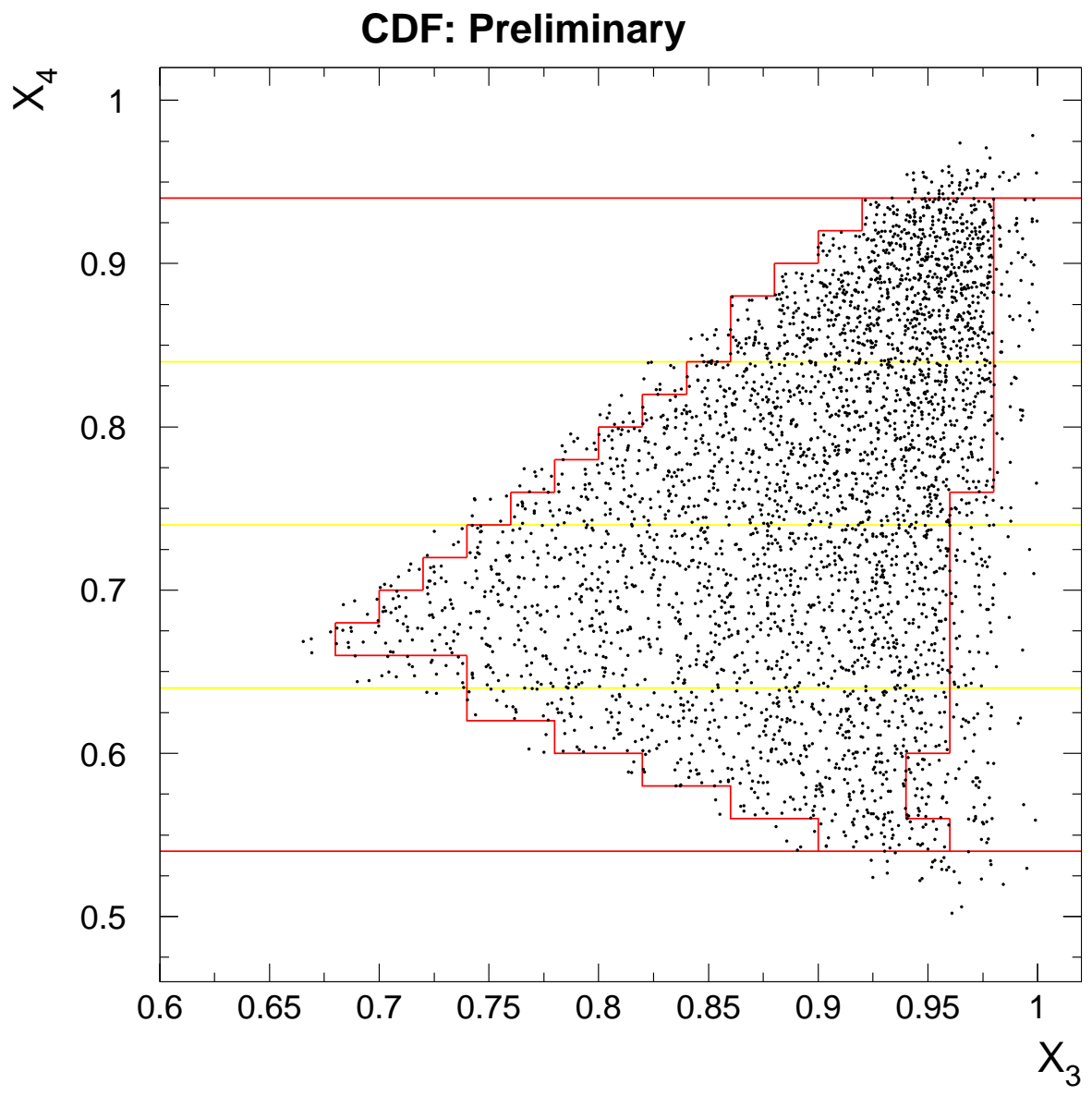

Figure 6.18: The area enclosed by the lines indicates the bins in $\mathrm{X}_{3}-\mathrm{X}_{4}$ space that are used in the analysis and plotted in Figures 6.14 through 6.17. Bins with a theoretical error $>0.2$ are excluded. 


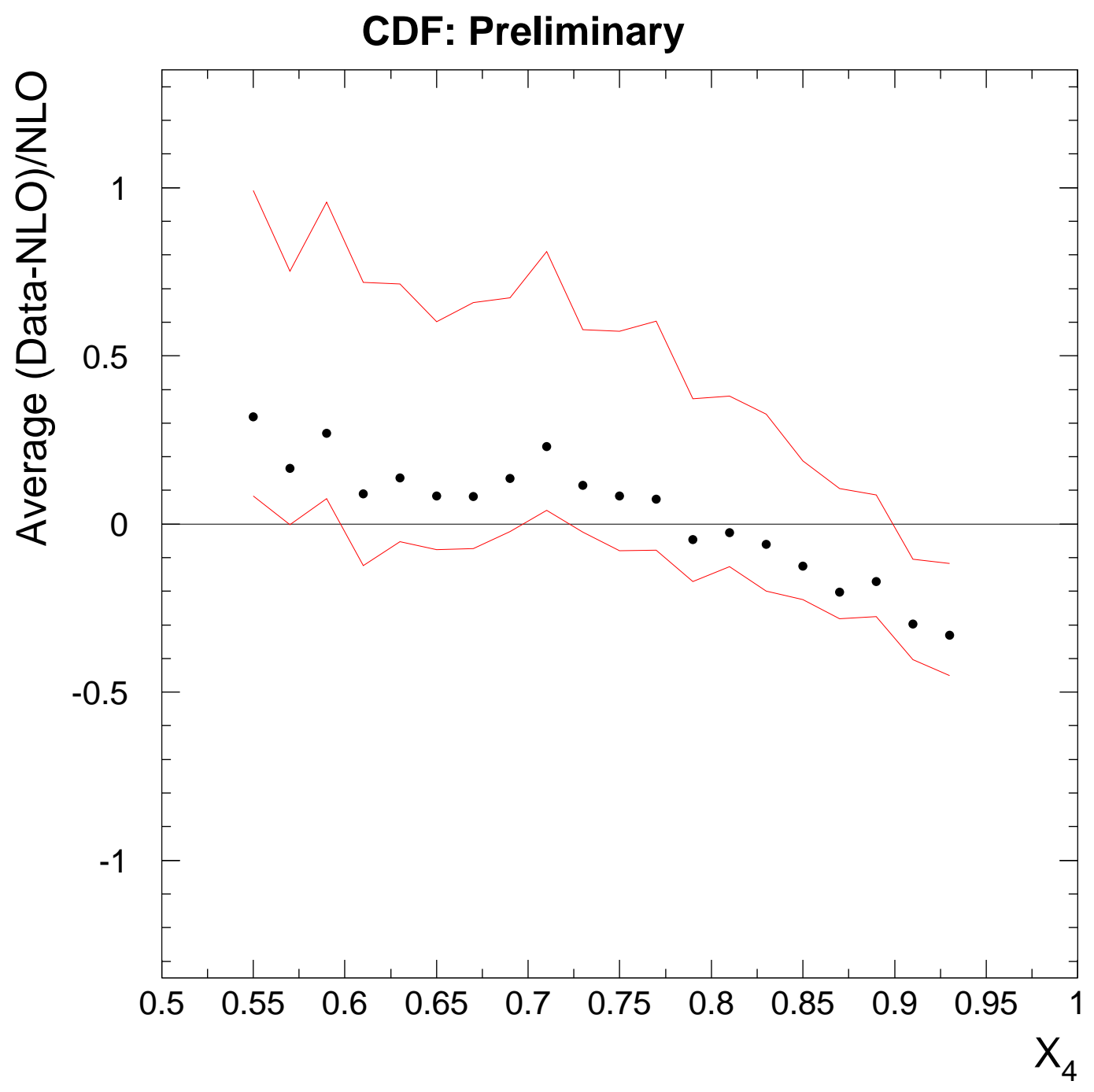

Figure 6.19: The average fractional difference for the three-jet sample as a function of $\mathrm{X}_{4}$. 


\begin{tabular}{|c|c|c|c|}
\hline Cut Parameter & Cut Parameter Value & $\chi^{2}$ & $\chi^{2}$ per bin \\
\hline \hline cone overlap & -0.05 & 1.47 & $6.58 \times 10^{-3}$ \\
cone overlap & +0.05 & 1.58 & $7.06 \times 10^{-3}$ \\
$\eta$ & -0.075 & 1.58 & $7.07 \times 10^{-3}$ \\
$\eta$ & +0.075 & 1.60 & $7.15 \times 10^{-3}$ \\
Jet $\mathrm{E}_{\mathrm{T}}$ & $-5 \mathrm{GeV}$ & 0.999 & $4.61 \times 10^{-3}$ \\
Jet $\mathrm{E}_{\mathrm{T}}$ & $+5 \mathrm{GeV}$ & 33.1 & 0.149 \\
geometrical acceptance & $-0.01 \mathrm{in}\left|\cos \theta_{3}^{*}\right|$ & 1.59 & $7.11 \times 10^{-3}$ \\
geometrical acceptance & $+0.01 \mathrm{in}\left|\cos \theta_{3}^{*}\right|$ & 1.60 & $7.15 \times 10^{-3}$ \\
$\sum \mathrm{E}_{\mathrm{T}}$ & $-10 \mathrm{GeV}$ & $5.07 \times 10^{-4}$ & $2.26 \times 10^{-6}$ \\
$\sum \mathrm{E}_{\mathrm{T}}$ & $+10 \mathrm{GeV}$ & $1.53 \times 10^{-2}$ & $6.81 \times 10^{-5}$ \\
number of tracks & -1 & $8.40 \times 10^{-2}$ & $3.75 \times 10^{-4}$ \\
number of tracks & +1 & $7.61 \times 10^{-4}$ & $3.40 \times 10^{-6}$ \\
distance between vertices & $-1 \mathrm{~cm}$ & $9.95 \times 10^{-3}$ & $4.44 \times 10^{-5}$ \\
distance between vertices & $+1 \mathrm{~cm}$ & $1.78 \times 10^{-2}$ & $7.93 \times 10^{-5}$ \\
$\sigma_{\mathrm{NLO}}$ & $<0.14$ & $8.29 \times 10^{-3}$ & $3.70 \times 10^{-5}$ \\
$\sigma_{\mathrm{NLO}}$ & $<0.27$ & $7.37 \times 10^{-3}$ & $3.29 \times 10^{-5}$ \\
\hline \multicolumn{2}{|c}{}
\end{tabular}

Table 6.2: The $\chi^{2}$ between the data and the scaled Dalitz distributions after the introduction of the various analysis cuts.

The $\chi^{2}$ between the unsmeared data and the NLO calculation is plotted in Figure 6.20 for several different values of the strong coupling constant, $\alpha_{\mathrm{S}}$, each with the appropriate parton distribution function from the CTEQ3 family. Table 6.3 summarizes the results that are plotted in Figure 6.20. The error shown consists of the contributions from the statistical uncertainty on the data and the error on the theoretical calculation. The present statistics result from one $16 \mathrm{CPU}$-week run of the theoretical calculation at each $\alpha_{\mathrm{S}}$ value. With this level of uncertainty, the $\chi^{2}$ does not appear to be changing systematically with $\alpha_{\mathrm{S}}$, so no measurement of $\alpha_{\mathrm{S}}$ can be extracted. The sensitivity of the result to the cut on the NLO uncertainty is small. The uncertainties in the bins rejected by this cut are significantly larger than 0.20.

A difference between the data and the theoretical calculation (for any assumed value of $\alpha_{\mathrm{S}}$ ) is a difference in the three-jet production cross section, which for the data is defined as:

$$
\sigma \equiv \frac{\mathrm{N}}{\epsilon \int \mathcal{L} \mathrm{dt}}
$$




\section{CDF: Preliminary}

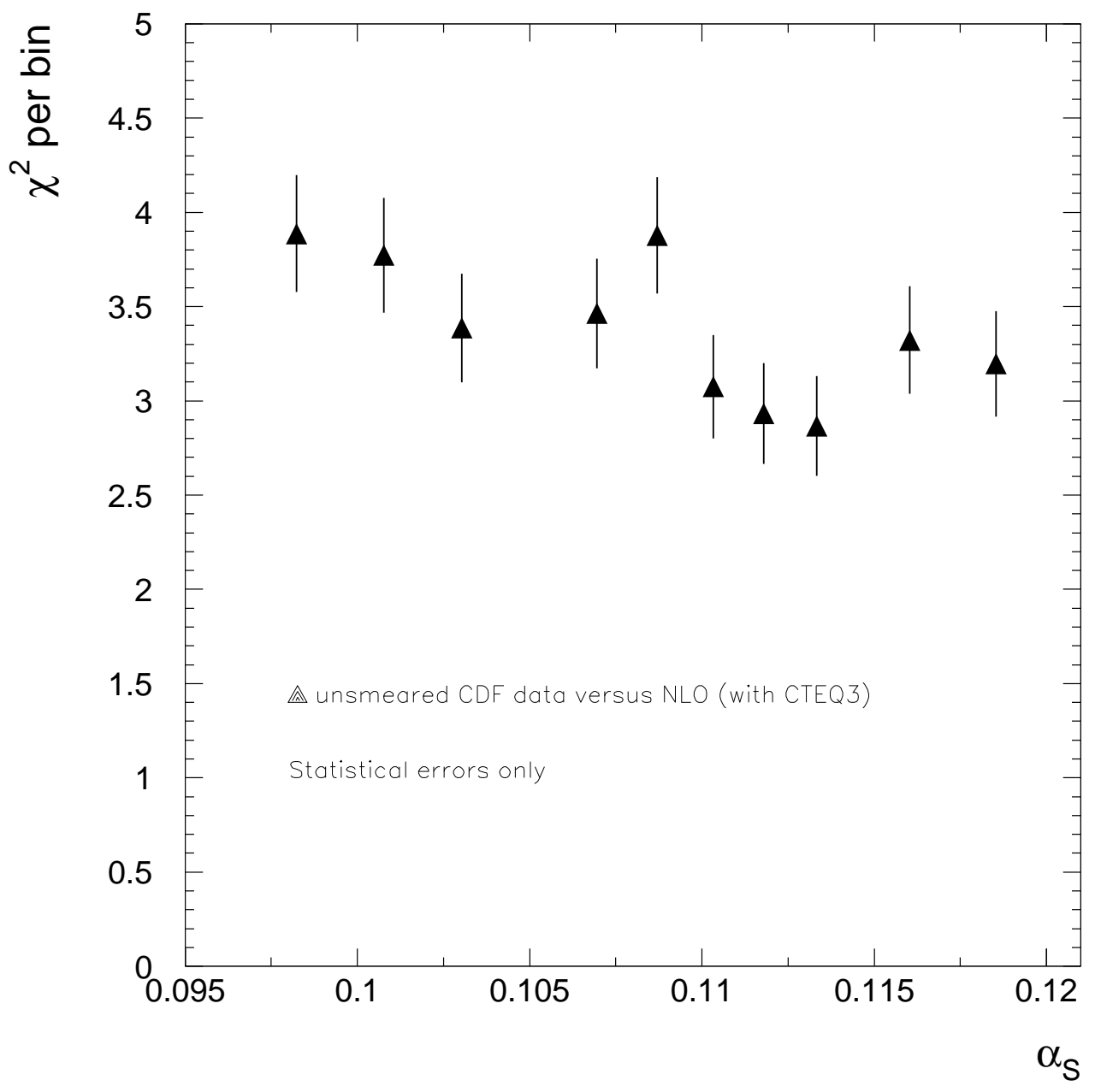

Figure 6.20: $\chi^{2}$ between the data and the NLO calculation for different values of $\alpha_{\mathrm{S}}$, using appropriate parton distribution functions from the CTEQ3 family. 


\begin{tabular}{|c|c|c|}
\hline$\alpha_{\mathrm{S}}$ & $\chi^{2}$ & $\chi^{2}$ per bin \\
\hline \hline 0.0982 & $654 \pm 51$ & $4.01 \pm 0.314$ \\
0.1008 & $632 \pm 50$ & $3.88 \pm 0.308$ \\
0.1030 & $567 \pm 48$ & $3.48 \pm 0.292$ \\
0.1070 & $582 \pm 48$ & $3.57 \pm 0.296$ \\
0.1087 & $676 \pm 52$ & $4.15 \pm 0.319$ \\
0.1103 & $515 \pm 45$ & $3.16 \pm 0.279$ \\
0.1118 & $479 \pm 44$ & $2.94 \pm 0.268$ \\
0.1133 & $481 \pm 44$ & $2.95 \pm 0.269$ \\
0.1160 & $564 \pm 48$ & $3.46 \pm 0.292$ \\
0.1185 & $543 \pm 47$ & $3.33 \pm 0.286$ \\
\hline
\end{tabular}

Table 6.3: The $\chi^{2}$ between the data and the NLO calculation for different values of $\alpha_{\mathrm{S}}$, using appropriate parton distribution functions from the CTEQ3 family.

where $\mathrm{N}$ is the total number of three-jet events after all cuts have been applied $\left(30,325\right.$ in our data sample), $\int \mathcal{L} \mathrm{dt}$ is the effective total integrated luminosity, and $\epsilon=0.93 \pm 0.02[66]$ is the $z$-vertex cut efficiency. For the NLO calculation, the predicted cross sections in all $\mathrm{X}_{3}-\mathrm{X}_{4}$ bins are added. The result is given in units of $\mathrm{nb}^{-1}$ area ${ }^{-1}$. Given the size of the bins in $\mathrm{X}_{3}-\mathrm{X}_{4}$ space, the cross section calculated in each bin is multiplied by a scale factor of 0.0004 to find the true three-jet production cross section in all individual bins. Table 6.4 summarizes the cross section predicted by the calculation for each $\alpha_{\mathrm{S}}$. The cross section uncertainty quoted in this table is statistical. The total three-jet production cross section, given the selection criteria discussed in Chapters 3 and 6, in CDF Run 1b is $419 \pm 2$ (stat.) ${ }_{-64}^{+185}$ (syst.) pb. This is consistent with an earlier CDF measurement of $627_{-168}^{+205}$ (syst.) pb, after the efficiencies of additional cuts introduced in our analysis are considered. The total multi-jet production cross section measured without these cuts in Reference [56] is $1340_{-360}^{+440}$ (syst.) pb. The cuts that are applied in this analysis in addition to the work in Reference [56] are the rejection of two-jet events (efficiency about 0.68), the $\sum \mathrm{E}_{\mathrm{T}}$ cut on the three leading jets (efficiency about 0.93 ), the cut on bins with NLO uncertainties $>20 \%$ (efficiency about 0.90), the cone overlap cut (efficiency about 0.93), the geometrical acceptance cut (efficiency about 0.93), the rejection of runs 
with malfunctioning detector components (efficiency about 0.97), and the cut on the jet pseudo-rapidity (efficiency about 0.98.) Uncertainties of approximately 1-2\% are assigned to these efficiency estimates. Statistical uncertainties in this earlier analysis are not reported because the result was obtained from a data set which also included two-jet events, significantly increasing the sample size. For the three-jet contribution, the statistical uncertainties are expected to be of the same magnitude as the statistical uncertainties in our analysis. If we do not restrict our analysis to bins with NLO uncertainties $<20 \%$, the total three-jet production cross section is $466 \pm 2$ (stat.) ${ }_{-71}^{+206}$ (syst.) pb. This again is consistent with the earlier measurement which found a total three-jet production cross section of $697_{-187}^{+228}$ (syst.) pb. The total three-jet production cross section for the NLO calculation, using the CTEQ3 parton distribution function with $\alpha_{\mathrm{S}}=0.1160$, is $402 \pm 3 \mathrm{pb}$ when all bins in the Dalitz plane are included. The cross section in each individual kinematically allowed bin in the Dalitz plane in tabulated in Tables F.1 through F.14 in Appendix F. The total threejet production cross sections predicted by the NLO calculation as a function of $\alpha_{\mathrm{S}}$ are compared to the data in Figure 6.21.

\begin{tabular}{|c|c|}
\hline$\alpha_{\mathrm{S}}$ & Three-jet Production Cross Section $(\mathrm{pb})$ \\
\hline \hline 0.0982 & $372 \pm 3$ \\
0.1008 & $367 \pm 3$ \\
0.1030 & $371 \pm 3$ \\
0.1070 & $386 \pm 3$ \\
0.1087 & $390 \pm 3$ \\
0.1103 & $387 \pm 4$ \\
0.1118 & $438 \pm 4$ \\
0.1133 & $395 \pm 3$ \\
0.1160 & $373 \pm 3$ \\
0.1185 & $366 \pm 3$ \\
\hline
\end{tabular}

Table 6.4: The three-jet production cross section of events in the NLO templates, for different values of $\alpha_{\mathrm{S}}$, using the appropriate parton distribution functions from the CTEQ3 family. The uncertainties are statistical only. 


\subsection{Discussion and Cross Checks}

We have defined several related $\chi^{2}$ factors to cross-check the results. First, as a final check on the unsmearing, we define a function called $\left(\chi^{\prime}\right)^{2}$, in which $\mathrm{n}_{\mathrm{D}_{\mathrm{i}}}$ of Equation 3.15 is replaced by $n_{H_{E R W I G}}$, the number of events generated by HERWIG in bin i. A plot of $\left(\chi^{\prime}\right)^{2}$ versus $\alpha_{\mathrm{S}}$ is shown in Figure 6.22. HERWIG events that have passed QFL and have been unsmeared using the correction factors described in Chapter 4 are plotted as circles. Hadron-level HERWIG events are plotted as squares. The points are consistent, confirming the validity of the unsmearing correction. The behavior of $\left(\chi^{\prime}\right)^{2}$ as a function of $\alpha_{\mathrm{S}}$ in Figure 6.22 is similar to the $\chi^{2}$ behavior in Figure 6.20. The error shown consists of the contributions from the statistical uncertainty on the HERWIG data sample and the error on the theoretical calculation.

The self-consistency of the results is further checked by defining a $\left(\chi^{\prime \prime}\right)^{2}$ in which the $\mathrm{n}_{\mathrm{D}_{\mathrm{i}}}$ of Equation 3.15 is replaced by $\mathrm{n}_{\mathrm{NLO}_{\mathrm{i}}}$, the number of events generated by the NLO prediction with $\alpha_{\mathrm{S}}=0.1160$. A plot of $\left(\chi^{\prime \prime}\right)^{2}$ versus $\alpha_{\mathrm{S}}$ is shown in Figure 6.23. The $\left(\chi^{\prime \prime}\right)^{2}$ in Figure 6.23 is constant for the whole range of $\alpha_{\mathrm{S}}$, indicating that the Dalitz variables are not sensitive to changes in $\alpha_{\mathrm{S}}$ at this level of accuracy. A systematic change of $\left(\chi^{\prime \prime}\right)^{2}$ with changes in $\alpha_{\mathrm{S}}$ is not apparent. Slight deviations of $\left(\chi^{\prime \prime}\right)^{2}$ from 1.0 are possibly due to real, non-systematic differences in the Dalitz distributions predicted by the NLO calculation, or due to an underestimate of the errors in the prediction. The error shown due to the error on the theoretical calculation.

As a final cross check on the potential for a measurement of $\alpha_{S}$, Figures 6.20 and 6.23 are duplicated using the complete set of bins in the Dalitz plane where the cut on the statistical uncertainty on the NLO calculation is not applied. Figures 6.24 and 6.25 show $\chi^{2}$ and $\left(\chi^{\prime \prime}\right)^{2}$ for that case, respectively. As both plots are very similar to Figures 6.20 and 6.23, they also indicate that with the computing resources currently available to us, the CTEQ3 parton distribution function is not adequately sensitive to the gluon distribution to measure $\alpha_{\mathrm{S}}$ by this method. The error shown consists of the contributions from the statistical uncertainty on the data and the error on the 
theoretical calculation. 


\section{CDF: Preliminary}

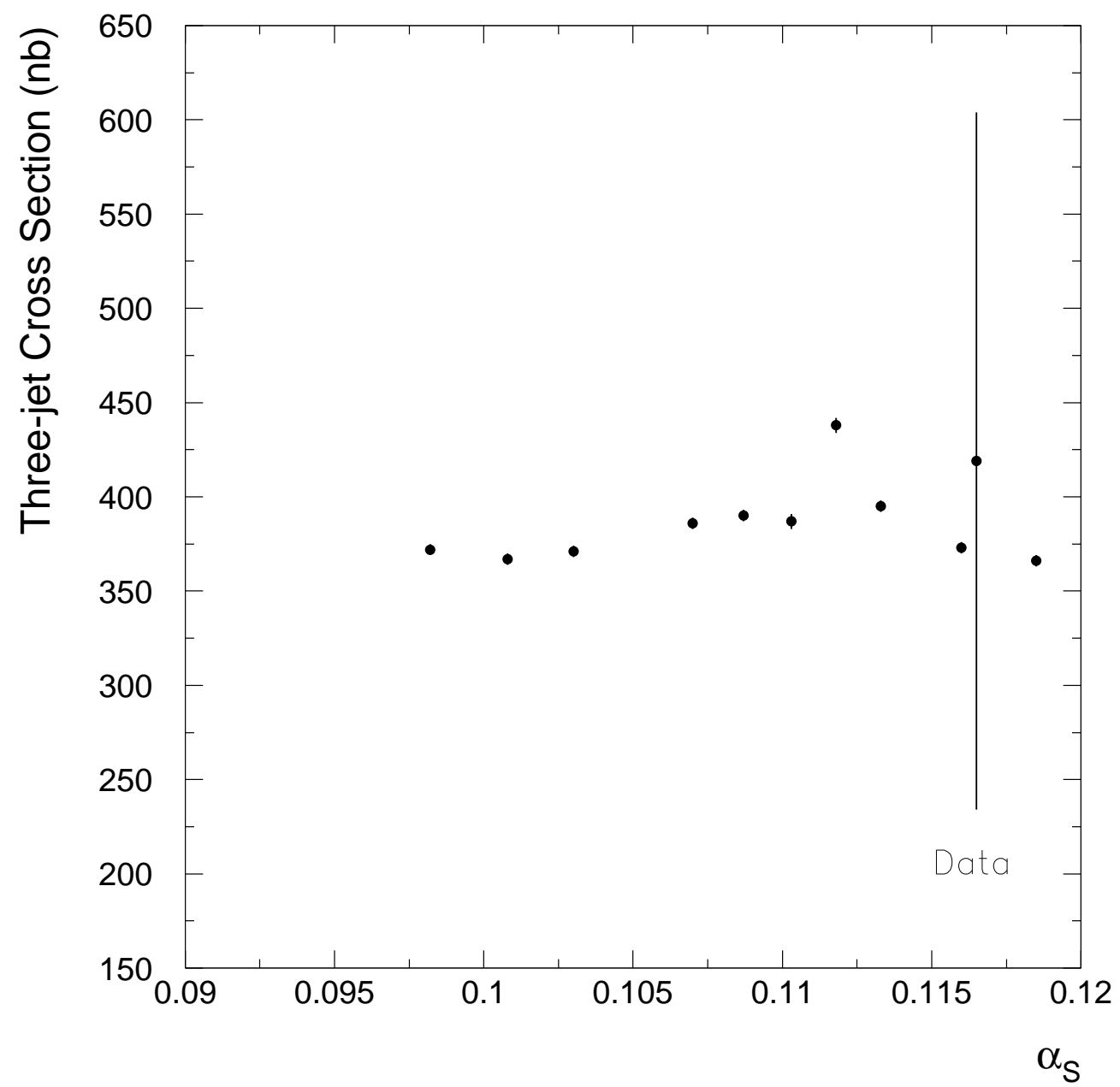

Figure 6.21: The total three-jet production cross section predicted by the NLO calculation as a function of $\alpha_{\mathrm{S}}$ compared to the data. 


\section{CDF: Preliminary}

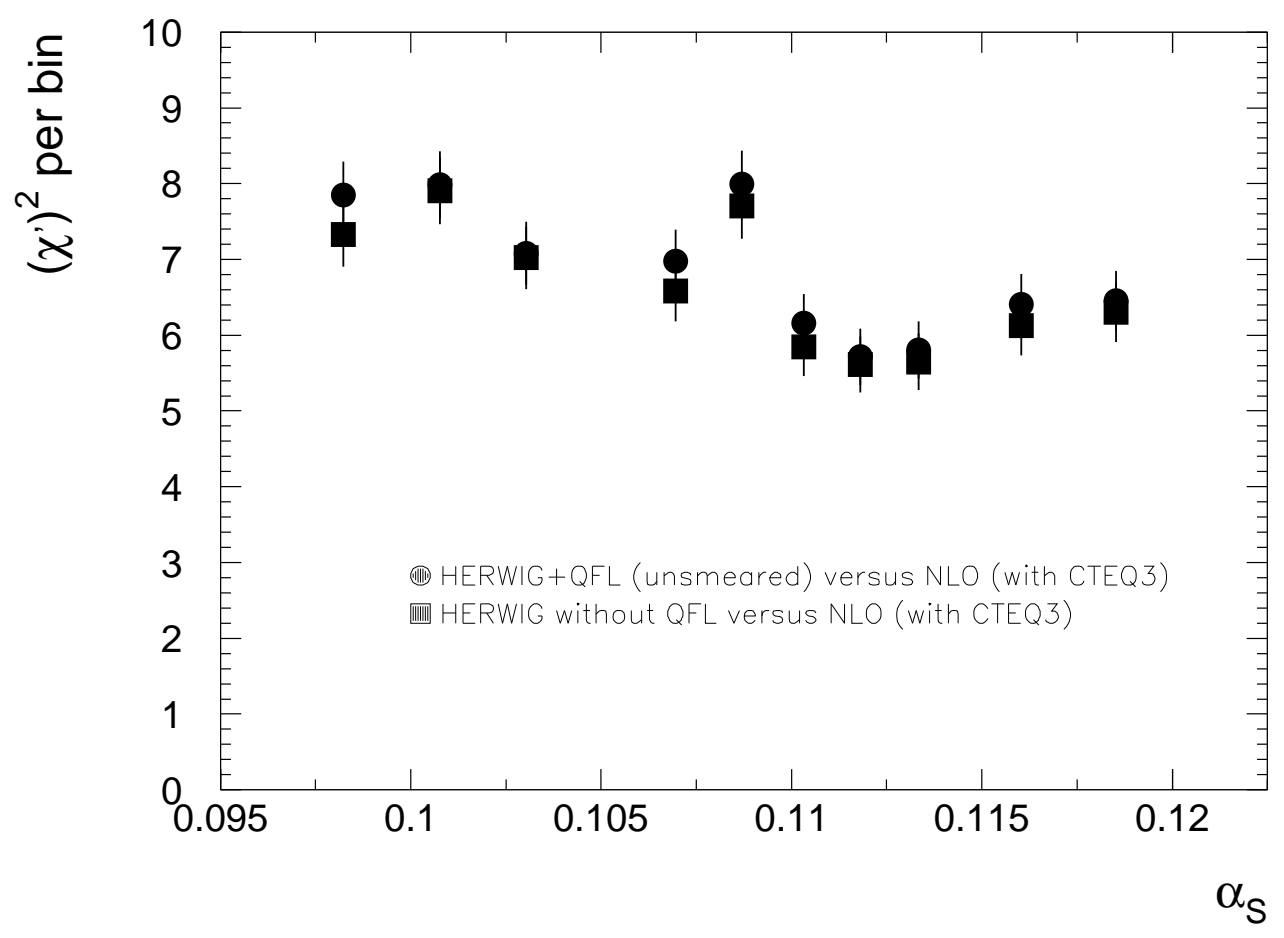

Figure 6.22: The circles represent $\left(\chi^{\prime}\right)^{2}$ between the NLO calculation and events that have been generated by HERWIG, passed through QFL, and then unsmeared by the Monte Carlo approach. The squares represent $\left(\chi^{\prime}\right)^{2}$ between the NLO calculation and hadron-level HERWIG events. The results are consistent, a confirmation of the validity of the unsmearing procedure. The $\left(\chi^{\prime}\right)^{2}$ factor is defined in Section 6.2. 
CDF: Preliminary

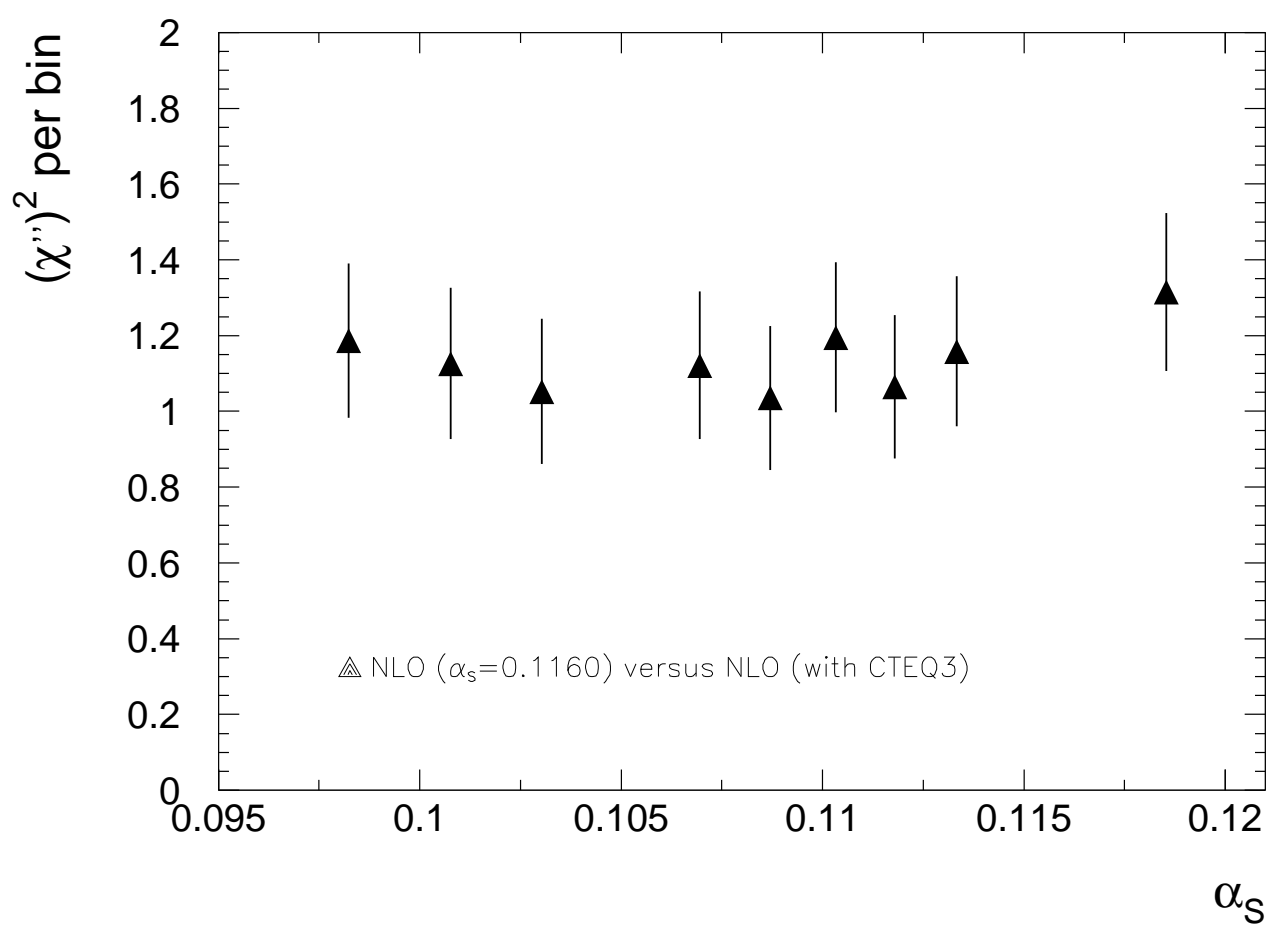

Figure 6.23: $\left(\chi^{\prime \prime}\right)^{2}$ between the NLO calculation with $\alpha_{\mathrm{S}}=0.1160$ and the NLO calculation for other values of $\alpha_{\mathrm{S}}$, using appropriate parton distribution functions from the CTEQ3 family. The $\left(\chi^{\prime \prime}\right)^{2}$ factor is defined in Section 6.2. 
CDF: Preliminary

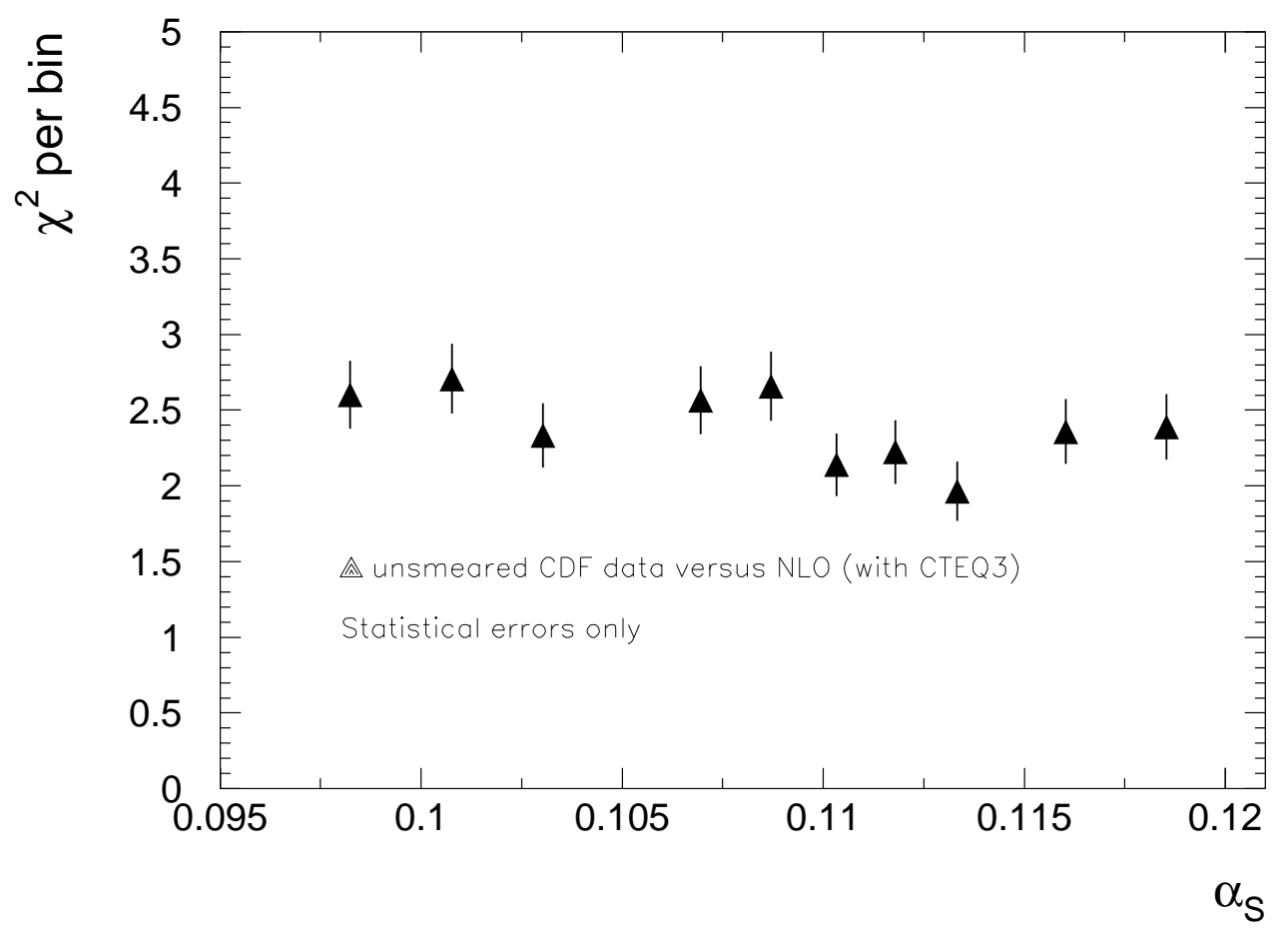

Figure 6.24: $\chi^{2}$ between the data and the NLO calculation for different values of $\alpha_{\mathrm{S}}$, using appropriate parton distribution functions from the CTEQ3 family. The cut on the statistical uncertainty on the NLO calculation is not applied. 
CDF: Preliminary

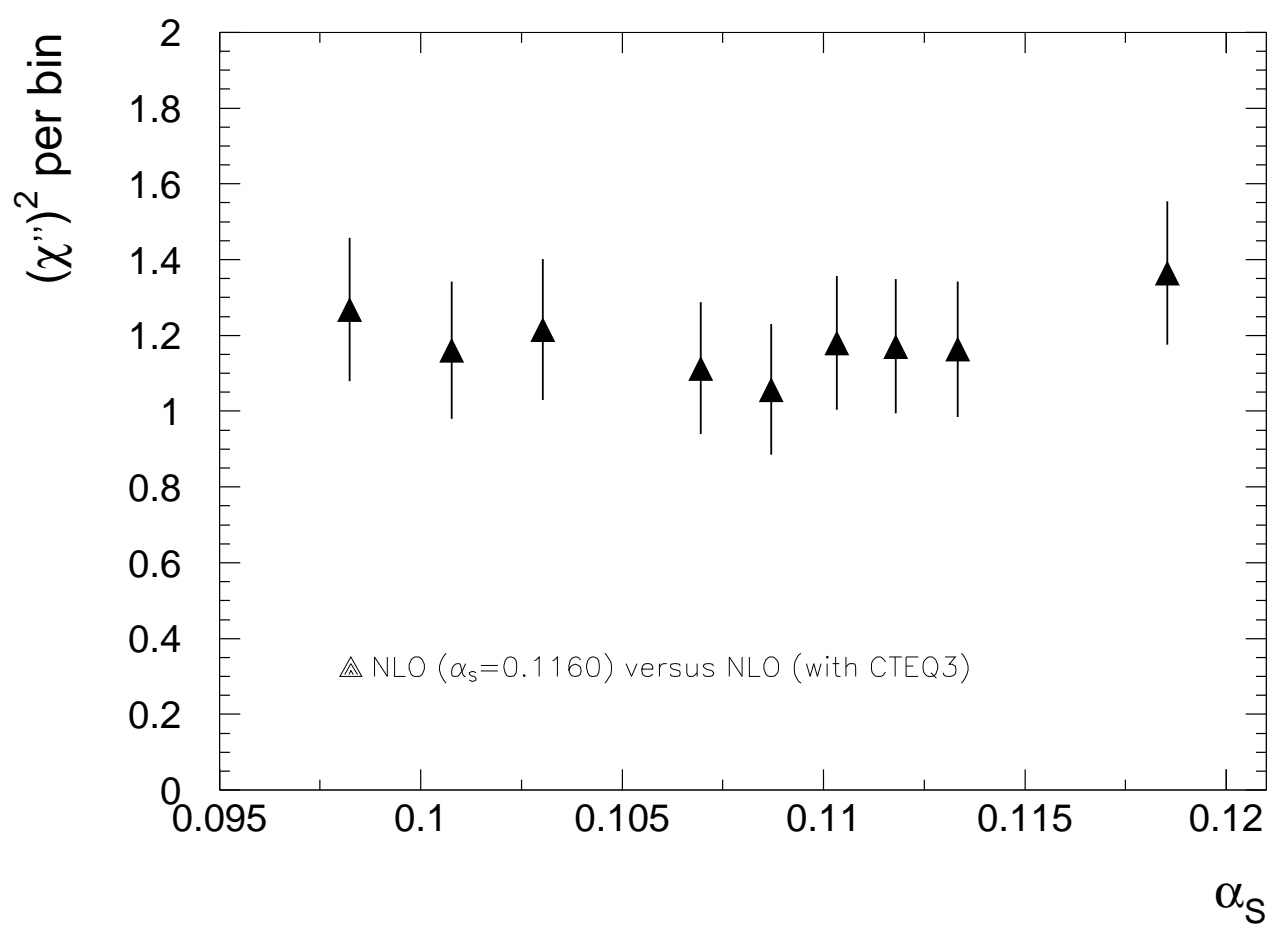

Figure 6.25: $\left(\chi^{\prime \prime}\right)^{2}$ between the NLO calculation with $\alpha_{\mathrm{S}}=0.1160$ and the NLO calculation for other values of $\alpha_{\mathrm{S}}$, using appropriate parton distribution functions from the CTEQ3 family. The $\left(\chi^{\prime \prime}\right)^{2}$ factor is defined in Section 6.2. The cut on the statistical uncertainty on the NLO calculation is not applied. 


\section{Chapter 7}

\section{Results from CTEQ4 Parton Distribution Functions}

To investigate the variability and validity of the results shown in the previous chapter, the main analysis has been repeated using the CTEQ4 parton distribution function family. Results and conclusions from this analysis are shown in this chapter. A comparison of the CTEQ3 and CTEQ4 parton distribution functions is shown in Figures 7.1 and 7.2 for the quarks and the gluons, respectively. The gluon distribution function varies by a few per cent at $\mathrm{x} \leq 0.1$; at larger $\mathrm{x}$, the CTEQ3 and the CTEQ4 distribution functions for quarks and gluons look rather similar.

\subsection{CTEQ4A Results}

The transverse energy distributions of all three jets for unsmeared data and the NLO predictions, using the CTEQ4M parton distribution function, are plotted in Figure 7.3 and compared to HERWIG. The HERWIG event sample has to be adjusted for systematic errors in the QFL energy scale (see Reference [35].) A minimum $\mathrm{p}_{\mathrm{T}}$ of $60 \mathrm{GeV}$ is used in the generation of the HERWIG sample. The three jet distributions are normalized to the same number of events to allow comparison of their shapes. 


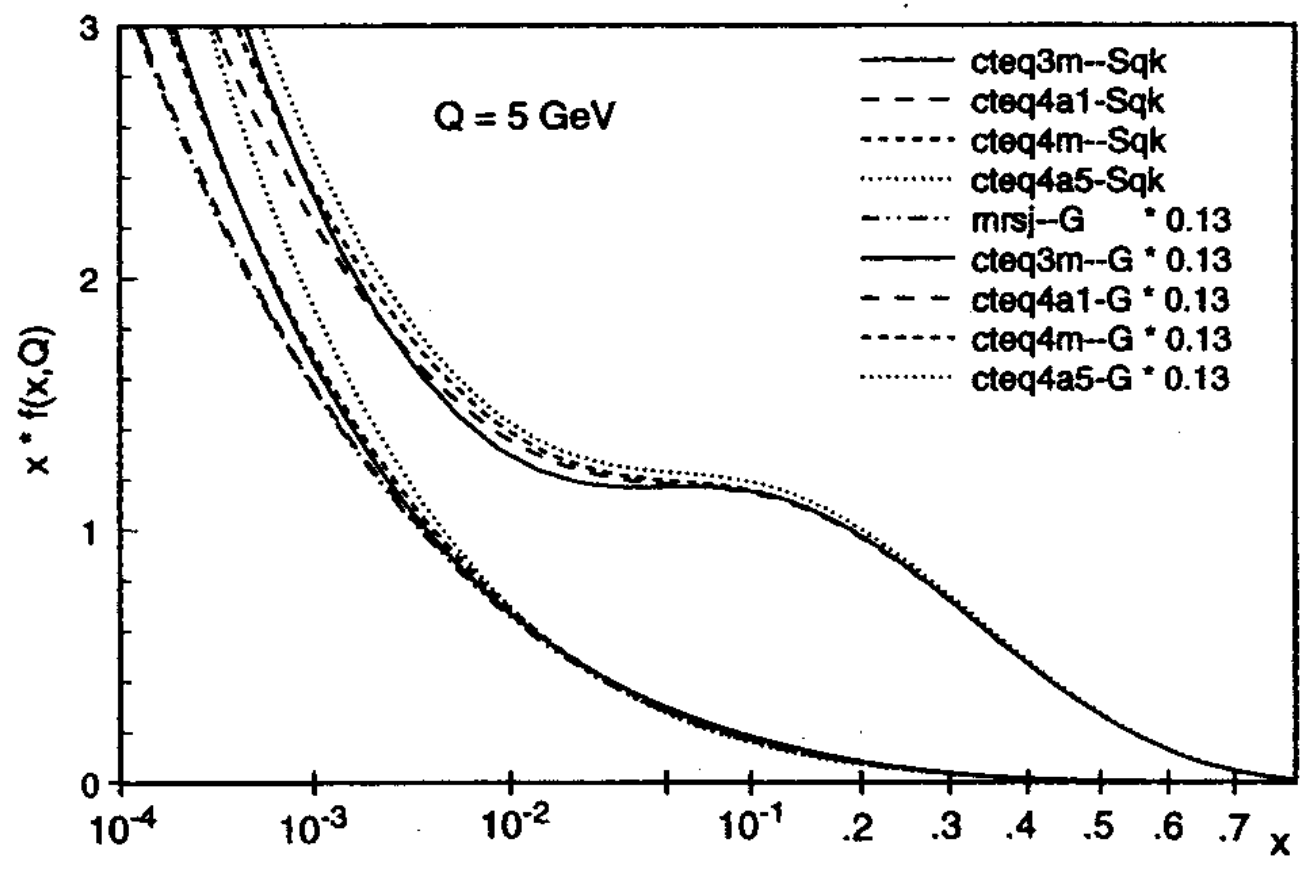

Figure 7.1: Comparison between the CTEQ3 and CTEQ4 parton distribution functions for the quarks (Figure taken from Reference [67].) 


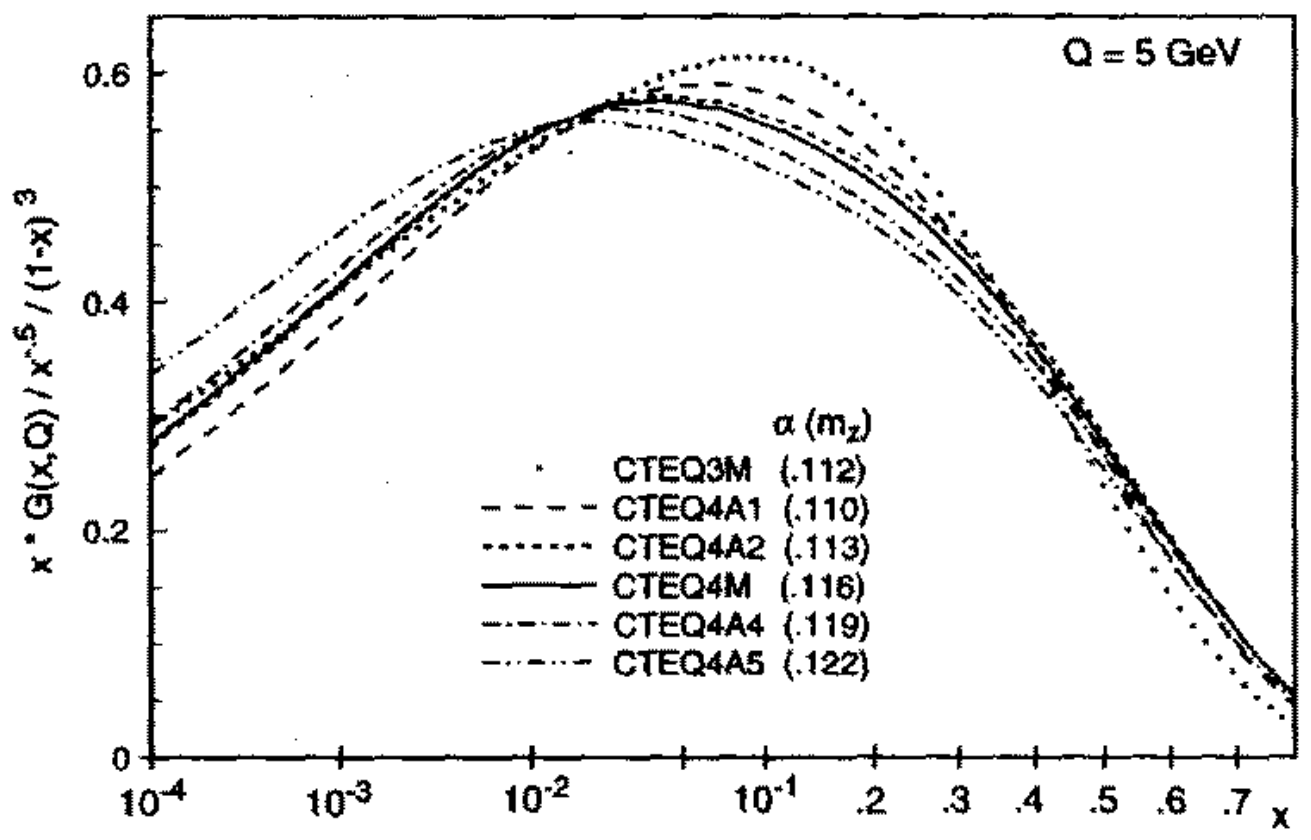

Figure 7.2: Comparison between the CTEQ3 and CTEQ4 parton distribution functions for the gluons (Figure taken from Reference [67].) 
To demonstrate the difference between the jet $\mathrm{E}_{\mathrm{T}}$ distributions obtained by using the CTEQ3 and CTEQ4M parton distribution functions, the ratio of the two jet $\mathrm{E}_{\mathrm{T}}$ distributions is shown in Figure 7.4. At the level of uncertainty achieved by the NLO calculation at this time, deviations of this ratio from the value 1.0 in the $\mathrm{E}_{\mathrm{T}}$ distributions using CTEQ3 and CTEQ4 could be attributed to systematic changes. In the HERWIG data sample, the third jet is calculated to be significantly more energetic than the corresponding distributions for the data and the NLO calculation. It is possible that this is due to the fact that HERWIG does not correctly model the jet multiplicities, as shown in Figure 6.2. For a given value of $\sum E_{\mathrm{T}}$ in an event, the third jet is more energetic if the total number of jets in the event is less than in the data sample or in the NLO prediction.

As an independent check on the data, the jet $\mathrm{E}_{\mathrm{T}}$ distributions obtained by the Jet100 trigger are investigated. Data obtained from the $\sum \mathrm{E}_{\mathrm{T}}$ trigger and from the Jet100 trigger agree very well. The jet $\mathrm{E}_{\mathrm{T}}$ distributions in both cases do not differ significantly. Figure 7.5 shows the transverse energy distributions for the three jets in unsmeared data from the Jet100 trigger, the NLO prediction using the CTEQ4M parton distribution function, and HERWIG with $\mathrm{p}_{\mathrm{T}}=60 \mathrm{GeV}$.

For comparison with the NLO results from the CTEQ3 parton distribution function family, the Dalitz variables are shown in Figures 7.6 through 7.10 for the prediction using CTEQ4A1 (Figure 7.6), CTEQ4A2 (Figure 7.7), CTEQ4M (Figure 7.8), CTEQ4A4 (Figure 7.9), and CTEQ4A5 (Figure 7.10.)

The data and the NLO prediction, using the CTEQ4M parton distribution function, are compared by computing the difference between the observed and predicted number of events, scaled by the number of predicted events, in each bin. This fractional difference is plotted in Figures 7.11 through 7.14 as a function of $\mathrm{X}_{3}$, spanning the entire range in $\mathrm{X}_{4}$. Figure 7.11 shows the region with $0.84<\mathrm{X}_{4} \leq 0.94$, $0.74<\mathrm{X}_{4} \leq 0.84$ in Figure 7.12, and Figures 7.13 and 7.14 span $0.64<\mathrm{X}_{4} \leq 0.74$ and $0.54<\mathrm{X}_{4} \leq 0.64$, respectively. Also shown in Figures 7.11 through 7.14 are the total 


\section{CDF: Preliminary}

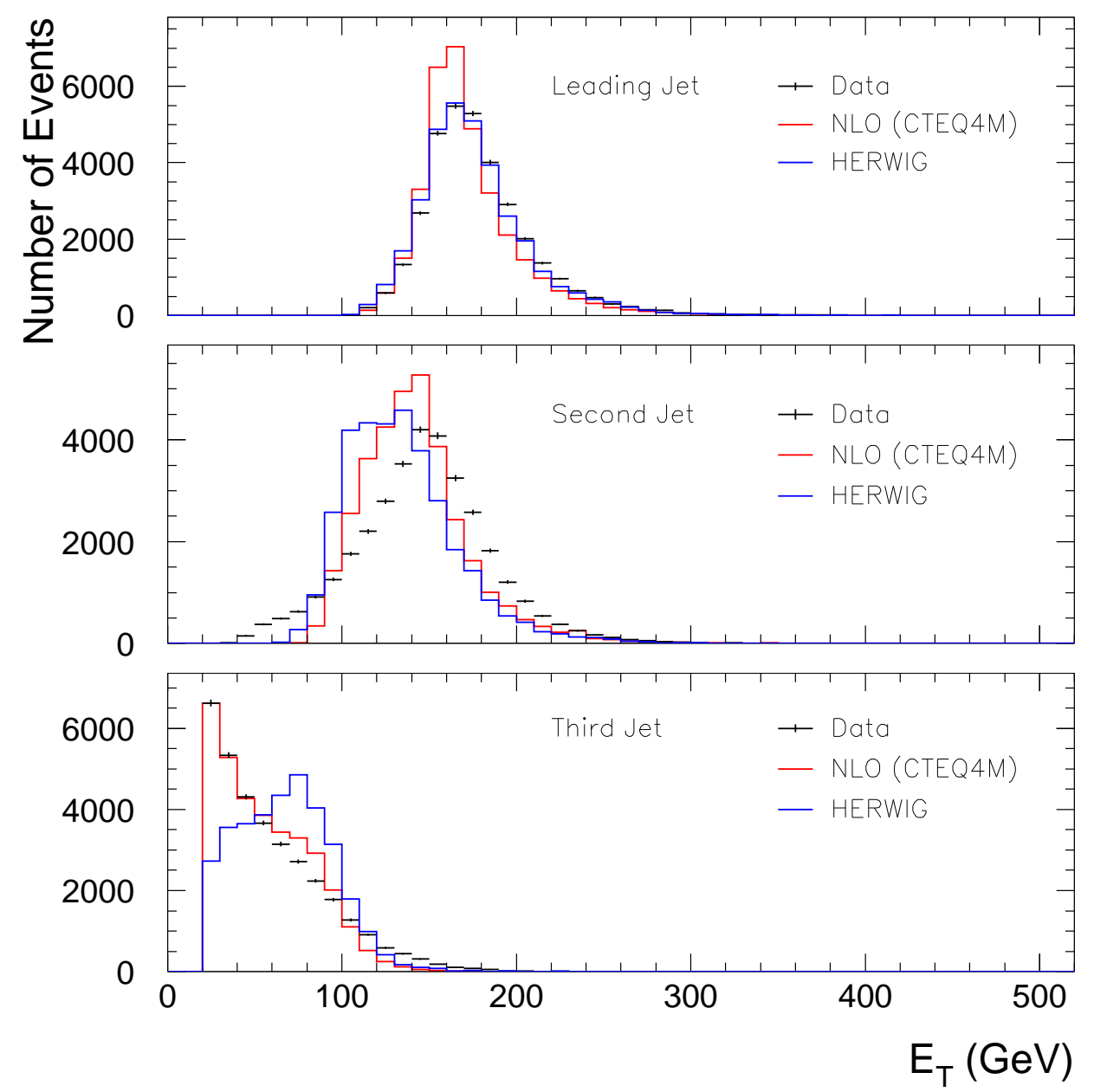

Figure 7.3: The transverse energy distributions for the three jets in unsmeared data, the NLO prediction using the CTEQ4M parton distribution function, and HERWIG. 


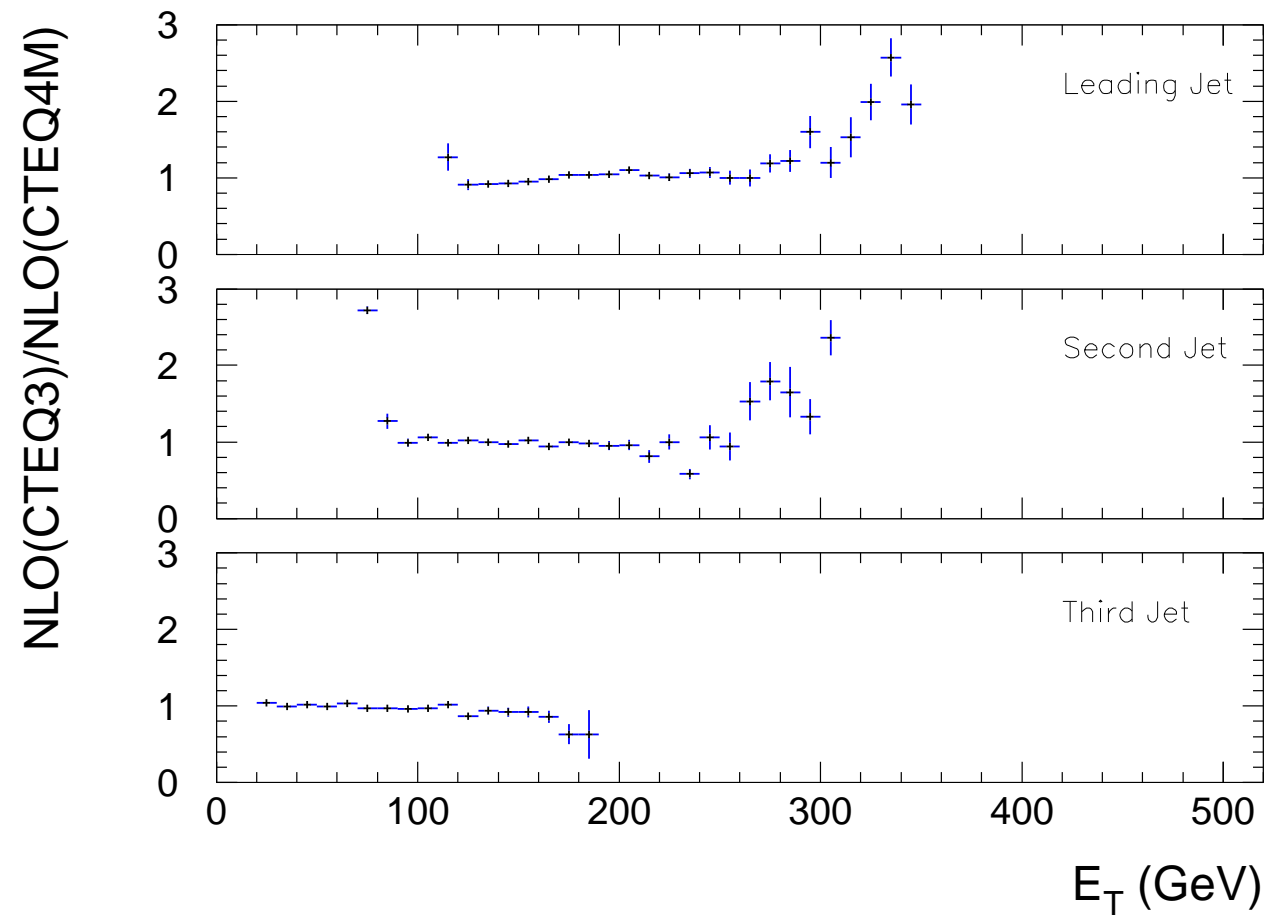

Figure 7.4: The ratio of the jet $\mathrm{E}_{\mathrm{T}}$ distributions obtained by using CTEQ3 and CTEQ4M. 
CDF: Preliminary

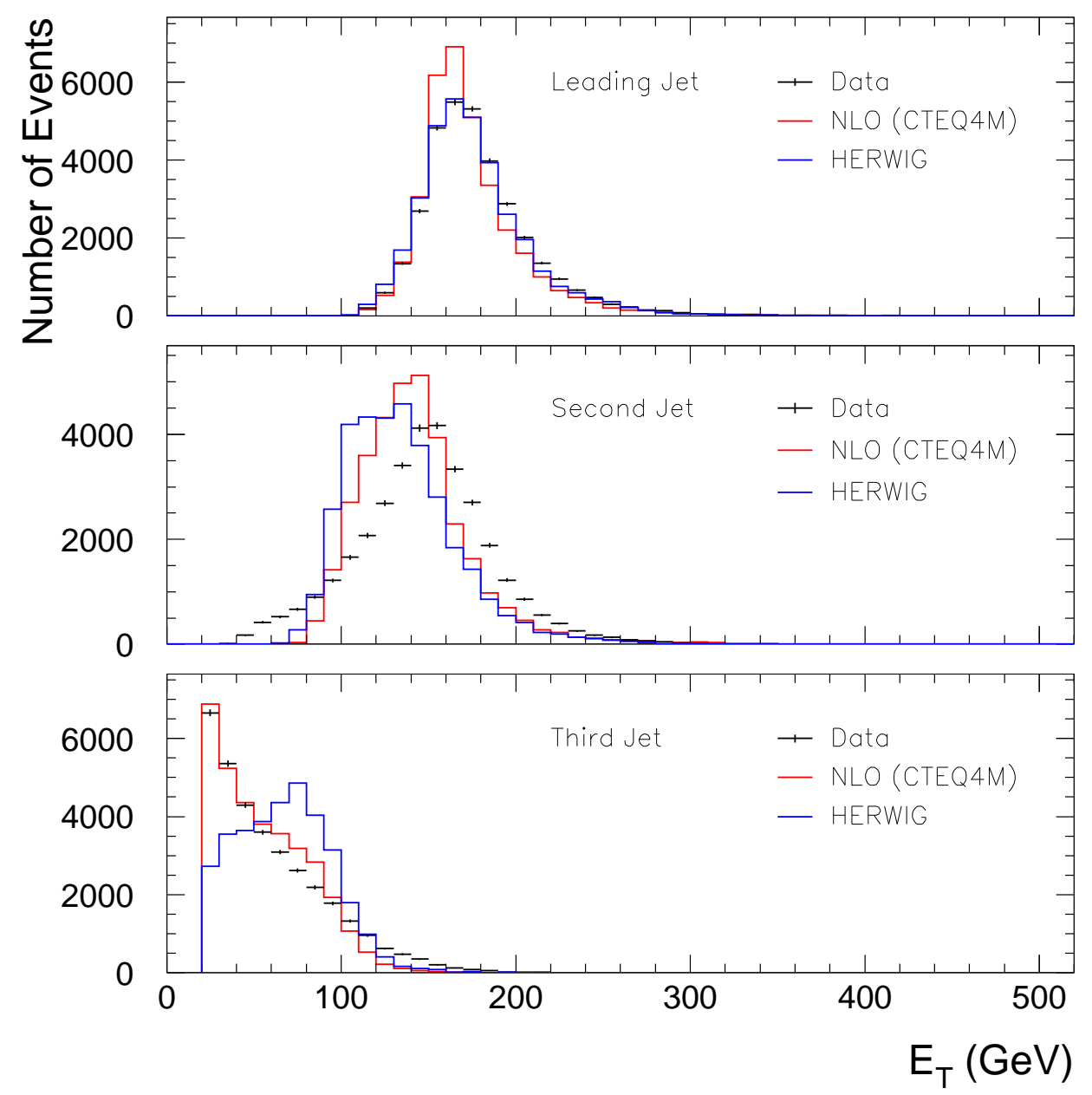

Figure 7.5: The transverse energy distributions for the three jets in unsmeared data from the Jet100 trigger, the NLO prediction using the CTEQ4M parton distribution function, and HERWIG with $\mathrm{p}_{\mathrm{T}}=60 \mathrm{GeV}$. 


\section{CDF: Preliminary}

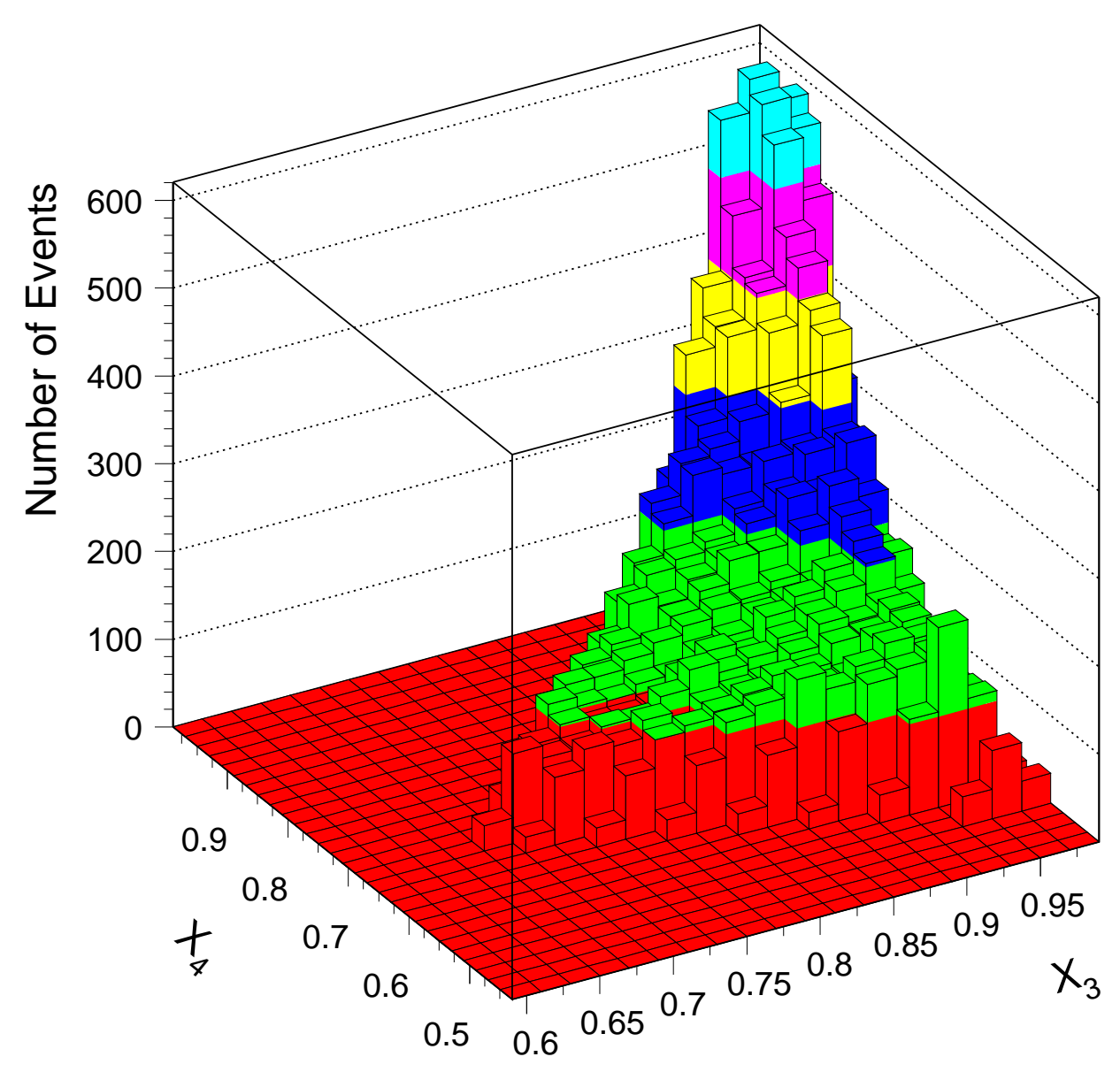

Figure 7.6: The event density in the Dalitz plane as predicted by the NLO calculation with CTEQ4A1. 


\section{CDF: Preliminary}

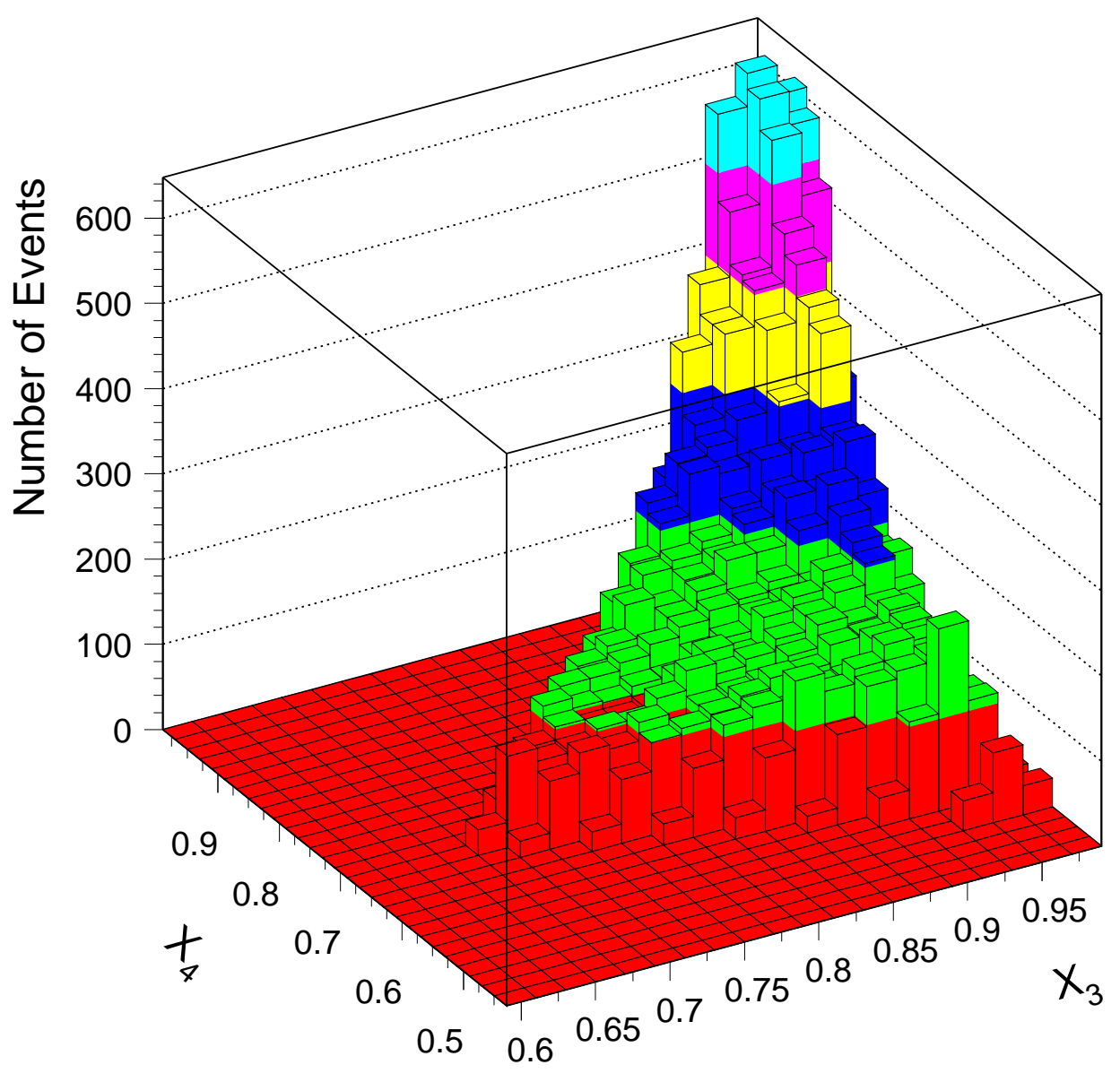

Figure 7.7: The event density in the Dalitz plane as predicted by the NLO calculation with CTEQ4A2. 


\section{CDF: Preliminary}

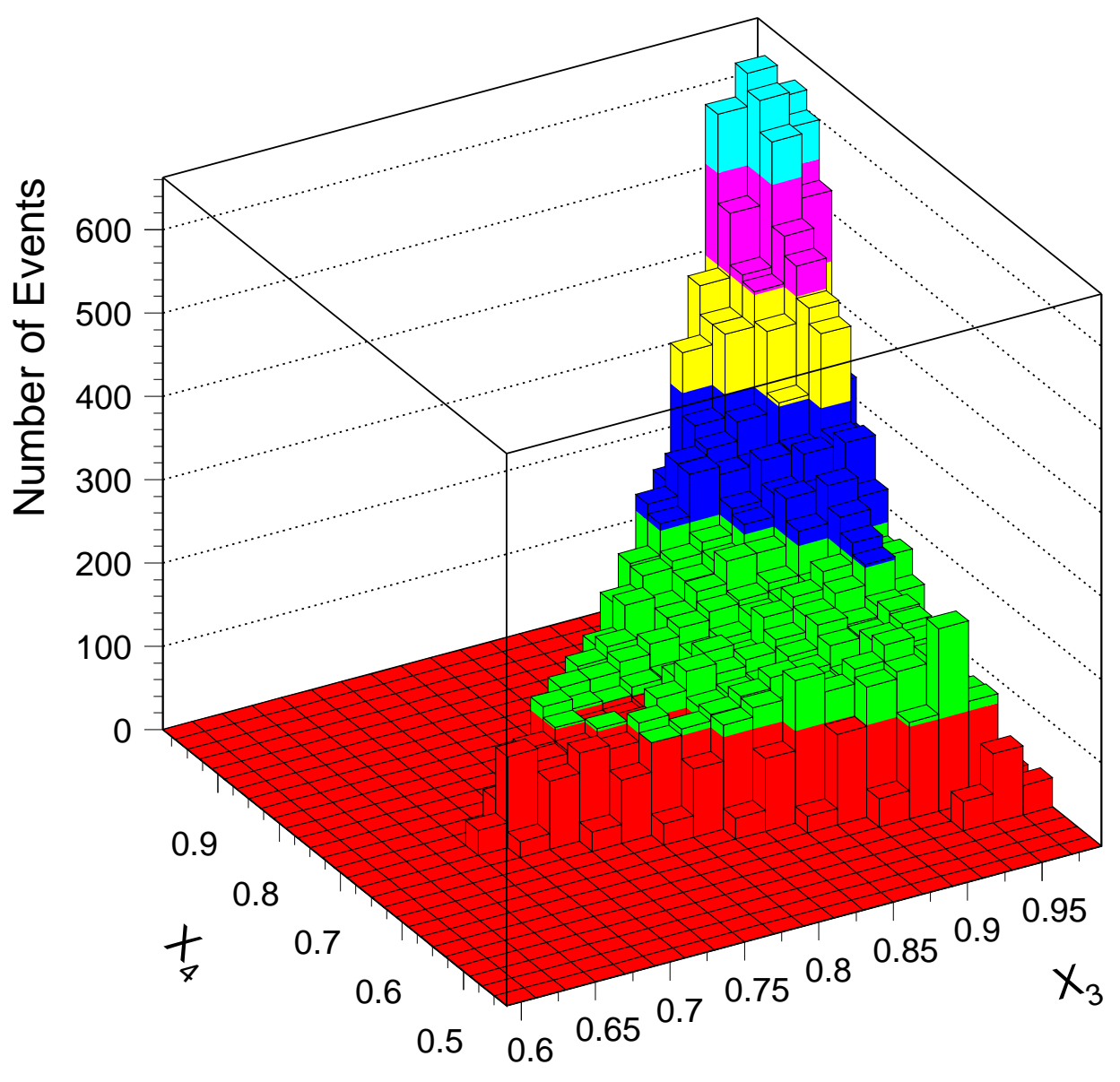

Figure 7.8: The event density in the Dalitz plane as predicted by the NLO calculation with CTEQ4M. 


\section{CDF: Preliminary}

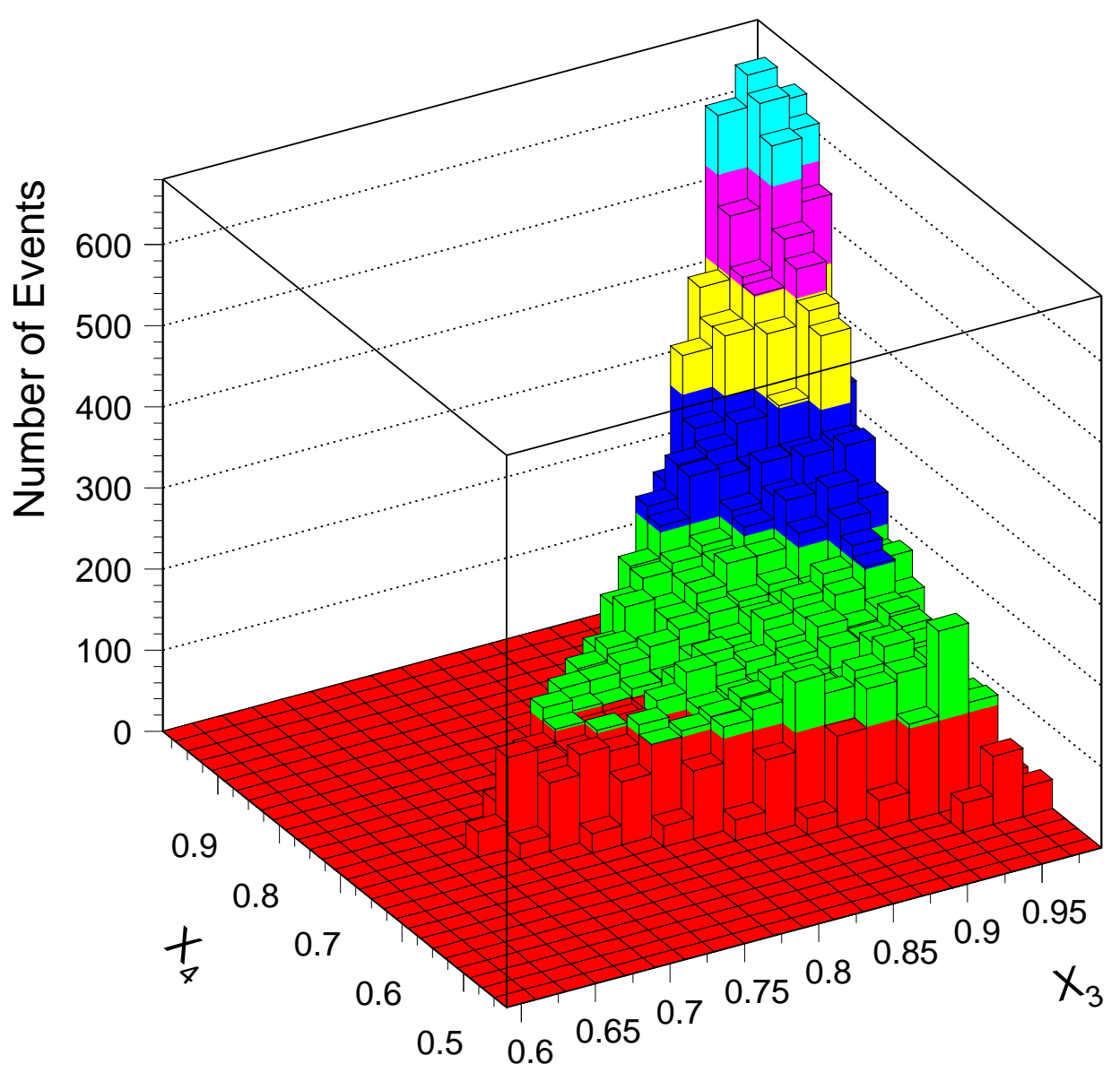

Figure 7.9: The event density in the Dalitz plane as predicted by the NLO calculation with CTEQ4A4. 


\section{CDF: Preliminary}

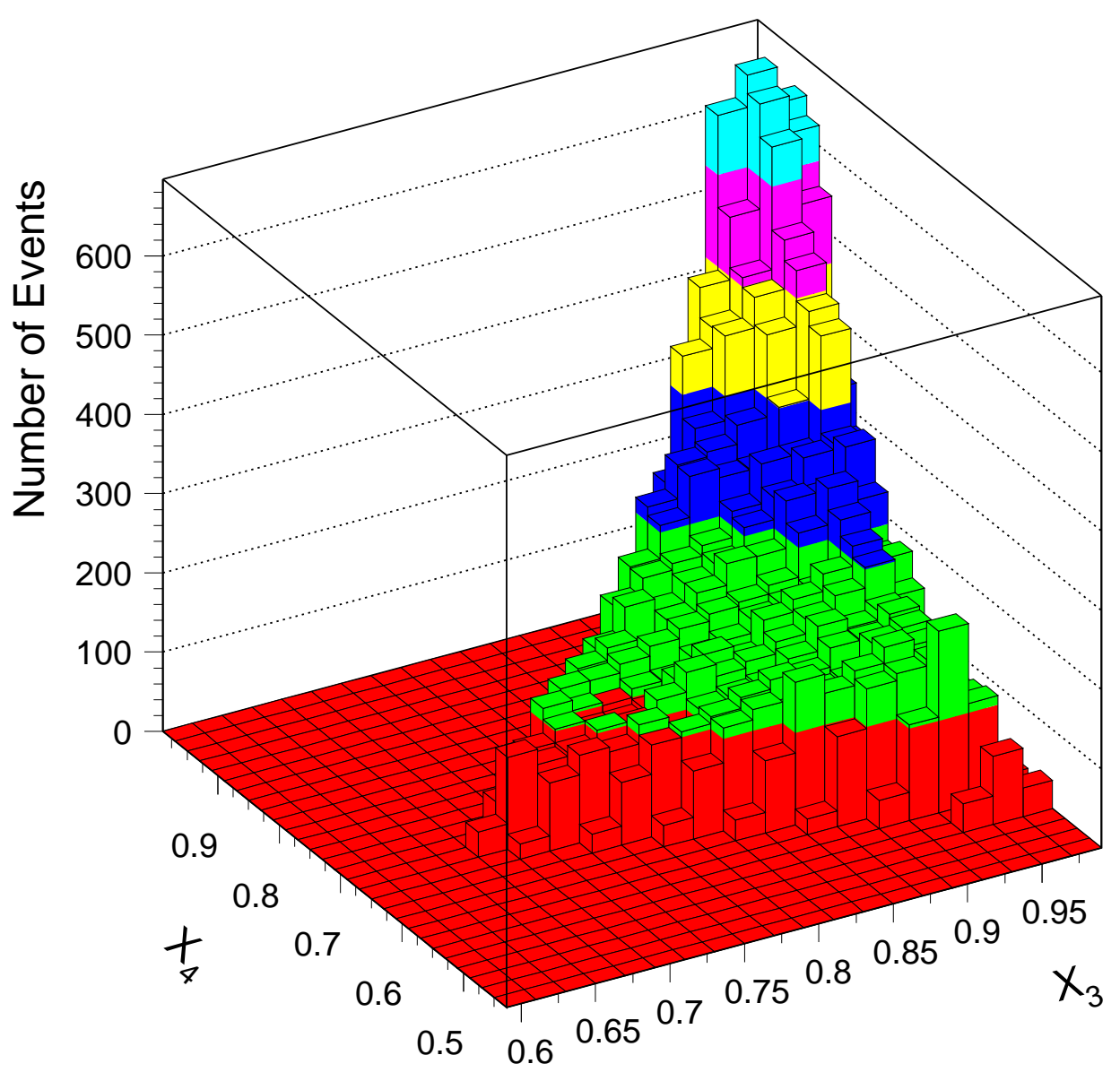

Figure 7.10: The event density in the Dalitz plane as predicted by the NLO calculation with CTEQ4A5. 
CDF: Preliminary

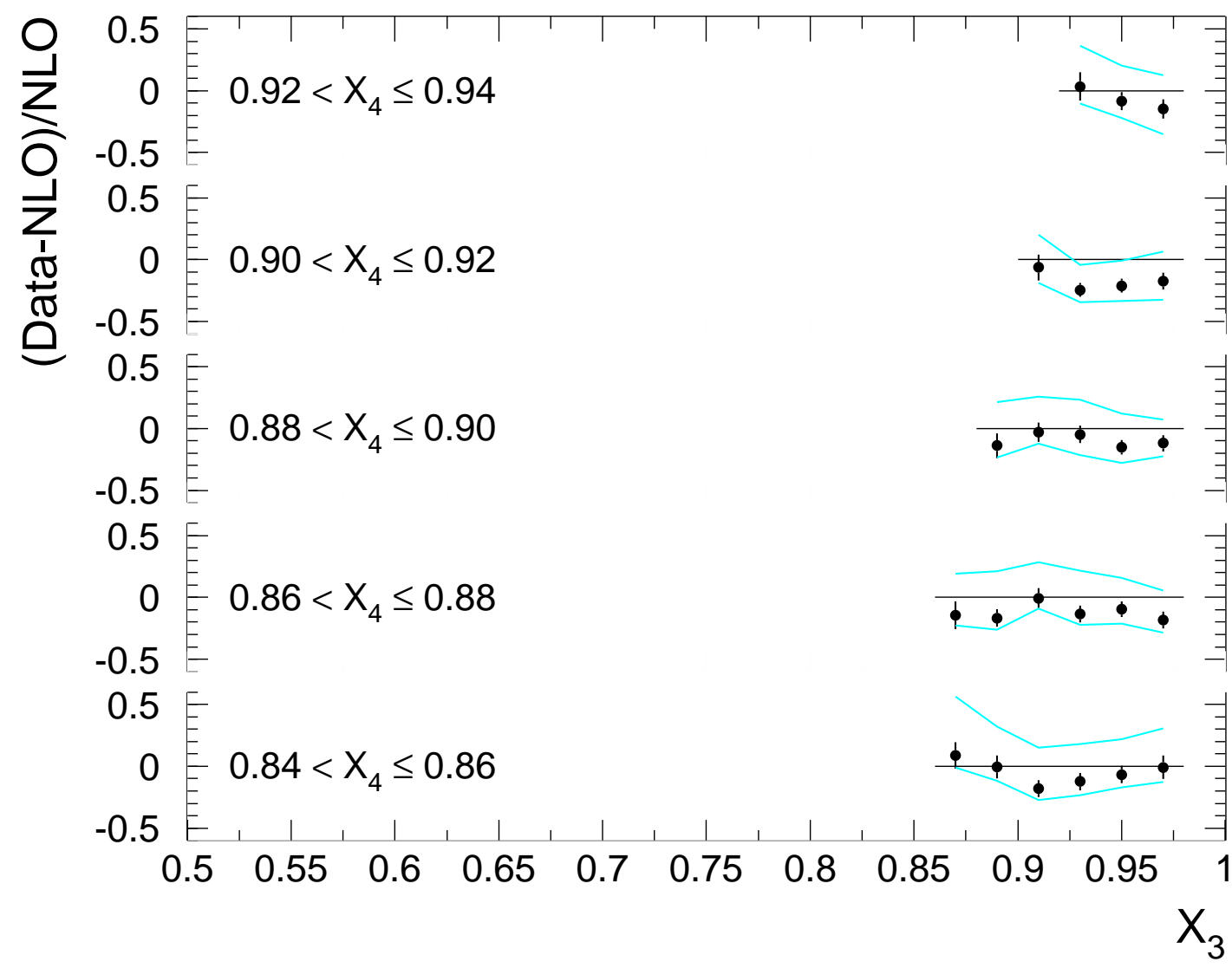

Figure 7.11: The fractional difference between unsmeared data and the NLO prediction, using the CTEQ4M parton distribution function, as a function of $\mathrm{X}_{3}$ for $0.84<\mathrm{X}_{4} \leq 0.94$.

systematic uncertainties on the fractional difference due to systematic uncertainties in the data (see Chapter 5). We limit the analysis to entry bins for which $\mathrm{X}_{3}<0.98$ to avoid event geometries that approach the singular case of two-jet events in the theoretical calculation; this leaves 217 bins for the comparison between the data and the calculation. The regions in $\mathrm{X}_{3}-\mathrm{X}_{4}$ space that are used in the comparison between the data and the NLO prediction, and which are plotted in Figures 7.11 through 7.14, are shown in Figure 7.15. The data and the NLO prediction are consistent.

The average fractional difference for the three-jet sample as a function of $\mathrm{X}_{4}$ 
CDF: Preliminary

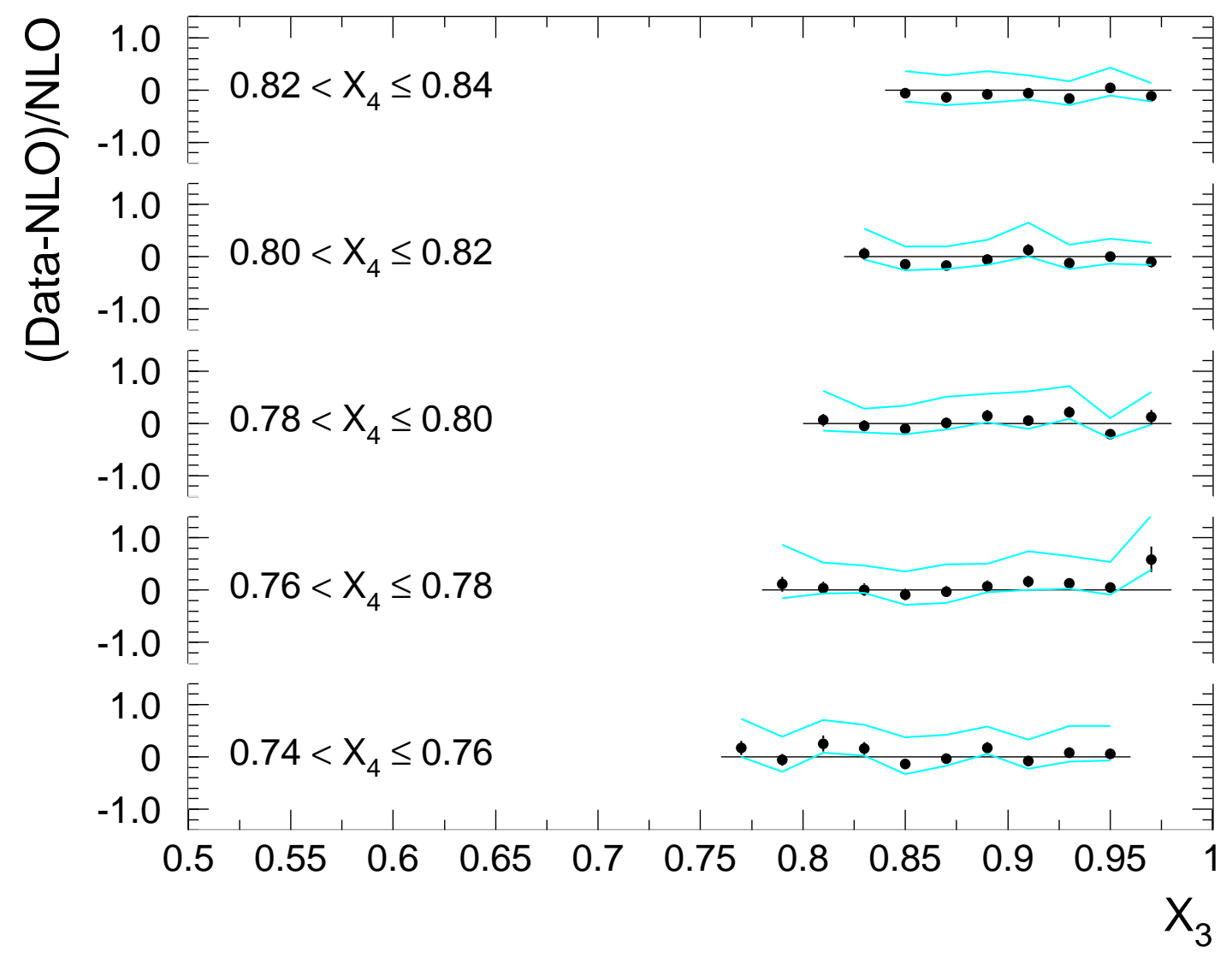

Figure 7.12: The fractional difference between unsmeared data and the NLO prediction, using the CTEQ4M parton distribution function, as a function of $\mathrm{X}_{3}$ for $0.74<\mathrm{X}_{4} \leq 0.84$. 
CDF: Preliminary

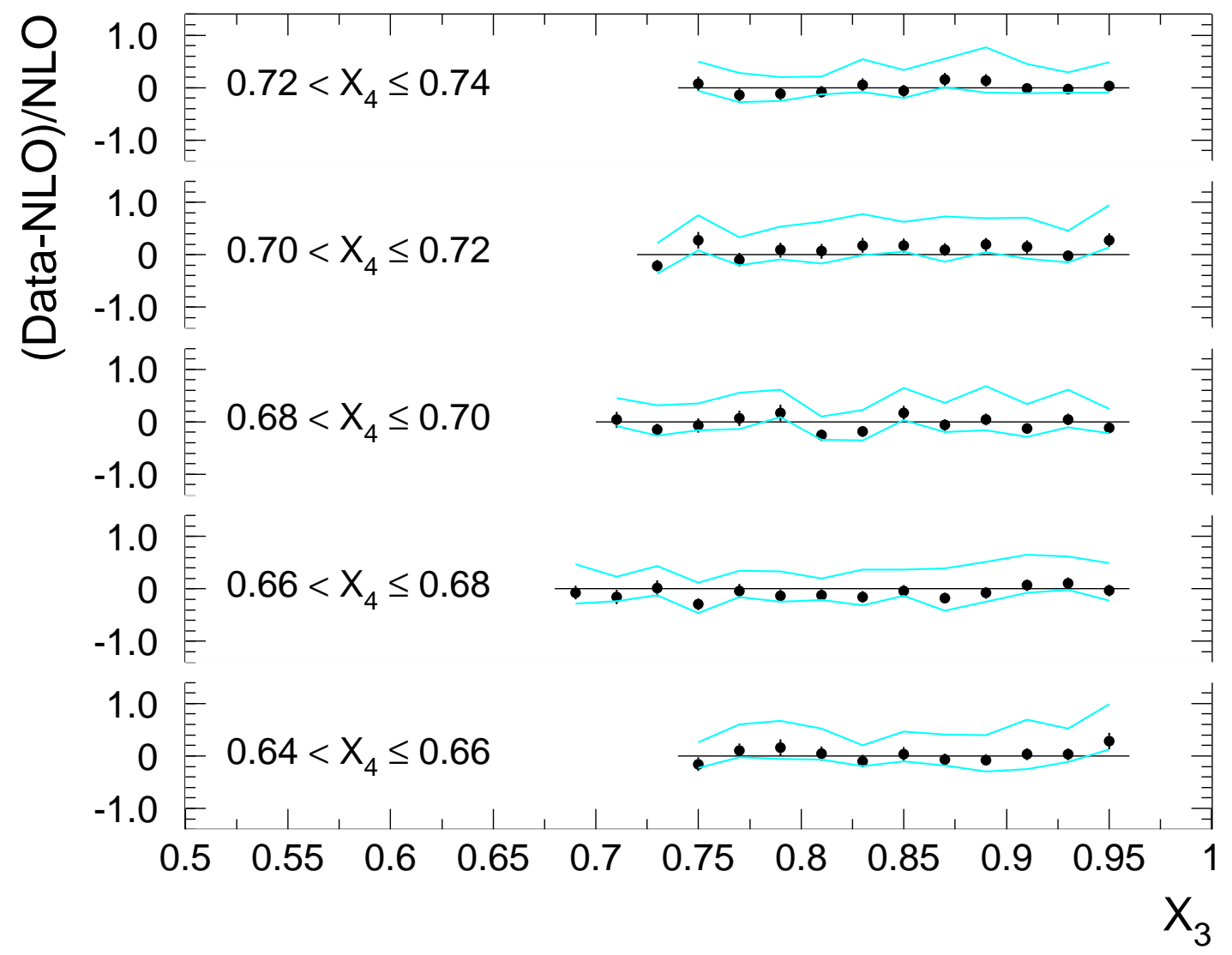

Figure 7.13: The fractional difference between unsmeared data and the NLO prediction, using the CTEQ4M parton distribution function, as a function of $\mathrm{X}_{3}$ for $0.64<\mathrm{X}_{4} \leq 0.74$. 
CDF: Preliminary

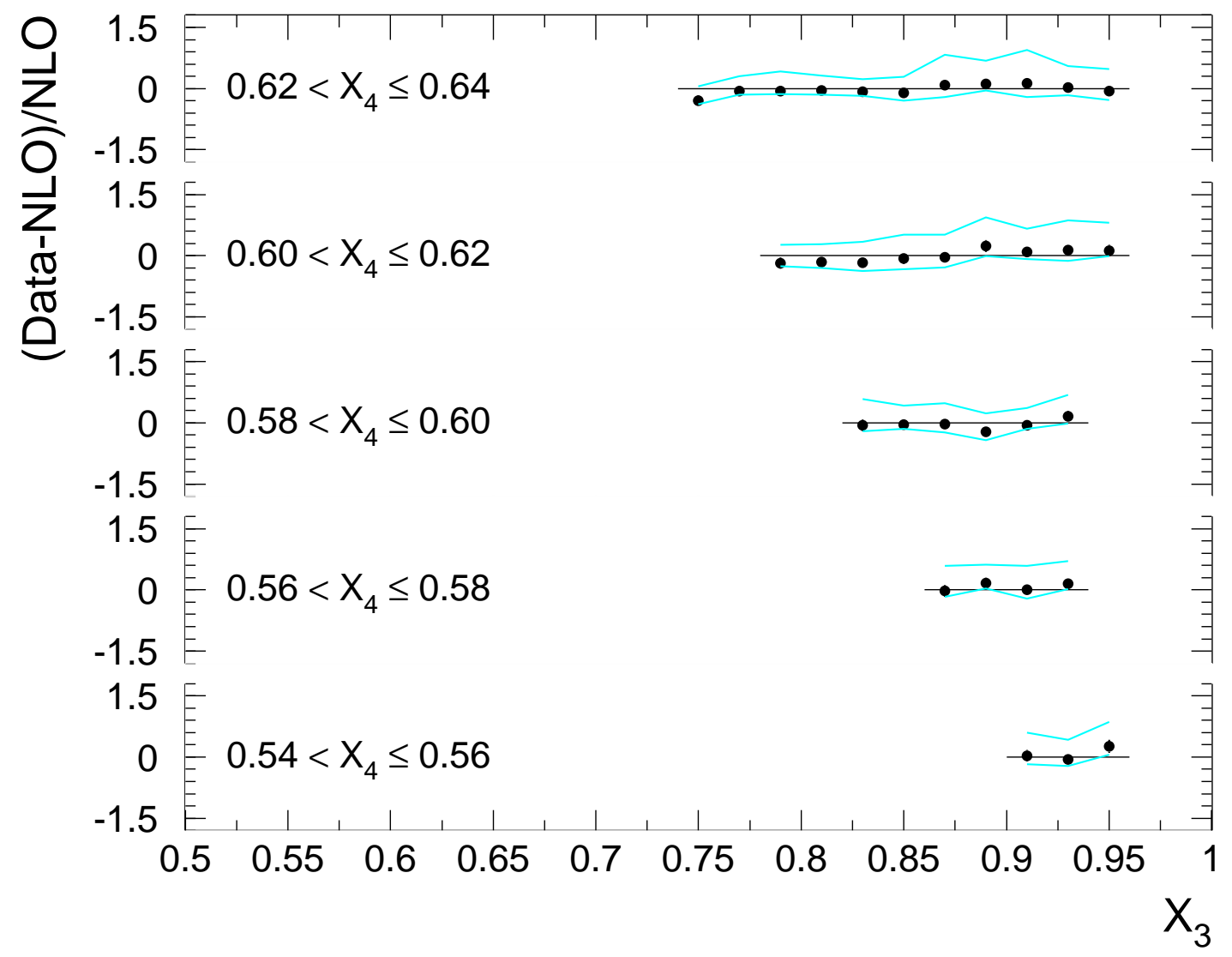

Figure 7.14: The fractional difference between unsmeared data and the NLO prediction, using the CTEQ4M parton distribution function, as a function of $\mathrm{X}_{3}$ for $0.54<\mathrm{X}_{4} \leq 0.64$. 


\section{CDF: Preliminary}

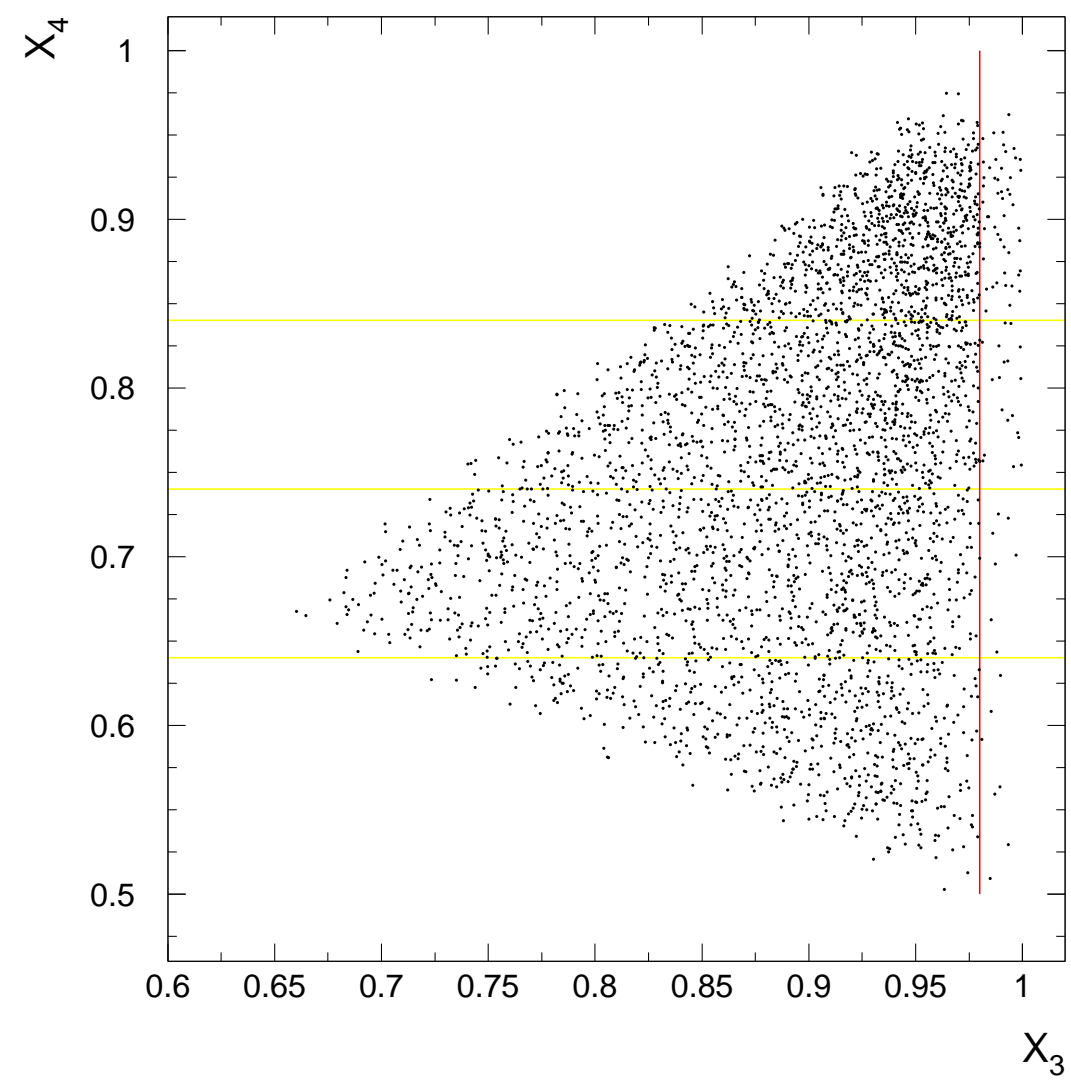

Figure 7.15: The area left of the line indicates the bins in $\mathrm{X}_{3}-\mathrm{X}_{4}$ space that are used in the analysis and plotted in Figures 7.11 through 7.14. 
is shown in Figure 7.16.

The $\chi^{2}$ between the unsmeared data and the NLO calculation is plotted in Figure 7.17 for several different values of the strong coupling constant, $\alpha_{\mathrm{S}}$, each with the appropriate parton distribution function from the CTEQ4 family. Table 7.1 summarizes the results that are plotted in Figure 7.17. The $\chi^{2}$ does not appear to be changing systematically with $\alpha_{\mathrm{S}}$, so no measurement of $\alpha_{\mathrm{S}}$ can be extracted. The error shown consists of the contributions from the statistical uncertainty on the data and the error on the theoretical calculation.

\begin{tabular}{|c|c|c|}
\hline$\alpha_{\mathrm{S}}$ & $\chi^{2}$ & $\chi^{2}$ per bin \\
\hline \hline 0.1097 & $744 \pm 59$ & $3.43 \pm 0.27$ \\
0.1126 & $736 \pm 59$ & $3.39 \pm 0.27$ \\
0.1155 & $731 \pm 59$ & $3.37 \pm 0.27$ \\
0.1184 & $727 \pm 59$ & $3.35 \pm 0.27$ \\
0.1214 & $723 \pm 59$ & $3.33 \pm 0.27$ \\
\hline
\end{tabular}

Table 7.1: The $\chi^{2}$ between the data and the NLO calculation for different values of $\alpha_{\mathrm{S}}$, using appropriate parton distribution functions from the CTEQ4 family.

Table 7.2 summarizes the cross section predicted by the calculation for each $\alpha_{\mathrm{S}}$. The cross section uncertainty quoted in this table is statistical and theoretical, due to the change of renormalization and factorization scales. To estimate the contribution of these changes in scales, they were varied by factors of 2 . If we use all data quality and offline selection cuts described in Chapters 3 and 6 , with the exception of the cut on the NLO uncertainty, and restrict our analysis to bins with $\mathrm{X}_{3}<0.98$, the total threejet production cross section is $456 \pm 2$ (stat.) ${ }_{-68}^{+202}$ (syst.) pb. For this particular set of cuts, this is consistent with the earlier measurement used for comparison in Chapter 6 where the total three-jet production cross section after the cut on $\mathrm{X}_{3}<0.98$ (efficiency about 0.99 ) is $690_{-185}^{+226} \mathrm{pb}$. The total three-jet production cross section for the NLO calculation, using the CTEQ4M parton distribution function with $\alpha_{\mathrm{S}}=0.1155$, is $482 \pm 2$ (stat.) ${ }_{-72}^{+31}$ (theor.) pb. The cross section in each individual kinematically allowed bin in the Dalitz plane in tabulated in Tables F.1 through F.14 in Appendix F. 


\section{CDF: Preliminary}

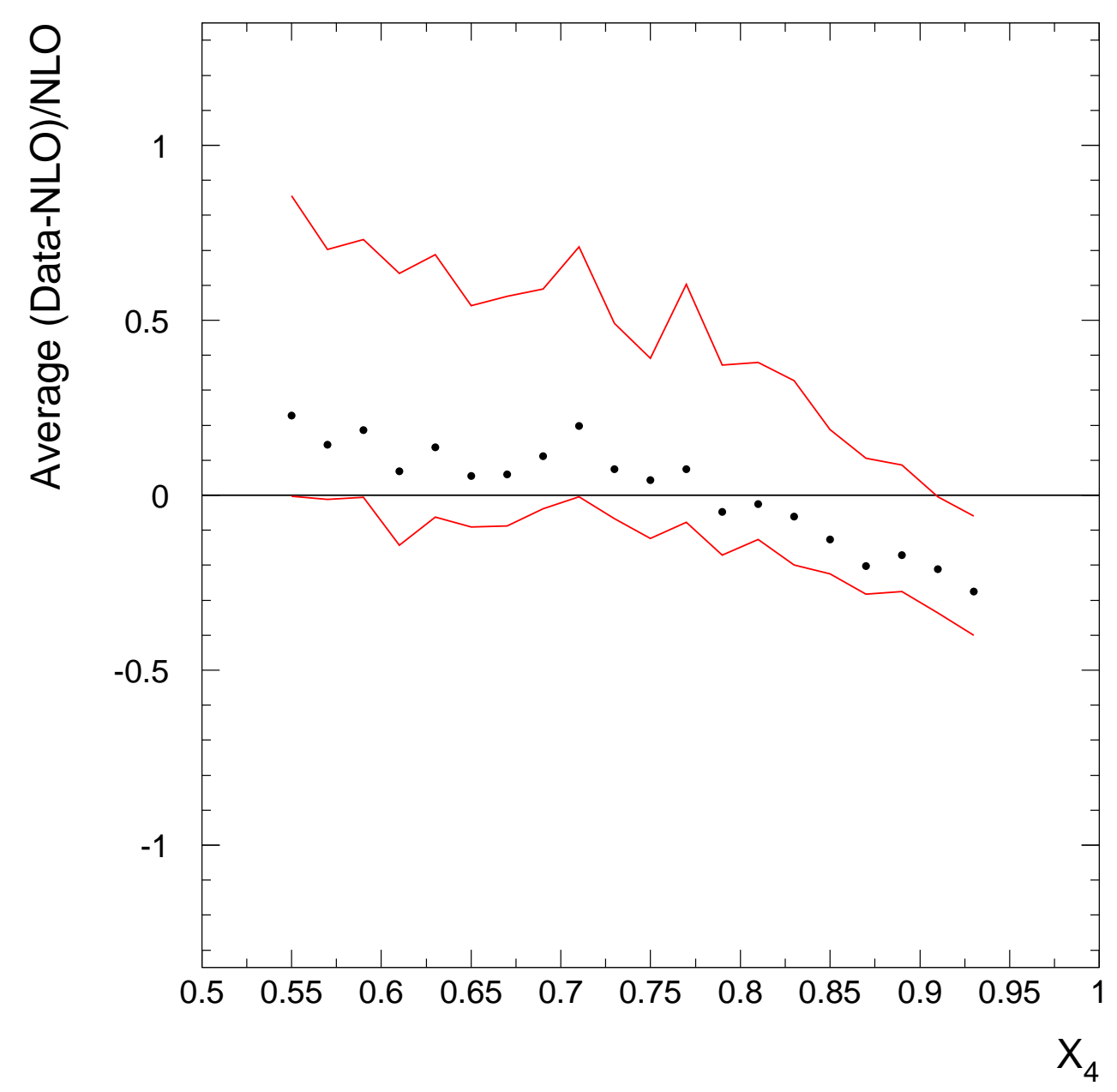

Figure 7.16: The average fractional difference for the three-jet sample as a function of $\mathrm{X}_{4}$. 


\section{CDF: Preliminary}

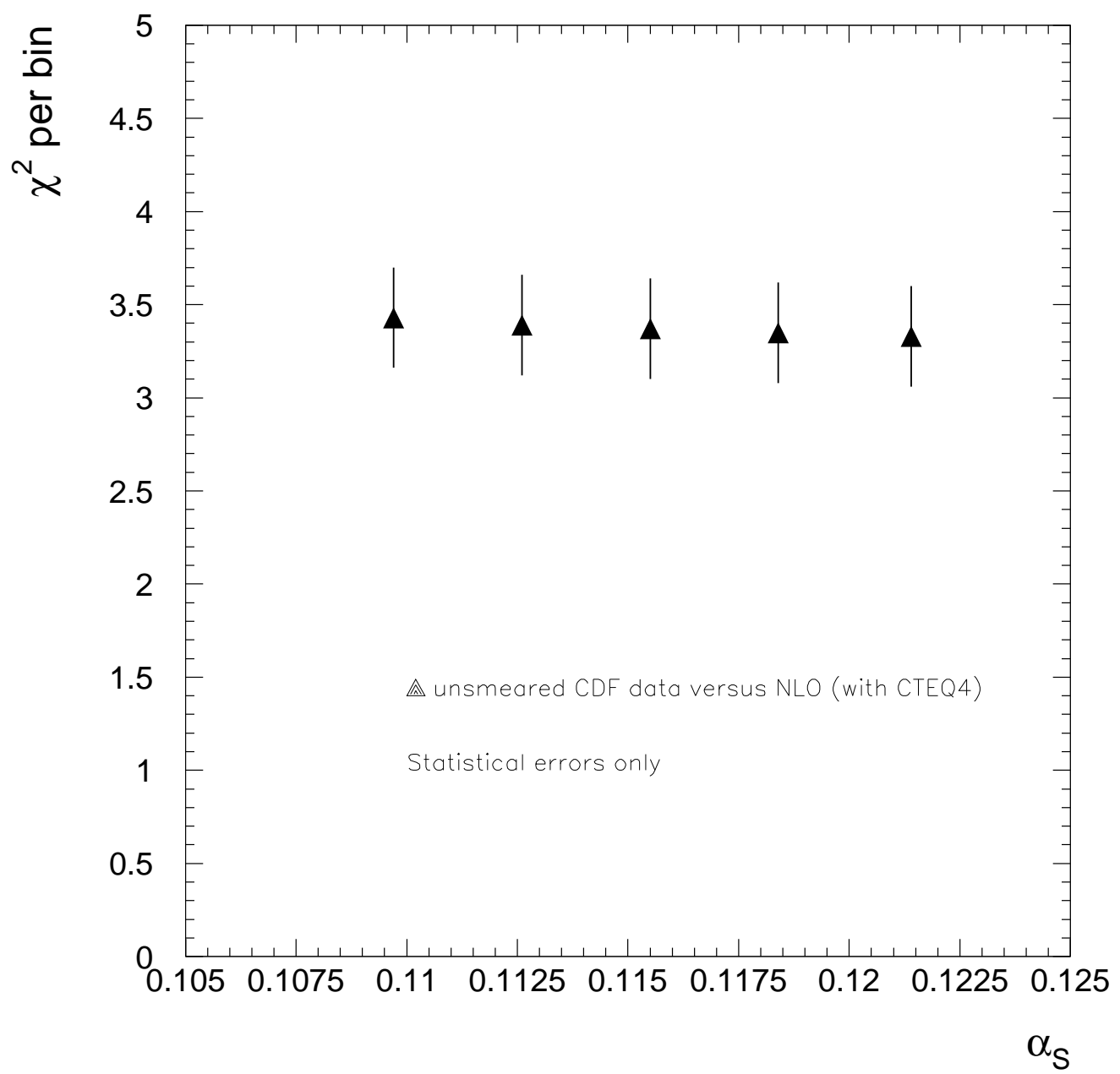

Figure 7.17: $\chi^{2}$ between the data and the NLO calculation for different values of $\alpha_{\mathrm{S}}$, using appropriate parton distribution functions from the CTEQ4 family. 


\begin{tabular}{|c|c|c|}
\hline Parton Distribution Function & $\alpha_{\mathrm{S}}$ & Three-jet Production Cross Section (pb) \\
\hline \hline CTEQ4A1 & 0.1097 & $453 \pm 2$ (stat.) $)_{-63}^{+27}$ (theor.) \\
CTEQ4A2 & 0.1126 & $473 \pm 2$ (stat.) $)_{-68}^{+29}$ (theor.) \\
CTEQ4M & 0.1155 & $482 \pm 2$ (stat.) $)_{-72}^{+31}$ (theor.) \\
CTEQ4A4 & 0.1184 & $493 \pm 3$ (stat.) $)_{-76}^{+34}$ (theor.) \\
CTEQ4A5 & 0.1214 & $503 \pm 3$ (stat.) $)_{-80}^{+37}$ (theor.) \\
CTEQ4HJ & 0.1159 & $501 \pm 3$ (stat.) $)_{-73}^{+27}$ (theor.) \\
\hline
\end{tabular}

Table 7.2: The three-jet production cross section of events in the NLO templates, for different values of $\alpha_{S}$, using the appropriate parton distribution functions from the CTEQ4 family. The uncertainties are statistical and theoretical, due to the changes of the renormalization and factorization scales.

We calculate $\left(\chi^{\prime}\right)^{2}$ between HERWIG and the NLO calculation using the CTEQ4 parton distribution functions. A plot of $\left(\chi^{\prime}\right)^{2}$ versus $\alpha_{\mathrm{S}}$ is shown in Figure 7.18. HERWIG events that have passed QFL and have been unsmeared using the correction factors described in Chapter 4 are plotted as circles. Hadron-level HERWIG events are plotted as squares. The behavior of $\left(\chi^{\prime}\right)^{2}$ as a function of $\alpha_{\mathrm{S}}$ in Figure 7.18 is similar to the $\chi^{2}$ behavior in Figure 7.17. The numerical value of $\left(\chi^{\prime}\right)^{2}$ in Figure 7.18 is larger than the one shown in Figure 6.22. This is due to the significantly reduced statistical uncertainties in the NLO calculation using the CTEQ4 parton distribution function family and a HERWIG data set with reduced statistical uncertainties. The error shown consists of the contributions from the statistical uncertainty on the HERWIG data sample and the error on the theoretical calculation. As the numerical value of $\left(\chi^{\prime}\right)^{2}$ increases from the analysis using CTEQ3 to the analysis with CTEQ4, the associated error also increases slightly. The smaller individual error contributions are balanced against the larger absolute value of $\left(\chi^{\prime}\right)^{2}$ for this slight increase in the error.

A plot of $\left(\chi^{\prime \prime}\right)^{2}$ between the NLO result using CTEQ4M with $\alpha_{\mathrm{S}}=0.1155$ and the other results from the CTEQ4A parton distribution function family versus $\alpha_{\mathrm{S}}$ is shown in Figure 7.19. The $\left(\chi^{\prime \prime}\right)^{2}$ in Figure 7.19 is constant for the whole range of $\alpha_{\mathrm{S}}$, indicating that the Dalitz variables are not sensitive to changes in $\alpha_{\mathrm{S}}$. The error 


\section{CDF: Preliminary}

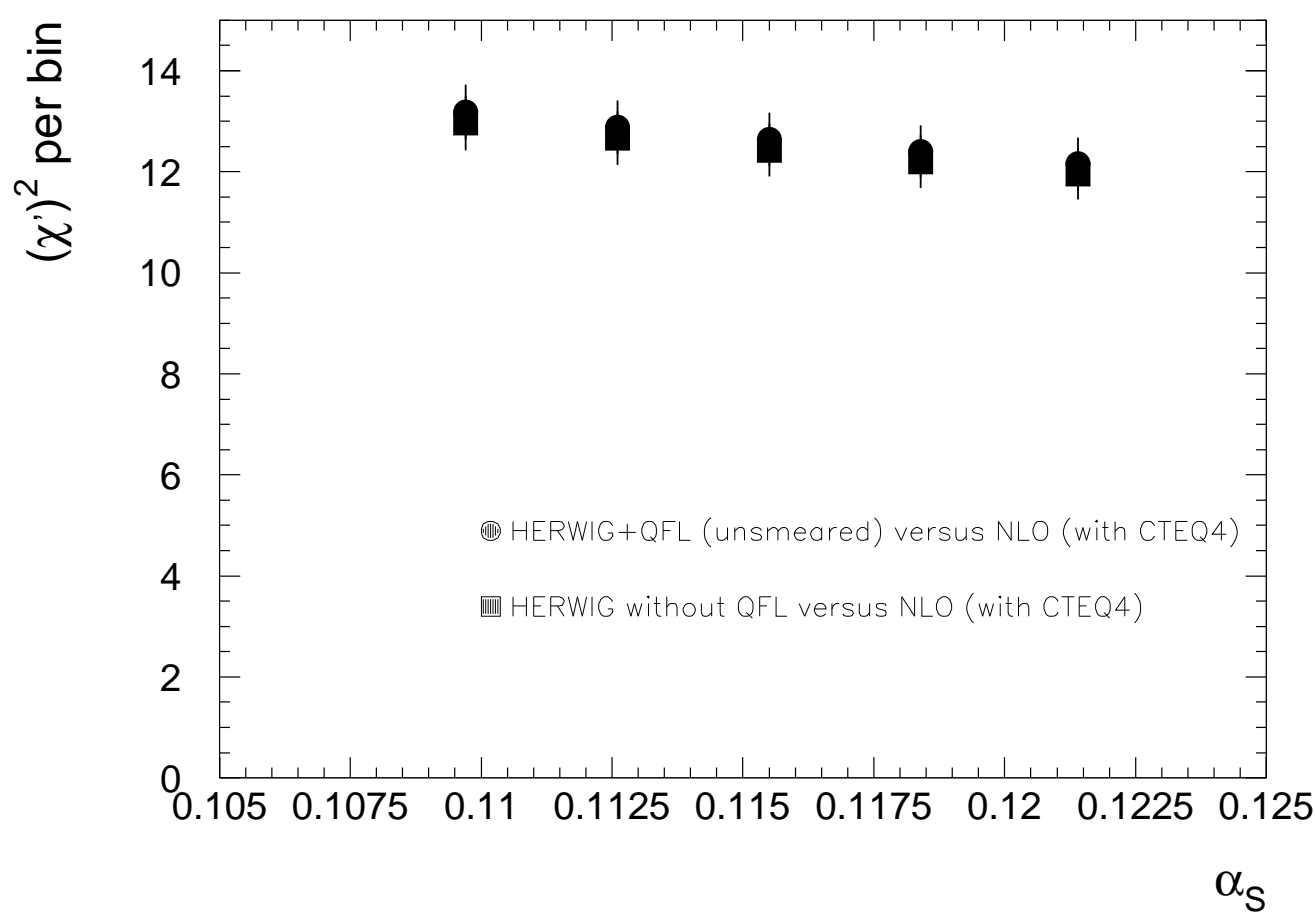

Figure 7.18: The circles represent $\left(\chi^{\prime}\right)^{2}$ between the NLO calculation and events that have been generated by HERWIG, passed through QFL, and then unsmeared by the Monte Carlo approach. The squares represent $\left(\chi^{\prime}\right)^{2}$ between the NLO calculation and hadron-level HERWIG events.

shown is due to the error on the theoretical calculation.

\subsection{CTEQ4HJ Results}

Similar analyses as for CTEQ4A have been performed for CTEQ4HJ. The transverse energy distributions of all three jets for unsmeared data and the NLO predictions, using the CTEQ4HJ parton distribution function, are plotted in Figure 7.20 and compared to HERWIG. The HERWIG event sample has to be adjusted for systematic errors in the QFL energy scale (see Reference [35].) A minimum $\mathrm{p}_{\mathrm{T}}$ of $60 \mathrm{GeV}$ is used 


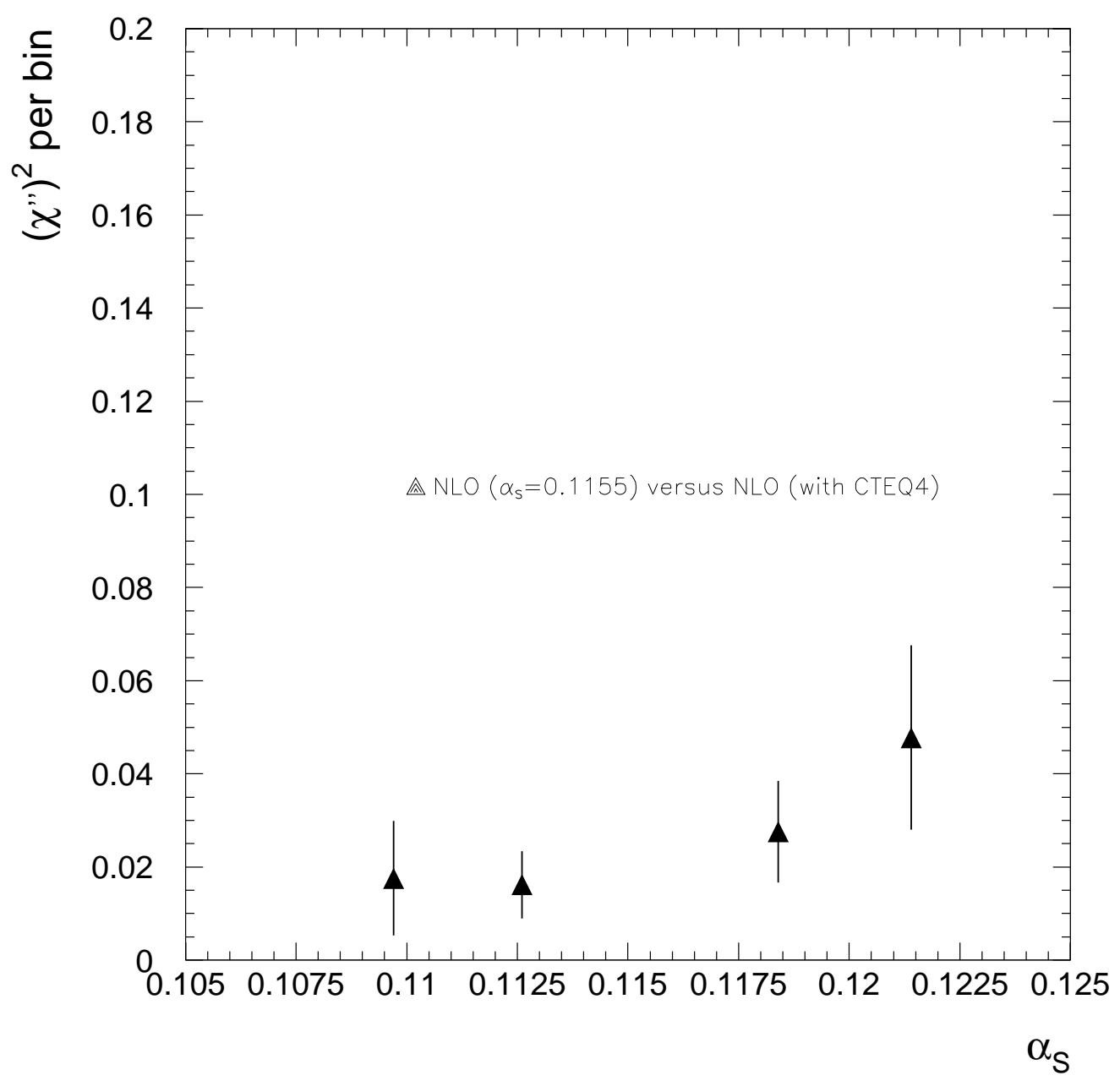

Figure 7.19: $\left(\chi^{\prime \prime}\right)^{2}$ between the NLO calculation with $\alpha_{\mathrm{S}}=0.1155$ and the NLO calculation for other values of $\alpha_{\mathrm{S}}$, using appropriate parton distribution functions from the CTEQ4 family. 
in the generation of the HERWIG sample. The three jet distributions are normalized to the same number of events to allow comparison of their shapes. To demonstrate the difference between the jet $\mathrm{E}_{\mathrm{T}}$ distributions obtained by using the CTEQ3 and CTEQ4HJ parton distribution functions, the ratio of the two jet $\mathrm{E}_{\mathrm{T}}$ distributions is shown in Figure 7.21. At the level of uncertainty achieved by the NLO calculation at this time, deviations of this ratio from the value 1.0 in the $\mathrm{E}_{\mathrm{T}}$ distributions using CTEQ3 and CTEQ4 could be attributed to systematic changes. In the HERWIG data sample, the third jet is calculated to be significantly more energetic than the corresponding distributions for the data and the NLO calculation. This appears to be due to the fact that HERWIG does not correctly model the jet multiplicities, as shown in Figure6.2. For a given value of $\sum \mathrm{E}_{\mathrm{T}}$ in an event, the third jet is more energetic if the total number of jets in the event is less than in the data sample or in the NLO prediction.

As an independent check on the data, the jet $\mathrm{E}_{\mathrm{T}}$ distributions obtained by the Jet100 trigger are investigated. Data obtained from the $\sum \mathrm{E}_{\mathrm{T}}$ trigger and from the Jet100 trigger agree very well. The jet $\mathrm{E}_{\mathrm{T}}$ distributions in both cases do not differ significantly. Figure 7.22 shows the transverse energy distributions for the three jets in unsmeared data from the Jet100 trigger, the NLO prediction using the CTEQ4HJ parton distribution function, and HERWIG with $\mathrm{p}_{\mathrm{T}}=60 \mathrm{GeV}$.

The Dalitz variables obtained by the NLO calculation using CTEQ4HJ are shown in Figure 7.23.

The data and the NLO prediction, using the CTEQ4HJ parton distribution function, are compared by computing the difference between the observed and predicted number of events, scaled by the number of predicted events, in each bin. This fractional difference is plotted in Figures 7.24 through 7.27 as a function of $\mathrm{X}_{3}$, spanning the entire range in $\mathrm{X}_{4}$. Figure 7.24 shows the region with $0.84<\mathrm{X}_{4} \leq 0.94$, $0.74<\mathrm{X}_{4} \leq 0.84$ in Figure 7.25, and Figures 7.26 and 7.27 span $0.64<\mathrm{X}_{4} \leq 0.74$ and $0.54<\mathrm{X}_{4} \leq 0.64$, respectively. Also shown in Figures 7.24 through 7.27 are the total 


\section{CDF: Preliminary}

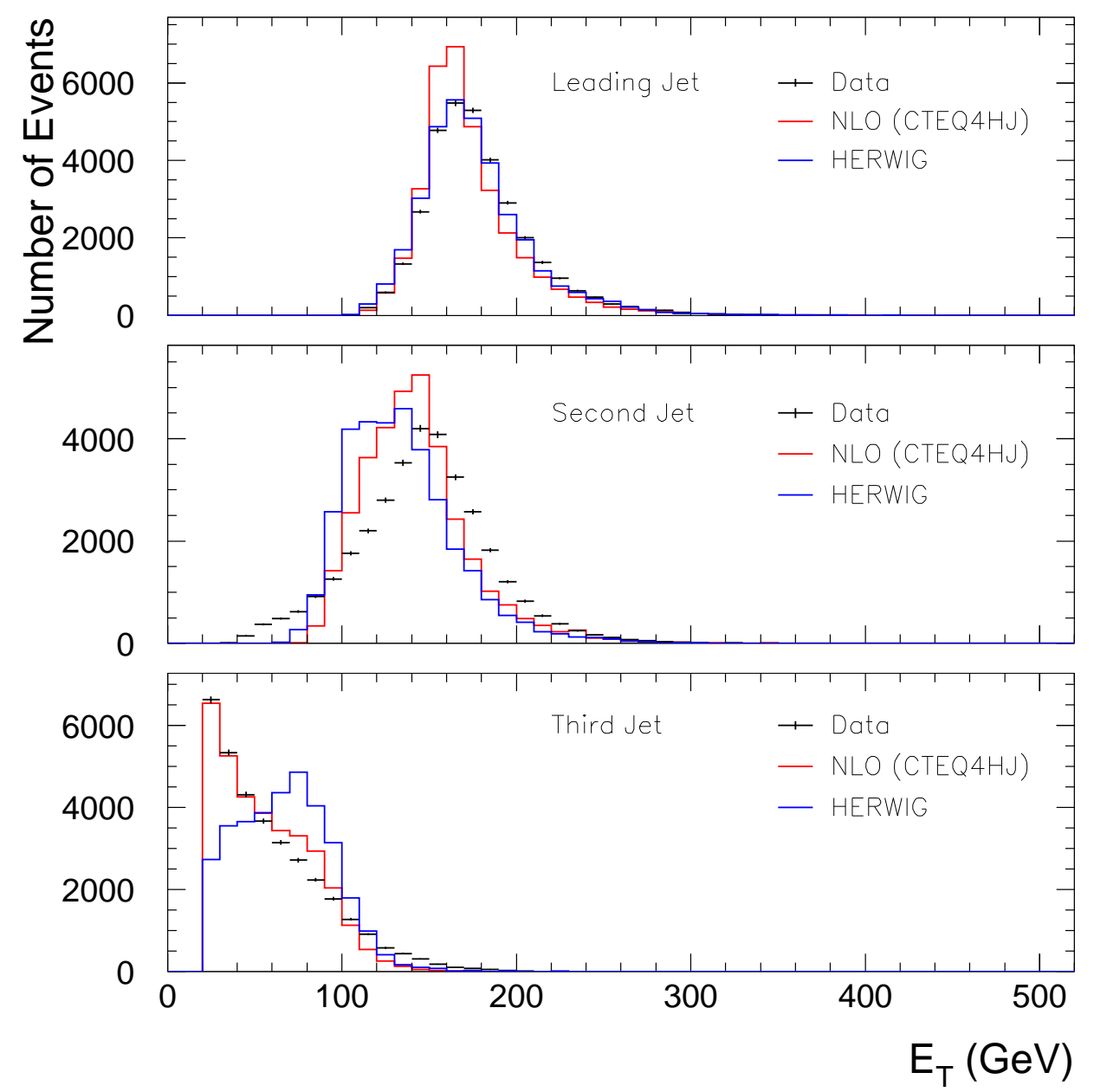

Figure 7.20: The transverse energy distributions for the three jets in unsmeared data, the NLO prediction using the CTEQ4HJ parton distribution function, and HERWIG. 


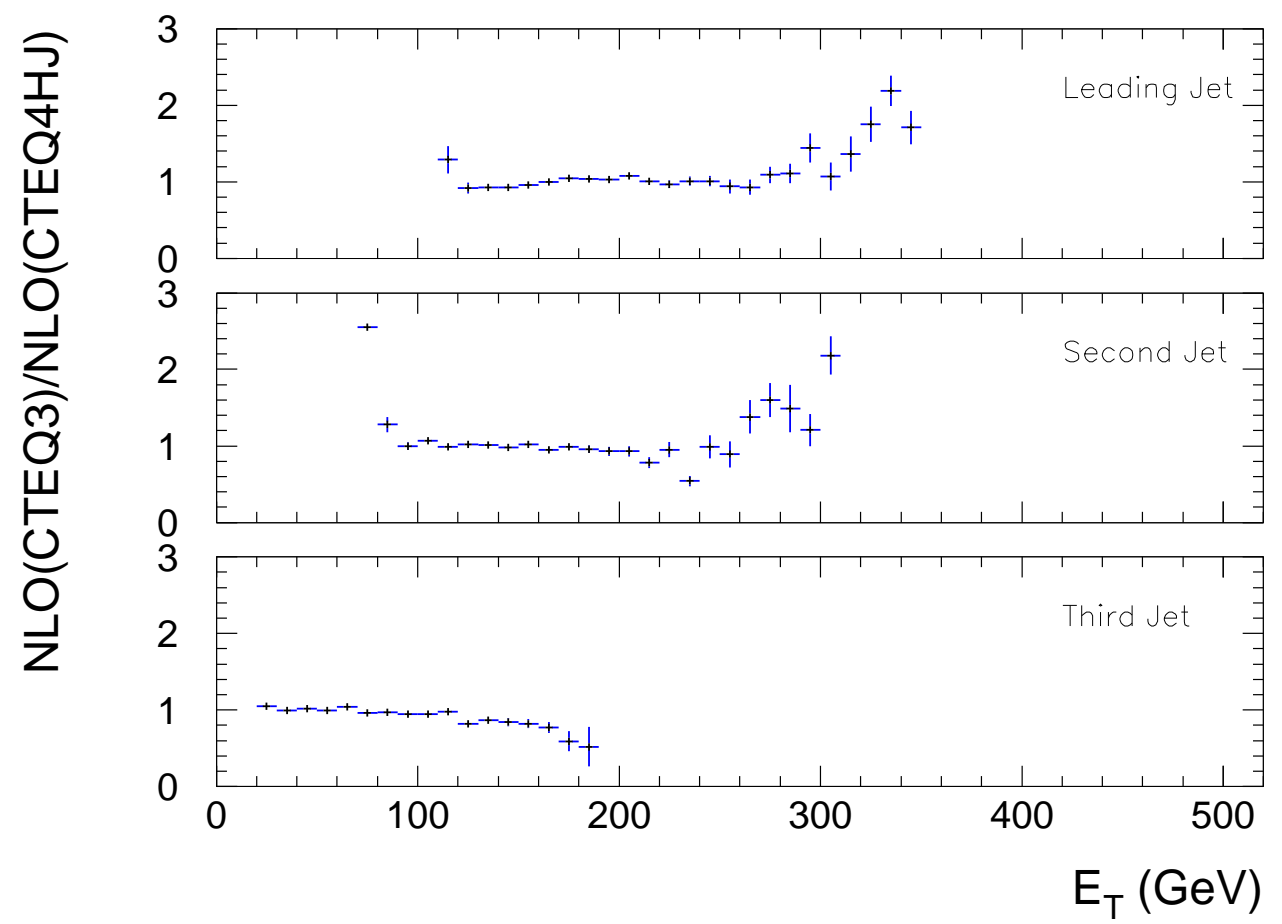

Figure 7.21: The ratio of the jet $\mathrm{E}_{\mathrm{T}}$ distributions obtained by using CTEQ3 and CTEQ4HJ. 
CDF: Preliminary

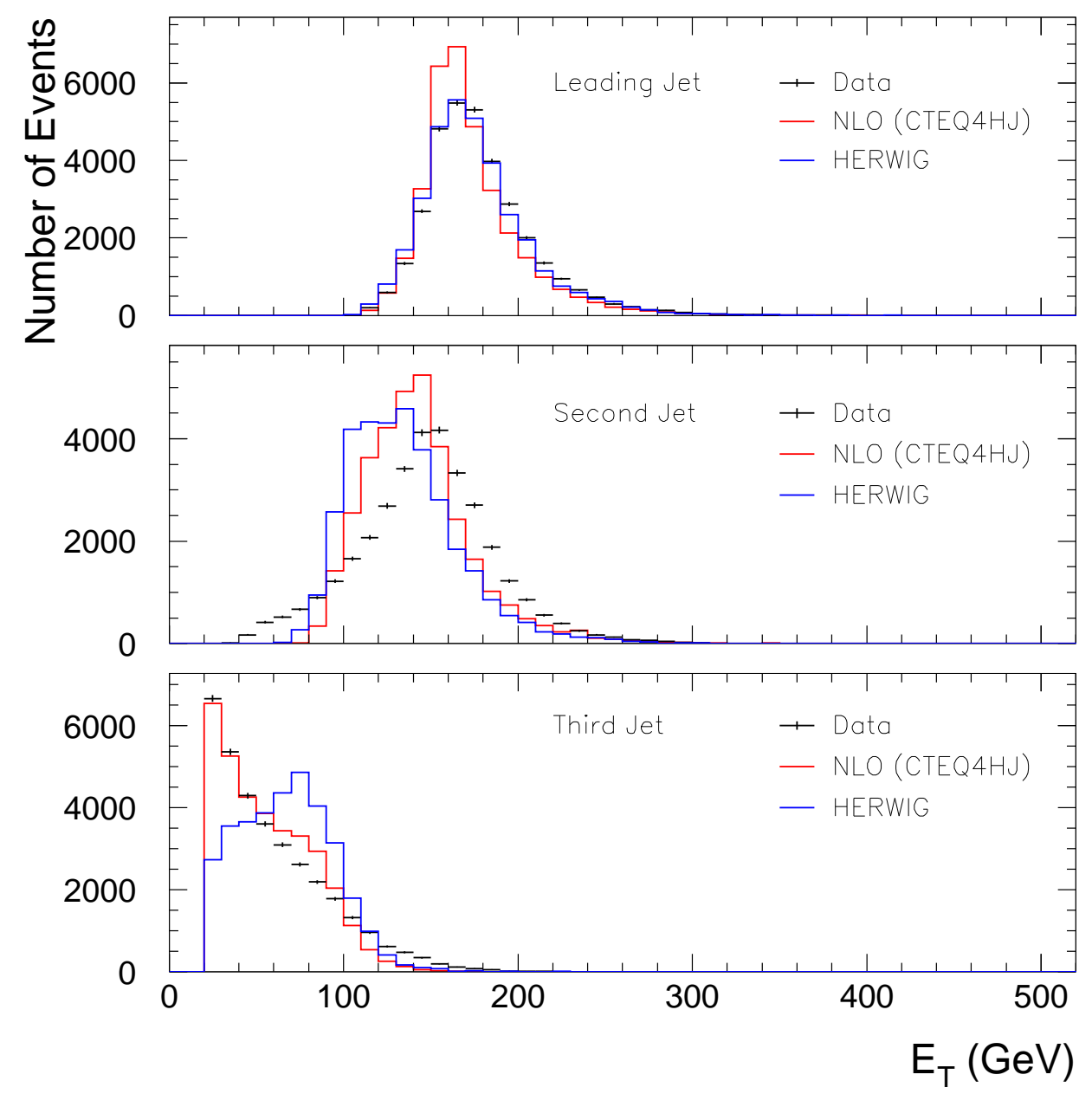

Figure 7.22: The transverse energy distributions for the three jets in unsmeared data from the Jet100 trigger, the NLO prediction using the CTEQ4HJ parton distribution function, and HERWIG with $\mathrm{p}_{\mathrm{T}}=60 \mathrm{GeV}$. 


\section{CDF: Preliminary}

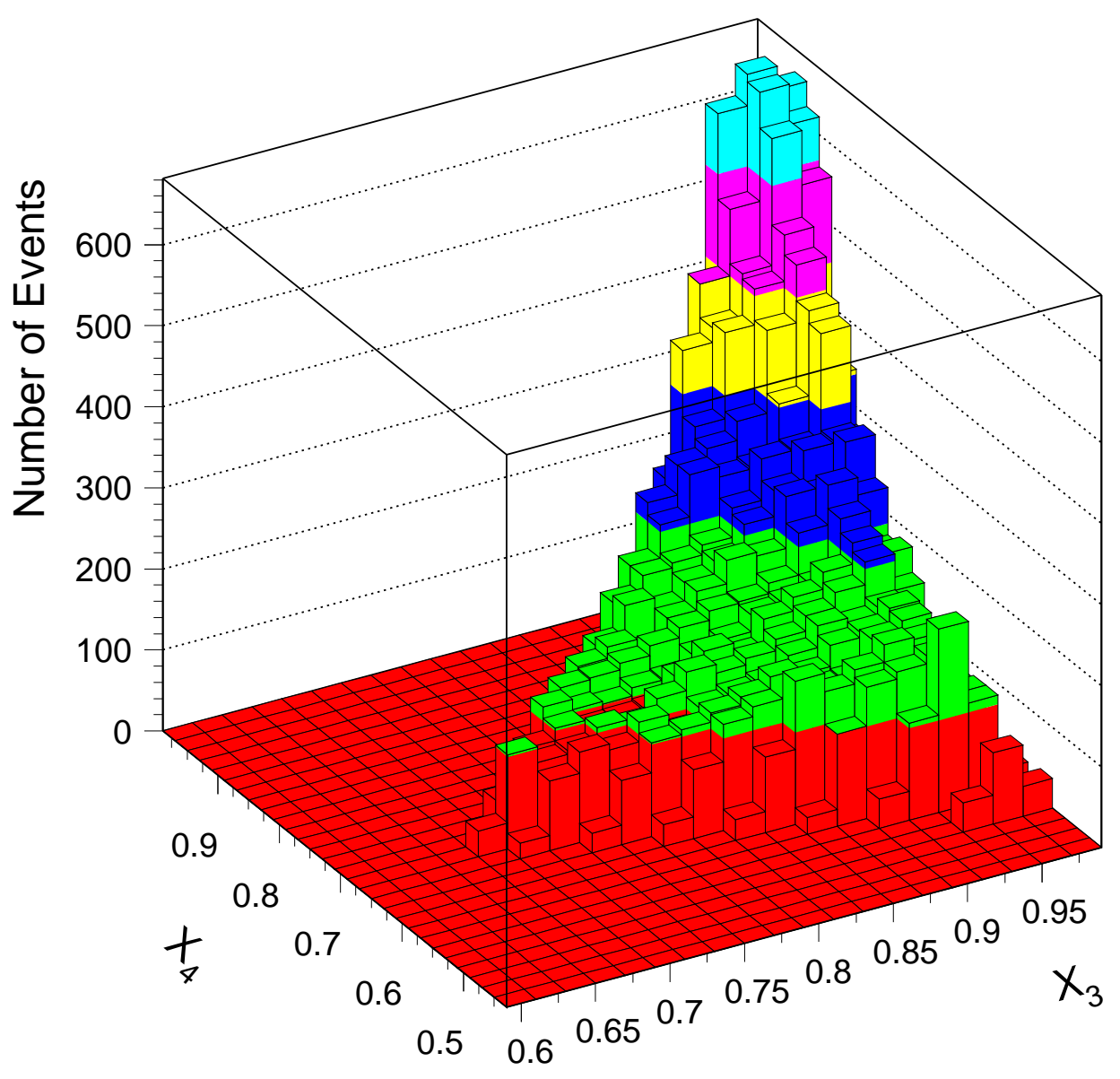

Figure 7.23: The event density in the Dalitz plane as predicted by the NLO calculation with CTEQ4HJ. 
CDF: Preliminary

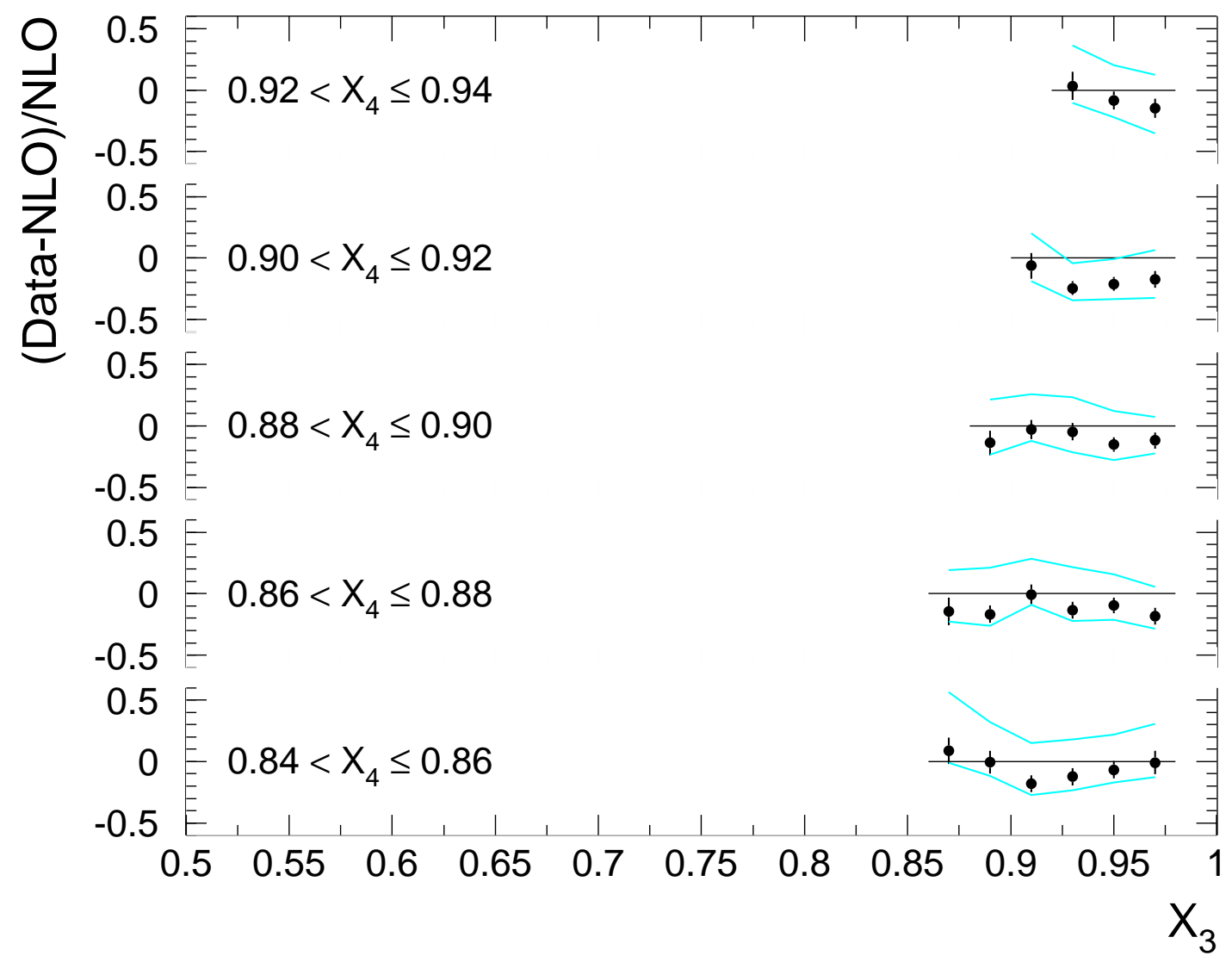

Figure 7.24: The fractional difference between unsmeared data and the NLO prediction, using the CTEQ4HJ parton distribution function, as a function of $\mathrm{X}_{3}$ for $0.84<\mathrm{X}_{4} \leq 0.94$.

systematic uncertainties on the fractional difference due to systematic uncertainties in the data (see Chapter 5).

The average fractional difference for the three-jet sample as a function of $\mathrm{X}_{4}$ is shown in Figure 7.28.

The $\chi^{2}$ between the unsmeared data and the NLO calculation using CTEQ4HJ is $725 \pm 59$ and $\chi^{2}$ per bin is $3.34 \pm 0.27$.

The total three-jet production cross section for the NLO calculation, using the CTEQ4HJ parton distribution function, is $501 \pm 3$ (stat.) ${ }_{-73}^{+27}$ (theor.) pb. 
CDF: Preliminary

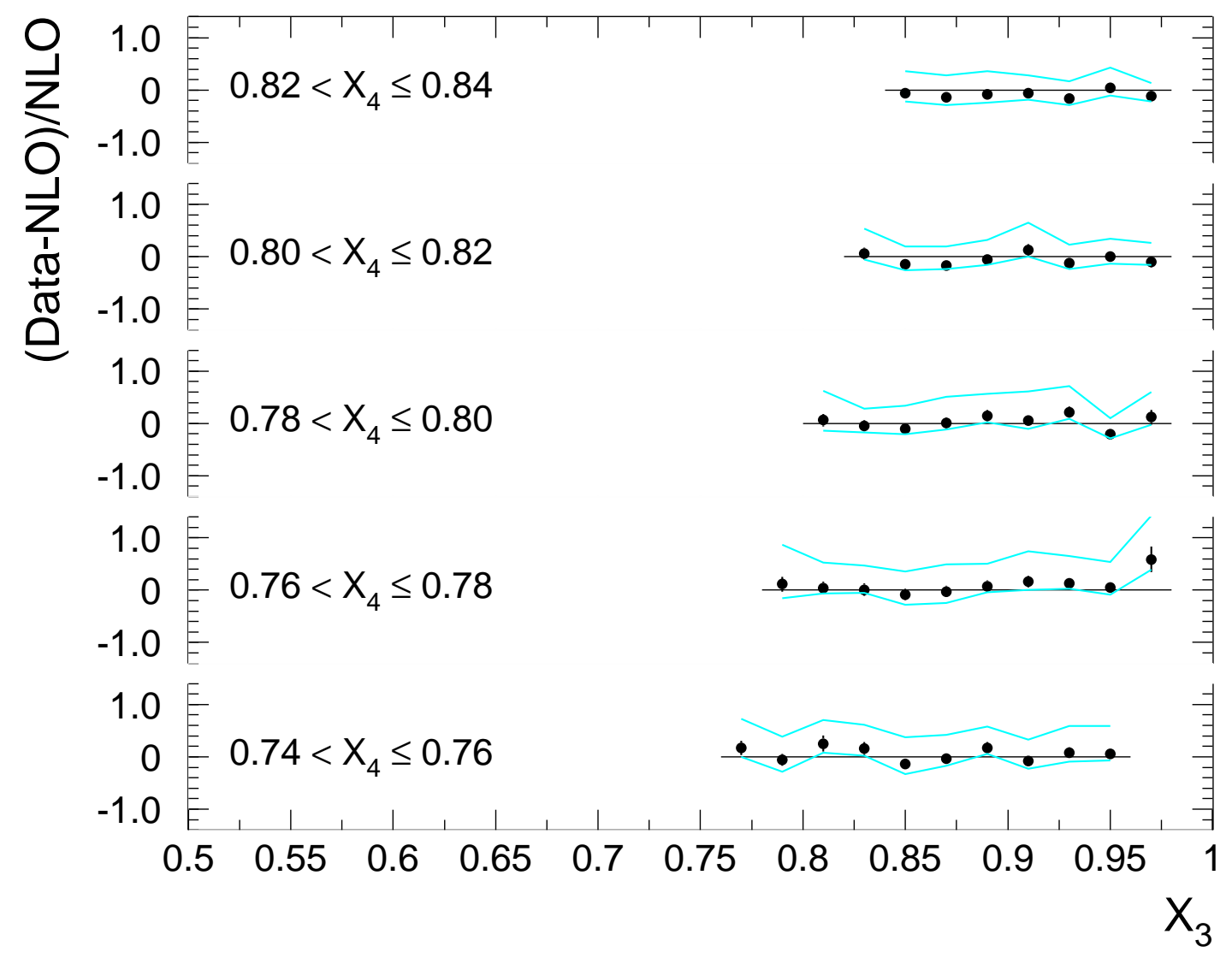

Figure 7.25: The fractional difference between unsmeared data and the NLO prediction, using the CTEQ4HJ parton distribution function, as a function of $\mathrm{X}_{3}$ for $0.74<\mathrm{X}_{4} \leq 0.84$. 
CDF: Preliminary

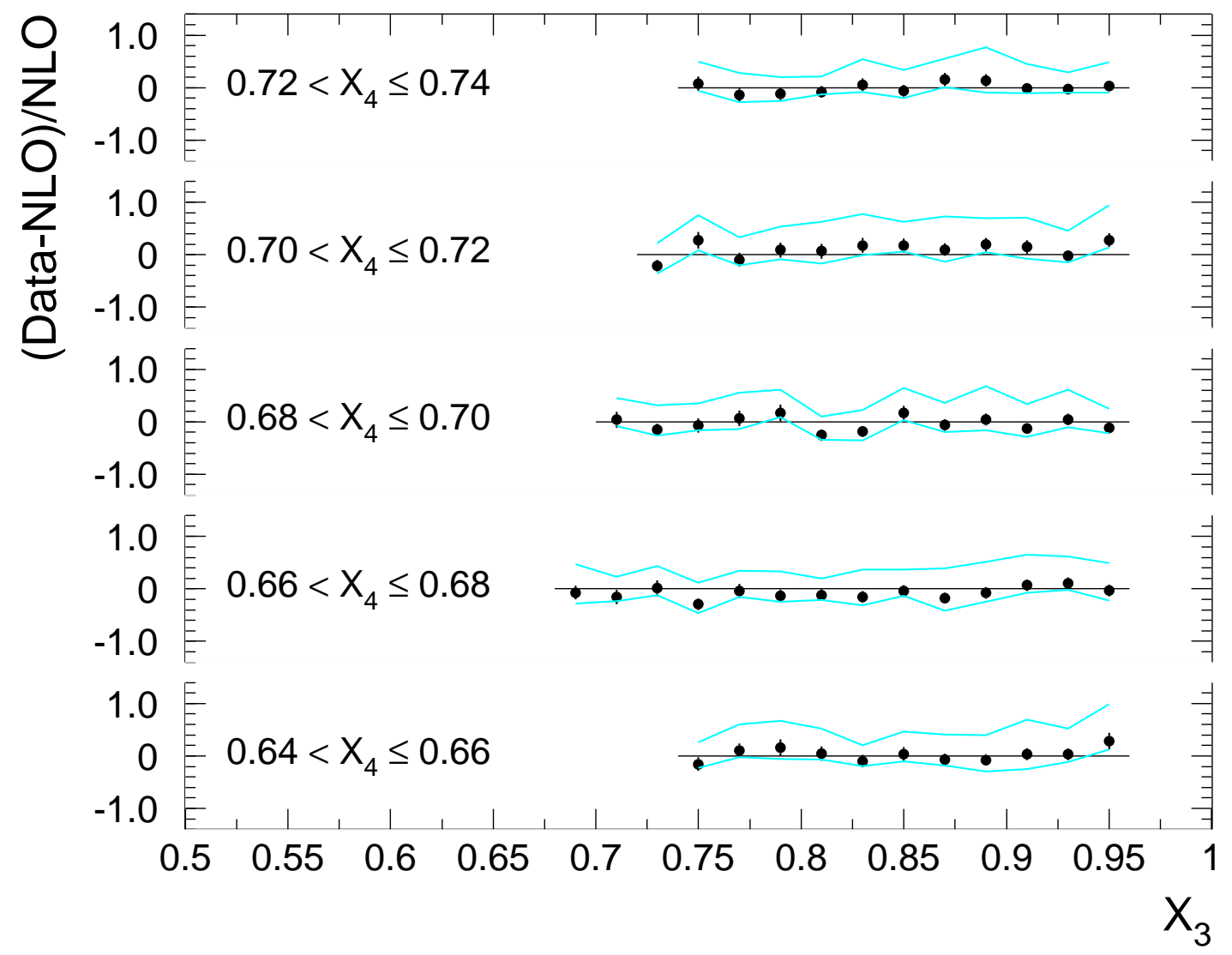

Figure 7.26: The fractional difference between unsmeared data and the NLO prediction, using the CTEQ4HJ parton distribution function, as a function of $\mathrm{X}_{3}$ for $0.64<\mathrm{X}_{4} \leq 0.74$. 
CDF: Preliminary

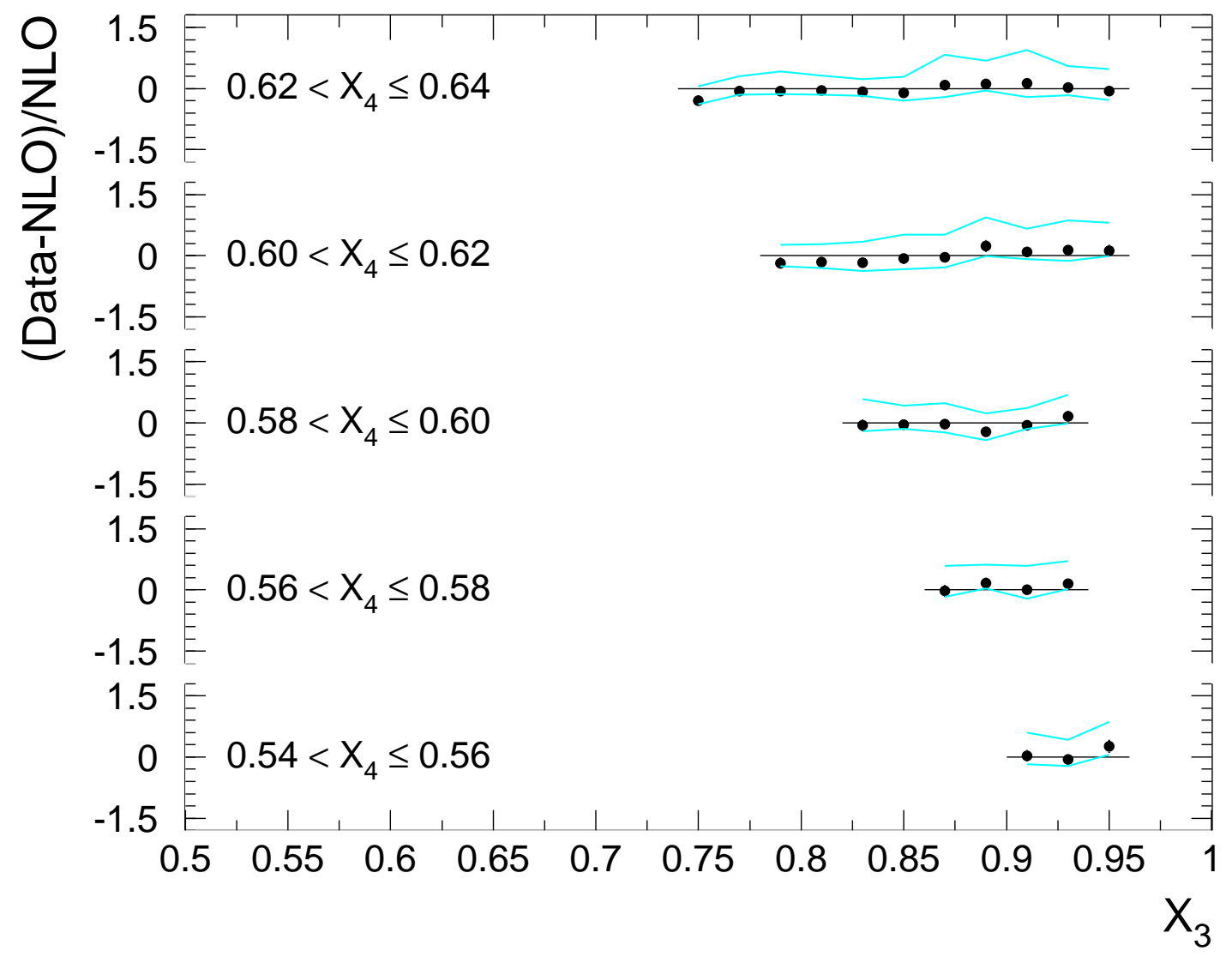

Figure 7.27: The fractional difference between unsmeared data and the NLO prediction, using the CTEQ4HJ parton distribution function, as a function of $\mathrm{X}_{3}$ for $0.54<\mathrm{X}_{4} \leq 0.64$. 


\section{CDF: Preliminary}

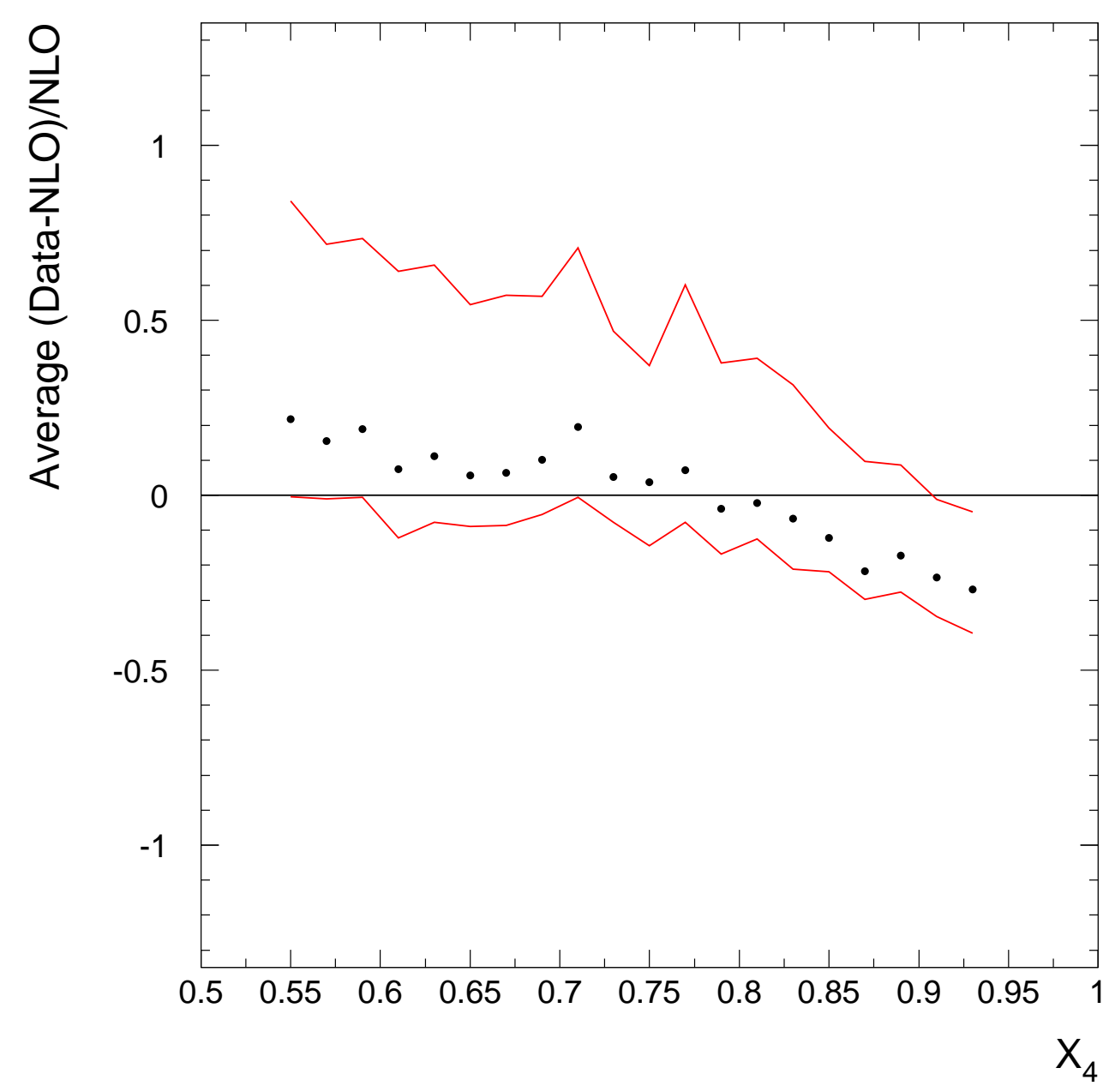

Figure 7.28: The average fractional difference for the three-jet sample as a function of $\mathrm{X}_{4}$. 


\section{Conclusions}

We use the CDF Run 1b data sample with a total integrated luminosity of $86 \mathrm{pb}^{-1}$ to compare multi-jet events to a prediction from a next-to-leading order QCD calculation. In the process, the data are corrected for calorimeter response and for detector resolution effects. The two independent approaches to this unsmearing procedure are not consistent; the procedure with the smaller systematic error, the Monte Carlo approach, is used to find the smearing correction factors. The large systematic uncertainties in the analytic unsmearing are due to our use of a piecewise-continuous parameterization of the Dalitz distributions.

The total systematic uncertainties on the data are determined. Given these uncertainties, the data and the NLO predictions are found to be consistent. Slight differences in the $\mathrm{E}_{\mathrm{T}}$ and Dalitz distributions are attributed to the neglect of higher jet multiplicities in the NLO calculation. The data are found to be stable with respect to the cuts introduced in this analysis. Small changes in the cut parameters do not result in significant changes in the Dalitz distributions.

The total inclusive three-jet production cross section is measured. It is consistent with the cross section predicted by the NLO calculation. A measurement of the strong coupling constant, $\alpha_{\mathrm{S}}$, is not possible. At the level of Monte Carlo statistics achieved in this analysis, the method explored is not sufficiently sensitive to changes of $\alpha_{\mathrm{S}}$ to extract a measurement.

Calculation of the NLO prediction requires extensive computing resources. Our analysis uses 10 different NLO templates in order to investigate the proposed method 
to measure $\alpha_{\mathrm{S}}$. With the computing resources currently available to us, the parton distribution functions do not constrain the gluon distribution sufficiently to allow a measurement of $\alpha_{\mathrm{S}}$. If large-scale computing was accessible, the method introduced in this thesis could be explored further. As discussed in Chapter 3, only a limited amount of measurements of $\alpha_{\mathrm{S}}$ are available from hadron-hadron colliders. New innovative methods to determine the strong coupling are important, especially if they have the potential to reduce current systematic uncertainties. The availability of a Monte Carlo generator to calculate three-jet events to NLO in perturbation theory also allows for the first direct measurement of the ratio of the three-jet and two-jet production cross sections. The results of the data analysis and the NLO Monte Carlo data obtained in our study could serve as basis for that measurement.

In the next run at the Tevatron (Run 2), data of a total integrated luminosity of $2 \mathrm{fb}^{-1}$ are expected. This increase in statistics by a factor of 20 , compared to Run $1 b$, will reduce the statistical uncertainties in the data significantly. Combined with the attempt to reduce the systematic uncertainty due to detector effects in Run 2, new data could allow further precision comparisons to predictions from QCD, especially as theorists are already thinking about an extension of the current NLO calculation to next-to-next-to-leading order (NNLO) [68]. Even though the reduction of experimental and theoretical errors provides a challenging task, it is hoped that it will lead to a deeper understanding of the strong interaction. 


\section{Appendix A}

\section{The Smearing Correction Factor in All Kinemati- cally Allowed Bins in the Dalitz Plane}

In Chapter 4, the unsmearing of the data is discussed. For complete information, the fractional difference between the unsmeared measured and the true Dalitz distributions in all individual kinematically allowed bins in the Dalitz plane is tabulated in this appendix. The unsmearing correction factor, $\mathrm{K}$, and its uncertainty are also shown. Bins that are not used in the analysis because the uncertainty on the NLO calculation in every data set is $>0.2$ are marked with an asterisk. The smearing correction factors are obtained by the Monte Carlo method. 


\begin{tabular}{|c|c|c|c|c|c|}
\hline $\mathrm{X}_{3}$ & $\mathrm{X}_{4}$ & (Unsmeared-True)/True & (Unsmeared-True) $/ \sigma$ & $\mathrm{K}$ & $\sigma_{\mathrm{NLO}}$ \\
\hline \hline 0.67 & 0.67 & $-0.024 \pm 0.19$ & -0.13 & $0.93 \pm 0.067$ & $0.392^{*}$ \\
0.69 & 0.65 & $0.23 \pm 0.28$ & 0.88 & $1.02 \pm 0.083$ & $0.191^{*}$ \\
0.69 & 0.67 & $-0.0017 \pm 0.11$ & -0.016 & $0.91 \pm 0.037$ & 0.106 \\
0.69 & 0.69 & $0.13 \pm 0.18$ & 0.73 & $0.94 \pm 0.053$ & $0.255^{*}$ \\
0.71 & 0.65 & $-0.20 \pm 0.099$ & -1.9 & $0.94 \pm 0.044$ & 0.108 \\
0.71 & 0.67 & $-0.14 \pm 0.094$ & -1.4 & $0.93 \pm 0.037$ & 0.126 \\
0.71 & 0.69 & $-0.18 \pm 0.089$ & -1.9 & $0.96 \pm 0.039$ & 0.118 \\
0.71 & 0.71 & $-0.065 \pm 0.14$ & -0.44 & $0.92 \pm 0.050$ & $0.193^{*}$ \\
0.73 & 0.63 & $0.45 \pm 0.30$ & 1.7 & $0.97 \pm 0.077$ & $0.394^{*}$ \\
0.73 & 0.65 & $0.16 \pm 0.12$ & 1.5 & $0.89 \pm 0.035$ & $0.225^{*}$ \\
0.73 & 0.67 & $-0.099 \pm 0.092$ & -1.0 & $0.98 \pm 0.038$ & 0.148 \\
0.73 & 0.69 & $0.013 \pm 0.10$ & 0.12 & $0.92 \pm 0.036$ & 0.130 \\
0.73 & 0.71 & $0.071 \pm 0.11$ & 0.68 & $0.94 \pm 0.036$ & 0.123 \\
0.73 & 0.73 & $-0.13 \pm 0.12$ & -1.0 & $0.88 \pm 0.045$ & $0.171^{*}$ \\
0.75 & 0.63 & $0.20 \pm 0.14$ & 1.5 & $0.95 \pm 0.043$ & 0.138 \\
0.75 & 0.65 & $-0.12 \pm 0.088$ & -1.3 & $0.96 \pm 0.037$ & 0.141 \\
\hline
\end{tabular}

Table A.1: The fractional difference between the unsmeared measured and the true Dalitz distributions in every kinematically allowed bin in the Dalitz plane as a function of $\mathrm{X}_{3}$ and $\mathrm{X}_{4}$.

\begin{tabular}{|c|c|c|c|c|c|}
\hline $\mathrm{X}_{3}$ & $\mathrm{X}_{4}$ & (Unsmeared-True)/True & (Unsmeared-True) $/ \sigma$ & $\mathrm{K}$ & $\sigma_{\mathrm{NLO}}$ \\
\hline \hline 0.75 & 0.67 & $0.14 \pm 0.11$ & 1.3 & $0.87 \pm 0.033$ & 0.117 \\
0.75 & 0.69 & $0.25 \pm 0.12$ & 2.2 & $0.87 \pm 0.033$ & 0.135 \\
0.75 & 0.71 & $0.13 \pm 0.11$ & 1.2 & $0.91 \pm 0.034$ & 0.118 \\
0.75 & 0.73 & $-0.0093 \pm 0.097$ & -0.10 & $0.95 \pm 0.034$ & 0.134 \\
0.75 & 0.75 & $-0.058 \pm 0.13$ & -0.43 & $0.95 \pm 0.048$ & $0.210^{*}$ \\
0.77 & 0.61 & $0.36 \pm 0.29$ & 1.4 & $0.86 \pm 0.064$ & $0.3099^{*}$ \\
0.77 & 0.63 & $-0.042 \pm 0.097$ & -0.42 & $0.93 \pm 0.035$ & 0.113 \\
0.77 & 0.65 & $0.028 \pm 0.10$ & 0.28 & $0.92 \pm 0.034$ & 0.127 \\
0.77 & 0.67 & $-0.0014 \pm 0.096$ & -0.015 & $0.92 \pm 0.035$ & 0.119 \\
0.77 & 0.69 & $0.097 \pm 0.10$ & 0.96 & $0.92 \pm 0.033$ & 0.110 \\
0.77 & 0.71 & $0.035 \pm 0.097$ & 0.37 & $0.94 \pm 0.033$ & 0.147 \\
0.77 & 0.73 & $-0.16 \pm 0.083$ & -1.8 & $0.92 \pm 0.033$ & 0.103 \\
0.77 & 0.75 & $0.042 \pm 0.096$ & 0.44 & $0.94 \pm 0.032$ & 0.145 \\
0.77 & 0.77 & $-0.038 \pm 0.12$ & -0.32 & $0.97 \pm 0.045$ & $0.209 *$ \\
0.79 & 0.61 & $-0.16 \pm 0.094$ & -1.5 & $0.99 \pm 0.041$ & 0.139 \\
0.79 & 0.63 & $-0.10 \pm 0.087$ & -1.1 & $0.91 \pm 0.033$ & 0.143 \\
\hline
\end{tabular}

Table A.2: The fractional difference between the unsmeared measured and the true Dalitz distributions in every kinematically allowed bin in the Dalitz plane as a function of $\mathrm{X}_{3}$ and $\mathrm{X}_{4}$. 


\begin{tabular}{|c|c|c|c|c|c|}
\hline $\mathrm{X}_{3}$ & $\mathrm{X}_{4}$ & (Unsmeared-True)/True & (Unsmeared-True) $/ \sigma$ & $\mathrm{K}$ & $\sigma_{\mathrm{NLO}}$ \\
\hline \hline 0.79 & 0.65 & $-0.020 \pm 0.093$ & -0.22 & $0.92 \pm 0.033$ & 0.136 \\
0.79 & 0.67 & $0.035 \pm 0.097$ & 0.37 & $0.93 \pm 0.033$ & 0.138 \\
0.79 & 0.69 & $-0.017 \pm 0.088$ & -0.20 & $0.92 \pm 0.032$ & 0.128 \\
0.79 & 0.71 & $-0.052 \pm 0.088$ & -0.58 & $0.99 \pm 0.034$ & 0.144 \\
0.79 & 0.73 & $-0.16 \pm 0.077$ & -1.9 & $1.00 \pm 0.034$ & 0.104 \\
0.79 & 0.75 & $-0.018 \pm 0.088$ & -0.20 & $0.95 \pm 0.032$ & 0.104 \\
0.79 & 0.77 & $0.11 \pm 0.092$ & 1.3 & $0.94 \pm 0.031$ & 0.106 \\
0.79 & 0.79 & $0.012 \pm 0.12$ & 0.10 & $0.99 \pm 0.044$ & $0.184^{*}$ \\
0.81 & 0.59 & $-0.29 \pm 0.13$ & -1.9 & $0.93 \pm 0.066$ & $0.284^{*}$ \\
0.81 & 0.61 & $0.19 \pm 0.11$ & 1.8 & $0.94 \pm 0.033$ & 0.120 \\
0.81 & 0.63 & $-0.042 \pm 0.086$ & -0.47 & $0.96 \pm 0.034$ & 0.0978 \\
0.81 & 0.65 & $0.071 \pm 0.098$ & 0.74 & $0.94 \pm 0.032$ & 0.136 \\
0.81 & 0.67 & $-0.081 \pm 0.083$ & -0.94 & $0.96 \pm 0.033$ & 0.108 \\
0.81 & 0.69 & $-0.012 \pm 0.088$ & -0.14 & $1.00 \pm 0.034$ & 0.143 \\
0.81 & 0.71 & $0.087 \pm 0.098$ & 0.92 & $0.93 \pm 0.031$ & 0.122 \\
0.81 & 0.73 & $-0.031 \pm 0.084$ & -0.37 & $1.02 \pm 0.033$ & 0.116 \\
\hline
\end{tabular}

Table A.3: The fractional difference between the unsmeared measured and the true Dalitz distributions in every kinematically allowed bin in the Dalitz plane as a function of $\mathrm{X}_{3}$ and $\mathrm{X}_{4}$.

\begin{tabular}{|c|c|c|c|c|c|}
\hline $\mathrm{X}_{3}$ & $\mathrm{X}_{4}$ & (Unsmeared-True)/True & (Unsmeared-True) $/ \sigma$ & $\mathrm{K}$ & $\sigma_{\mathrm{NLO}}$ \\
\hline \hline 0.81 & 0.75 & $0.064 \pm 0.090$ & 0.73 & $0.95 \pm 0.030$ & 0.0636 \\
0.81 & 0.77 & $0.036 \pm 0.085$ & 0.42 & $0.95 \pm 0.030$ & 0.116 \\
0.81 & 0.79 & $0.053 \pm 0.086$ & 0.64 & $0.92 \pm 0.028$ & 0.0953 \\
0.81 & 0.81 & $0.025 \pm 0.11$ & 0.23 & $1.00 \pm 0.042$ & $0.109{ }^{*}$ \\
0.83 & 0.59 & $0.081 \pm 0.11$ & 0.73 & $0.92 \pm 0.037$ & 0.134 \\
0.83 & 0.61 & $-0.087 \pm 0.083$ & -1.0 & $0.96 \pm 0.033$ & 0.122 \\
0.83 & 0.63 & $-0.097 \pm 0.082$ & -1.1 & $0.93 \pm 0.032$ & 0.139 \\
0.83 & 0.65 & $-0.067 \pm 0.084$ & -0.77 & $0.98 \pm 0.033$ & 0.134 \\
0.83 & 0.67 & $0.066 \pm 0.095$ & 0.71 & $0.89 \pm 0.030$ & 0.129 \\
0.83 & 0.69 & $-0.095 \pm 0.080$ & -1.1 & $0.96 \pm 0.032$ & 0.129 \\
0.83 & 0.71 & $0.070 \pm 0.093$ & 0.77 & $0.93 \pm 0.030$ & 0.145 \\
0.83 & 0.73 & $0.099 \pm 0.093$ & 1.1 & $0.94 \pm 0.030$ & 0.124 \\
0.83 & 0.75 & $0.033 \pm 0.085$ & 0.39 & $0.97 \pm 0.030$ & 0.106 \\
0.83 & 0.77 & $0.061 \pm 0.086$ & 0.73 & $0.94 \pm 0.029$ & 0.118 \\
0.83 & 0.79 & $0.016 \pm 0.081$ & 0.21 & $0.96 \pm 0.028$ & 0.105 \\
0.83 & 0.81 & $-0.0024 \pm 0.074$ & -0.033 & $0.96 \pm 0.028$ & 0.112 \\
\hline
\end{tabular}

Table A.4: The fractional difference between the unsmeared measured and the true Dalitz distributions in every kinematically allowed bin in the Dalitz plane as a function of $\mathrm{X}_{3}$ and $\mathrm{X}_{4}$. 


\begin{tabular}{|c|c|c|c|c|c|}
\hline $\mathrm{X}_{3}$ & $\mathrm{X}_{4}$ & (Unsmeared-True)/True & (Unsmeared-True) $/ \sigma$ & $\mathrm{K}$ & $\sigma_{\mathrm{NLO}}$ \\
\hline \hline 0.83 & 0.83 & $0.17 \pm 0.12$ & 1.5 & $0.99 \pm 0.038$ & $0.143^{*}$ \\
0.85 & 0.57 & $-0.055 \pm 0.17$ & -0.32 & $0.88 \pm 0.058$ & $0.621^{*}$ \\
0.85 & 0.59 & $0.19 \pm 0.10$ & 2.0 & $0.89 \pm 0.030$ & 0.149 \\
0.85 & 0.61 & $0.028 \pm 0.088$ & 0.33 & $0.96 \pm 0.031$ & 0.132 \\
0.85 & 0.63 & $0.055 \pm 0.093$ & 0.60 & $0.91 \pm 0.030$ & 0.0950 \\
0.85 & 0.65 & $0.16 \pm 0.10$ & 1.7 & $0.97 \pm 0.032$ & 0.104 \\
0.85 & 0.67 & $0.022 \pm 0.088$ & 0.25 & $0.94 \pm 0.031$ & 0.113 \\
0.85 & 0.69 & $-0.012 \pm 0.085$ & -0.14 & $0.95 \pm 0.030$ & 0.117 \\
0.85 & 0.71 & $-0.16 \pm 0.070$ & -2.1 & $1.00 \pm 0.031$ & 0.120 \\
0.85 & 0.73 & $-0.025 \pm 0.080$ & -0.31 & $0.95 \pm 0.029$ & 0.120 \\
0.85 & 0.75 & $-0.17 \pm 0.066$ & -2.4 & $0.99 \pm 0.030$ & 0.107 \\
0.85 & 0.77 & $0.044 \pm 0.081$ & 0.55 & $0.97 \pm 0.029$ & 0.125 \\
0.85 & 0.79 & $0.054 \pm 0.079$ & 0.70 & $0.94 \pm 0.027$ & 0.0937 \\
0.85 & 0.81 & $-0.11 \pm 0.065$ & -1.6 & $1.03 \pm 0.028$ & 0.0963 \\
0.85 & 0.83 & $0.056 \pm 0.078$ & 0.74 & $0.97 \pm 0.026$ & 0.0996 \\
0.85 & 0.85 & $-0.080 \pm 0.091$ & -0.84 & $1.01 \pm 0.037$ & $0.1511^{*}$ \\
\hline
\end{tabular}

Table A.5: The fractional difference between the unsmeared measured and the true Dalitz distributions in every kinematically allowed bin in the Dalitz plane as a function of $\mathrm{X}_{3}$ and $\mathrm{X}_{4}$.

\begin{tabular}{|c|c|c|c|c|c|}
\hline $\mathrm{X}_{3}$ & $\mathrm{X}_{4}$ & (Unsmeared-True)/True & (Unsmeared-True) $/ \sigma$ & $\mathrm{K}$ & $\sigma_{\mathrm{NLO}}$ \\
\hline \hline 0.87 & 0.57 & $-0.14 \pm 0.086$ & -1.5 & $0.98 \pm 0.036$ & 0.137 \\
0.87 & 0.59 & $-0.085 \pm 0.078$ & -1.1 & $0.96 \pm 0.030$ & 0.122 \\
0.87 & 0.61 & $-0.046 \pm 0.080$ & -0.57 & $0.94 \pm 0.030$ & 0.124 \\
0.87 & 0.63 & $-0.094 \pm 0.075$ & -1.2 & $0.94 \pm 0.030$ & 0.123 \\
0.87 & 0.65 & $-0.065 \pm 0.077$ & -0.83 & $1.01 \pm 0.032$ & 0.127 \\
0.87 & 0.67 & $0.11 \pm 0.091$ & 1.3 & $0.94 \pm 0.029$ & 0.104 \\
0.87 & 0.69 & $0.068 \pm 0.086$ & 0.81 & $0.98 \pm 0.029$ & 0.128 \\
0.87 & 0.71 & $0.082 \pm 0.086$ & 0.98 & $0.96 \pm 0.028$ & 0.124 \\
0.87 & 0.73 & $0.18 \pm 0.090$ & 2.1 & $0.88 \pm 0.026$ & 0.117 \\
0.87 & 0.75 & $0.038 \pm 0.080$ & 0.48 & $0.97 \pm 0.028$ & 0.104 \\
0.87 & 0.77 & $0.059 \pm 0.080$ & 0.76 & $0.97 \pm 0.027$ & 0.126 \\
0.87 & 0.79 & $-0.055 \pm 0.069$ & -0.77 & $1.03 \pm 0.028$ & 0.0978 \\
0.87 & 0.81 & $-0.087 \pm 0.066$ & -1.3 & $0.98 \pm 0.026$ & 0.123 \\
0.87 & 0.83 & $-0.085 \pm 0.065$ & -1.3 & $0.99 \pm 0.026$ & 0.110 \\
0.87 & 0.85 & $-0.061 \pm 0.064$ & -0.92 & $1.01 \pm 0.026$ & 0.0961 \\
0.87 & 0.87 & $0.14 \pm 0.11$ & 1.4 & $0.95 \pm 0.034$ & 0.125 \\
\hline
\end{tabular}

Table A.6: The fractional difference between the unsmeared measured and the true Dalitz distributions in every kinematically allowed bin in the Dalitz plane as a function of $\mathrm{X}_{3}$ and $\mathrm{X}_{4}$. 


\begin{tabular}{|c|c|c|c|c|c|}
\hline $\mathrm{X}_{3}$ & $\mathrm{X}_{4}$ & (Unsmeared-True)/True & (Unsmeared-True) $/ \sigma$ & $\mathrm{K}$ & $\sigma_{\mathrm{NLO}}$ \\
\hline \hline 0.89 & 0.55 & $-0.0057 \pm 0.15$ & -0.037 & $0.97 \pm 0.058$ & $0.240^{*}$ \\
0.89 & 0.57 & $-0.016 \pm 0.080$ & -0.20 & $0.96 \pm 0.029$ & $\mathrm{y} 0.146$ \\
0.89 & 0.59 & $-0.071 \pm 0.075$ & -0.93 & $0.97 \pm 0.029$ & 0.118 \\
0.89 & 0.61 & $-0.072 \pm 0.075$ & -0.94 & $0.98 \pm 0.029$ & 0.122 \\
0.89 & 0.62 & $0.048 \pm 0.082$ & 0.59 & $0.96 \pm 0.028$ & 0.112 \\
0.89 & 0.65 & $-0.043 \pm 0.075$ & -0.56 & $0.92 \pm 0.027$ & 0.111 \\
0.89 & 0.67 & $-0.039 \pm 0.074$ & -0.52 & $0.92 \pm 0.027$ & 0.135 \\
0.89 & 0.69 & $0.017 \pm 0.078$ & 0.22 & $0.94 \pm 0.027$ & 0.113 \\
0.89 & 0.71 & $0.031 \pm 0.078$ & 0.41 & $0.94 \pm 0.027$ & 0.127 \\
0.89 & 0.73 & $-0.12 \pm 0.064$ & -1.7 & $0.96 \pm 0.027$ & 0.104 \\
0.89 & 0.75 & $-0.031 \pm 0.070$ & -0.44 & $0.96 \pm 0.026$ & 0.106 \\
0.89 & 0.77 & $-0.013 \pm 0.068$ & -0.19 & $0.96 \pm 0.026$ & 0.119 \\
0.89 & 0.79 & $-0.053 \pm 0.065$ & -0.80 & $1.00 \pm 0.026$ & 0.108 \\
0.89 & 0.81 & $0.11 \pm 0.076$ & 1.5 & $0.99 \pm 0.026$ & 0.103 \\
0.89 & 0.83 & $0.093 \pm 0.074$ & 1.3 & $0.95 \pm 0.024$ & 0.104 \\
0.89 & 0.85 & $-0.011 \pm 0.064$ & -0.17 & $1.03 \pm 0.025$ & 0.105 \\
\hline
\end{tabular}

Table A.7: The fractional difference between the unsmeared measured and the true Dalitz distributions in every kinematically allowed bin in the Dalitz plane as a function of $\mathrm{X}_{3}$ and $\mathrm{X}_{4}$.

\begin{tabular}{|c|c|c|c|c|c|}
\hline $\mathrm{X}_{3}$ & $\mathrm{X}_{4}$ & (Unsmeared-True)/True & (Unsmeared-True) $/ \sigma$ & $\mathrm{K}$ & $\sigma_{\mathrm{NLO}}$ \\
\hline \hline 0.89 & 0.87 & $0.031 \pm 0.067$ & 0.46 & $1.00 \pm 0.024$ & 0.0992 \\
0.89 & 0.89 & $-0.031 \pm 0.088$ & -0.34 & $1.02 \pm 0.034$ & 0.116 \\
0.91 & 0.55 & $0.0071 \pm 0.086$ & 0.083 & $0.95 \pm 0.031$ & 0.131 \\
0.91 & 0.57 & $0.040 \pm 0.076$ & 0.53 & $0.96 \pm 0.027$ & 0.115 \\
0.91 & 0.59 & $0.12 \pm 0.084$ & 1.5 & $0.98 \pm 0.028$ & 0.126 \\
0.91 & 0.61 & $-0.0092 \pm 0.073$ & -0.12 & $0.93 \pm 0.026$ & 0.122 \\
0.91 & 0.63 & $-0.14 \pm 0.063$ & -2.0 & $0.99 \pm 0.027$ & 0.105 \\
0.91 & 0.65 & $-0.010 \pm 0.072$ & -0.14 & $0.97 \pm 0.026$ & 0.117 \\
0.91 & 0.67 & $0.18 \pm 0.083$ & 2.3 & $0.96 \pm 0.026$ & 0.124 \\
0.91 & 0.69 & $0.10 \pm 0.078$ & 1.4 & $0.91 \pm 0.024$ & 0.110 \\
0.91 & 0.71 & $0.032 \pm 0.072$ & 0.45 & $0.97 \pm 0.026$ & 0.105 \\
0.91 & 0.73 & $0.0067 \pm 0.069$ & 0.10 & $0.97 \pm 0.025$ & 0.118 \\
0.91 & 0.75 & $-0.081 \pm 0.064$ & -1.2 & $0.99 \pm 0.025$ & 0.0945 \\
0.91 & 0.77 & $-0.020 \pm 0.065$ & -0.30 & $0.98 \pm 0.025$ & 0.0822 \\
0.91 & 0.79 & $0.058 \pm 0.071$ & 0.84 & $0.93 \pm 0.023$ & 0.0967 \\
0.91 & 0.81 & $-0.013 \pm 0.064$ & -0.20 & $1.01 \pm 0.025$ & 0.0917 \\
\hline
\end{tabular}

Table A.8: The fractional difference between the unsmeared measured and the true Dalitz distributions in every kinematically allowed bin in the Dalitz plane as a function of $\mathrm{X}_{3}$ and $\mathrm{X}_{4}$. 


\begin{tabular}{|c|c|c|c|c|c|}
\hline $\mathrm{X}_{3}$ & $\mathrm{X}_{4}$ & (Unsmeared-True)/True & (Unsmeared-True) $/ \sigma$ & $\mathrm{K}$ & $\sigma_{\mathrm{NLO}}$ \\
\hline \hline 0.91 & 0.83 & $-0.10 \pm 0.058$ & -1.7 & $1.03 \pm 0.025$ & 0.0930 \\
0.91 & 0.85 & $0.048 \pm 0.068$ & 0.72 & $1.03 \pm 0.025$ & 0.0990 \\
0.91 & 0.87 & $0.025 \pm 0.064$ & 0.40 & $1.03 \pm 0.024$ & 0.0865 \\
0.91 & 0.89 & $-0.051 \pm 0.060$ & -0.83 & $1.06 \pm 0.025$ & 0.120 \\
0.91 & 0.91 & $-0.025 \pm 0.086$ & -0.29 & $1.13 \pm 0.038$ & 0.114 \\
0.93 & 0.53 & $0.066 \pm 0.16$ & 0.44 & $1.01 \pm 0.056$ & $0.321^{*}$ \\
0.93 & 0.55 & $0.12 \pm 0.080$ & 1.5 & $0.90 \pm 0.025$ & 0.149 \\
0.93 & 0.57 & $-0.11 \pm 0.064$ & -1.6 & $1.01 \pm 0.027$ & 0.131 \\
0.93 & 0.59 & $0.082 \pm 0.077$ & 1.1 & $0.94 \pm 0.025$ & 0.0996 \\
0.93 & 0.61 & $0.021 \pm 0.071$ & 0.30 & $0.94 \pm 0.025$ & 0.107 \\
0.93 & 0.63 & $-0.11 \pm 0.063$ & -1.7 & $1.02 \pm 0.027$ & 0.142 \\
0.93 & 0.65 & $-0.043 \pm 0.066$ & -0.64 & $0.96 \pm 0.025$ & 0.0911 \\
0.93 & 0.67 & $-0.050 \pm 0.062$ & -0.79 & $0.94 \pm 0.024$ & 0.103 \\
0.93 & 0.69 & $-0.0070 \pm 0.064$ & -0.11 & $0.94 \pm 0.023$ & 0.109 \\
0.93 & 0.71 & $-0.060 \pm 0.061$ & -0.96 & $0.95 \pm 0.023$ & 0.0910 \\
0.93 & 0.73 & $0.10 \pm 0.072$ & 1.5 & $0.96 \pm 0.023$ & 0.108 \\
\hline
\end{tabular}

Table A.9: The fractional difference between the unsmeared measured and the true Dalitz distributions in every kinematically allowed bin in the Dalitz plane as a function of $\mathrm{X}_{3}$ and $\mathrm{X}_{4}$.

\begin{tabular}{|c|c|c|c|c|c|}
\hline $\mathrm{X}_{3}$ & $\mathrm{X}_{4}$ & (Unsmeared-True)/True & (Unsmeared-True) $/ \sigma$ & $\mathrm{K}$ & $\sigma_{\mathrm{NLO}}$ \\
\hline \hline 0.93 & 0.75 & $-0.10 \pm 0.058$ & -1.6 & $0.99 \pm 0.024$ & 0.0873 \\
0.93 & 0.77 & $0.086 \pm 0.068$ & 1.3 & $0.97 \pm 0.023$ & 0.102 \\
0.93 & 0.79 & $0.047 \pm 0.064$ & 0.74 & $0.98 \pm 0.023$ & 0.0899 \\
0.93 & 0.81 & $-0.016 \pm 0.060$ & -0.26 & $1.02 \pm 0.023$ & 0.0992 \\
0.93 & 0.83 & $0.029 \pm 0.062$ & 0.47 & $1.02 \pm 0.023$ & 0.0935 \\
0.93 & 0.85 & $-0.062 \pm 0.058$ & -1.0 & $1.02 \pm 0.023$ & 0.0858 \\
0.93 & 0.87 & $0.024 \pm 0.062$ & 0.39 & $1.07 \pm 0.025$ & 0.0863 \\
0.93 & 0.89 & $0.12 \pm 0.070$ & 1.8 & $1.12 \pm 0.026$ & 0.0721 \\
0.93 & 0.91 & $-0.0060 \pm 0.065$ & -0.092 & $1.13 \pm 0.027$ & 0.0790 \\
0.93 & 0.93 & $0.037 \pm 0.093$ & 0.40 & $1.13 \pm 0.040$ & 0.103 \\
0.95 & 0.53 & $-0.0042 \pm 0.094$ & -0.045 & $1.04 \pm 0.037$ & $0.202 *$ \\
0.95 & 0.55 & $0.028 \pm 0.084$ & 0.34 & $1.03 \pm 0.032$ & 0.149 \\
0.95 & 0.57 & $0.056 \pm 0.084$ & 0.68 & $1.02 \pm 0.031$ & $0.169 *$ \\
0.95 & 0.59 & $0.0094 \pm 0.081$ & 0.12 & $1.04 \pm 0.031$ & $0.158^{*}$ \\
0.95 & 0.61 & $-0.014 \pm 0.077$ & -0.18 & $1.03 \pm 0.030$ & 0.126 \\
0.95 & 0.63 & $-0.032 \pm 0.075$ & -0.42 & $1.07 \pm 0.031$ & 0.149 \\
\hline
\end{tabular}

Table A.10: The fractional difference between the unsmeared measured and the true Dalitz distributions in every kinematically allowed bin in the Dalitz plane as a function of $\mathrm{X}_{3}$ and $\mathrm{X}_{4}$. 


\begin{tabular}{|c|c|c|c|c|c|}
\hline $\mathrm{X}_{3}$ & $\mathrm{X}_{4}$ & (Unsmeared-True)/True & (Unsmeared-True) $/ \sigma$ & $\mathrm{K}$ & $\sigma_{\mathrm{NLO}}$ \\
\hline \hline 0.95 & 0.65 & $-0.056 \pm 0.071$ & -0.77 & $1.01 \pm 0.028$ & 0.129 \\
0.95 & 0.67 & $-0.064 \pm 0.068$ & -0.91 & $1.02 \pm 0.028$ & 0.130 \\
0.95 & 0.69 & $0.027 \pm 0.072$ & 0.37 & $1.05 \pm 0.028$ & 0.125 \\
0.95 & 0.71 & $0.075 \pm 0.072$ & 1.1 & $1.00 \pm 0.026$ & 0.117 \\
0.95 & 0.73 & $0.0053 \pm 0.066$ & 0.081 & $1.02 \pm 0.025$ & 0.121 \\
0.95 & 0.75 & $-0.023 \pm 0.059$ & -0.39 & $1.01 \pm 0.024$ & 0.109 \\
0.95 & 0.77 & $0.038 \pm 0.062$ & 0.61 & $1.00 \pm 0.023$ & 0.0941 \\
0.95 & 0.79 & $-0.0085 \pm 0.058$ & -0.15 & $1.03 \pm 0.023$ & 0.0831 \\
0.95 & 0.81 & $-0.0033 \pm 0.057$ & -0.057 & $1.03 \pm 0.023$ & 0.0823 \\
0.95 & 0.83 & $0.013 \pm 0.058$ & 0.22 & $1.01 \pm 0.022$ & 0.0859 \\
0.95 & 0.85 & $-0.029 \pm 0.057$ & -0.51 & $1.08 \pm 0.024$ & 0.0880 \\
0.95 & 0.87 & $0.0023 \pm 0.059$ & 0.039 & $1.08 \pm 0.025$ & 0.0774 \\
0.95 & 0.89 & $-0.055 \pm 0.058$ & -0.91 & $1.14 \pm 0.027$ & 0.0920 \\
0.95 & 0.91 & $0.017 \pm 0.066$ & 0.26 & $1.20 \pm 0.030$ & 0.0924 \\
0.95 & 0.93 & $-0.080 \pm 0.064$ & -1.2 & $1.21 \pm 0.033$ & 0.0809 \\
0.95 & 0.95 & $0.10 \pm 0.14$ & 0.76 & $1.28 \pm 0.059$ & $0.214 *$ \\
\hline
\end{tabular}

Table A.11: The fractional difference between the unsmeared measured and the true Dalitz distributions in every kinematically allowed bin in the Dalitz plane as a function of $\mathrm{X}_{3}$ and $\mathrm{X}_{4}$.

\begin{tabular}{|c|c|c|c|c|c|}
\hline $\mathrm{X}_{3}$ & $\mathrm{X}_{4}$ & (Unsmeared-True)/True & (Unsmeared-True) $/ \sigma$ & $\mathrm{K}$ & $\sigma_{\mathrm{NLO}}$ \\
\hline \hline 0.97 & 0.51 & $-0.035 \pm 0.24$ & -0.14 & $1.04 \pm 0.10$ & $1.82^{*}$ \\
0.97 & 0.53 & $0.080 \pm 0.12$ & 0.66 & $1.04 \pm 0.046$ & $0.282^{*}$ \\
0.97 & 0.55 & $-0.072 \pm 0.11$ & -0.64 & $1.11 \pm 0.050$ & $0.220^{*}$ \\
0.97 & 0.57 & $-0.15 \pm 0.10$ & -1.4 & $1.07 \pm 0.047$ & $0.272^{*}$ \\
0.97 & 0.59 & $0.023 \pm 0.11$ & 0.19 & $1.06 \pm 0.045$ & $0.318^{*}$ \\
0.97 & 0.61 & $-0.031 \pm 0.10$ & -0.29 & $1.06 \pm 0.045$ & $0.278^{*}$ \\
0.97 & 0.63 & $-0.0021 \pm 0.11$ & -0.019 & $1.01 \pm 0.040$ & $0.302^{*}$ \\
0.97 & 0.65 & $-0.055 \pm 0.10$ & -0.53 & $1.03 \pm 0.040$ & $0.176^{*}$ \\
0.97 & 0.67 & $0.082 \pm 0.11$ & 0.78 & $1.09 \pm 0.040$ & $0.282^{*}$ \\
0.97 & 0.69 & $0.11 \pm 0.11$ & 1.1 & $1.06 \pm 0.038$ & $0.304^{*}$ \\
0.97 & 0.71 & $0.066 \pm 0.099$ & 0.69 & $1.04 \pm 0.036$ & $0.222^{*}$ \\
0.97 & 0.73 & $-0.13 \pm 0.076$ & -1.6 & $1.04 \pm 0.034$ & $0.234^{*}$ \\
0.97 & 0.75 & $0.017 \pm 0.084$ & 0.21 & $1.04 \pm 0.033$ & $0.129^{*}$ \\
0.97 & 0.77 & $0.025 \pm 0.080$ & 0.31 & $1.07 \pm 0.033$ & 0.127 \\
0.97 & 0.79 & $0.023 \pm 0.079$ & 0.29 & $1.15 \pm 0.033$ & 0.149 \\
0.97 & 0.81 & $-0.14 \pm 0.066$ & -1.9 & $1.12 \pm 0.031$ & 0.143 \\
\hline
\end{tabular}

Table A.12: The fractional difference between the unsmeared measured and the true Dalitz distributions in every kinematically allowed bin in the Dalitz plane as a function of $\mathrm{X}_{3}$ and $\mathrm{X}_{4}$. 


\begin{tabular}{|c|c|c|c|c|c|}
\hline $\mathrm{X}_{3}$ & $\mathrm{X}_{4}$ & (Unsmeared-True)/True & (Unsmeared-True) $/ \sigma$ & $\mathrm{K}$ & $\sigma_{\mathrm{NLO}}$ \\
\hline \hline 0.97 & 0.83 & $0.034 \pm 0.072$ & 0.49 & $1.14 \pm 0.030$ & 0.117 \\
0.97 & 0.85 & $-0.056 \pm 0.063$ & -0.86 & $1.16 \pm 0.029$ & 0.0921 \\
0.97 & 0.87 & $0.048 \pm 0.069$ & 0.71 & $1.15 \pm 0.028$ & 0.0911 \\
0.97 & 0.89 & $-0.037 \pm 0.063$ & -0.58 & $1.22 \pm 0.030$ & 0.0873 \\
0.97 & 0.91 & $0.010 \pm 0.070$ & 0.14 & $1.30 \pm 0.035$ & 0.100 \\
0.97 & 0.93 & $-0.052 \pm 0.079$ & -0.64 & $1.26 \pm 0.041$ & 0.144 \\
0.97 & 0.95 & $0.058 \pm 0.12$ & 0.48 & $1.24 \pm 0.055$ & $0.223^{*}$ \\
0.97 & 0.97 & $0.13 \pm 0.31$ & 0.46 & $1.32 \pm 0.13$ & $8.16^{*}$ \\
0.99 & 0.51 & $-0.16 \pm 0.36$ & -0.41 & $1.11 \pm 0.20$ & $0.607^{*}$ \\
0.99 & 0.53 & $-0.086 \pm 0.33$ & -0.25 & $1.16 \pm 0.16$ & $-^{*}$ \\
0.99 & 0.55 & $0.27 \pm 0.50$ & 0.62 & $1.09 \pm 0.15$ & $0.238^{*}$ \\
0.99 & 0.57 & $0.32 \pm 0.45$ & 0.78 & $1.03 \pm 0.14$ & $0.932^{*}$ \\
0.99 & 0.59 & $0.14 \pm 0.35$ & 0.43 & $1.07 \pm 0.14$ & $-{ }^{*}$ \\
0.99 & 0.61 & $-0.33 \pm 0.20$ & -1.3 & $1.34 \pm 0.17$ & $-{ }^{*}$ \\
0.99 & 0.63 & $0.41 \pm 0.44$ & 1.1 & $0.91 \pm 0.10$ & $-{ }^{*}$ \\
0.99 & 0.65 & $0.082 \pm 0.29$ & 0.29 & $1.10 \pm 0.12$ & $-^{*}$ \\
\hline
\end{tabular}

Table A.13: The fractional difference between the unsmeared measured and the true Dalitz distributions in every kinematically allowed bin in the Dalitz plane as a function of $\mathrm{X}_{3}$ and $\mathrm{X}_{4}$.

\begin{tabular}{|c|c|c|c|c|c|}
\hline $\mathrm{X}_{3}$ & $\mathrm{X}_{4}$ & (Unsmeared-True)/True & (Unsmeared-True) $/ \sigma$ & $\mathrm{K}$ & $\sigma_{\mathrm{NLO}}$ \\
\hline \hline 0.99 & 0.67 & $0.32 \pm 0.33$ & 1.1 & $1.17 \pm 0.12$ & $4.08^{*}$ \\
0.99 & 0.69 & $-0.060 \pm 0.23$ & -0.25 & $1.15 \pm 0.11$ & $-^{*}$ \\
0.99 & 0.71 & $0.15 \pm 0.27$ & 0.59 & $0.87 \pm 0.077$ & $-^{*}$ \\
0.99 & 0.73 & $0.13 \pm 0.25$ & 0.52 & $1.06 \pm 0.084$ & $-^{*}$ \\
0.99 & 0.75 & $0.033 \pm 0.21$ & 0.16 & $1.13 \pm 0.087$ & $1.36^{*}$ \\
0.99 & 0.77 & $0.14 \pm 0.23$ & 0.62 & $1.08 \pm 0.077$ & $0.432^{*}$ \\
0.99 & 0.79 & $-0.0042 \pm 0.19$ & -0.022 & $1.08 \pm 0.078$ & $-{ }^{*}$ \\
0.99 & 0.81 & $0.0029 \pm 0.18$ & 0.016 & $1.01 \pm 0.069$ & $0.325^{*}$ \\
0.99 & 0.83 & $0.035 \pm 0.18$ & 0.20 & $1.18 \pm 0.074$ & $0.385^{*}$ \\
0.99 & 0.85 & $-0.010 \pm 0.16$ & -0.063 & $1.11 \pm 0.067$ & $0.497^{*}$ \\
0.99 & 0.87 & $-0.14 \pm 0.13$ & -0.96 & $1.25 \pm 0.072$ & $0.340^{*}$ \\
0.99 & 0.89 & $-0.066 \pm 0.13$ & -0.48 & $1.19 \pm 0.068$ & $0.178^{*}$ \\
0.99 & 0.91 & $0.29 \pm 0.20$ & 1.6 & $1.27 \pm 0.073$ & $0.368^{*}$ \\
0.99 & 0.93 & $-0.014 \pm 0.15$ & -0.088 & $1.30 \pm 0.081$ & $0.470^{*}$ \\
0.99 & 0.95 & $-0.18 \pm 0.19$ & -0.85 & $1.49 \pm 0.13$ & $-{ }^{*}$ \\
0.99 & 0.97 & $-0.11 \pm 0.49$ & -021 & $1.44 \pm 0.32$ & $-^{*}$ \\
\hline
\end{tabular}

Table A.14: The fractional difference between the unsmeared measured and the true Dalitz distributions in every kinematically allowed bin in the Dalitz plane as a function of $\mathrm{X}_{3}$ and $\mathrm{X}_{4}$. 


\section{Appendix B}

\section{The Analytical Unsmearing Procedure}

In this appendix, we describe the analytical method to unsmear the data. The description of the procedure involves the generic variable $\mathrm{X}$ which in our case stands for either one of the Dalitz variables $\mathrm{X}_{3}$ and $\mathrm{X}_{4}$, but it can generally be replaced by any variable that is a function of the jet energies.

Due to the smearing in the detector, the response to signals of one specific energy is not at Delta-function. This measured distribution is called the detector response function. The observed spectrum of the variable $\mathrm{X}$ can be described such that the contents of each bin are given by

$$
\mathrm{O}^{\text {bin }} \equiv \frac{\int^{\text {bin }} \mathrm{C} \mathrm{dX^{ \text {meas } }}}{\text { bin width }}
$$

where $\mathrm{C}$ is the convolution of the true spectrum and the detector response function,

$$
\mathrm{C} \equiv \int_{-\infty}^{\infty} \mathrm{R}\left(\mathrm{X}^{\text {meas }}, \mathrm{X}^{\text {true }}, \mathrm{Y}\left(\mathrm{X}^{\mathrm{meas}}\right)\right) \mathrm{T}\left(\mathrm{X}^{\text {true }}, \mathrm{Z}\right) \mathrm{d} \mathrm{X}^{\text {true }}
$$

$\mathrm{O}^{\text {bin }}$ is the observed number of entries in an $\mathrm{X}$ bin. When we refer to the values of the convolution in all bins, we call the resulting function $\mathrm{O}$. $\mathrm{R}$ represents the detector response function which depends on the measured value of $\mathrm{X}, \mathrm{X}^{\text {meas }}$, the true value of $\mathrm{X}, \mathrm{X}^{\text {true }}$, and a set of parameters, $\mathrm{Y}$. The true spectrum, $\mathrm{T}$, depends on $\mathrm{X}^{\text {true }}$ and another set of parameters, Z, which are explained below.

The integration over $\mathrm{X}^{\text {meas }}$ and the division by the bin width account for the fact that the events are distributed over the whole bin and do not simply assume the 
value at the center of the bin. It is performed over the data bin that corresponds to $\mathrm{O}^{\text {bin }}$. Effectively, we are calculating the mean of $\mathrm{C}$ in that specific bin.

We perform the convolution over all possible values of $\mathrm{X}^{\text {true }}$ in order to account for the contributions from all $\mathrm{X}^{\text {true }}$ to the number of observed events in a specific data bin. In the case of the Dalitz variables, the boundaries of integration are the intervals from $\frac{2}{3}$ to 1 for $\mathrm{X}_{3}$ and from $\frac{1}{2}$ to 1 for $\mathrm{X}_{4}$, since any other values of $\mathrm{X}^{\text {true }}$ are kinematically forbidden (see the previous chapter). Parameterization of the true spectrum does not make sense outside these ranges.

The data are unsmeared by using the procedure outlined in this appendix. A detailed description of the individual steps of the procedure can be found below. This method was adapted from Reference [35] and generalized to suit the analysis of the generic variable $\mathrm{X}$.

1. The jet energies for the $\mathrm{X}^{\text {meas }}$ distribution are corrected using JTC96. Corrections for errors in the absolute and relative energy scales are applied, in addition to adjustments that are necessary to account for the underlying event, out-of-cone corrections are not used.

2. We choose a set of values of $X^{\text {true }}$ and find the corresponding distributions for $\mathrm{X}^{\text {meas }}$. These are the detector response distributions which we then normalize to unit area since the detector only smears X, but it does not create or destroy events.

3. We find the function $\mathrm{R}$, depending on $\mathrm{X}^{\text {true }}, \mathrm{X}^{\text {meas }}$, and a set of parameters $\mathrm{Y}$, that best describes all detector response distributions, the detector response function.

4. We define the $\chi^{2}$ between the detector response distributions and $\mathrm{R}$, and call it $\chi_{1}^{2}$. By minimizing it, we obtain the values of the parameters $Y$. The fitting program MINUIT is used for this task. 
5. We parameterize the true spectrum, T.

6. We fix the values of the parameters $\mathrm{Y}$ in $\mathrm{R}$ and define as $\chi_{2}^{2}$ the $\chi^{2}$ between the data distributions in $\mathrm{X}$ and the value of the convolution integral, $\mathrm{O}$, in all bins. We minimize $\chi_{2}^{2}$ to find the parameters $\mathrm{Z}$ in the true spectrum, T. MINUIT is used to perform this fit.

7. With this particular choice of parameters $\mathrm{Z}$ and $\mathrm{Y}$, we calculate the values of the true spectrum and the convolution integral in each bin. The smeared spectrum is given by the values of the convolution integral for the chosen set of parameters. We expect the smeared spectrum to reproduce the data distribution.

8. To quantify the factor that needs to be applied to the data to correct for the smearing, we define a correction factor, $\mathrm{K}$, for each bin:

$$
\mathrm{K}\left(\mathrm{X}^{\text {meas }}\right) \equiv \frac{\text { smeared spectrum }\left(\mathrm{X}^{\text {meas }}\right)}{\text { true spectrum }\left(\mathrm{X}^{\text {meas }}\right)} \text {. }
$$

The data are divided by $\mathrm{K}$ to give us the unsmeared, fully corrected, values:

$$
\text { corrected data }\left(\mathrm{X}^{\text {meas }}\right) \equiv \frac{\operatorname{data}\left(\mathrm{X}^{\text {meas }}\right)}{\mathrm{K}\left(\mathrm{X}^{\text {meas }}\right)}
$$

\section{The Detector Response Function}

In order to parameterize the detector response, several HERWIG Monte Carlo samples are generated. For our analysis of the unsmearing of the Dalitz variables, we used a total of 439,313 HERWIG events. The values of $\mathrm{X}^{\text {true }}$ and $\mathrm{X}^{\text {meas }}$ are calculated for all these Monte Carlo events. The internal consistency of the unsmearing procedure is checked by an independent HERWIG sample, containing 72,990 events.

To find the detector response function for a given value of $\mathrm{X}^{\text {true }}$, we begin by selecting events with a well defined $\mathrm{X}^{\text {true }}$. Values of $\mathrm{X}^{\text {true }}$ are chosen, ranging over all values possible for that variable, such that the whole range of $\mathrm{X}^{\text {meas }}$ can be parameterized simultaneously. We call these $\mathrm{X}_{j}^{\text {true }}$. In the case of the Dalitz variables, 
$\mathrm{X}_{3}$ and $\mathrm{X}_{4}, \mathrm{X}_{j}^{\mathrm{true}}=0.72,0.75,0.80,0.85,0.90,0.93$ and $\mathrm{X}_{j}^{\text {true }}=0.53,0.60,0.67,0.74$, $0.81,0.88,0.95$, respectively, spanning all of the kinematically allowed values. The range of true values of $\mathrm{X}$ is $\pm 0.5 \%$ of the nominal value. The detector simulation, QFL, is applied to these events to produce smeared distributions for $\mathrm{X}^{\text {meas }}$. We next fit several trial functions to the $\mathrm{X}^{\text {meas }}$ distributions to find the best parameterization for the detector response function. We define $\chi_{3}^{2} /$ d.o.f. to be the $\chi^{2} /$ d.o.f. between a trial function and the $\mathrm{X}^{\text {meas }}$ distribution. The $\chi_{3}^{2} /$ d.o.f. is computed for all functions for each sample, and the lowest average $\chi_{3}^{2} /$ d.o.f. indicates the best parameterization.

An acceptable parameterization of the detector response for the study of the Dalitz variables turns out to be the sum of three Gaussians:

$$
\mathrm{R} \equiv \sum_{i=1}^{3} \frac{\mathrm{n}_{i}}{\sqrt{2 \pi} \sigma_{i}} \exp \left(\frac{-\left(\mu_{i}-\mathrm{X}^{\text {meas }}\right)^{2}}{2 \sigma_{i}^{2}}\right) .
$$

Each individual Gaussian has to be normalized to unity and multiplied by a relative weight, $\mathrm{n}_{i}$. The nine parameters, $\mathrm{n}_{i}, \sigma_{i}$, and $\mu_{i}$ (which were collectively called $\mathrm{Y}$ in the earlier discussion), in turn are parameterized as functions of $\mathrm{X}^{\text {true }}$ to provide some adjustability in $\mathrm{R}$ when it is simultaneously fit to all samples. We parameterize them by:

$$
\begin{aligned}
\mathrm{n}_{i} & \equiv \mathrm{n}_{i 1}\left(\mathrm{X}^{\text {true }}\right)^{3}+\mathrm{n}_{i 2}\left(\mathrm{X}^{\text {true }}\right)^{2}+\mathrm{n}_{i 3}\left(\mathrm{X}^{\text {true }}\right)+\mathrm{n}_{i 4}, \\
\sigma_{i} & \equiv \sigma_{i 1}\left(\mathrm{X}^{\text {true }}\right)^{3}+\sigma_{i 2}\left(\mathrm{X}^{\text {true }}\right)^{2}+\sigma_{i 3}\left(\mathrm{X}^{\text {true }}\right)+\sigma_{i 4}, \text { and } \\
\mu_{i} & \equiv \mu_{i 1}\left(\mathrm{X}^{\text {true }}\right)^{3}+\mu_{i 2}\left(\mathrm{X}^{\text {true }}\right)^{2}+\mu_{i 3}\left(\mathrm{X}^{\text {true }}\right)+\mu_{i 4},
\end{aligned}
$$

so a total of 36 parameters is used in the parameterization of $\mathrm{R}$.

Figures B.1 and B.2 show the distributions of $\mathrm{X}^{\text {meas }}$ for the samples ranging from $\frac{2}{3}$ to 1 in $\mathrm{X}_{3}$ and from $\frac{1}{2}$ to 1 in $\mathrm{X}_{4}$ at the nominal values of $\mathrm{X}^{\text {true }}$. Also shown in the plots are the fits of the parameterized detector response to the histograms.

The 36 parameters $\left(\mathrm{n}_{i j}, \sigma_{i j}\right.$, and $\left.\mu_{i j}\right)$ are found by a simultaneous fit of $\mathrm{R}$ to the sample $\mathrm{X}^{\text {meas }}$ distributions. The $\chi_{1}^{2}$ to be minimized is given by:

$$
\chi_{1}^{2} \equiv \sum_{j=1}^{\mathrm{N}} \frac{\left(\mathrm{R}_{j}-\eta_{j}\right)^{2}}{\nu_{j}}
$$



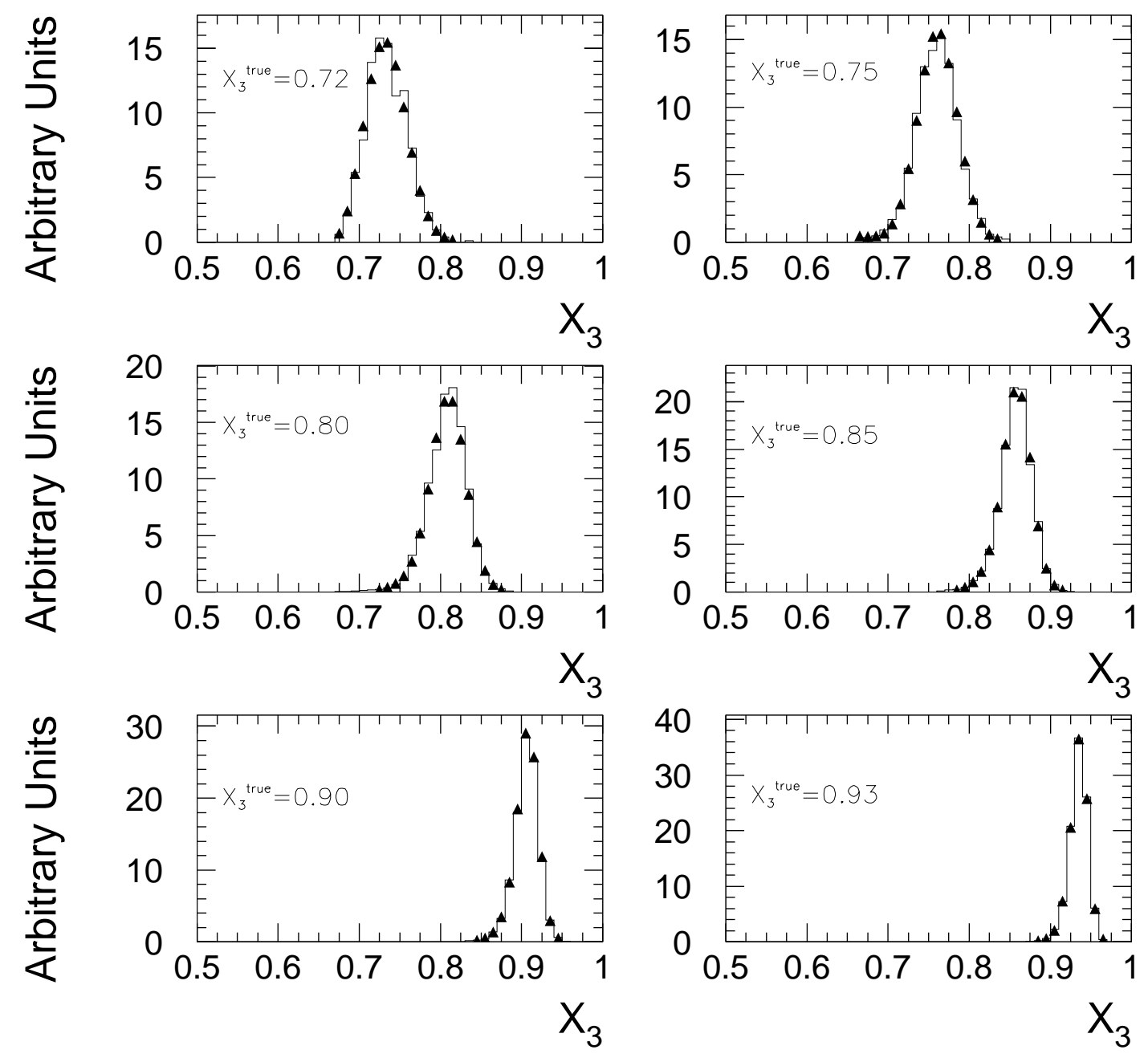

Figure B.1: The measured $\mathrm{X}_{3}$ distributions for $\mathrm{X}_{j}^{\text {true }}=0.72,0.80,0.85,0.90$, and 0.93 , and the detector response function, $\mathrm{R}$. 

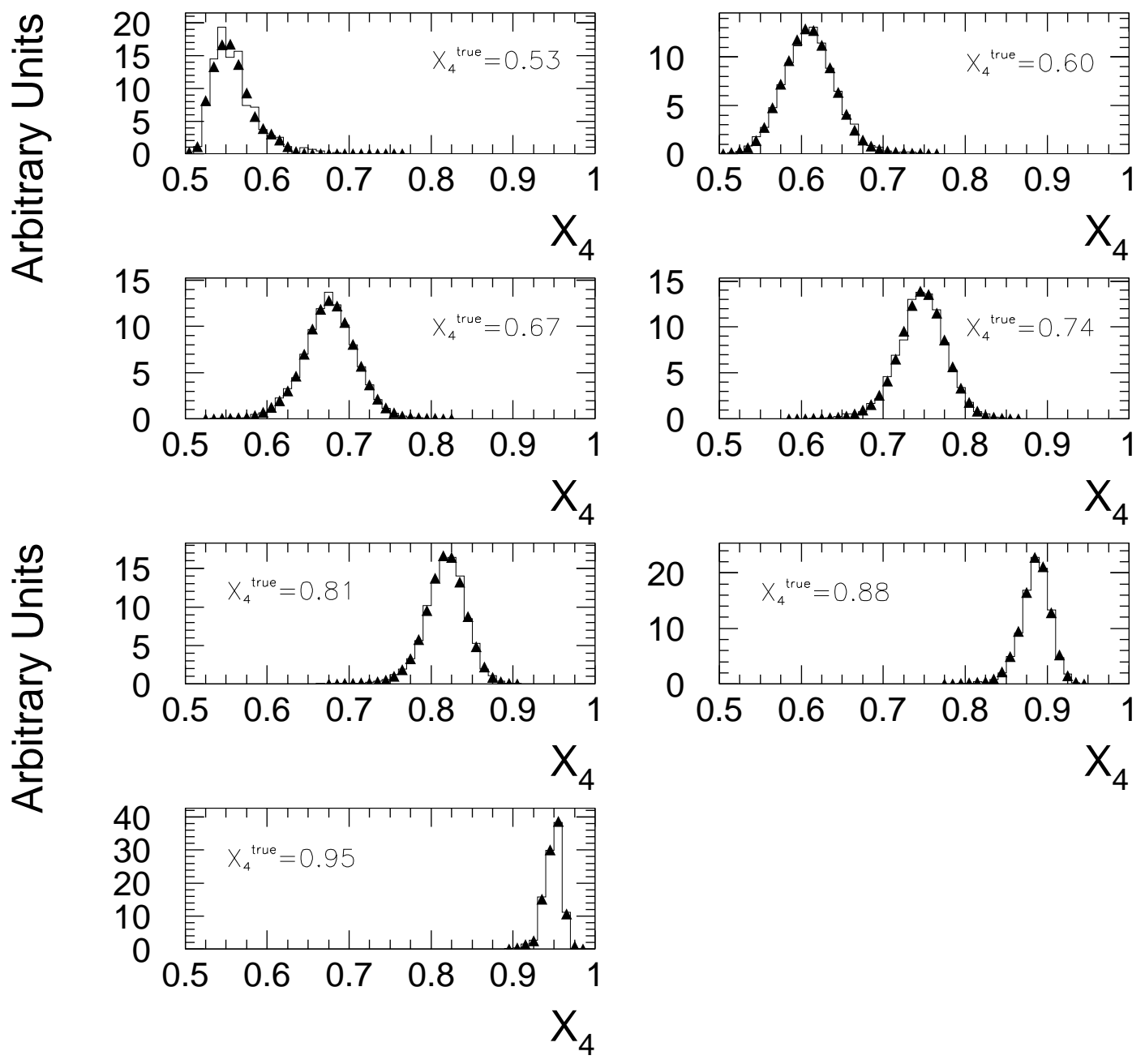

Figure B.2: The measured $\mathrm{X}_{4}$ distributions for $\mathrm{X}_{j}^{\text {true }}=0.53,0.60,0.67,0.74,0.81$, 0.88 , and 0.95 , and the detector response function, $\mathrm{R}$. 
where $j$ indexes the number of samples with different $\mathrm{X}^{\text {true }}$ (in our case $\mathrm{N}=6$ for $\mathrm{X}_{3}$ and 7 for $\left.\mathrm{X}_{4}\right), \mathrm{R}_{j}$ is $\mathrm{R}$ for a given value of $\mathrm{X}_{j}^{\mathrm{true}}, \eta_{j}$ is the smeared distribution of $\mathrm{X}_{j}^{\mathrm{true}}$, and $\nu_{j}$ the number of events generated at $\mathrm{X}_{j}^{\text {true }} \cdot \chi_{1}^{2}=1.2$ and 2.0 for the fits to the measured $\mathrm{X}_{3}$ and $\mathrm{X}_{4}$ distributions, respectively. The higher $\chi_{1}^{2}$ for $\mathrm{X}_{4}$ is solely due to the shape of the response function $\mathrm{X}_{j}^{\text {true }}=0.53$, close to the limit of the kinematically allowed region. At this limit the response function is not a simple analytic function that could be parameterized.

\section{Parameterization of the $\mathrm{X}^{\text {true }}$ Spectrum}

The $\mathrm{X}^{\text {true }}$ spectrum is parameterized by a function that minimizes the $\chi_{4}^{2}$ between $\mathrm{T}$ and the true spectrum histogram. Several trial functions for $\mathrm{T}$ were examined. To parameterize $\mathrm{X}_{3}$ and $\mathrm{X}_{4}$, the true distributions are divided into several regions. The parameterizations in these regions are polynomials of varying degrees:

$$
\begin{gathered}
\mathrm{T}_{\mathrm{X}_{3}} \equiv \begin{cases}\mathrm{Z}_{1}\left(\mathrm{X}^{\text {true }}\right)^{3}+\mathrm{Z}_{2}\left(\mathrm{X}^{\text {true }}\right)^{2}+\mathrm{Z}_{3}\left(\mathrm{X}^{\text {true }}\right)+\mathrm{Z}_{4} & \text { if } \frac{2}{3} \leq \mathrm{X}_{3} \leq 0.84 \\
\mathrm{Z}_{5}\left(\mathrm{X}^{\text {true }}\right)^{2}+\mathrm{Z}_{6}\left(\mathrm{X}^{\text {true }}\right)+\mathrm{Z}_{7} & \text { if } 0.84<\mathrm{X}_{3} \leq 0.94, \text { and }(\mathrm{B} .8) \\
\mathrm{Z}_{8}\left(\mathrm{X}^{\text {true }}\right)^{2}+\mathrm{Z}_{9}\left(\mathrm{X}^{\text {true }}\right)+\mathrm{Z}_{10} & \text { if } 0.94<\mathrm{X}_{3} \leq 1\end{cases} \\
\mathrm{T}_{\mathrm{X}_{4}} \equiv \begin{cases}\mathrm{Z}_{1}\left(\mathrm{X}^{\text {true }}\right)^{2}+\mathrm{Z}_{2}\left(\mathrm{X}^{\text {true }}\right)+\mathrm{Z}_{3} & \text { if } 0.50 \leq \mathrm{X}_{4} \leq 0.56 \\
\mathrm{Z}_{4}\left(\mathrm{X}^{\text {true }}\right)^{2}+\mathrm{Z}_{5}\left(\mathrm{X}^{\text {true }}\right)+\mathrm{Z}_{6} & \text { if } 0.68<\mathrm{X}_{4} \leq 0.92 \\
\mathrm{Z}_{7}\left(\mathrm{X}^{\text {true }}\right)^{4}+\mathrm{Z}_{8}\left(\mathrm{X}^{\text {true }}\right)^{3}+\mathrm{Z}_{9}\left(\mathrm{X}^{\text {true }}\right)^{2}+\mathrm{Z}_{10}\left(\mathrm{X}^{\text {true }}\right)+\mathrm{Z}_{11} \\
\mathrm{Z}_{12}\left(\mathrm{X}^{\text {true }}\right)^{3}+\mathrm{Z}_{13}\left(\mathrm{X}^{\text {true }}\right)^{2}+\mathrm{Z}_{14}\left(\mathrm{X}^{\text {true }}\right)+\mathrm{Z}_{15} & \text { if } 0.92 \leq \mathrm{X}_{4} \leq 1\end{cases}
\end{gathered}
$$

The functions $\mathrm{T}_{\mathrm{X}_{3}}$ and $\mathrm{T}_{\mathrm{X}_{4}}$ are the parameterizations for the true $\mathrm{X}_{3}$ and $\mathrm{X}_{4}$ distributions, respectively, and the $Z_{i}$ are the parameters $\mathrm{Z}$ in these cases. The $\mathrm{X}_{3}$ distribution can be described by 10 parameters, the $\mathrm{X}_{4}$ by 15 . Figures B.3 and B.4 show the true spectra for $\mathrm{X}_{3}$ and $\mathrm{X}_{4}$ and the functions $\mathrm{T}_{\mathrm{X}_{3}}$ and $\mathrm{T}_{\mathrm{X}_{4}}$ that parameterize the distributions. 


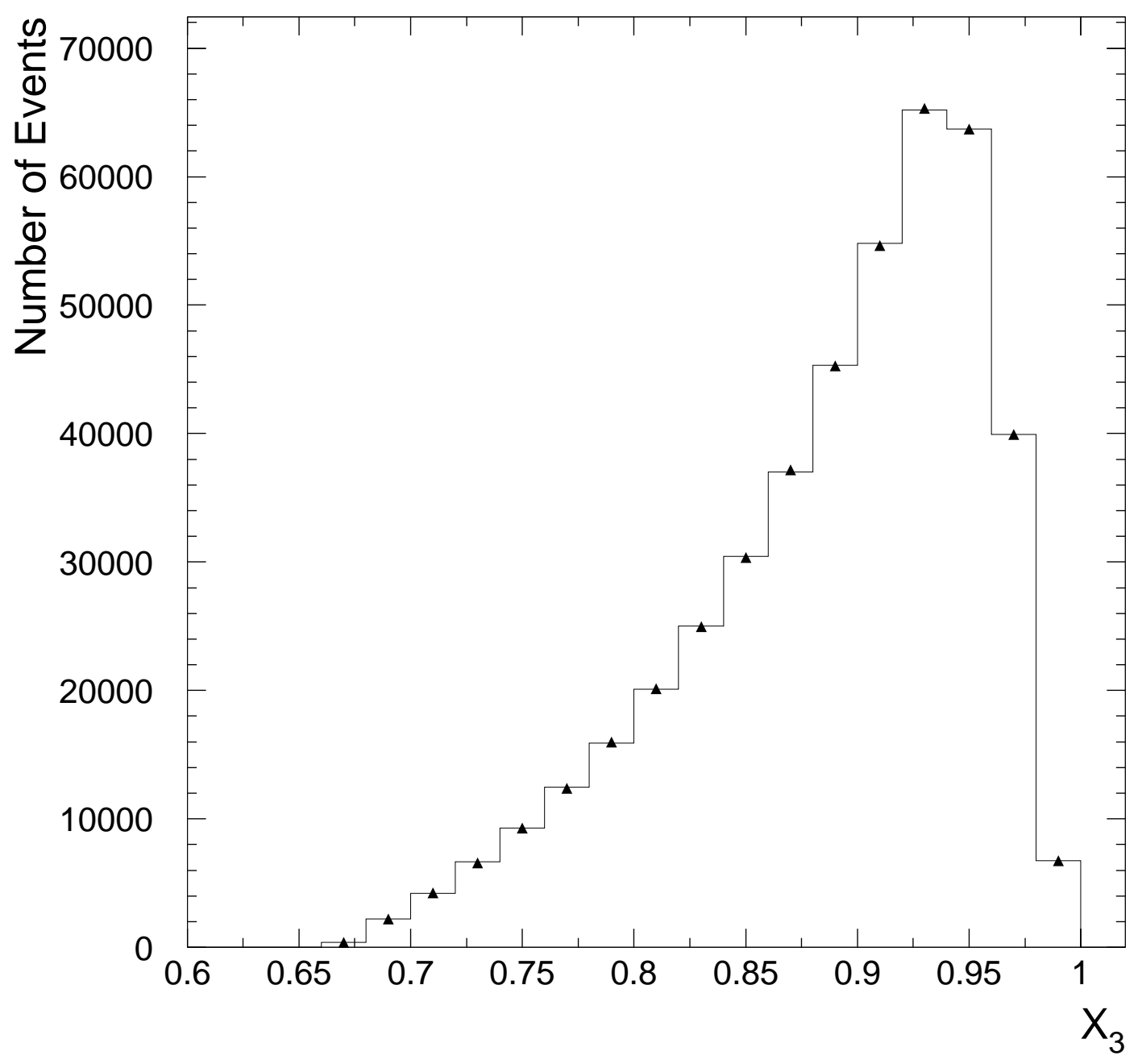

Figure B.3: The $\mathrm{X}_{3}^{\text {true }}$ distribution and $\mathrm{T}$, which is parameterized by polynomials of varying degrees. 


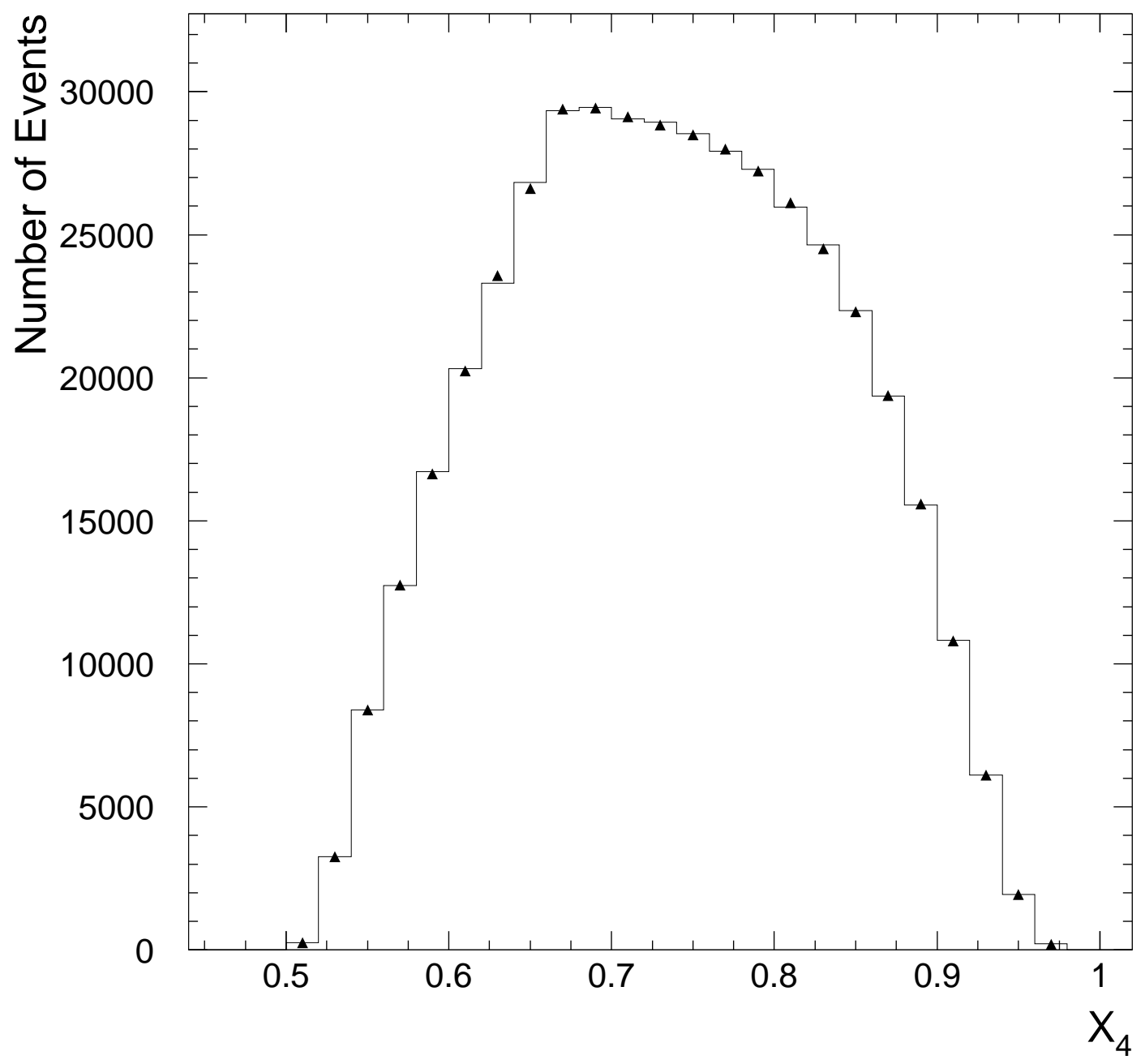

Figure B.4: The $\mathrm{X}_{4}^{\text {true }}$ distribution and $\mathrm{T}$, which is parameterized by polynomials of varying degrees. 


\section{The Unsmearing Factor}

To obtain the unsmearing factor, $\mathrm{K}$, the parameters $\mathrm{Z}$ in the parameterization of the true spectrum have to be determined, and the ratio of the (smeared) measured distribution over the true distribution has to be computed for each bin in X.

The parameters in $\mathrm{T}$ are found by minimizing the $\chi_{2}^{2}$ between the $\mathrm{O}^{\text {bin }}$ integral (Equation B.2) and the data X spectrum:

$$
\chi_{2}^{2} \equiv \sum_{\text {bin }} \frac{\left(\mathrm{O}^{\text {bin }}-o^{\text {bin }}\right)^{2}}{o^{\text {bin }}},
$$

where $o^{\text {bin }}$ is the number of events in the data spectrum in the specific bin.

In principle, $\mathrm{O}$ is given by Equation B.1. When it is computed, the integration limits for integral "C" change to the kinematically allowed region for the variable $\mathrm{X}$. The integral that has to be fitted to the data $\mathrm{X}$ spectrum then assumes the form:

$$
\mathrm{O}^{\text {bin }}=\frac{1}{\text { bin width }} \int^{\text {bin }} \int_{\mathrm{X}_{\text {lower }}}^{\mathrm{X}_{\text {upper }}} \mathrm{R}\left(\mathrm{X}^{\text {meas }}, \mathrm{X}^{\text {true }}\right) \mathrm{T}\left(\mathrm{X}^{\text {true }}, \mathrm{Z}\right) \mathrm{dX^{ \text {true } }} \mathrm{dX}^{\text {meas }} \text {, }
$$

where $\mathrm{X}_{\text {lower }}$ and $\mathrm{X}_{\text {upper }}$ are the lower and the upper bounds of the kinematically allowed region for X. Figures B.5 and B.6 show the data spectra for $\mathrm{X}_{3}$ and $\mathrm{X}_{4}$, respectively, and the convolution integral, $\mathrm{O}$, fitted to the histograms. $\chi_{2}^{2}=1.6$ and 1.4 for $\mathrm{X}_{3}$ and $\mathrm{X}_{4}$, respectively, in our analysis.

The smearing correction factor, $\mathrm{K}^{\text {bin }}$, in each bin of the measured spectrum is found by computing $\frac{\mathrm{O}^{\text {bin }}}{\mathrm{T}^{\text {bin }}}$.

\section{Results of the Analytical Unsmearing Procedure}

The results of the analytical unsmearing procedure are obtained by taking the ratio between the predicted smeared and the true X spectra. The unsmearing factor, $\mathrm{K}$, calculated by this method is shown in Figures B.7 and B. 8 for $\mathrm{X}_{3}$ and $\mathrm{X}_{4}$, respectively. The error on $\mathrm{K}$ is estimated by computing the fractional difference between the true and the unsmeared measured HERWIG X distributions. 


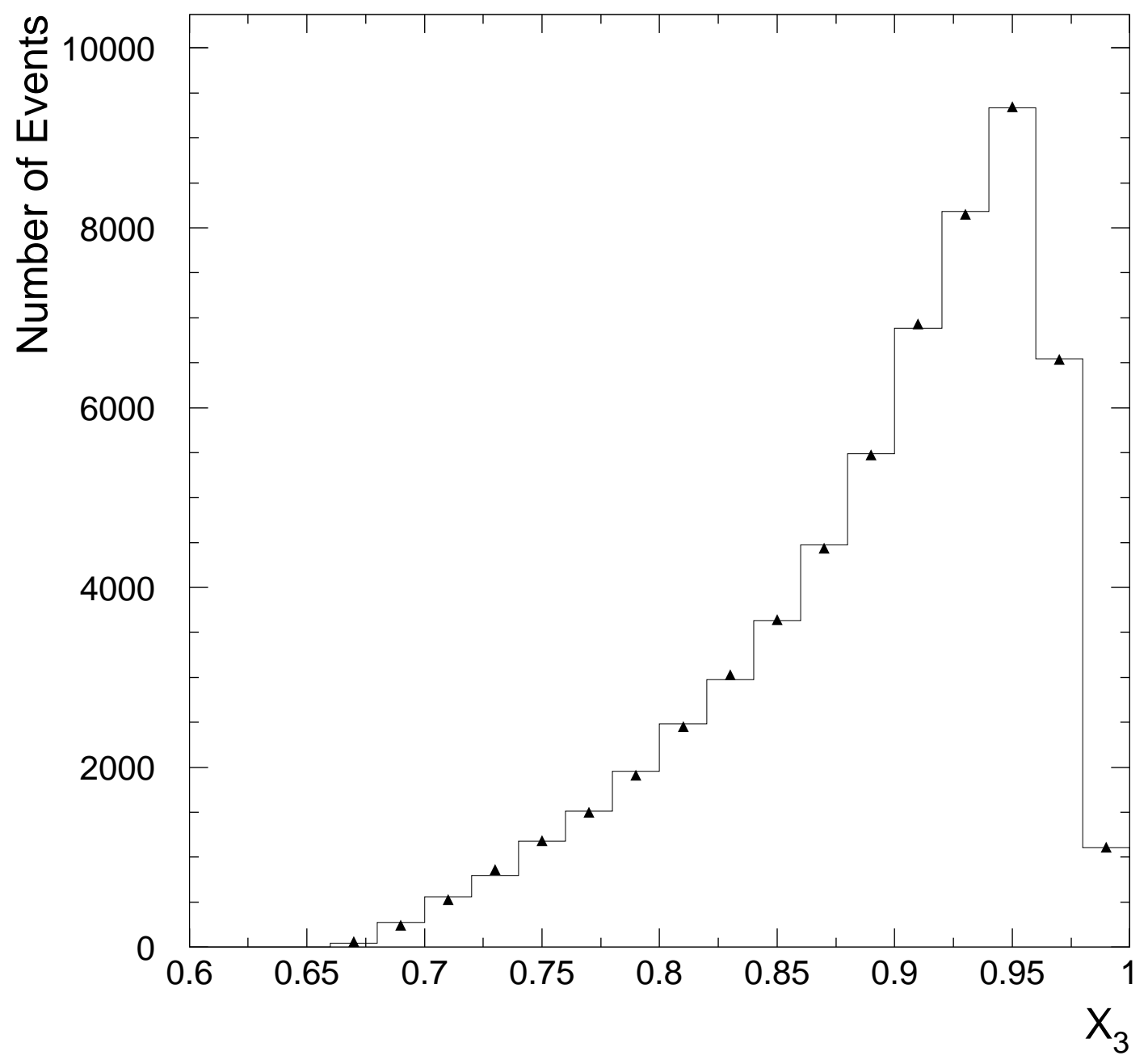

Figure B.5: The $\mathrm{X}_{3}$ data distribution and the convolution integral, $\mathrm{O}$. 


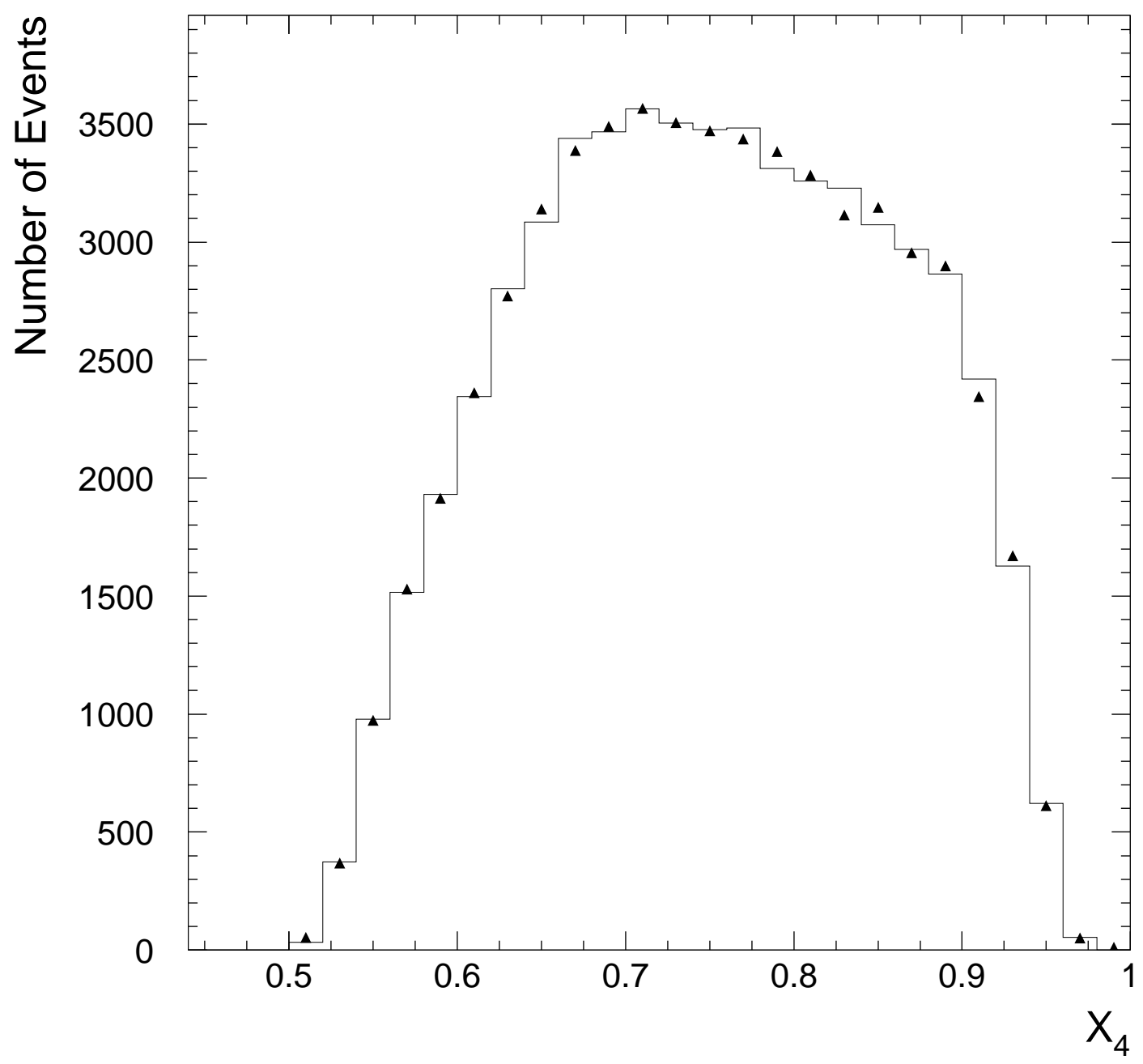

Figure B.6: The $\mathrm{X}_{4}$ data distribution and the convolution integral, O. 


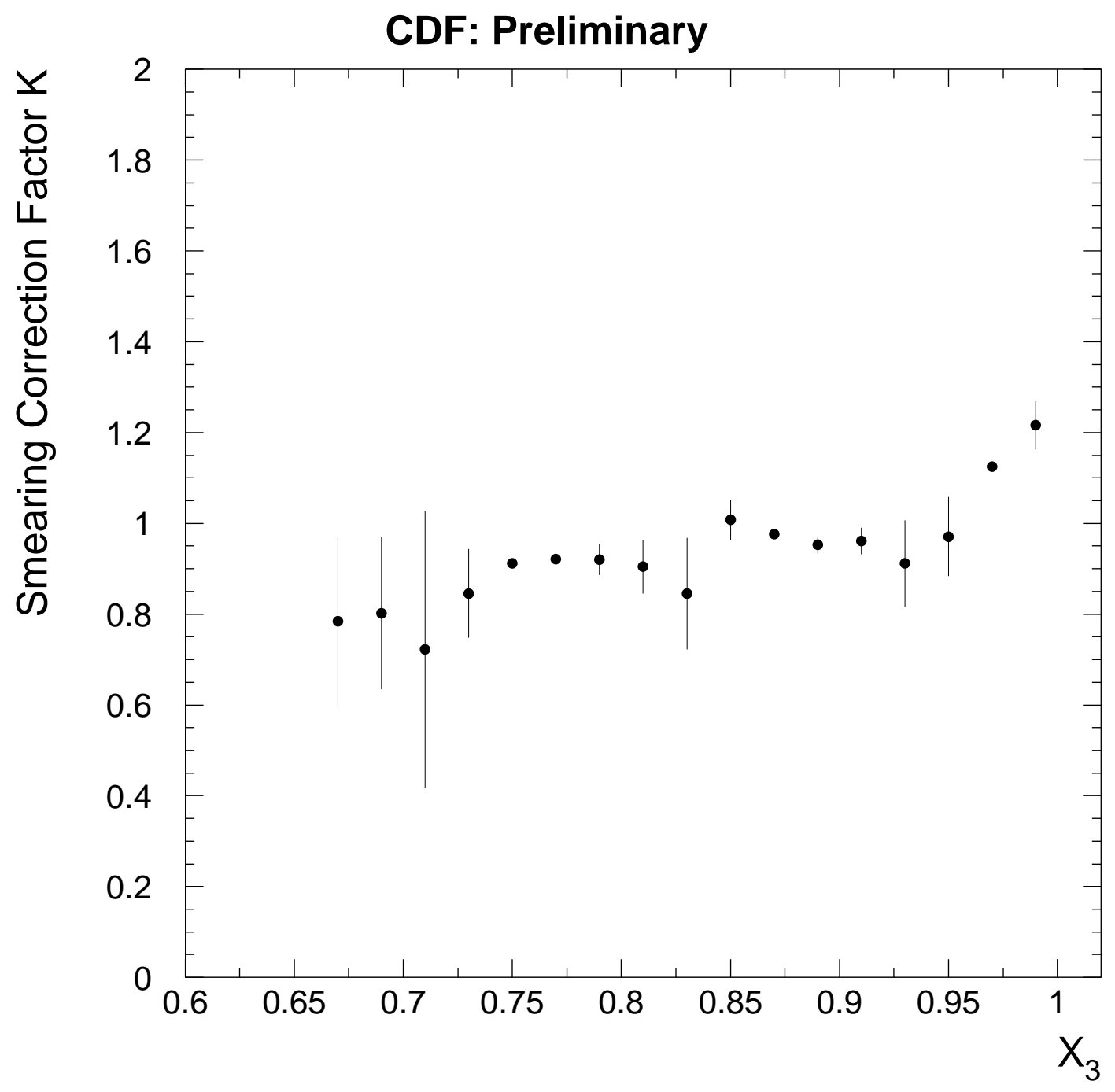

Figure B.7: The smearing correction factor, $\mathrm{K}$, for bins of size 0.02 in $\mathrm{X}_{3}$, as calculated with the analytical approach. 


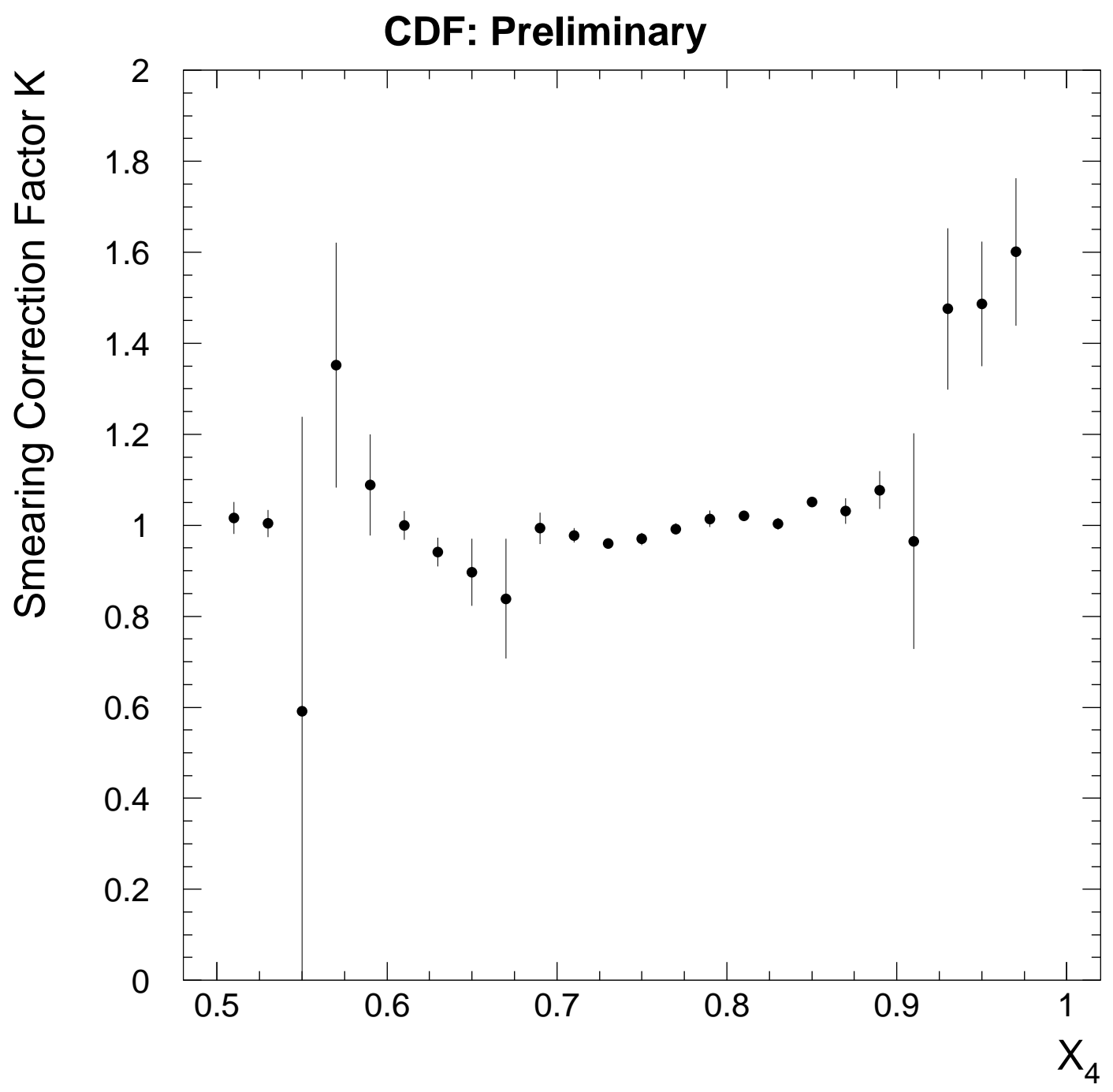

Figure B.8: The smearing correction factor, $\mathrm{K}$, for bins of size 0.02 in $\mathrm{X}_{4}$, as calculated with the analytical approach. 
The smearing correction factors for the first and the last two bins in both distributions are obtained by extrapolation. The parameterization of $\mathrm{R}$ does not preserve the normalization to unity in these bins, as R extends beyond the kinematically allowed region. Thus, K computed by the analytical method in these bins is corrected by extrapolation from bins where $\mathrm{R}$ fully preserves the normalization.

The procedure to unsmear data for detector resolution effects, provided the variables under investigation are functions of jet energies, is completed. The correction factors by which the CDF data have to be adjusted are found to be of the order of a few percent. The unsmearing factor is less than 1.00 in regions where $\mathrm{X}_{3}$ or $\mathrm{X}_{4}$ are low, and it is greater than 1.00 at high $\mathrm{X}_{3}$ or $\mathrm{X}_{4}$.

\section{Internal Consistency Check}

To check the internal consistency of the analytical unsmearing procedure, we apply the correction factors, $\mathrm{K}^{\text {bin }}$, to the measured spectrum of an independent sample of Monte Carlo events for which we know both the true and the measured spectrum. We expect the corrected spectrum to match the true Monte Carlo spectrum perfectly.

Figures B.9 and B.10 show the fractional differences between the unsmeared measured and the true $\mathrm{X}_{3}$ and $\mathrm{X}_{4}$ spectra, respectively. The analytical unsmearing procedure is not internally consistent. Deviations from 0 in these plots that show systematic behavior are due mainly to the large systematic uncertainty associated with this analytic unsmearing procedure which are caused by our choice of parameterization. It was not possible to find single continuous analytic functions that describe the true $\mathrm{X}_{3}$ and $\mathrm{X}_{4}$ spectra. Instead, piecewise-continuous functions are used to parameterize them. During the computation of the convolution integrals, the functions calculated by the fitting routine do not exactly match at the boundaries of the intervals for the piecewise-continuous functions. The smearing correction factors become discontinuous at these boundaries (see Figures B.7 and B.8), and large systematic 
uncertainties have to be assigned to their numerical values. As a consequence, the fractional differences between the measured unsmeared and the true spectra in Figures B.9 and B.10 appear to have systematic distributions about 0 . The $\mathrm{X}_{3}$ and $\mathrm{X}_{4}$ distributions studied in this analysis do not allow continuous parameterizations. 


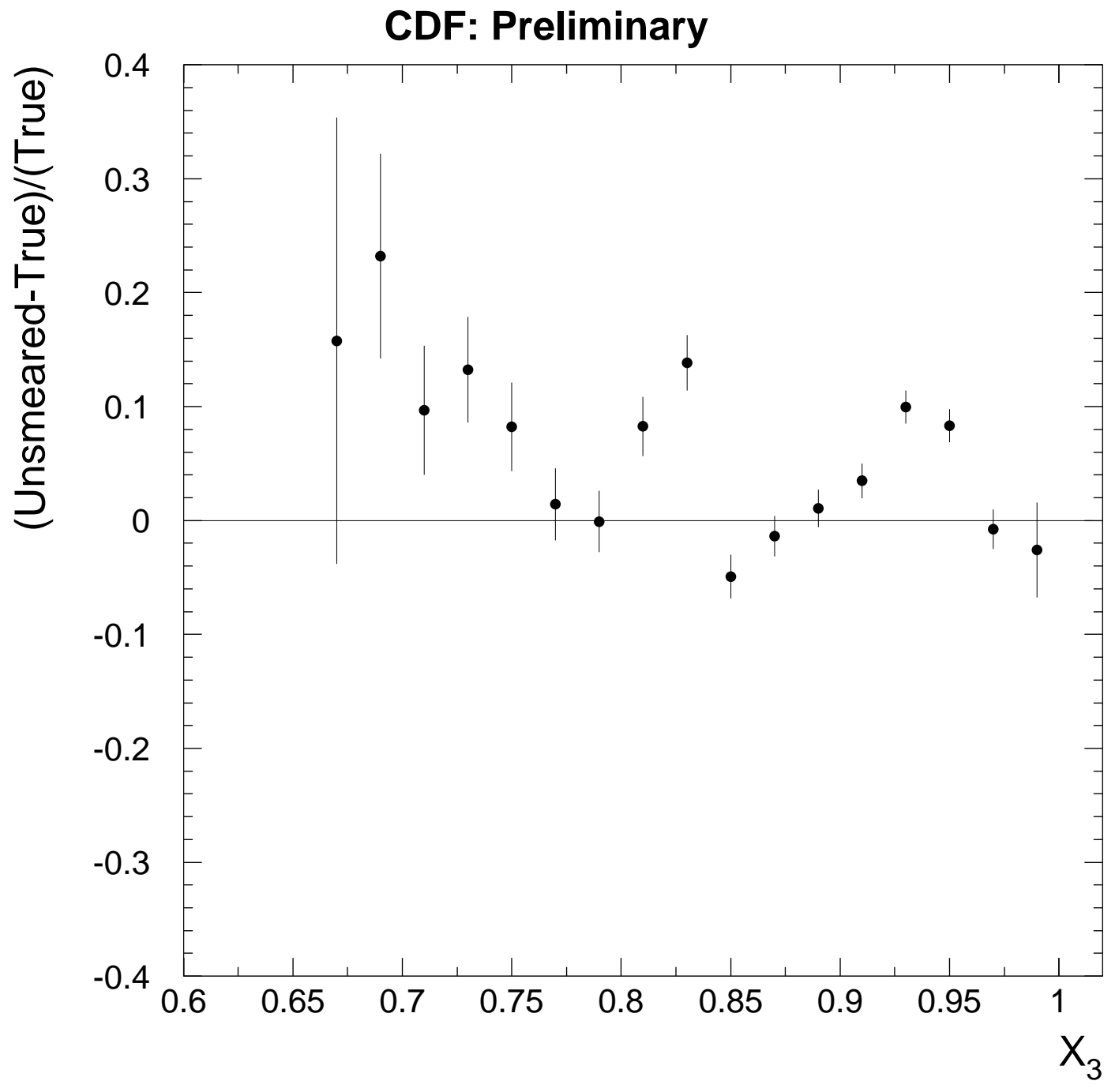

Figure B.9: The fractional difference between the number of Monte Carlo events (which have been generated by HERWIG, propagated through QFL, then unsmeared) and their true number, as calculated with the analytical approach, versus $\mathrm{X}_{3}$. 


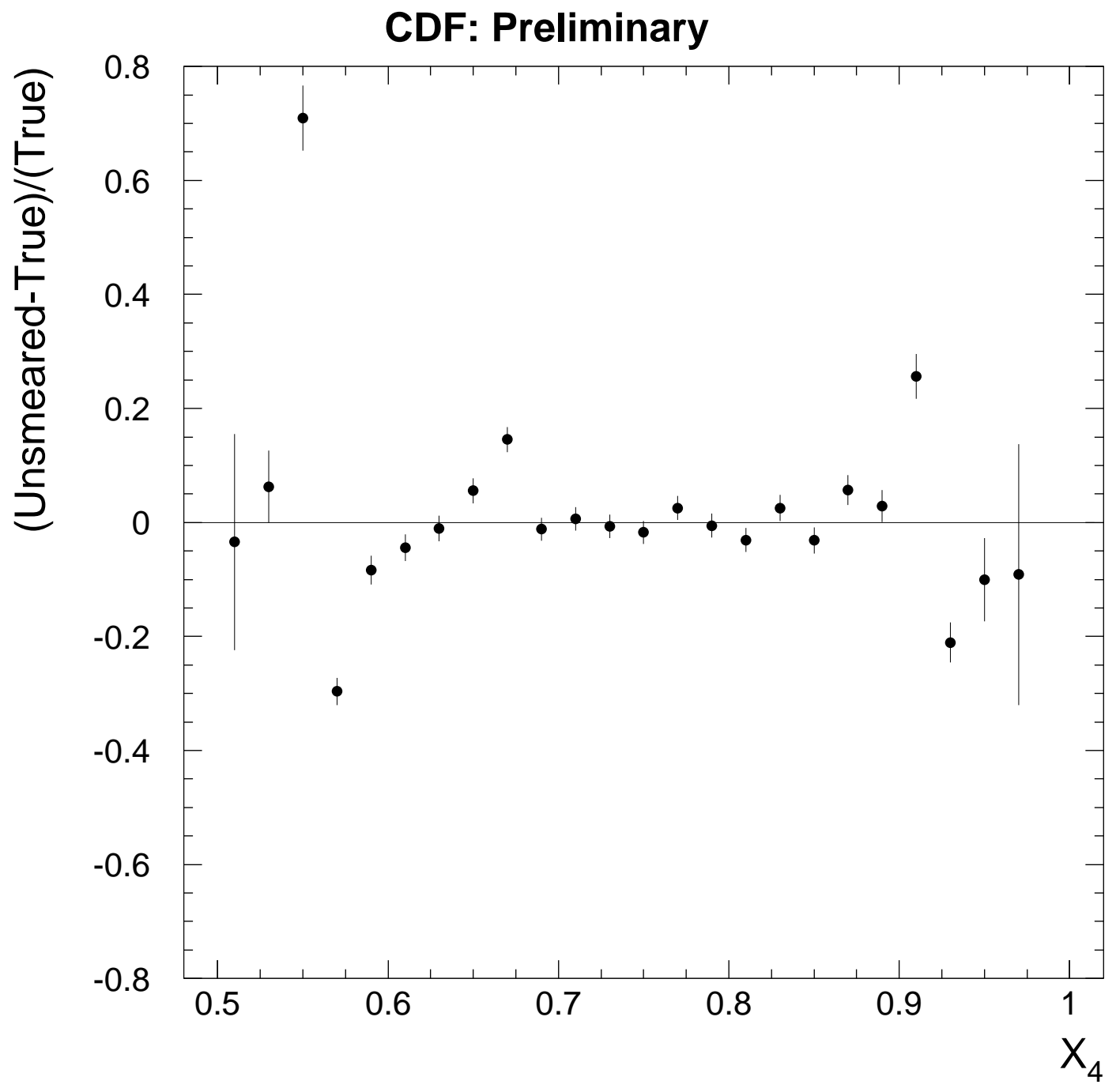

Figure B.10: The fractional difference between the number of Monte Carlo events (which have been generated by HERWIG, propagated through QFL, then unsmeared) and their true number, as calculated with the analytical approach, versus $\mathrm{X}_{4}$. 


\section{Appendix C}

\section{Summary of the $\mathrm{E}_{\mathrm{T}}$ Smearing Correction Factors}

In Chapter 4, the smearing correction factor for the $\mathrm{E}_{\mathrm{T}}$ distributions of the three leading jets in the analysis are discussed. For complete information on the smearing correction factors which are obtained by the Monte Carlo method, they are tabulated in this appendix.

\begin{tabular}{|c|c|c|}
\hline $\mathrm{E}_{\mathrm{T}}(\mathrm{GeV})$ & Unsmearing Correction Factor & Uncertainty \\
\hline \hline 25 & 1.48 & 0.00534 \\
35 & 1.01 & 0.00323 \\
45 & 0.99 & 0.00323 \\
55 & 0.98 & 0.00337 \\
65 & 0.99 & 0.00362 \\
75 & 0.99 & 0.00384 \\
85 & 1.05 & 0.00425 \\
95 & 1.09 & 0.00445 \\
105 & 1.18 & 0.00473 \\
115 & 1.21 & 0.00458 \\
125 & 1.16 & 0.00399 \\
135 & 1.10 & 0.00333 \\
145 & 1.01 & 0.00274 \\
155 & 0.96 & 0.00244 \\
165 & 0.92 & 0.00230 \\
175 & 0.89 & 0.00232 \\
\hline
\end{tabular}

Table C.1: The unsmearing correction factor for the $\mathrm{E}_{\mathrm{T}}$ distributions and their uncertainties for $20 \mathrm{GeV}<\mathrm{E}_{\mathrm{T}}<180 \mathrm{GeV}$. 


\begin{tabular}{|c|c|c|}
\hline $\mathrm{E}_{\mathrm{T}}(\mathrm{GeV})$ & Unsmearing Correction Factor & Uncertainty \\
\hline \hline 185 & 0.88 & 0.00254 \\
195 & 0.89 & 0.00290 \\
205 & 0.90 & 0.00341 \\
215 & 0.91 & 0.00401 \\
225 & 0.93 & 0.00487 \\
235 & 0.92 & 0.00570 \\
245 & 0.97 & 0.00711 \\
255 & 0.98 & 0.00846 \\
265 & 0.97 & 0.0100 \\
275 & 1.00 & 0.0123 \\
285 & 1.04 & 0.0150 \\
295 & 1.01 & 0.0171 \\
305 & 1.02 & 0.0206 \\
315 & 1.16 & 0.0280 \\
325 & 1.14 & 0.0321 \\
335 & 1.08 & 0.0363 \\
\hline
\end{tabular}

Table C.2: The unsmearing correction factor for the $\mathrm{E}_{\mathrm{T}}$ distributions and their uncertainties for $180 \mathrm{GeV}<\mathrm{E}_{\mathrm{T}}<340 \mathrm{GeV}$.

\begin{tabular}{|c|c|c|}
\hline $\mathrm{E}_{\mathrm{T}}(\mathrm{GeV})$ & Unsmearing Correction Factor & Uncertainty \\
\hline \hline 345 & 1.09 & 0.0432 \\
355 & 1.15 & 0.0527 \\
365 & 1.17 & 0.0653 \\
375 & 1.08 & 0.0713 \\
385 & 1.17 & 0.0926 \\
395 & 1.48 & 0.134 \\
405 & 1.22 & 0.131 \\
415 & 0.88 & 0.115 \\
425 & 0.98 & 0.141 \\
435 & 1.11 & 0.202 \\
445 & 1.51 & 0.297 \\
455 & 1.67 & 0.460 \\
465 & 1.95 & 0.512 \\
475 & 0.95 & 0.304 \\
485 & 3.00 & 1.15 \\
495 & 3.33 & 2.19 \\
\hline
\end{tabular}

Table C.3: The unsmearing correction factor for the $\mathrm{E}_{\mathrm{T}}$ distributions and their uncertainties for $340 \mathrm{GeV}<\mathrm{E}_{\mathrm{T}}<500 \mathrm{GeV}$. 


\section{Appendix D}

\section{Summary of the Systematic Uncertainties on the Dalitz Variables}

In Chapter 5, the study of the systematic uncertainty on the Dalitz variables is discussed. To facilitate extraction of the information provided in Figures 5.1 through 5.12, the total systematic uncertainties and their main contributions in every bin are tabulated in this appendix.

\begin{tabular}{|c|c|c|c|c|c|c|}
\hline $\mathrm{X}_{3}$ & $\begin{array}{c}\text { Det. } \\
\text { Cal. }(\%)\end{array}$ & $\begin{array}{c}\text { Jet } \\
\text { Frag. }(\%)\end{array}$ & $\begin{array}{c}\text { Cal. } \\
\text { Stab. }(\%)\end{array}$ & $\begin{array}{c}\text { Und. } \\
\text { Ev. }(\%)\end{array}$ & $\begin{array}{l}\text { Rel. En. } \\
\text { Sc. }(\%)\end{array}$ & Total (\%) \\
\hline$\overline{0.96-0.98}$ & +28.4 & +18.3 & +14.0 & +51.2 & +25.5 & +68.9 \\
\hline $0.98-1.00$ & $+20.5-20.0$ & $+11.9-20.0$ & +10.0 & +50.0 & $+36.1 \quad-24.5$ & $+67.9-71.8$ \\
\hline
\end{tabular}

Table D.1: The total systematic uncertainty, and the main contributions, on the Dalitz variables for $0.96<\mathrm{X}_{4} \leq 0.98$.

\begin{tabular}{|c|c|c|c|c|c|c|c|c|c|c|c|c|}
\hline $\mathrm{X}_{3}$ & \multicolumn{2}{|c|}{$\begin{array}{c}\text { Det. } \\
\text { Cal. (\%) }\end{array}$} & \multicolumn{2}{|c|}{$\begin{array}{c}\text { Jet } \\
\text { Frag. }(\%)\end{array}$} & \multicolumn{2}{|c|}{$\begin{array}{c}\text { Cal. } \\
\text { Stab. }(\%)\end{array}$} & \multicolumn{2}{|c|}{$\begin{array}{c}\text { Und. } \\
\text { Ev. (\%) }\end{array}$} & \multicolumn{2}{|c|}{$\begin{array}{l}\text { Rel. En. } \\
\text { Sc. (\%) }\end{array}$} & \multicolumn{2}{|c|}{ Total (\%) } \\
\hline$\overline{0.94-0.96}$ & $\begin{array}{l}+18.5 \\
\end{array}$ & -3.8 & +17.3 & $\overline{-4.6}$ & $\begin{array}{l}+10.7 \\
\end{array}$ & 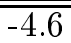 & +29.1 & $\begin{array}{l}-16.1 \\
-16\end{array}$ & $\overline{c+11.6}$ & $\overline{-6.1}$ & +41.9 & $\overline{-19.2}$ \\
\hline $0.96-0.98$ & +22.5 & -12.5 & $\begin{array}{r}+16.9 \\
\end{array}$ & -12.5 & +13.1 & -7.1 & +39.1 & -25.6 & +9.9 & -11.9 & +51.1 & -34.2 \\
\hline $0.98-1.00$ & +15.7 & -13.9 & +8.8 & -12.5 & +6.9 & -8.3 & +38.9 & -30.6 & +10.9 & -11.3 & +45.0 & -38.7 \\
\hline
\end{tabular}

Table D.2: The total systematic uncertainty, and the main contributions, on the Dalitz variables for $0.94<\mathrm{X}_{4} \leq 0.96$. 


\begin{tabular}{|c|c|c|c|c|c|c|c|c|c|c|c|c|}
\hline $\mathrm{X}_{3}$ & \multicolumn{2}{|c|}{$\begin{array}{c}\text { Det. } \\
\text { Cal. (\%) }\end{array}$} & \multicolumn{2}{|c|}{$\begin{array}{c}\text { Jet } \\
\text { Frag. }(\%)\end{array}$} & \multicolumn{2}{|c|}{$\begin{array}{c}\text { Cal. } \\
\text { Stab. }(\%)\end{array}$} & \multicolumn{2}{|c|}{$\begin{array}{c}\text { Und. } \\
\text { Ev. }(\%)\end{array}$} & \multicolumn{2}{|c|}{$\begin{array}{l}\text { Rel. En. } \\
\text { Sc. (\%) }\end{array}$} & \multicolumn{2}{|c|}{ Total (\%) } \\
\hline $0.92-0.94$ & +15.8 & -4.6 & +14.3 & -7.0 & +11.7 & -2.7 & +17.1 & -7.7 & +11.0 & -6.6 & +31.8 & -13.7 \\
\hline $0.94-0.96$ & +16.0 & -5.8 & +15.8 & -6.0 & +10.5 & -3.6 & +17.3 & -11.9 & +8.0 & -2.5 & +31.4 & -15.3 \\
\hline $0.96-0.98$ & +14.4 & -9.3 & +11.1 & -9.5 & +7.9 & -6.6 & +23.6 & -17.8 & +7.9 & -5.9 & +32.0 & -24.0 \\
\hline $0.98-1.00$ & +18.8 & -9.9 & +11.7 & -11.3 & +9.9 & -9.2 & +27.5 & -15.5 & +7.6 & -13.5 & +37.5 & -27.1 \\
\hline
\end{tabular}

Table D.3: The total systematic uncertainty, and the main contributions, on the Dalitz variables for $0.92<\mathrm{X}_{4} \leq 0.94$.

\begin{tabular}{|c|c|c|c|c|c|c|c|c|c|c|c|c|}
\hline $\mathrm{X}_{3}$ & \multicolumn{2}{|c|}{$\begin{array}{c}\text { Det. } \\
\text { Cal. }(\%) \\
\end{array}$} & \multicolumn{2}{|c|}{$\begin{array}{c}\text { Jet } \\
\text { Frag. }(\%)\end{array}$} & \multicolumn{2}{|c|}{$\begin{array}{c}\text { Cal. } \\
\text { Stab. }(\%) \\
\end{array}$} & \multicolumn{2}{|c|}{$\begin{array}{c}\text { Und. } \\
\text { Ev. }(\%)\end{array}$} & \multicolumn{2}{|c|}{$\begin{array}{l}\text { Rel. En. } \\
\text { Sc. (\%) }\end{array}$} & \multicolumn{2}{|c|}{ Total (\%) } \\
\hline$\overline{0.90-0 .}$ & $\overline{13.4}$ & $\overline{-5.2}$ & $\overline{11.1}$ & $\begin{array}{c}-7.4 \\
\end{array}$ & 8.5 & -4.8 & $\overline{13.7}$ & -7 . & -15.1 & $\begin{array}{l}-3.2 \\
\end{array}$ & .1 & $\overline{13}$ \\
\hline $82-0.94$ & 4.3 & -6. & & -7 & +9.7 & -4 & 14.2 & -6 & -8.0 & -3 & 0 & 13 \\
\hline .96 & 2.3 & -6.2 & 0.8 & -7.5 & +9.4 & -5 & 4.6 & -9 & -8.6 & -4 & +25.5 & -15 \\
\hline 0.98 & 15.6 & -5.9 & 2.6 & -8.4 & +10.2 & -3.9 & -17.7 & -11.8 & +5.2 & -7.9 & +29.1 & -18.1 \\
\hline $0.98-1.00$ & +11.1 & -6.4 & +6.8 & -5.7 & +6.4 & -5.7 & +18.6 & -15.0 & +6.6 & -9.2 & +24.6 & -20.5 \\
\hline
\end{tabular}

Table D.4: The total systematic uncertainty, and the main contributions, on the Dalitz variables for $0.90<\mathrm{X}_{4} \leq 0.92$.

\begin{tabular}{|c|cc|cc|cc|cc|cc|rr|}
\hline $\mathrm{X}_{3}$ & \multicolumn{2}{|c|}{$\begin{array}{c}\text { Det. } \\
\text { Cal. (\%) }\end{array}$} & \multicolumn{2}{c|}{ Jet } & \multicolumn{2}{c|}{ Cal. } & \multicolumn{2}{c|}{ Und. } & \multicolumn{2}{c|}{ Rel. En. } & \multicolumn{2}{c|}{ Total (\%) } \\
& \multicolumn{2}{|c|}{ Stab. (\%) } & \multicolumn{2}{c|}{ Ev. (\%) } & \multicolumn{1}{c|}{ Sc. (\%) } & & \\
\hline \hline $0.88-0.90$ & +18.7 & -2.3 & +19.8 & -0.5 & +12.3 & -4.1 & +16.9 & -2.3 & +21.9 & -9.9 & +40.7 & -11.3 \\
$0.90-0.92$ & +16.3 & -4.8 & +13.6 & -5.0 & +11.9 & -1.1 & +11.7 & -3.6 & +12.3 & -5.6 & +29.7 & -9.8 \\
$0.92-0.94$ & +15.3 & -8.6 & +14.9 & -9.1 & +9.8 & -3.7 & +13.2 & -10.5 & +11.6 & -5.5 & +29.3 & -17.6 \\
$0.94-0.96$ & +18.0 & -8.0 & +16.4 & -7.9 & +10.8 & -3.8 & +13.8 & -7.7 & +11.9 & -3.5 & +32.3 & -14.6 \\
$0.96-0.98$ & +11.5 & -5.8 & +9.8 & -5.0 & +7.1 & -3.7 & +11.7 & -6.5 & +8.1 & -4.9 & +22.0 & -11.9 \\
$0.98-1.00$ & +13.1 & -3.9 & +6.6 & -1.0 & +9.7 & -1.9 & +23.3 & -3.9 & +16.9 & -6.8 & +33.7 & -9.1 \\
\hline
\end{tabular}

Table D.5: The total systematic uncertainty, and the main contributions, on the Dalitz variables for $0.88<\mathrm{X}_{4} \leq 0.90$. 


\begin{tabular}{|c|cc|cc|cc|cc|cr|rr|}
\hline $\mathrm{X}_{3}$ & \multicolumn{2}{|c|}{$\begin{array}{c}\text { Det. } \\
\text { Cal. (\%) }\end{array}$} & \multicolumn{2}{c|}{ Jet } & \multicolumn{2}{c|}{ Cal. } & \multicolumn{2}{c|}{ Und. } & \multicolumn{2}{c|}{ Rel. En. } & \multicolumn{2}{c|}{ Total (\%) } \\
& Frag. (\%) & Stab. (\%) & Ev. (\%) & Sc. (\%) & & \\
\hline \hline $0.86-0.88$ & +20.6 & -3.4 & +16.3 & -4.6 & +14.9 & -1.1 & +16.7 & -4.6 & +18.3 & -6.3 & +39.1 & -9.9 \\
$0.88-0.90$ & +22.7 & -2.0 & +20.2 & -3.7 & +16.2 & -1.0 & +21.6 & -2.2 & +20.3 & -10.2 & +45.5 & -11.4 \\
$0.90-0.92$ & +15.4 & -3.7 & +14.8 & -3.7 & +11.3 & -0.4 & +12.7 & -3.7 & +10.4 & -5.5 & +29.2 & -8.6 \\
$0.92-0.94$ & +22.5 & -4.5 & +18.7 & -5.9 & +14.3 & -2.0 & +18.5 & -4.3 & +16.0 & -5.4 & +40.7 & -10.4 \\
$0.94-0.96$ & +15.0 & -6.5 & +11.5 & -6.5 & +10.5 & -2.8 & +14.1 & -7.8 & +10.4 & -3.0 & +27.8 & -12.7 \\
$0.96-0.98$ & +15.4 & -5.1 & +13.0 & -6.3 & +9.7 & -3.1 & +14.4 & -7.1 & +12.3 & -4.7 & +29.4 & -12.2 \\
$0.98-1.00$ & +31.2 & -5.3 & +28.8 & -2.7 & +18.7 & -1.3 & +40.0 & -2.7 & +27.6 & -6.1 & +67.2 & -9.1 \\
\hline
\end{tabular}

Table D.6: The total systematic uncertainty, and the main contributions, on the Dalitz variables for $0.86<\mathrm{X}_{4} \leq 0.88$.

\begin{tabular}{|c|cc|cc|cc|cc|cr|rr|}
\hline $\mathrm{X}_{3}$ & \multicolumn{2}{|c|}{ Det. } & \multicolumn{2}{c|}{ Jet } & \multicolumn{2}{c|}{ Cal. } & \multicolumn{2}{c|}{ Und. } & \multicolumn{2}{c|}{ Rel. En. } & \multicolumn{2}{c|}{ Total (\%) } \\
& \multicolumn{2}{c|}{ Cal. (\%) } & \multicolumn{2}{c|}{ Frag. (\%) } & Stab. (\%) & Ev. (\%) & \multicolumn{2}{c|}{ Sc. (\%) } & \\
\hline \hline $0.84-0.86$ & +37.5 & -5.1 & +35.6 & -2.9 & +24.6 & -3.6 & +25.4 & -5.8 & +25.0 & -16.0 & +67.5 & -18.4 \\
$0.86-0.88$ & +19.7 & -1.4 & +18.9 & -1.1 & +15.8 & -0.6 & +18.7 & -1.7 & +24.0 & -8.6 & +43.8 & -9.1 \\
$0.88-0.90$ & +18.5 & -6.2 & +14.9 & -6.5 & +12.3 & -1.7 & +13.0 & -4.3 & +13.9 & -4.4 & +32.8 & -11.0 \\
$0.90-0.92$ & +18.8 & -2.6 & +18.9 & -4.8 & +12.9 & -1.0 & +18.1 & -2.1 & +21.1 & -9.6 & +40.6 & -11.3 \\
$0.92-0.94$ & +17.4 & -7.5 & +15.7 & -7.5 & +12.7 & -2.8 & +16.5 & -3.9 & +14.3 & -4.6 & +34.4 & -12.5 \\
$0.94-0.96$ & +16.4 & -4.2 & +15.7 & -5.0 & +9.4 & -3.4 & +13.6 & -4.7 & +12.7 & -7.1 & +30.8 & -11.3 \\
$0.96-0.98$ & +16.5 & -5.8 & +13.7 & -5.8 & +11.5 & -3.2 & +15.6 & -7.9 & +13.8 & -2.9 & +32.0 & -12.1 \\
$0.98-1.00$ & +19.2 & -12.5 & +17.6 & -12.5 & +14.1 & -6.3 & +20.3 & -20.3 & +23.9 & -15.8 & +43.1 & -31.8 \\
\hline
\end{tabular}

Table D.7: The total systematic uncertainty, and the main contributions, on the Dalitz variables for $0.84<\mathrm{X}_{4} \leq 0.86$. 


\begin{tabular}{|c|cc|cc|cc|cc|cc|cc|}
\hline $\mathrm{X}_{3}$ & \multicolumn{2}{|c|}{ Det. } & \multicolumn{2}{c|}{ Jet } & \multicolumn{2}{c|}{ Cal. } & \multicolumn{2}{c|}{ Und. } & \multicolumn{2}{c|}{ Rel. En. } & \multicolumn{2}{c|}{ Total (\%) } \\
& Cal. (\%) & Frag. (\%) & Stab. (\%) & \multicolumn{2}{c|}{ Ev. (\%) } & \multicolumn{2}{c|}{ Sc. (\%) } & & \\
\hline \hline $0.82-0.84$ & +22.1 & -2.7 & +23.7 & -0.7 & +14.9 & -2.0 & +16.2 & -5.4 & +17.9 & -8.8 & +43.0 & -10.8 \\
$0.84-0.86$ & +20.7 & -6.6 & +15.5 & -7.0 & +17.4 & -1.3 & +19.6 & -7.6 & +27.2 & -10.2 & +45.8 & -16.0 \\
$0.86-0.88$ & +24.3 & -4.0 & +23.8 & -6.4 & +19.0 & -3.1 & +17.4 & -2.8 & +25.5 & -14.3 & +49.7 & -16.7 \\
$0.88-0.90$ & +21.6 & -3.6 & +20.7 & -3.6 & +18.9 & -5.6 & +19.8 & -7.5 & +23.5 & -14.8 & +46.9 & -18.2 \\
$0.90-0.92$ & +19.7 & -5.0 & +16.4 & -6.2 & +12.2 & -2.5 & +14.7 & -5.5 & +16.7 & -8.3 & +36.1 & -13.0 \\
$0.92-0.94$ & +21.1 & -7.6 & +20.9 & -7.8 & +15.6 & -4.6 & +17.4 & -6.1 & +14.3 & -4.3 & +40.4 & -14.0 \\
$0.94-0.96$ & +19.3 & -6.9 & +17.0 & -7.1 & +13.8 & -3.5 & +15.6 & -3.1 & +17.0 & -9.0 & +37.2 & -14.2 \\
$0.96-0.98$ & +14.2 & -3.4 & +11.7 & -4.8 & +9.3 & -3.2 & +16.1 & -6.1 & +13.4 & -5.4 & +29.4 & -10.5 \\
$0.98-1.00$ & +13.6 & -1.9 & +9.4 & -0.0 & +11.3 & -0.0 & +15.1 & -9.4 & +26.8 & -4.6 & +36.7 & -10.7 \\
\hline
\end{tabular}

Table D.8: The total systematic uncertainty, and the main contributions, on the Dalitz variables for $0.82<\mathrm{X}_{4} \leq 0.84$.

\begin{tabular}{|c|c|c|c|c|c|c|c|c|c|c|c|c|}
\hline $\mathrm{X}_{3}$ & \multicolumn{2}{|c|}{$\begin{array}{c}\text { Det. } \\
\text { Cal. (\%) }\end{array}$} & \multicolumn{2}{|c|}{$\begin{array}{c}\text { Jet } \\
\text { Frag. }(\%)\end{array}$} & \multicolumn{2}{|c|}{$\begin{array}{c}\text { Cal. } \\
\text { Stab. }(\%)\end{array}$} & \multicolumn{2}{|c|}{$\begin{array}{c}\text { Und. } \\
\text { Ev. }(\%)\end{array}$} & \multicolumn{2}{|c|}{$\begin{array}{l}\text { Rel. En. } \\
\text { Sc. (\%) }\end{array}$} & \multicolumn{2}{|c|}{ Total (\%) } \\
\hline$\overline{0.80-0.82}$ & 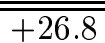 & 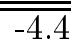 & " & 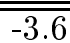 & 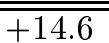 & 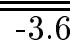 & 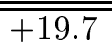 & $\overline{-\overline{-3.6}}$ & " +23.3 & "-12.9 & ("+50.4 & $\overline{-15.0}$ \\
\hline $0.82-0.84$ & +24.0 & -0.0 & +18.4 & -1.8 & +17.1 & -1.1 & -17.1 & -1.1 & +22.5 & -10.7 & +44.8 & -11.0 \\
\hline $0.84-0.86$ & +19.1 & -1.0 & +18.9 & -3.0 & +14.6 & -2.3 & +21.9 & -2.3 & +15.1 & -11.9 & +40.6 & -12.8 \\
\hline $0.86-0.88$ & +22.4 & -0.9 & +21.5 & -1.9 & +15.7 & -2.8 & +20.1 & -0.9 & +18.2 & -6.8 & +44.1 & -7.8 \\
\hline $0.88-0.90$ & +20.8 & -2.9 & +18.5 & -2.2 & +12.7 & -1.6 & +16.2 & -2.5 & +20.1 & -9.3 & +40.1 & -10.4 \\
\hline $0.90-0.92$ & +22.8 & -0.3 & +21.9 & -1.1 & +17.0 & -2.1 & +19.9 & -1.9 & +21.9 & -11.0 & +46.5 & -11.4 \\
\hline $0.92-0.94$ & +20.2 & -3.7 & +18.0 & -4.8 & +14.7 & -1.1 & +17.9 & -0.8 & +19.0 & -10.7 & +40.3 & -12.4 \\
\hline $0.94-0.96$ & +18.1 & -7.0 & +15.3 & -7.5 & +11.8 & -3.2 & +15.4 & -6.8 & +15.9 & -4.1 & +34.6 & -13.3 \\
\hline $0.96-0.98$ & +19.8 & -0.9 & +16.4 & -0.6 & +14.4 & -0.3 & +19.3 & -0.0 & +19.6 & -6.1 & +40.3 & -6.3 \\
\hline $0.98-1.00$ & +19.1 & -5.1 & +17.2 & -8.5 & +11.9 & -0.0 & +18.6 & -10.2 & +11.4 & -13.8 & +35.8 & -19.8 \\
\hline
\end{tabular}

Table D.9: The total systematic uncertainty, and the main contributions, on the Dalitz variables for $0.80<\mathrm{X}_{4} \leq 0.82$. 


\begin{tabular}{|c|cc|cc|cc|cc|cr|rr|}
\hline $\mathrm{X}_{3}$ & \multicolumn{2}{|c|}{ Det. } & \multicolumn{2}{c|}{ Jet } & \multicolumn{2}{c|}{ Cal. } & \multicolumn{2}{c|}{ Und. } & \multicolumn{2}{c|}{$\begin{array}{c}\text { Rel. En. } \\
\text { Ca. }\end{array}$} & \multicolumn{2}{c|}{ Total (\%) } \\
& Cal. (\%) & Frag. (\%) & Stab. (\%) & Ev. (\%) & Sc. (\%) & \\
\hline \hline $0.78-0.80$ & +13.4 & -7.3 & +15.8 & -9.8 & +13.0 & -4.1 & +9.8 & -1.6 & +11.8 & -9.8 & +28.8 & -16.3 \\
$0.80-0.82$ & +24.9 & -5.0 & +24.6 & -2.7 & +16.8 & -5.0 & +18.7 & -2.3 & +31.0 & -16.8 & +53.1 & -18.5 \\
$0.82-0.84$ & +17.7 & -4.9 & +17.6 & -4.5 & +10.7 & -0.0 & +13.2 & -1.2 & +15.3 & -12.2 & +33.9 & -14.0 \\
$0.84-0.86$ & +21.8 & -1.3 & +23.8 & -0.8 & +18.8 & -4.2 & +20.9 & -2.5 & +25.2 & -10.6 & +49.7 & -11.8 \\
$0.86-0.88$ & +23.5 & -0.7 & +21.6 & -3.8 & +18.9 & -2.7 & +21.6 & -1.4 & +25.2 & -10.8 & +49.8 & -11.9 \\
$0.88-0.90$ & +20.3 & -6.6 & +19.4 & -7.5 & +12.9 & -2.7 & +12.9 & -0.9 & +12.3 & -4.8 & +35.7 & -11.4 \\
$0.90-0.92$ & +24.1 & -0.0 & +23.2 & -0.3 & +17.6 & -1.6 & +22.2 & -0.5 & +27.2 & -15.8 & +51.6 & -15.9 \\
$0.92-0.94$ & +21.0 & -2.5 & +19.8 & -0.5 & +15.4 & -2.5 & +17.1 & -2.8 & +19.1 & -8.9 & +41.5 & -10.0 \\
$0.94-0.96$ & +19.9 & -4.1 & +16.5 & -3.6 & +13.0 & -0.2 & +16.5 & -2.6 & +17.3 & -8.0 & +37.5 & -10.0 \\
$0.96-0.98$ & +22.2 & -3.9 & +18.7 & -3.9 & +14.9 & -1.4 & +17.8 & -1.1 & +20.7 & -11.3 & +42.6 & -12.7 \\
$0.98-1.00$ & +27.8 & -3.0 & +29.4 & -6.1 & +24.2 & -12.1 & +33.3 & -18.2 & +33.5 & -17.4 & +66.8 & -28.7 \\
\hline
\end{tabular}

Table D.10: The total systematic uncertainty, and the main contributions, on the Dalitz variables for $0.78<\mathrm{X}_{4} \leq 0.80$.

\begin{tabular}{|c|cc|cc|cc|cc|cr|rr|}
\hline $\mathrm{X}_{3}$ & \multicolumn{2}{|c|}{ Det. } & \multicolumn{2}{c|}{ Jet } & \multicolumn{2}{c|}{ Cal. } & \multicolumn{2}{c|}{ Und. } & \multicolumn{2}{c|}{$\begin{array}{c}\text { Rel. En. } \\
\text { Sc. }\end{array}$} & \multicolumn{2}{c|}{ Total (\%) } \\
& Cal. (\%) & Frag. (\%) & Stab. (\%) & Ev. (\%) & Sc. $\%$ & \\
\hline \hline $0.76-0.78$ & +19.2 & -0.9 & +20.8 & -0.9 & +17.9 & -0.9 & +21.4 & -4.9 & +26.9 & -8.3 & +48.0 & -8.4 \\
$0.78-0.80$ & +31.1 & -3.4 & +31.8 & -1.0 & +22.3 & -7.3 & +26.2 & -0.8 & +38.2 & -22.6 & +68.0 & -24.5 \\
$0.80-0.82$ & +22.9 & -2.4 & +22.5 & -1.6 & +17.7 & -3.5 & +15.0 & -1.2 & +23.4 & -8.7 & +46.0 & -9.8 \\
$0.82-0.84$ & +22.0 & -1.2 & +22.4 & -2.0 & +17.2 & -0.0 & +18.8 & -1.6 & +22.3 & -5.7 & +46.2 & -6.3 \\
$0.84-0.86$ & +26.2 & -1.2 & +19.5 & -1.6 & +17.1 & -4.3 & +16.7 & -5.7 & +26.8 & -20.3 & +48.6 & -20.9 \\
$0.86-0.88$ & +24.6 & -6.0 & +25.5 & -5.0 & +19.1 & -7.1 & +22.0 & -1.2 & +27.0 & -19.1 & +53.3 & -22.5 \\
$0.88-0.90$ & +22.0 & -4.8 & +17.8 & -6.9 & +16.7 & -0.6 & +16.4 & -0.0 & +14.5 & -6.8 & +39.5 & -10.9 \\
$0.90-0.92$ & +24.4 & -0.3 & +24.1 & -1.7 & +18.5 & -2.5 & +20.7 & -1.2 & +22.5 & -13.3 & +49.5 & -13.6 \\
$0.92-0.94$ & +23.6 & -3.3 & +22.1 & -2.6 & +16.8 & -1.9 & +18.9 & -0.7 & +20.3 & -8.1 & +45.9 & -9.4 \\
$0.94-0.96$ & +22.3 & -0.2 & +21.0 & -2.4 & +16.5 & -1.7 & +20.1 & -0.0 & +23.1 & -12.8 & +46.4 & -13.2 \\
$0.96-0.98$ & +24.0 & -0.9 & +22.5 & -0.4 & +19.6 & -0.9 & +24.9 & -14.3 & +26.2 & -12.0 & +52.7 & -12.0 \\
$0.98-1.00$ & +40.5 & -5.7 & +33.7 & -5.7 & +22.9 & -2.9 & +28.6 & -0.0 & +43.4 & -26.2 & +77.4 & -31.0 \\
\hline
\end{tabular}

Table D.11: The total systematic uncertainty, and the main contributions, on the Dalitz variables for $0.76<\mathrm{X}_{4} \leq 0.78$. 


\begin{tabular}{|c|c|c|c|c|c|c|c|c|c|c|c|c|}
\hline $\mathrm{X}_{3}$ & \multicolumn{2}{|c|}{$\begin{array}{c}\text { Det. } \\
\text { Cal. (\%) }\end{array}$} & \multicolumn{2}{|c|}{$\begin{array}{c}\text { Jet } \\
\text { Frag. }(\%)\end{array}$} & \multicolumn{2}{|c|}{$\begin{array}{c}\text { Cal. } \\
\text { Stab. }(\%)\end{array}$} & \multicolumn{2}{|c|}{$\begin{array}{c}\text { Und. } \\
\text { Ev. (\%) }\end{array}$} & \multicolumn{2}{|c|}{$\begin{array}{l}\text { Rel. En. } \\
\text { Sc. (\%) }\end{array}$} & \multicolumn{2}{|c|}{ Total (\%) } \\
\hline$\overline{00.74-0.76}$ & +20.5 & 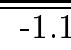 & 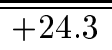 & $\overline{-2.2}$ & 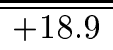 & $\overline{-1.1}$ & +23.3 & 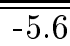 & " +32.5 & - & $\begin{array}{l}+54.5 \\
\end{array}$ & 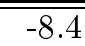 \\
\hline $0.76-0.78$ & +23.7 & -1.0 & +21.9 & -1.9 & +20.3 & -1.9 & -19.3 & -2.4 & +20.7 & -14.0 & +47.5 & -14.5 \\
\hline $0.78-0.80$ & +22.4 & -7.1 & +22.0 & -5.7 & +18.6 & -6.2 & +21.0 & -2.9 & +21.7 & -20.7 & +47.3 & -23.6 \\
\hline $0.80-0.82$ & +20.7 & -6.8 & +16.4 & -7.7 & +12.6 & -4.1 & +11.3 & -6.3 & +18.2 & -6.7 & +36.3 & -14.3 \\
\hline $0.82-0.84$ & +20.5 & -3.3 & +16.1 & -3.3 & +15.0 & -1.7 & +20.8 & -2.1 & +15.8 & -10.1 & +39.8 & -11.5 \\
\hline $0.84-0.86$ & +25.7 & -5.9 & +26.1 & -2.0 & +22.0 & -5.5 & +24.0 & -7.9 & +34.6 & -18.1 & +60.0 & -21.4 \\
\hline $0.86-0.88$ & +23.8 & -4.2 & +23.3 & -3.8 & +18.8 & -2.4 & +17.1 & -2.8 & +22.9 & -11.8 & +47.8 & -13.6 \\
\hline $0.88-0.90$ & +14.9 & -1.3 & +16.7 & -2.5 & +12.2 & -0.6 & +15.4 & -3.1 & +19.2 & -8.9 & +35.4 & -9.9 \\
\hline $0.90-0.92$ & +21.3 & -0.6 & +18.2 & -0.3 & +15.9 & -4.1 & +17.1 & -0.3 & +25.0 & -15.7 & +44.3 & -16.3 \\
\hline $0.92-0.94$ & +22.0 & -0.6 & +21.8 & -0.3 & +16.3 & -5.2 & +18.3 & -2.9 & +25.9 & -14.0 & +47.3 & -15.2 \\
\hline $0.94-0.96$ & +23.2 & -1.3 & +20.9 & -2.0 & +19.1 & -1.3 & +20.4 & -2.3 & +27.9 & -12.1 & +50.3 & -12.7 \\
\hline $0.96-0.98$ & +25.6 & -1.4 & +23.5 & -1.4 & +21.4 & -3.3 & +20.0 & -5.6 & +26.8 & -15.1 & +52.8 & -16.5 \\
\hline $0.98-1.00$ & +19.9 & -8.3 & +13.0 & -11.1 & +8.3 & -11.1 & +19.4 & -8.3 & +8.3 & -3.9 & +32.9 & -20.0 \\
\hline
\end{tabular}

Table D.12: The total systematic uncertainty, and the main contributions, on the Dalitz variables for $0.74<\mathrm{X}_{4} \leq 0.76$.

\begin{tabular}{|c|c|c|c|c|c|c|c|c|c|c|c|c|}
\hline $\mathrm{X}_{3}$ & \multicolumn{2}{|c|}{$\begin{array}{c}\text { Det. } \\
\text { Cal. (\%) }\end{array}$} & \multicolumn{2}{|c|}{$\begin{array}{c}\text { Jet } \\
\text { Frag. }(\%)\end{array}$} & \multicolumn{2}{|c|}{$\begin{array}{c}\text { Cal. } \\
\text { Stab. }(\%)\end{array}$} & \multicolumn{2}{|c|}{$\begin{array}{c}\text { Und. } \\
\text { Ev. }(\%)\end{array}$} & \multicolumn{2}{|c|}{$\begin{array}{l}\text { Rel. En. } \\
\text { Sc. (\%) }\end{array}$} & \multicolumn{2}{|c|}{ Total (\%) } \\
\hline $0.72-0.74$ & -20.2 & -13.6 & +21.8 & -8.6 & +14.8 & -7.4 & +9.9 & -4.9 & +21.9 & -19.5 & $\overline{41.0}$ & 26.8 \\
\hline $0.74-0.76$ & +20.1 & -5.6 & 6.0 & -5.1 & 15.0 & -0.5 & +14.5 & -2.3 & +20.9 & -9.1 & 9.1 & -12.1 \\
\hline $0.76-0.78$ & +25.8 & -3.3 & +22.4 & -2.2 & 17.0 & -1.1 & +19.2 & -1.6 & +23.5 & -14.5 & 3.8 & -15.2 \\
\hline $0.78-0.80$ & +18.8 & -7.7 & +18.4 & -10.6 & +12.1 & -2.9 & +14.0 & -4.3 & +16.7 & -5.2 & +36.3 & -15.1 \\
\hline $0.80-0.82$ & +14.4 & -1.0 & +17.0 & -1.5 & +12.0 & -1.0 & +13.0 & -15 & +14.7 & -5.0 & +32.0 & -5.7 \\
\hline $0.82-0.84$ & +22.8 & -4.9 & +23.2 & -5.7 & +17.5 & -0.8 & +17.9 & -4.1 & +21.4 & -10.7 & +46.3 & -13.7 \\
\hline $0.84-0.86$ & +21.5 & -6.9 & +21.5 & -8.0 & +14.5 & -2.7 & +18.3 & -1.1 & +19.7 & -9.2 & +43.1 & -14.3 \\
\hline $0.86-0.88$ & +19.0 & -4.1 & +17.4 & -4.5 & +12.7 & -0.3 & +11.6 & -2.4 & +16.7 & -10.5 & +35.2 & -12.4 \\
\hline $0.88-0.90$ & +26.4 & -4.8 & +23.9 & -3.1 & +22.8 & -5.9 & +23.2 & -3.5 & +27.6 & -18.7 & +55.6 & -20.7 \\
\hline $0.90-0.92$ & +24.8 & -1.2 & +21.4 & -0.9 & +16.0 & -2.2 & +21.5 & -1.5 & +18.7 & -9.4 & +46.3 & -9.9 \\
\hline $0.92-0.94$ & +18.4 & -2.1 & +16.2 & -4.6 & +12.6 & -5.2 & +10.7 & -0.8 & +12.8 & -4.7 & +32.2 & -7.1 \\
\hline $0.94-0.96$ & +21.6 & -0.8 & +18.4 & -0.6 & +13.9 & -1.6 & +15.9 & -0.0 & +24.7 & -12.0 & +43.2 & -12.1 \\
\hline $0.96-0.98$ & +21.9 & -4.9 & +19.6 & -6.0 & +15.9 & -5.5 & +24.2 & -9.9 & +29.2 & -16.1 & +50.5 & -21.2 \\
\hline $0.98-1.00$ & +30.5 & -6.7 & +32.2 & -6.7 & +26.7 & -3.3 & +26.7 & -10.0 & +34.3 & -23.3 & +67.6 & -27.3 \\
\hline
\end{tabular}

Table D.13: The total systematic uncertainty, and the main contributions, on the Dalitz variables for $0.72<\mathrm{X}_{4} \leq 0.74$. 


\begin{tabular}{|c|cc|cc|cc|cc|cc|cc|}
\hline $\mathrm{X}_{3}$ & \multicolumn{2}{|c|}{ Det. } & \multicolumn{2}{c|}{ Jet } & \multicolumn{2}{c|}{ Cal. } & \multicolumn{2}{c|}{ Und. } & \multicolumn{2}{c|}{ Rel. En. } & \multicolumn{2}{c|}{ Total (\%) } \\
& \multicolumn{2}{|c|}{ Cal. (\%) } & \multicolumn{2}{c|}{ Frag. (\%) } & Stab. (\%) & \multicolumn{2}{c|}{ Ev. (\%) } & Sc. (\%) & & \\
\hline \hline $0.70-0.72$ & +19.5 & -1.2 & +23.5 & -3.6 & +14.3 & -1.2 & +21.4 & -3.6 & +19.5 & -13.1 & +44.4 & -14.1 \\
$0.72-0.74$ & +25.6 & -4.8 & +23.6 & -0.6 & +20.4 & -7.8 & +25.7 & -6.6 & +25.5 & -14.8 & +54.3 & -18.6 \\
$0.74-0.76$ & +18.9 & -7.5 & +19.9 & -10.0 & +11.4 & -2.5 & +10.9 & -2.0 & +19.3 & -8.9 & +37.1 & -15.6 \\
$0.76-0.78$ & +19.7 & -2.7 & +20.7 & -1.1 & +17.0 & -4.9 & +17.0 & -4.9 & +28.7 & -9.0 & +47.1 & -11.8 \\
$0.78-0.80$ & +23.0 & -2.8 & +20.8 & -0.9 & +14.6 & -4.7 & +15.0 & -2.3 & +16.6 & -14.9 & +41.0 & -16.1 \\
$0.80-0.82$ & +22.3 & -3.3 & +22.3 & -0.0 & +18.6 & -4.4 & +23.5 & -4.9 & +29.0 & -21.0 & +52.3 & -22.2 \\
$0.82-0.84$ & +26.7 & -0.9 & +27.0 & -0.4 & +19.6 & -2.2 & +20.0 & -3.1 & +20.3 & -15.5 & +51.4 & -16.0 \\
$0.84-0.86$ & +22.6 & -1.9 & +17.3 & -3.4 & +16.9 & -3.4 & +13.9 & -2.6 & +15.2 & -7.3 & +38.9 & -9.3 \\
$0.86-0.88$ & +26.9 & -6.1 & +25.3 & -6.1 & +19.1 & -6.9 & +22.8 & -7.3 & +31.8 & -16.9 & +57.1 & -21.5 \\
$0.88-0.90$ & +21.7 & -1.7 & +19.5 & -4.5 & +13.7 & -1.0 & +17.1 & -1.4 & +20.8 & -10.8 & +42.0 & -11.9 \\
$0.90-0.92$ & +22.0 & -4.2 & +24.2 & -0.7 & +19.2 & -5.2 & +22.5 & -1.0 & +21.8 & -18.0 & +49.2 & -19.3 \\
$0.92-0.94$ & +25.4 & -1.7 & +22.7 & -0.9 & +17.8 & -1.7 & +21.5 & -3.2 & +22.3 & -12.0 & +49.3 & -12.7 \\
$0.94-0.96$ & +26.5 & -2.0 & +25.5 & -4.0 & +19.1 & -0.9 & +20.8 & -0.6 & +22.8 & -10.4 & +51.7 & -11.4 \\
$0.96-0.98$ & +27.1 & -7.7 & +24.0 & -8.3 & +21.9 & -8.3 & +21.9 & -4.7 & +28.8 & -14.7 & +55.7 & -20.9 \\
$0.98-1.00$ & +30.5 & -13.3 & +25.4 & -3.3 & +20.0 & -0.0 & +20.0 & -10.0 & +28.3 & -38.6 & +56.4 & -42.2 \\
\hline
\end{tabular}

Table D.14: The total systematic uncertainty, and the main contributions, on the Dalitz variables for $0.70<\mathrm{X}_{4} \leq 0.72$.

\begin{tabular}{|c|cc|cc|cc|cc|cc|rr|}
\hline $\mathrm{X}_{3}$ & \multicolumn{2}{|c|}{$\begin{array}{c}\text { Det. } \\
\text { Cal. (\%) }\end{array}$} & \multicolumn{2}{c|}{ Jet } & \multicolumn{2}{c|}{ Cal. } & \multicolumn{2}{c|}{ Und. } & \multicolumn{2}{c|}{$\begin{array}{c}\text { Rel. En. } \\
\text { Fc. (\%) }\end{array}$} & \multicolumn{2}{c|}{ Total (\%) } \\
& Stab. (\%) & Ev. (\%) & Sc. & \\
\hline \hline $0.68-0.70$ & +30.2 & -1.6 & +35.1 & -3.1 & +23.4 & -3.1 & +25.0 & -1.6 & +22.0 & -11.2 & +61.6 & -12.2 \\
$0.70-0.72$ & +20.2 & -3.5 & +16.5 & -5.8 & +15.1 & -2.3 & +15.7 & -0.6 & +19.8 & -9.5 & +39.4 & -11.9 \\
$0.72-0.74$ & +30.9 & -7.0 & +26.8 & -7.6 & +20.9 & -1.3 & +19.0 & -0.6 & +23.7 & -7.7 & +55.1 & -13.0 \\
$0.74-0.76$ & +24.2 & -1.1 & +26.4 & -2.3 & +15.3 & -1.7 & +16.4 & -0.6 & +18.9 & -9.3 & +46.3 & -9.8 \\
$0.76-0.78$ & +22.2 & -3.5 & +19.1 & -4.1 & +17.6 & -4.1 & +15.9 & -2.4 & +26.4 & -17.9 & +46.0 & -19.3 \\
$0.78-0.80$ & +18.3 & -5.4 & +18.7 & -2.2 & +11.9 & -0.5 & +14.6 & -0.5 & +20.0 & -6.1 & +38.0 & -6.6 \\
$0.80-0.82$ & +25.7 & -1.0 & +22.2 & -1.5 & +16.0 & -3.1 & +19.6 & -3.6 & +22.5 & -10.6 & +47.9 & -11.8 \\
$0.82-0.84$ & +23.1 & -3.8 & +21.8 & -0.0 & +18.3 & -2.4 & +16.8 & -0.5 & +28.6 & -20.4 & +49.4 & -20.9 \\
$0.84-0.86$ & +18.6 & -0.0 & +19.8 & -3.5 & +13.4 & -0.9 & +16.9 & -3.0 & +21.3 & -10.6 & +40.7 & -11.6 \\
$0.86-0.88$ & +21.6 & -4.0 & +21.6 & -4.8 & +18.3 & -2.4 & +20.3 & -2.4 & +19.8 & -12.1 & +45.5 & -14.1 \\
$0.88-0.90$ & +30.6 & -1.5 & +28.6 & -0.4 & +21.6 & -4.8 & +24.2 & -4.5 & +31.4 & -18.1 & +61.5 & -19.3 \\
$0.90-0.92$ & +25.3 & -0.0 & +23.0 & -0.3 & +18.5 & -0.7 & +19.6 & -3.1 & +30.6 & -18.0 & +53.3 & -18.3 \\
$0.92-0.94$ & +24.4 & -0.6 & +22.2 & -3.1 & +19.8 & -1.6 & +23.6 & -0.0 & +29.5 & -13.5 & +53.9 & -14.0 \\
$0.94-0.96$ & +20.4 & -2.4 & +19.6 & -1.0 & +15.2 & -0.3 & +18.6 & -0.3 & +17.8 & -10.9 & +41.1 & -11.3 \\
$0.96-0.98$ & +20.9 & -4.7 & +21.3 & -4.1 & +18.1 & -0.6 & +17.5 & -0.6 & +24.5 & -6.7 & +46.1 & -9.2 \\
$0.98-1.00$ & +29.1 & -9.5 & +25.9 & -9.5 & +14.3 & -4.8 & +23.8 & -4.8 & +14.3 & -32.3 & +49.9 & -35.6 \\
\hline
\end{tabular}

Table D.15: The total systematic uncertainty, and the main contributions, on the Dalitz variables for $0.68<\mathrm{X}_{4} \leq 0.70$. 


\begin{tabular}{|c|cc|cc|cc|cc|cc|cc|}
\hline $\mathrm{X}_{3}$ & \multicolumn{2}{|c|}{ Det. } & \multicolumn{2}{c|}{ Jet } & \multicolumn{2}{c|}{ Cal. } & \multicolumn{2}{c|}{ Und. } & \multicolumn{2}{c|}{ Rel. En. } & Total (\%) \\
& Cal. (\%) & Frag. (\%) & \multicolumn{2}{c|}{ Stab. (\%) } & Ev. (\%) & \multicolumn{2}{c|}{ Sc. (\%) } & & \\
\hline \hline $0.66-0.68$ & +20.0 & -2.4 & +26.5 & -12.2 & +9.8 & -4.9 & +7.3 & -2.4 & +39.4 & -20.7 & +52.9 & -24.8 \\
$0.68-0.70$ & +30.5 & -1.3 & +30.2 & -0.7 & +22.0 & -4.0 & +26.0 & -2.0 & +19.0 & -22.4 & +58.0 & -22.9 \\
$0.70-0.72$ & +17.5 & -0.6 & +21.0 & -3.8 & +17.1 & -3.2 & +20.9 & -5.1 & +22.9 & -7.3 & +44.8 & -10.2 \\
$0.72-0.74$ & +25.8 & -0.0 & +19.1 & -1.9 & +17.7 & -0.6 & +16.5 & -1.3 & +11.7 & -13.1 & +41.9 & -13.3 \\
$0.74-0.76$ & +26.6 & -0.0 & +28.2 & -1.4 & +18.1 & -2.2 & +23.2 & -2.9 & +30.3 & -23.9 & +57.3 & -24.2 \\
$0.76-0.78$ & +21.1 & -6.5 & +17.3 & -5.9 & +16.5 & -1.2 & +15.9 & -4.7 & +20.5 & -6.4 & +41.0 & -11.9 \\
$0.78-0.80$ & +27.1 & -4.6 & +25.8 & -4.0 & +15.6 & -0.6 & +19.1 & -3.5 & +27.2 & -11.4 & +52.4 & -13.4 \\
$0.80-0.82$ & +18.3 & -1.8 & +13.8 & -2.8 & +14.7 & -0.5 & +12.4 & -3.7 & +19.6 & -8.9 & +35.8 & -10.2 \\
$0.82-0.84$ & +31.4 & -0.5 & +32.1 & -1.6 & +23.0 & -3.1 & +23.0 & -5.2 & +28.0 & -17.0 & +62.1 & -18.1 \\
$0.84-0.86$ & +20.0 & -3.5 & +18.1 & -3.9 & +16.1 & -0.9 & +16.5 & -1.7 & +23.2 & -8.3 & +42.4 & -10.0 \\
$0.86-0.88$ & +30.7 & -3.1 & +32.0 & -0.9 & +23.6 & -8.4 & +28.0 & -6.7 & +37.4 & -26.3 & +68.6 & -28.6 \\
$0.88-0.90$ & +35.0 & -1.7 & +35.0 & -2.5 & +22.0 & -6.2 & +22.8 & -4.6 & +27.2 & -16.3 & +64.8 & -18.2 \\
$0.90-0.92$ & +25.6 & -1.9 & +25.3 & -1.3 & +19.7 & -4.5 & +21.6 & -4.2 & +29.1 & -12.2 & +54.8 & -13.8 \\
$0.92-0.94$ & +23.6 & -3.0 & +22.5 & -3.0 & +15.7 & -2.4 & +17.5 & -0.0 & +21.9 & -10.3 & +45.8 & -11.4 \\
$0.94-0.96$ & +26.4 & -2.4 & +23.6 & -1.6 & +17.1 & -2.8 & +19.5 & -7.2 & +29.7 & -18.8 & +53.0 & -20.5 \\
$0.96-0.98$ & +27.9 & -1.6 & +21.4 & -4.8 & +19.4 & -0.0 & +15.3 & -2.4 & +27.5 & -13.9 & +51.0 & -15.0 \\
$0.98-1.00$ & +65.6 & -20.0 & +62.7 & -20.0 & +50.0 & -25.0 & +65.0 & -20.0 & +74.4 & -33.9 & +143 & -54.5 \\
\hline
\end{tabular}

Table D.16: The total systematic uncertainty, and the main contributions, on the Dalitz variables for $0.66<\mathrm{X}_{4} \leq 0.68$.

\begin{tabular}{|c|c|c|c|c|c|c|c|c|c|c|c|c|}
\hline $\mathrm{X}_{3}$ & \multicolumn{2}{|c|}{$\begin{array}{c}\text { Det. } \\
\text { Cal. (\%) }\end{array}$} & \multicolumn{2}{|c|}{$\begin{array}{c}\text { Jet } \\
\text { Frag. }(\%)\end{array}$} & \multicolumn{2}{|c|}{$\begin{array}{c}\text { Cal. } \\
\text { Stab. }(\%)\end{array}$} & \multicolumn{2}{|c|}{$\begin{array}{c}\text { Und. } \\
\text { Ev. }(\%)\end{array}$} & \multicolumn{2}{|c|}{$\begin{array}{l}\text { Rel. En. } \\
\text { Sc. (\%) }\end{array}$} & \multicolumn{2}{|c|}{ Total (\%) } \\
\hline$\overline{00.68-0.70}$ & $\overline{c+7.6}$ & $\overline{-19.0}$ & 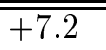 & $\overline{-21.4}$ & 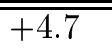 & $-\overline{-4.8}$ & +4.8 & -16.7 & +25.6 & -20.8 & $\overline{c+27.6}$ & $\overline{-39.4}$ \\
\hline $0.70-0.72$ & +26.0 & -1.9 & +24.7 & -2.8 & +17.9 & -2.8 & +17.9 & -1.9 & +24.8 & -12.2 & +50.4 & -13.1 \\
\hline $0.72-0.74$ & +22.8 & -0.7 & +22.2 & -0.7 & +17.3 & -2.2 & +12.2 & -2.2 & +23.8 & -12.4 & +45.0 & -12.8 \\
\hline $0.74-0.76$ & +21.4 & -1.3 & +26.3 & -2.6 & +16.3 & -2.0 & +17.6 & -2.0 & +26.2 & -7.6 & +49.1 & -8.6 \\
\hline $0.76-0.78$ & +25.2 & -5.7 & +23.9 & -6.3 & +16.7 & -0.6 & +14.4 & -4.0 & +18.2 & -6.7 & +45.0 & -11.6 \\
\hline $0.78-0.80$ & +21.1 & -3.3 & +22.2 & -6.5 & +17.4 & -0.5 & +22.3 & -2.2 & +15.7 & -16.2 & +44.5 & -18.0 \\
\hline $0.80-0.82$ & +21.9 & -1.5 & +24.0 & -5.0 & +13.4 & -0.5 & +20.4 & -1.5 & +22.0 & -9.2 & +46.2 & -10.7 \\
\hline $0.82-0.84$ & +16.0 & -5.7 & +14.9 & -6.2 & +11.4 & -0.0 & +12.4 & -3.6 & +18.4 & -4.8 & +33.2 & -10.3 \\
\hline $0.84-0.86$ & +19.5 & -3.5 & +20.5 & -7.0 & +12.0 & -1.0 & +17.5 & -0.5 & +21.7 & -10.5 & +41.5 & -13.2 \\
\hline $0.86-0.88$ & +23.6 & -2.2 & +23.0 & -2.6 & +21.4 & -4.4 & +21.8 & -0.4 & +23.3 & -11.6 & +50.6 & -12.9 \\
\hline $0.88-0.90$ & +23.3 & -8.2 & +24.5 & -4.6 & +19.6 & -7.3 & +20.1 & -2.7 & +26.7 & -20.5 & +51.4 & -23.9 \\
\hline $0.90-0.92$ & +28.3 & -6.3 & +27.2 & -5.5 & +23.5 & -10.2 & +25.5 & -5.5 & +36.2 & -22.8 & +63.7 & -26.9 \\
\hline $0.92-0.94$ & +24.6 & -5.1 & +23.5 & -5.8 & +17.7 & -3.7 & +19.7 & -3.4 & +18.9 & -11.1 & +47.1 & -14.4 \\
\hline $0.94-0.96$ & +27.6 & -0.4 & +24.6 & -2.5 & +21.1 & -2.9 & +19.6 & -0.4 & +27.8 & -11.5 & +54.5 & -12.1 \\
\hline $0.96-0.98$ & +32.6 & -0.7 & +34.3 & -1.5 & +27.6 & -7.5 & +33.6 & -3.7 & +41.9 & -23.2 & +76.7 & -24.7 \\
\hline $0.98-1.00$ & +31.3 & -7.7 & +17.4 & -7.7 & +23.1 & -15.4 & +23.1 & -7.7 & +82.8 & -63.9 & +96.0 & -67.1 \\
\hline
\end{tabular}

Table D.17: The total systematic uncertainty, and the main contributions, on the Dalitz variables for $0.64<\mathrm{X}_{4} \leq 0.66$. 


\begin{tabular}{|c|cc|cc|cc|cc|cc|cc|}
\hline $\mathrm{X}_{3}$ & \multicolumn{2}{|c|}{$\begin{array}{c}\text { Det. } \\
\text { Cal. }\end{array}$} & \multicolumn{2}{c|}{$\begin{array}{c}\text { Jet } \\
\text { Frag. }\end{array}$} & \multicolumn{2}{c|}{$\begin{array}{c}\text { Cal. } \\
\text { Stab. }(\%)\end{array}$} & \multicolumn{2}{c|}{$\begin{array}{c}\text { Und. } \\
\text { Ev. }(\%)\end{array}$} & \multicolumn{2}{c|}{$\begin{array}{c}\text { Rel. En. } \\
\text { Sc. }(\%)\end{array}$} & \multicolumn{2}{c|}{ Total (\%) } \\
\hline \hline $0.72-0.74$ & +21.7 & -9.1 & +20.2 & -9.1 & +12.1 & -6.1 & +30.3 & -0.0 & +32.5 & -17.4 & +54.8 & -22.5 \\
$0.74-0.76$ & +24.2 & -3.4 & +27.6 & -5.1 & +16.1 & -0.8 & +14.4 & -3.4 & +25.7 & -11.4 & +49.7 & -13.4 \\
$0.76-0.78$ & +25.5 & -1.2 & +19.1 & -3.7 & +15.9 & -1.8 & +12.8 & -2.4 & +11.0 & -7.1 & +39.4 & -8.6 \\
$0.78-0.80$ & +28.6 & -3.9 & +25.7 & -3.9 & +16.3 & -0.6 & +19.1 & -0.0 & +23.1 & -5.6 & +51.4 & -7.9 \\
$0.80-0.82$ & +19.6 & -1.1 & +14.5 & -0.0 & +13.1 & -1.6 & +19.7 & -7.7 & +17.0 & -7.0 & +38.0 & -10.5 \\
$0.82-0.84$ & +16.3 & -0.5 & +17.3 & -3.5 & +12.9 & -1.5 & +13.9 & -0.0 & +15.1 & -10.7 & +33.9 & -11.4 \\
$0.84-0.86$ & +23.6 & -4.5 & +21.6 & -4.0 & +18.6 & -7.0 & +15.1 & -7.0 & +21.1 & -17.3 & +45.2 & -20.9 \\
$0.86-0.88$ & +30.6 & -4.3 & +33.8 & -3.8 & +22.0 & -7.3 & +28.7 & -7.7 & +35.4 & -25.0 & +68.2 & -27.8 \\
$0.88-0.90$ & +26.1 & -4.2 & +25.4 & -4.6 & +19.1 & -1.5 & +18.3 & -0.8 & +25.1 & -12.5 & +51.5 & -14.1 \\
$0.90-0.92$ & +35.2 & -6.6 & +33.8 & -7.6 & +27.8 & -12.2 & +29.5 & -11.5 & +34.4 & -23.2 & +72.2 & -30.3 \\
$0.92-0.94$ & +24.6 & -4.8 & +25.2 & -8.2 & +18.7 & -1.4 & +19.7 & -0.0 & +24.7 & -15.0 & +50.9 & -17.8 \\
$0.94-0.96$ & +27.7 & -8.4 & +26.9 & -4.8 & +22.4 & -6.0 & +22.4 & -2.0 & +25.9 & -21.6 & +56.3 & -24.5 \\
$0.96-0.98$ & +31.5 & -1.6 & +26.9 & -0.0 & +17.8 & -3.1 & +24.0 & -3.9 & +30.3 & -11.6 & +59.4 & -12.7 \\
$0.98-1.00$ & +35.8 & -1.8 & +37.6 & -5.9 & +35.3 & -17.6 & +47.1 & -11.8 & +65.8 & -34.3 & +102 & -44.4 \\
\hline
\end{tabular}

Table D.18: The total systematic uncertainty, and the main contributions, on the Dalitz variables for $0.62<\mathrm{X}_{4} \leq 0.64$. 


\begin{tabular}{|c|c|c|c|c|c|c|c|c|c|c|c|c|}
\hline $\mathrm{X}_{3}$ & \multicolumn{2}{|c|}{$\begin{array}{c}\text { Det. } \\
\text { Cal. (\%) } \\
\end{array}$} & \multicolumn{2}{|c|}{$\begin{array}{c}\text { Jet } \\
\text { Frag. }(\%) \\
\end{array}$} & \multicolumn{2}{|c|}{$\begin{array}{c}\text { Cal. } \\
\text { Stab. }(\%) \\
\end{array}$} & \multicolumn{2}{|c|}{$\begin{array}{c}\text { Und. } \\
\text { Ev. (\%) }\end{array}$} & \multicolumn{2}{|c|}{$\begin{array}{l}\text { Rel. En. } \\
\text { Sc. }(\%)\end{array}$} & \multicolumn{2}{|c|}{ Total (\%) } \\
\hline $0.76-0.78$ & +18.0 & -7.5 & +24.6 & -7.5 & +5.0 & -0.0 & +20.0 & -10.0 & +48.8 & -30.5 & +61.1 & "-33.8 \\
\hline $0.78-0.80$ & +23.0 & -0.8 & +25.4 & -3.3 & +19.2 & -2.5 & +19.2 & -0.0 & +34.7 & -7.7 & +55.8 & -8.9 \\
\hline $0.80-0.82$ & +29.3 & -0.5 & +27.1 & -0.0 & +20.1 & -5.4 & +17.4 & -2.2 & +20.2 & -16.8 & +52.0 & -17.8 \\
\hline $0.82-0.84$ & +28.8 & -3.8 & +26.6 & -0.5 & +22.3 & -7.6 & +25.5 & -6.0 & +30.9 & -22.0 & +60.3 & -24.4 \\
\hline $0.84-0.86$ & +28.2 & -4.5 & +29.3 & -4.5 & +23.2 & -7.3 & +22.6 & -6.8 & +34.8 & -26.2 & +62.5 & -28.8 \\
\hline $0.86-0.88$ & +27.4 & -5.2 & +26.5 & -4.7 & +20.7 & -7.8 & +22.3 & -6.2 & +32.8 & -22.5 & +58.8 & -25.5 \\
\hline $0.88-0.90$ & +26.5 & -3.1 & +24.9 & -1.6 & +21.7 & -5.9 & +22.8 & -4.7 & +29.4 & -18.8 & +56.3 & -20.5 \\
\hline 0.90-0.92 & +24.8 & -1.4 & +22.0 & -2.9 & +19.6 & -3.6 & +20.3 & -2.5 & +27.1 & -15.3 & +51.3 & -16.2 \\
\hline $0.92-0.94$ & +29.3 & -1.5 & +30.7 & -1.5 & +23.4 & -4.7 & +27.0 & -8.0 & +35.0 & -20.9 & +65.6 & -23.0 \\
\hline $0.94-0.96$ & +29.3 & -3.0 & +27.6 & -0.8 & +21.2 & -2.5 & +22.9 & -3.8 & +31.8 & -11.1 & +60.0 & -12.4 \\
\hline $0.96-0.98$ & +34.7 & -1.7 & +32.1 & -1.7 & +23.9 & -3.4 & +34.2 & -4.3 & +40.2 & -18.5 & +74.8 & -19.5 \\
\hline $0.98-1.00$ & +35.5 & -5.0 & +32.2 & -5.0 & +25.0 & -5.0 & +25.0 & -10.0 & +32.8 & -16.6 & +68.0 & -21.2 \\
\hline
\end{tabular}

Table D.19: The total systematic uncertainty, and the main contributions, on the Dalitz variables for $0.60<\mathrm{X}_{4} \leq 0.62$.

\begin{tabular}{|c|c|c|c|c|c|c|c|c|c|c|c|c|}
\hline $\mathrm{X}_{3}$ & \multicolumn{2}{|c|}{$\begin{array}{c}\text { Det. } \\
\text { Cal. }(\%)\end{array}$} & \multicolumn{2}{|c|}{$\begin{array}{c}\text { Jet } \\
\text { Frag. }(\%) \\
\end{array}$} & \multicolumn{2}{|c|}{$\begin{array}{c}\text { Cal. } \\
\text { Stab. }(\%)\end{array}$} & \multicolumn{2}{|c|}{$\begin{array}{c}\text { Und. } \\
\text { Ev. (\%) }\end{array}$} & \multicolumn{2}{|c|}{$\begin{array}{l}\text { Rel. En. } \\
\text { Sc. (\%) }\end{array}$} & \multicolumn{2}{|c|}{ Total (\%) } \\
\hline $0.80-0.82$ & $\overline{c+19.1}$ & $\begin{array}{l}-4.7 \\
\end{array}$ & $\overline{c+15.9}$ & $\overline{c-7.0}$ & $\begin{array}{c}+16.3 \\
\end{array}$ & $\overline{c-4.7}$ & -9.3 & $\overline{-0.0}$ & $\begin{array}{c}+54.7 \\
\end{array}$ & $\bar{~}-20.9$ & $\begin{array}{c}+63.0 \\
\end{array}$ & $\begin{array}{l}-23.0 \\
\end{array}$ \\
\hline $0.82-0.84$ & +32.2 & -4.2 & +34.8 & -3.3 & +23.3 & -0.8 & 0.8 & -2.5 & +30.8 & -13.7 & 68.5 & -15.0 \\
\hline $0.84-0.86$ & +25.4 & -2.4 & +22.6 & -1.9 & +18.2 & -2.9 & +17.7 & -1.4 & +25.5 & -9.8 & +49.5 & -10.9 \\
\hline $0.86-0.88$ & +26.7 & -4.9 & +25.4 & -5.3 & +20.9 & -0.0 & +20.4 & -1.0 & +27.0 & -18.9 & +54.2 & -20.3 \\
\hline $0.88-0.90$ & +26.0 & -1.4 & +28.1 & -0.5 & +20.2 & -3.8 & +26.4 & -7.7 & +28.7 & -25.1 & +58.2 & -26.6 \\
\hline $0.90-0.92$ & +24.2 & -3.0 & +22.0 & -5.2 & +17.8 & -2.6 & +14.8 & -3.3 & +20.6 & -5.9 & +45.1 & -9.5 \\
\hline $0.92-0.94$ & +22.5 & -1.0 & +20.3 & -1.4 & +18.3 & -2.4 & +16.9 & -0.7 & +23.6 & -14.6 & +45.8 & -14.9 \\
\hline $0.94-0.96$ & +30.7 & -8.3 & +28.6 & -6.2 & +24.4 & -9.1 & +24.8 & -7.4 & +36.3 & -25.4 & +65.5 & -29.8 \\
\hline $0.96-0.98$ & +30.9 & -5.1 & +28.2 & -3.6 & +25.4 & -8.0 & +25.4 & -6.5 & +24.4 & -16.0 & +60.3 & -20.1 \\
\hline $0.98-1.00$ & +47.6 & -11.8 & +43.6 & -5.9 & +41.2 & -5.9 & +41.2 & -11.8 & +74.7 & -39.5 & +115 & -43.6 \\
\hline
\end{tabular}

Table D.20: The total systematic uncertainty, and the main contributions, on the Dalitz variables for $0.58<\mathrm{X}_{4} \leq 0.60$.

\begin{tabular}{|c|cc|cc|cc|cc|cc|cc|}
\hline $\mathrm{X}_{3}$ & \multicolumn{2}{|c|}{ Det. } & \multicolumn{2}{c|}{ Jet } & \multicolumn{2}{c|}{ Cal. } & \multicolumn{2}{c|}{ Und. } & \multicolumn{2}{c|}{ Rel. En. } & \multicolumn{2}{c|}{ Total (\%) } \\
& Cal. (\%) & Frag. (\%) & Stab. (\%) & Ev. (\%) & Sc. (\%) & \\
\hline \hline $0.84-0.86$ & +26.2 & -17.1 & +30.8 & -8.6 & +11.4 & -5.7 & +25.7 & -20.0 & +55.3 & -25.7 & +74.0 & -38.2 \\
$0.86-0.88$ & +29.9 & -1.9 & +29.7 & -3.1 & +23.8 & -5.0 & +23.1 & -1.9 & +31.9 & -13.3 & +62.4 & -14.8 \\
$0.88-0.90$ & +21.2 & -0.8 & +17.6 & -1.2 & +12.9 & -3.1 & +16.0 & -5.1 & +18.8 & -9.3 & +39.1 & -11.2 \\
$0.90-0.92$ & +26.8 & -3.1 & +27.3 & -0.8 & +21.7 & -4.7 & +21.7 & -4.3 & +31.7 & -20.8 & +58.4 & -22.0 \\
$0.92-0.94$ & +22.6 & -1.8 & +21.3 & -2.9 & +18.6 & -1.4 & +22.5 & -0.7 & +21.3 & -11.0 & +47.7 & -11.7 \\
$0.94-0.96$ & +25.3 & -4.3 & +26.9 & -3.0 & +17.5 & -5.1 & +18.4 & -6.0 & +26.9 & -13.4 & +52.3 & -16.4 \\
$0.96-0.98$ & +26.9 & -3.6 & +27.6 & -3.6 & +22.7 & -10.0 & +25.5 & -5.5 & +43.8 & -28.4 & +67.6 & -31.0 \\
$0.98-1.00$ & +25.5 & -0.0 & +27.1 & -0.0 & +18.8 & -0.0 & +18.8 & -6.3 & +44.2 & -23.4 & +63.6 & -24.2 \\
\hline
\end{tabular}

Table D.21: The total systematic uncertainty, and the main contributions, on the Dalitz variables for $0.56<\mathrm{X}_{4} \leq 0.58$. 


\begin{tabular}{|c|cc|cc|cc|cc|cc|cc|}
\hline $\mathrm{X}_{3}$ & \multicolumn{2}{|c|}{ Det. } & \multicolumn{2}{c|}{ Jet } & \multicolumn{2}{c|}{ Cal. } & \multicolumn{2}{c|}{ Und. } & \multicolumn{2}{c|}{ Rel. En. } & \multicolumn{2}{c|}{ Total (\%) } \\
& \multicolumn{2}{c|}{ Cal. (\%) } & \multicolumn{2}{c|}{ Frag. (\%) } & \multicolumn{2}{c|}{ Stab. (\%) } & Ev. (\%) & Sc. (\%) & & \\
\hline \hline $0.88-0.90$ & +7.8 & -1.5 & +6.2 & -2.9 & +8.8 & -1.5 & +10.3 & -4.4 & +24.2 & -17.7 & +39.1 & -18.7 \\
$0.90-0.92$ & +27.4 & -7.0 & +24.7 & -7.0 & +19.9 & -6.5 & +21.5 & -6.5 & +27.1 & -15.7 & +58.4 & -20.8 \\
$0.92-0.94$ & +24.8 & -2.2 & +23.8 & -0.4 & +21.0 & -5.5 & +19.2 & -1.1 & +26.4 & -16.6 & +47.7 & -17.8 \\
$0.94-0.96$ & +20.1 & -3.9 & +17.7 & -4.8 & +17.0 & -1.3 & +20.5 & -1.3 & +28.9 & -14.5 & +52.3 & -16.0 \\
$0.96-0.98$ & +34.5 & -3.8 & +33.4 & -1.9 & +28.3 & -6.6 & +33.0 & -8.5 & +31.8 & -20.4 & +67.6 & -23.4 \\
$0.98-1.00$ & +47.2 & -13.3 & +35.6 & -13.3 & +33.3 & -20.0 & +33.3 & -13.3 & +42.7 & -22.1 & +63.6 & -37.7 \\
\hline
\end{tabular}

Table D.22: The total systematic uncertainty, and the main contributions, on the Dalitz variables for $0.54<\mathrm{X}_{4} \leq 0.56$.

\begin{tabular}{|c|c|c|c|c|c|c|c|c|c|c|c|c|}
\hline $\mathrm{X}_{3}$ & \multicolumn{2}{|c|}{$\begin{array}{c}\text { Det. } \\
\text { Cal. (\%) } \\
\end{array}$} & \multicolumn{2}{|c|}{$\begin{array}{c}\text { Jet } \\
\text { Frag. }(\%) \\
\end{array}$} & \multicolumn{2}{|c|}{$\begin{array}{c}\text { Cal. } \\
\text { Stab. (\%) }\end{array}$} & \multicolumn{2}{|c|}{$\begin{array}{c}\text { Und. } \\
\text { Ev. (\%) }\end{array}$} & \multicolumn{2}{|c|}{$\begin{array}{l}\text { Rel. En. } \\
\text { Sc. (\%) }\end{array}$} & \multicolumn{2}{|c|}{ Total (\%) } \\
\hline $0.92-0.94$ & +28.4 & -3.3 & +25.0 & -4.9 & +23.0 & -1.6 & +19.7 & -1.6 & +34.4 & -32.2 & +59.5 & -32.9 \\
\hline $0.94-0.96$ & +24.3 & -0.0 & +22.0 & -1.3 & +18.7 & -5.2 & +18.1 & -2.6 & +27.7 & -17.2 & +50.3 & -18.4 \\
\hline $0.96-0.98$ & +23.0 & -3.9 & +23.6 & -2.9 & +19.6 & -8.8 & +18.6 & -8.8 & +49.0 & -25.8 & +65.0 & -29.2 \\
\hline $0.98-1.00$ & +78.5 & -22.2 & +69.5 & -22.2 & +55.6 & -22.2 & +77.8 & -12.3 & +42.7 & -72.9 & +188 & -85.3 \\
\hline
\end{tabular}

Table D.23: The total systematic uncertainty, and the main contributions, on the Dalitz variables for $0.52<\mathrm{X}_{4} \leq 0.54$.

\begin{tabular}{|c|c|c|c|c|c|c|c|c|c|c|c|c|}
\hline $\mathrm{X}_{3}$ & \multicolumn{2}{|c|}{$\begin{array}{c}\text { Det. } \\
\text { Cal. (\%) } \\
\end{array}$} & \multicolumn{2}{|c|}{$\begin{array}{c}\text { Jet } \\
\text { Frag. }(\%) \\
\end{array}$} & \multicolumn{2}{|c|}{$\begin{array}{c}\text { Cal. } \\
\text { Stab. (\%) }\end{array}$} & \multicolumn{2}{|c|}{$\begin{array}{c}\text { Und. } \\
\text { Ev. }(\%)\end{array}$} & \multicolumn{2}{|c|}{$\begin{array}{l}\text { Rel. En. } \\
\text { Sc. (\%) }\end{array}$} & \multicolumn{2}{|c|}{ Total (\%) } \\
\hline $0.96-0.98$ & $\overline{c+37.4}$ & 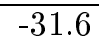 & $\begin{array}{c}+28.5 \\
\end{array}$ & -31.6 & $\begin{array}{l}+26.3 \\
\end{array}$ & $\begin{array}{l}-21.1 \\
\end{array}$ & +36.8 & $\begin{array}{l}-21.1 \\
\end{array}$ & +37.2 & $\overline{-51.0}$ & +75.7 & $\begin{array}{l}-74.6 \\
\end{array}$ \\
\hline $0.98-1.00$ & +75.7 & -12.5 & +78.0 & -12.5 & +62.5 & -12.5 & +50.0 & -12.5 & +65.0 & -30.6 & +150 & -40.6 \\
\hline
\end{tabular}

Table D.24: The total systematic uncertainty, and the main contributions, on the Dalitz variables for $0.50<\mathrm{X}_{4} \leq 0.52$. 


\section{Appendix E}

\section{The Dalitz Distributions After Variation of the Cut Parameters}

In Chapter 6, the stability of the data with respect to changes in the cut parameters is discussed. For complete information, the Dalitz distributions after variation of the cut parameters by $\pm \sigma$ are shown in this appendix. Only the jet $\mathrm{E}_{\mathrm{T}}$ cut is varied by approximately $5 \sigma$. 


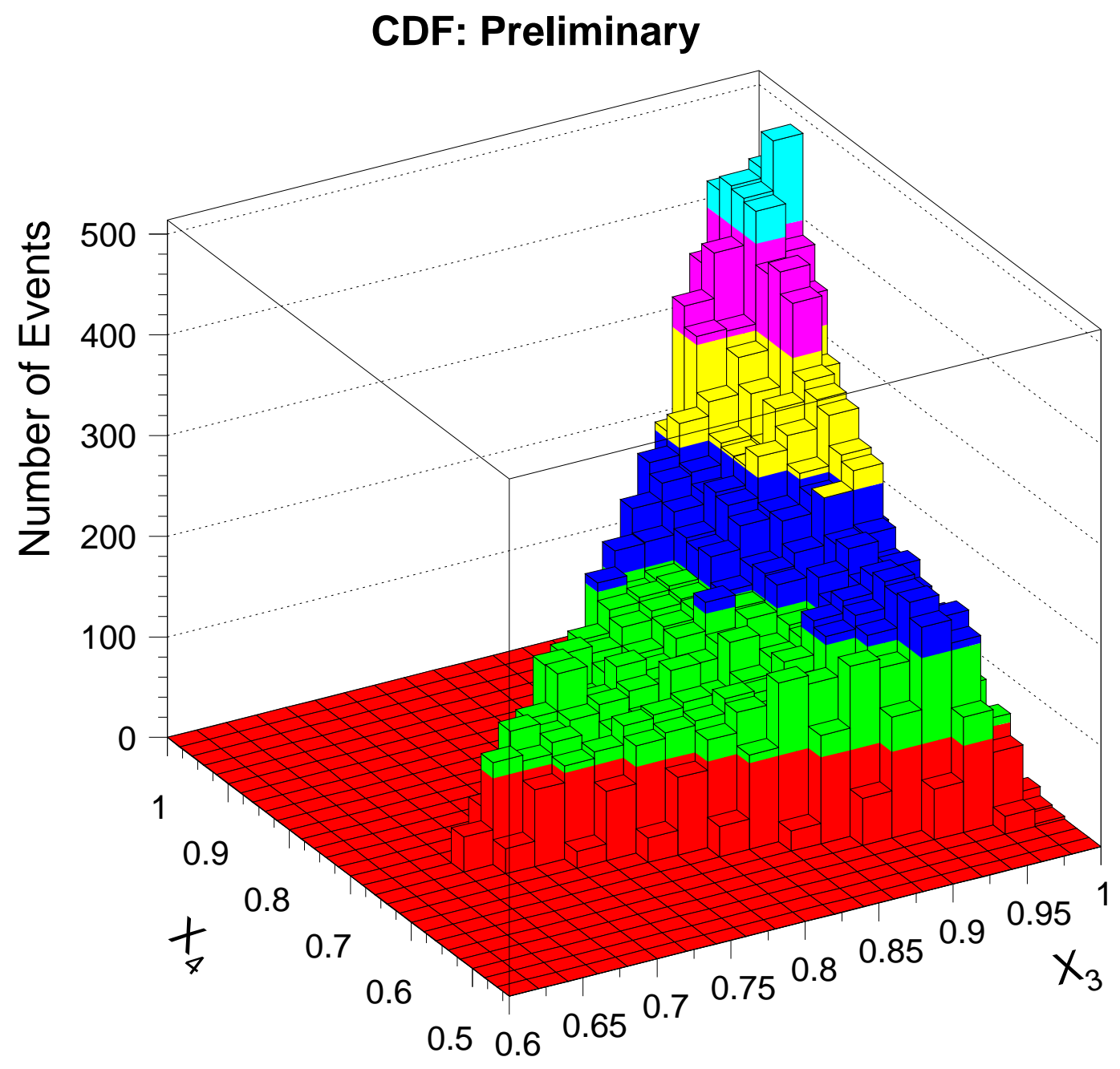

Figure E.1: The Dalitz distribution after variation of the cone overlap cut by -0.05 . 


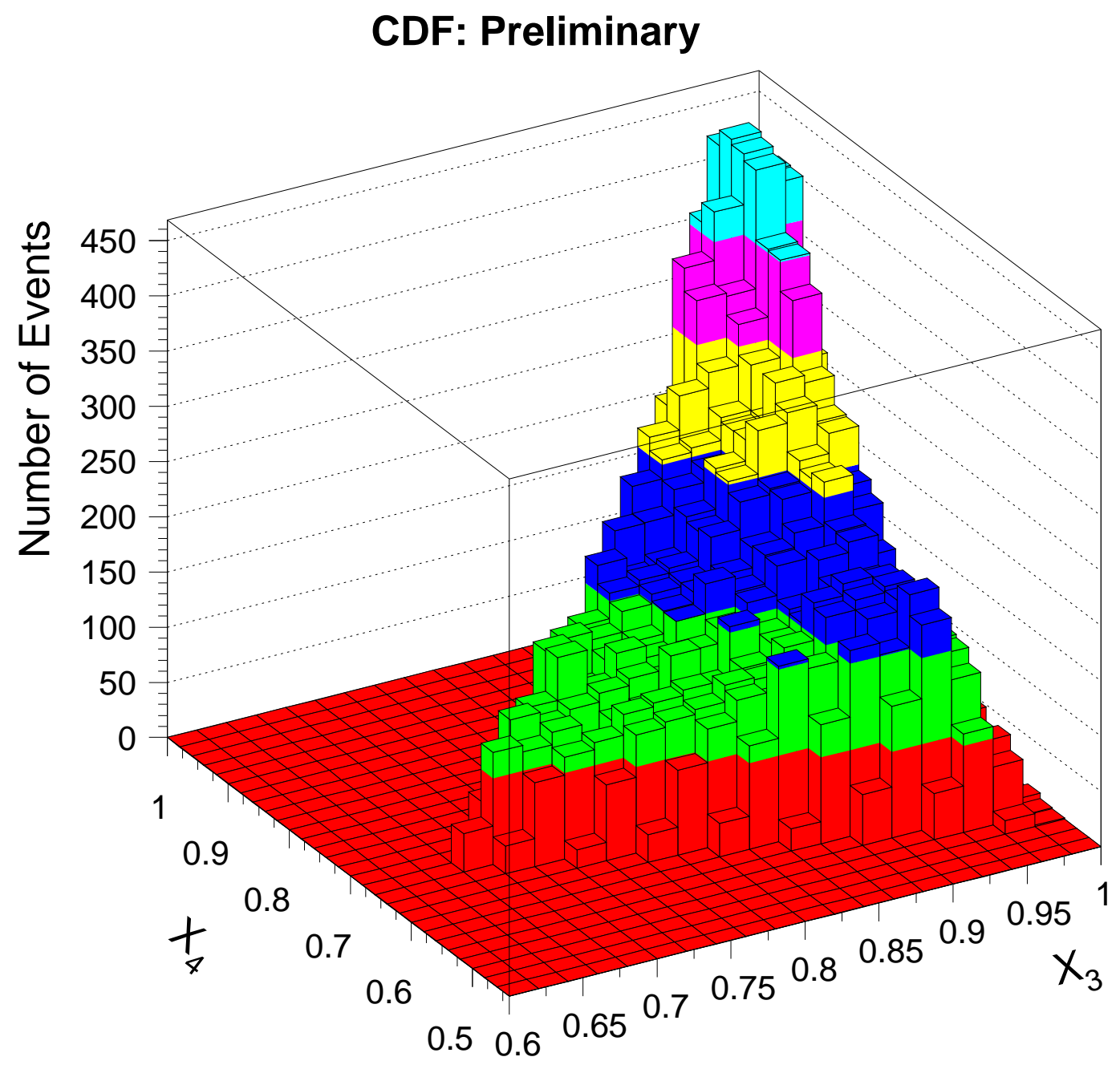

Figure E.2: The Dalitz distribution after variation of the cone overlap cut by +0.05 . 


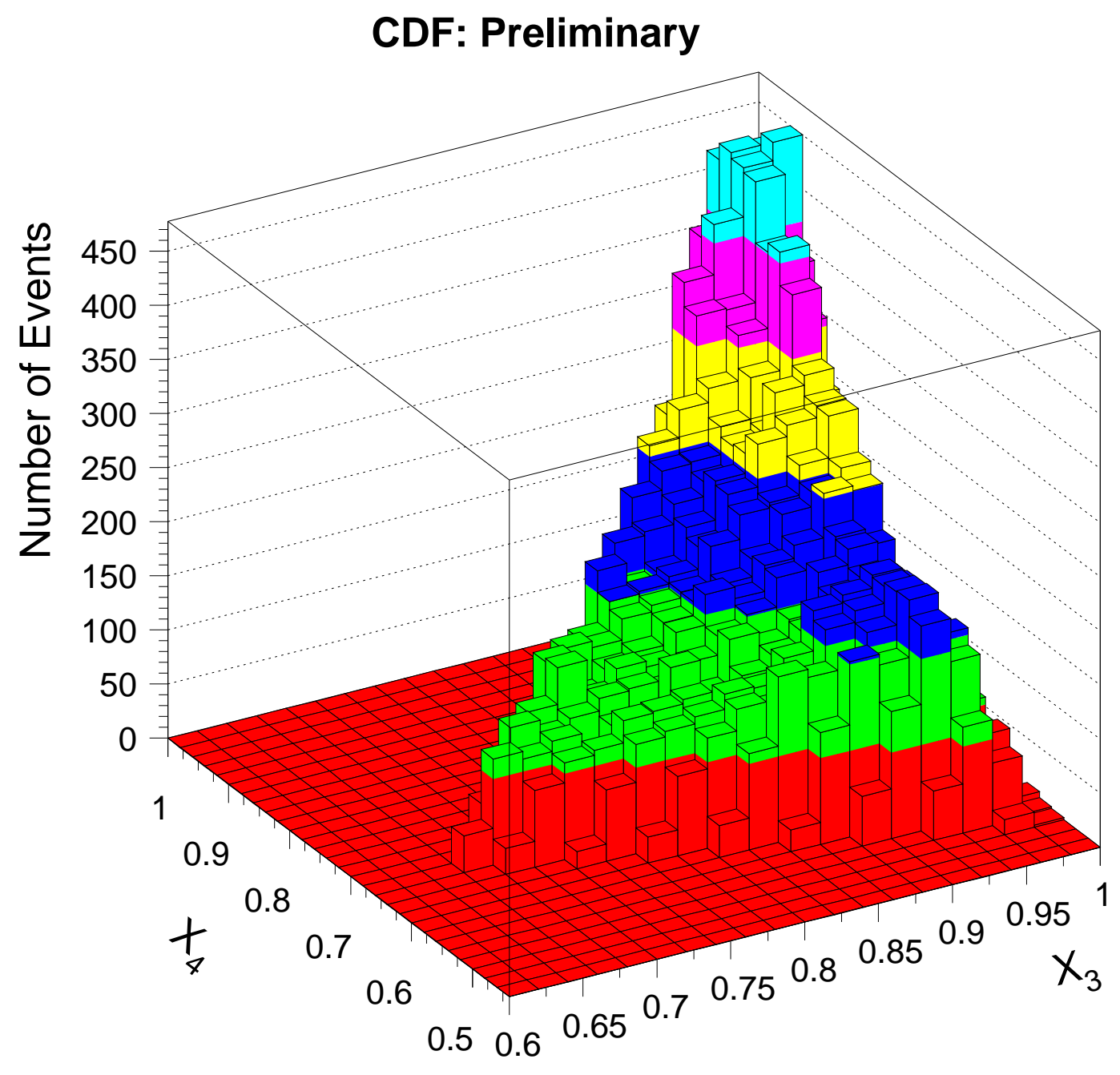

Figure E.3: The Dalitz distribution after variation of the $\eta$ cut by -0.075 . 


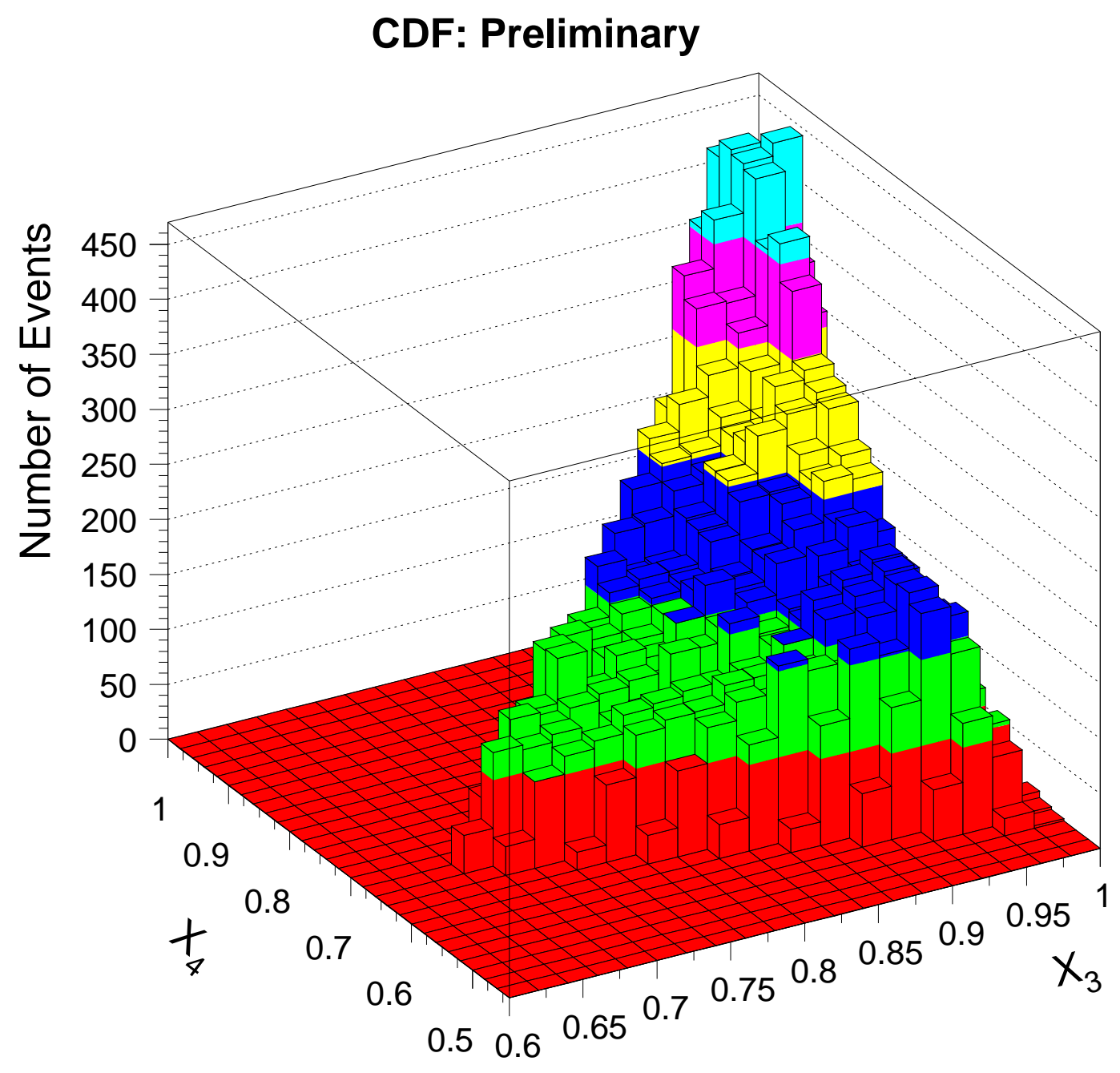

Figure E.4: The Dalitz distribution after variation of the $\eta$ cut by +0.075 . 


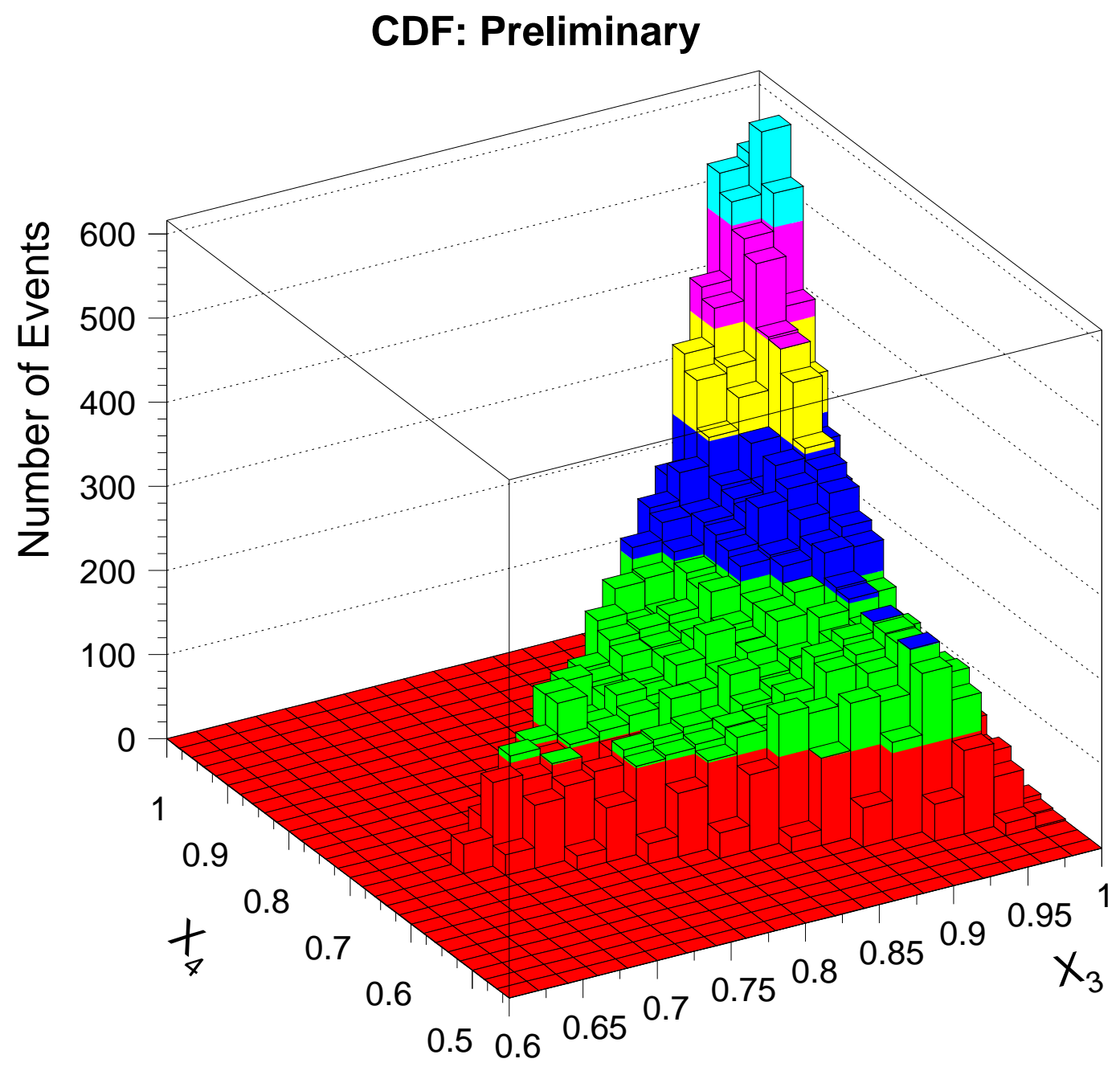

Figure E.5: The Dalitz distribution after variation of the jet $\mathrm{E}_{\mathrm{T}}$ cut by $-5 \mathrm{GeV}$. 


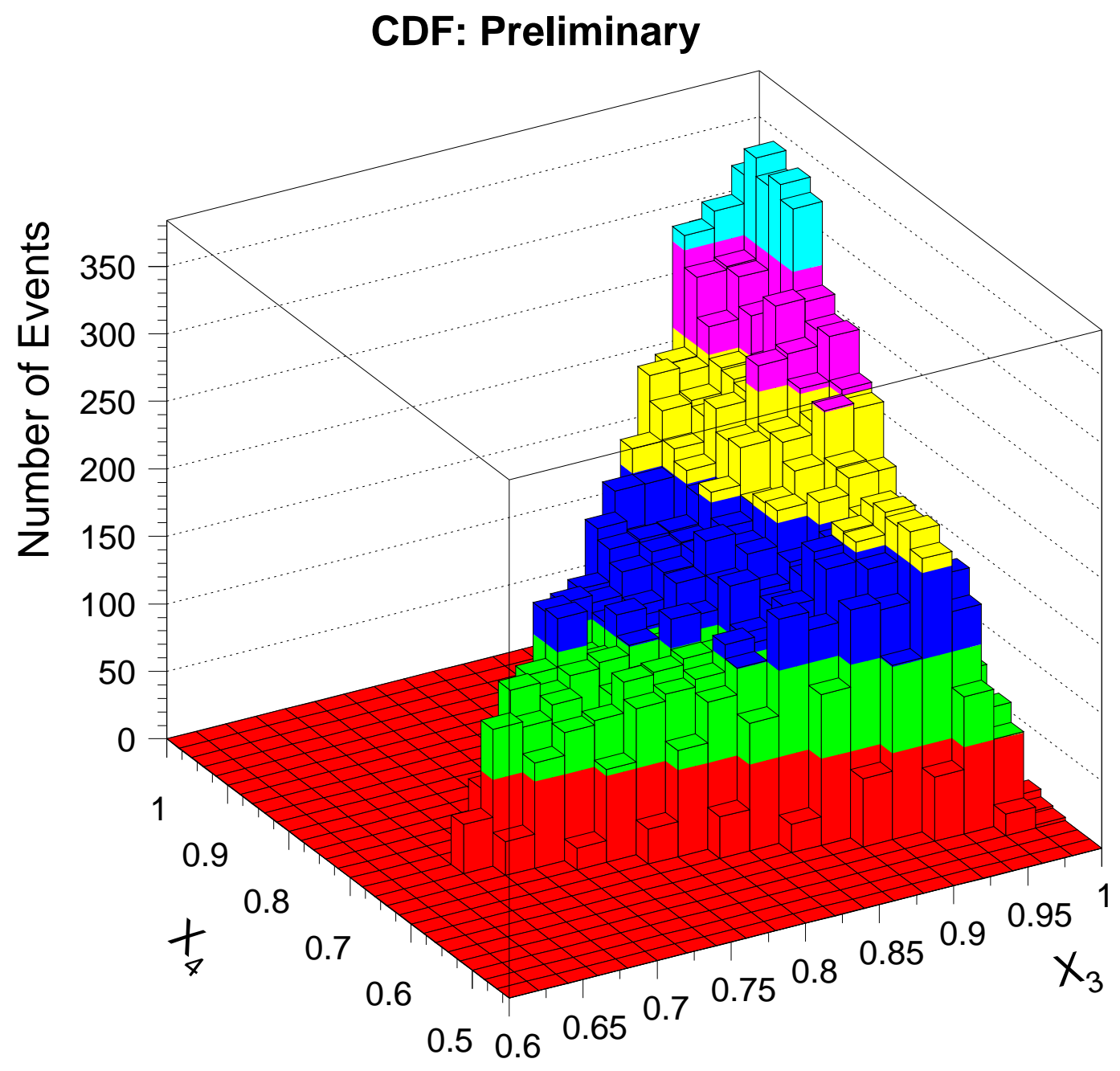

Figure E.6: The Dalitz distribution after variation of the jet $\mathrm{E}_{\mathrm{T}}$ cut by $+5 \mathrm{GeV}$. 


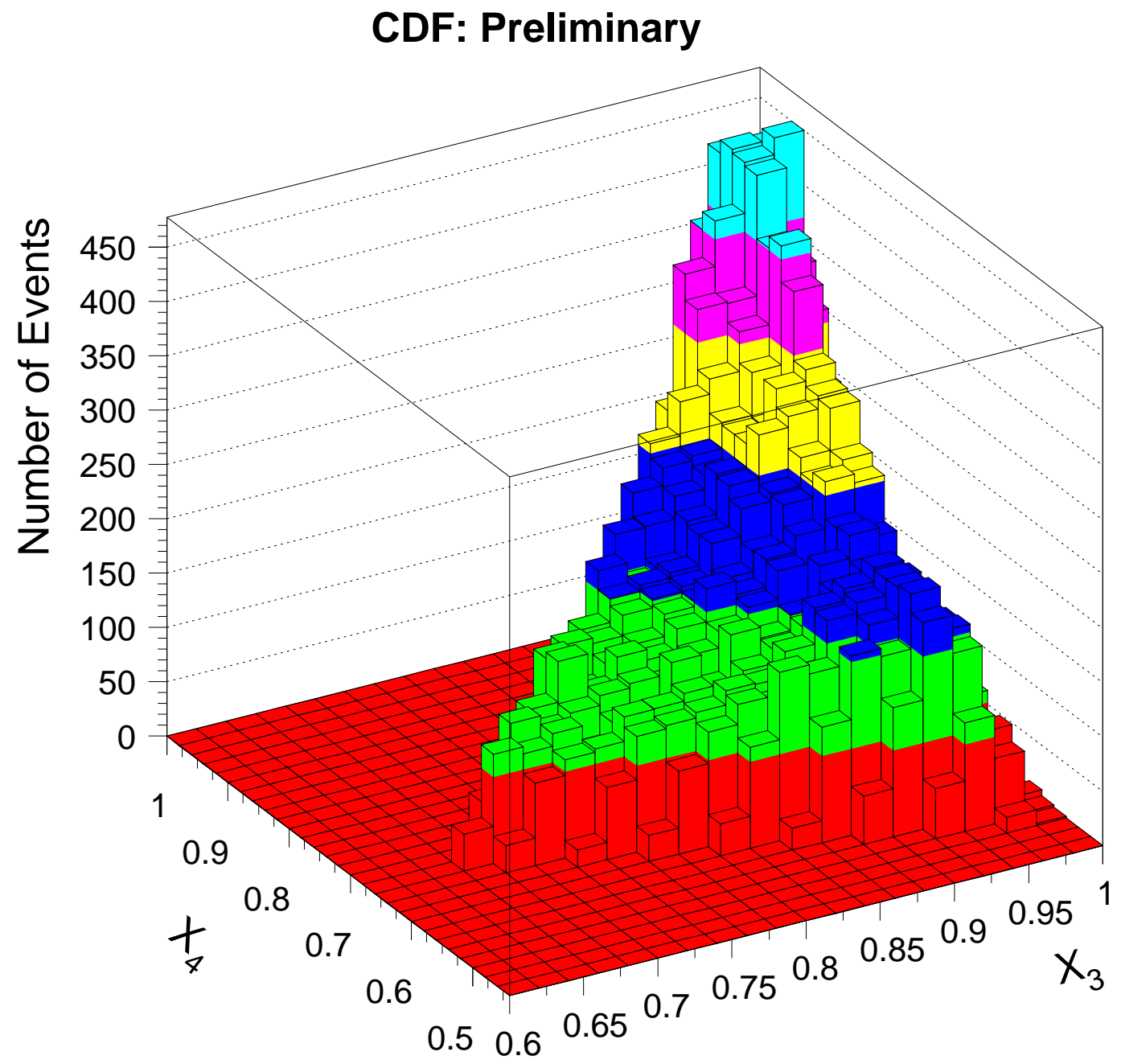

Figure E.7: The Dalitz distribution after variation of the geometrical acceptance cut by -0.01 in $\left|\cos \theta_{3}^{*}\right|$. 


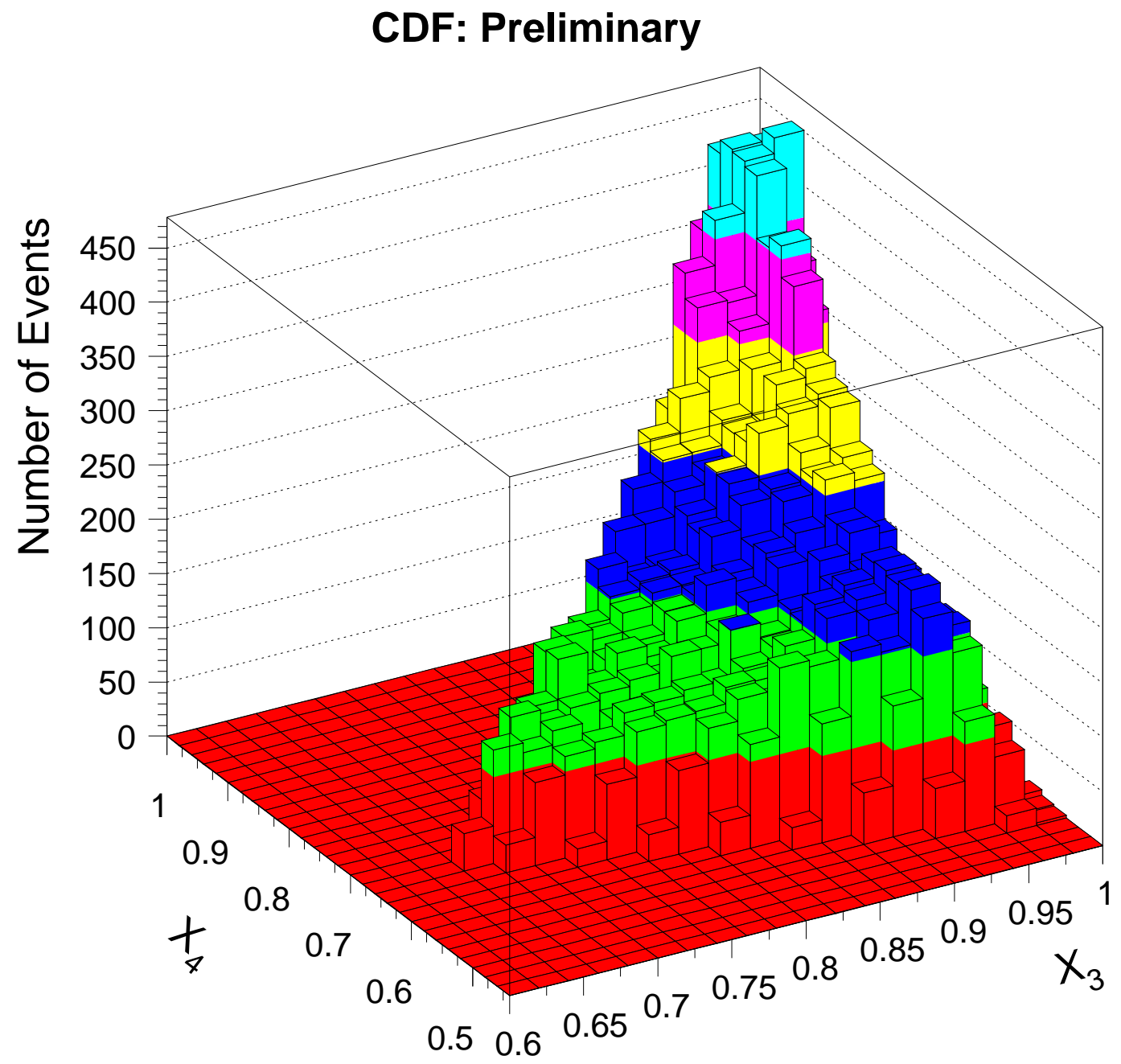

Figure E.8: The Dalitz distribution after variation of the geometrical acceptance cut by +0.01 in $\left|\cos \theta_{3}^{*}\right|$. 


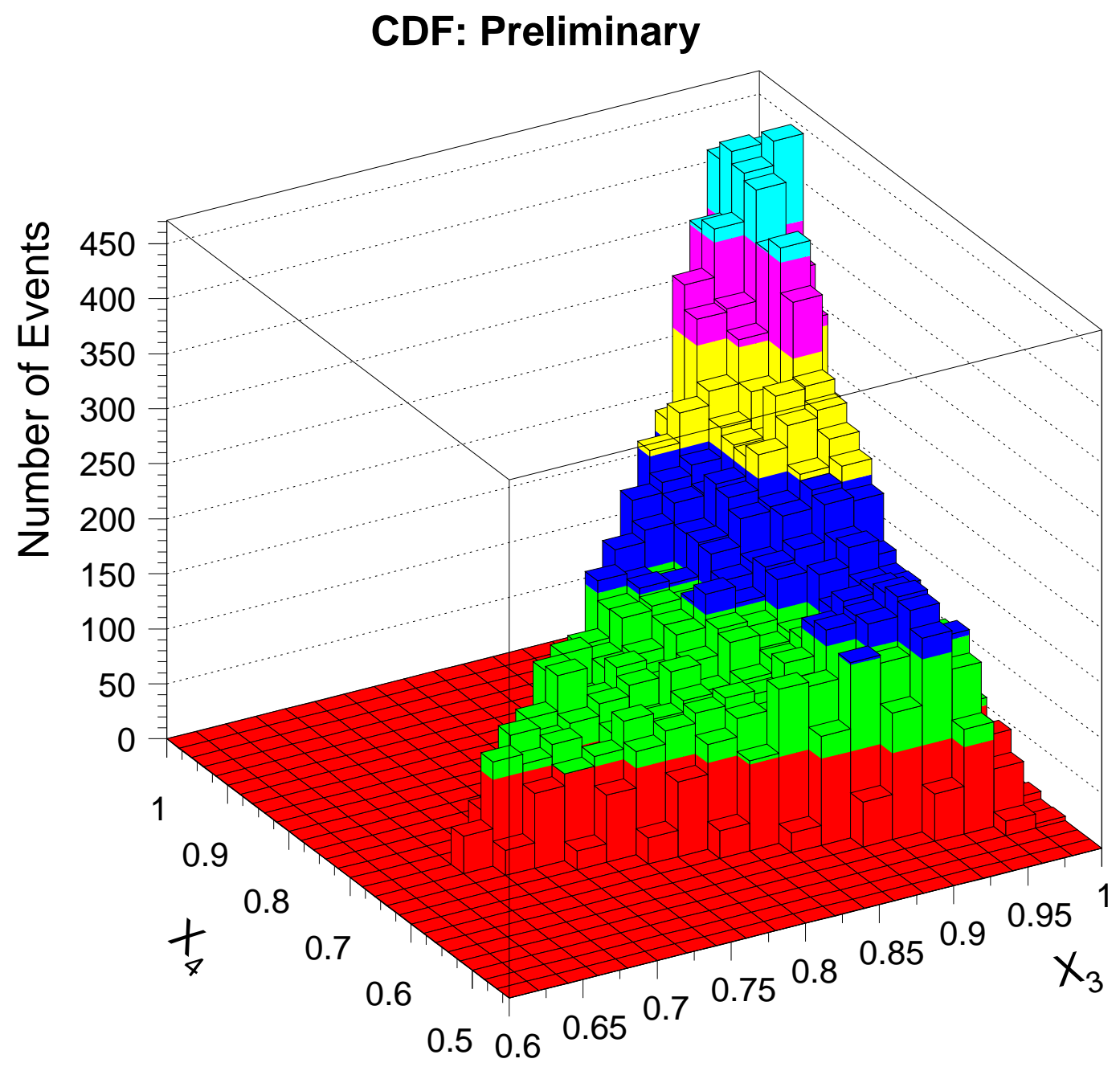

Figure E.9: The Dalitz distribution after variation of the $\sum \mathrm{E}_{\mathrm{T}}$ cut by $-10 \mathrm{GeV}$. 


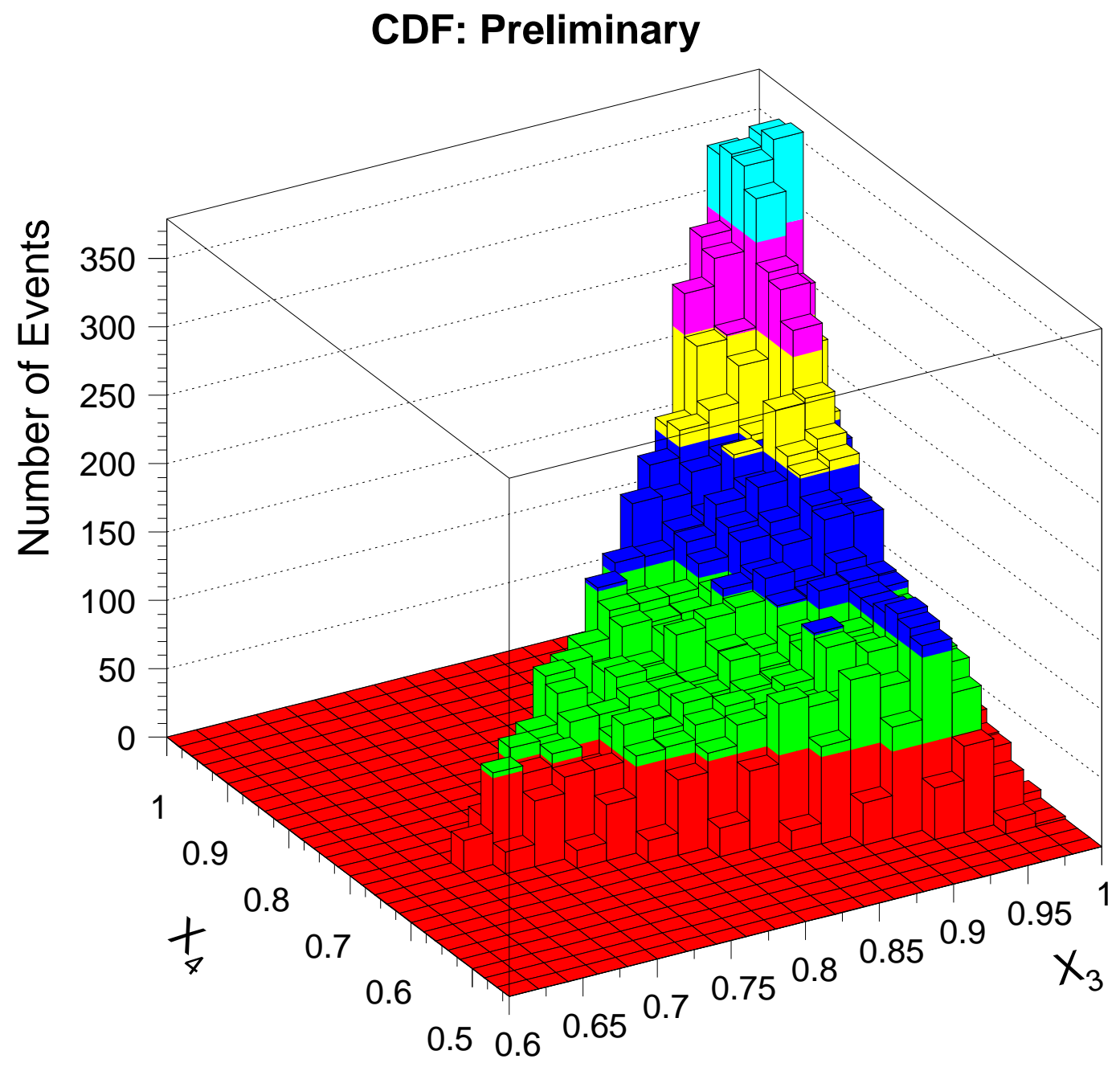

Figure E.10: The Dalitz distribution after variation of the $\sum \mathrm{E}_{\mathrm{T}}$ cut by $+10 \mathrm{GeV}$. 


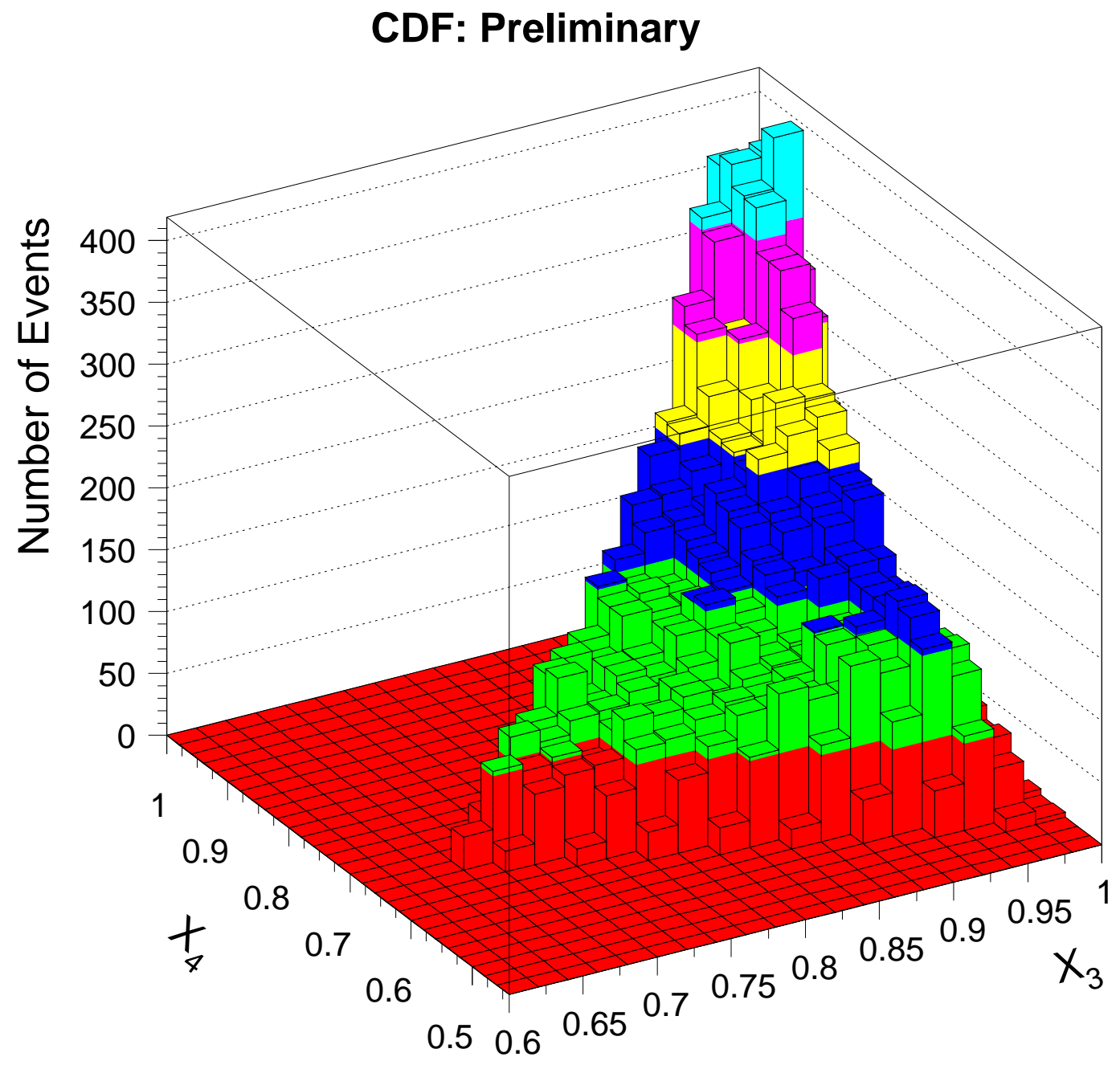

Figure E.11: The Dalitz distribution after variation of the number of tracks per vertex by -1 . 


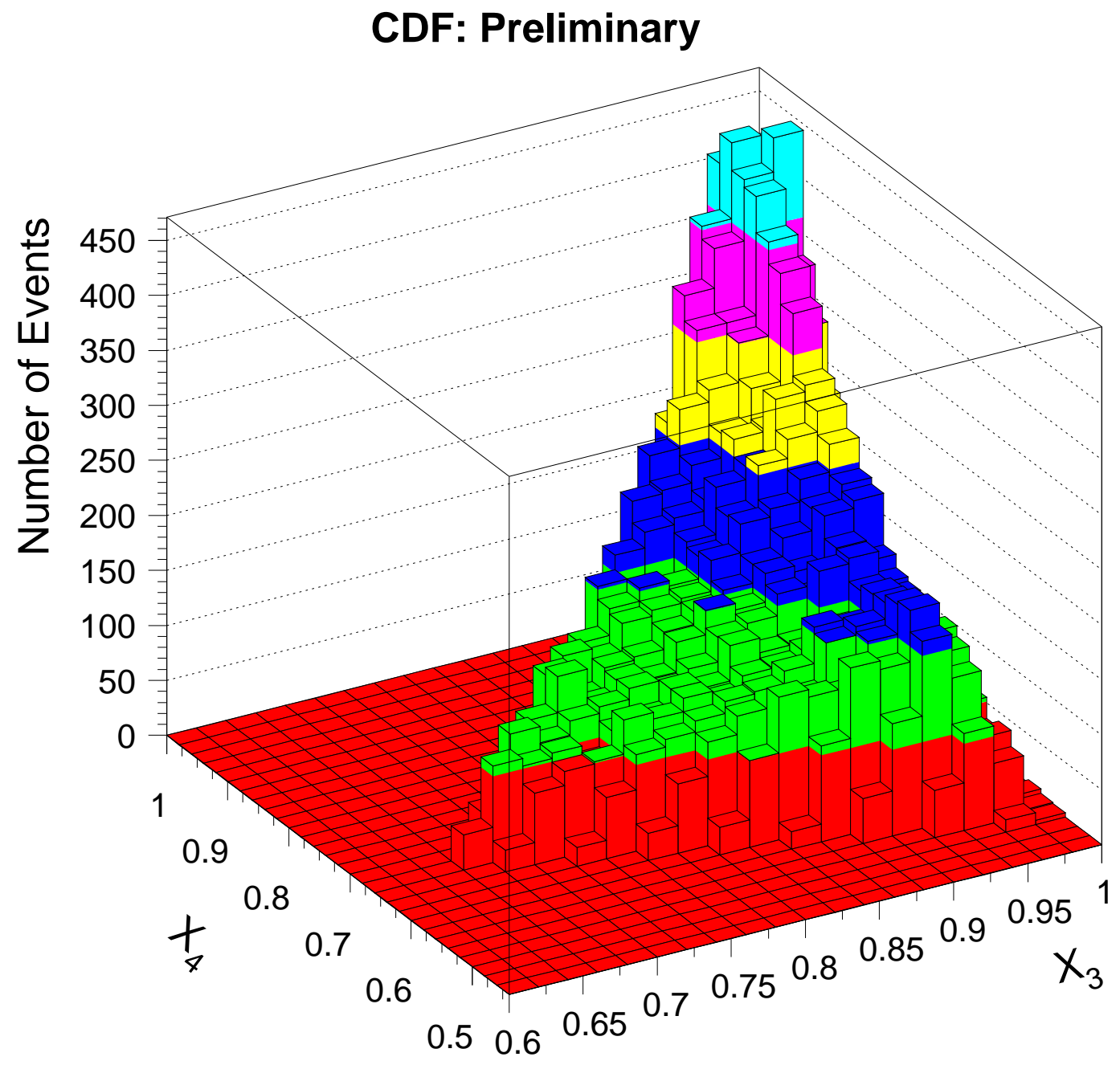

Figure E.12: The Dalitz distribution after variation of the number of tracks per vertex by +1 . 


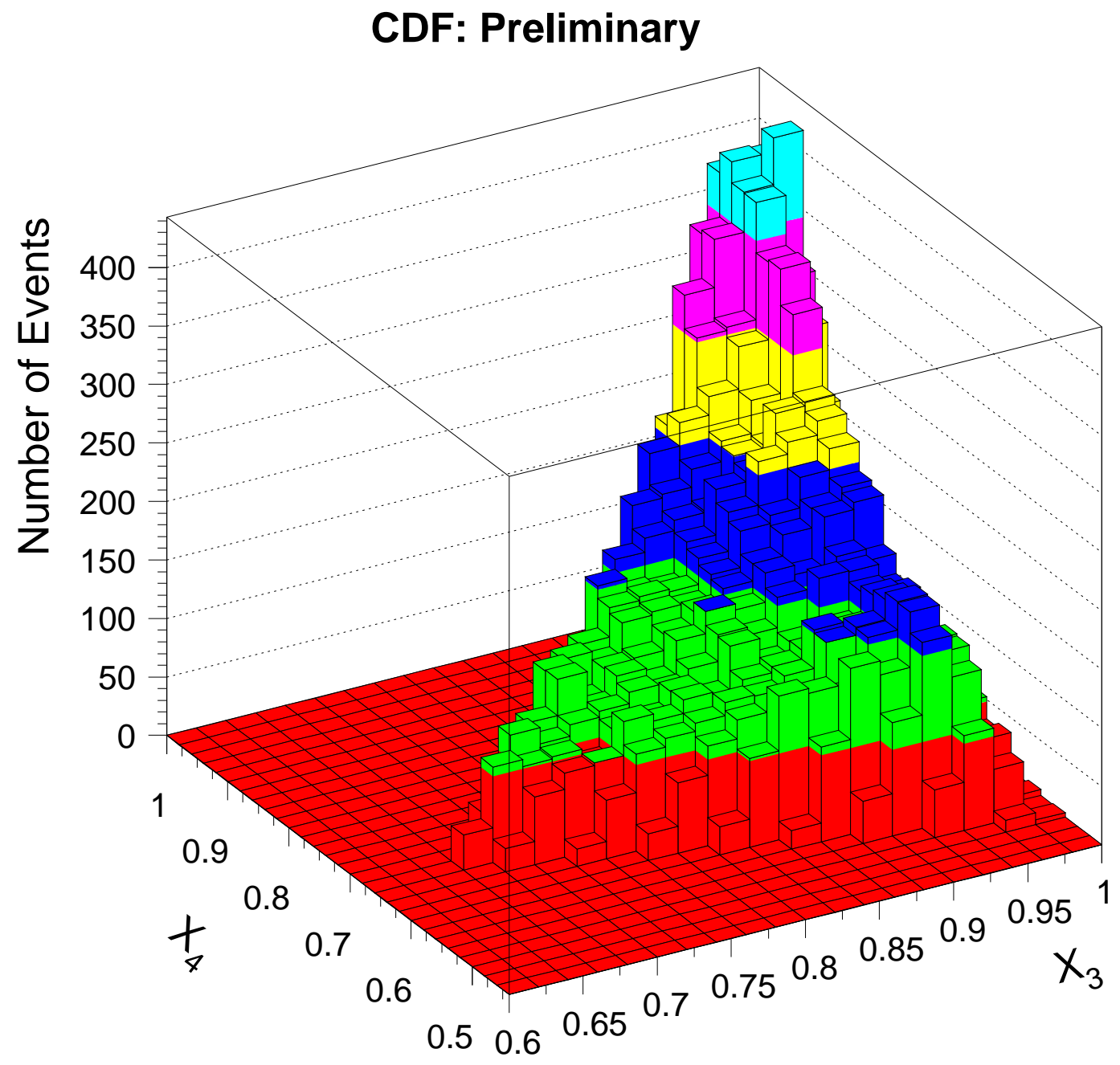

Figure E.13: The Dalitz distribution after variation of the distance between two resolved vertices by $-1 \mathrm{~cm}$. 


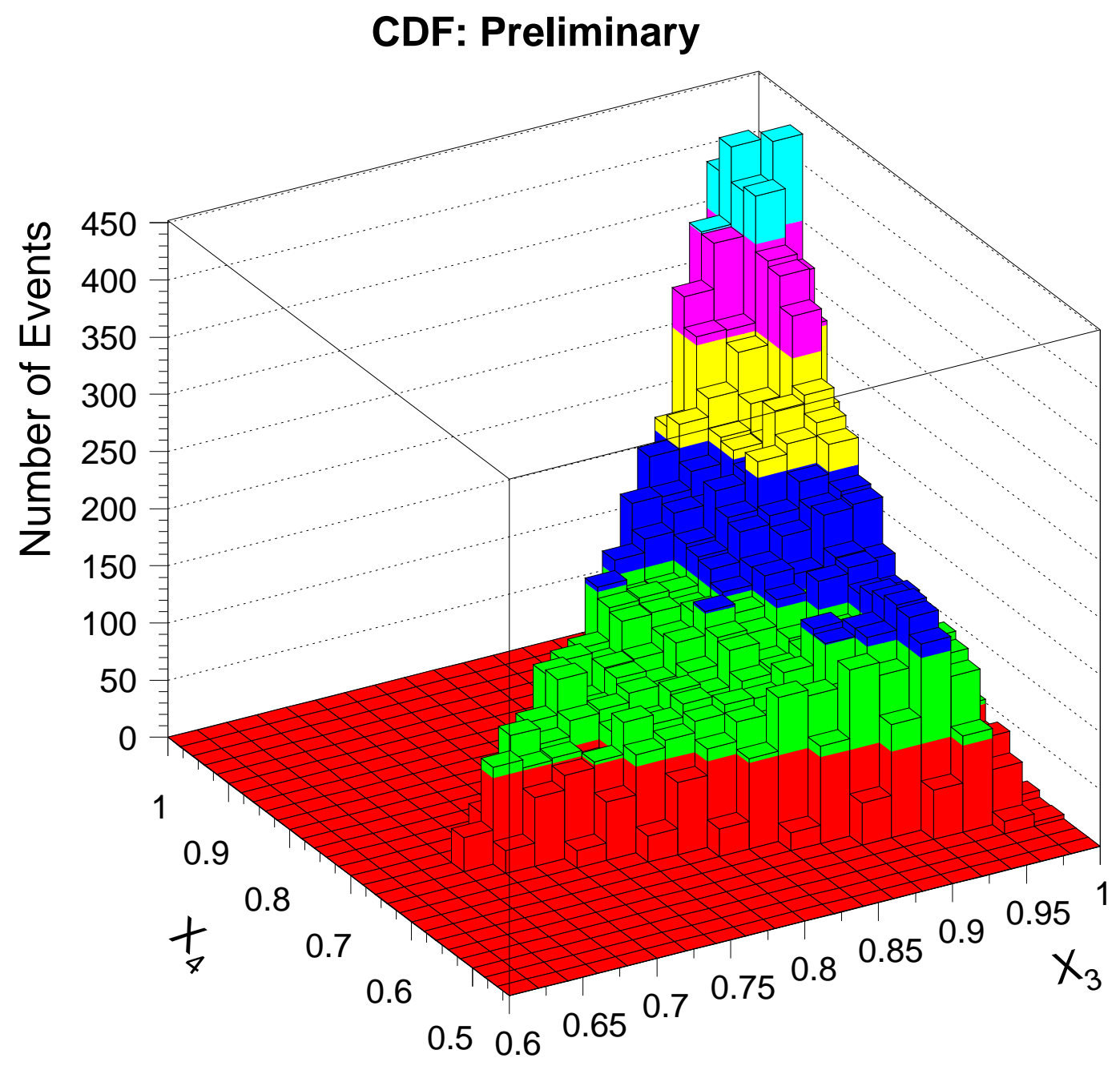

Figure E.14: The Dalitz distribution after variation of the distance between two resolved vertices by $+1 \mathrm{~cm}$. 


\section{Appendix F}

\section{The Cross Section in All Kinematically Allowed Bins in the Dalitz Plane}

In Chapter 6, the three-jet production cross section is discussed. For complete information, the cross section in all individual kinematically allowed bins in the Dalitz plane is tabulated in this appendix. To normalize the three-jet production cross sections calculated by the NLO calculation using CTEQ3 at $\alpha_{\mathrm{S}}=0.1160$ and CTEQ4M, the numerical values stated in Columns 5 and 6 have to be multiplied by 1.16 and 0.946 , respectively. 


\begin{tabular}{|c|c|c|cc|c|c|}
\hline $\mathrm{X}_{3}$ & $\mathrm{X}_{4}$ & $\begin{array}{c}\text { Cross Section } \\
(\mathrm{pb})\end{array}$ & \multicolumn{2}{|c|}{$\begin{array}{c}\text { Uncertainty } \\
(\mathrm{pb})\end{array}$} & $\begin{array}{c}\text { NLO Cross Section } \\
\text { CTEQ3 }(\mathrm{pb})\end{array}$ & $\begin{array}{c}\text { NLO Cross Section } \\
\text { CTEQ4M }(\mathrm{pb})\end{array}$ \\
\hline \hline 0.67 & 0.67 & 0.505 & -0.125 & +0.267 & $0.192 \pm 0.0753$ & $0.421 \pm 0.0531$ \\
0.69 & 0.65 & 0.325 & -0.128 & +0.090 & $0.345 \pm 0.0658$ & $0.260 \pm 0.0456$ \\
0.69 & 0.67 & 1.43 & -0.327 & +0.827 & $1.44 \pm 0.152$ & $1.51 \pm 0.105$ \\
0.69 & 0.69 & 0.661 & -0.0807 & +0.408 & $0.392 \pm 0.100$ & $0.636 \pm 0.0661$ \\
0.71 & 0.65 & 1.03 & -0.135 & +0.519 & $1.19 \pm 0.128$ & $1.13 \pm 0.0898$ \\
0.71 & 0.67 & 1.29 & -0.131 & +0.579 & $1.19 \pm 0.150$ & $1.29 \pm 0.102$ \\
0.71 & 0.69 & 1.55 & -0.185 & +0.611 & $1.26 \pm 0.149$ & $1.34 \pm 0.101$ \\
0.71 & 0.71 & 0.886 & -0.125 & +0.394 & $0.560 \pm 0.108$ & $0.754 \pm 0.0738$ \\
0.73 & 0.63 & 0.230 & -0.0517 & +0.126 & $0.172 \pm 0.0678$ & $0.321 \pm 0.0444$ \\
0.73 & 0.65 & 1.32 & -0.170 & +0.594 & $0.658 \pm 0.148$ & $1.47 \pm 0.108$ \\
0.73 & 0.67 & 1.41 & -0.188 & +0.590 & $0.823 \pm 0.122$ & $1.35 \pm 0.106$ \\
0.73 & 0.69 & 1.43 & -0.185 & +0.785 & $1.21 \pm 0.157$ & $1.56 \pm 0.104$ \\
0.73 & 0.71 & 1.48 & -0.276 & +0.805 & $1.29 \pm 0.159$ & $1.75 \pm 0.118$ \\
0.73 & 0.73 & 0.730 & -0.196 & +0.300 & $0.654 \pm 0.112$ & $0.594 \pm 0.0698$ \\
0.75 & 0.63 & 0.916 & -0.123 & +0.456 & $0.967 \pm 0.133$ & $1.05 \pm 0.0877$ \\
0.75 & 0.65 & 1.30 & -0.111 & +0.637 & $1.13 \pm 0.159$ & $1.39 \pm 0.107$ \\
\hline
\end{tabular}

Table F.1: The three-jet production cross section in every kinematically allowed bin in the Dalitz plane as a function of $\mathrm{X}_{3}$ and $\mathrm{X}_{4}$.

\begin{tabular}{|c|c|c|cc|c|c|}
\hline $\mathrm{X}_{3}$ & $\mathrm{X}_{4}$ & $\begin{array}{c}\text { Cross Section } \\
(\mathrm{pb})\end{array}$ & \multicolumn{2}{|c|}{$\begin{array}{c}\text { Uncertainty } \\
(\mathrm{pb})\end{array}$} & $\begin{array}{c}\text { NLO Cross Section } \\
\text { CTEQ3 }(\mathrm{pb})\end{array}$ & $\begin{array}{c}\text { NLO Cross Section } \\
\text { CTEQ4M }(\mathrm{pb})\end{array}$ \\
\hline \hline 0.75 & 0.67 & 1.22 & -0.296 & +0.700 & $1.39 \pm 0.162$ & $1.57 \pm 0.106$ \\
0.75 & 0.69 & 1.64 & -0.160 & +0.757 & $1.21 \pm 0.163$ & $1.43 \pm 0.109$ \\
0.75 & 0.71 & 2.13 & -0.332 & +0.789 & $1.41 \pm 0.166$ & $1.58 \pm 0.114$ \\
0.75 & 0.73 & 2.00 & -0.242 & +0.781 & $1.24 \pm 0.166$ & $1.77 \pm 0.123$ \\
0.75 & 0.75 & 0.800 & -0.067 & +0.436 & $0.558 \pm 0.117$ & $1.03 \pm 0.0894$ \\
0.77 & 0.61 & 0.386 & -0.130 & +0.236 & $0.234 \pm 0.0723$ & $0.355 \pm 0.0462$ \\
0.77 & 0.63 & 1.52 & -0.130 & +0.597 & $1.42 \pm 0.161$ & $1.54 \pm 0.108$ \\
0.77 & 0.65 & 1.67 & -0.193 & +0.750 & $1.25 \pm 0.159$ & $1.70 \pm 0.120$ \\
0.77 & 0.67 & 1.44 & -0.171 & +0.591 & $1.38 \pm 0.164$ & $1.53 \pm 0.110$ \\
0.77 & 0.69 & 1.64 & -0.317 & +0.754 & $1.56 \pm 0.171$ & $1.43 \pm 0.106$ \\
0.77 & 0.71 & 1.53 & -0.180 & +0.720 & $1.14 \pm 0.168$ & $1.53 \pm 0.113$ \\
0.77 & 0.73 & 1.49 & -0.226 & +0.725 & $1.72 \pm 0.177$ & $1.78 \pm 0.129$ \\
0.77 & 0.75 & 1.95 & -0.282 & +0.924 & $1.30 \pm 0.188$ & $1.82 \pm 0.118$ \\
0.77 & 0.77 & 1.05 & -0.0885 & +0.504 & $0.608 \pm 0.127$ & $0.948 \pm 0.0987$ \\
0.79 & 0.61 & 0.977 & $-0.0862+0.545$ & $0.720 \pm 0.100$ & $1.13 \pm 0.0907$ \\
0.79 & 0.63 & 1.58 & -0.125 & +0.811 & $0.968 \pm 0.168$ & $1.63 \pm 0.120$ \\
\hline
\end{tabular}

Table F.2: The three-jet production cross section in every kinematically allowed bin in the Dalitz plane as a function of $\mathrm{X}_{3}$ and $\mathrm{X}_{4}$. 


\begin{tabular}{|c|c|c|cr|c|c|}
\hline $\mathrm{X}_{3}$ & $\mathrm{X}_{4}$ & $\begin{array}{c}\text { Cross Section } \\
(\mathrm{pb})\end{array}$ & \multicolumn{2}{|c|}{$\begin{array}{c}\text { Uncertainty } \\
(\mathrm{pb})\end{array}$} & $\begin{array}{c}\text { NLO Cross Section } \\
\text { CTEQ3 }(\mathrm{pb})\end{array}$ & $\begin{array}{c}\text { NLO Cross Section } \\
\text { CTEQ4M }(\mathrm{pb})\end{array}$ \\
\hline \hline 0.79 & 0.65 & 1.70 & -0.305 & +0.756 & $1.24 \pm 0.169$ & $1.40 \pm 0.109$ \\
0.79 & 0.67 & 1.49 & -0.200 & +0.779 & $1.21 \pm 0.167$ & $1.80 \pm 0.117$ \\
0.79 & 0.69 & 1.81 & -0.119 & +0.686 & $1.39 \pm 0.178$ & $1.50 \pm 0.116$ \\
0.79 & 0.71 & 1.81 & -0.291 & +0.743 & $1.24 \pm 0.178$ & $1.67 \pm 0.124$ \\
0.79 & 0.73 & 1.67 & -0.252 & +0.606 & $1.85 \pm 0.192$ & $1.91 \pm 0.135$ \\
0.79 & 0.75 & 1.79 & -0.423 & +0.849 & $1.81 \pm 0.188$ & $2.02 \pm 0.138$ \\
0.79 & 0.77 & 1.81 & -0.444 & +1.23 & $1.86 \pm 0.197$ & $1.87 \pm 0.141$ \\
0.79 & 0.79 & 0.973 & -0.158 & +0.281 & $0.786 \pm 0.145$ & $1.07 \pm 0.0966$ \\
0.81 & 0.59 & 0.405 & -0.0933 & +0.255 & $0.280 \pm 0.0795$ & $0.350 \pm 0.0497$ \\
0.81 & 0.61 & 1.38 & -0.246 & +0.719 & $1.39 \pm 0.167$ & $1.72 \pm 0.113$ \\
0.81 & 0.63 & 1.60 & -0.168 & +0.608 & $1.86 \pm 0.182$ & $1.74 \pm 0.116$ \\
0.81 & 0.65 & 1.73 & -0.185 & +0.801 & $1.32 \pm 0.180$ & $1.70 \pm 0.122$ \\
0.81 & 0.67 & 1.99 & -0.203 & +0.713 & $1.73 \pm 0.186$ & $2.13 \pm 0.130$ \\
0.81 & 0.69 & 1.54 & -0.181 & +0.736 & $1.28 \pm 0.183$ & $1.88 \pm 0.135$ \\
0.81 & 0.71 & 1.72 & -0.383 & +0.900 & $1.58 \pm 0.192$ & $1.69 \pm 0.130$ \\
0.81 & 0.73 & 1.74 & -0.0983 & +0.556 & $1.71 \pm 0.198$ & $2.08 \pm 0.128$ \\
\hline
\end{tabular}

Table F.3: The three-jet production cross section in every kinematically allowed bin in the Dalitz plane as a function of $\mathrm{X}_{3}$ and $\mathrm{X}_{4}$.

\begin{tabular}{|c|c|c|c|c|c|c|}
\hline $\mathrm{X}_{3}$ & $\mathrm{X}_{4}$ & $\begin{array}{c}\text { Cross Section } \\
(\mathrm{pb})\end{array}$ & \multicolumn{2}{|c|}{$\begin{array}{l}\text { Uncertainty } \\
(\mathrm{pb})\end{array}$} & $\begin{array}{c}\text { NLO Cross Section } \\
\text { CTEQ3 }(\mathrm{pb})\end{array}$ & $\begin{array}{c}\text { NLO Cross Section } \\
\text { CTEQ4M (pb) }\end{array}$ \\
\hline 0.81 & 0.75 & 2.15 & $\begin{array}{c}-0.308 \\
\end{array}$ & +0.781 & $1.62 \pm 0.103$ & $1.82 \pm 0.128$ \\
\hline 0.81 & 0.77 & 2.12 & -0.209 & +0.977 & $1.81 \pm 0.210$ & $2.41 \pm 0.144$ \\
\hline 0.81 & 0.79 & 2.48 & -0.459 & +1.32 & $2.35 \pm 0.224$ & $2.29 \pm 0.149$ \\
\hline 0.81 & 0.81 & 1.08 & -0.162 & +0.544 & $1.51 \pm 0.165$ & $1.27 \pm 0.106$ \\
\hline 0.83 & 0.59 & 1.26 & -0.189 & +0.864 & $0.762 \pm 0.102$ & $1.21 \pm 0.101$ \\
\hline 0.83 & 0.61 & 1.60 & -0.390 & +0.964 & $1.48 \pm 0.180$ & $1.88 \pm 0.126$ \\
\hline 0.83 & 0.63 & 1.80 & -0.205 & +0.610 & $1.32 \pm 0.183$ & $1.91 \pm 0.134$ \\
\hline 0.83 & 0.65 & 1.48 & -0.153 & +0.492 & $1.36 \pm 0.182$ & $1.78 \pm 0.121$ \\
\hline 0.83 & 0.67 & 1.63 & -0.296 & +1.01 & $1.51 \pm 0.195$ & $1.95 \pm 0.132$ \\
\hline 0.83 & 0.69 & 1.77 & -0.370 & +0.875 & $1.48 \pm 0.191$ & $2.15 \pm 0.136$ \\
\hline 0.83 & 0.71 & 2.12 & -0.340 & +1.09 & $1.39 \pm 0.202$ & $1.95 \pm 0.140$ \\
\hline 0.83 & 0.73 & 2.12 & -0.290 & +0.980 & $1.65 \pm 0.204$ & $2.23 \pm 0.147$ \\
\hline 0.83 & 0.75 & 2.19 & -0.251 & +0.871 & $1.95 \pm 0.207$ & $2.37 \pm 0.142$ \\
\hline 0.83 & 0.77 & 2.29 & -0.143 & +1.06 & $1.88 \pm 0.222$ & $2.23 \pm 0.153$ \\
\hline 0.83 & 0.79 & 2.19 & -0.306 & +0.741 & $2.22 \pm 0.234$ & $2.59 \pm 0.169$ \\
\hline 0.83 & 0.81 & 2.58 & -0.283 & +1.15 & $2.19 \pm 0.245$ & $2.63 \pm 0.166$ \\
\hline
\end{tabular}

Table F.4: The three-jet production cross section in every kinematically allowed bin in the Dalitz plane as a function of $\mathrm{X}_{3}$ and $\mathrm{X}_{4}$. 


\begin{tabular}{|c|c|c|c|c|c|c|}
\hline $\mathrm{X}_{3}$ & $\mathrm{X}_{4}$ & $\begin{array}{c}\text { Cross Section } \\
(\mathrm{pb})\end{array}$ & \multicolumn{2}{|c|}{$\begin{array}{c}\text { Uncertainty } \\
(\mathrm{pb})\end{array}$} & $\begin{array}{c}\text { NLO Cross Section } \\
\text { CTEQ3 (pb) }\end{array}$ & $\begin{array}{l}\text { NLO Cross Section } \\
\text { CTEQ4M (pb) }\end{array}$ \\
\hline$\overline{\overline{0.83}}$ & $\overline{0.83}$ & $\overline{1.25}$ & 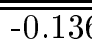 & 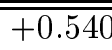 & $\bar{~} 1.23 \pm 0.176$ & $\bar{~} 1.40 \pm 0.119$ \\
\hline 0.85 & 0.57 & 0.283 & -0.108 & +0.209 & $0.137 \pm 0.0851$ & $0.275 \pm 0.0525$ \\
\hline 0.85 & 0.59 & 2.19 & -0.236 & +1.08 & $1.20 \pm 0.179$ & $2.36 \pm 0.144$ \\
\hline 0.85 & 0.61 & 1.53 & -0.43 & +0.954 & $1.43 \pm 0.189$ & $1.71 \pm 0.125$ \\
\hline 0.85 & 0.63 & 1.79 & -0.37 & +0.809 & $2.01 \pm 0.191$ & $2.08 \pm 0.131$ \\
\hline 0.85 & 0.65 & 1.77 & -0.23 & +0.732 & $1.91 \pm 0.199$ & $1.84 \pm 0.136$ \\
\hline 0.85 & 0.67 & 2.19 & -0.21 s & +0.928 & $1.80 \pm 0.203$ & $2.06 \pm 0.135$ \\
\hline 0.85 & 0.69 & 2.02 & -0.23 & +0.823 & $1.74 \pm 0.203$ & $1.94 \pm 0.140$ \\
\hline 0.85 & 0.71 & 2.33 & $-0.21\rangle$ & +0.909 & $1.74 \pm 0.209$ & $2.07 \pm 0.140$ \\
\hline 0.85 & 0.73 & 2.28 & -0.326 & +0.982 & $1.84 \pm 0.220$ & $2.37 \pm 0.154$ \\
\hline 0.85 & 0.75 & 2.09 & -0.447 & +1.25 & $2.10 \pm 0.224$ & $2.53 \pm 0.159$ \\
\hline 0.85 & 0.77 & 2.23 & -0.46 & +1.08 & $1.94 \pm 0.242$ & $2.55 \pm 0.169$ \\
\hline 0.85 & 0.79 & 2.24 & -0.26 & +1.12 & $2.70 \pm 0.253$ & $2.71 \pm 0.175$ \\
\hline 0.85 & 0.81 & 2.49 & $-0.31 s$ & +1.01 & $2.72 \pm 0.262$ & $3.17 \pm 0.177$ \\
\hline 0.85 & 0.83 & 2.93 & -0.470 & +1.34 & $2.81 \pm 0.280$ & $3.33 \pm 0.189$ \\
\hline 0.85 & 0.85 & 1.11 & -0.206 & +0.755 & $1.34 \pm 0.203$ & $1.64 \pm 0.143$ \\
\hline
\end{tabular}

Table F.5: The three-jet production cross section in every kinematically allowed bin in the Dalitz plane as a function of $\mathrm{X}_{3}$ and $\mathrm{X}_{4}$.

\begin{tabular}{|c|c|c|c|c|c|c|}
\hline $\mathrm{X}_{3}$ & $\mathrm{X}_{4}$ & $\begin{array}{c}\text { Cross Section } \\
(\mathrm{pb})\end{array}$ & \multicolumn{2}{|c|}{$\begin{array}{l}\text { Uncertainty } \\
(\mathrm{pb})\end{array}$} & $\begin{array}{c}\text { NLO Cross Section } \\
\text { CTEQ3 }(\mathrm{pb})\end{array}$ & $\begin{array}{c}\text { NLO Cross Section } \\
\text { CTEQ4M (pb) }\end{array}$ \\
\hline 0.87 & 0.57 & 1.32 & $\overline{-2-0.196}$ & +0.826 & $1.27 \pm 0.174$ & $\overline{1.50 \pm 0.126}$ \\
\hline 0.87 & 0.59 & 1.96 & -0.398 & +1.06 & $1.71 \pm 0.209$ & $2.04 \pm 0.138$ \\
\hline 0.87 & 0.61 & 1.75 & -0.447 & +1.03 & $1.68 \pm 0.208$ & $1.94 \pm 0.139$ \\
\hline 0.87 & 0.63 & 1.95 & -0.543 & +1.33 & $1.73 \pm 0.212$ & $2.04 \pm 0.138$ \\
\hline 0.87 & 0.65 & 1.82 & -0.234 & +0.921 & $1.64 \pm 0.209$ & $2.15 \pm 0.151$ \\
\hline 0.87 & 0.67 & 1.87 & -0.533 & +1.28 & $2.08 \pm 0.216$ & $2.26 \pm 0.154$ \\
\hline 0.87 & 0.69 & 2.13 & -0.299 & +0.969 & $1.70 \pm 0.218$ & $2.45 \pm 0.157$ \\
\hline 0.87 & 0.71 & 2.20 & -0.473 & +1.26 & $1.76 \pm 0.219$ & $2.32 \pm 0.148$ \\
\hline 0.87 & 0.73 & 2.60 & -0.322 & +0.916 & $2.04 \pm 0.238$ & $2.42 \pm 0.161$ \\
\hline 0.87 & 0.75 & 2.61 & -0.355 & +1.25 & $2.35 \pm 0.245$ & $2.90 \pm 0.169$ \\
\hline 0.87 & 0.77 & 2.56 & -0.577 & +1.36 & $2.07 \pm 0.261$ & $2.75 \pm 0.178$ \\
\hline 0.87 & 0.79 & 2.52 & -0.299 & +1.26 & $2.74 \pm 0.268$ & $2.88 \pm 0.176$ \\
\hline 0.87 & 0.81 & 2.89 & -0.222 & +1.28 & $2.24 \pm 0.275$ & $3.86 \pm 0.218$ \\
\hline 0.87 & 0.83 & 3.03 & -0.505 & +1.51 & $2.71 \pm 0.298$ & $3.87 \pm 0.211$ \\
\hline 0.87 & 0.85 & 3.21 & -0.288 & +1.41 & $3.33 \pm 0.320$ & $3.45 \pm 0.208$ \\
\hline 0.87 & 0.87 & 1.48 & -0.145 & +0.579 & $1.85 \pm 0.232$ & $2.12 \pm 0.163$ \\
\hline
\end{tabular}

Table F.6: The three-jet production cross section in every kinematically allowed bin in the Dalitz plane as a function of $\mathrm{X}_{3}$ and $\mathrm{X}_{4}$. 


\begin{tabular}{|c|c|c|cr|c|c|}
\hline $\mathrm{X}_{3}$ & $\mathrm{X}_{4}$ & $\begin{array}{c}\text { Cross Section } \\
(\mathrm{pb})\end{array}$ & \multicolumn{2}{|c|}{$\begin{array}{c}\text { Uncertainty } \\
(\mathrm{pb})\end{array}$} & $\begin{array}{c}\text { NLO Cross Section } \\
\text { CTEQ3 }(\mathrm{pb})\end{array}$ & $\begin{array}{c}\text { NLO Cross Section } \\
\text { CTEQ4M }(\mathrm{pb})\end{array}$ \\
\hline \hline 0.89 & 0.55 & 0.627 & -0.117 & +0.184 & $0.401 \pm 0.0962$ & $0.475 \pm 0.0637$ \\
0.89 & 0.57 & 2.23 & -0.247 & +0.871 & $1.47 \pm 0.214$ & $2.16 \pm 0.150$ \\
0.89 & 0.59 & 1.78 & -0.474 & +1.04 & $1.86 \pm 0.219$ & $2.29 \pm 0.160$ \\
0.89 & 0.61 & 2.28 & -0.468 & +1.28 & $1.79 \pm 0.219$ & $1.98 \pm 0.147$ \\
0.89 & 0.63 & 2.44 & -0.344 & +1.26 & $1.97 \pm 0.220$ & $2.35 \pm 0.159$ \\
0.89 & 0.65 & 2.13 & -0.510 & +1.10 & $2.07 \pm 0.229$ & $2.53 \pm 0.167$ \\
0.89 & 0.67 & 2.18 & -0.397 & +1.41 & $1.71 \pm 0.231$ & $2.51 \pm 0.164$ \\
0.89 & 0.69 & 2.60 & $-0.501+1.60$ & $2.14 \pm 0.242$ & $2.66 \pm 0.163$ \\
0.89 & 0.71 & 2.73 & $-0.325+1.15$ & $1.91 \pm 0.243$ & $2.44 \pm 0.162$ \\
0.89 & 0.73 & 2.50 & $-0.519+1.39$ & $2.47 \pm 0.256$ & $2.57 \pm 0.162$ \\
0.89 & 0.75 & 3.07 & $-0.303+1.08$ & $2.47 \pm 0.263$ & $2.90 \pm 0.179$ \\
0.89 & 0.77 & 3.18 & $-0.346+1.26$ & $2.43 \pm 0.289$ & $3.21 \pm 0.187$ \\
0.89 & 0.79 & 3.15 & $-0.360+1.12$ & $2.67 \pm 0.289$ & $3.31 \pm 0.197$ \\
0.89 & 0.81 & 3.03 & $-0.315+1.22$ & $2.98 \pm 0.307$ & $3.71 \pm 0.215$ \\
0.89 & 0.83 & 3.24 & $-0.590+1.52$ & $3.13 \pm 0.327$ & $3.98 \pm 0.224$ \\
0.89 & 0.85 & 3.53 & $-0.389+1.16$ & $3.20 \pm 0.336$ & $4.20 \pm 0.255$ \\
\hline
\end{tabular}

Table F.7: The three-jet production cross section in every kinematically allowed bin in the Dalitz plane as a function of $\mathrm{X}_{3}$ and $\mathrm{X}_{4}$.

\begin{tabular}{|c|c|c|cr|c|c|}
\hline $\mathrm{X}_{3}$ & $\mathrm{X}_{4}$ & $\begin{array}{c}\text { Cross Section } \\
(\mathrm{pb})\end{array}$ & \multicolumn{2}{|c|}{$\begin{array}{c}\text { Uncertainty } \\
(\mathrm{pb})\end{array}$} & $\begin{array}{c}\text { NLO Cross Section } \\
\text { CTEQ3 }(\mathrm{pb})\end{array}$ & $\begin{array}{c}\text { NLO Cross Section } \\
\text { CTEQ4M }(\mathrm{pb})\end{array}$ \\
\hline \hline 0.89 & 0.87 & 3.41 & -0.387 & +1.55 & $3.65 \pm 0.362$ & $5.24 \pm 0.264$ \\
0.89 & 0.89 & 1.90 & -0.214 & +0.775 & $2.41 \pm 0.279$ & $2.84 \pm 0.195$ \\
0.91 & 0.55 & 1.57 & -0.325 & +0.853 & $1.54 \pm 0.202$ & $1.60 \pm 0.132$ \\
0.91 & 0.57 & 2.04 & -0.448 & +1.19 & $1.98 \pm 0.228$ & $2.28 \pm 0.152$ \\
0.91 & 0.59 & 2.38 & -0.223 & +1.07 & $1.83 \pm 0.231$ & $2.56 \pm 0.165$ \\
0.91 & 0.61 & 2.60 & -0.422 & +1.33 & $1.96 \pm 0.239$ & $2.48 \pm 0.158$ \\
0.91 & 0.63 & 2.40 & -0.727 & +1.73 & $2.35 \pm 0.247$ & $2.38 \pm 0.155$ \\
0.91 & 0.65 & 2.35 & -0.631 & +1.49 & $2.09 \pm 0.244$ & $2.41 \pm 0.153$ \\
0.91 & 0.67 & 2.62 & $-0.363+1.44$ & $2.03 \pm 0.252$ & $2.76 \pm 0.173$ \\
0.91 & 0.69 & 2.47 & $-0.452+1.32$ & $2.38 \pm 0.262$ & $2.96 \pm 0.193$ \\
0.91 & 0.71 & 2.66 & $-0.513+1.31$ & $2.59 \pm 0.272$ & $2.57 \pm 0.180$ \\
0.91 & 0.73 & 3.03 & $-0.300+1.40$ & $2.40 \pm 0.283$ & $3.30 \pm 0.199$ \\
0.91 & 0.75 & 2.93 & $-0.477+1.30$ & $3.28 \pm 0.300$ & $3.68 \pm 0.214$ \\
0.91 & 0.77 & 3.52 & $-0.479+1.74$ & $3.60 \pm 0.296$ & $3.59 \pm 0.217$ \\
0.91 & 0.79 & 3.57 & $-0.566+1.84$ & $3.35 \pm 0.324$ & $3.97 \pm 0.227$ \\
0.91 & 0.81 & 3.31 & $-0.378+1.54$ & $3.84 \pm 0.352$ & $3.73 \pm 0.236$ \\
\hline
\end{tabular}

Table F.8: The three-jet production cross section in every kinematically allowed bin in the Dalitz plane as a function of $\mathrm{X}_{3}$ and $\mathrm{X}_{4}$. 


\begin{tabular}{|c|c|c|cr|c|c|}
\hline $\mathrm{X}_{3}$ & $\mathrm{X}_{4}$ & $\begin{array}{c}\text { Cross Section } \\
(\mathrm{pb})\end{array}$ & \multicolumn{2}{|c|}{$\begin{array}{c}\text { Uncertainty } \\
(\mathrm{pb})\end{array}$} & $\begin{array}{c}\text { NLO Cross Section } \\
\text { CTEQ3 }(\mathrm{pb})\end{array}$ & $\begin{array}{c}\text { NLO Cross Section } \\
\text { CTEQ4M }(\mathrm{pb})\end{array}$ \\
\hline \hline 0.91 & 0.83 & 3.31 & -0.430 & +1.19 & $3.72 \pm 0.346$ & $4.30 \pm 0.241$ \\
0.91 & 0.85 & 3.75 & -0.423 & +1.52 & $3.84 \pm 0.380$ & $5.58 \pm 0.279$ \\
0.91 & 0.87 & 4.53 & -0.384 & +1.33 & $4.88 \pm 0.422$ & $5.57 \pm 0.298$ \\
0.91 & 0.89 & 4.68 & -0.453 & +1.39 & $3.68 \pm 0.442$ & $6.07 \pm 0.314$ \\
0.91 & 0.91 & 2.23 & -0.296 & +0.628 & $2.92 \pm 0.332$ & $3.12 \pm 0.227$ \\
0.93 & 0.53 & 0.580 & -0.190 & +0.345 & $0.355 \pm 0.114$ & $0.489 \pm 0.0713$ \\
0.93 & 0.55 & 2.64 & -0.468 & +1.37 & $1.70 \pm 0.253$ & $3.02 \pm 0.188$ \\
0.93 & 0.57 & 2.59 & $-0.299+1.23$ & $2.06 \pm 0.270$ & $2.41 \pm 0.163$ \\
0.93 & 0.59 & 2.81 & $-0.719+1.28$ & $2.72 \pm 0.271$ & $2.49 \pm 0.177$ \\
0.93 & 0.61 & 2.72 & $-0.625+1.78$ & $2.47 \pm 0.265$ & $2.61 \pm 0.170$ \\
0.93 & 0.63 & 2.56 & $-0.455+1.30$ & $1.85 \pm 0.263$ & $2.66 \pm 0.170$ \\
0.93 & 0.65 & 2.60 & $-0.376+1.23$ & $3.05 \pm 0.278$ & $2.57 \pm 0.177$ \\
0.93 & 0.67 & 2.98 & $-0.339+1.37$ & $2.81 \pm 0.289$ & $3.11 \pm 0.198$ \\
0.93 & 0.69 & 2.94 & $-0.411+1.59$ & $2.70 \pm 0.294$ & $3.30 \pm 0.221$ \\
0.93 & 0.71 & 3.42 & $-0.435+1.69$ & $3.33 \pm 0.303$ & $3.57 \pm 0.213$ \\
0.93 & 0.73 & 3.39 & $-0.242+1.09$ & $2.97 \pm 0.320$ & $3.92 \pm 0.218$ \\
\hline
\end{tabular}

Table F.9: The three-jet production cross section in every kinematically allowed bin in the Dalitz plane as a function of $\mathrm{X}_{3}$ and $\mathrm{X}_{4}$.

\begin{tabular}{|c|c|c|c|c|c|c|}
\hline $\mathrm{X}_{3}$ & $\mathrm{X}_{4}$ & $\begin{array}{c}\text { Cross Section } \\
(\mathrm{pb})\end{array}$ & \multicolumn{2}{|c|}{$\begin{array}{l}\text { Uncertainty } \\
(\mathrm{pb})\end{array}$} & $\begin{array}{c}\text { NLO Cross Section } \\
\text { CTEQ3 }(\mathrm{pb})\end{array}$ & $\begin{array}{l}\text { NLO Cross Section } \\
\text { CTEQ4M (pb) }\end{array}$ \\
\hline 0.93 & 0.75 & 3.29 & -0.501 & +1.56 & $3.86 \pm 0.337$ & $3.65 \pm 0.218$ \\
\hline 0.93 & 0.77 & 3.82 & -0.358 & +1.75 & $3.63 \pm 0.371$ & $4.12 \pm 0.237$ \\
\hline 0.93 & 0.79 & 4.02 & -0.402 & +1.67 & $4.06 \pm 0.365$ & $4.01 \pm 0.238$ \\
\hline 0.93 & 0.81 & 3.35 & -0.415 & +1.35 & $3.78 \pm 0.375$ & $4.65 \pm 0.269$ \\
\hline 0.93 & 0.83 & 3.93 & -0.548 & +1.59 & $4.32 \pm 0.404$ & $5.66 \pm 0.282$ \\
\hline 0.93 & 0.85 & 4.34 & -0.543 & +1.49 & $5.15 \pm 0.442$ & $6.16 \pm 0.309$ \\
\hline 0.93 & 0.87 & 4.35 & -0.450 & +1.77 & $5.39 \pm 0.465$ & $6.27 \pm 0.323$ \\
\hline 0.93 & 0.89 & 4.98 & -0.878 & +1.46 & $7.03 \pm 0.507$ & $7.13 \pm 0.350$ \\
\hline 0.93 & 0.91 & 4.85 & -0.643 & +1.31 & $6.90 \pm 0.545$ & $8.61 \pm 0.415$ \\
\hline 0.93 & 0.93 & 2.74 & -0.369 & +0.868 & $4.31 \pm 0.445$ & $3.54 \pm 0.285$ \\
\hline 0.95 & 0.53 & 1.19 & -0.217 & +0.600 & $0.937 \pm 0.189$ & $1.14 \pm 0.118$ \\
\hline 0.95 & 0.55 & 1.88 & -0.299 & +0.896 & $1.61 \pm 0.240$ & $1.69 \pm 0.134$ \\
\hline 0.95 & 0.57 & 2.08 & -0.340 & +1.09 & $1.37 \pm 0.232$ & $1.66 \pm 0.149$ \\
\hline 0.95 & 0.59 & 1.97 & -0.587 & +1.29 & $1.45 \pm 0.229$ & $1.59 \pm 0.143$ \\
\hline 0.95 & 0.61 & 1.86 & -0.230 & +1.11 & $1.98 \pm 0.250$ & $1.89 \pm 0.153$ \\
\hline 0.95 & 0.63 & 1.91 & -0.467 & +1.08 & $1.51 \pm 0.225$ & $2.13 \pm 0.179$ \\
\hline
\end{tabular}

Table F.10: The three-jet production cross section in every kinematically allowed bin in the Dalitz plane as a function of $\mathrm{X}_{3}$ and $\mathrm{X}_{4}$. 


\begin{tabular}{|c|c|c|cr|c|c|}
\hline $\mathrm{X}_{3}$ & $\mathrm{X}_{4}$ & $\begin{array}{c}\text { Cross Section } \\
(\mathrm{pb})\end{array}$ & \multicolumn{2}{|c|}{$\begin{array}{c}\text { Uncertainty } \\
(\mathrm{pb})\end{array}$} & $\begin{array}{c}\text { NLO Cross Section } \\
\text { CTEQ3 }(\mathrm{pb})\end{array}$ & $\begin{array}{c}\text { NLO Cross Section } \\
\text { CTEQ4M }(\mathrm{pb})\end{array}$ \\
\hline \hline 0.95 & 0.65 & 2.27 & -0.274 & +1.24 & $2.11 \pm 0.272$ & $1.96 \pm 0.164$ \\
0.95 & 0.67 & 2.20 & -0.452 & +1.17 & $2.08 \pm 0.270$ & $2.51 \pm 0.186$ \\
0.95 & 0.69 & 2.45 & -0.275 & +1.01 & $2.32 \pm 0.289$ & $2.95 \pm 0.201$ \\
0.95 & 0.71 & 3.11 & -0.354 & +1.61 & $2.63 \pm 0.309$ & $2.83 \pm 0.205$ \\
0.95 & 0.73 & 3.24 & $-0.392+1.40$ & $2.86 \pm 0.346$ & $3.61 \pm 0.224$ \\
0.95 & 0.75 & 3.66 & $-0.463+1.84$ & $3.01 \pm 0.358$ & $4.34 \pm 0.261$ \\
0.95 & 0.77 & 3.81 & $-0.503+1.77$ & $4.08 \pm 0.384$ & $4.23 \pm 0.257$ \\
0.95 & 0.79 & 3.96 & $-0.397+1.48$ & $5.09 \pm 0.423$ & $5.82 \pm 0.311$ \\
0.95 & 0.81 & 4.88 & $-0.651+1.69$ & $5.42 \pm 0.446$ & $6.08 \pm 0.299$ \\
0.95 & 0.83 & 5.40 & $-0.766+2.01$ & $5.53 \pm 0.475$ & $6.62 \pm 0.336$ \\
0.95 & 0.85 & 5.01 & $-0.564+1.54$ & $5.65 \pm 0.497$ & $6.95 \pm 0.347$ \\
0.95 & 0.87 & 5.72 & $-0.727+1.59$ & $6.94 \pm 0.537$ & $8.35 \pm 0.405$ \\
0.95 & 0.89 & 5.45 & $-0.797+1.76$ & $6.41 \pm 0.590$ & $8.87 \pm 0.400$ \\
0.95 & 0.91 & 5.31 & $-0.827+1.35$ & $7.07 \pm 0.653$ & $9.15 \pm 0.435$ \\
0.95 & 0.93 & 5.09 & $-0.774+1.59$ & $8.05 \pm 0.651$ & $7.87 \pm 0.442$ \\
0.95 & 0.95 & 1.90 & $-0.358+0.794$ & $1.91 \pm 0.408$ & $2.18 \pm 0.255$ \\
\hline
\end{tabular}

Table F.11: The three-jet production cross section in every kinematically allowed bin in the Dalitz plane as a function of $\mathrm{X}_{3}$ and $\mathrm{X}_{4}$.

\begin{tabular}{|c|c|c|c|c|c|c|}
\hline $\mathrm{X}_{3}$ & $\mathrm{X}_{4}$ & $\begin{array}{c}\text { Cross Section } \\
(\mathrm{pb})\end{array}$ & \multicolumn{2}{|c|}{$\begin{array}{c}\text { Uncertainty } \\
(\mathrm{pb})\end{array}$} & $\begin{array}{c}\text { NLO Cross Section } \\
\text { CTEQ3 (pb) }\end{array}$ & $\begin{array}{c}\text { NLO Cross Section } \\
\text { CTEQ4M (pb) }\end{array}$ \\
\hline 0.97 & 0.51 & 0.161 & $\begin{array}{l}-0.119 \\
\end{array}$ & +0.121 & $0.0272 \pm 0.0496$ & $0.163 \pm 0.0221$ \\
\hline 0.97 & 0.53 & 0.698 & -0.203 & +0.453 & $0.482 \pm 0.136$ & $0.317 \pm 0.0813$ \\
\hline 0.97 & 0.55 & 0.854 & -0.200 & +0.616 & $0.619 \pm 0.136$ & $0.376 \pm 0.0824$ \\
\hline 0.97 & 0.57 & 0.749 & -0.232 & +0.506 & $0.508 \pm 0.138$ & $0.395 \pm 0.0892$ \\
\hline 0.97 & 0.59 & 1.04 & -0.209 & +0.629 & $0.472 \pm 0.150$ & $0.282 \pm 0.0837$ \\
\hline 0.97 & 0.61 & 0.855 & -0.166 & +0.640 & $0.544 \pm 0.151$ & $0.365 \pm 0.0871$ \\
\hline 0.97 & 0.63 & 0.985 & -0.125 & +0.586 & $0.539 \pm 0.163$ & $0.970 \pm 0.101$ \\
\hline 0.97 & 0.65 & 1.06 & -0.261 & +0.811 & $1.06 \pm 0.187$ & $0.709 \pm 0.117$ \\
\hline 0.97 & 0.67 & 1.04 & -0.155 & +0.528 & $0.664 \pm 0.187$ & $0.889 \pm 0.143$ \\
\hline 0.97 & 0.69 & 1.37 & -0.126 & +0.631 & $0.655 \pm 0.199$ & $0.828 \pm 0.139$ \\
\hline 0.97 & 0.71 & 1.53 & -0.319 & +0.851 & $1.00 \pm 0.222$ & $0.891 \pm 0.145$ \\
\hline 0.97 & 0.73 & 1.53 & -0.324 & +0.773 & $1.06 \pm 0.248$ & $1.02 \pm 0.151$ \\
\hline 0.97 & 0.75 & 1.92 & -0.317 & +1.01 & $2.17 \pm 0.279$ & $1.71 \pm 0.186$ \\
\hline 0.97 & 0.77 & 1.91 & -0.230 & +1.01 & $1.68 \pm 0.201$ & $1.58 \pm 0.196$ \\
\hline 0.97 & 0.79 & 2.27 & -0.289 & +0.970 & $2.04 \pm 0.303$ & $2.43 \pm 0.233$ \\
\hline 0.97 & 0.81 & 2.83 & -0.175 & +1.14 & $2.70 \pm 0.387$ & $3.75 \pm 0.277$ \\
\hline
\end{tabular}

Table F.12: The three-jet production cross section in every kinematically allowed bin in the Dalitz plane as a function of $\mathrm{X}_{3}$ and $\mathrm{X}_{4}$. 


\begin{tabular}{|c|c|c|cr|c|c|}
\hline $\mathrm{X}_{3}$ & $\mathrm{X}_{4}$ & $\begin{array}{c}\text { Cross Section } \\
(\mathrm{pb})\end{array}$ & \multicolumn{2}{|c|}{$\begin{array}{c}\text { Uncertainty } \\
(\mathrm{pb})\end{array}$} & $\begin{array}{c}\text { NLO Cross Section } \\
\text { CTEQ3 }(\mathrm{pb})\end{array}$ & $\begin{array}{c}\text { NLO Cross Section } \\
\text { CTEQ4M }(\mathrm{pb})\end{array}$ \\
\hline \hline 0.97 & 0.83 & 3.14 & -0.330 & +0.923 & $3.68 \pm 0.432$ & $4.51 \pm 0.292$ \\
0.97 & 0.85 & 3.84 & -0.466 & +1.23 & $5.67 \pm 0.522$ & $5.11 \pm 0.352$ \\
0.97 & 0.87 & 4.31 & -0.528 & +1.27 & $6.42 \pm 0.585$ & $7.28 \pm 0.416$ \\
0.97 & 0.89 & 5.35 & -0.629 & +1.17 & $7.79 \pm 0.680$ & $8.29 \pm 0.449$ \\
0.97 & 0.91 & 4.87 & -0.876 & +1.42 & $6.96 \pm 0.699$ & $8.61 \pm 0.500$ \\
0.97 & 0.93 & 3.79 & -0.906 & +1.21 & $4.63 \pm 0.666$ & $6.33 \pm 0.428$ \\
0.97 & 0.95 & 2.12 & -0.723 & +1.08 & $2.22 \pm 0.496$ & $3.38 \pm 0.347$ \\
0.97 & 0.97 & 0.262 & -0.118 & +0.178 & $0.0185 \pm 0.151$ & no data \\
0.99 & 0.51 & 0.0251 & -0.00993 & +0.0376 & $0.0275 \pm 0.0167$ & $0.0611 \pm 0.00673$ \\
0.99 & 0.53 & 0.0360 & -0.0308 & +0.0676 & no data & no data \\
0.99 & 0.55 & 0.102 & -0.0386 & +0.0889 & $0.0957 \pm 0.0228$ & $0.148 \pm 0.00900$ \\
0.99 & 0.57 & 0.0542 & -0.0131 & +0.0344 & $0.0280 \pm 0.0261$ & $0.200 \pm 0.0117$ \\
0.99 & 0.59 & 0.0521 & -0.0227 & +0.0598 & no data & no data \\
0.99 & 0.61 & 0.0727 & -0.0154 & +0.0495 & no data & $0.133 \pm 0.0116$ \\
0.99 & 0.63 & 0.0911 & -0.0405 & +0.0933 & no data & $0.0416 \pm 0.0183$ \\
0.99 & 0.65 & 0.0879 & -0.0896 & +0.0843 & no data & $0.306 \pm 0.0173$ \\
\hline
\end{tabular}

Table F.13: The three-jet production cross section in every kinematically allowed bin in the Dalitz plane as a function of $\mathrm{X}_{3}$ and $\mathrm{X}_{4}$.

\begin{tabular}{|c|c|c|cr|c|c|}
\hline $\mathrm{X}_{3}$ & $\mathrm{X}_{4}$ & $\begin{array}{c}\text { Cross Section } \\
(\mathrm{pb})\end{array}$ & \multicolumn{2}{|c|}{$\begin{array}{c}\text { Uncertainty } \\
(\mathrm{pb})\end{array}$} & $\begin{array}{c}\text { NLO Cross Section } \\
\text { CTEQ3 }(\mathrm{pb})\end{array}$ & $\begin{array}{c}\text { NLO Cross Section } \\
\text { CTEQ4M }(\mathrm{pb})\end{array}$ \\
\hline \hline 0.99 & 0.67 & 0.106 & -0.0580 & +0.152 & $0.0109 \pm 0.0445$ & $0.00608 \pm 0.0245$ \\
0.99 & 0.69 & 0.0961 & -0.0342 & +0.0480 & no data & no data \\
0.99 & 0.71 & 0.273 & -0.115 & +0.154 & no data & $0.338 \pm 0.0316$ \\
0.99 & 0.73 & 0.235 & -0.0640 & +0.159 & no data & $0.737 \pm 0.0331$ \\
0.99 & 0.75 & 0.247 & -0.0494 & +0.0812 & $0.0644 \pm 0.0879$ & no data \\
0.99 & 0.77 & 0.258 & -0.0801 & +0.200 & $0.243 \pm 0.105$ & $1.35 \pm 0.0611$ \\
0.99 & 0.79 & 0.230 & -0.0662 & +0.154 & no data & no data \\
0.99 & 0.81 & 0.629 & -0.124 & +0.225 & $0.400 \pm 0.130$ & $0.511 \pm 0.0703$ \\
0.99 & 0.83 & 0.472 & -0.0504 & +0.173 & $0.390 \pm 0.150$ & $0.0123 \pm 0.0812$ \\
0.99 & 0.85 & 0.498 & -0.158 & +0.215 & $0.320 \pm 0.159$ & $0.270 \pm 0.102$ \\
0.99 & 0.87 & 0.613 & -0.0554 & +0.412 & $0.567 \pm 0.193$ & $0.223 \pm 0.116$ \\
0.99 & 0.89 & 0.866 & -0.0780 & +0.292 & $1.32 \pm 0.235$ & $0.488 \pm 0.146$ \\
0.99 & 0.91 & 1.11 & -0.226 & +0.271 & $0.823 \pm 0.303$ & $3.98 \pm 0.171$ \\
0.99 & 0.93 & 1.08 & -0.293 & +0.406 & $0.770 \pm 0.362$ & $0.863 \pm 0.189$ \\
0.99 & 0.95 & 0.519 & -0.200 & +0.232 & no data & $0.145 \pm 0.0155$ \\
0.99 & 0.97 & 0.0576 & -0.0407 & +0.0844 & no data & no data \\
\hline
\end{tabular}

Table F.14: The three-jet production cross section in every kinematically allowed bin in the Dalitz plane as a function of $\mathrm{X}_{3}$ and $\mathrm{X}_{4}$. 


\section{Appendix G}

\section{The CDF Collaboration}

T. Affolder, ${ }^{23}$ H. Akimoto, ${ }^{45}$ A. Akopian, ${ }^{38}$ M. G. Albrow, ${ }^{11}$ P. Amaral, ${ }^{8}$

S. R. Amendolia, ${ }^{34}$ D. Amidei, ${ }^{26}$ K. Anikeev, ${ }^{24}$ J. Antos, ${ }^{1}$ G. Apollinari, ${ }^{11}$

T. Arisawa, ${ }^{45}$ T. Asakawa, ${ }^{43}$ W. Ashmanskas, ${ }^{8}$ F. Azfar, ${ }^{31}$ P. Azzi-Bacchetta, ${ }^{32}$

N. Bacchetta, ${ }^{32}$ M. W. Bailey, ${ }^{28}$ S. Bailey, ${ }^{16}$ P. de Barbaro, ${ }^{37}$ A. Barbaro-Galtieri, ${ }^{23}$

V. E. Barnes ${ }^{36}$ B. A. Barnett,${ }^{19}$ S. Baroiant,${ }^{5}$ M. Barone,${ }^{13}$ G. Bauer,${ }^{24}$ F. Bedeschi, ${ }^{34}$

S. Belforte, ${ }^{42}$ W. H. Bell, ${ }^{15}$ G. Bellettini, ${ }^{34}$ J. Bellinger, ${ }^{46}$ D. Benjamin, ${ }^{10}$

J. Bensinger ${ }^{4}$ A. Beretvas, ${ }^{11}$ J. P. Berge,${ }^{11}$ J. Berryhill,${ }^{8}$ B. Bevensee, ${ }^{33}$ A. Bhatti,${ }^{38}$

M. Binkley, ${ }^{11}$ D. Bisello, ${ }^{32}$ M. Bishai, ${ }^{11}$ R. E. Blair, ${ }^{2}$ C. Blocker, ${ }^{4}$ K. Bloom,${ }^{26}$

B. Blumenfeld, ${ }^{19}$ S. R. Blusk,${ }^{37}$ A. Bocci, ${ }^{34}$ A. Bodek,${ }^{37}$ W. Bokhari, ${ }^{33}$ G. Bolla,${ }^{36}$

Y. Bonushkin, ${ }^{6}$ D. Bortoletto,${ }^{36}$ J. Boudreau, ${ }^{35}$ A. Brandl, ${ }^{28}$ S. van den Brink, ${ }^{19}$

C. Bromberg, ${ }^{27}$ M. Brozovic, ${ }^{10}$ N. Bruner, ${ }^{28}$ E. Buckley-Geer, ${ }^{11}$ J. Budagov, ${ }^{9}$

H. S. Budd, ${ }^{37}$ K. Burkett, ${ }^{16}$ G. Busetto, ${ }^{32}$ A. Byon-Wagner, ${ }^{11}$ K. L. Byrum, ${ }^{2}$

P. Calafiura, ${ }^{23}$ M. Campbell, ${ }^{26}$ W. Carithers,${ }^{23}$ J. Carlson, ${ }^{26}$ D. Carlsmith,${ }^{46}$

W. Caskey, ${ }^{5}$ J. Cassada, ${ }^{37}$ A. Castro, ${ }^{32}$ D. Cauz,${ }^{42}$ A. Cerri, ${ }^{34}$ A. W. Chan, ${ }^{1}$

P. S. Chang, ${ }^{1}$ P. T. Chang, ${ }^{1}$ J. Chapman, ${ }^{26}$ C. Chen,${ }^{33}$ Y. C. Chen, ${ }^{1}$ M. -

T. Cheng, ${ }^{1}$ M. Chertok, ${ }^{40}$ G. Chiarelli, ${ }^{34}$ I. Chirikov-Zorin,${ }^{9}$ G. Chlachidze ${ }^{9}$

F. Chlebana, ${ }^{11}$ L. Christofek, ${ }^{18}$ M. L. Chu, ${ }^{1}$ Y. S. Chung, ${ }^{37}$ C. I. Ciobanu, ${ }^{29}$

A. G. Clark, ${ }^{14}$ A. Connolly, ${ }^{23}$ J. Conway, ${ }^{39}$ M. Cordelli, ${ }^{13}$ J. Cranshaw, ${ }^{41}$ D. Cronin- 
Hennessy, ${ }^{10}$ R. Cropp,${ }^{25}$ R. Culbertson,,${ }^{11}$ D. Dagenhart,${ }^{44}$ S. D'Auria,${ }^{15}$ F. DeJongh,${ }^{11}$ S. Dell'Agnello, ${ }^{13}$ M. Dell'Orso, ${ }^{34}$ L. Demortier ${ }^{38}$ M. Deninno, ${ }^{3}$ P. F. Derwent,${ }^{11}$ T. Devlin, ${ }^{39}$ J. R. Dittmann, ${ }^{11}$ S. Donati, ${ }^{34}$ J. Done,${ }^{40}$ T. Dorigo, ${ }^{16}$ N. Eddy,${ }^{18}$ K. Einsweiler, ${ }^{23}$ J. E. Elias, ${ }^{11}$ E. Engels, Jr., ${ }^{35}$ R. Erbacher, ${ }^{11}$ D. Errede, ${ }^{18}$ S. Errede, ${ }^{18}$ Q. Fan,${ }^{37}$ R. G. Feild, ${ }^{47}$ J. P. Fernandez,${ }^{11}$ C. Ferretti, ${ }^{34}$ R. D. Field, ${ }^{12}$ I. Fiori, ${ }^{3}$ B. Flaugher, ${ }^{11}$ G. W. Foster,${ }^{11}$ M. Franklin, ${ }^{16}$ J. Freeman,,${ }^{11}$ J. Friedman, ${ }^{24}$ Y. Fukui, ${ }^{22}$ I. Furic, ${ }^{24}$ S. Galeotti, ${ }^{34}$ M. Gallinaro, ${ }^{38}$ T. Gao, ${ }^{33}$ M. Garcia-Sciveres, ${ }^{23}$ A. F. Garfinkel, ${ }^{36}$ P. Gatti, ${ }^{32}$ C. Gay, ${ }^{47}$ D. W. Gerdes,${ }^{26}$ P. Giannetti, ${ }^{34}$ P. Giromini, ${ }^{13}$ V. Glagolev, ${ }^{9}$ D. Glenzinski, ${ }^{11}$ M. Gold, ${ }^{28}$ J. Goldstein, ${ }^{11}$ A. Gordon, ${ }^{16}$ I. Gorelov, ${ }^{28}$ A. T. Goshaw, ${ }^{10}$ Y. Gotra, ${ }^{35}$ K. Goulianos,${ }^{38}$ C. Green, ${ }^{36}$ G. Grim,${ }^{5}$ P. Gris,${ }^{11}$ L. Groer, ${ }^{39}$ C. Grosso-Pilcher, ${ }^{8}$ M. Guenther, ${ }^{36}$ G. Guillian, ${ }^{26}$ J. Guimaraes da Costa, ${ }^{16}$ R. M. Haas, ${ }^{12}$ C. Haber, ${ }^{23}$ E. Hafen, ${ }^{24}$ S. R. Hahn, ${ }^{11}$ C. Hall,${ }^{16}$ T. Handa, ${ }^{17}$ R. Handler, ${ }^{46}$ W. Hao ${ }^{41}$ F. Happacher, ${ }^{13}$ K. Hara,${ }^{43}$ A. D. Hardman,${ }^{36}$ R. M. Harris,${ }^{11}$ F. Hartmann, ${ }^{20}$ K. Hatakeyama, ${ }^{38}$ J. Hauser ${ }^{6}$ J. Heinrich,,${ }^{33}$ A. Heiss,${ }^{20}$ M. Herndon,,${ }^{19}$ C. Hill, ${ }^{5}$ K. D. Hoffman,${ }^{36}$ C. Holck,${ }^{33}$ R. Hollebeek, ${ }^{33}$ L. Holloway, ${ }^{18}$ R. Hughes,${ }^{29}$ J. Huston, ${ }^{27}$ J. Huth, ${ }^{16}$ H. Ikeda, ${ }^{43}$ J. Incandela, ${ }^{11}$ G. Introzzi, ${ }^{34}$ J. Iwai, ${ }^{45}$ Y. Iwata, ${ }^{17}$ E. James,${ }^{26}$ H. Jensen, ${ }^{11}$ M. Jones ${ }^{33}$ U. Joshi, ${ }^{11}$ H. Kambara,${ }^{14}$ T. Kamon, ${ }^{40}$ T. Kaneko, ${ }^{43}$ K. Karr, ${ }^{44}$ H. Kasha, ${ }^{47}$ Y. Kato, ${ }^{30}$ T. A. Keaffaber, ${ }^{36}$ K. Kelley, ${ }^{24}$ M. Kelly, ${ }^{26}$ R. D. Kennedy, ${ }^{11}$ R. Kephart,${ }^{11}$ D. Khazins,,${ }^{10}$ T. Kikuchi,${ }^{43}$ B. Kilminster, ${ }^{37}$ B. J. Kim, ${ }^{21}$ D. H. Kim,${ }^{21}$ H. S. Kim,${ }^{18}$ M. J. Kim, ${ }^{21}$ S. H. Kim,${ }^{43}$ Y. K. Kim ${ }^{23}$ M. Kirby, ${ }^{10}$ M. Kirk ${ }^{4}$ L. Kirsch, ${ }^{4}$ S. Klimenko, ${ }^{12}$ P. Koehn,${ }^{29}$ A. Köngeter, ${ }^{20}$ K. Kondo, ${ }^{45}$ J. Konigsberg, ${ }^{12}$ K. Kordas, ${ }^{25}$ A. Korn, ${ }^{24}$ A. Korytov, ${ }^{12}$ E. Kovacs, ${ }^{2}$ J. Kroll, ${ }^{33}$ M. Kruse,${ }^{37}$ S. E. Kuhlmann, ${ }^{2}$ K. Kurino, ${ }^{17}$ T. Kuwabara, ${ }^{43}$ A. T. Laasanen, ${ }^{36}$ N. Lai ${ }^{8}$ S. Lami, ${ }^{38}$ S. Lammel,,${ }^{11}$ J. I. Lamoureux,${ }^{4}$ J. Lancaster,${ }^{10}$ M. Lancaster, ${ }^{23}$ R. Lander,${ }^{5}$ G. Latino,${ }^{34}$ T. LeCompte, ${ }^{2}$ A. M. Lee IV,${ }^{10}$ K. Lee,${ }^{41}$ S. Leone,${ }^{34}$ J. D. Lewis, ${ }^{11}$ M. Lindgren,${ }^{6}$ T. M. Liss,${ }^{18}$ J. B. Liu ${ }^{37}$ Y. C. Liu,${ }^{1}$ N. Lockyer ${ }^{33}$ J. Loken, ${ }^{31}$ M. Loreti,${ }^{32}$ D. Lucchesi ${ }^{32}$ P. Lukens, ${ }^{11}$ S. Lusin, ${ }^{46}$ L. Lyons, ${ }^{31}$ J. Lys,${ }^{23}$ R. Madrak,${ }^{16}$ K. Maeshima,${ }^{11}$ P. Maksimovic,${ }^{16}$ L. Malferrari, ${ }^{3}$ 
M. Mangano, ${ }^{34}$ M. Mariotti, ${ }^{32}$ G. Martignon, ${ }^{32}$ A. Martin,,${ }^{47}$ J. A. J. Matthews,${ }^{28}$ J. Mayer, ${ }^{25}$ P. Mazzanti, ${ }^{3}$ K. S. McFarland,${ }^{37}$ P. McIntyre ${ }^{40}$ E. McKigney,${ }^{33}$ M. Menguzzato, ${ }^{32}$ A. Menzione,${ }^{34}$ C. Mesropian,${ }^{38}$ A. Meyer,${ }^{11}$ T. Miao, ${ }^{11}$ R. Miller,${ }^{27}$ J. S. Miller, ${ }^{26}$ H. Minato, ${ }^{43}$ S. Miscetti, ${ }^{13}$ M. Mishina, ${ }^{22}$ G. Mitselmakher ${ }^{12}$ N. Moggi, ${ }^{3}$ E. Moore,${ }^{28}$ R. Moore ${ }^{26}$ Y. Morita, ${ }^{22}$ T. Moulik, ${ }^{24}$ M. Mulhearn,${ }^{24}$ A. Mukherjee, ${ }^{11}$ T. Muller,${ }^{20}$ A. Munar,${ }^{34}$ P. Murat,${ }^{11}$ S. Murgia, ${ }^{27}$ J. Nachtman,${ }^{6}$ S. Nahn, ${ }^{47}$ H. Nakada, ${ }^{43}$ T. Nakaya, ${ }^{8}$ I. Nakano, ${ }^{17}$ C. Nelson,,${ }^{11}$ T. Nelson, ${ }^{11}$ C. Neu, ${ }^{29}$ D. Neuberger ${ }^{20}$ C. Newman-Holmes,${ }^{11}$ C.-Y. P. Ngan,${ }^{24}$ H. Niu, ${ }^{4}$ L. Nodulman, ${ }^{2}$ A. Nomerotski, ${ }^{12}$ S. H. Oh, ${ }^{10}$ T. Ohmoto,${ }^{17}$ T. Ohsugi, ${ }^{17}$ R. Oishi,${ }^{43}$ T. Okusawa,${ }^{30}$ J. Olsen, ${ }^{46}$ W. Orejudos,${ }^{23}$ C. Pagliarone, ${ }^{34}$ F. Palmonari, ${ }^{34}$ R. Paoletti,${ }^{34}$ V. Papadimitriou, ${ }^{41}$ S. P. Pappas,${ }^{47}$ D. Partos,${ }^{4}$ J. Patrick, ${ }^{11}$ G. Pauletta,${ }^{42}$ M. Paulini, ${ }^{(*)}{ }^{23}$ C. Paus, ${ }^{24}$ L. Pescara,${ }^{32}$ T. J. Phillips, ${ }^{10}$ G. Piacentino, ${ }^{34}$ K. T. Pitts,${ }^{18}$ A. Pompos,${ }^{36}$ L. Pondrom, ${ }^{46}$ G. Pope,${ }^{35}$ M. Popovic,${ }^{25}$ F. Prokoshin, ${ }^{9}$ J. Proudfoot,${ }^{2}$ F. Ptohos ${ }^{13}$ O. Pukhov, ${ }^{9}$ G. Punzi ${ }^{34}$ K. Ragan,${ }^{25}$ A. Rakitine,${ }^{24}$ D. Reher,${ }^{23}$ A. Reichold,${ }^{31}$ A. Ribon, ${ }^{32}$ W. Riegler,${ }^{16}$ F. Rimondi, ${ }^{3}$ L. Ristori, ${ }^{34}$ M. Riveline,${ }^{25}$ W. J. Robertson, ${ }^{10}$ A. Robinson,${ }^{25}$ T. Rodrigo ${ }^{7}$ S. Rolli, ${ }^{44}$ L. Rosenson,${ }^{24}$ R. Roser,${ }^{11}$ R. Rossin, ${ }^{32}$ A. Roy ${ }^{24}$ A. Safonov, ${ }^{38}$ R. St. Denis, ${ }^{15}$ W. K. Sakumoto, ${ }^{37}$ D. Saltzberg, ${ }^{6}$ C. Sanchez, ${ }^{29}$ A. Sansoni, ${ }^{13}$ L. Santi, ${ }^{42}$ H. Sato,${ }^{43}$ P. Savard, ${ }^{25}$ P. Schlabach,,${ }^{11}$ E. E. Schmidt ${ }^{11}$ M. P. Schmidt, ${ }^{47}$ M. Schmitt,${ }^{16}$ L. Scodellaro, ${ }^{32}$ A. Scott,${ }^{6}$ A. Scribano, ${ }^{34}$ S. Segler, ${ }^{11}$ S. Seidel,${ }^{28}$ Y. Seiya, ${ }^{43}$ A. Semenov, ${ }^{9}$ F. Semeria,${ }^{3}$ T. Shah, ${ }^{24}$ M. D. Shapiro, ${ }^{23}$ P. F. Shepard, ${ }^{35}$ T. Shibayama, ${ }^{43}$ M. Shimojima, ${ }^{43}$ M. Shochet,${ }^{8}$ J. Siegrist, ${ }^{23}$ G. Signorelli, ${ }^{34}$ A. Sill, ${ }^{41}$ P. Sinervo, ${ }^{25}$ P. Singh, ${ }^{18}$ A. J. Slaughter, ${ }^{47}$ K. Sliwa, ${ }^{44}$ C. Smith, ${ }^{19}$ F. D. Snider,${ }^{11}$ A. Solodsky, ${ }^{38}$ J. Spalding,,${ }^{11}$ T. Speer, ${ }^{14}$ P. Sphicas, ${ }^{24}$ F. Spinella, ${ }^{34}$ M. Spiropulu,${ }^{16}$ L. Spiegel,${ }^{11}$ J. Steele,${ }^{46}$ A. Stefanini, ${ }^{34}$ J. Strologas, ${ }^{18}$ F. Strumia, ${ }^{14}$ D. Stuart, ${ }^{11}$ K. Sumorok,${ }^{24}$ T. Suzuki, ${ }^{43}$ T. Takano, ${ }^{30}$ R. Takashima, ${ }^{17}$ K. Takikawa ${ }^{43}$ P. Tamburello, ${ }^{10}$ M. Tanaka, ${ }^{43}$ B. Tannenbaum,${ }^{6}$ W. Taylor, ${ }^{25}$ M. Tecchio, ${ }^{26}$ R. Tesarek, ${ }^{11}$ P. K. Teng, ${ }^{1}$ K. Terashi, ${ }^{38}$ S. Tether ${ }^{24}$ A. S. Thompson, ${ }^{15}$ R. Thurman-Keup, ${ }^{2}$ P. Tipton, ${ }^{37}$ S. Tkaczyk,${ }^{11}$ 
K. Tollefson,${ }^{37}$ A. Tollestrup, ${ }^{11}$ H. Toyoda,${ }^{30}$ W. Trischuk, ${ }^{25}$ J. F. de Troconiz, ${ }^{16}$ J. Tseng, ${ }^{24}$ N. Turini, ${ }^{34}$ F. Ukegawa,${ }^{43}$ T. Vaiciulis,${ }^{37}$ J. Valls,${ }^{39}$ S. Vejcik III, ${ }^{11}$ G. Velev, ${ }^{11}$ R. Vidal,${ }^{11}$ R. Vilar,${ }^{7}$ I. Volobouev,${ }^{23}$ D. Vucinic,${ }^{24}$ R. G. Wagner, ${ }^{2}$ R. L. Wagner, ${ }^{11}$ J. Wahl, ${ }^{8}$ N. B. Wallace,${ }^{39}$ A. M. Walsh,${ }^{39}$ C. Wang,,${ }^{10}$ M. J. Wang, ${ }^{1}$ T. Watanabe, ${ }^{43}$ D. Waters, ${ }^{31}$ T. Watts,${ }^{39}$ R. Webb,${ }^{40}$ H. Wenzel,${ }^{20}$ W. C. Wester III,${ }^{11}$ A. B. Wicklund, ${ }^{2}$ E. Wicklund, ${ }^{11}$ T. Wilkes,${ }^{5}$ H. H. Williams, ${ }^{33}$ P. Wilson, ${ }^{11}$ B. L. Winer, ${ }^{29}$ D. Winn, ${ }^{26}$ S. Wolbers, ${ }^{11}$ D. Wolinski, ${ }^{26}$ J. Wolinski, ${ }^{27}$ S. Wolinski, ${ }^{26}$ S. Worm,${ }^{28}$ X. Wu,${ }^{14}$ J. Wyss,${ }^{34}$ A. Yagil,${ }^{11}$ W. Yao,${ }^{23}$ G. P. Yeh, ${ }^{11}$ P. Yeh, ${ }^{1}$ J. Yoh,${ }^{11}$ C. Yosef,,${ }^{27}$ T. Yoshida, ${ }^{30}$ I. Yu, ${ }^{21}$ S. Yu, ${ }^{33}$ Z. Yu, ${ }^{47}$ A. Zanetti, ${ }^{42}$ F. Zetti, ${ }^{23}$ and S. Zucchelli ${ }^{3}$

\section{(CDF Collaboration)}

1 Institute of Physics, Academia Sinica, Taipei, Taiwan 11529, Republic of China

2 Argonne National Laboratory, Argonne, Illinois 60439

3 Istituto Nazionale di Fisica Nucleare, University of Bologna, I-40127 Bologna, Italy

4 Brandeis University, Waltham, Massachusetts 02254

5 University of California at Davis, Davis, California 95616

6 University of California at Los Angeles, Los Angeles, California 90024

7 Instituto de Fisica de Cantabria, CSIC-University of Cantabria, 39005 Santander, Spain

8 Enrico Fermi Institute, University of Chicago, Chicago, Illinois 60637

9 Joint Institute for Nuclear Research, RU-141980 Dubna, Russia

10 Duke University, Durham, North Carolina 27708

11 Fermi National Accelerator Laboratory, Batavia, Illinois 60510

12 University of Florida, Gainesville, Florida 32611

13 Laboratori Nazionali di Frascati, Istituto Nazionale di Fisica Nucleare, I-00044 Frascati, Italy

14 University of Geneva, CH-1211 Geneva 4, Switzerland

15 Glasgow University, Glasgow G12 8QQ, United Kingdom

16 Harvard University, Cambridge, Massachusetts 02138 
17 Hiroshima University, Higashi-Hiroshima 724, Japan

18 University of Illinois, Urbana, Illinois 61801

19 The Johns Hopkins University, Baltimore, Maryland 21218

20 Institut für Experimentelle Kernphysik, Universität Karlsruhe, 76128 Karlsruhe, Germany

21 Center for High Energy Physics: Kyungpook National University, Taegu 702-701; Seoul National University, Seoul 151-742; and SungKyunKwan University, Suwon 440-746; Korea

22 High Energy Accelerator Research Organization (KEK), Tsukuba, Ibaraki 305, Japan

23 Ernest Orlando Lawrence Berkeley National Laboratory, Berkeley, California 94720

24 Massachusetts Institute of Technology, Cambridge, Massachusetts 02139

25

Institute of Particle Physics: McGill University, Montreal H3A 2T8; and University of Toronto, Toronto M5S

1A7; Canada

26 University of Michigan, Ann Arbor, Michigan 48109

27 Michigan State University, East Lansing, Michigan 48824

28 University of New Mexico, Albuquerque, New Mexico 87131

29 The Ohio State University, Columbus, Ohio 43210

30 Osaka City University, Osaka 588, Japan

31 University of Oxford, Oxford OX1 3RH, United Kingdom

32 Universita di Padova, Istituto Nazionale di Fisica Nucleare, Sezione di Padova, I-35131 Padova, Italy

33 University of Pennsylvania, Philadelphia, Pennsylvania 19104

34 Istituto Nazionale di Fisica Nucleare, University and Scuola Normale Superiore of Pisa, I-56100 Pisa, Italy

35 University of Pittsburgh, Pittsburgh, Pennsylvania 15260

36 Purdue University, West Lafayette, Indiana 47907

37 University of Rochester, Rochester, New York 14627

38 Rockefeller University, New York, New York 10021

39 Rutgers University, Piscataway, New Jersey 08855

40 Texas A $8 M$ University, College Station, Texas 77843

41 Texas Tech University, Lubbock, Texas 79409

42 Istituto Nazionale di Fisica Nucleare, University of Trieste/ Udine, Italy 
43 University of Tsukuba, Tsukuba, Ibaraki 305, Japan

44 Tufts University, Medford, Massachusetts 02155

45 Waseda University, Tokyo 169, Japan

46 University of Wisconsin, Madison, Wisconsin 53706

47 Yale University, New Haven, Connecticut 06520

(*) Now at Carnegie Mellon University, Pittsburgh, Pennsylvania 15213 


\section{References}

[1] Mendeleev, D. I., Principles of Chemistry (1869).

[2] Maxwell, J. C., A Treatise on Electricity and Magnetism (1873).

[3] Thompson, J. J., Phil. Mag. 44, 293 (1897).

[4] Rutherford, E., Phil. Mag. 21, 669 (1911).

[5] Chadwick, J., Proc. Roy. Soc. A136, 692 (1932).

[6] Dirac, P. A. M., Proc. Roy. Soc. A117, 610 (1928).

[7] Anderson, C. D., Phys. Rev. 43, 491 (1933).

[8] Neddermeyer, S. H. and C. D. Anderson, Phys. Rev. 51, 884 (1937).

[9] Reines, F. and C. L. Cowan, Jr., Phys. Rev. 92, 830 (1953).

[10] Gell-Mann, M., Phys. Lett. 8, 214 (1964).

[11] Zweig, G., CERN Report 8419/Th 412 (1964).

[12] Arnison, G., et al. (UA1 Collaboration), Phys. Lett. B122, 103 (1983).

[13] Arnison, G., et al. (UA1 Collaboration), Phys. Lett. B126, 398 (1983).

[14] Abe, F., et al. (CDF Collaboration), Phys. Rev. Lett. 74, 2626 (1995).

[15] Abachi, S., et al. (D0 Collaboration), Phys. Rev. Lett. 74, 2632 (1995). 
[16] Noether, E., Nachr. kgl. Ges. Wiss. Göttingen, 235 (1918).

[17] Weinberg, S., The Quantum Theory of Fields I, Cambridge University Press, Cambridge (1995).

[18] Englert, F. and R. Brout, Phys. Rev. Lett. 13, 321 (1964);

Higgs, P., Phys. Rev. Lett. 13, 508 (1964).

[19] Glashow, S. L., Nucl. Phys. 22, 579 (1961);

Salam, A., Proc. 8th NOBEL Symposium, N. Svartholm, Ed., 367, Stockholm (1968);

Weinberg, S., Phys. Rev. Lett. 19, 1264 (1967).

[20] Roe, B., Particle Physics at the New Millenium, Springer, New York (1996).

[21] Groom, D., et al. (Particle Data Group), European Physical Journal C15, 1 $(2000)$.

[22] Drees, J., Proc. of the 1981 International Symposium on Lepton and Photon Interactions at High Energies, W. Pfeil, Ed., 474, Bonn (1981).

[23] Barger, V. and R. Phillips, Collider Physics, Addison-Wesley, New York (1997).

[24] Halzen, F. and A. Martin, Quarks and Leptons, John Wiley and Sons, New York (1984).

[25] Quigg, C., Gauge Theories of the Strong, Weak, and Electromagnetic Interactions, Benjamin-Cummings, New York (1983).

[26] 't Hooft, G., Nucl. Phys. B33, 173 (1971);

't Hooft, G., Nucl. Phys. B35, 167 (1971).

[27] Ellis, R., W. Sterling, and B. Webber, QCD and Collider Physics, Cambridge University Press, Cambridge (1996). 
[28] Ellis, R., Z. Kunszt, and D. Soper, Phys. Rev. Lett. 64, 2121 (1990).

[29] Aitchison, I. and A. Hey, Gauge Theories in Particle Physics, Adam Hilger, Bristol (1989).

[30] Altarelli, G. and G. Parisi, Nucl. Phys. B126, 298 (1977);

Gribov, V. and L. Lipatov, Sov. J. Nucl. Phys. 15, 438 (1972);

Dokshitzer, Y., Sov. Phys. JETP 46, 641 (1972).

[31] Hanson, G., et al., Phys. Rev. Lett. 35, 1609 (1975).

[32] Andersson, B., The Lund Model, Cambridge University Press, Cambridge (1998).

[33] Newman, H. (Mark-J Collaboration), Ch. Berger (PLUTO Collaboration), G. Wolf (TASSO Collaboration), and S. Orito (JADE Collaboration), Proc. of the 1979 International Symposium on Lepton and Photon Interactions at High Energies, T. B. W. Kirk and H. D. I. Abarbanel, Ed., 3, 19, 34, and 52, Batavia (1979).

[34] Möhl, D., G. Petrucci, L. Thorndahl, and S. van der Meer, Phys. Rep. 58, 73 (1980).

[35] Hinrichsen, B., A Measurement of the Dijet Differential Cross Section in $p \bar{p}$ Collisions at $\sqrt{s}=1.8$ TeV, Ph.D. Thesis, University of Toronto (1999).

[36] Abe, F., et al. (CDF Collaboration), Nucl. Inst. Meth. A271, 387 (1988).

[37] Azzi, P., et al. (CDF Collaboration), Nucl. Inst. Meth. A360, 73 (1995).

[38] Balka, L., et al. (CDF Collaboration), Nucl. Inst. Meth. A267, 272 (1988).

[39] Bertolucci, S., et al. (CDF Collaboration), Nucl. Inst. Meth. A267, 301 (1988).

[40] Fukui, Y., et al. (CDF Collaboration), Nucl. Inst. Meth. A267, 280 (1988). 
[41] Brandenburg, G., et al. (CDF Collaboration), Nucl. Inst. Meth. A267, 257 (1988).

[42] Cihangir, S., et al. (CDF Collaboration), Nucl. Inst. Meth. A267, 249 (1988).

[43] Ascoli, G., et al. (CDF Collaboration), Nucl. Inst. Meth. A268, 33 (1988).

[44] Lewis, J. D., et al., The 1992 CDF Muon System Upgrade, CDF Collaboration Internal Note 2858 (1994).

[45] Byrum, K., et al. (CDF Collaboration), Nucl. Inst. Meth. A268, 46 (1988).

[46] Abe F., et al. (CDF Collaboration), Phys. Rev. D50, 2966 (1994).

[47] Kilgore, W. and W. Giele, Hadronic Three Jet Production at Next-to-Leading Order, LANL-HEP-PH/9903361 (1999).

[48] Asakawa, T. and S. Geer, A Study of Three-jets Events with Large Total Transverse Energies at the Fermilab Proton-Antiproton Collider, CDF Collaboration Internal Note 2449 (1994).

[49] Buckley, E. and S. Geer, A Study of Events with the Highest Total Transverse Energy in CDF, CDF Collaboration Internal Note 2160 (1993).

[50] Abe, F., et al. (CDF Collaboration), Phys. Rev. D45, 1448 (1992).

[51] Abe, F., et al. (CDF Collaboration), Phys. Rev. Lett. 68, 1104 (1992).

[52] Abe, F., et al. (CDF Collaboration), Phys. Rev. D45, 2249 (1992).

[53] Abe, F., et al. (CDF Collaboration), Phys. Rev. Lett. 62, 1825 (1989).

[54] Kilgore, W. and W. Giele, Phys. Rev. D55, 7183 (1997).

[55] Geer, S., Properties of Multijet Events with Large Total Transverse Energies at the Fermilab Proton-Antiproton Collider, CDF Collaboration Internal Note 2443 (1994). 
[56] Abe, F., et al. (CDF Collaboration), Phys. Rev. Lett. 80, 3461 (1998).

[57] Dittmann, J., Studies of Multiple Interactions and Jet Corrections, CDF Collaboration Internal Note 4001 (1997).

[58] Geer, S., Proc. of the $10^{\text {th }}$ Topical Workshop on Proton-Antiproton Collider Physics, Batavia (1995);

Abbott, B., et al. (D0 Collaboration), Phys. Rev. Lett. 86, 1955 (2001).

[59] Giele, W. and E. Glover, Phys. Rev. D46, 1980 (1992);

Giele, W., E. Glover, and D. Kosower, Nucl. Phys. B403, 633 (1993).

[60] Del Duca, D., and Frixione, S., Proc. of the Workshop on Standard Model Physics (and More) at the LHC, G. Altarelli and M. L. Mangano, Ed., 47ff, Geneva (2000).

[61] Bjorken, J., Phys. Rev. 148, 1467 (1966).

[62] Gross, D. and C. Llewellyn Smith, Nucl. Phys. B14, 337 (1969).

[63] Behrends, S., A Plot of the Systematic Uncertainty on Jet $E_{T}$, CDF Collaboration Internal Note 3550 (1996).

[64] Wei, C. and R. Harris, Dijet Angular Distribution from Run 1a+1b, CDF Collaboration Internal Note 3637 (1996).

[65] Cronin-Hennessy, D., et al., Nucl. Inst. and Meth. 37, 443 (2000).

[66] Bhatti, A., Inclusive Jet Cross Section Measurement for Run 1b, CDF Collaboration Internal Note 4678 (1998).

[67] Lai, H.L., et al., Phys. Rev. D55, 1280 (1997).

[68] Kilgore, W., private communication. 